\title{
Endogenous technological change : the influence of information technology : theoretical considerations and empirical results
}

Citation for published version (APA):

van Meijl, J. C. M. (1995). Endogenous technological change : the influence of information technology : theoretical considerations and empirical results. [Doctoral Thesis, Maastricht University]. Datawyse / Universitaire Pers Maastricht. https://doi.org/10.26481/dis.19950921jm

Document status and date:

Published: 01/01/1995

DOI:

10.26481/dis.19950921jm

Document Version:

Publisher's PDF, also known as Version of record

Please check the document version of this publication:

- A submitted manuscript is the version of the article upon submission and before peer-review. There can be important differences between the submitted version and the official published version of record.

People interested in the research are advised to contact the author for the final version of the publication, or visit the DOI to the publisher's website.

- The final author version and the galley proof are versions of the publication after peer review.

- The final published version features the final layout of the paper including the volume, issue and page numbers.

Link to publication

\footnotetext{
General rights rights.

- You may freely distribute the URL identifying the publication in the public portal. please follow below link for the End User Agreement:

www.umlib.nl/taverne-license

Take down policy

If you believe that this document breaches copyright please contact us at:

repository@maastrichtuniversity.nl

providing details and we will investigate your claim.
}

Copyright and moral rights for the publications made accessible in the public portal are retained by the authors and/or other copyright owners and it is a condition of accessing publications that users recognise and abide by the legal requirements associated with these

- Users may download and print one copy of any publication from the public portal for the purpose of private study or research.

- You may not further distribute the material or use it for any profit-making activity or commercial gain

If the publication is distributed under the terms of Article $25 \mathrm{fa}$ of the Dutch Copyright Act, indicated by the "Taverne" license above, 


\section{Endogenous Technological Change The Influence of Information Technology}

Theoretical Considerations and Empirical Results

Hans van Meijl 



\section{Endogenous Technological Change The Influence of Information Technology}

Theoretical Considerations and Empirical Results

PROEFSCHRIFT

ter verkrijging van de graad van doctor aan de Rijksuniversiteit Limburg te Maastricht, op gezag van de Rector Magnificus, Prof. mr. M.J. Cohen, volgens het besluit van het College van Dekanen, in het openbaar te verdedigen op donderdag 21 september 1995 om 16.00 uur

door

Johannes Christianus Maria van Meijl

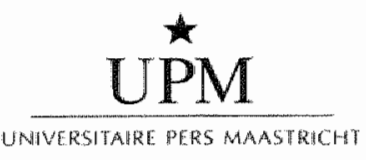




\section{Promotor:}

Prof. dr. L.L.G. Soete

\section{Co-promotor:}

Dr. A.H. van Zon

\section{Leden van de beoordelingscommissie:}

Prof. d.. J. Muysken (voorzitter)

Prof. dr. P.A. Mohnen (Université du Québec, CAN)

Prof. dr. W.E. Steinmueller

\section{CP-DATA KONINKLIJKE BIBLIOTHEEK, DEN HAAG}

Meijl, Johannes Christianus Maria van

Endogenous technological change: the case of information technology, theoretical considerations and empirical results /

Johannes Christianus Maria van Meijl. -

Maastricht : Universitaire Pers Maastricht. - Ill.

Thesis Rijksuniversiteit Limburg Maastricht. - With ref. With summary in Dutch.

ISBN 90-5278-197-4

Subject headings: technological innovation / information technology / R\&D spillover effects.

(1995, J.C.M. van Meijl, Maastricht 


\section{Contents}

Preface

Vii

PART I INTRODUCTION

1. General Introduction

1.1 The Current General Purpose Technology: Information Technology

1.2 The IT Productivity Paradox

1.3 Everywhere Around Us We See the Computer Except in Knowledge Accumulation?

1.4 Outline of the Thesis

2. Technological Change and Growth: A Selective Survey

2.1 Endogenous Growth Models

2.2 Information Technology as a Key or General Purpose Technology 29

2.3 Basic Ingredients for an Endogenous Technological Change Model 35

PART II ENDOGENOUS TECHNOLOGICAL CHANGE MODELS

3. Endogenous Technological Change by Cost Reducing and Demand Creating Innovations

3.1 Demand Creating R\&D

3.2 Cost Reducing R\&D

3.3 Demand Creating and Cost Reducing R\&D

3.4 Conclusion

4. General Purpose Technologies and Technological Change 81

4.1. Information Technology and Technological Progress 81

4.2 Endogenous Technological Change by Investments in Information Technology and R\&D

4.3 The Influence of Market Segmentation on Technological Change 93

4.4 Conclusion 96

5. Inter- and Intra-Industry Spillovers, Product Differentiation and Entry 103

5.1 Derivation of Demand Functions with Product Differentiation

5.2 Cost Reducing and Demand Creating $\mathbb{R} \& D$ with Inter- and Intraindustry R\&D Spillovers 
5.3 An Endogenous Technological Change Model

5.4 Steady State Growth Rates

5.5 Dynamics

5.6 Steady State

5.7 Conclusion

PART III MEASUREMENT

6. The Influence of $R \& D$ and R\&D Spillovers on Productivity Growth: A Survey of Econometric Studies

6.1 R\&D Investment and Productivity Growth

6.2 "Pure" Rent and Knowledge Spillover Effects

6.3 Process R\&D, Product R\&D and R\&D Spillover Effects 168

$\begin{array}{lr}6.4 \text { Conclusion } & 171\end{array}$

7. R\&D and R\&D Spillovers

7.1 A Model of Technology Generation by Cost Reduction and Demand Creation with Spillovers

7.2 Data

7.3 Estimation Results

7.4 The Role of Technology in Different Groups of Sectors: High-tech, Services and Other Sectors.

8. Information Technology Spillovers: Empirical Evidence

8.1 An Empirical Model of Technological Change with Spillovers related to IT-goods and non-IT goods

8.4 The Importance of Spillover Effects over Time 250

8.5 Reconsidering the "IT Productivity Paradox" 253

8.6 Conclusion $\quad 255$

PART IV SUMMARY 259

9. Summary 261

9.1 The Process of Technological Progress is Changing 261

9.2 Theoretical Considerations on Endogenous Technological Change 263

9.3 Empirical Results 266

References $\quad 271$

Samenvatting (Summary in Dutch) $\quad 283$

$\begin{array}{lr}\text { Curriculum Vitae } & 289\end{array}$ 


\section{Preface}

During the last two years of my study of economics at the Faculty of Economics and Business Administration at the State University of Limburg I worked as a student assistant on the HERMT project at the Maastricht Economic Research Institute on Innovation and Technology (MERIT). The HERMIT project investigated the influence of Information Technology (IT) on the European economy. However, the results indicated that the influences of $\mathrm{IT}$ on the European economy were negligible. This result was disappointing as the so-called "information technology revolution" promised huge economy-wide impacts. The underlying HERMES framework turned out to be too rigid and not suited to investigate the economic influence of IT. Together with Luc Soete and Adriaan van Zon I decided to writte a proposal for a Ph.D. thesis which attempted to develop a framework that would consider the main influences of IT. This proposal was approved by the scientific committee in 1990 and at the beginning of 1991 I started the research at MERIT, although I was formally employed by the faculty.

As extensively elaborated in this thesis, research is a process where externalities are important. Although it is only my name on the cover of this thesis, and I take full responsibility for the views expressed here or any remaining errors, I have received many positive externalities from several persons for which I am most grateful. First of all I would like to thank my supervisors, Luc Soete and Adriaan van Zon, for their trust, enthusiastic encouragement and valuable comments. Luc Soete concentrated on the general framework of this thesis and provided me with original views on the influence of IT on society while Adriaan van Zon provided guidance on the various practical and mathematical problems.

My special thanks goes to Thomas Ziesemer who helped me to study the optimal control theory in a relatively fast and efficient way and who made many insightful comments on the theoretical chapters of my thesis. Furthermore, I thank Elhanan Helpman who provided me with valuable comments on chapter 4 and 8 . Next, I would like to thank my former classmates, especially Frank Bunte and Maurice Oude Wansink, for their critical remarks and support.

I am also grateful to the members of the thesis examining committee, Joan Muysken, Ed Steinmueller and Pierre Mohnen, who offered constructive suggestions for improvement of this work. I feel particullarly indebted to Pierre 
Mohnen and Ed Steinmueller who also provided comments on earlier papers and encouraged and inspired me to go on with my research.

I would also like to thank all my colleagues who made MERIT a very stimulating and enjoyable environment to do my research. First of all I want to thank Wilma Coenegrachts who acted more or less like a second "mother". I also want to thank Mieke Donders, Ellen Nelissen and Silvana de Sanctis for their secretarial assistance, Karin Kamp who did an outstanding job of correcting my English and Huub Meijers for his advice on the lay-out of this thesis.

A special word of thanks is reserved for my mother who encouraged and enabled me to do the things I liked; also her moral advice and support during bad times stimulated me to finish this thesis. Finally, I want to thank Ria for her love and support and making my efforts worthwhile. 


\section{PART I}

\section{INTRODUCTION}





\section{General}

Introduction

For almost four decades economists have known that technological change is the single most important determinant of economic growth (Abramovitz 1956, Solow 1957). For example Solow (1957) concluded that $87.5 \%$ of the increase in gross output per capita was attributable to technological change and the remaining $12.5 \%$ to increased use of capital. However, little progress has been made in unveiling the contents of the "residual" of aggregate production functions, or in describing technological change as an economic process. According to Abramovitz, the residual is at best, "a measure of our ignorance" (1956, p. 11) and technological change itself has remained more or less an empty concept. As Freeman (1994, p. 463) states: "One of the continuing paradoxes in economic theory has been the contrast between the general consensus that technical change is the most important source of dynamism in capitalist economies and its relative neglect in most mainstream literature".

In this study we focus on the dual nature of technological change. In particular, we accept the view that the generation and distribution of knowledge are critical factors in the process of technological change. Technological change can, from this perspective, best be compared with the movements of a snake. For the snake to progress it can either bring its tail section forward or advance its head and leaving its tail lagging behind. The pushing forward of the head corresponds to the generation of new knowledge advancing the technological frontier. The catching-up of the tail section to the head of the snake is more akin to the process of knowledge distribution which diffuses the newest technologies throughout the economy advancing the average technology level to or near to the best practice or frontier level.

Knowledge has of course a number of uncommon characteristics, among which are its "non-rival" good properties; i.e. it is a good that is infinitely expansible without loss of its intrinsic qualities, so that it can be possessed and used jointly by as many as care to do so (Nelson 1959, Arrow 1962, Romer 1993). A part of the knowledge created by an innovating firm therefore spills over to other firms 
(public good feature of knowledge). This naturally creates difficulties in appropriating rents (private aspect of knowledge) in the generation of knowledge.

At the empirical level, many studies have attempted to measure the influence of knowledge generation and distribution on the economic performance of firms, industries and countries. However, knowledge is an intangible asset and therefore generally is not directly visible and measurable. These studies therefore used inputs in the knowledge generation process such as R\&D expenditures, which are visible and measurable, to study their influence on economic performance. Most empirical studies on the generation of knowledge find a positive and significant influence of "own" R\&D expenditures on technological change (for example Griliches 1980, Hall and Mairesse 1992). Empirical studies that attempt to measure the relationship between the distribution of knowledge and technological change examine the influence of R\&D expenditures performed by one firm (industry/country) on another firm"s (industry"s/country"s) level of technological change. However, this relationship is hard to measure because it depends on the "economic" or "technological" distance between sectors, which is difficult to determine (Grilliches 1979). Despite this problem, a general conclusion of these studies is that $\mathbb{R} \& D$ performed by other firms (for example laffe 1986, Bernstein 1988), industries (for example Terleckyj 1980, Wolff and Nadiri 1993) or countries (for example Mohnen 1992b, Coe, Helpman and Hoffmaister 1995) has a significant influence on the rate of technological change. In this thesis we examine inter-firm and inter-industry spillovers and neglect the international dimension of knowledge spillovers. ${ }^{2}$

In the present study, we develop a modell in which knowledge is generated endogenously. Firms "invest" in knowledge creation to raise their technological level and this to improve their competitive position relative to rivals. In this context, we identify two different types of knowledge. The first type is knowledge that is directed at increasing the efficiency of the production process or "castreducing" knowledge. It can be expected that this kind of knowledge is generated by performing process-oriented R\&D. The second type is knowledge that is directed at enhancing the quality level of the products which is often called "product" innovation. I call this "demand-creating" knowledge to encompass both the creation of new products and the enhancement of existing product characteristics. Demand creating knowledge can be generated by engaging in product R\&D.

Furthermore, and in order to integrate the dual nature of technological change mentioned above, we will include, besides these internal knowledge sources (knowledge generation), external knowledge sources i.e. knowledge created by other

1 For an more extensive review of the literthure, see Griliches (1992), Mohnen (1992), Mairesse and Mohmen (1994) or chapter 6.

2 For international R\&D apillovers see e.g. Bernstein and Mohnen (1994), Coe and Helpman (1994), Bemstein (1995b), and the two previously mentioned contributions. For at survey on this subject see Mohnen (1994), 
firms. With regard to distribution of knowledge features and the realisation of potential spillover effects, an important change has arisen with the emergence and diffusion of information technology (IT). The main advantages of IT are, not surprisingly, related to the processing of information. IT increases the speed and accuracy of processing, storing and transmitting information. An important effect of IT is linked to the "nature" of knowledge during the IT era (see David and Foray 1994, Ergas 1994, Arora and Gambardella 1994). It is possible to distinguish between "tacit" or "private" knowledge on the one hand, and "codified" or "public" knowledge on the other. The first type of knowledge is context dependent and costly to transfer, and it enables a firm to profit when they perform $\mathbb{R} \& D$, the second kind is public scientific or technological knowledge that cannot be kept inside the firm and spills over to other firms. ${ }^{3}$ A rapid increase in the computational power is related to a better theoretical understanding of problems and advances in mathematics and computer sciences resulted in an increased ability to codify knowledge (Ergas 1994, Arora and Gambardella 1994). Knowledge becomes more of the "codified" nature than of the "tacit" nature, which increases the importance of spillower effects.

Furthermore, IT also causes an increased degree of integration between firms by generating networks between firms and imcreasing the accessability of large knowledge sources, such as patent publications and databank networks. In addition to the increased codification of knowledge, these aspects of IT also increase the value of spillover effects. Foray and David (1994) go as far as arguing that the distribution and accessability of knowledge are becoming relatively more important than the generation of knowledge for the technology generation process.

The influence of IT on spillover effects is, however, only one aspect of the "pervasive" influence of IT. Pervasive is not just a question of a widespread variety of new products or industries but of a technology generic (pervasive) in use, one which affects every industry and every service (Freeman and Soete 1993). "The same type of computer (hardware) can be used to perform regressions for economists, monitor the production process in the glass industry, or entertain children with computer games. However, to enable these totally different functions, computer programs (software) have to be developed.

The appearance of information technollogy promised huge productivity gains. However, studies that measured the influence of IT on productivity found disappointing results. For example Baily and Gordon (1988) noticed that "official

\footnotetext{
${ }^{3}$ Section 1.1 explans the tacit and codifhed nature of knowledge in a more elaborated way.

4 According to Freenan and Soete (1993) the most impontant adwantages based on the use of IT can be divided into fout groups: 1) speed of processing and transmiting infornation, 2) storage capacily for large quantities of information, 3) fle xbility in oganising manufacturing, dosign, marketing and administration, and

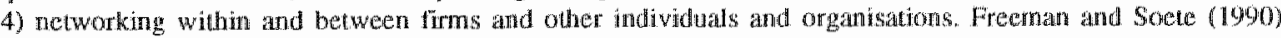
argue that "information techtology can indeed be identitied as a pervasive, generic technollogy with often dramatic potentials for productivity gains with respect to all producton factors ${ }^{\text {th }}, \mathrm{p}, 225$.
} 
data show enormous productivity gains in the manufacture of computers but apparently little improvement in their use" and they posed the following question; "What has all that computer power been doing, and where is the "black hole" into which all those computers are disappearing?" (pp. 350-351). Despite the fact that all firms invest heavily in information technology, these investments do not seem to be productive. Robert Solow commented upon this situation by stating: "we see computers everywhere except in the economic statistics". This became known as the "IT productivity paradox" or the "Solow-paradox".

Brynjolfsson (1993) identifies four main explanations for this paradox: measurement errors, lags, redistribution and mis-management. ${ }^{5}$ The first two explanations are optimistic and are based on the fact that the benefits of IT investments are difficult to measure or will show up in the future. The other two explanations are pessimistic and assume that there are no major benefits related to IT.

The pervasiveness of IT requires the total economic and social infrastructure to be changed before the potential benefits of IT can be translated into actual benefits. Such a change is certainly not instantaneous but may take decades (Freeman and Perez 1988). IT has to be diffused in the economy and user-specific computer programs have to be developed before potential benefits can be obtained and measured. Diffusion of IT is necessary for the codification of knowledge and to exploit the benefits of networks. The last decades are characterised by large investments in IT goods and therefore a rapid diffusion of IT goods. It can therefore be expected that the IT paradox will eventually be solved because the benefits of IT may "finally" become measurable.

In this thesis we develop a model that incorporates the knowledge generation and distribution aspects of technological change mentioned above. Furthermore, by separating $R \& D$ effects from IT effects we are able to address these two issues using a single conceptual framework.

In the remainder of the introduction we elaborate on two previously mentioned issues. Section 1.1 examines the characteristics of information technologies and section 1.2 describes the "Information Technology Paradox". Section 1.3 investigates the often "neglected" influence of IT on the knowledge creation process itself. The last section provides an outline of the thesis.

\subsection{The Current General Purpose Technology: Information Technology}

In any given "era" a few technologies exist that have an important influence on the process of techmological change in a large part of the economy; the so called "general purpose technologies" (Bresnahan and Trajtenberg 1994). The steam engine during the first industrial revolution and electricity in the early part of this century

\footnotetext{
${ }^{5}$ Section 1,2 examines these four explanations in more detall.
} 
are widely thought to have played such a role. When considering a technology that has a widespread influence in almost all sectors in the current "era", there is widespread agreement that "information" technologies play this role. Besides their" widespread influence, a rapid rate of technological change in the technology itself is a characteristic of a general purpose technology (GPT).

Table 1.1 Constant quality investment price deflators

\begin{tabular}{cccccc}
\hline \hline Year & GDP & Investment & OCAM $^{1}$ & Computers & Processors \\
\hline 1958 & 0.329 & 0.362 & 10.237 & 168.370 & 204.270 \\
1960 & 0.339 & 0.370 & 10.285 & 173.930 & 173.930 \\
1965 & 0.370 & 0.380 & 6.467 & 22.591 & 54.245 \\
1970 & 0.439 & 0.443 & 3.891 & 8.962 & 12.598 \\
1975 & 0.598 & 0.608 & 2.434 & 3.956 & 7.019 \\
1980 & 0.861 & 0.869 & 1.177 & 1.258 & 1.438 \\
1985 & 1.106 & 1.032 & 0.641 & 0.562 & 0.486 \\
1989 & 1.249 & 1.127 & 0.453 & 0.357 & 0.349 \\
\hline
\end{tabular}

Source: Survey of Current Business, BEA and Jorgenson and Stiroh's calculation (Jorgenson and Stiroh 1995, table 2). ${ }^{\circ}$ OCAM = Office, Computing, and Accounting Machinery.

An example to illustrate the enormous level of technological change in the IT sector is the number of components on a single chip. This number increased from 32 in the mid-1960s to about 100,000 in the early 1980 s to over 1 million in the early 1990s, almost conforms to "Moores Law", which states that the number of components on a single chip would double every year. Advances in IT have caused a dramatic decline in information processing costs. For example the constant quality price of computers declined by $19.9 \%$ per year, while inflation rates for GDP and investments were $4.3 \%$ and 3.7\%, respectively, for the $1958-1989$ period (see Jorgenson and Stiroh, 1995). During this period the quality-adjusted price index for investments generally had increased by a factor of 3.1 while the price index for computers decreased by a factor of 471 (see table 1.1). The quality-adjusted price index for processors declined by a factor of 585 (see table 1.1). A graphical illustration of this dramatic price decline is given in figure 1.1. This dramatic decline in information costs is at the core of the pervasive or economy-wide impact of IT. The continuous fall in the quality adjusted IT prices has led to the further

\footnotetext{
${ }^{6}$ See for example Rosenberg (1982), Forester (1985), Freeman and Soete (1990) and David (1992).

7 These NIPA price indices for computers utilize a "hedonic" methodology that standardizes computers for changes in technical characteristics, such as the speed of processing and main memory, by incorporating these characteristics into an econometric model of computer prices.
} 


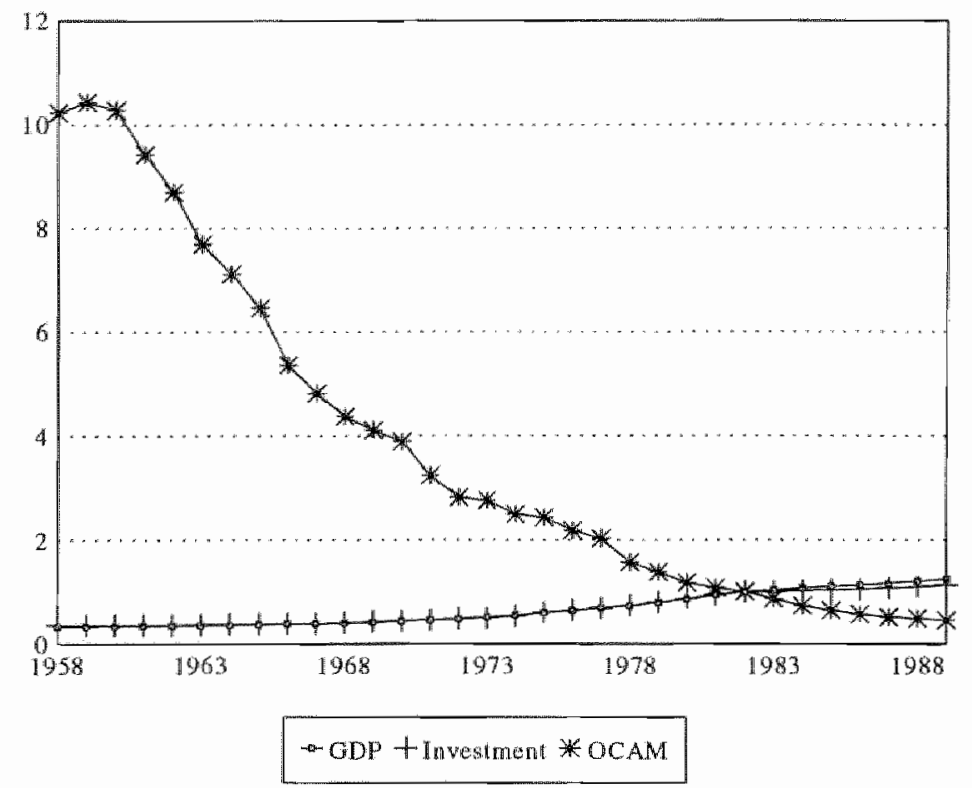

Figure 1.1. The constant quality investment price deflators of IT Source: Jorgenson and Stiroh, 1995.

opening up of areas of application. Following Freeman and Soete (1990), the most import characteristics of information technologies can be summarized as follows.

(i) There exist a continuing high rate of technical change in the cluster of electronics-related industries themselves, as well as in a wide range of applications. Underlying this process is the continuing dramatic improvement in large-scale integration of electronic circuits, and the continuing fall in costs which this permits. The revolutionary developments in integrated circuits have their parallel in communication technology, where fibre optics have made possible similar drastic improvements in costs and performance. The convergence of these technological advances means that the capability for communicating, processing and storing information is still rapaly improwing and is becoming eten cheaper.

(ii) $\mathrm{IT}^{\mathrm{s}} \mathrm{s}$ capability for an improvement in the quality of products, processes, and services. On-line monitoring and quality control of output has already led to dramatic improvements in industries as diverse as colour television and passenger cars. It leads to capital-saving, labour-saving, material-saving and energy saving improvements in production processes since it reduces the number of rejects and wasted components both for intermediate and final output.

(iii) Advances in linking wetworks of firms, such as for example, networks between component and material suppliers in assembly-type firms (as in the automobile industry) or in service firms (as in hotel and catering industries). 
Furthermore, the new flexibility and speed of communication systems and their links with data banks creates the possibility for a wider variety of new "ValueAdded Networks" (VANS) and new types of information services.

(iv) Greater flexibilty and speed in model changes and design changes. Some analysts have referred to this phenomenon as "economies of scope" replacing to some extent, "economies of scale". Whereas previous assembly line and flow" production systems were relatively inflexible and based on the continuous replication of vast numbers of standardized, homogenous products, the new flexibility conferred by IT permits more rapid changes of tooling and dies, so that small production runs become economic and the prospects for small and mediumsized firms are greatly improved, reducing barriers to entry and permitting major changes in industrial structure.

(v) A reduction in electro-mechanical components and in various stages of component transformation as a result of the redesign of products and processes. This leads to substantial savings of materials and energy, as well as to a process of structural change which involves the loss of jobs in some sectors of metals and metal goods and an increase in jobs in some sectors of electronic component industry, ellectronic products, and producer services.

(vi) A strong tendency towatds a more rapid rate of product and process change and more intense technological competition (resulting from above factors and the continuing rapid improvement in micro-electronic performance). In turn, this strengthens the demand for new skills and services in design, development, software engineering, and microelectronics products generally, affecting both inhouse skill and employment profiles and the growth of new services, information flows, and supplies of equipment. The more rapid rate of product and process change is itself facilitated and stimulated by the diffusion of computer aided design systems (CAD).

All of this means that IT is not only affecting the structure of the econony in terms of new industries and services but that it is also profoundly affecting the internal structure and management of all enterprises and the relationships between them. These characteristics of IT indicate that IT is indeed a pervasive on general purpose technology because it affects the whole economic system. Furthermore, the diffusion of IT throughout the whole economic system generates a large potential for productivity gains with respect to all production factors (Freeman and soete $1990)$.

\subsection{The IT Productivity Paradox}

Since the 1970 s, one of the main issues for economists has been the productivity slowdown, a world-wide fall in the productivity growth rate since the mid-1960s as 
compared with the first half of the postwar period. ${ }^{B}$ However, this productivity slowdown coincided with major technological innovations in the area of microelectronics and communication technologies and a rapid increase in the wse of these information technologies. These broad developments are the basis of the "IT productivity paradox": IT seems to be unproductive, despite the expected huge productivity gains of IT (as described in the previous section). Studies that measured the relationship between IT and productivity supported the paradox. For example Loveman (1988) found that IT had no impact on output or productivity growth. Morrison and Berndt (1991) concluded that the marginal benefits of IT were less $(80 \%)$ than marginal costs which indicated over-investments in IT. Berndt, Morrison and Rosenblum (1992) found that a relatively larger high-tech capital stock was negatively related to labour productivity growth. The empirical findings of other studies that measured the relationship between IT and productivity are shown in table 1.2, which is based on an extensive review of Brynjolfsson (1993). Brynjolfsson concluded that "research on IT and productivity has been disappointing" because its findings have been disappointing.

Many explanations of the IT productivity paradox exist. Brynjolfsson (1993) identified four main explanations."

1. Measurement error: Outputs (and inputs) of information-using industries are not being properly measured by conventional approaches. The sorts of benefits ascribed by managers to information technology-increased quality, variety, customer service, speed and responsiveness--are precisely the aspects of output measurement that are poorly accounted for in productivity statistics. Rapid innovation has made IT-intensive industries particularly susceptible to the problems associated with measuring quality changes and valuing new products.

2. Lags: Time lags in the pay-offs to IT make analysis of current costs vs. current benefits misleading. This would be the case if extensive learning by both individuals and organisations were needed to exploit IT, as it is for most radically new technollogies.

3. Redistribution: it is especially likely that IT is used in redistributive activities among firms, making it privately beneficial without adding to total output: IT rearranges the shares of the pie without making it any bigger. Firms with inadequate IT budgets would lose market share and profits to high IT spenders.

4. Mis-management: The lack of explicit measures of the value of information makes

\footnotetext{
For an early contribution to this issue see Nordhans (1972). For an extensive treatment of the productivity slowdown sed for example Grilichss (1980), Link (1986), Baily and Giordon (1988) or Meijers (1994).

9 Problems in the creation of soltwane is another explanation for the TT productivity paradox. Howewer, Steinmueller (1994) states that "while software costs have grown rapidly, it is a mis-characterization to assign the responsible for low measured productivity growth from the use of computers to productivity problems in software creation. Instead, the cormous expansion in the variety of problems for which computer solutions have been amployed have driven an increasiog prolifenation of software solutions, many of which are not yet
nilized".
} 
Table 1.2 Principal empirical studies of $\mathrm{m}$ and productivity

\begin{tabular}{|c|c|}
\hline Study & Findings \\
\hline \multicolumn{2}{|l|}{ Manufacturing } \\
\hline Loveman (1988) & IT investments added nothing to output \\
\hline Morrison and Berndt (1991) & IT marginal bernefit is only 80 cents per dollar invested \\
\hline Siegel and Griliches (1992) & IT using industries tend to be more productive \\
\hline Brymolfsson and Hitt (1993) & $\begin{array}{l}\text { The return on investments in } 17 \text { capital is over } 50 \% \text { a year } \\
\text { (Period } 1987.1991 \text { ) }\end{array}$ \\
\hline \multicolumn{2}{|l|}{ Services } \\
\hline Cron and Sobol (1983) & Bimodal distribution among high IT immestors: either good or bad \\
\hline Strassman $(1990)$ & $\begin{array}{l}\text { No correlation between various IT ratios and performanos } \\
\text { measures }\end{array}$ \\
\hline $\operatorname{Roach}(1991)$ & $\begin{array}{l}\text { Vast increase in IT capital per information worket while } \\
\text { measured output decreased }\end{array}$ \\
\hline Harris and Katz (1989) & $\begin{array}{l}\text { Weak positive relationship between IT and various performance } \\
\text { measures }\end{array}$ \\
\hline Parsons et all. (1990) & $\begin{array}{l}\text { IT coeffucient in translog production function small and often } \\
\text { negative }\end{array}$ \\
\hline Brymjolfsson and Hitt (1993) & $\begin{array}{l}\text { The return on IT investments is over } 60 \% \text { a year } \\
\text { (Period } 1987-1991 \text { ) }\end{array}$ \\
\hline
\end{tabular}

Source: Brynjolfssion 1993, table 2 and table 3, p. 71 .

it particularly vulnerable to mis-allocation and over consumption by managers.

The last two explanations propose that there really are no major benefits, presentlly or in the future. The first explanation assumes that there are benefits related to IT, while these benefits are not captured using conventional measurement methods. However, some recent studies, based on detailed firm data and a very recent data base, measured a positive influence of IT on productivity growth (see table 1.2), and therefore point toward the second explanation; the lag hypothesis. ${ }^{116}$ This lag hypothesis is optimistic predicting that the benefits of IT will show up in the future.

Next some evidence from recent empirical studies, there are other arguments that favour the lag hypothesis. In the previous section we describe seven impacts of IT. These impacts imply that the influence of IT is pervasive. Perez $(1983,1985)$ argues

${ }^{10}$ Oher recent empirical studies that found a positive relationstup between IT and producaivity, such as lot example Bresnahan (1986), Lichtenberg (1993) and, Lat and Tohutsu (1992) are dechibed in chapter 8 (section 8.5). 
that these impacts involve a new "best practise" set of rules and customs for designers, engineers, entrepreneurs and managers, which differ in many important respects. Freeman and Soete argue that "such changes make possible a "quantum leap" in potential productivity, which, however, is at first only realized in a few leading sectors. It takes decades before the productivity gains to be realized throughout the economy as a result of learning, adaption, incremental innovation and institutional change" (1987, p. 57).

Freeman and Soete (1987) state that an interesting historical analogy to the case of information technology is provided by the experience of electric power. The key technical innovations were made between the 1870s and 1880s (Mensch 1975) while the first economic effects of electric power only showed up after 1900 and the major economic effects after 1920. One of the main reasons for this delay was that the required complementary changes necessary (for example organisational) to fully exploit the advantages of electric power over steam power took a long time. Devine (1983) has described this change in some detail: "Replacing a steam engine with one or more electric motors, leaving the power distribution system unchanged, appears to have been the usual juxtaposition of a new technology upon the framework of an old one...shaft and belt power distribution systems were in place, and manufacturers were familiar with their problems. Turning line shafts with motors was an improvement that required modifying only the front end of the system....As long as the electric motors were simply used in place of steam engines to turn long line shafts, the shortcomings of mechanical power distribution systems remained" (p. 357). Only after 1900 did manufacturers begin to realize that the indirect benefits of using unit electric drives, such as flexibility in factor layout, were far greater than the direct energy saving benefits. David (1992) stressed that this analogy between electrification and computerization is useful as it illustrates the importance of keeping an appropriately long time-frame in mind when discussing the connections between the information revolution and productivity growth. Helpman and Trajtenberg (1994) modelled the productivity path linked to a new genera purpose technology, and emphasised that besides the plain diffusion or the continuous improvements in the general purpose technology itself, a wide array of different types of complementary investments (including organisational and institutional changes), both within firms and between firms, are important.

According to these arguments the diffusion of a general purpose technology is not an easy process but it may take decades while economic and social processes have to be adjusted. Only when IT has diffused on a sufficient scale and complementary investments are made can its potential benefits be translated into actual benefits and the influence of IT becomes measurable. Following these arguments the "IT productivity paradox" is not surprising because the diffusion of a general purpose technology takes a long time.

The dramatic decline in IT prices caused a rapid diffusion of IT. At the end of the 1980 s and beginning of the 1990 s IT had diffused on a large scale. For example 
the share of IT investments in total investments increased from $4.7 \%$ in 1978 to $9.4 \%$ in 1992 in France. ${ }^{11}$ Benefits connected to a large diffusion of $\mathrm{IT}$, such as networks and access to large databanks, emerged. Therefore, rapid economy-wide diffusion of IT products and adjustments of the economic and social system is a prerequisite for the influence of IT to become measurable in the future.

\subsection{Everywhere Around Us We See the Computer Except in Knowledge Accumulation? ${ }^{12}$}

We accept the view that knowledge about technological and scientific opportunities and constraints is a critical factor in remaining competitive through successful innovation. Firms "invest" in knowledge creation to enhance their technological level and to improve their competitiveness. The knowledge stock of a firm is created by performing R\&D, knowledge spillovers from other firms in the same or other industries, joint development programs, purchasing technology, learning by doing, etc. For a long time internal knowledge generation or "own" R\&D activities were the main source of acquired knowledge. David and Foray (1994) argue that knowledge distribution and access to external knowledge sources will become more important in the future.

Economic activities use various types of knowledge and many methods of classifying these varieties exists. We use the taxonomic scheme developed by Dasgupta and David (1987, 1994) which focuses on the characteristics of knowledge that affect the ways in which it can be generated, stored, accessed and transferred among economic agents. Like Dasgupta and David, we find it useful to draw a distinction between knowledge and information. The term information will be used here to refer to knowledge that has been reduced and converted into messages that can easily be communicated among economic agents. Transformation of knowledge into information is a necessary condition for the exchange of knowledge as a commodity. Codificalion of knowledge is a step in the process of reduction and conversion, which renders the transmission, verification, storage and the reproduction of information less costly. ${ }^{1 / 3}$ This transformation makes knowledge more of what is described as a non-rival gaod.

In contrast to codified knowledge, tacit knowledge, as conceptualized by Polyani (1966), refers to a fact of common perception: that we all are aware of certain objects without being focused on them. Examples of tacit knowledge are skills, "scientific expertise" or "engineering expertise" that are acquired experientially and transferred by demonstration, by personal instructions and the provision of expert services. The transfer process itself, as a rule, is a comparatively costly affair (in

\footnotetext{
1 SOUTCe, INSEE data, see chapter 8.

12 Soete $(1995), 0.5$

1.3. C. Fordy and David (1994), p. 2 .
} 
contrast to codified knowledge) for both the provider and the recipient of tacit knowledge. In so far as codified and tacit knowledge are substitutable inputs (at the margin) in the production of further knowledge, the relative proportions in which they are used is likely to reflect their relative access and transmission costs to the users. ${ }^{14}$ According to Dasgupta and David (1992) this relative proportion has to be explained endogenously; "What gets brought into focus (and codified) and what remains in the background (as tacit knowledge) is to be explained endogenously, by considering the structure(s) of pecuniary and non-pecuniary rewards and costs facing the agents involved".

Some contemporary developments shift the relative proportion of tacit and codified knowledge in the knowledge generation process to the advantage of the latter (Ergas 1994, Arora and Gambardella 1994). According to Arora and Gambardella (1994), the increased ablity to codify knowledge is caused by advances in three highly complementary areas: (1) a better theoretical understanding of problems, (2) improved instrumentation, and (3) increased computational capability. Adwances in computer power induce more sophisticated instruments which enable researchers to test theories more rapidly and effectively. For an effective use of computational power researchers also have to formalise problems in abstract terms, which depends on a sound theoretical understanding of the problems themselves. The analysis of protein structures is an example of the latter. ${ }^{15}$ A protein chain of 150 amino acids can give rise to $5^{150}$ possible molecular structures, a number that is impossible to investigate even with supercomputers. $A$ recently developed theorem, using the principle of energy minimisation, cut the number of valid alternatives to $150^{2}$. This is still too large a number of possibilities to handle with "pen and paper", but not with supercomputers or personal computers for that matter.

Ergas (1994) stresses the rapid developments in mathematics and computer science, which significantly changed the problem-solving techniques available for R\&D. He illustrates this with the power of algorithms. It is, in effect, a common feature of optimisation problems that the number of possible solutions increases as an exponential function of the number of independent variables, so that extrema rapidly become too numerous to handle. The ability to solve these problems depends on the availability of algorithms that provide reasonable approximations of the analytical optimum. A characteristic of algorithms is that the computational requirement of the algorithm increase by some linear function of the problem's size, while the analytical complexity of the problems increases exponentially. These algorithms, together with the enormous processing abilities of current computers, make it possible to model more complex problems.

\footnotetext{
1.4 Mid. p. 22 .

15.5 This example is borrowed from Arota and Gambardella (1994), p. 525 .
} 
Important here is the fact that the advances in the tools have increased the ability to codify knowledge. According to Ergas (1994) this increased codification of the knowledge stock has at least six consequences:

First, it brings science into tighter and quicker interaction with technology. Advances in mathematics, such as those associated with work on fractals or on efficient codes, are more rapidly integrated into the practising tools of engineers as they are translated into computational algorithms for problem solving.

Second, formalisation allows the new tools to spread far more rapidly than their earlier counterparts. In effect, these tools embodied in computer software, rather than in the knowledge base of practising engineers, means that the pace of their diffusion no longer depends on the rate at which new cohorts enter into the engineering labour market, but rather on the existing generations' ability to upgrade their skills as rapidly as the tools advance.

Third, it increases the pace of new product and process development. The ability to test through simulation rather than through practice, and to do so on the basis of an arbitrarily large range of assumed conditions, allows for more focused and hence more productive search, thereby cutting the delay involved in going from the initial specification to the agreed-upon prototype. Moreover, by reducing the time and cost required for new product and process design, these tools encourage producers to experiment across a broad front - to develop and test many variants of a new product rather than only one or a few. As a result, products and processes become more differentiated and are renewed more quickly - accelerating the rate of technical obsolescence.

Fourth, it allows the deployment of products and systems which would otherwise be too complex and risky to use. This is because it enables simulation of the performance of equipment such as nuclear reactors or large commercial airplanes in regimes far removed from what would be expected in normal operation.

Fifth, codification changes the costs of technology diffusion. There is an important and irreducible element of tacitness to "learning by doing" and "learning by using". But as more and more products and processes are translated into formal models, knowledge about them moves closer to being of the "blueprint" kind, than to the tacit, experiential variety long dominant in most industrial settings. This increases the ease with which knowledge can be transferred; both from the laboratory or research centre to production (and back), and among producers.

Sixth, it increases the degree to which practical knowledge can be patented - and the incentives for doing so. The more formalised knowledge is the less effectively it can be protected by trade secret - and the more readily accepted it is as a basis for protection to patent and copyright.

Codification and computerisation have changed the nature of knowledge knowledge has become more of the "blueprint" type than of the tacit type. The increased codification of knowledge tends to reduce the transaction cost advantages 
of in-house $R \& D$, and it therefore encourages a shift to a more decentralised $R \& D$ system.' External knowledge sources (spillover effects) are becoming more important in the knowledge generation process of firms.

\subsection{Outline of the Thesis}

The remainder of the thesis is organised as follows. Part I gives a general introduction. Chapter 2 gives a selective survey of the literature on technological change and growth. We concentrate on the "new" or endogenous growth theories, as these theories try to endogenise technological change. Furthermore, we discuss some contributions that model the special influence of general purpose technologies. From these contributions we deduce some basic elements for an endogenous technological change model to be specified in part II.

A schematic representation of this model is given in figure 1.2. In chapter 3 we develop a traditional model in which knowledge is created by engaging in internal $\mathbb{R} \& D$ (in this chapter all the arrows with a number are neglected in figure 1.2). The change in the knowledge stock is dependent on the amount of resources devoted to internal $R \& D$ and the productivity of these $R \& D$ expenditures. A higher knowledge stock induces technological change and dependent on the appropriability of the innovation, leads to improved economic performance (see figure 1.2). We identify two types of technological change: efficiency and quality improvements.

In chapters 4 and 5 we expand this model with some "public" features of knowledge. Knowledge created in one firm can also be used by other firms. Ideas generated by other firms increase the productivity of internal R\&D efforts. This represents an incentive effect to invest in $\mathbb{R} \& D$. However, higher spillovers also mean that the appropriability of innovations declines, which is a disincentive effect for innovations.

With regard to the incorporation of IT, we have assumed 1) IT investment goods are not like other capital goods used together with labour to produce output but are used to "smooth" the production process. IT goods therefore have a "direct" productivity enhancing effect (arrow 1 in figure 1.2).2) IT influences the working methods and direction of the knowledge creation process itself. It stimulates the creation of knowledge that is linked to the general purpose-technology and it improves the productivity of the knowledge generation or R\&D process (see arrow 2). An example is when expensive and time consuming physical experiments can be replaced by computer simulations. 3 ) information technology increases the value of spillovers and therefore the public aspect of knowledge. This codification of knowledge means that knowledge generated in a particular firm can be more productive in other firms (arrow 3 in combination with arrow 4). The increase in the usefulness of spillovers in the IT era also has a negative side effect. If one firm.

${ }^{16}$ C.f. Ergas (1994), p.7 and Arora and Gambardella (1994), p. 528. 


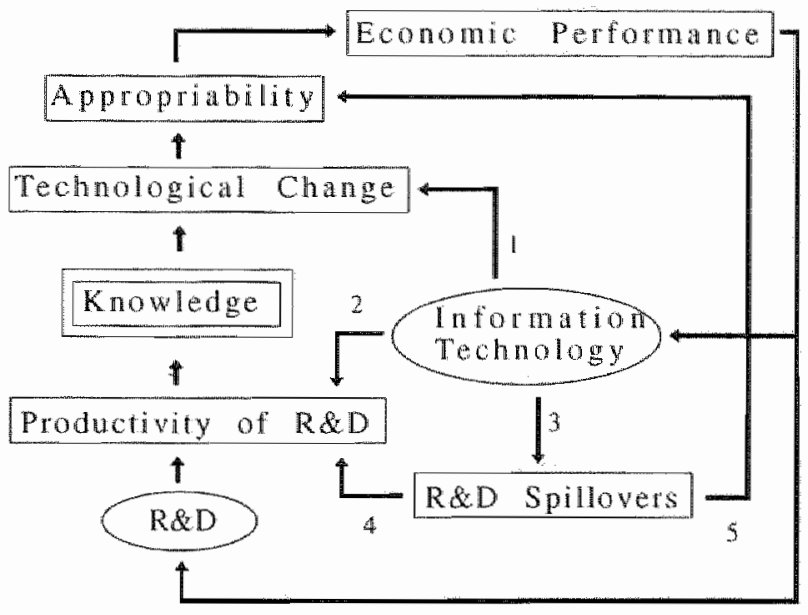

Figure 1.2. The technological change generation process.

is able to use knowledge created by other firms more effectively then this implies that the rewards to the innovating firm diminish. The appropriability or private aspects of created knowledge is reduced, so that firms incentives to invest in technological progress diminish (see arrow 3 and arrow 5). This is considered in chapter 5, which examines the appropriability diminishing effect of knowledge spillovers. In this chapter we also investigate the influence of the incentive and disincentive effect of spillovers on technological change. Furthermore, we investigate the influence of product differentiation and entry on technological change.

Part III is concerned with the measurement of technological change within an endogenous technological change model. This section investigates the influence of inputs to the knowledge generation process on the economic performance of firms or industries. We measure the relationship between R\&D expenditures, (IT-related or non-IT-related) R\&D spillovers and technological change. In particular, chapter 6 gives a survey of the results of previous empirical studies on the relationship between inputs to the knowledge generation process and economic performance. We study the influence of internal R\&D and $R \& D$ spillovers on (mainly) the productivity performance of firms or sectors. We distinguish between "pure" knowledge and rent spillovers. Rent spillovers are embodied in goods and exist because quality improvements are poorly reflected in prices or because some knowledge comes along with the purchased goods. "Pure" knowledge spillovers mainly reflect that a firm can use ideas developed by other firms and that these knowledge fllows are unrelated to input flows.

In chapter 7 we estimate the influence of internal $R \& D$, rent spillovers and knowledge spillovers on technological change. We investigate whether the estimated influence differs among groups of sectors. The model will be estimated. 
for France. We have chosen France for four reasons. First, France is a well developed OECD country with a substantial IT sector. Second, all other studies on the relationship between IT and productivity are done for the United States. Third, some studies already exist on the relationship between $R \& D$ and productivity to compare estimation results. Fourth, the data availability for France is good. With regard to the latter we require input-output matrices and investment matrices on at sufficiently detailed level for a recent period. The low desegregation level is necessary to get a meaningful classification of IT producing sectors and we need data for a recent period to test our main hypotheses.

Chapter 8 concentrates on the estimation of the influence of information technology on technological progress. In this thesis we concentrate on sectoral spillover effects and are interested in spillover effects from the IT producing sectors to the rest of the economy. This means that we examine the spillover effects between sectors and not within sectors. The model developed in chapter 5 includes both types of spillovers and will therefore not be estimated. In chapters 7 and 8 we estimate an adjusted version of the endogenous technological change model developed in chapters 3 and 4 . The version is adjusted in the sense that we will also consider data problems, such as quality improvements that are poorly reflected in prices. Furthermore, in chapter 8 we test our two main hypotheses. First, "extemal knowledge sources become more important in the knowledge generation process. The walue of spillover effects increases in this IT era". Second, "the IT productivity paradox is loosing its "paradoxical" characteristics" or "does the information technology paradox still exist or is there a positive influence of IT on technological change?"

Finally, part IV, which consists of chapter 9 , summarizes the main findings of this thesis. 


\section{Technological}

\section{Change and Growth: A Selective Survey}

This chapter will provide a selective survey of some recent developments in the field of technological change and growth. ${ }^{17}$ If there is one common feature in these developments it is the central role of technological change. The neoclassical growth models showed that technological change was the most important factor contributing to economic growth (e.g. Solow 1957). However, a serious drawback of these models was that they treated this important factor as exogenous to the system. Arrow characterises this situation as follows: "A view of Economic Growth that depends so heavily on an exogenous variable, let alone one so difficult to measure as the quantity of knowledge, is hardly intellectually satisfactory. From a quantitative, empirical point of view, we are left with time as an explanatory variable. Now trend projections, however necessary they may be in practice, are basically a confession of ignorance, and, what is worse from a practical viewpoint, are not policy variables" (Arrow 1962, p. 155). Hence, the need has arisen to make technological change endogenous. In this chapter we discuss some recent theories that make technological change endogenous.

Section 2.1, the main part of our discussion, will survey the "new" growth or endogenous growth models. In this survey we concentrate on the way these models treat technological change and enable endogenous growth. A new strand of the literature attempts to model the special influence of "general purpose technologies" on economic growth. A brief discussion of some contributions to this field is given in section 2.2. The last section infers some general elements for the endogenous technological change model to be developed in chapters 3 to 5 .

\subsection{Endogenous Growth Models}

The "new" growth theories stress the role played by technical progress and the determinants of technological change. These theories found several ways to

\footnotetext{
${ }^{17}$ Selective as we only survey the main contributions in a few hidds which are related to this thesis. For a more elaborated treaument of this subject or a survey of the older contributions on the field of technological change and growth see, for example, Jones (1975), Link (1987), Gomulka (1990) or Barro and Sala-i-Martun (1995).
} 
endogenize technological change in a general equilibrium model. In this selective survey we concentrate on the mechanics that make growth endogenous and whether these mechanisms capture the key characteristics of the knowledge generation process.

The model that will be used to highlight the various growth mechanisms is the simple Cobb-Douglas production function with two production factors.

$Y=A \cdot K^{\beta} \cdot L^{\alpha}$

The first factor, $K$ (stands for Capital, knowledge or human skills), can be accumulated. The second factor, $L$ (Labour), cannot be accumulated or has an exogenous growth rate. The crucial feature in all growth models is the marginal product of the factor which can be accumulated $(K)$. Diminishing returns to this factor decreases the incentive to invest when this factor accumulates. This implies that growth vanishes in the long run. A positive growth rate only can be achieved if the marginal product to the factor that can be accumulated is constant over time so that the incentive to invest in this factor does not vanish. With the usual assumption of diminishing returns to capital there has to be "something" that keeps the marginal return to capital from falling. This "something" is exogenous in the neoclassical growth models and made endogenous in the "new" growth theories.

In our discussion of the new growth theories we therefore concentrate on the marginal product of capital:

$$
\frac{\partial Y}{\partial K}=\frac{\beta \cdot A \cdot L^{\alpha}}{K^{1-\beta}}
$$

It is clear that there are only three possibilities for constant returns to capital. First, $\beta=1$, simply assuming constant returns to capital and no labour growth $(\hat{L}=0$, Romer 1986) or labour is not productive ( $\alpha=0$, Rebelo 1991). Second, $\vec{A}>0$, assuming that productivity of capital increases. This increase in productivity can be exogenous (neo-classical growth models) or endogenous (Romer 1990, Helpman and Grossman 1991b). Third, $\hat{L}>0$, labour growth is positive. Before discussing whether or not labour growth makes endogenous growth possible we take the growth rate of equation (2.2) and assume that the marginal product has to be constant. This key equation will be called "constant marginal product of capital condition".

$0=\hat{A}-(1-\beta) \cdot(R-L)+(\alpha+\beta-1) \cdot C$

Models that assume a positive growth rate of labour examine the growth rate of capital per capita or income per capita. A characteristic of models with positive labour growth is that they assume constant returns to scale $(\alpha+\beta=1)$ which implies 
that labour growth alone cannot achieve a positive growth rate of capital per capita (see equation (2.3)).

The relationship between capital growth and income growth can be derived from the production function. Putting equation (2.1) in growth rates yields:

$$
(\hat{Y}-\hat{C})=\hat{A}+\beta \cdot(\hat{K}-\hat{L})+(\alpha+\beta-1) \cdot \hat{L}
$$

Substitution of equation (2.3) in this equation shows that the growth rate of income has to be equal. to the growth rate of capital $\hat{Y}=k$ in order to obtain a constant value of the marginal product of capital.

One of the characteristics of endogenous growth is that savings behaviour has an influence on long-run growth. In this chapter we assume that consumers maximize the Constant-Intertemporal-Elasticity-of-Substitution utility function (Ramsey 1928).

$$
U(c)=\int_{i}^{\infty} e^{-p \tau} \frac{c^{1-\sigma}}{1-\sigma} \cdot d \tau
$$

where $\rho$ is the rate of time preference and $\sigma$ the intertemporal elasticity of substitution. Utility maximization yields the well known Ramsey formula for the growth rate of consumption.

$$
\hat{c}=\frac{r-p}{\sigma}
$$

where $r$ is the interest rate.

For simplicity, investments are equal to the difference between production and consumption. The former is dependent on the technology and the latter on consumer preferences.

$\dot{K}=Y-C$

Balanced growth together with the finding that $\hat{Y}=R$ implies that the steady state growth rate of consumption is equal to the steady state growth rate of income and capital $\hat{C}=\hat{Y}=\hat{R}$.

We use the following procedure to describe the various new growth theories. First we discuss the constant marginal product of capital condition (equation (2.3)) which will be the central equation to compare the various growth theories and to determine the main mechanisms for endogenous growth of these theories. There are only two mechanism to achieve a positive growth of income per capita. First, the "constant return to capital" approach $(\beta=1)$ and the "increase of total factor productivity" approach $(\hat{A}>0)$. Second, we determine the steady state growth rate of consumption. We only discuss the main contributions to the endogenous growth models (for a more elaborated survey of these theories see for example Sala-i- 
Martin 1990, van de Klundert and Smulders 1992. Verspagen 1992a or Schneider and Ziesemer 1994). Before we turn to the endogenous growth models we discuss the neoclassical model with and without exogenous technological change as the endogenous grow th models are based on these models.

\section{Original Neoclassical Model}

The original neoclassical model assumes constant returns to scale $(\alpha+\beta=1)$, decreasing returns to capital $(\beta<1)$, and no exogenous technological progress $A=0$. Substitution of these three assumptions in the central growth equation (equation (2.3)) implies that the steady state growth rate of capital, and therefore income, has to be equal to the exogenous labour growth rate $\mathbb{K}=\hat{L}$. The steady state growth rate of income per capita is therefore zero which contradicts one of the stylized facts on economic growth, i.e. the growth of income per capita is positive (Kaldor 1961).

\section{Neaclassical Growth Model with Exogenous Technological Change}

The most simple solution to the problem of a zero growth rate of income per capita is given by Tinbergen (1942) and Solow (1956). They assume a constant exogenous growtlin rate of technological change $(\hat{A}=\gamma)$ and hold the other wo assumptions of the neoclassical grow th model. The central growth equation shows that the growth rate of the capital-labour ratio is equal to:

$$
\hat{K}-\hat{L}=\hat{Y}-\mathcal{L}=\frac{\hat{A}}{1-\beta}
$$

The growth rates of the capitallabour ratio and income per head are therefore positive and totally dependent on the exogenous growth rate of technological change. ${ }^{\prime \prime}$ This neoclassical growth model therefore uses the "increase in total factor productivity" approach. Exogenous technological change increases the productivity of capital which keeps the marginal product of capital from falling.

One can also interpret $A$ as the stock of knowledge available in the economy which is used as a third production factor in the production process. However, a fundamental difference exists between the traditional inputs of labour and capital on the one hand and knowledge on the other. Labour and capital are "rival" inputs in the sense that the use of labour or capital by one firm prevents other firms from using this input. Knowledge is a "non-rival" good meaning that the use of a piece of knowledge by one firm does not prevent other firms from using this piece of knowledge (see chapter 1). This implies that the neoclassical model exhibits

\footnotetext{
183 If we hat assumed Hitrod neutrat or habon angmenting lechnological change instead of Hicks neutral technological change the exogenons growth ate would huve been $A_{\text {thias }}=(1-\beta), A_{\text {harrof }}$. The growth of income per capita would have been equal to the exogenous hamod growth rate of technological change.
} 
constant returns to scale of rival inputs, which allows perfect competition at the firm level. However, with respect to all three inputs, the model exhibits increasing returns to scale. This non-convexity caused by the non-rivalry of knowledge makes grow th possible. The Solow model interpreted in this manner therefore includes the non-rivalry aspect of knowledge, however, the increase in this knowledge stock is exogenous.

\section{1 .1 : Endogenous Growth Models: "Constant Retums fo Capital"}

The "constant returns to capital" approach keeps the marginal product from falling by assuming constant return to capital; $\beta=1$. Rebelo (1991) used the most elementary application of this approach. Next to the assumption of constant returns to capital he assumed no returns to other production factors that could not be accumulated $(\alpha=0)$. He maintained a constant returns to scale model $(\alpha+\beta=1)$. The production function becomes:

$Y=A . K$

The marginal product to capital is constant $(\partial Y / \partial K=A)$ and equal to the interest rate $(r)$. Equation (2.6) shows that the steady state grow th rate can be positive and because this growth rate is determined within the model (dependent on savings behaviour) this is an endogenous growth model. The steady state growth rate is:

$g=\frac{A-\rho}{\sigma}$

So when $(A>\rho)$ we obtain a positive growth rate of $\gamma, C$ and $K$.

This so called AK model makes growth endogenous which is often interpreted as an improvement to the Solow model. However, there is no technological progress in this model and the technology or knowledge stock possesses no nonrival goods characteristics. Because these characteristics are different from the general perception that knowledge increases over time and possesses some nonrival elements this $A K$ theory is a step back to the Solow model. Making growth endogenous should be no device for its own purpose, but description of the factors that really determine growth should be the objective.

Romer (1986) showed a second direction of the constant returns to capital approach. It differs from the $A K$ model of Rebelo in that the elasticity of the nonreproducible inputs is not equal to zero: $\alpha>0$. This implies increasing return to scale $(\alpha+\beta>1)$. Increasing returns to scale are normally incompatible with perfect market competition. The "new" trade theory showed some directions to cope with this problem. The oldest departure from the standard trade model is the 
"Marshallian" approach." This approach assumes that increasing returns are wholly external to the firm which allows perfect competition to remain (external economies of scale). Romer (1986) followed this Marshallian approach by assuming increasing returns to scale at the aggregated level and constant returns to scale at the firm level. These increasing returns to scale are generated by externalities or spillover effects from the production of knowledge. A firm's decision influences the output of all firms while the individual firm does not take this effect into account. The production function for each firm $i$ becomes:

$$
Y_{i}=A \cdot K_{i}^{\beta} \cdot L_{i}{ }^{1-\beta} \cdot K^{W}
$$

Where $K_{j}$ is the private capital stock of firm $i$ and $k$ is the aggregate capital stock in the economy. Individual firms take $k$ as given. The aggregated production function becomes:

$$
Y=A \cdot K^{\beta+v L^{1-B}}
$$

Like most other growth models with increasing returns Romer assumes no population growth $(\mathcal{L}=0)$. The central growth equation (2.3) becomes:

$$
0=(\mathbb{1}-\beta-\omega) \cdot \hat{R}
$$

It is immediately clear that we can obtain only a positive steady state growth rate of capital (and income) with "correct" externalities $(\beta+\psi=1)$. Spillover effects or externalities keep the marginal return of capital from falling. We obtain constant returns to capital, increasing returns to scalle, and a constant growth rate of capital and income $\left(g=\left(A \cdot L^{i-\beta}-p\right) / \sigma\right)$. In this way the problem of market imperfections is avoided. Because individual producers do not consider their knowledge spillovers on other producers a competitive economy achieves a lower growth rate than socially optimal. With $\beta+\psi<1$, we again have diminishing returns to capitall and we are back to the neoclassical model with $(R=0)$. Therefore increasing returns to scale are not sufficient to generate a constant positive growth ratio. With $\beta+\psi>1$ we get increasing growth rates (Romer 1986).

Learning by doing (Arrow 1962) is an important theoretical explanation for these externalities since the acquisition of knowledge is positively related to experience. An index of experience and therefore the knowledge stock is cumulative investment or the capital stock (Arrow 1962, Sheshinski 1967, Romer 1986).

This approach creates endogenous growth by positive spillover effects linked to the accumulation of a particular production factor. It therefore recognises that knowledge has some non-rival good characteristics. However, technological

${ }^{19}$ See e.g. Eaton and Panagariya (1974), Ethier $(1979,1982)$. 
progress or the growth of the knowledge stock appears as a side product to activities that are not specifically directed to the creation of knowledge.

\section{1 .2 :Endogenous Growth Models: "Increase of Total Factor Prodnctivity"}

The second approach makes a positive steady state growth possible by increasing the total factor productivity, $\hat{A}>0$. A first example of this approach is the neoclassical growth model that assumed an exogenous rate of technological change. The new growth models in this tradition make the rate of technological change endogenous. Technological change is generated by "investments" in knowledge. Resources, like for example R\&D expenditures, must be devoted to expand the amount of knowledge and not only to activities that are directly productive. This requires imperfect markets, because in these markets rewards to all production factors are less than total production. ${ }^{20}$ The "new" trade theory showed again a direction to cope with this problem. Chamberlinian monopolistic competition caused by internal economies of scale (increasing returns to scale at the firm level) possesses the required characteristics and treats market imperfections as simple as possible (no strategic interactions between actors on the market). ${ }^{21}$ Models that used the Chamberlinian approach to generate endogenous growth are Judd (1985), Romer (1987), Barro and Sala-i-Martin (1992).

The dominating strand of the "new" growth literature uses a combination of the Marshallian and Chamberimian approach. This approach combines imperfect competition with extermalities. An explicit R\&D sector produces blueprints for new goods, but also has some spillover effects on the aggregate stock of knowledge (Romer 1990, Helpman \& Grossman 1991b, Aghion and Howitt 1992). Returns to R\&D come in the form of monopoly rents in imperfectly competitive product markets. This R\&D sector produces new varieties of products or higher quality products. As a side product (externality), R\&D imcreases the general stock of knowledge which has two effects. Furst, it increases the efficiency of the R\&D sector in the future and second it increases the productivity of inputs in the manufacturing sector or the quality of consumer products.

We will illustrate this approach using the main ideas from Romer"s (1990) paper on "Endogenous Technological Change". Romer identifies three sectors: final good, investment goods and $\mathrm{R} \& \mathrm{D}$ sector. Technological change takes the form of the introduction of new investment goods for use in final goods production. A larger number of investment goods used in final good production increases the level of

20 Aln exception is discussed in Ziesemer (1991). If knowledge produced by thouseholds as in Lucas (1988) and Rebelo (199), perfect competition is possible.

\footnotetext{
21 See for example Krugman (1979, 1980), Helpman and Krugman (1985). Another direction concentuates on imperfect competition and uses internal econontics of scale to case this market imperfection. The main market structure they use is Cournot ofigopoly (Brander atnd Spencer 1985, Helpman and Krogman 1989).
} 
productivity. Blueprints ${ }^{22}$ for new investment goods are created in the R\&D sector, so technological progress is the intentional outcome of economic behaviour.

The adjusted Cobb-Douglas production function for the final good $Y$ becomes:

$$
Y=A^{\prime} \cdot H_{y}^{\alpha} \cdot L^{\beta} \cdot \sum_{i=1}^{k i} k_{i}^{1-\alpha-\beta}
$$

where $H_{y}$ is the amount of skilled labour devoted to the production of good $Y, L$ is unskilled labour $k_{i}$ represent the quantities of different varieties of investment goods and $n$ represents the number of different varieties of investment goods.

Capital is not a homogenous good but a collection of differentiated investment goods in the production function. Romer (1990) used the Ethier (1982) specification which implies that an increase in the number of varieties increases productivity. The intuition behind this result is that more specialised investment goods increase the productivity level.

For simplicity, the model has a symmetric structure, implying that all investment goods are supplied at the same level, $k_{i}=k^{23}$ It is assumed that one unit of investment goods presents $\sigma$ units of capital. Aggregate capital then becomes: $K=\sigma . n . k$. The production function becomes:

$$
Y=A^{\prime} \cdot H_{y}^{\alpha \alpha} \cdot L^{\beta} \cdot n \cdot K^{1-\alpha-\beta}=\frac{A^{\prime}}{\sigma^{1-\alpha-\beta}} \cdot n^{\alpha+\beta} \cdot H_{y}^{\alpha} \cdot L^{\beta} \cdot K^{1-\alpha-\beta}
$$

The Total Factor Productivity is equal to $A=A^{n} \cdot n^{\alpha+\beta} / \sigma^{1-\alpha-\beta}$. The productivity of the production factors labour and capital therefore is dependent on the number of investment varieties $(n)$. The marginal product of capital is:

$$
\frac{\partial Y}{\partial K}=\left[\frac{A^{\prime}}{\sigma^{1-\alpha-\beta}} \cdot n^{\alpha+\beta}\right] \cdot H_{y}^{\alpha} \cdot L^{\beta} \cdot(1-\alpha-\beta) \cdot K^{-\alpha-\beta}
$$

The constant marginal product of capital condition becomes:

$$
0=(\alpha+\beta) \cdot \hat{n}+\alpha \cdot \hat{H}_{y}+\beta \cdot \hat{L}-(\alpha+\beta) \cdot \hat{R}
$$

22 Blueprints are guidelines to produce a given type of imvesument good.

${ }^{23}$ In the case of the more general production tumction, $Y=A^{4} \cdot H_{p}^{\alpha} \cdot L^{\beta} \cdot \sum C_{i} \cdot k_{i}^{1-\alpha-\beta}$, cost minimization yields:

$$
\frac{k_{i}}{k_{j}}=\left(\frac{c_{i}}{c_{j}}\right)^{\frac{1}{\text { खणश }}}
$$

The structure in the Romer model is symmetric because all investment goods are equally productive while $\mathrm{c}_{\mathrm{i}}$ is equal to 1 . for all investment goods. 
Romer assumes that the number of unskilled (L) people is exogenously determined and constant over time $(\hat{L}=0)$. The number of skilled people $(\vec{H})$ is constant and in the equilibrium the number of people that works in the production sector $\left(H_{y}\right)$ is also constant (see equation (2.20)). The constant marginal product of capital condition becomes in this case:

$R=\hat{n}$

The only way to obtain a constant positive growth rate in this model is to assume a constant growth rate of the number of investment varieties. We now discuss how Romer achieved this result.

The new varieties (blue-prints) of investment goods are produced in the R\&D sector.

$\dot{n}=\gamma \cdot n^{\phi} \cdot H_{s}$

where $n$ represents the number of investment varieties. $H_{n}$ is the number of skilled people that work in the R\&D sector.

In an equilibrium the number of people that work in the R\&D sector will be constant $\left(H_{i z}\right.$ is constant). A constant positive growth rate is only possible when $\phi$ is equal to 1 while $n=\gamma \cdot H_{n} n^{\phi-1}$. When $\phi$ is smaller than 1 , growth will eventually vanish and there will be unbounded growth when $\phi$ is Jarger than 1. Crucial for endogenous growth is therefore the presence of $n$ (power 1) at the right hand side of equation (2.19) which implies an externality (non-convexity) of a certain magnitude in the production of blueprints.

Romer justifies this by pointing at the non-rionl characteristics of knowledge. Together with the production of new varieties the R\&D sector generates some gemeral knowledge as a side product. This knowledge cannot be appropriated by the producers of the specific investment variety while it "spills over" to other inventors of investment goods. Other producers of blueprints can use this general knowledge. The number of investment goods can therefore be interpreted as a knowledge stock.

The productivity level of skilled people in the R\&D sector is equal to $\gamma \cdot n$ (substitute $\phi$ is 1 in equation (2.19)). The larger the general knowledge stock $(n)$ the more productive $\mathbb{R} \& D$ expenditures become. An invention of a new investment variety in a research sector therefore increases the productivity in all research sectors. These positive knowledge spillover effects cause a constant incentive to invest in R\&D (if the number of skilled people working in the R\&D sector is constant, $H_{* t}$ is constant).

Besides the general knowledge (a side-product), the research sectors produce blueprints for new investment goods. Blueprints consist of specific technological. knowledge that is fully appropriable. The research sector gets patents for blueprints 
and sells these to the investment sector. We conclude by pointing to the private and social returns by the production of knowledge. Private return to knowledge exists by the sale of blueprints and a social return is linked to the positive externality in the creation of these blueprints.

For a constant growth rate, the number of skilled people that work in the research sector has to be constant (see equation (2.18) and equation (2.19)). Equation $(2,19)$ implies the larger this number, the higher the endogenous growth rate. The number of skilled people that work in the research sector $\left(H_{n}\right)$ is endogenously determined in the model and equal to: ${ }^{24}$

$$
H_{n}=\frac{\vec{H}-\rho \cdot \frac{\lambda}{\gamma}}{\lambda \cdot \sigma+I}, \quad \lambda=\frac{\alpha}{(1-\alpha-\beta) \cdot(\alpha+\beta)}
$$

The endogenous growth rate is higher if 1) the productivity of the $R \& D$ sector is higher $(\gamma)$. 2) the larger the human capital force $\bar{H}$. 3) the more patient households are (small value of $p$ ). 4) the more willing to substitute inter-temporally (low $\sigma$ ) 5) the greater the perceived differentiation of products.

We conclude that endogenous growth in the Romer model is caused by two special features. First, the Ethier specification of the production function of the final good. This implies that an increasing number of varieties enhances the productivity of all other production factors. Second, the non-convexity in the varieties or knowledge generation function, in particular the correct knowledge spillover effect $(\phi=1)$.

The knowledge generated by $R \& D$ activities creates positive steady state growth because it increases the marginal productivity of all factors. This causes a constant incentive to invest in $\mathrm{R} \& \mathrm{D}$. Manufacturing will therefore also be growing at a constant ratio. Important for the generation of endogenous growth is that the incentive to invest in $R \& D$ does not decline. The growing stock of knowledge as a side product of $R \& D$ generates this constant incentive. It does not matter if $R \& D$ firms are expanding product variety (Romer 1990, Grossman and Helpman 1991b) or increase the quality level of a constant number of varieties goods (Aghion and Howitt 1990, Grossman and Helpman 1991a).

\subsubsection{Conchuding Remarks on Endogenous Growth Theories}

Our survey of the main contributions to the endogenous growth theory identified two main possibilities to generate endogenous growth. Both possibilities require that the incentives to the production factor that could be accumulated, do not

\footnotetext{
24 See the original paper for a derivation of this result (Romer 1990).
} 
decline. The first approach requires a "constant return to capital". Besides the unrealistic Rebelo $A K$ specification, this approach assumes that knowledge is created as a side product (externality) to the accumulation of a particular production factor. Knowledge has some non-rival good characteristics because all firms can use knowledge that is created (as a side product) by a particular firm. The second approach generates endogenous growth by intentional investments in technological change that create an "increase in the total factor productivity". The dominating strand in the literature assumes that technology investments are worthwhile because a part of the research output is appropriable. Besides these private aspects of knowledge creation they also consider some public features of knowledge. The main ideas of the new growth theories on technological change can be summarized as:

- Technological change has to be endogenous and is the main driving force of economic growth.

- The creation of knowledge requires the diversion of resources from other activities.

- A part of the created knowledge should be appropriable to earn back investments in knowledge.

- Positive externalities or spill-over effects exist in the generation of knowledge (non-rival aspects of knowledge).

- The general knowledge stock increases the efficiency of the R\&D generation process and the production process.

These ideas will be the basic elements in our endogenous technological change model to be developed in chapters 3 to 5 . The Romer (1990) model contains all these elements and could therefore be a starting point for our model. However, in this thesis we want to examine the influence of the magnitude of spillover effects on the knowledge generation process in a theoretical and empirical way while one of our main hypotheses is that spillovers are becoming increasingly important in the IT era. Endogenous growth in the Romer (1990) model, however, requires a precise magnitude of the spillover effects $(\phi=1)$, which has to be constant over time. This assumption is too rigid for measuring the magnitude of spillover effects. This implies that we have to depart from the Romer (1990) framework, otherwise the steady state growth will be zero or grows without bound. We therefore concentrate on the knowledge generation process in a partial equilibrium model, while maintaining the main thoughts of the Romer (1990) model.

\subsection{Information Technology as a Key or General Purpose Technology}

At any time a few technologies exist that play an important role in fostering technical change in a wide range of sectors, thereby bringing about sustained and pervasive productivity gains (Landes 1969, Rosenberg 1982). We discuss a few 
recent contributions which modelled some of the main influences of a general purpose technology.

\section{Partial Equilibrium Model}

Bresnahan and Trajtenberg (1992) assume that economic growth appears to be driven by a few general purpose technologies (GPT). They emphasize three characteristics of GPT's. First, a GPT is pervasive, what means that it can be used in almost every sector in the economy. Second, large technological opportunities exist in the GPT itself, and third, innovations in the GPT enhances productivity of R\&D in downstream sectors, therefore "innovational complementarities" exist. These three characteristics make a GPT an "engine of growth". An improved GPT will be adopted by an increasing number of user sectors and it increases the incentives to develop complementary advances that raise the incentives to adopt. The demand for a GPT will therefore increase which leads to more innovation in the GPT sector, which results again in more adoption and more complementary advances, and so forth. Because of the pervasiveness, the GPT will spread throughout the whole economy and it affects the overall growth rate.

Bresnahan and Trajtenberg (B-T) develop a partial equilibrium model for two sectors, the GPT producing sector and the GPT using sector. An actual or potential GPT using firm is engaged in R\&D of its own and its rents increase when the quality level $\left(Q_{\text {cpp }}\right)$ of the GPT increases. The using firm maximizes its rents by choosing its own technological level $\left(T_{n}\right){ }^{25}$

$$
\underset{T_{u}}{\operatorname{Max}} \pi^{u}\left(P_{G P T_{T}} Q_{G P T^{\prime}} T_{t}\right)-R_{u}\left(T_{u}\right)
$$

The price level of a GPT ( $P_{\text {GPT }}$ ) has a negative effect, and the quality level of the GPT $\left(Q_{G \times P}\right)$ and the technological level of the user firm itself $\left(T_{*}\right)$ have a positive influence on the gross pay off $\left(\pi_{1}\right)$ of the user firm. The technological level of the user firm also has a positive influence on the $R \& D$ costs $\left(R_{u}\right)$. The crucial assumption in their model is the presence of "innovational complementarities".

$$
\frac{\partial^{2} \pi^{\prime \prime}\left(P_{G P T} Q_{C P T} T_{H}\right)}{\partial Q_{G M} \cdot \partial T_{n}}=0
$$

This assumption reflects one side of the "dual inducement mechanism" in the Bresnahan-Trajtenberg model; the user firm has an incentive to increase its equilibrium technology level in response to a quality improvement in the GPT

\footnotetext{
25 The firm affects the quality level of the product is sells, and/or the efficiency of its production pracess.
} 
input. Another assumption is that the number of user firms expands as the quality level of the GPT improves and its price decreases.

The GPT producing industry consists of one firm which may behave as a monopoly vis a vis the user firms. It chooses its price in this case according to:

$$
P_{G P T}^{m}=\operatorname{Max}_{P_{G r}}\left(P_{G P T}-c\right) \cdot \sum_{d \varepsilon A} X^{a}\left(P_{G P T}, Q_{C P T^{*}} T_{z}\right)
$$

where $c$ is the constant marginal costs and $X^{\prime \prime}$ is the demand function from the user firms. The total number of possible user firms of the GPT is given by $\mathrm{A}$. The GPT producing sector maximizes its profits by choosing its quality level $\left(Q_{G P T}\right)$ :

$$
\underset{Q_{G P r}}{\operatorname{Max}}\left(P_{G P Y}^{m}-C\right) \cdot \sum_{a \in A} X^{a}\left(P_{G P T^{m}}^{m}, Q_{G P T^{\prime}} T_{u}\right)-R_{G P T}\left(Q_{G P T}\right)
$$

An important assumption is that an increase in the technology level of one of the user firms $\left(T_{u}\right)$ induces the GPT producing firm to enhance the quality level of the GPT. This mechanism is the other part of the "dual inducement mechanism". In a growth setting it is this mechanism that causes endogenous growth.

This "dual inducement mechanism" causes a vertical externality. The pay-offs of the GPT producing and using firms are linked. The GPT producing firm innovates only when it can appropriate some of the social returns $\left(P_{\mathrm{GP}}>\mathrm{C}\right)$. Although, this implies that the incentive to innovate for the user firm is too low while the user firm gets the right incentive to innovate when price is equal to marginal costs $\left(P_{C P T}=c\right)$. This, however, implies no innovation in the GPT producing firms. In the usual range of appropriability, it is clear that neither firm get the right incentives to innovate.

A second externality is linked with the pervasiveness of the GPT. A larger demand by one user induces the GPT producer to enhance the quality level of its product which enhances the gross pay-offs of all user firms. From the viewpoint of the user firms, the quality level of the GPT is a public good, which implies that the equilibrium is characterised by too little innovation.

Three conclusions can be drawn from this analysis. First, the externalities imply that the private incentives are too low in relation to the social incentives. Second, government policy to correct for the vertical externality is not simply increasing the appropriabilities of the general purpose industries as is usually done, because this decreases the incentives of users to invent complementary assets. The right policy also considers the feedbacks and trade-offs involved. Third, the second externality implies that early adopters of a GPT are extremely important for the whole economy, they create a demand which induces improvements in the GPT and this technological change is useful for most sectors. 


\section{General Equilibrium Models}

Amable (1993) distinguishes two types of innovations. Incremental innovations are a continuous stream of improvements of a given technological system. These improvements are limited given a certain technological system. Radical innovations represent a change in the technological system and create new possibilities for incremental innovations (these radical innovations can be interpreted as new general purpose technologies). Amable formalizes these two types of innovations by combining the Romer (1990) and Aghion and Howitt (1992) concepts of technological change. The Romer concept represents incremental innovations and the Aghion and Howitt concept symbolises radical innovations. Researchers engage either in radical or incremental innovation. Radical innovations are uncertain and are considered as a discrete stochastic process whereas incremental innovations are not subject to uncertainty and described by a deterministic and continuous process. Growth turns out to be an irregular process in this model, phases of growth at a decreasing rate alternate with sudden jumps in the productivity level.

Helpman and Trajtenberg (1994) introduce (like Amable) the GPT in a general equilibrium model, but stress that complementary inputs first have to be developed before a new GPT becomes an engine of growth. While radical innovations or general purpose technologies appear endogenous in the Amable (1993) model, Helpman and Trajtenberg treat these innovations exogenously. "Since a full-fledged general equilibrium model of GPT"s proves to be exceedingly complex and intractable, however, we analyze a scaled back version in which advances in the GPT's are exogenous. As a results we ignore the feedback from user sectors to the GPT's" (Helpman and Trajtenberg 1994, p. 2). Another main difference is that in the Amable model new goods compatible with the new radical innovation can be directly produced while in the Helpman and Trajtenberg model complementary assets first have to be developed.

Helpman and Trajtenberg ( $\mathrm{H}-\mathrm{T})$ assume that new GPT's arrive at predetermined time intervals of equal length, $\beta=T_{i}-T_{i-1}$, where $T_{i}$ represents the time at which the $i^{\text {th }}$ GPT becomes available. A final good $\left(Y_{i}\right)$ has to be produced with the aid of the GPT $_{i}$ and an assembly of a continuum of components specially developed for this $\mathrm{GPT}_{\mathrm{i}}\left(D_{i}\right)$. The production function is:

$$
\begin{aligned}
& \gamma_{i}=\lambda^{i} \cdot D_{i}, \quad \lambda>1 \\
& D_{i}=\left[\int_{0}^{n_{i}} x_{i}(j)^{\alpha} \cdot d j\right]^{\frac{1}{\alpha}}=n_{i}^{\frac{1-\alpha}{\alpha}} \cdot X_{i}, \quad 0<\alpha<1
\end{aligned}
$$

where $\lambda^{i}$ is the productivity level of the $\mathrm{i}^{\text {th }}$ GPT, $x_{i}$ is a component that is compatible with GPT $T_{i}$ and $n_{i}$ the number of components compatible with GPT $T_{i}$ In 
a symmetric equilibrium the demand for each component is the same, $X_{i}=n_{i} x_{i}$. It is assumed that a unit of components is manufactured with a unit of labour; $X_{j}$ therefore represents the aggregate employment level of users of GPT ${ }^{*}$ The productivity level of a certain GPT therefore is:

$$
\frac{Y_{i}}{X_{i}}=\lambda^{i} \cdot n_{i}^{\frac{1-\alpha x}{\alpha}}
$$

As in the Romer (1990) model, the productivity level of a certain GPT is higher the more components are available for this GPT (this result is caused by the use of the Ethier (1982) specification for the components in equation (2.25)). This characteristic, linked to a new GPT is one of the main messages of the H-T paper: "evidently, a general purpose technology is more valuable the more compatible components have been developed for its use" (p. 5). A producer of final goods will use the GPT with the highest productivity level.

New components compatible with GPT are developed in the R\&D sector:

$\ddot{n}_{i}=a \cdot l_{i}$

$l_{i}$ is the number of people devoted to the development of components compatible with GPT; and the parameter a represents their productivity level. New components are developed for those GPT's that have the highest valued components. This implies that whenever it is profitable to develop components for the $i^{\text {th }}$ GPT it is not profitable to develop components for older GPT's.

We use figure 2.1 to highlight the main mechanisms in the two phases that characterise this model. We assume that at time $t_{0}$ the GPT $\mathrm{GP}_{i=1}$ is the best GPT and that compatible components have already been developed. Then at $T$, the $i^{\text {th }}$ GPT will be introduced and "phase 1 " begins. The R\&D sectors immediately switch to the production of compatible components of the new technology $\left(i_{i+1}=0, n_{i}>0\right)$ and more resources are devoted to this sector. This causes an initial reduction in productivity output. The new GPT will only be used in the production of the final goods if its is higher than that of the old $\mathrm{GPT}_{\mathrm{i}-1}$. This is the case when:

$$
\frac{Y_{i}}{X_{i}}>\frac{Y_{i-1}}{X_{i-1}} \Rightarrow n_{i} \cdot \lambda^{\frac{\alpha}{i-\alpha i}}>n_{i-1}
$$

Therefore, producers only will switch to the new GPT, when enough complementary inputs for this new GPT, have been developed. In the meantime they will continue to produce with the old $\mathrm{GPT}_{i-1}\left(Y_{i-1}>0\right.$ and $\left.Y_{i}=0\right)$. Growth of GDP is negative during this first phase because more resources will be devoted to develop compatible components for the new $\mathrm{GPT}_{i}$. Say, that at time $T_{i}+d_{1}$ the condition stated in equation (2.28) is satisfied and the productivity level that can be achieved with the new GPT is higher than that of the old GPT G. $_{i-1}$. At this time ends 


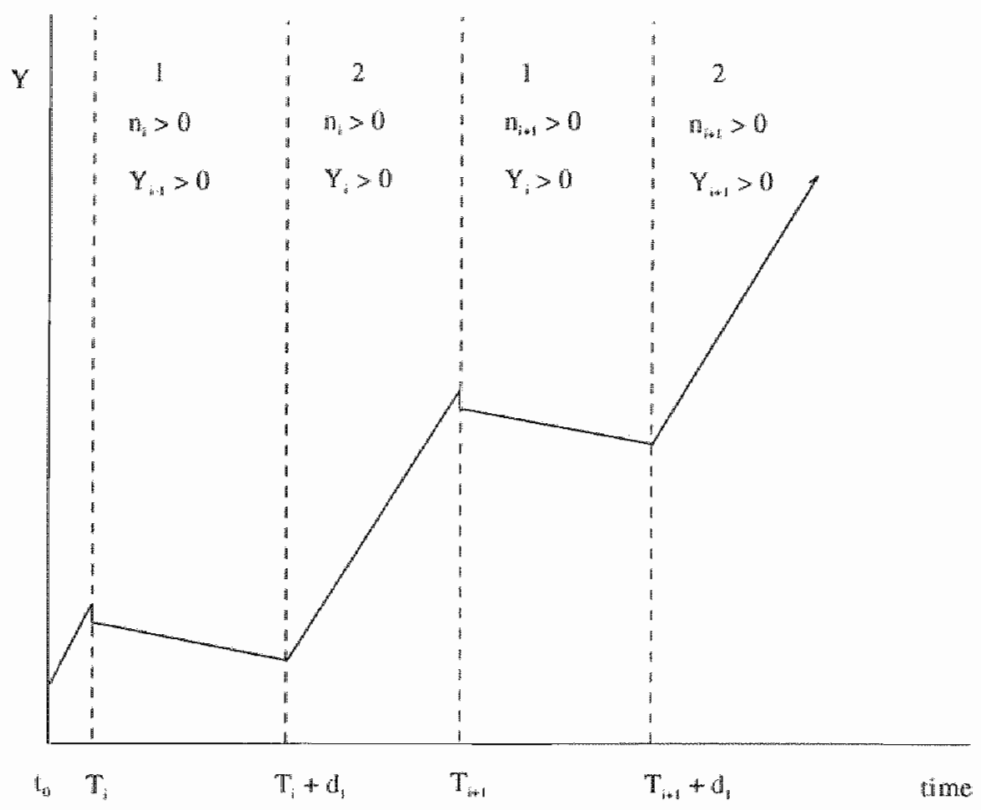

Figure 2.1. The two phases in Helpman-Trajtenberg model

"phase 1" ends. "Phase 2" starts when producers of final goods shift to produce with the new GPT since R\&D is directed at components compatible with this technology, the number of compatible components increases which causes the productivity to increase (see equation (2.26)). The aggregate output level increases. However, "phase 2" ends when a new GPT G $_{i+1}$ appears and the process starts all over. Yet, all the R\&D will be directed to the new technology $\left(\dot{n}_{i}=0, n_{j+1}>0\right)$ and the number of varieties of the old technology remains the same. The average growth rate over an entire cycle is equal to $g=\log \lambda /\left(T_{j+1}-T_{j}\right)$.

Some interesting conclusions can be drawn from the H-T model. First, a recurrent cycle exists, associated with each new and ever improving generation of GPT's. This is in accordance with the empirical findings of David (1992). This cycle contains two phases. Directly after the introduction of a new GPT phase 1 begins; output, productivity and profits decline and real wages stagnate. The benefits from a better GPT show up only in the second phase after enough complementary components have been developed. Output, productivity, real wages and profits increase. Second, measurement of the ex post rates of return in the first phase of a new important technology may generate disappointing results because it may seem this new technology appears to create no productivity gains (this could also be the case with the current "TT productivity paradox"). Third, government policy is dependent on where along a cycle one stands and the time horizon of a policy 
maker. For example, when a policy maker has a short time horizon and wants to shorten the first phase of negative output growth, increasing competition among component producers (increase $\alpha$ ) is the correct policy. A higher $\alpha$ implies that a smaller number of new components is necessary to make the new GPT more productive than the old one (see equation (2.28)). However, this policy in the second phase will reduce grow th because the appropriability in the new component sector is lower and this sector obtains less incentives to innovate. Policies that increase $\lambda$ are always desirable. One of the determinants of $\lambda$ may be basic research. Basic research could be justified in this way.

\section{Elements to Remember}

- GPT's are special because they are pervasive, characterised by high technological opportunities themselves and induce other sectors to innovate.

- The quality level of a GPT is a type of public good.

- GPT's can only be effective if sufficient complementary inputs are developed.

- GPT's can be an "engine of growth".

\subsection{Basic Ingredients for an Endogenous Technological Change Model}

In this chapter we investigated recent growth theories. These theories showed some general elements of the process of technological change. We can identify six elements for an endogenous technological change model. A model that has to deal with growth should first of all treat technology endogenously. Second, technology appears as the intentional outcome of economic behaviour and not as a sideproduct of other activities. Resources have to be invested to create knowledge and technological change. Third, a part of the knowledge created should be appropriable to earn back the resources of knowledge creation. Fourth, knowledge also has some public features. A part of the knowledge spilis over to other firms (externalities). Spillover effects are an important characteristic of the knowledge creation process. Fifth, some dominant, key or general purpose technologies exist that influence all sectors of the economy. Sixth, these key technologies are special because they are characterised by large technological opportunities and they may create incentives for other sectors to invest in technological change. 


\section{PART II}

\section{ENDOGENOUS TECHNOLOGICAL CHANGE MODELS}





\section{Endogenous}

\section{Technological Change by Cost Reducing and Demand Creating Innovations}

The survey of the literature provided six elements of a model that analyses technological change. In this chapter we deal with the first three elements. Contrary to what standard empirical implementations of technological change suggest, technological change is the intentional outcome of the behaviour of economic agents rather than an "exogenous" residual. Economic agents "invest" in technological change in order to reap its future benefits. They invest in the creation of knowledge and this knowledge is used to enhance their technological level. As is argued in the first chapter, knowledge has some private and public characteristics. In this chapter we examine the private properties of knowledge while the public properties of knowledge (the fourth element) will be investigated in chapters 4 and 5. Private aspects of knowledge enable firms to reap the benefits of their investments in knowledge. In Schumpeters' model of technical change innovators obtain a temporary monopoly position in which they can gain profits from their investments in knowledge (Schumpeter 1942). However, Schumpeter stressed that the benefits were temporary because sooner or later other firms would imitate or improve the technology. Schumpeter asserted that this was an important mechanism through which technology spreads and technological progress keeps on going because innovators cannot become complacent after a successful irnovation, but have to keep on innovating to hold or improve their market positions.

In this chapter we develop a simple model that makes technological change endogenous. Firms invest in the creation of knowledge to enhance their technological level. Research and Development (R\&D) expenditures are an important component of such investments in knowlledge. The R\&D process, directed at creating knowledge, can therefore be seen as an imperfect approximation of the technology generation process of a firm.

Figure 3.1 shows a schematic outline of the model which we develop in this chapter. We identify two sorts of technological change. First, the productivity level of the production process and second, the quality level of the product. For 


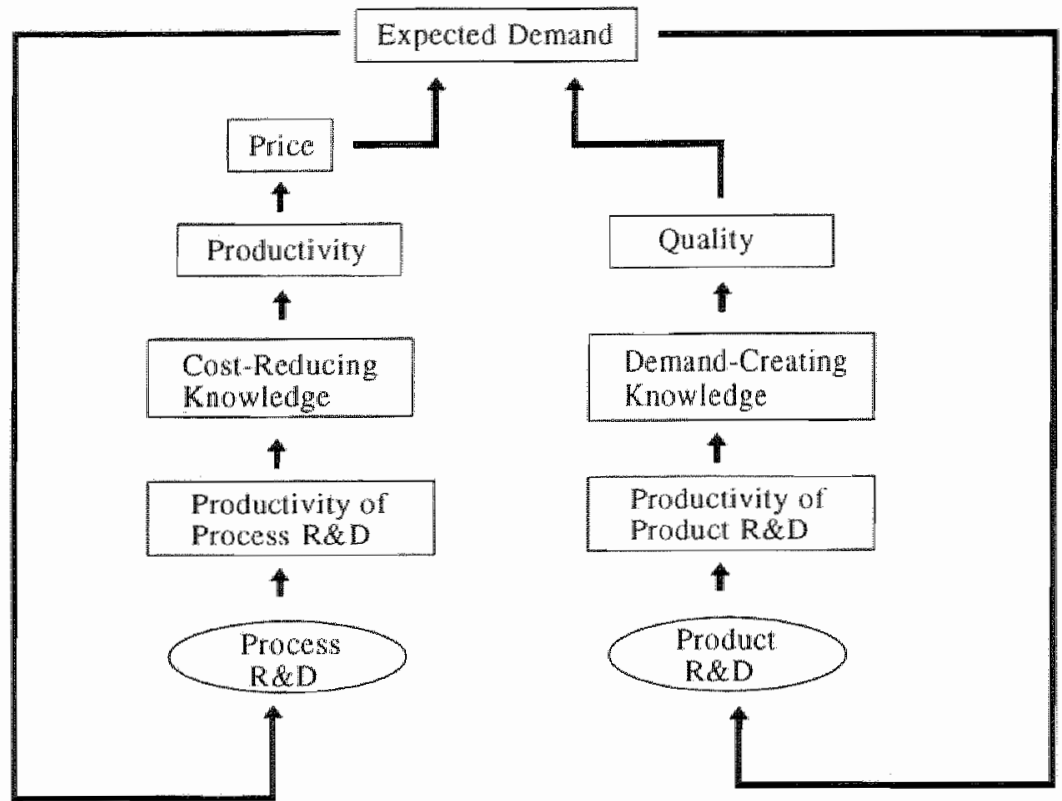

Figure 3.1. Schematic outline of the endogenous technological change model

conceptual convenience we also split the knowledge stock of a firm into two categories that relate to the two types of technological change. One is "costreducing" knowledge which is linked to the productivity level; second is "demandcreating" knowledge which is knowledge about quality improvements. Firms invest in $R \& D$ to create these two types of knowledge. Process $R \& D$ is directed toward obtaining cost-saving benefits in the future while product $R \& D$ is directed toward improving the quality levels of a firm's products which increases expected demand and helps to obtain demand creating benefits in the future. It is important to recognise that in both cases the benefits of current $R \& D$ occur in the future.

The main aim of this model is to determine factors that enhance or temper the incentives to invest in knowledge creation and which therefore influence the rate of technological change. We determine the optimal level of R\&D directed at costreducing innovations and demand-creating innovations given initial quality and productivity levels. Furthermore, we investigate whether product and process $R \& D$ are substitutes or complements.

Our model is a partial equilibrium model and related to the static model of Levin and Reiss (1988) in which the amount of product and process R\&D were endogenously determined. Kamien and Schwartz (1969), Sato and Ramachandran (1974), Sato and Suzawa (1983) also considered the dynamic effects of process R\&D but neglected the effects of product $R \& D$. We develop a model that considers the dynamic effects of both process $R \& D$ and product $R \& D$. Because of the inter- 
temporal effects of $R \& D$, we will use optimal control theory to solve the models in this chapter. The latter usually implies that the models themselves are (over-) simplified representations of economic behaviour/constraints. In this chapter we extensively study both the mathematical detail of the dynamics of the back-bone model and the solutions to the back-bone model. This enables us to omit these details in chapters 4 and 5 .

In section 3.1 we discuss the case in which a firm can engage in product $R \& D$ in order to achieve a higher expected demand (demand creating R\&D). In section 3.2 we consider the case in which a firm can engage in process $R \& D$ in order to achieve efficiency gains in the production process (cost reducing R\&D). Section 3.3 discusses the case in which a firm can engage in both demand creating R\&D as well as cost-reducing R\&D. Section 3.4 contains a summary and some concluding remarks.

\subsection{Demand Creating $R \& D$}

In this section we discuss the case in which an entrepreneur can engage in product R\&D in order to increase the quality of his products. We distinguish between two different types of quality: intrinsic quality and perceived quality. The former can be regarded as a weighted score on a number of relevant aspects of the product in question, while the latter can be thought of as the intrinsic quality of some product relative to the intrinsic quality of other similar/competing products. Instead of specifying the R\&D behaviour of competitors in detail, we simply assume that everyone engages in product $R \& D$ directed at improving the perceived quality of ones product by increasing its intrinsic quality. We disregard the possible role of marketing here, which might increase the perceived quality of a product without a corresponding rise in its intrinsic quality characteristics.

The main effect of increasing the perceived quality of a product is to raise (expected) demand. Perceived quality is the level of quality experienced by people. Without any R\&D activities, the perceived quality level will decline over time, because other products, which serve as a reference group, are, for example, improving. When perceived quality influences expected demand, a firm must engage in "defensive" product R\&D activities in order to maintain its market share.

An entrepreneur can therefore influence his expected demand not only by the standard price instrument, but also through the perceived quality level of a product which serves as an additional "marketing" instrument. When the perceived quality levell of a product is enhanced by means of advertising, this approach resembles the treatment of advertising by Dorfman and Steiner (1954) .

Hoos (1959), Nerlove and Arrow (1962) and Gould (1970) assumed that current advertising expenditures can be seen as a form of investment in a stock of goodwill which affects the present and future demand for a product. We use a related approach in which current product R\&D expenditures influence the quality level 
not only in the current period but also in the future. A firm which maximises profits must therefore take into account the inter-temporal benefits of product $R \& D$ which result from current $R \& D$ expenditures. The inter-temporal effects of product R\&D, which changes both the intrinsic and the perceived quality level, could be more important than the inter-temporal effects of advertising which shifts only the perceived quality level. ${ }^{26}$

We assume that the expected demand for a product is negatively influenced by the price of the product and positively influenced by the perceived quality of the product. We postulate the following expected demand function:

$$
Y_{1}^{d}=X_{0} \cdot Q_{p, p^{b}}^{b} P_{i}^{-i t}
$$

where $Y_{t}^{\prime}$ is the expected demand at time $t, P_{i}$ is the price level at time $t, X_{0}$ is a positive scale parameter and $Q_{p, t}$ is the perceived quality level at time $t$. The parameters $a$ and $b$ reflect the price and quality elasticity of demand, respectively.

The perceived quality level of a product can be raised by performing product R\&D. Without any product $R \& D$ activities, the level of perceived quality will decrease due to the $\mathrm{R} \& \mathrm{D}$ activities of competitors. This effect represents the Schumpeterian idea that profits from technical advantage are temporary and that innovators have to invest in $R \& D$ to keep their positions. For reasons of simplicity we assume a constant exponential decay of perceived quality. ${ }^{27}$

$$
\frac{d Q_{p}}{d t}=\gamma \cdot R_{d, t}^{\delta}-w \cdot Q_{p, t}, \quad 0 \leq \delta<1
$$

where $R_{u, l}$ is the amount of product $R \& D$ at time $t, w$ is the perceived quality depreciation rate and $\delta$ is the $R \& D$ elasticity of the quality generation process. We assume decreasing marginal quality retum to product $R \& D$ expenditures $(0 \leq \delta<1)$.

A high value of $w$ implies that the perceived quality depreciates quickly and that firms must invest in a significant amount of product R\&D to maintain their market share. A high value of $w$ may be caused by vigourous quality competition in a market. When $w=0$, the perceived quality level coincides with the intrinsic quality level. When we interpret $Q_{p}$ as Goodwill and $R_{i f}$ as advertising expenditures and assume that the costs of adding to goodwill are linear $(\delta=1)$ we obtain the dynamic Nerlove/Arrow equation. As in Gould (1970) we assume non-linear costs, which implies different dynamic behaviour than linear costs.

We assume that a firm will choose its price level and product R\&D budget so as to maximize the present value of its expected profits $(\pi)$, given its initially

\footnotetext{
26 People eventually learn that they are fooled by marketers.

27 "The threat of the competitor"s product R\&D will be made endogenous in chapter 5 where we introduce competitors explicitly.
} 
perceived quality level. We can specify the inter-temporal profit maximisation problem of a producer by:

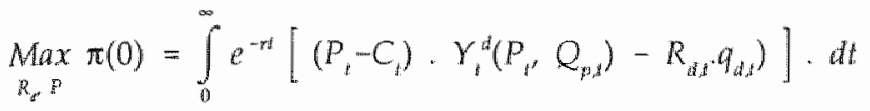

$$
\begin{aligned}
& \text { s.t. } Y_{v}^{d}=X_{0, t} \cdot Q_{p a t}^{b} \cdot P^{-a}, \quad 0 \leq b<1 \\
& \partial Q_{p} / \partial t=\gamma \cdot R_{i, t}^{8}-w \cdot Q_{p, t}, \quad 0 \leq \delta<1 \\
& Q_{p, y-1)}=Q_{p, 0}
\end{aligned}
$$

where $C_{t}$ is the unit production cost at time $t^{26}, r$ is the discount rate and $q_{a, t}$ is the price of product $\mathrm{R} \& \mathrm{D}$ at time $t, a, b$ and $\delta$ are non-negative parameters.

By using the current value Hamiltonian, we can in effect disregard the discount factor (Chiang 1992). The current value Hamiltonian of this problem is:

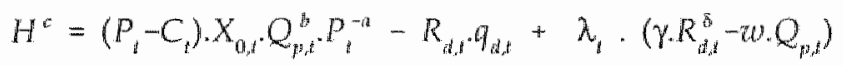

where $P_{f}$ and $R_{d,}$ are control variables. $Q_{p, A}$ is the state variable and $\lambda_{k}$ is its costate variable. The first two components on the right hand side of equation (3.4) are simply the profit function at time $t$ which depends on the values of the control variables to be chosen. The third component is rewritten as $\lambda_{0} Q_{p}$. Where $\lambda$ is interpreted as an increase in future profits resulting from an increase in $Q_{p}$ at time t. This third term therefore represents the amount of additional profits in the future due to an increase in the state variable $Q_{y, t}$. The Hamiltonian maximizes, therefore, the value of current and future profits (see also Dorfman 1969).

The conditions ${ }^{29}$ necessary for a path to be optimal under these assumptions are the following first order conditions: ${ }^{30}$

$$
\frac{\partial H_{c}}{\partial P}=\gamma^{d}-(P-C) \cdot \frac{a \cdot Y^{a}}{P}=0 \Rightarrow P \cdot\left[1-\frac{1}{a}\right]=C ; a>1
$$

${ }^{28}$ In this chapter we assume that product R\&D increases the intrinsic quality of the product without affecting unit costs. A logical extension of this model is that a higher quality level can only be obtained at the expense of higher unit costs: $C=C(Q)$ where $C>0$. This extension is incorporated in chapters 4 and 5 .

29 The necessary conditions are also sufficient for global maximization if the Mangarasian Sufficiency Theorem is satisfied. This theorem contains two conditions. First, the profit function and the perceved quality generation function are differentiable and concave in the variables $\left(Q_{p^{\prime}}, R_{d}\right)$ and second, in the optimal solution it is true that $\lambda \geq 0$, for all $t \in\left[0, \infty>\right.$, if $d Q$, d is nonlinear in $Q_{0}$ or $R_{d}$ (see Chiang (1992), pp. 21.5-221). Our model satisfies the first condition; the second condition has to be checked for any optimal solution because $d Q_{p} / d$ is nonlinear in $R_{d}$.

30 For reasons of simplicity we have dropped time subscripts. 


$$
\begin{aligned}
& \frac{\partial H_{c}}{\partial R_{d}}=-q_{d}+\lambda \cdot \gamma \cdot \delta \cdot R_{d i}^{\delta-1}=0 \\
& \frac{\partial H_{c}}{\partial Q_{p}}=-\frac{d \lambda}{d t}+\lambda \cdot r=(P-C) \cdot X_{0} \cdot P^{-u-u} \cdot b \cdot Q_{p}^{b-1}-w \cdot \lambda \\
& \frac{\partial H_{c}}{\partial \lambda}=\frac{d Q_{p}}{d t}=\gamma \cdot R_{d}^{\delta}-w \cdot Q_{p} \\
& T V C: \lim _{i \rightarrow m} \lambda_{p} e^{-t t}=0
\end{aligned}
$$

The first equation shows that the price will be determined as a mark-up over marginal costs. This is the familiar Amoroso-Robinson relationship, which also holds in this case, where we have assumed a linear homogenous production function and instantaneous adjustment of labour and capital, i.e. the standard factors of production. The mark-up depends on the price elasticity of demand (a). Because the price has to be positive the price elasticity of demand has to be greater than one.

The second equation implies that in the optimum situation, a marginal increase in the amount of product $R \& D$, which decreases current profits with its price $\left(q_{i}\right)$, has to be counterbalanced by an increase in future profits; the marginal increase in quality times its marginal value $(\lambda)$.

In the third equation $d \lambda / d t$ denotes the rate at which the marginal value of a unit of quality is depreciating when $d \lambda / d t<0$ and appreciating when $d \lambda / d t>0$. In the optimum situation the marginal value of quality depreciates at the rate at which quality is contributing to the current profits (this is represented by the first term on the left hand side). The marginal value of capital appreciates at the rate of the discount rate and the perceived quality depreciation factor (respectively the second term on the right hand side and the second term on the left hand side).

The fourth equation is the dynamic constraint, i.e. the quality generation function. The fifth equation is the Transversality Condition (TVC). This condition implies that the marginal present value of quality is zero when $t \rightarrow \infty$. The intuition of the latter condition is that the firm maximizes profits in the interval $[t=0, t=\infty]$, so the firm has to exhaust all possible profit possibilities during this interval. This is only the case when at the end of the interval the marginal present value of increasing the quality level is equal to zero.

A firm has to form expectations about the future course of input prices and the autonomous scale of demand in order to make decisions about its output price level and its product R\&D level. Gould (1970) in his related dynamic model of advertisements assumed constant prices. We will assume that prices and the exogenous scale of demand increase at given constant rates: 


$$
\begin{aligned}
& q_{d, i}=q_{a, 0} \cdot e^{1, i t} \\
& C_{1}=C_{0,} \cdot e^{m i t} \\
& X_{0, i}=X_{0,0} \cdot e^{n i t}
\end{aligned}
$$

We assume that the marginal cost $(C)$ is increasing at rate $m$, the price of product $\operatorname{R\& D}\left(q_{d}\right)$ at rate $l_{d}$ and the autonomous scale of demand $\left(X_{0}\right)$ at rate $n$.

\section{Stendy State Growth Rates}

We will differentiate the first order conditions with respect to time to obtain the equilibrium (steady state) rates of growth:

$$
\begin{aligned}
& \hat{p}=\hat{C} \\
& \hat{\lambda}+(\delta-1) \cdot \hat{R}_{d}=\hat{q}_{d} \\
& \hat{\lambda}=\hat{C}-(1-b) \cdot \hat{Q}_{p}-a \cdot \hat{P}+\hat{X}_{a} \\
& \hat{Q}_{p}=\delta \cdot \hat{R}_{d}
\end{aligned}
$$

where we assume that the growth rates of the system will be constant in the steady state. The changes of growth rates over time are therefore equal to zero. After making a few straightforward substitutions we obtain the following steady state growth rates:

$$
\begin{aligned}
& \hat{R}_{a}^{*}=\frac{1}{1-b . \delta} \cdot\left(X_{0}-\hat{C} \cdot(a-1)-\hat{q}_{a}\right) \\
& \hat{Q}_{p}^{*}=\delta . \hat{R}_{d}^{*} \\
& \hat{Y}^{a *}=\frac{1}{1-b . \delta} \cdot\left(X_{0}-\hat{C} \cdot(a-b . \delta)-a_{a}\right)
\end{aligned}
$$

where $1 /(1-b . \delta)$ is the grow th rate multiplier. The growth rate multiplier is larger the greater the technological opportunities in a sector. The technological opportunities are represented in this model by the quality elasticity of demand (b) and the R\&D elasticity of the quality generation process ( $\delta$ ).

Technological progress, which in this model is reflected in the quality level, in the steady state is higher in sectors which possess a higher exogenous growth rate of demand and is lower in sectors that face higher cost increases. Higher costs show up through higher marginal production costs (variable costs; wages and rental price of capital) and a higher price level of $\mathrm{R} \& \mathrm{D}$ (fixed costs). The elasticity of demand (a) has a negative impact on the steady state growth rate of product $\mathrm{R} \& \mathrm{D}$ when the unit costs are increasing and has a positive impact when unit costs 
are decreasing. The steady state growth rates are constant because we assume that the growth rates of $X_{0}, q_{i}$ and $C$ are also constant (see equation (3.10)).

\section{Transversality Condition}

The growth rate of product $R \& D$ is constant in the steady state, which in combination with equation (3.12) implies that the steady state growth rate of the marginal value of perceived quality $(\lambda)$ is also constant. We can use this information when we put the transversality condition in growth rates:

$$
\text { TVC } \lim _{t \rightarrow \infty+\infty} \lambda \cdot e^{-r t}=0 \Rightarrow \lambda-r<0 \Rightarrow r>\hat{q}_{d t}+(1-\delta) \cdot \hat{R}_{i t}^{*}
$$

The transversality condition is satisfied if $r \hat{q}_{d}+(1-0) . \hat{R}_{d}^{*}$. The economic rational for this sufficiency condition is simple: the growth rate of the marginal value of perceived quality has to be less than the discount rate, otherwise one would continuously invest in perceived quality.

\section{Elimination of Time}

The differential equations which describe the dynamics of the systems which we study are non-linear. This makes these systems analytically less tractable. In this study we will therefore use a qualitative-graphic (phase diagram) analysis to study its dynamics." The two differential equations which describe the dynamics of the system are $d Q_{p} / d t=f\left(Q_{y^{\prime}} R_{n}\right)$ and $d R_{d} / d t=g\left(Q_{y^{\prime}} R_{2}\right)$ can be calculated from the first order conditions. Because we assume that imput prices and the autonomous scale of demand grow at a constant rate, the first order conditions and therefore the differential equations depend on time. For the application of the phase-diagram technique it is a prerequisite that the variable $t$ (time) does not enter into the differential equations as a separate argument (the system thas to be autonomous) because otherwise each point in a phase space car imply different directions of the system over time. When this is the case, it is not possible to make qualitative statements about the characteristics of a possible equilibrium (see Chiang 1984).

In order to apply the phase diagram analysis to the case with exponentially growing input prices, we have to remove the time component from the profit maximization problem by a suitable redefinition of the variables of the system. Equation (3.15) showed that both perceived quality and the product R\&D budgets grow at constant rates which are directly linked to the constant growth rates of the

an For details of the qualitative-graphic analysis of a monlinear differential-equation, see A.C. Chiang (1984). Concerming the value of this approach Chang says: "The two variable phase dilagram,... is limited in that it can only answer qualitative questions those concerning the location and dymamic stability of the intertemporal equilibrim(s). But , it has the compensating advantages of being able to handle nonilinear systems as comfortable as linear ones and to address problems couched in terms of general functions as readily as those in terms of specific ones" (Cliang (1984), p. 629). 
exogenous variables of the system and to the various elasticities of the system. When we deflate $Q_{p}$ and $R_{d}$ with their steady state growth rates, we will get an autonomous system of differential equations (see Lucas 1988). To this end, we define the following new variables:

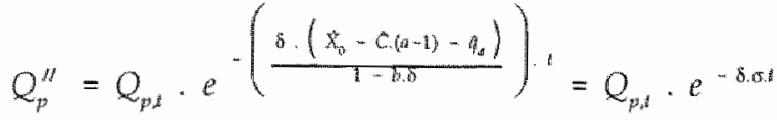

$$
\begin{aligned}
& R_{d t}^{\prime \prime}=R_{d, t} \cdot e^{-\left(\frac{x_{a}-C(n-1)-\hat{t}_{d}}{1-b .0}\right) \cdot t}=R_{d, t} \cdot e^{-\sigma . t} \\
& p^{\prime \prime}=P_{i} \cdot e^{-m a l}
\end{aligned}
$$

where $\sigma=\left(\hat{X}_{0}-(a-1) \cdot \hat{C}-\hat{q}_{d}\right) /(1-b . \delta)$. We will redefine the profit maximization problem given by equation (3.3) in terms of these three new variables. In order to get a new dynamic constraint in terms of $Q_{p}^{\prime \prime}$, we can differentiate equation (3.17) with respect to time. Together with the old constraint (3.2) and equation (3.18) we obtain:

$$
\frac{d Q_{p}^{\prime \prime}}{d t}=\gamma \cdot\left(R_{y}^{\prime \prime}\right)^{\delta}-(w+\delta \cdot \sigma) \cdot Q_{p}^{\prime \prime}
$$

The depreciation factor has increased with the steady state growth rate of $Q_{p}$ in comparison with the old constraint (equation (3.2)). Using the assumption that all exogenous variables grow at a constant rate (see equation (3.10)) and the equations $((3.17)-(3.20))$, we can rewrite the current value Hamiltonian as:

$$
H_{c}=\left(P^{\prime \prime}-C_{0}\right) \cdot X_{0,0} \cdot\left(Q_{p}^{\prime \prime}\right)^{b} \cdot\left(P^{\prime \prime}\right)^{-a}-R_{d}^{\prime \prime} \cdot q_{d, 0}+\lambda^{\prime \prime} \cdot\left(\gamma \cdot\left(R_{d}^{\prime \prime}\right)^{\delta}-(w+\delta \cdot \sigma) \cdot Q_{p}^{\prime \prime}\right)
$$

where $\lambda^{\prime \prime}=\lambda \cdot e^{-\left((1-\delta) \sigma+f_{d} \cdot t\right.}$. The associated discount factor of this problem is $\sigma+\hat{\eta}_{i t}-r$, where $\sigma+\phi_{i l}$ represents the steady state growth rate of the revenues, costs and product $R \& D$ budget.

We have now reached the stage where every explicit time dependency has been removed. We can calculate the first order conditions associated with this problem:

$$
\frac{\partial H_{c}}{\partial P^{\prime \prime}}=0 \Rightarrow P^{\prime \prime}=\left[\frac{a}{a-1}\right] \cdot C_{0} ; \quad a>1
$$




$$
\begin{aligned}
& \frac{\partial H_{c}}{\partial R_{d}^{\prime \prime}}=-q_{i, 0}+\lambda^{\prime \prime} \cdot \gamma \cdot \delta\left(R_{i d}^{\prime \prime}\right)^{\delta-1}=0 \\
& \frac{\partial H_{c}}{\partial Q_{p}^{\prime \prime}}=-\frac{d \lambda^{\prime \prime}}{d t}+\lambda^{\prime \prime} \cdot\left(r-\sigma-\hat{q}_{d}\right)=\left(P^{\prime \prime}-C_{0}\right) \cdot X_{0, r^{\prime}} b \cdot\left(P^{\prime \prime}\right)^{-d} \cdot\left(Q_{p}^{\prime \prime}\right)^{b-1}-(w+\delta \cdot \sigma) \cdot \lambda^{\prime \prime} \\
& \frac{\partial H_{c}}{\partial \lambda^{\prime \prime}}=\frac{d Q_{p}^{\prime \prime}}{d t}=\gamma\left(R_{d}^{\prime \prime}\right)^{\delta}-(w+\delta \cdot \sigma) \cdot Q_{p}^{\prime \prime} \\
& \text { TVC: } \lim _{t \rightarrow \infty} \lambda_{t}^{\| t} \cdot e^{\left(\sigma+g_{t}-r\right) t}=0
\end{aligned}
$$

When we put these first order conditions in growth rates and calculate the steady state growth rates of these redefined variables we find that the steady state growth rates of $Q_{p}^{\prime \prime}, R_{d \prime}^{\prime \prime}$ and $\lambda^{\prime \prime}$ are zero. A zero growth rate for $\lambda^{\prime \prime}$ in the steady state implies that the transversality condition is satisfied when $r>\sigma+\hat{q}_{d}$ in the steady state.

When we combine equation (3.22)-(3.24) and set $d \lambda / d t=0$ in the steady state we can derive the following expression for the quality/sales ratio:

$$
\frac{Q_{p}^{\prime \prime}}{P^{\prime \prime} \cdot Y^{\prime \prime}}=\frac{b}{a \cdot\left(\gamma+w-\hat{q}_{d}-(1-\delta) \cdot \sigma\right)} \cdot \frac{\gamma^{\prime} \delta \cdot R_{d}^{\prime \prime} \delta-1}{q_{d, 0}}
$$

This equation contains the familiar Dorfman/Steiner (1954) and Nerlove/Arrow (1962) advertising to sales ratio as a special case. The Nerlove/Arrow ratio can be obtained when there are constant returns to $R \& D(\delta=1)$, when the exogenous growth rates of all variables are zero $\left(\sigma=\hat{q}_{d}=0\right)$ and when we use R\&D expenditures $\left(q_{d, 0}=1\right)$. In addition, the static Dorfman/Steiner result can be obtained when the discount rate and depreciation factor are zero.

\section{Dynarics}

To study the dynamics of the autonomous system we need the two differential equations, $d Q_{p}^{\prime \prime} / d t=f\left(Q_{p}^{\prime \prime}, R_{d i}^{\prime \prime}\right)$ and $d R_{d i}^{\prime \prime} / d t=g\left(Q_{p}^{\prime \prime}, R_{d \prime}^{\prime \prime}\right)$, which describe the movements in the ( $\left.Q_{p}^{\prime \prime}, R_{t}^{\prime \prime}\right)$ space. The first differential equation is equation (3.25), i.e. the dynamic constraint. The second differential equation can be calculated from the other first order conditions. We can solve equation (3.23) for $\lambda^{\prime \prime}$, and differentiate this result with respect to time. Now we substitute $d \lambda^{\prime \prime} / d t$ and $\lambda^{\prime \prime}$ in equation (3.24) and obtain the differential equation for $d R_{d i}^{\prime \prime} / d t$ :

$$
\frac{q_{0} \cdot\left(r+w-\theta_{d}-(1-\delta) \cdot \sigma\right)}{\gamma \cdot \delta} \cdot\left(R_{d}^{\prime \prime}\right)^{1-\delta}-\frac{q_{t, 0^{0}}(1-\delta) \cdot\left(R_{d}^{\prime \prime}\right)^{-\delta}}{\gamma \cdot \delta} \cdot \frac{d R_{d}^{\prime \prime}}{d t}=\frac{C_{0} X_{0,0} b}{(a-1) \cdot\left(P^{\prime \prime}\right)^{a}} \cdot\left(Q_{p}^{\prime \prime}\right)^{b-1}
$$

Note that, in this redefined system, $d Q_{k}^{\prime \prime} / d t$ and $d R_{i}^{\prime \prime} / d t$ depend only on $Q_{p}^{\prime \prime}$ and $R_{d \prime}^{\prime \prime}$ and that the time variable no longer enters into these equations as a separate argument. 


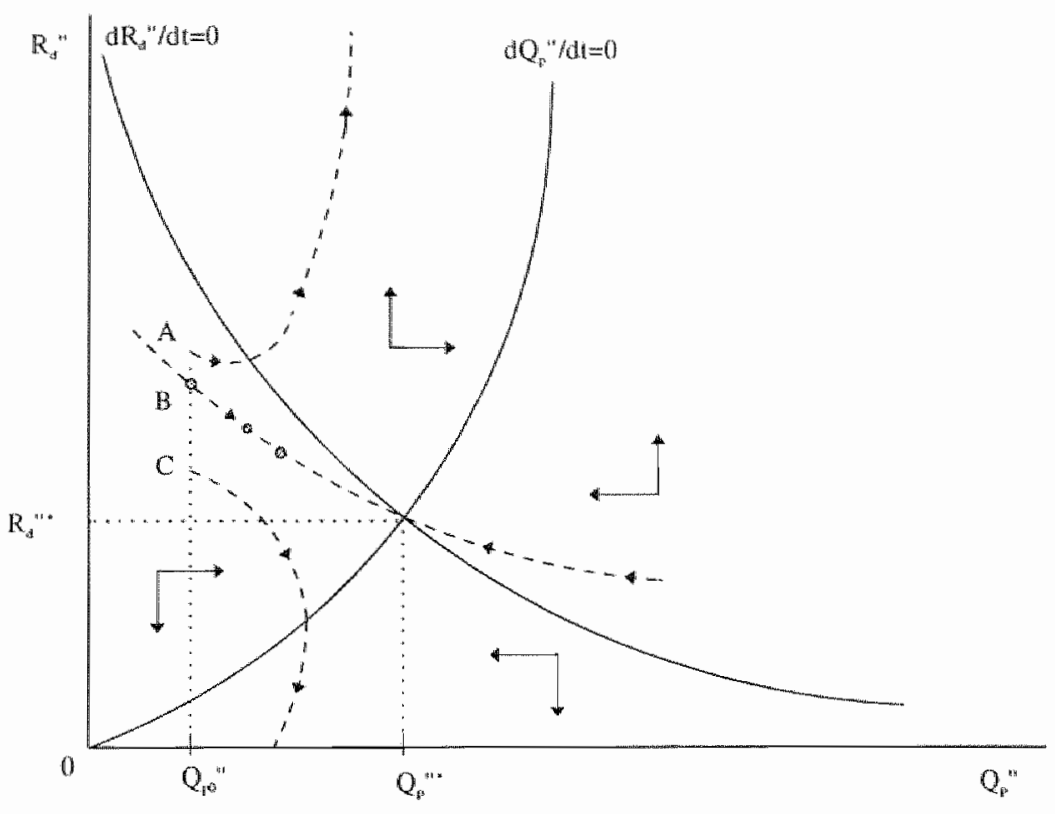

Figure 3.2. The dynamics of perceived quality and product $R \& D$

To construct the phase diagram, we need two demarcation loci, the $d Q_{p}^{\prime \prime} / d t=0$ and $d R_{d i}^{\prime \prime} / d t=0$ loci. Each demarcation locus provides the location for any prospective equilibrium and separates the phase space into two regions, one characterised by $d i / d t<0$ and the other by $d i / d t>0$, where $i=Q_{p}^{\prime \prime}, R_{d i}^{\prime \prime}{ }^{32}$ An implication of the autonomous character of this system is that these demarcation lines have positions which remain fixed over time. The two demarcation lines represent the graphs of the two equations of (zero) motion:

$\frac{d Q_{p}^{\prime \prime}}{d t}=0 \Rightarrow R_{u}^{\prime \prime}=\left(\frac{w+\delta \cdot \sigma}{\gamma}\right)^{\frac{1}{\delta}} \cdot\left(Q_{p}^{\prime \prime}\right)^{\frac{1}{3}}$

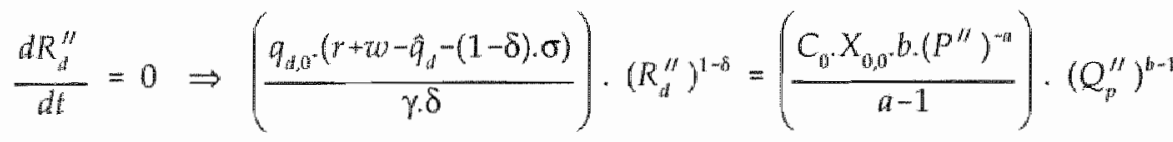

The perceived quality level and product $R \& D$ level have to be positive in equation (3.30), which implies $r+w>(1-\delta), \sigma+\hat{\theta}_{d}$. This condition is automatically satisfied when the transversality condition is met (see equation (3.26)). The two demarcation lines

${ }^{32}$ See Chiang (1984), p. 629. 
are drawn in figure 3.2, they constitute a "phase diagram" drawn in the $\left(Q_{\mathrm{p}}^{\prime \prime}, R_{a t}\right)$ space. The $d R_{d}^{\prime \prime} / d t=0$ locus is negatively sloped because $0 \leq b<1,0 \leq 8<1$ and $a>1$. The locus $d Q_{p}^{\prime \prime} / d t=0$ is positively sloped.

The economic interpretation of $d R_{d}^{* t} / d t=0$ can best be seen from equation (3.30), The right hand side of equation (3.30) can be rewritten as $\left(P^{\prime \prime}-C_{0}\right) . \partial Y^{3} / \partial Q_{p}^{*}$, which is the marginal increase of profits due to an expansion of $Q_{p}^{\prime \prime}$. The left hand side can be rewritten as $\left(r+w-\hat{q}_{t}-(1-\delta) \cdot \sigma\right) \cdot q_{i} / \partial\left(d Q_{p}^{\prime f} / d t\right) / \partial R_{d}^{\prime \prime}$, which are the marginal costs of obtaining an extra unit of $Q_{p}^{\prime \prime}$. So, on the $d R_{d}^{\prime \prime} / d t=0$ locus, the marginal cosks of increasing $Q_{p}^{\prime \prime}$ are equal to the associated marginal benefits. The negative slope of this locus can be explained as follows. On the right hand side of equation (3.30) the marginal benefits are declining when $Q_{p}^{\prime \prime}$ increases because $\partial^{2} Y^{\prime \prime} / \partial Q_{j, 2}^{\prime \prime}$ is negative $(b<1)$. On the $d R_{d /}^{\prime \prime} / d t=0$ locus marginal benefits are equal to marginal costs, so when marginal benefits are declining, marginal costs must also dectine. The matginal costs of 'producing' $Q_{p}^{f \prime}$ are increasing when $R_{d}^{\prime \prime}$ increases because $\partial^{2}\left(d Q_{p}^{\prime \prime} / d t\right) / \partial R_{v i}^{\prime \prime 2}$ is negative. Therefore, when the lower benefits associated with a higher $Q_{p}^{\prime \prime}$ level have to be met with lower marginal costs on the $d R_{d}^{\prime \prime} / d t=0$ locus, the level of $R_{d}^{\prime \prime}$ has to decline. This implies a negative slope of the $d R_{i d}^{\prime \prime} / d t=0$ locus.

The economic interpretation of the $d Q_{p}^{\prime \prime} / d t=0$ locus is simpler. On this locus the depreciation of the perceived quality level is equal to the generation of perceived quality. This locus has a positive slope because a higher perceived quality level implies more depreciation which has to be met with more quality generation which can only be achieved by doing more product R\&D.

Using the $d Q_{p}^{\prime \prime} / d t$ differential equation (equation (3.25)) to describe the horizontal movements in the phase diagram we find that anywhere below the $d Q_{p}^{\prime \prime} / d t=0$ locus, the perceived quality level $Q_{p}^{\prime \prime}$ is decreasing: the amount of product $R \& D$ is not enough to maintain the perceived quality level. Similarly, $Q_{p}^{\prime \prime}$ is increasing above the $d Q_{p}^{\prime \prime} / d t=0$ locus. The horizontal arrows demonstrate these directions of motion. According to the $d R_{d}^{\prime \prime} / d t$ differential equation (equation (3.28)) above the $d R_{d f}^{\prime \prime} / d t=0$ locus, product $R \& D R_{d}^{\prime \prime}$ will increase and below this locus, $R_{d}^{\prime \prime}$ will decrease. The vertical arrows demonstrate these directions of motion. The equilibrium (steady state) is at the intersection of the two curves. The steady state levels of the perceived quality and product $R \& D$ are given by $Q_{p}^{\prime \prime} \cdot$ and $R_{d j}^{\prime \prime}$ ", respectively. This configuration of arrows surrounding the equilibrium suggests a saddle point equilibrium. ${ }^{33}$

${ }^{33}$ The characteristics of a saddle point equilibrium are described in Chiang: "A saddle point is an equilibrium with a clouble personality it is stable in some directions, but unstable in others. More accurately, a saddle point has exactly one pair of streamlines-the stable branches of the saddle pointthat flow directly and consistently toward the equilibrium, and exactly one pair of streamlines-the unstable bronches-that flow directly and consistently away from it. All the other trajectories head toward the saddle point initially but sooner or later turn away from it"(Chiang (1984), p. 633). 
The configuration of arrows in figure 3.2 leads us to conclude that the equilibrium at $\left(Q_{*}^{*}, R_{*}^{*}\right)$ is a saddle point. This conclusion is made through a qualitative judgement of the phase diagram. Because the positions of the curves are drawn with considerable latitude, we examined the validity of this conclusion by an investigation of the local stability characteristics around the steady state. We applied a linearisation (first order Taylor expansion) of the nonlinear differentialequation system near the steady state and this analysis showed us that the equilibrium is indeed locally saddle point stable ${ }^{34}$

In order to describe the dynamics, we now assume that the firm has the initial perceived quality level $Q_{p}^{\prime \prime}$ and that it is furthermore free to choose the amount of product R\&D. The figure shows three possible trajectories which start from this initial level $Q_{p 0}^{\prime \prime}$ and an arbitrarily chosen level of product R\&D. ${ }^{35}$ Along each of these trajectories the necessary static and dynamic first order conditions are satisfied. Moreover, the trajectories fulfil the directional requirements imposed by the horizontal and vertical arrows.

Let us first consider what happens if a firm chooses a level of product $\mathbb{R} \& D$ which is higher than the product $R \& D$ level which is associated with point $B_{r}$ say at $\mathrm{A}$. The assumed location of the saddle path through $\mathrm{B}$ and the general directional requirements imply that the level of product R\&D declines until one gets an intersection with the $d R^{\prime \prime} / d t=0$ locus. After this intersection the level of product $R \& D$ will increase without bound. All the trajectories which start above point $B$ will therefore end up with an infinitively high level of $R_{d}^{\prime \prime}$ and $Q_{p}^{\prime \prime}$. When $Q_{p}^{\prime \prime} \rightarrow \infty$, however, the marginal increase of expected demand is approaching zero. This makes the left hand side of equation (3.24) negative which implies a violation of the transversality condition, as $\lambda^{\prime \prime}, \hat{\lambda}^{\prime \prime}>>0$. Because this path violates the transversality condition it camnot have been optimal to start at point $A$.

Consider any trajectory that starts below point B, say at $C$. The level of the perceived quality increases until the intersection with the $d Q_{p}^{\prime \prime} / d t=0$ locus, and then decreases. The level of product $\mathrm{R} \& \mathrm{D}$ is so low that the perceived quality level decreases. The level of product $R \& D$ continually decreases and eventually becomes zero or negative. When $R_{d}^{\prime \prime}$ is zero, $\lambda^{\prime \prime}$ will also be zero and in combination with equation (3.24) $d \lambda^{\prime \prime} / d t<0$ and $\lambda$ becomes negative. A negative $R_{d \prime}^{\prime \prime}$ level implies by equation (3.23) also a negative value for $\lambda^{\prime \prime}$. A negative marginal value for perceived quality cannot be optimal because the last $R \& D$ expenditures are inefficient and it violates the Mangasarian sufficiency theorem. Therefore, it cannot have been optimal to start from $C$.

Because on all these trajectories either the transversality condition or the Mangarasian sufficiency theorem are violated the unique saddlepath which leads to

\footnotetext{
3 The mathematical description of this analysis is given in Appendix $3 \mathrm{~A}$. For more details of the procedure of linearization of a non-linear system, see A.C. Chiang (1984), section 18.6.

${ }^{35}$ Given the initial quality level $Q_{\text {pe }}$ a firm can choose from an infinite number of values of product $R \& D$. All these possibilities are located on the vertical $Q_{p} A B C$ locus.
} 
the steady state equilibrium is the only possible candidate for an optimal solution. In the equilibrium the values for $Q_{p}^{\prime \prime}$ and $R_{d /}^{\prime \prime}$ are constant and positive. A positive constant equilibrium product R\&D level $\left(R_{\text {fl }}^{\prime \prime}\right)$ also implies a positive constant level for $\lambda$ (see equation (3.23)). This satisfies the transversality condition and the Mangarasian sufficiency theorem. The saddle path is therefore the unique optimal path. For each initial value of the perceived quality level of a firm, this implies a unique optimal product $\mathbb{R} \& D$ level. In our case with $Q_{\text {pin }}^{\prime \prime}$ the optimal initial level of product $\mathrm{R} \& \mathrm{D}$ corresponds to point $\mathrm{B}$. In the long rum the perceived quality level converges to the steady state level $Q_{p}^{\prime \prime}$ along the saddle path.

\section{Steady State}

We get the steady state values for $R_{n^{\prime}}^{\prime \prime}$ and $Q_{p}^{\prime \prime}$ if we solve equations (3.29) and (3.30) for these variables:

$$
\begin{aligned}
& R_{d}^{\prime \prime}=\left[\frac{\gamma^{b} \cdot \delta \cdot(w+\delta \cdot \sigma)^{\eta-b} \cdot X_{0,0} b \cdot(a-1)^{a-1}}{q_{d, a} \cdot\left(\gamma+w-q_{\alpha}-(1-\delta) \cdot \sigma\right) \cdot C_{0}^{a-1} \cdot a^{a}}\right]^{\frac{1}{T-b, \sigma}} \\
& Q_{p}^{\prime \prime *}=\left[\frac{X_{0,0} \cdot b \cdot \delta \cdot \gamma^{\frac{1}{\delta}} \cdot(w+\delta \cdot \sigma)^{\frac{\delta-1}{\delta}} \cdot(a-1)^{\alpha-1}}{q_{a, 0^{*}}\left(\gamma+w-q_{d}-(1-\delta) \cdot \sigma\right) \cdot C_{a}^{a-1} \cdot a^{n}}\right]^{\frac{\delta}{1-\beta \delta}}
\end{aligned}
$$

The steady state values of the quality level and the amount of product R\&D and therefore technological change are positively influenced by the autonomous scale of demand $\left(X_{0,0}\right)$, the productivity of the quality generation process $(\gamma)$, the product R\&D elasticity of the quality generation process ( $\delta$ ), and the quality elasticity of demand (b). The rate of technical change due to product $R \& D$ is negatively influenced by the unit cost of production $(C)$, the price of product $R \& D\left(q_{d}\right)$, the discount rate $(r)$ and the elasticity of demand (a). The perceived quality depreciation factor ( $w$ ) has a negative influence on the steady state quality level. The influence of $w$ on the steady state product $R \& D$ level is ambiguous. On the one hand a higher level of $R \& D$ is required to maintain a given perceived quality R\&D level, while on the other hand less R\&D is needed because the associated steady state quality level will be lower.

Notice that the discounted steady state level of product R\&D should be just enough to maintain the discounted perceived quality level. It should be stressed that in the equilibrium only the redefined (discounted) variables $\left(R_{d}^{\prime \prime}, Q_{p}^{\prime \prime}\right)$ are constant but that the "original" variables $R_{d}$ and $Q_{p}$, have a constant steady state growth rate (see equation (3.15)). 


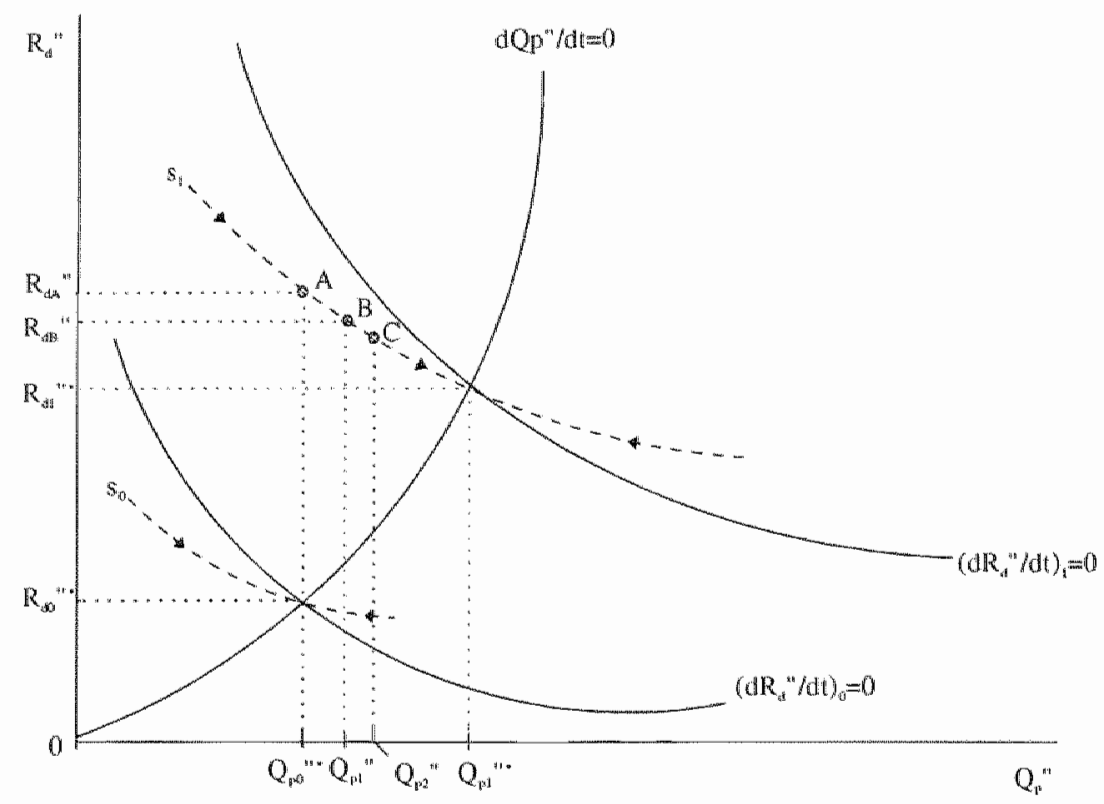

Figure 3.3. Increase of autonomous scale of demand

We can derive the following simple expression for the product R\&D intensity (product $R \& D /$ sales ratio) in the steady state:

$\phi_{\delta}=\frac{R_{d}^{\prime \prime}{ }^{*} q_{d, 0}}{p^{\prime \prime *}{ }^{*} Y^{\prime \prime *}}=\frac{\delta \cdot b \cdot(w+\delta \cdot \sigma)}{a \cdot\left(r+w-\hat{q}_{d i}-(1-\delta) \cdot \sigma\right)}$

The product $R \& D$ intensity $\left(\phi_{f}\right)$ is larger when the: technological opportunities are Larger ( $\delta)$, impact of quality on demand is larger $(b)$, depreciation rate is larger $(w+\delta, \sigma)_{s}$ price elasticity of demand smaller $(a)$ and marginal opportunity costs are lower $\left(r+w-\hat{q}_{d}-(1-\delta) \cdot \sigma\right)$. A closer look at the determinants of the depreciation rate and marginal opportunity costs reveal that the interest rate has a negative influence on the product $R \& D$ intensity. The perceived quality depreciation factor $(w)$, which reflects the level of competition in an industry, has a positive influence on the product $R \& D$ intensity. ${ }^{36}$ This is an interesting result because it implies that

\footnotetext{
${ }^{36}$ The partial derivative of $\phi_{d}$ with regard to $w$ is $\frac{\partial \phi_{a}}{d w}=\frac{\delta b a\left(r-q_{a}-\sigma\right)}{\left(a \cdot\left(r+w-q_{d}-(1-\delta) \sigma\right)\right)^{2}}>0$
}

The value of this derivative is positive because the TVC has to be satisfied in the steady state equilibrium, $r>q_{d}+\sigma$ (see equation (3.26)). 
industries with more vigourous qualicy competition have a higher product R\&D intensity.

\section{Graphical Mustration of Transtional Dynamics}

What happens if the value of an exogenous variable or a parameter changes? First. equation (3.31) and equation (3.32) show us that the steady state values of $R_{j}^{\prime \prime}$ and $Q_{p}^{\prime \prime}$ will change. Second, the loci in the phase diagram will move. We will illustrate this case with figure 3.3 .

We assume the following intial situation: the firm is in the steady state, it has the perceived quality level $Q_{p:}^{\prime \prime}$ " and the corresponding product $R \& D$ level is $R_{w 0}^{\prime \prime}$. The $\left(d R_{d}^{\prime \prime} / d t\right)_{0}=0$ and the $d Q_{p}^{\prime \prime} / d t=0$ loci of this situation are also drawn in figure 3.3. Their mathematical expressions are given by equation (3.29) and equation (3.30).

Suppose that there is a rise of the autonomous scale of demand $\left(X_{00}\right)$. The first locus $d Q_{j}^{\prime \prime} / d t=0$ will not be affected by this change. The second locus $d R_{d \prime}^{\prime \prime} / d t=0$ will change. This curve will move upwards from $\left(d R_{d / d}^{\prime \prime} / d\right)_{0}=0$ to $\left(d R_{d /}^{\prime \prime} / d t\right)_{1}=0$ in figure 3.3. The steady state will change from $R_{d i}^{\prime \prime}$ and $Q_{j 0}^{\prime \prime}$ to $R_{n 1}^{\prime \prime *}$ and $Q_{p 1}^{\prime \prime *}$. The saddle path which belongs to this new equilibrium is given by the locus with arrows $s_{1}$. An increase in the autonomous demand therefore moves the steady state including the saddle path to the north east. The firm is no longer in a steady state equilibrium. Given its initial quality level $\left(Q_{m}^{\prime \prime}\right)$, a firm will choose the corresponding optimal level of product $R \& D$ on the new saddle path $s_{1}$, in point $A$ $\left(R_{u A}^{\prime \prime}\right)$. Its new quality level $\left(Q_{p}^{\prime \prime}\right)$ will be (approximately):

$$
Q_{p, 1}^{\prime \prime}=Q_{p a}^{\prime \prime}+\gamma\left(R_{d, A}^{\prime \prime}\right)^{\prime \prime}-70 \cdot Q_{p h}^{\prime \prime}
$$

With this new perceived quality level $Q_{p}^{\prime \prime}$ the firm will choose the product $\mathrm{R} \& \mathrm{D}$ level in point $B\left(R_{d f}^{\prime \prime}\right)$ on the saddlepath $s_{1}$. When we repeat this process we find that the new quality level will be $Q_{p 2}^{\prime \prime}$ and the corresponding $\mathbb{R} \& D$ level is given by point C. Note that $R_{d A}^{\prime \prime}>R_{d B}^{\prime \prime}>R_{d C}^{\prime \prime}$ implying that an increasing quality level, $\Delta Q_{p, A}^{\prime \prime}>\Delta Q_{p g}^{\prime \prime}>\Delta Q_{p}^{\prime \prime}$, causes the adjustment process to slow down.

With figure 3.4 we illustrate two characteristics of the adjustment process. First, a firm does not immediately jump to the steady state by choosing a higher R\&D level but adjusts its quality level gradually. Second, the adjustment process slows down when one gets closer to the steady state. In the left part of figure 3.4 (figure a), we have drawn the relationship between marginal costs ( $R \& D$ expenditures: $f\left(Q_{z^{\prime \prime}}^{z \prime}\right)$ ) and the gross change in the perceived quality level $d Q_{p}{ }^{\prime \prime} / d t^{37}$ The quality

37 The gross change in $Q_{p}^{f t}\left(d Q_{p} z^{*} / d t\right)$ is the net change in $Q_{p}^{\prime \prime}\left(d Q_{p}, / d t\right)$ plus the depreciation of perceived quality $\left(w^{\prime} \cdot Q_{j}^{\prime \prime}\right): \frac{d Q_{p}^{s^{n}}}{d t}=Q_{p}^{s^{\prime \prime}}=\frac{d Q_{* t}^{d \prime}}{d t} * w^{\prime} \cdot Q_{p}^{\prime \prime}$. 
generation function, i.e. equation (3.20), implies a convex relationship between the marginal costs and $d Q_{p}^{g^{\prime \prime}} / d t$. This convex relationship explains the first characteristic because it implies that it is always cheaper to achieve a certain increase in quality in two smaller steps instead of one big step. We can also demonstrate this with figure (a). The marginal costs of achieving $Q_{p c}^{g / n}$ in one time are $f\left(Q_{p c}^{g}\right)$, which is more than two times larger than the marginal costs $f\left(Q_{p k i s c}^{z}\right)$ of achieving $Q_{r 0.5 c}^{8}$. Another implication of this process is that the steady state level will only be approached in the long run.

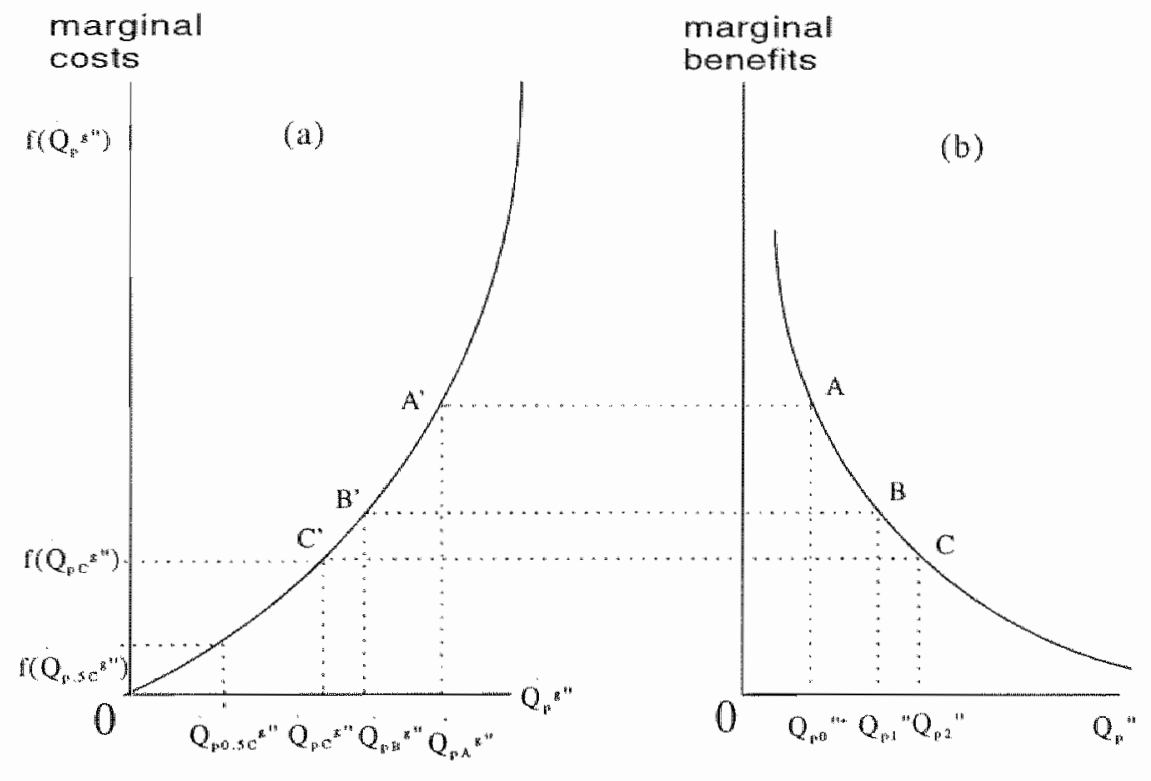

Figure 3.4. Marginal costs and marginal benefits

In figure (b), we depict the relationship between the marginal benefits with respect to the perceived quality level and the perceived quality level itself. The marginal benefits (increase in expected demand) decline when the level of $Q_{p}^{\prime \prime}$ increases (see the demand function, $0 \leq b<1$ (equation (3.1)).

On the optimal quality generation plan the marginal costs involved in generating one extra unit of quality must always balance the marginal benefits of the generation of the extra unit of quality. Figure (a) and (b) together can therefore explain the second characteristic ${ }^{38}$ Given the initial quality level $Q_{p 0}^{\prime \prime}$, the

38 We discuss the same situation that considered in figure 3.3. We postulate that the increase in the scale of the autonomous demand has already taken place in figure 3.4. Incidently, the effect of an increase in the scale of the autonomous demand in figure 3.4 is an upward shift of the marginal benefit curve in figure (b). 
marginal benefits of an increase in the quality level are given by point $\mathrm{A}$ in figure (b). Since marginal costs have to be equal to marginal benefits, the $R \& D$ expenditures are given by point $A^{\prime}$ in figure (a). The corresponding gross change in the quality level is $Q_{p A}^{z}$. The initial perceived quality level $\left(Q_{p 0}^{\prime \prime}{ }^{\prime \prime}\right)$ and the net change in the quality level gives the new quality level $Q_{p 7}^{\prime \prime}{ }^{39}$ We can repeat this process and see that given this new quality level the firm has $R \& D$ expenditures in point $B^{\prime}$ and a change in gross quality of $Q_{p d}^{3 \prime \prime}$. When we compare $Q_{p 0}^{\prime \prime}$ " $A A^{\prime}$ ' with $Q_{p 1}^{\prime \prime} B B^{\prime}$, we can conclude that a higher perceived quality level implies lower $R \& D$ expenditures and a smaller gross change in $Q_{p}^{\prime \prime}$ and therefore implicitly net change in perceived quality. This implies that the adjustment process slows down when the perceived quality level gets closer to the steady state value.

Figure 3.4 is also suitable for illustrating the dynamic behaviour of the system with the Nerlove and Arrow dynamic equation which describes the case with constant returns to $R \& D$. With constant returns to $R \& D$ the marginal costs of $R \& D$ are constant (independent of $d Q_{p}^{\prime \prime} / d t$ level). We can imagine them as a horizontal line in (a) of figure 3.4, say through point $B^{\prime}$. This implies that the steady state quality level is given by $Q_{j,}^{\prime \prime}$ because marginal costs are equal to marginal benefits in the steady state. Let us first consider what this implies for the transitional dynamics when the initial quality level is lower than this steady state level, say $Q_{p 0}^{\prime \prime}$. The marginal benefits of $R \& D$ in this situation are equal to $A$ and the marginal costs are equal to $B$. The marginal benefits of $R \& D$ are greater than the marginal costs and the firm will invest in $R \& D$ until marginal benefits are equal to marginal costs, which is the case by $Q_{p 1}^{\prime \prime}$. The firm will jump from $Q_{p 0}^{\prime \prime}$ to $Q_{p 1}^{\prime \prime}$ at $t=0$. There are no transitional dynamics in this case because the firm jumps immediately to the steady state. Let us now consider the outcome if the initial quality level is higher than the steady state level, say at $Q_{p 2}^{\prime \prime}$. The marginal benefits are equal to $C$ in this situation which are lower than the marginal costs. This implies that there will be no investment in R\&D and that the perceived quality level decreases in proportion with the depreciation rate until the steady state perceived quality level is met.

We can conclude that changes in exogenous variables or parameters will shift the steady state optimal behaviour by firms. This, however, implies that firms will only gradually adjust to this new steady state with decreasing marginal returns to $R \& D$ and jump immediately to the steady state with constant marginal returns to R\&D.

\footnotetext{
${ }^{39}$ The net change in the perceived quality level is the gross change in the perceived quality level less the depreciation of perceived quality. The net change in the perceived quality level is positive because the points $A$ and $A^{\prime}$ in figure 3.4 correspond to point $A$ in figure 3.3, which implies at positive change in the perceived quality level as it is situated to the left of the $d Q_{p}^{n} / d t=0$ locus.
} 


\subsection{Cost Reducing $R \& D$}

In this section we discuss the case in which an entrepreneur can engage in process R\&D in order to increase the efficiency of his production process. As in the case of product $R \& D$, the benefits of process $\mathbb{R} \& D$ spread into the future. Current process R\&D expenditures increase the productivity of the production process in a permanent fashion. A pioneering attempt to deal with this problem is the work of Kamien and Schwarz (1969) which is based on a situation with constant prices. Sato and Ramachandran (1974) discussed this kind of problem in a situation with exponentially growing prices which from the beginning could be removed from the dynamic optimization problem. Sato and Suzawa (1983) developed a similar model in which a firm can invest in applied and basic R\&D with exponentially growing prices. In this section we discuss this kind of problem with cost reducing $R \& D$ in the presence of exponentially growing prices and output which, unfortunately, cannot be immediately removed from the optimization problem.

To keep things as simple as possible, we assume a Cobb-Douglas production function with constant returns to scale in which two factors of production, capital and labour, are combined.

$$
Y_{i}=F\left(A_{t^{r}} L_{t^{\prime}} K_{i}\right)=A_{i} \cdot K_{1}^{\alpha} \cdot L_{i}^{1-\alpha}
$$

where $X_{t}$ is the level of production at time $t, K_{i}$ is the capital stock, $L_{t}$ is the amount of labour and $A$, is the total factor productivity index or TFP variable.

In this section we will use the total cost function which is associated with the Cobb-Douglas production function ${ }^{40}$.

$$
T C_{1}=\frac{Y_{1}}{A_{1}} \cdot\left(\frac{w_{1}}{1-\alpha}\right)^{1-\alpha} \cdot\left(\frac{w_{1}}{\alpha}\right)^{\alpha}=Y_{1} \cdot C_{1}
$$

where $w_{t}$ is the expected wage level at time $1, \tau$, is the expected user cost of capital at time $t$ and $C_{j}$ is the unit costs at time $t$. The advantage of this total cost function approach in comparison with a direct production function approach is that the dual cost function approach automatically implies the optimum allocation of labour and capital. In this way we can save two control variables, capital and labour. Another advantage is that the optimisation problem can be specified in the same way as the profit-maximization problem of section 3.1.

Producers may engage in cost reducing $R \& D$, which we call process $R \& D$. Process R\&D is aimed at increasing the efficiency of the production process, by

40 Sato and Suzawa (1983) also used this cost function approach. 
producing more output with the same amount of inputs. ${ }^{41}$ We assume decreasing marginal productivity increases with respect to process $R \& D$ expenditures. We can specify the following productivity generation function: ${ }^{42}$

$$
\frac{d A}{d t}=\eta \cdot R_{c, t}^{\theta}, \quad 0 \leq \theta<1
$$

where $R_{c t}$ is the amount of process $R \& D$ at time $t$. We assume that the expected demand is negatively influenced by the price level of the product. We will use the following expected demand curve:

$$
Y_{t}^{d}=X_{0, s} \cdot P_{t}^{-a}
$$

We now assume that a firm will maximize the present value of its expected profits by choosing the optimal price and process $R \& D$ level, given its initial productivity level. The inter-temporal profit maximisation problem becomes:

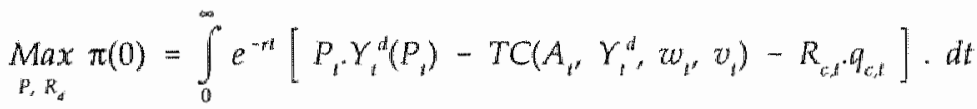

$$
\begin{aligned}
& \text { s.t. } Y_{i}^{d}=X_{0,1} P_{i}^{-4 A} \\
& T C_{i}=\frac{1}{A} \cdot Y_{i}^{d} \cdot w_{t}^{1-\alpha} \cdot v_{i}^{\alpha} \cdot(1-\alpha)^{\alpha-1} \cdot \alpha^{-\alpha} \\
& d A / d t=\eta \cdot R_{c, t}^{\theta} \\
& A_{i=0}=A_{0}
\end{aligned}
$$

where $q_{i,}$ is the expected process $R \& D$ price at time $t$. We assume that the exogenous scale of demand $\left(X_{0}\right)$, the price of process $\operatorname{R\& D}\left(q_{c}\right)$, the wage rate $(w)$ and the user cost of capital $(v)$ all grow with an exogenous constant growth rate. The current value Hamiltonian of this problem is:

$$
H^{e}=X_{0, t} \cdot P_{t}^{1-4}-\frac{1}{A_{t}} \cdot X_{0, f} \cdot P_{!}^{-a} \cdot w_{l}^{1-\alpha} \cdot v_{1}^{\alpha} \cdot Z_{1}-R_{c, t} \cdot q_{c, t}+\mu \cdot \eta \cdot R_{c, t}^{\theta}
$$

\footnotetext{
4 C.t. Kennedy (1964).
}

42 Note that Sato and Ramachandran (1974) and Sato and Suzawa (1983) use a different dynamic constraint. Their specification is: $A=f\left(R_{t, g}\right)$. This specification implies that a constant level of process $R \& D$ is enough to obtain a constant growth rate of the productivity level. Similar specifications in the context of human capital accumulation can be found in Lucas (1988) and Romer (1990). 
where $Z_{1}=(1-\alpha)^{\alpha-1} \cdot \alpha^{-\alpha}$. The first order condition with respect to the price level is:

$$
P_{i}=\left(\frac{a}{a-1}\right) \cdot \frac{w_{t}^{1-\alpha} \cdot v_{1}^{\alpha} \cdot Z_{1}}{A_{i}}=\left[\frac{a}{a-1}\right] \cdot C_{i}
$$

Notice that this equation again shows that the price will be determined by a markup over unit costs which depends on the price elasticity of demand. However, unit costs are not given for an entrepreneur as in section 3.1, but can be influenced by process R\&D activities which influence total factor productivity $(A)$. Differentiation of all the first order conditions with respect to time gives rise to the following equilibrium (steady state) rates of growth:

$$
\begin{aligned}
& \hat{R}_{v}^{*}=\frac{1}{1-(a-1) \cdot \theta} \cdot\left[\hat{X}_{0}-(a-1) \cdot(\alpha \cdot \hat{v}+(1-\alpha) \cdot \hat{w})-\hat{q}_{c}\right] \\
& \hat{A}^{*}=\theta \cdot \hat{R}_{*}^{*} \\
& \hat{P}^{*}=\frac{1}{1-(a-1) \cdot \theta} \cdot\left[-\theta \cdot \hat{X}_{0}+\alpha \cdot \hat{v}+(1-\alpha) \cdot \hat{w}+\theta \cdot \hat{\theta}_{0}\right]
\end{aligned}
$$

where $1 /(1-(a-1), \theta)$ is the grow th rate multiplier. This growth rate multiplier is, as in section 3.1, positively dependent on the technological opportunities $(\theta)$ and on demand characteristics, i.e the price elasticity of demand $(a)$. The higher the price elasticity of demand, the greater the influence of price reducing productivity improvements.

As in the case of demand creating innovations, the growth rate of the exogenous scale of demand has a positive influence and the growth rates of the prices of labour, capital and process $R \& D$ have a negative influence on the steady state growth rates of $R_{c}$ and $A$.

To determine the influence of the negatively sloped demand curve on the steady state growth rates of $R_{c}$ and $A$, we will put the price elasticity of demand equal to zero $(a=0)$. When $a=0$, we have an exogenous level of production and the firm minimizes costs to meet this exogenous production level. When we put $a=0$ in equation (3.42), it follows that the grow th rates of the prices of labour and capital have a positive influence on the steady state growth rates of $A$ and $R_{\mathrm{f}}$. This is because higher growth rates of the prices of labour and capital imply that the potential benefits of process R\&D per unit of output increase. The level of output is given, and therefore the total amount of potential benefits increases, which justifies a higher growth rate of process R\&D. When we have an elastic demand curve $(a>1)$ we have the opposite result; a higher growth rate of input prices decreases the steady state growth rates of $A$ and $R_{r}$. The reason for this result is that the level of output is not exogenous but endogenously determined. In this situation $(a>1)$ higher input prices not only mean that the potential benefits per unit of output of engaging in process $R \& D$ are higher, but also that the output level will 
decrease because the price level will increase. In a situation with an elastic demand curve $(a>1)$ the level of output decreases more than proportionally, which implies that the total potential benefits for process R\&D are smaller. Smaller potential benefits imply a lower steady state growth rate of $R_{c}$ and $\dot{A}$.

When one puts the price elasticity of demand equal to zero one gets another striking result; the influence of the R\&D elasticity of the productivity generation process $(\theta)$ becomes negative on the steady state growth rate of process R\&D and stays positive on the steady state growth rate of $A$. The process $R \& D$ elasticity of the productivity generation process $(\theta)$ has a positive influence on the steady state growth rate of $A$. The reason for this is that the costs of achieving a certain productivity increase declines when $\theta$ rises. But $\theta$ has a negative influence on the steady state growth rate of $R_{r}$. In this case there are two opposite effects. First, as we have seen, productivity production costs decline which results in a higher growth rate of the productivity level implying a higher process $R \& D$ growth rate. Second, the amount of process $R \& D$ necessary to achieve a certain level of productivity growth is less. In this particular situation the second effect dominates the first effect. With a negatively sloped demand curve, $\theta$ has a positive influence on the growth rate of $R_{c}$. The reason for this is besides the two opposite influences of the situation when $a=0$, we have a third effect. A higher $\theta$ means a higher productivity growth rate, which lowers unit costs. In the case of an elastic demand curve this implies a more than proportionally higher output level. The total effect in this case is a higher growth of process $R \& D$.

\section{Time Elimination Method}

As in section 3.1 we first redefine the variables of the system. Because we found constant steady state growth rates for $A, R_{c}$ and $P$, we normalize the productivity level, the process R\&D level, and the price level with their corresponding constant steady state growth rates. We obtain the following new variables:

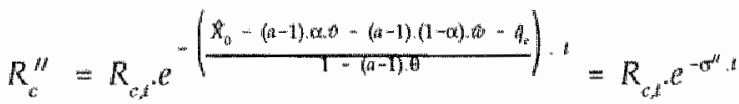

$$
\begin{aligned}
& A^{\prime \prime}=A_{i} \cdot e^{-\theta \cdot 0^{\prime \prime \prime}}
\end{aligned}
$$

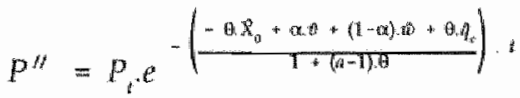

where $\sigma^{\prime \prime}=\left(\hat{X}_{0}-(a-1) \cdot \alpha \cdot \hat{0}-(a-1) \cdot(1-\alpha) \cdot \hat{w}-\hat{q}_{0}\right) /(1+\theta)$. Again we obtain the new dynamic constraint in terms of $A^{\prime \prime}$ by differentiating the second equation of (3.43) with respect to time. When we use this result in combination with the old constraint (equation (3.37)) we can solve for $d A^{\prime \prime} / d t$ and obtain: 


$$
\frac{d A^{\prime \prime}}{d t}=\eta \cdot\left(R_{c}^{\prime \prime}\right)^{\theta}-\theta \cdot \sigma^{\prime \prime} \cdot A^{\prime \prime}
$$

The profit maximization problem (equation (3.39)) can be rewritten in terms of these new variables. The current value Hamiltonian of this redefined problem is:

$$
H^{c}=X_{0,0^{0}}\left(P_{t}^{\prime \prime}\right)^{1-\alpha}-\frac{X_{0,0}}{A^{\prime \prime}} \cdot\left(P_{1}^{\prime \prime}\right)^{-a} \cdot w_{0}^{1-\alpha} \cdot v_{0}^{\alpha} \cdot Z_{1}-R_{e}^{\prime \prime} \cdot q_{c, 0}+\mu \cdot\left(\eta \cdot\left(R_{c}^{\prime \prime}\right)^{\theta}-\theta \cdot \sigma^{\prime \prime} \cdot A^{\prime \prime}\right)
$$

The discount rate is $\sigma^{\prime \prime}+\hat{q}_{c}-r$.

Using the first order conditions, we are able to acquire the two differential equations, $d A^{\prime \prime} / d t=f\left(A^{\prime \prime}, R_{c}^{\prime \prime}\right)$ and $d R_{c}^{\prime \prime} / d t=g\left(A^{\prime \prime}, R_{c}^{\prime \prime}\right)$, which are necessary to study the dynamics of the system. The $d A^{\prime \prime} / d t$ locus is equation (3.44) and the $d R_{c}^{\prime \prime} / d t$ locus can be obtained from the other first order conditions. We can calculate the steady state levels of $A^{\prime \prime}$ and $R_{c}^{\prime \prime}$ by putting these two differential equations equal to zero:

$$
\begin{aligned}
& \frac{d A^{\prime \prime}}{d t}=0 \Rightarrow R_{c}^{\prime \prime}=\left(\frac{\theta \cdot \sigma^{\prime \prime}}{\eta}\right)^{\frac{1}{\theta}} \cdot\left(A^{\prime \prime}\right)^{\frac{1}{\theta}} \\
& \frac{d^{\prime \prime} R_{c}^{\prime \prime}}{d t}=0 \Rightarrow \frac{q_{c, 0}\left(r-\hat{q}_{c}-(1-\theta) \cdot \sigma^{\prime \prime}\right)}{\eta \cdot \theta} \cdot\left(R_{c}^{\prime \prime}\right)^{1-\theta}= \\
& X_{0,0} \cdot\left(\frac{1-\alpha}{w_{0}}\right)^{(\alpha-1) \cdot(1-\alpha)} \cdot\left(\frac{\alpha}{v_{0}}\right)^{(a-1) \cdot \alpha} \cdot\left(\frac{a-1}{a}\right)^{a} \cdot\left(A^{\prime \prime \prime}\right)^{a-2}
\end{aligned}
$$

To get an economically meaningful situation we require that $r-\hat{q}_{i}-(1-\theta) \cdot \sigma^{\prime \prime}>0$, which is automatically satisfied when the transversality condition is met. ${ }^{43}$

\section{Steady State}

When we solve equations (3.46) and (3.47) for $A^{\prime \prime}$ and $R_{c}^{\prime \prime}$ we get the following steady state values:

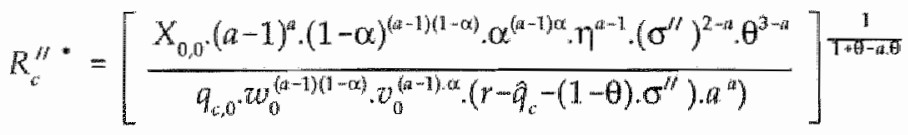

${ }^{43}$ The transversality condition is satisfied when $r>q_{i}+\sigma^{\prime \prime}$ 


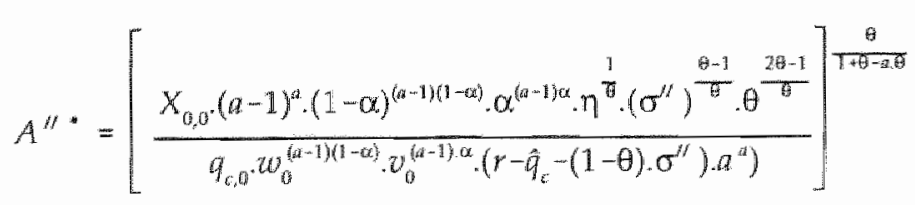

First, as in the case of steady state growth rates, the influence of labour and capital prices is negative when demand is elastic $(a>1)$ and positive when demand is totally inelastic $(a=0)$.

Second, with an elastic demand curve, the productivity of the productivity generation process ( $\eta$ ) has a positive influence on $R_{c}^{\prime \prime}$ and a negative influence when demand is totally inelastic. This result can be explained by considering the same three influences that caused a similar effect from $\theta$ on the steady state growth rate of the process $\mathrm{R} \& \mathrm{D}$ level.

As in the case with demand creating innovations we can derive a simple equation for the process RSD intensity in the steady state:

$$
\phi_{c}=\frac{R_{c}^{\prime \prime} q_{u, a}}{P^{\prime \prime} \cdot Y^{\prime / *}}=\frac{\theta \cdot(a-1) \cdot(\theta \cdot \sigma)}{a \cdot\left(r-q_{c}-(1-\theta) \cdot \sigma\right)}
$$

The process R\&D intensity $\left(\phi_{c}\right)$ is greater when technological opportunities are larger $(\theta)$, the depreciation rate is larger $(\theta . \sigma)$, the price elasticity of demand larger (a) and the marginal opportunity costs are less $\left(r-\hat{q}_{c}-(1-\theta) \sigma\right)$.

\section{Dymannics}

The two demarcation lines which divide the $\left(A^{\prime \prime}, R_{c}^{\prime \prime}\right)$ space into four principally different areas are given by equations (3.46) and (3.47). The first demarcation line, which is given by equation (3.46), has the same general form as in section 3.1. The shape of the second demarcation line, as given by equation (3.47), is dependent on the value of the process R\&D elasticity $(\theta)$ and the price elasticity of demand $(a)$. To illustrate these statements we can simplify these two equations by collecting all constant variables and parameters in one term. Equations (3.46) and (3.47) become:

$$
\begin{aligned}
& R_{c}^{a^{\prime \prime}}=Z_{2^{2}}\left(A^{\prime \prime}\right)^{\frac{1}{\theta}} \\
& R_{i}^{p^{n}}=Z_{3}\left(A^{\prime \prime}\right)^{\frac{a-2}{\sqrt{-1}}}
\end{aligned}
$$

As in the previous case, note that the first first order condition implies that $a>1$ and that we assume $0 \leq \theta<1$. In order to obtain both loci we take the first and second derivative with respect to $A^{\prime \prime}$ : 


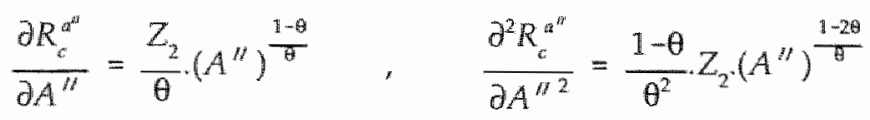

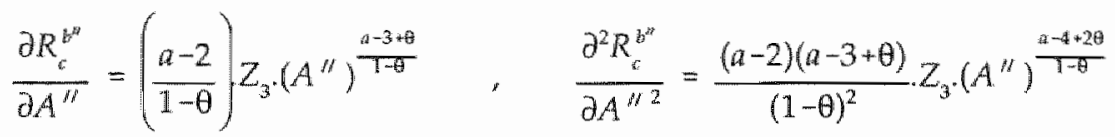

The first and second derivative of $R_{c}{ }^{4 \prime \prime}$ are positive. This means that the slope of the $d A$ " $/ d t=0$ locus is increasing at an ever increasing rate and the locus is convex. When we look at the first and second derivative of $R_{r}^{b / \prime}$ with respect to $A^{\prime \prime}$ " we can identify four different situations. We now describe the dynamics in these four situations.

\section{A. Low Price Elasticity of Denand: $1<a \leq 2$}

In this situation the first derivative of $R_{c}^{b / i}$ has a negative value and the second derivative has a positive value. This is the situation we derive in section 3.1 . The $d R_{c}^{\prime \prime} / d t=0$ has a negative slope which becomes less negative as $A$ " becomes larger. The phase diagram has already been given in figure 3.2. The steady state level is the only viable path in the long run and can only be reached by means of the unique saddlepath. The optimal decisions of a firm regarding of the amount of process $R \& D$ is given by this saddle path when the initial productivity level is not equal to the steady state level.

When $a=2$, the $d R_{c}^{\prime \prime} / d t=0$ locus is independent of $A^{\prime \prime}$. This demarcation line becomes a horizontal line at $R_{c}^{\prime \prime}=Z_{3}$. We have drawn this situation in figure 3.5 . If we draw some trajectories which fulfil the directional requirements implied by the arrows, we again see a saddlepoint configuration. In this situation, the saddle path is the $d R_{c}^{\prime \prime} / d t=0$ locus. We assume that the firm has an initial productivity level of $A_{0}^{* \prime}$. The firm will choose a constant level of discounted process R\&D which is equal to the constant discounted steady state level of process R\&D. This implies that the change in the productivity level is the same. Because the initial discounted process R\&D level of the firm is lower than the constant discounted steady state process R\&D level and the change in the productivity level is the same, the growth rate of the discounted productivity level of this firm will be larger than the constant steady state grow th rate. Its new discounted productivity level in the next period $\left(A_{1}^{\prime \prime}\right)$ is therefore located to the right of $A_{0}^{\prime \prime}$. Given $A_{1}^{\prime \prime}$, the firm will choose the same discounted process. R\&D level which results in a higher growth rate than the constant steady state growth rate. The difference with the constant steady state growth rate is less, because the initially discounted productivity level is higher. This implies that the adjustment speed slows down.

When we compare the adjustment speed between the situation with $1<a<2$ and the situation in which $a=2$, we notice that the adjustment speed is slower in the last 
situation. The reason for this is that the positive saddle path of the first situation implies a faster adjustment than the horizontal saddle path in the second situation. We can condude that a higher price elasticity of demand decreases the adjustment speed.

\section{$B_{1}$. Moderate Price Elasticity of Demand: $2<a<3-\theta$}

We illustrate the dynamics of this situation in figure 3.6 . The $d A^{\prime \prime} / d t=0$ is again convex. The first derivative of the $d R_{c}^{\prime \prime} / d t=0$ locus $\left(R_{c}^{b / l}\right)$ is positive which differs from all of the previous situations. The second derivative of $R_{c}^{b / h}$ is negative. The $d R^{\prime \prime} / d t=0$ has a decreasing positive slope and is concave (see figure 3.6 ). The economic interpretation of the positive slope of the $d R_{v}^{\prime \prime} / d t=0$ locus can be seen in equation (3.47). We noticed in section 2 that on the $d R_{c}^{\prime \prime} / d t=0$ locus the marginal costs are equal to the marginal benefits of uncreasing the state variable, which is in this case the productivity level. The left hand side of equation (3.47) shows that nothing important has changed on the cost side. The right hand side of equation (3.47), i.e. the marginal benefits, has changed. When $a>2$ an increase in the productivity level has a positive influence on the marginal benefits as opposed to the negative influence it has when $a<2$. When prices are constant, a higher productivity level implies declining marginal benefits in terms of cost savings. The marginal cost savings, $\partial T C / \partial A$, per unit of output, are declining as $A$ " increases. With an elastic demand curve a higher productivity level also implies a lower price, which results in a more than proportional increase in output. As a result, the benefits increase as the productivity level increases. Since marginal benefits, as well as marginal costs, increase as the productivity level gets higher we get a positive slope of the $d R_{c}^{\prime \prime} / d t=0$ locus. We have drawn the two demarcation loci in figure 3.6. The convex nature of $d A^{\prime \prime} / d t=0$ and the concave nature of $d R_{*}^{\prime \prime} / d t=0$ imply a positive point of intersection. This intersection point is again the steady state level in which the redefined variables $\left(A^{\prime \prime}, R_{c}^{\prime \prime}\right)$ are constant and $R_{c, t}$ and $A$, grow at a constant rate. The dynamics again imply a saddlepoint stable equilibrium. This saddlepath has a positive slope, while in all the previous saddle paths the slope was negative.

When $A_{0}^{\prime \prime}<A^{\| \prime}$, a positively sloped adjustment path implies that the discounted process R\&D level which a firm chooses is lower than the discounted steady state level of process R\&D. The productivity grow th rate is still higher than the steady state growth rate of such a firm because of its lower initial productivity level. The adjustment speed implied by a positive saddlepath is slower than the adjustment path implied by a negative or horizontal saddle path.

\section{B. Moderate Price Elasticity of Demand: $3-\theta \leq a<(1+\theta) / \theta$}

The first and second derivative of $R_{c}^{b / t}$ are positive. This implies that the amount of process $R \& D$ rises at an ever increasing rate as the productivity level increases. 

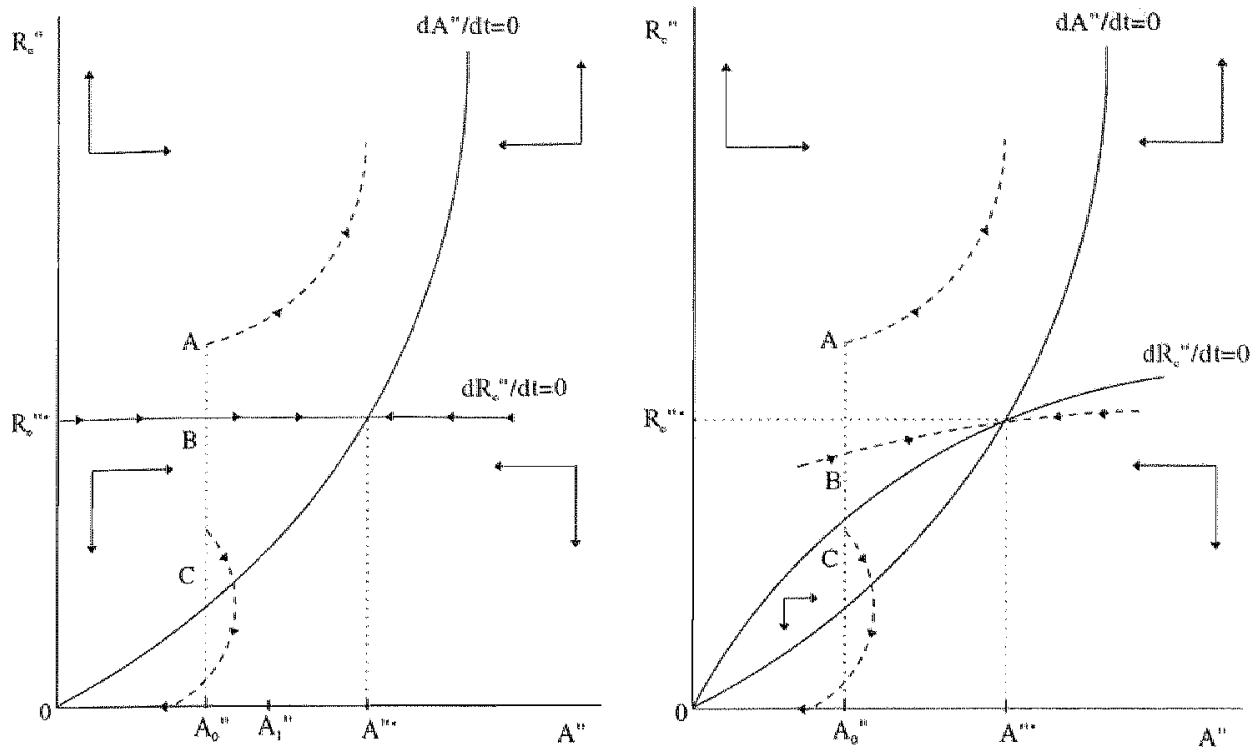

Figure 3.5. Low price elasticity of demand: $a=2$

Figure 3.6. Moderate price elasticity of demand $2<a<3 m \theta$
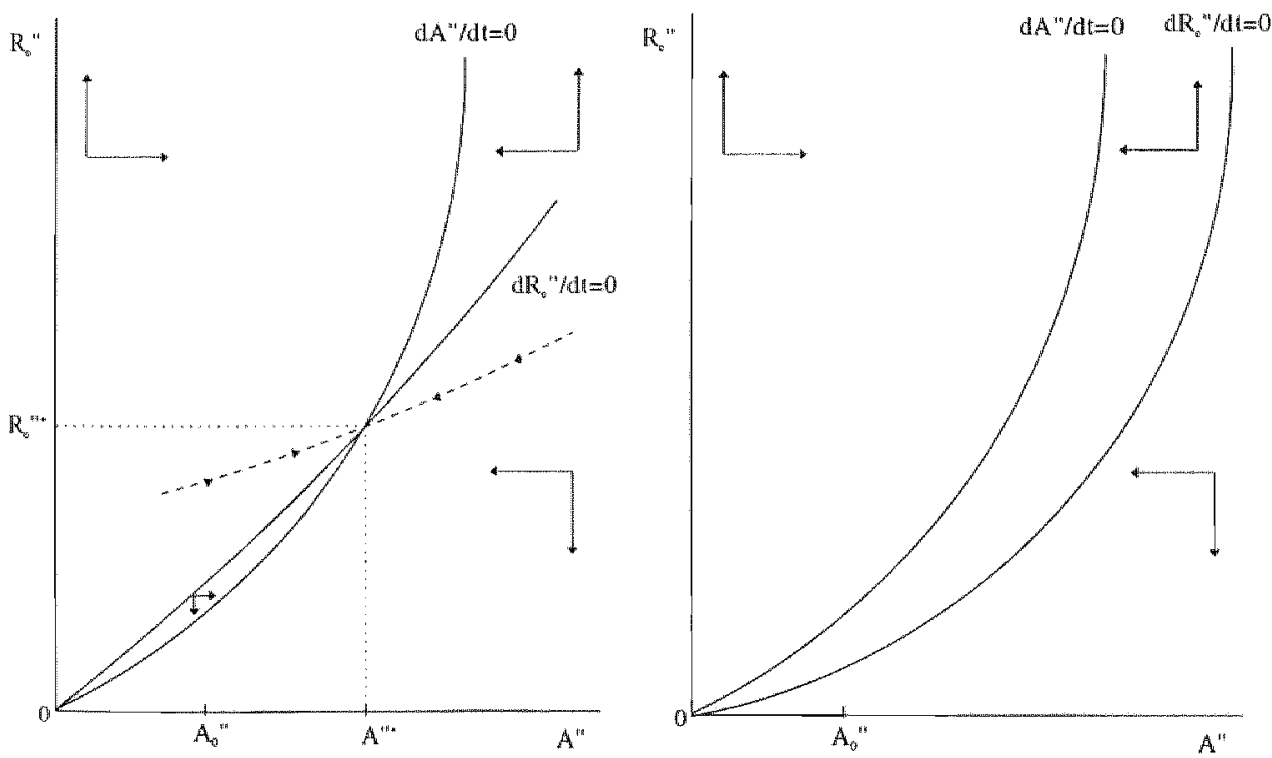

Figure 3.7. Moderate price elasticity of demand $30<a<(1+\theta) / \theta$

Figure 3.8. High price elasticity of demand $a=(1+\theta) / \theta$ 
The $d R_{c}^{\prime \prime} / d t=0$ locus is, like the $d A^{\prime \prime} / d t=0$ locus, convex. As long as the price elasticity of demand is not too high, $a<(1+\theta) / \theta$, we get a positive point of intersection in which the $d R_{c}^{\prime \prime} / d t=0$ locus intersects the $d A^{\prime \prime} / d t=0$ from above. Two demarcation lines which fulfil these conditions are drawn in figure 3.7. The directional requirements are again given by the horizontal and vertical arrows. The implied dynamics of these arrows are very similar to situation $B_{1}$.

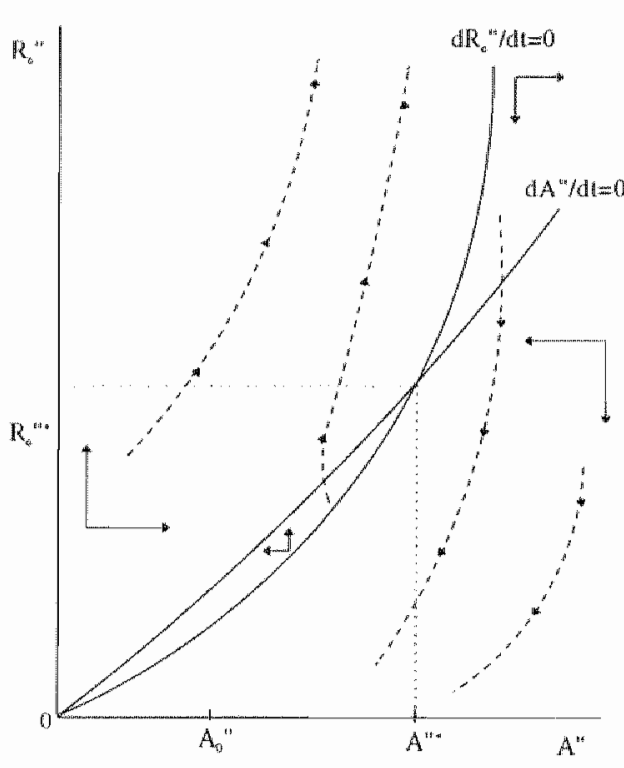

Figure 3.9. Unstable node

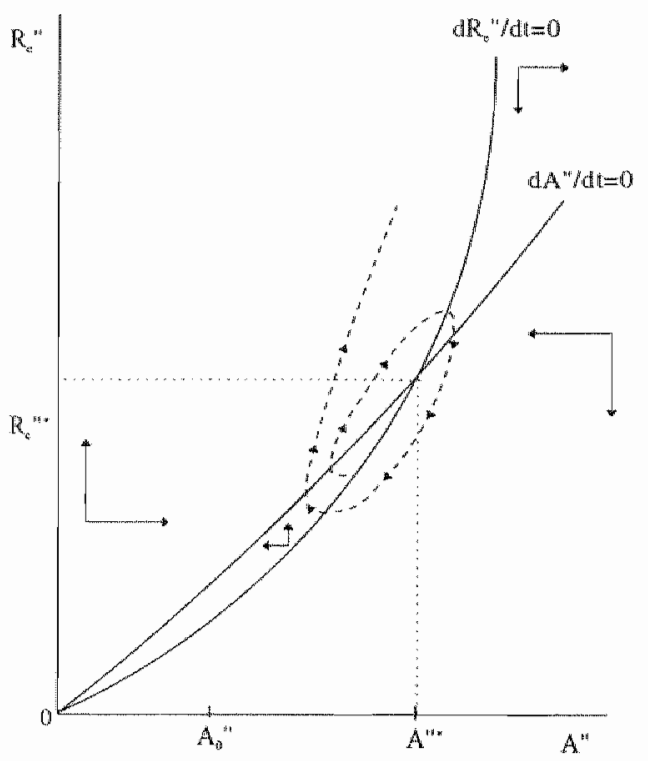

Figure 3.10. Unstable focus

\section{High Price Elastic Demand: $a \geq(1+\theta) / \theta$}

When $a=(1+\theta) / \theta$ both curves are again convex. In this special situation, illustrated in figure 3.8, there is no intersection point. The trajectories, which are drawn in accordance with the directional requirements of the arrows, show us that this system is unstable. The only point which lies on both demarcation lines is $(0,0)$. This point is not an economically viable point because the productivity level is zero at this point. When $a>(1+\theta) / \theta$, we obtain a positive intersection point, in which the $d A^{\prime \prime} / d t=0$ locus cuts the $d R_{c}^{\prime \prime} / d t=0$ from above. If we draw a phase diagram, we cannot easily define the characteristics of the equilibrium. We postpone our graphical analysis until we have checked the local stability of the steady state.

In appendix $3 B$ we checked our qualitative conclusions about the characteristics of the equilibria in the four situations. We examined the local stability around the steady state in the four situations with a linearisation of the non-linear differential system around the steady state. This analysis confirmed that the equilibria in 
situations $A, B_{1}$ and $B_{2}$ were locally saddle point stable and showed that situation $C$ is characterised by an unstable equilibrium. The latter can be an unstable node of an unstable focus. ${ }^{44}$ When the price elasticity of demand $(a)$ is not too large then the steady state is an unstable node. This situation is depicted in figure 3.9. The steady state is an unstable focus, as $a$ is very large (figure 3.10 ).

\subsection{Demand Creating and Cost Reducing R\&D}

In this section we will integrate sections 3.1 and 3.2. As in Levin and Reiss (1988), a firm can perform product $R \& D$ to enhance its perceived product quality level and can perform process $\mathbb{R} \& D$ to increase the efficiency of its production process. Our approach differs from Levin and Reiss in that we treat this problem in a dynamic setting as opposed to a static setting. In this section we allow $R \& D$ prices and technological opportunities to differ between product and process $R \& D$. We will examine the effects of exogenous variables and parameters on the amount and composition of $\mathbb{R} \& D$. Moreover, we attempt to discover whether product and process $\mathbb{R} \& D$ are complements or substitutes

By integrating the profit maximisation problems of sections 3.1 and 3.2 , the profit maximization problem for a producer becomes:

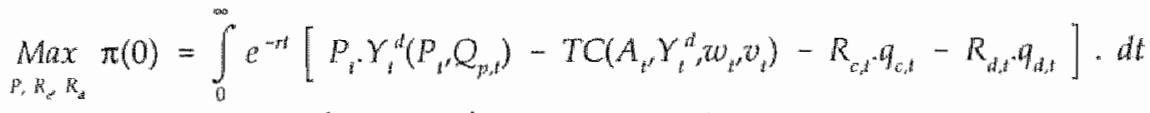

$$
\begin{aligned}
& \text { s.t. } Y_{t}^{d}=X_{0,1} Q_{p}^{b} P_{t}^{-a}, \quad 0 \leq b<1 \\
& T C_{i}=\frac{1}{A} \cdot Y_{i}^{d} \cdot w_{1}^{1-\alpha} \cdot v_{1}^{\alpha} \cdot(1-\alpha)^{\alpha-1} \cdot \alpha^{-\alpha \alpha}, \quad 0 \leq \alpha \leq 1 \\
& d A / d t=\eta \cdot R_{c, t}^{\theta}, \quad 0 \leq \theta<1 \\
& d Q_{\eta} / d t=\gamma \cdot R_{d, t}^{\delta}-w \cdot Q_{p, t}, \quad 0 \leq \delta<\mathbb{1} \\
& A_{t=0}=A_{0}, Q_{p, t=0}=Q_{p, 0}
\end{aligned}
$$

As in other sections, we assume that prices and the exogenous scale of demand have a constant growth rate. ${ }^{45}$ The current value Hamiltonian associated with this problem is:

\footnotetext{
44 A node is an equilibrim where all trajectories associated with it either flow noncyclically toward it (stable node) or flow noncyclically away from it (unstable node). A focus is an equilibrium which is characterised by whirling trajectories, all of which flow either cyclically toward it (stable focus), or flow cyclically away from it (unstable focus) (Chiang (1984), pp. 633-634).

${ }^{45}$ Notice the difference between $w$ (with a time subscript) and $w$ : the first variable is the price of labour at time $t$ and the second is the perceived quality depreciation factor.
} 


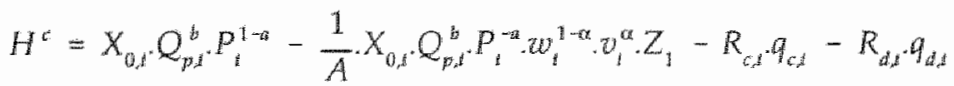

$$
\begin{aligned}
& +\mu \cdot \eta \cdot R_{c, t}^{\otimes}+\lambda \cdot\left(\gamma \cdot R_{d, j}^{\delta}-w \cdot Q_{p, t}\right)
\end{aligned}
$$

where $Z_{1}=(1-\alpha)^{\alpha-1}$. $\alpha{ }^{-\alpha}$. The technological opportunities of process $R \& D$ are given by the values of $\eta$ and $\theta$, and the technological opportunities of product R\&D are given by the values of 8 and $\gamma$. The higher the values of these parameters the higher the technological opportunities.

\section{Steady State Growoth Rates}

When we assume that the growth rates are constant in the steady state we can derive the values of these steady state rates of growth stated in equation (3.57).

$$
\begin{aligned}
& \hat{R}_{c}^{*}=\frac{1}{1-(a-1) \cdot \theta-\delta \cdot b} \cdot\left[\hat{X}_{0}-(a-1) \cdot \alpha \cdot \hat{b}-(a-1) \cdot(1-\alpha) \cdot \hat{w}-b \cdot \delta \cdot \hat{q}_{d}-(1-b \cdot \delta) \cdot \hat{q}_{c}\right] \\
& \hat{R}_{d}^{*}=\frac{\hat{X}_{0}-(a-1) \cdot \alpha \cdot \hat{\theta}-(a-1) \cdot(1-\alpha) \cdot \hat{w}-(a-1) \theta \cdot \hat{q}_{c}-(1-(a-1) \theta) \cdot \hat{q}_{d}}{1-(a-1) \cdot \theta-\delta \cdot b} \\
& \hat{A}^{*}=\theta \cdot \hat{R}_{c}^{*} \\
& \hat{Q}_{p}^{*}=\delta \cdot \hat{R}_{d}^{*} \\
& \hat{P}^{*}=\frac{-\theta \cdot \hat{X}_{0}+(1-b \cdot \delta) \cdot(\alpha \cdot \hat{\nabla}+(1-\alpha) \cdot \hat{w})+\theta \cdot b \cdot \delta \cdot \hat{q}_{c}+\theta \cdot(1-b \cdot \delta) \cdot \hat{q}_{d}}{1-(a-1) \cdot \theta-\delta \cdot b}
\end{aligned}
$$

The value of the growth rate multiplier is $1 /(1-(a-1) \cdot \theta-\delta . b)$. The value of this multiplier is higher than in the case where one can only engage in product $R \& D$ or process R\&D. The $(a-1) \cdot \theta$ term characterises the cost-reducing effect in the presence of a negatively sloped demand curve (section 3.2). The b. 8 term characterises the demand creating effect (section 3.1). These cost-reducing and demand-creation effects reduce both the denominator and therefore increase the influence of the various exogenous growth rates. The directions of the grow th rate influences the exogenous scale of demand. The various input prices on the steady growth rates of $R_{4}, R_{d *} A$, $Q$ and $P$ are the same as in previous sections.

A distinction with the previous sections is the appearance of the price growth rate of process R\&D, as well as the price growth rate of product R\&D. Note that the influence of both prices of $R \& D$ are negative on both R\&D steady state growth rates. This implies that process and product $R \& D$ are complements rather than substitutes. Notice furthermore that $\hat{R}_{c}^{*}+\hat{q}_{c}=\hat{R}_{d d^{*}}^{*}+\hat{q}_{d^{*}}$ which implies that the growth tate of the process and product R\&D budget are equal. When $\hat{q}_{c}=\hat{q}_{\text {it }}$ the steady state growth rates of process and product $\mathbb{R} \& D$ are equal because of constant elasticities everywhere.

The steady state growth rates, when a firm can only engage in one particular type of R\&D, are special cases of this situation where a firm can do both kinds of 
$R \& D$. When there are no technological opportunities to perform process $R \& D, \theta=0$, the $\hat{R}_{d}^{*}, \hat{Q}_{p}^{*}$ become identical to the steady state growth rates of section 3.1 in which we treated only product R\&D (see equation (3.15)). When there are no technological opportunities to perform product $R \& D$, i.e. $\delta=0$, the steady state growth rates of $R_{c}$ and $A$ become identical to the steady state growth rates of section 3.2 (see equation (3.42))

\section{Time Elimination Method}

First we redefine the endogenous variables, $R_{w, z^{\prime}}, R_{d, t^{\prime}} A_{t^{r}}, Q_{p, t^{\prime}}$ in the same manner as in the previous sections; we normalize them using their corresponding steady state growth rates. The two dynamic constraints in terms of the redefined variables are:

$\frac{d A^{\prime \prime}}{d t}=\eta \cdot\left(R_{c}^{\prime \prime}\right)^{\theta}-\sigma_{z} \cdot A^{\prime \prime}$

where $\sigma_{a}$ is the steady state growth rate of the productivity level.

$\frac{d Q_{p}^{\prime \prime}}{d t}=\gamma \cdot\left(R_{d}^{\prime \prime}\right)^{\delta}-\left(w+\sigma_{g}\right) \cdot Q_{p}^{\prime \prime}$

where $\sigma_{q}$ is the steady state growth rate of the quality level. The current value Hamiltonian of the redefined problem is:

$$
\begin{gathered}
H^{c}=X_{0,0}\left(P^{\prime \prime}\right)^{1-a} \cdot\left(Q_{p}^{\prime \prime}\right)^{b}-\frac{X_{0,0}}{A^{\prime \prime}} \cdot\left(P^{\prime \prime}\right)^{-a} \cdot\left(Q_{p}^{\prime \prime}\right)^{b} \cdot w_{0}^{1-\alpha} \cdot v_{0}^{\alpha} \cdot Z_{1}-R_{c}^{\prime \prime} \cdot q_{c, 0}-R_{d}^{\prime \prime} \cdot q_{d, 0} \\
+\lambda^{\prime \prime} \cdot\left(\gamma \cdot\left(R_{d}^{\prime \prime}\right)^{\delta}-\left(w o+\sigma_{p}\right) \cdot Q_{p}^{\prime \prime}\right)+\mu^{\prime \prime} \cdot\left(\eta \cdot\left(R_{c}^{\prime \prime}\right)^{\theta}-\sigma_{a} \cdot A^{\prime \prime}\right)
\end{gathered}
$$

$\sigma_{1}=\left(\hat{X}_{0}-(a-1) \cdot \alpha \cdot \hat{\theta}-(a-1) \cdot(\mathbb{1}-a) \cdot \hat{a}-b \cdot \delta \cdot \hat{q}_{d}-(1-a) \cdot \theta \cdot \hat{q}_{c}\right) /(1-(a-1) \cdot \theta-b \cdot \delta)$ is the associated discount rate. ${ }^{47} \sigma_{\mathrm{i}}$ is again the steady state growth rate of revenues, costs and both R\&D budgets. The four differential equations which characterise this system can again be calculated from the first order conditions:

$\frac{d R_{d}^{\prime \prime}}{d t}=\phi_{1} \cdot\left(R_{d}^{\prime \prime}\right)^{\delta} \cdot\left(\phi_{2} \cdot\left(R_{d d}^{\prime \prime}\right)^{1-\delta}-\phi_{3} \cdot\left(Q_{p}^{\prime \prime}\right)^{b-1} \cdot\left(A^{\prime \prime}\right)^{a-1}\right)$

\footnotetext{
46 Note that $C=\alpha .0+(1-\alpha) \cdot \hat{x}$.

47 Similarities with the discount factors in other sections, after the application of the time elimination method is apparent when one notes that $\sigma_{1}=q_{c}+R_{c}^{\prime \prime}=\hat{q}_{d}+R_{d t}^{d /}$.
} 


$$
\frac{d R_{c}^{\prime \prime}}{d t}=\phi_{4}\left(R_{c}^{\prime \prime}\right)^{\theta} \cdot\left(\phi_{5^{n}}\left(R_{c}^{\prime \prime}\right)^{3-\theta}-\phi_{6} \cdot\left(Q_{y}^{\prime \prime}\right)^{b} \cdot\left(A^{\prime \prime}\right)^{a-2}\right)
$$

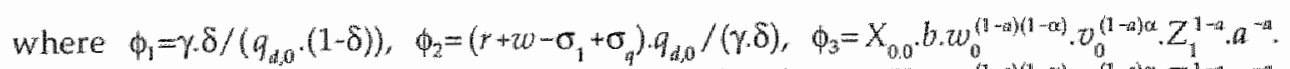

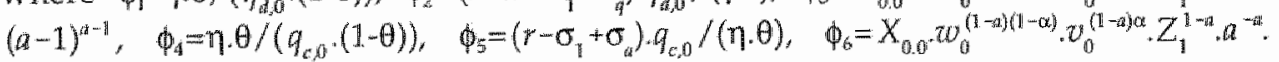
$(a-1)^{-a}$. The $d A^{\prime \prime} / d t$ and $d Q_{p}^{\prime \prime} / d t$ differential equations are given by equations (3.58) and (3.59) respectively.

\section{Steady State}

When we put $d A^{\prime \prime} / d t, d Q_{j p}^{\prime \prime} / d t, d R_{c}^{\prime \prime} / d t$ and $d R_{d t}^{\prime \prime} / d t$ equal to zero in the four differential equations and solve for $R_{c, t}^{\prime \prime}, R_{d,}^{\prime \prime}, A_{t}^{\prime \prime}, Q_{p, t}^{\prime \prime}$ we get the following steady state values:

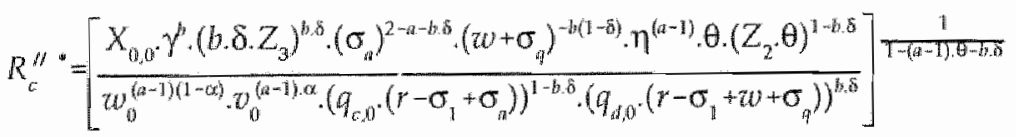

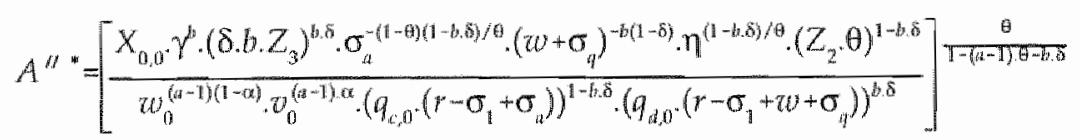

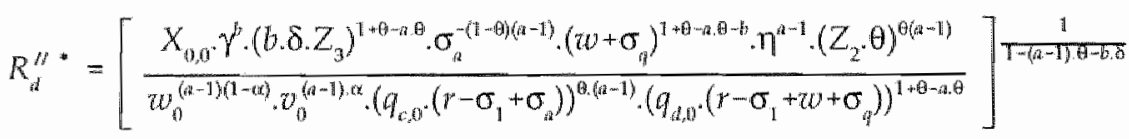

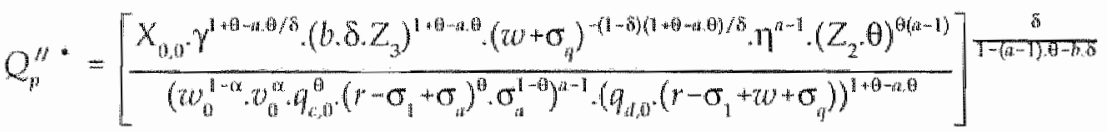

The denominator of the general exponent of all the steady state values again contains the cost-reducing $((a-1) \cdot \theta)$ and denand-creation (b. $\delta)$ effects. In comparison with the previous analyses, where these effects were treated separately, the value of the exponent has increased. The result is that the effects of exogenous variables and parameters are more pronounced.

In general the direction of the influences of the various parameters and exogenous variables, on the steady state levels, is the same as in the cases in which product and process R\&D are treated separately. The exogenous scale of demand has a positive influence on the steady state level, while the various costs variables have a negative influence on the steady state level. The productivity parameter and R\&D elasticity of the productivity (perceived quality) generation process again have 
a positive influence on the steady state productivity (perceived quality) level, and also on the steady state perceived quality (productivity) level. Factors which favour the productivity (quality) level also have a positive influence on the quality (productivity) level. Product and process R\&D are again complements instead of substitutes. We illustrate the intuition behind this result with a few examples. On the one hand, a higher perceived quality level increases the demand for the firms product. Cost-savings per unit of output remain constant but a higher output level implies an increase in the total cost-savings (benefits) of process $R \& D$ activities. Factors which favour perceived quality therefore also favour productivily. Factors which increase the productivity level on the other hand, reduce costs per unit of output which results in a lower price level. This lower price level implies a higher marginal increase in demand as $Q_{p}^{\prime \prime}$ increases. This justifies a higher level of product R\&D. Factors which favour the productivity level therefore also favour the perceived quality level.

The R\&D intensities of product and process R\&D in the steady state are similar to those intensities in sections 3.1 and 3.2 (respectively equation (3.33)) and equation (3.50)), the only difference is the changed steady state growth rates of $A$ and $Q_{p}$.

\section{Dynamics}

The dynamics of the system in which demand-creating and cost-reducing innovations are possible is characterised by four non-linear differential equations. To draw the dynamics of $R_{c, t}^{\prime \prime}, R_{d, t}^{\prime \prime}, A_{i}^{\prime \prime}$ and $Q_{p, t}^{\prime \prime}$ we need a four-dimensional space. Because this is not possible we will clarify the dynamics in two twodimensional phase diagrams to get a qualitative impression of the dynamic behaviour of the system.

First, we describe the dymamics of process $\mathbb{R} \& D$ and productivity in the ( $A$ " $\left.R^{\prime \prime}\right)$ space. The two demarcation lines, $d A^{\prime \prime} / d t=0$ and $d R_{v}^{\prime \prime} / d t=0$, can be calculated from equations $(3.58)$ and $(3.62)$ :

$$
\begin{aligned}
& \frac{d A^{\prime \prime}}{d t}=0 \Rightarrow R_{c}^{\prime \prime}=\left(\frac{\sigma_{t}}{\eta}\right)^{\frac{1}{\theta}} \cdot\left(A^{\prime \prime}\right)^{\frac{1}{\theta}} \\
& \frac{d R_{c}^{\prime \prime}}{d t}=0 \Rightarrow R_{c}^{\prime \prime}=\left(\frac{\phi_{b}}{\phi_{5}}\right)^{\frac{1}{1-\theta}} \cdot\left(Q_{p}^{\prime \prime}\right)^{\frac{1}{1-\theta}} \cdot\left(A^{\prime \prime}\right)^{\frac{1-\alpha 2}{1-\theta}}
\end{aligned}
$$

The only change to this system, in comparison with the "pure" process $\mathbb{R} \& D$ case of section 3.2, is the occurrence of the perceived quality level in equation (3.68). We first assume that the perceived quality level is constant. We then have the same situation as described in section 3.2 where the dynamics are described by the phase diagrams (3.5-3.10). The system is saddle point stable as long as the price ellasticity 
of demand is $1<a<(1+\theta) / \theta$ and unstable as $a>(1+\theta) / \theta$. If the value of $Q_{p}$ changes we see a corresponding shift in the $d R_{c}^{\prime \prime} / d t=0$ locus. This locus moves upwards (downwards) as the perceived quality level is higher (lower). These movements have no influence on the stability characteristics. The $d R_{c}^{\prime \prime} / d t=0$ locus shifts because on this locus the marginal costs of process $R \& D$ activities are equal to the marginal benefits of product $\mathbb{R} \& \mathrm{D}$ activities. A higher (lower) perceived quality level increases (decreases) the benefits while costs stay the same, which results in an upward (downwatd) shift of the $d R_{c}^{n t} / d t=0$ locus.

Second, we will describe the dynamics of product R\&D and perceived quality in the $\left(Q_{p}^{\prime \prime}, R_{d}^{\prime \prime}\right)$ space. Equation (3.69) and (3.71) give us the two demarcation lines, $\left(d Q_{p}^{\prime \prime} / d t\right)=0$ and $\left(d R_{t}^{\prime \prime} / d t\right)=0$ which divides this area into four parts:

$$
\begin{aligned}
& \frac{d Q_{p}^{\prime \prime}}{d t}=0 \Rightarrow R_{d}^{\prime \prime}=\left(\frac{w+\sigma_{a}}{\gamma}\right)^{\frac{1}{\delta}} \cdot\left(Q_{p}^{\prime \prime}\right)^{\frac{1}{b}} \\
& \frac{d R_{d}^{\prime \prime}}{d t}=0 \Rightarrow R_{d \prime}^{\prime \prime}=\left(\frac{\phi_{3}}{\phi_{2}}\right)^{\frac{1}{1-\delta}} \cdot\left(Q_{p}^{\prime \prime}\right)^{\frac{b-1}{1-\theta}} \cdot\left(A^{\prime \prime}\right)^{\frac{a-1}{1-\delta}}
\end{aligned}
$$

This system is similar to the system where one can only engage in product R\&D. The principal difference is the occurrence of the endogenous productivity level in the $\left(d R_{d}^{\prime \prime} / d t\right)=0$ demarcation line. If this productivity level is constant then we are back to the section 3.1 situation where the system is saddle point stable. A higher (lower) productivity level shifts the $\left(d R_{d i}^{\prime \prime} / d t\right)=0$ locus upwards (downwards) which does not influence the stability properties.

We now integrate these two parts and examine the dynamics when $A^{\prime \prime}$ and $Q_{p}^{\prime \prime}$ both change. We assume that the initially perceived quality level and productivity level are smaller than the steady state values; $A_{0}^{\prime \prime}<A^{\prime \prime}$ " and $Q_{p, 0}^{\prime \prime}<Q_{p}^{\prime \prime}$. Furthermore we assume that $1<a<(1+\theta) / \theta$ which implies that the first system is saddle point stable. In phase diagram 3.11 we have drawn the demarcation loci of the first system. The $d A^{\prime \prime} / d t=0$ locus is independent of the perceived quality level and the $d R^{\prime \prime} / d t=0$ locus depends on the perceived quality level. At time $t=0$, the initially perceived quality level is equal to $Q_{p b}^{\prime \prime}$ for the firm. The $\left(d R_{c}^{\prime \prime} / d t\right)_{0}=0$ locus depends on this level. We get a steady state which is located at point $E_{0}$ and an associated saddlepath $s_{0}$. Given its initial productivity level $\left(A_{0}^{\prime \prime}\right)$, the firm will choose the process $R \& D$ level of $R_{v, 0}^{\prime \prime}$ on this saddle path.

In phase diagram 3.12 we have drawn the demarcation loci of the second system. Only the $\left(d R^{\prime \prime} / d t\right)=0$ locus is dependent on the productivity level. The $\left(d R_{d}^{\prime \prime} / d t\right)_{0}=0$ locus is drawn dependent on the initially given productivity level $A_{0}^{\prime \prime}$. The steady state is located at point $E_{0}^{\prime}$ and the accompanying saddle path is $s_{0}^{\prime}$. A firm with an initially perceived quality level of $Q_{p, 0}^{\prime \prime}$ will choose the product R\&D level of $R_{d, k)}^{1 / 2}$. 


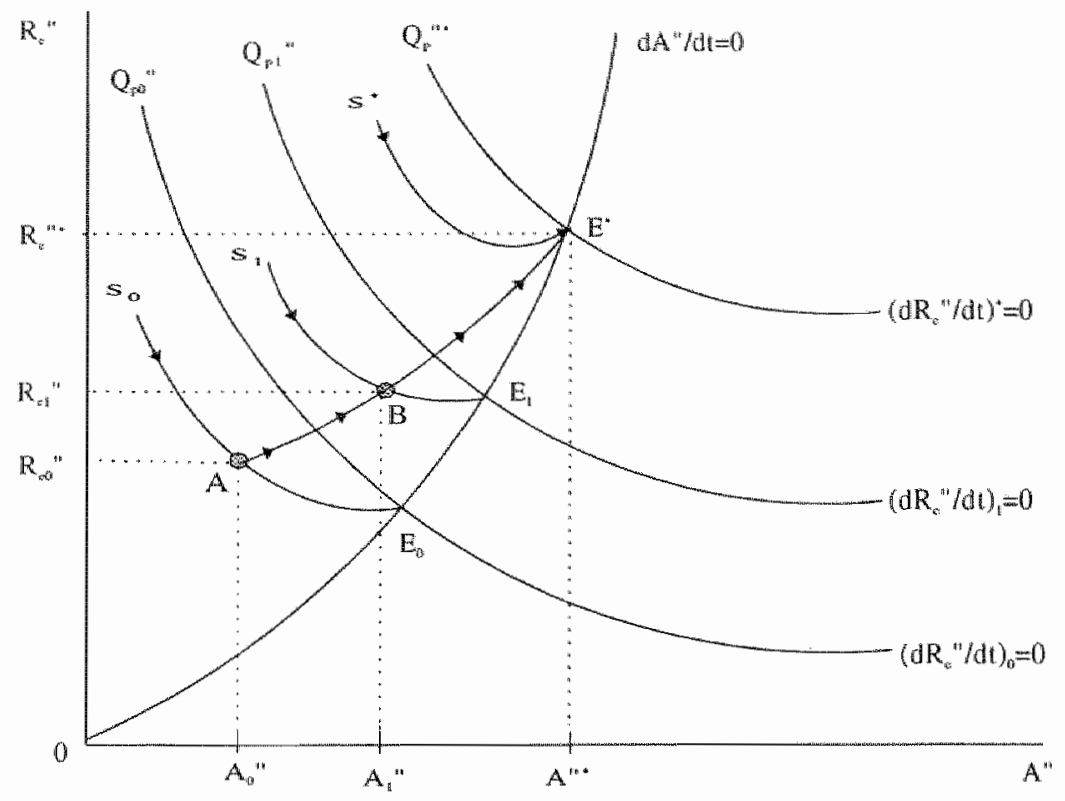

Figure 3.11. Productivity generation process

The firm has chosen its product and process R\&D level at time $t=0$. Given these values we can calculate the firm's new productivity level and perceived quality level (approximately):

$A_{1}^{\prime \prime}=A_{0}^{\prime \prime}+\eta \cdot\left(R_{c, 0}^{\prime \prime}\right)^{\theta}-\sigma_{a} \cdot A_{0}^{\prime \prime}$

$Q_{p, i}^{\prime \prime}=Q_{p, 0}^{i f}+\gamma \cdot\left(R_{d, 0}^{\prime \prime}\right)^{\delta}-\left(w+\sigma_{q}\right) \cdot Q_{p, 0}^{\prime \prime}$

The new productivity level $\left(A_{1}^{\prime \prime}\right)$ and the new perceived quality level $\left(Q_{\mu, 1}^{\prime \prime}\right)$ have both increased. The higher perceived quality level $\left(Q_{p, 1}^{\prime \prime}\right)$ shifts the $d R_{c}^{\prime \prime} / d t=0$ locus upwards to $\left(d R_{c}^{\prime \prime} / d t\right)_{1}=0$ in figure 3.11. The steady state equilibrium shifts from $E_{0}$ to $E_{1}$. A higher perceived quality level implies a higher steady state productivity level. The higher productivity level $\left(A_{1}^{\prime \prime}\right)$ shifts the $d R_{d}^{\prime \prime} / d t=0$ locus upwards to $\left(d R_{d d}^{\prime \prime} / d t\right)_{1}=0$ in figure 3.12. The steady state equilibrium shifts from $E_{0}^{\prime}$ to $E_{1}^{\prime} \cdot A$ higher initial productivity level results in a higher steady state perceived quality level. This analysis again shows that process and product $R \& D$ are complements instead of substitutes. Given the new productivity level $\left(A_{1}^{\prime \prime}\right)$ the firm will choose the amount of process $R \& D, R_{c, 1}^{\prime \prime}$ which is located on the new saddlepath $s_{\|}$in figure 3.11. Given its new perceived quality level $\left(Q_{p, 1}^{\prime \prime}\right)$ the firm will choose the level of product $R \& D, R_{d, 1}^{\prime \prime}$ on the new saddle path $s_{1}^{\prime}$ in figure 3.12. With these new product and process $R \& D$ levels we can calculate the new productivity and 


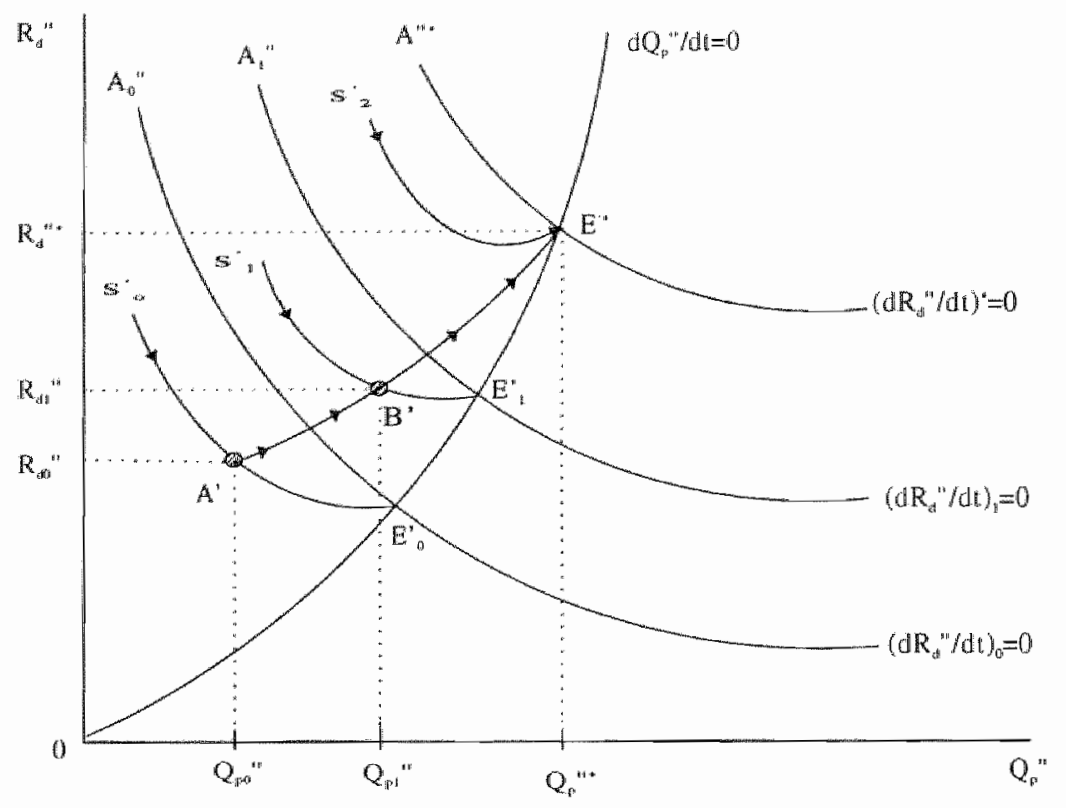

Figure 3.12. Perceived quality generation process

perceived quality levels, $Q_{p, 2}^{\prime \prime}$ and $A_{2}^{\prime \prime}$. The process repeats itself and continues until the steady state values $\left(Q_{p}^{\prime \prime}\right.$ and $A^{\prime \prime}$ ) ) are approached. When the productivity level and perceived quality level of the firm are equal to the steady state values, the firm will choose the process and product R\&D levels which are just enough to maintain the current perceived quality and productivity levels. The $d R_{c}^{\prime \prime} / d t=0$ and $d R_{d \prime}^{f l} / d t=0$ will not continue to shift and the firm will choose $R_{d}^{\prime \prime}$ * and $R_{v}^{\prime \prime}$ from now on. We have reached the steady state $\left(Q_{p}^{\prime \prime}, A^{\prime \prime *}\right)$ in which the discounted values of $Q_{p}$, and $A$ are constant and $Q_{p, t}$ and $A_{f}$ grow at a constant rate. When the initial productivity and perceived quality level are not equal to these steady state values we get a long-run adjustment path for the amount of process $R \& D$ and product $R \& D$ which is given by $A B E^{*}$ in figure 3.11 and $A^{\prime} B^{\prime} E^{\prime *}$ in figure 3.12 respectively.

We again examine the validity of the saddle point steady state property of this four dimensional differential equation system by a linearisation of the nonlinear differential-equation system around the steady state. Feichtinger and Hartl (1986) describe the conditions for saddle point stability of an optimal control system with two state and co-state variables. ${ }^{\$ 8}$ The system is saddle point stable when two characteristic roots are real and negative and the other two characteristic roots are

48 For details on the stability of a non-linear differential system with more than one state variable see Feichtinger and Hartl (1986), pp. 122-154. 
real and positive. In this case the system has a saddlepoint plane. When the two initial conditions $A_{0}{ }^{\prime \prime}$ and $Q_{p, 0}$ " are known, the beginning point of the unique dynamic path in this saddlepoint plane can be determined. We checked the conditions of Feichtinger and Hartl for a saddle point plane in appendix $3 \mathrm{C}_{2}$ and concluded that this system is saddle point stable when $1>(a-1) \cdot \theta+b . \delta$. So, when the growth rate multiplier is positive the system is saddle point stable.

\subsection{Conclusion}

In this chapter we developed an endogenous technological change model. Firms "invested" in technological change to obtain benefits in the future. Technological change showed up through productivity improvements or an enhanced quality level. The amount of technology investments turned out to be dependent on technological opportunities, demand characteristics and opportunity costs. Current R\&D expenditures have to be compared to future profits. We used optimal control theory to solve this problem because this method can deal with the inter-temporal benefits which characterise the technology generation process.

We determined the optimal amount of product and process R\&D chosen by a firm given its perceived quality and productivity level. We used a time elimination method to analyze prices with an arbitrary constant growth rate. The analyses showed that the various systems are characterised by saddle point stability. A unique saddle path exists which "prescribes" the optimal choices of the amount of product and/or process $R \& D$. The system will only become unstable when the growth rate multiplier is negative.

In general, technological progress (innovations) is favoured by factors which increase demand or by factors which increase the efficiency of the perceived quality or productivity generation process. Factors which temper technological progress are the wage rate and the user cost of capital if the price elasticity of demand is relatively high. However, these factors favour technological progress when demand is totally price inelastic. The price of $R \& D$ tempers technological progress independently of the price elasticity of demand.

An interesting result is that the R\&D intensities turned out to be constant and only dependent on a few structural parameters. Again high technological opportunities and favourable demand conditions (e.g. high quality elasticity of demand) lead to high $R \& D$ intensity. The Schumpeterian idea that rewards associated with private innovative efforts are not everlasting was reflected in our "perceived" quality concept. It turned out that vigourous quality competition, reflected in a faster depreciation of a firms" perceived quality, increases the product $R \& \mathbb{D}$ intensity.

Product and process R\&D turn out to be complements rather than substitutes in our analysis because they reinforce each other, while the total R\&D budget can be freely chosen. 
An elaboration of this analysis would include the effect of perceived quality on unit costs. A higher quality level of a product can only be obtained by increasing unit production costs. A profit maximizing firm that considers engaging in product R\&D to raise its quality level must therefore not only consider an increase in expected demand but also an increase in unit production costs. This extension of the model will be included in chapters 4 and 5 .

Other elaborations of this backbone model are the fourth to sixth element of an endogenous technological change model which we neglected in this chapter. In chapter 4 we discuss the special features of a General Purpose Technology and include the feature that a firm can use knowledge generated by other firms or sectors. 


\section{Appendix 3A. Checking the Saddle Point of Section 3.1 by Characteristic Roots}

The configuration of arrows in figure 3.2 leads us to conclude that the equilibrium at $\left(Q^{*}, R^{\prime \prime}\right)$ is a saddle point. This characteristic can be examined by a linearisation (first order Taylor expansion) of the nonlinear differential-equation system near the steady state. ${ }^{49}$

To keep this analysis as straight forward as possible, we simplify the two differential equations (3.25) and (3.28) somewhat:

$Q_{p}^{\prime \prime}=\frac{d Q_{p}^{\prime \prime}}{d t}=\gamma \cdot\left(\mathbb{R}_{d}^{u \prime}\right)^{\delta}-w^{\prime} \cdot Q_{p}^{\prime \prime}$

where $w^{\prime}=w+\delta . \sigma$

$\dot{R}_{d}^{\prime \prime}=\frac{d R_{d}^{d /}}{d t}=\phi_{1} \cdot\left(R_{d}^{\prime \prime}\right)^{b} \cdot\left(\phi_{2^{n}}\left(R_{d}^{\prime \prime}\right)^{1-\delta}-\phi_{3} \cdot\left(Q_{p}^{\prime \prime}\right)^{3-1}\right)$

where $\phi_{1}=\gamma \cdot \delta /\left(q_{a, 0} \cdot(1-\delta)\right), \phi_{2}=\left(r+w-q_{a}-(1-\delta) \cdot \sigma\right) \cdot q_{d, \delta} /(\gamma \cdot \delta)$ and $\phi_{3}=C_{0} \cdot X_{0,0} \cdot b \cdot\left(P^{\prime \prime}\right)^{-\alpha /} /(a-1)$. We first construct the Jacobean matrix and evaluate it at the steady-state point $\left(Q_{p}^{\prime \prime}, R_{*}^{\prime *}\right)$.

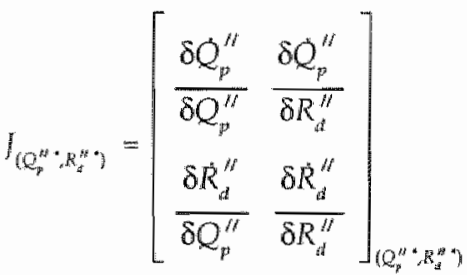

The four partial derivatives evaluated at $\left(Q_{p}^{\prime *}, R_{d}^{\prime \prime *}\right)$ are:

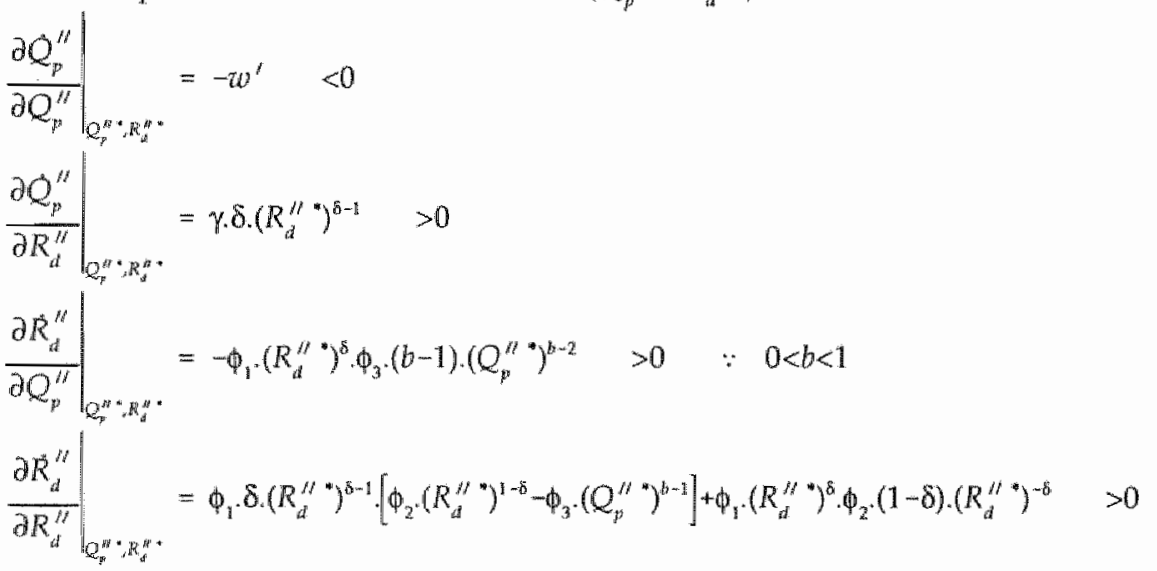

Note that the term between the brackets in the last equation is equal to zero in the steady state. The Jacobean matrix evaluated at $\left(Q_{p}^{\prime \prime}{ }^{*}, R_{i j}^{f \prime *}\right)$ is:

$f_{Q_{n}^{*} \cdot R_{x}^{\prime \prime} \cdot}=\left[\begin{array}{ll}<0 & >0 \\ >0 & >0\end{array}\right]$

To check the dynamic stability of the equilibrium, we have to know the signs of the two characteristic roots, $r_{1}$ and $r_{2}$. A saddle point stable equilibrium implies that the two roots

49 For more details of the procedure of linearization of a non-linear system, see A.C. Chiang (1984), section 18.6 . 
have opposite signs. Important information in this context can be derived from the determinant of the Jacobean matrix.

$r_{1} \cdot r_{2}=\left|q_{t}^{a} ; x_{z}^{\prime \prime}\right|=(-)(+)-(+)(+)<0$

The determinant of the Jacobean matrix is negative, which implies that the two characteristic roots have opposite signs. This immediately enables us to conclude that the equilibrium $\left(Q_{p}^{\prime \prime} * R_{d}^{\prime \prime}\right)$ is locally a saddle point.

\section{Appendix 3B. Checking the Saddle Points of Section 3.2 by Characteristic Roots}

The configuration of arrows in the various situations lead us to conclude that when $1<a<(1+\theta) / \theta$ we have a saddle point equilibrium and when $a>(1+\theta) / \theta$ the stability characteristics are not clear. We can examine the validity of these conclusions by examining the llocal stability characteristics around the steady state. We will again examine this using a linearisation of the nonlinear differential-equation system (Chiang 1984). We first simplify the two diflerential equations:

$$
\begin{aligned}
& A=\frac{d A^{\prime \prime}}{d t}=\eta \cdot\left(R_{c}^{\prime \prime}\right)^{\theta}-\theta \cdot \sigma^{\prime \prime} \cdot A^{\prime \prime} \\
& \dot{R}^{\prime \prime}=\frac{d R_{E}^{\prime \prime}}{d t}=\phi_{1} \cdot\left(R_{i}^{\prime \prime}\right)^{0} \cdot\left(\phi_{2} \cdot\left(R_{t}^{\prime \prime}\right)^{1-0}-\phi_{3} \cdot\left(A^{\prime \prime}\right)^{a-2}\right)
\end{aligned}
$$

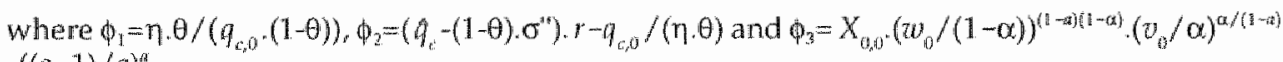
$((a-1) / a)^{d}$.

The Jacobean matrix evaluated at the steady-state point (E) is:

$$
I_{E}=\left[\begin{array}{cc}
-\theta \cdot \sigma^{\prime \prime} & \eta \cdot \theta \cdot\left(R_{c}^{\prime \prime *}\right)^{\beta-1} \\
-(a-2) \cdot \phi_{1} \cdot \phi_{3} \cdot\left(R_{c}^{\prime \prime}\right)^{\theta} \cdot\left(A^{*}\right)^{a-3} & \phi_{1} \cdot \phi_{2} \cdot(1-\theta)
\end{array}\right]
$$

The determinant of the Jacobean matrix becomes:

$$
r_{1} \cdot r_{2}=\left|I_{A^{\prime \prime}}, R_{2}^{\prime \prime}\right|=-\theta \cdot \sigma^{\prime \prime} \cdot \phi_{1} \cdot \phi_{2} \cdot(1-\theta)+(a-2) \cdot \eta \cdot \theta \cdot \phi_{1} \cdot \phi_{3} \cdot 2 \cdot\left(R_{c}^{\prime \prime \prime}\right)^{20-1} \cdot\left(A^{\prime \prime}\right)^{a-3}
$$

The value of the determinant of the Jacobeam matrix is dependent on the value of the price elasticity of demand. When $1<n \leq 2$ we can immediately conclude that the system is saddlcpoint stalle because the value of the determinant is negative. This werifies our conclusion in situation A. To examine the local stability around the steady state when a>2 we have to compute the sign of the determinant of the Jacobean matrix. We can simplity the determinant to:

$$
r_{1} \cdot r_{2}=\theta \cdot \sigma^{\prime \prime} \cdot \phi_{1} \cdot \phi_{2} \cdot(a \theta-1-\theta)
$$

The determinant of the lacobean matrix is negative as $a<(1+\theta) / \theta$ and positive as $a>(1+\theta) / \theta$. With a negative determinant the only possibility is again that the steady state is locally saddle point stable. This confirms our conclusion in situation $B_{1}$ and $B_{2}$ that the steady state is a saddle point if $a<(1+\theta) / \theta$. When the determinant is positive we are not able to make this inference directly about the local stability of the system. ${ }^{51}$ in order to make

51) We have used the fact that in the steady state the following two equalities should hold:

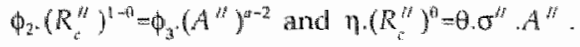

51 see A. C. Chiang (1984), p. 643, table 18.1. 
this inference we must calculate the trace of the Jacobean matrix (Ir $\int_{\mathbb{E}}$ ) ${ }^{52}$ The trace of this Jacobean matrix is:

$$
r_{1}+r_{2}=t r I_{E}=-\theta \cdot \sigma^{\prime \prime}+\phi_{1} \cdot(1-\theta)=r-q-\sigma^{\prime \prime}>0
$$

where $P q+\sigma^{\prime \prime}$ if the transwersality condition is satisfied. Relevant for the ability to make inference is the positive sign of the trace of the Jacobean. A positive determinant in combination with a positive trace implies an unstable equilibrium, which clarifies our discussion in situation $C$. Whether we are dealing with an unstable node or with an unstable focus depends on the values of the paramelers and exogenous growth rates. ${ }^{53}$

$$
\left(t r I_{\mathbb{E}}\right)^{2} \geq 4 \cdot\left|J_{E}\right|, \quad\left(r-\hat{q}-\sigma^{\prime \prime}\right)^{2} \geq 4 \cdot\left(\frac{\left(r-\hat{q}-(1-\theta) \cdot \sigma^{\prime \prime}\right) \cdot \theta \cdot \sigma^{\prime \prime}}{1-\theta}\right) \cdot(a-\theta-\theta-1)
$$

If this condition holds, for example when $a$ is slightly larger than $(\theta+1) / \theta$, we have an unstable node. When this condition is not satisfied we have an unstable focus.

\section{Appendix 3C. Checking the Saddle Point of Section 3.3 by Characteristic Roots}

Feichtinger and Hartl state that the general condition for a saddle point plane without loops is characterised by the following conditions: ${ }^{54}$
1) $\operatorname{det} I>0$
2) $K<0$
3) $0<\operatorname{det} \leq K^{2} / 4$

First, we have to compute the determinant of the Jacobean matrix evaluated at the steadystate point (E):

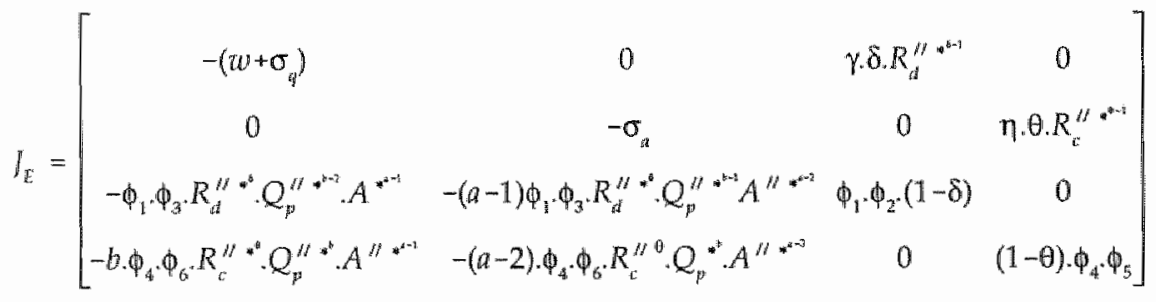

The determinant of the Jacobean matrix can be simplified to:

$\|=\phi_{1} \cdot \phi_{2} \cdot \phi_{4} \cdot \phi_{5} \cdot \sigma_{i} \cdot \sigma_{i j}[1-(a-1) \cdot \theta-b . \delta]$

The value of the determinant is positive when $1>(a-1), \theta+b .8$. This expression is equal to the denominator of the grow th rate multiplier (see equation $(3.57)$ ) and the denomirator of the general exponent of all steady state values (see e.g. equation (3.63)).

Second, we have to calculate the value of the following matrix $K$ :

52 The trace of $f_{E}$, symbolised by $t w J_{\mathbb{E}}$, is the sum of the principal-diagonal elements of the Jacobean. There is a simple relationship between the trace of $f_{R}$ and the characteristic roots: $r_{1}+r_{2}=$ ir I (Chiang 1984, p, 641).

53 A node is an equilibrium such that all the trajectories associated with it either flow noncyclically toward it (stable node) or flow noncyclically away from it (unstable node). A focus is an equilibrium which is characterised by whirling trajectories, all of which either flow cyclically toward it (stable focus), or flow cyclically away from it (unstable focus) (Chiang 1984, pp. 633-634).

${ }^{54}$ For details on the stability of a non-linear differential system with more than one state variable see Feichtinger and Hartl (1986), pp. 122-154. The matrix $K$ is defined at the end of this appendix. 


$$
K=\left[\begin{array}{cc}
\frac{\delta Q_{p}^{\prime \prime}}{\delta Q_{p}^{\prime \prime}} & \frac{\delta Q_{p}^{\prime \prime}}{\delta R_{d}^{\prime \prime}} \\
\frac{\delta R_{d}^{\prime \prime}}{\delta Q_{p}^{\prime \prime}} & \frac{\delta R_{d}^{\prime \prime}}{\delta R_{d}^{\prime \prime}}
\end{array}\right]+\left[\begin{array}{cc}
\frac{\delta A^{\prime \prime}}{\delta A^{\prime \prime}} & \frac{\delta A^{\prime \prime}}{\delta R_{c}^{\prime \prime}} \\
\frac{\delta R_{c}^{\prime \prime}}{\delta A^{\prime \prime}} & \frac{\delta R_{c}^{\prime \prime}}{\delta R_{c}^{\prime \prime}}
\end{array}\right]+2 \cdot\left[\begin{array}{cc}
\frac{\delta Q_{p}^{\prime \prime}}{\delta A^{\prime \prime}} & \frac{\delta \dot{Q}_{y}^{\prime \prime}}{\delta R_{c}^{\prime \prime}} \\
\frac{\delta R_{d}^{\prime \prime}}{\delta A^{\prime \prime}} & \frac{\delta R_{d}^{\prime \prime}}{\delta R_{c}^{\prime \prime}}
\end{array}\right]
$$

From the first matrix, on the right hand side, we calculate the determinant of this "demand creating sub-system" which turns out to be negative. From the second matrix, on the right hand side, we calculate the value of the determinant of this "cost-reducing sub-system" which has a negative value as long as $a<(\theta+1) / \theta$. The determinant of the third matrix, which describes cross influences is zero. Overall we can conclude that the second condition, a negative value for $K$, is met when the first condition is met. The third condition, $0<\operatorname{det} \int \leq \mathrm{K}^{2} / 4$ is also satisfied if the first condition is met. Hence, we can conclude that this system is saddle point stable when $1>(a-1) \cdot \theta+b . \delta$. 


\section{General Purpose Technologies and Technological Change}

In this chapter we introduce the influence of a general purpose technology on technological change and introduce the public properties of knowledge in the backbone model. As discussed in chapters 1 and 2 a general purpose technology differs from other technologies because it is pervasive, is characterised by high technological opportunities, and induces innovative efforts in many other sectors. These three characteristics may imply that the general purpose technology can create growth across the whole economy. Information technology (T) can be considered the current general purpose technology. We will investigate the special features of IT in this chapter and extend the endogenous technological change model developed in chapter 3 with these features.

In addition to the influences of IT and spillovers on technological progress, we also investigate the influence of the degree of quality and price competition in domestic and foreign markets on technological progress. An interesting question in this context is whether or not a competitive foreign market or sophisticated domestic demand stimulates technological progress.

In section 4.1 we discuss the direct and indirect influences of IT on technological progress. Section 4.2 elaborates the backbone model developed in chapter 3 with the cost-reducing and demand-creating role of information technology. Section 4.3 investigates the influence of market segmentation.

\subsection{Information Technology and Technological Progress}

The general purpose of IT goods is to process information (inclusive telecommunication). The role of IT investment goods (such as computers, CAM, fax, telephones, etc.) in the production process differs, therefore, from the role of other capital goods because IT investment goods are used to smooth the process of production while other capital goods are used together with labour to produce output (Van Zon 1991). The IT capital stock delivers services which therefore directly influence the efficiency of the production process. Therefore, IT goods possess an explicit cost-reducing role in the production process. However, IT 


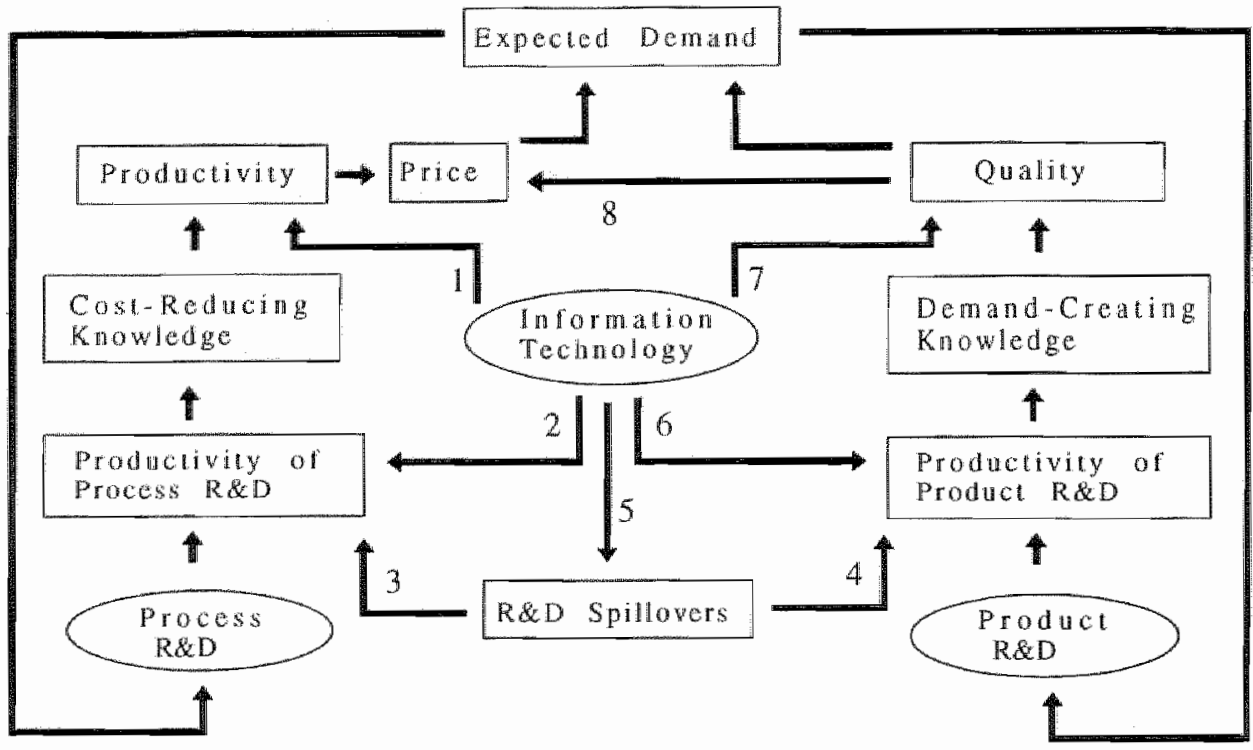

Figure 4.1. Schematic outline of the Endogenous Technological Change Model

capital goods also improve the perceived quality level. ${ }^{55}$ This happens when the "pure" quality increases (e.g. better design by using $\mathrm{CAD}$, better monitoring of manufacturing), service improves (better and faster communication channels), delivery time shortens (better communication and scheduling) and product variety increases (flexible production). It is expected that these impacts of IT stimulate demand, thus possessing demand-creating characteristics. Because IT investment goods differ from other investment goods they should be modelled in a different manner. The model developed in chapter 3 is suited to treat the special characteristics of IT, i.e the cost-reducing and demand-creating aspects of IT. The "direct" cost-reducing role of IT leads to a higher productivity level. This influence of IT is given by arrow (1) in figure 4.1. The "direct" demand-creating aspects of IT leads to an enhanced quality level (depicted by arrow 7 ).

Technological change, featured by productivity and quality improvements, can therefore not only be created by investments in knowledge (as was the case in chapter 3) but also by investments in IT goods. A general technology change generation function is:

55 The perceived quality level is the guality level of a product relative to the quality level of similarf competing products (see chapter 3 , 
$\frac{d T}{d t}=\tau \cdot\left(\frac{d K}{d t}\right)^{\delta_{k}} \cdot\left(I^{e}\right)^{\delta_{t,}}$

Where $T$ is the technological level (productivity or quality level), $K$ is the knowledge stock and $l_{e}$ are the IT investments in efficiency units.

We measure IT goods in efficiency units because high technological opportunities are one of the characteristics of a general purpose technology. It can therefore be expected that the performance or efficiency (quality) level of a unit of IT, say computers, increases rapidly over time. A present-day computer is much more efficient than a computer ten years ago.

The number of efficiency units can be formulated as the physical number of IT goods $(I)$ times an efficiency or quality index of IT goods $(E)$.

$I^{2}=1 . E$

Next to the "direct" effect of IT on technological change it also has an "indirect" effect via its influence on the knowledge generation process. This effect is mostly neglected in discussions about IT. Soete (1995) commented upon this situation by posing the following question "Everywhere around us we see the computer except in knowledge accumulation?". The knowledge generation process can be formulated as:

$\frac{d K}{d t}=\left[\eta \cdot\left(d^{e}\right)^{\delta_{1,2}} \cdot\left(S \cdot(1)^{\delta_{s}}\right)^{\delta_{s}}\right] \cdot R^{\delta}$

where $S$ represents the knowledge spillovers and $R$ represents the internal $R \& D$ expenditures. There is one significant difference with the knowledge generation process described in chapter 3 . The productivity level of own or internal $R \& D$, which is depicted by the term between brackets, is dependent on several variables while in chapter 3 this term was fully exogenous.

In chapter 3 all knowledge was created by internal R\&D activities. Knowledge was more or less considered as a "private" good that could only be used by the firm that created the knowledge. However, knowledge also has some public good features. The use of a piece of knowledge by one firm does not prevent another firm from using this piece of knowledge and is inexpensive to teach and to learn compared with the cost of an original invention or discovery. Therefore knowledge generated by other firms or sectors can be used in a firms' own knowledge generation process. Discoveries in other firms or sectors may generate new technological possibilities for a firm's own research efforts. Therefore knowledge generated in other firms or sectors increases the productivity of a firm's own R\&D efforts. However, in contrast to the neo-classical thought, in which all knowledge is 
public, we assume that only a part of the knowledge generated by other firms is accessible to a firm. The spillover knowledge stock can be modelled as:

$$
S_{i}=\sum_{i \neq i} \omega_{i, j} \cdot R_{i}
$$

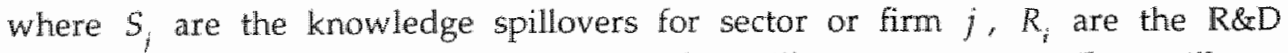
expenditures by firm or sector $i, \omega_{i,}$ is the spillover parameter. This spillover parameter depicts the part of the knowledge created by ather firms or sectors that can be used by firm or sector $j$. The height of the spillover parameter is determined by the technological closeness of sectors on the one hand and the degree of appropriability on the other hand. Sectors are technologically "close" when they are working in the same technological areas. A telecommunications industry is technologically "closer" to the computer or semi-conductor industry than to the food industry. It can be expected that as industries become "closer" in a technological sense the more useful will become the knowledge generated by the other firm or industry (higher value of $\left.\omega_{i, j}\right)$. The other determinant of $\omega_{i, j}$ is the degree of excludability or private aspects of the knowledge generated. If a firm can keep the generated knowledge inside the firm (full excludability), by means of secrecy for example, then $\omega_{i, j}$ will be zero. The larger the "public" content of the knowledge generated by a firm $i$, the higher the value of $\omega_{i, j}$, ceteris paribus. However if a firm can also use knowledge generated by other firms, these other firms can use the firm's knowledge which implies that generated knowledge is relatively less valuable for the firm itself. We discuss this aspect further in chapter 5. In this chapter we introduce the public nature of knowledge which causes knowledge created by other firms to increase the productivity level of internal $R \& D$ efforts. This is represented by the term $S^{\delta_{s}}$ in equation (4.3) and by arrow (3) and arrow (4) in figure 4.1.

We now turn to the effects of IT on the knowledge generation process (indirect influence on technological change). First, relatively inexpensive computer simulations substitute for expensive and time consuming physical experiments. Computational power enables researchers to test theories more rapidly and effectively than in the pre-computer era. These features of IT lead to an increase in the productivity level of $R \& D$. This effect is represented by $\left(I^{2}\right)^{s_{12}}$ in equation (4.3) and by arrow (2) or arrow (6) in figure 4. It It is important to notice that we used IT goods in efficiency units since the increased efficiency of, for example, computers, creates new and better research possibilities. Second, information technology makes knowledge generated by other firms or sectors more valuable for a firm. This is caused by the fact that for an effective use of computational power, researches must formalise problems in abstract terms, which depends on a sound theoretical understanding of the problems themselves. This implies that knowledge generated by other firms becomes more valuable to a firm. IT also improved the transmission and processing of information and created information networks. Investments in 
such networks may enhance the value of knowledge created by other firms. These features of information technology influence the walue of the spillover parameter $\left(\omega_{i j}\right)$. The usable part of the knowledge generated by other firms increases through the availability and use of $\mathrm{IT} .^{56}$

$$
S_{j}^{y}=\sum_{i \neq i} \omega_{i, j}\left(I_{i}^{g}\right), R_{i}=\sum_{i \times i} \omega_{s, i} \cdot\left(I_{i}^{g}\right)^{s_{s i}} \cdot R_{i}=\left(I_{j}^{g}\right)^{s_{s i}} \cdot \sum_{i \neq i} \omega_{i, j} \cdot R_{i}
$$

We introduced this effect by $\left(S .\left(I_{j}^{e}\right)^{\mathrm{s}_{\mathrm{s}}}\right)$ in equation (4.3) and by arrow (5) in figure 4.1. The appropriability of internal R\&D diminishes since IT increases the part of internal R\&D efforts that other firms can use. This reduces, therefore, incentives to invest in $R \& D$. We investigate this aspect of IT in chapter 5, where we introduce other firms that compete in the same market.

In conclusion we can say that the productivity of own R\&D in the knowledge generation effect is dependent on knowledge created by other firms and the physical and efficiency level of IT investments. IT investments increase the productivity level of internal R\&D directly and indirectly via the increased value of knowledge generated by other firms. Technological change, which is generated by "direct" IT effects and created knowledge, becomes (substitution of equation (4.3) in equation (4.1)):

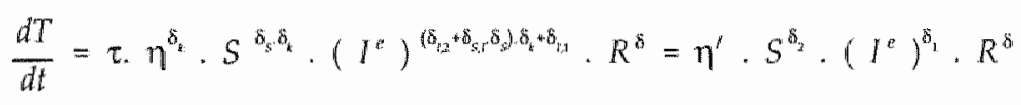

In this chapter we use the right most part of equation (4.6). We should keep in mind that all the mentioned effects are behind this simple formulation. So, $\delta_{1}$ represents the direct IT effect $\left(\delta_{i, 1}\right)$ and the two "indirect" IT effects $\left(\delta_{1,2}\right.$ and $\left.\delta_{s, \ell}\right)$ which increase the productivity of the knowledge generation process.

\subsection{Endogenous Technological Change by Investments in Information Technology and R\&D}

\subsubsection{Demand Creating Effects of IT}

In the literature on modelling the influence of IT (see e.g. Loveman 1988, Morrison and Berndt 1991), the productivity enhancing effect of IT is the central impact in the models. Besides this cost-reducing effect, people stress other important characteristics of IT which increase the perceived quality level. Through better monitoring and control of the production process, quality levels of products have

\footnotetext{
${ }^{56}$ For simplicity we assume that the IT influence on spillover parameter $\omega_{i j}$ is independent of $i$.
} 
improved. Computer Added Manufacturing (CAM) and Computer Added Design (CAD) increase the flexibility of the production process, which in combination with faster and better communication with suppliers and customers, tailor the products to the preferences of the users. Instead of one standard product, a whole range of product varieties are designed to satisfy the specific preferences of various groups of users. These impacts of IT all increase the perceived quality of a product directly. Firms can also increase their quality levels by performing product R\&D $\left(R_{A}\right)$. The productivity of own product $R \& D$ can be enhanced by the use of knowledge generated by other firms $\left(S_{d t}\right)$ and by information technology investments. The quality generation function is: ${ }^{57}$

$$
\frac{d Q_{p}}{d t}=\gamma \cdot S_{d}^{\tilde{s}_{2}} \cdot\left(I_{d} \cdot E_{d}\right)^{\tilde{\sigma}_{1}} \cdot R_{d}^{\delta}-w \cdot Q_{p} ; 0 \leq \delta, \delta_{1}, \delta_{2}<1
$$

The perceived quality level $\left(Q_{p}\right)$ can be enhanced by investments in productoriented IT goods $\left(I_{d}\right)$ and product-oriented $\operatorname{R\& D}\left(R_{d}\right) \cdot \gamma$ is the exogenous productivity level of the quality generation process. The parameter $w$ represents the perceived quality depreciation rate. Without any product-oriented R\&D or productoriented IT activities, the level of perceived quality will decrease due to the competitor's $\mathbb{R} \& D$ and IT efforts. Instead of specifying the behaviour of ones competitors, we assume a constant exponential decay of perceived quality for reasons of simplicity. ${ }^{5 / 5}$

The perceived quality level is expected to have a positive influence on demand. Next to this quality aspect of demand, we consider the traditional negative influence of the price level $(P)$ of the product. We postulate therefore the following expected demand function:

$$
\gamma^{\prime \prime}=X_{0} \cdot Q_{p}^{b(a-1)} \cdot P^{-i s}
$$

where $Y_{d}$ is the expected demand and $X_{0}$ is a positive scale parameter of demand. The parameter $a$ represents the price elasticity of demand and $b \cdot(a-1)$ is the quality elasticity of demand. Not performing product-oriented R\&D or product-oriented IT decreases a firm's perceived quality level because we assume that competitors will use in R\&D and IT to increase their quality levels. A lower perceived quality level leads to lower demand which implies that a firm must undertake defensive $R \& D$ or IT activities to maintain their market position.

However, we can expect that a higher quality level also effects production costs. According to the standard industrial organisation literature, a higher quality level can only be achieved at higher unit production costs. Dorfman and Steiner (1954) state that: "By quality we mean any aspect of a product, including the services

\footnotetext{
57 Where 81 embodies the dired $\left(\delta_{h,}\right)$ and indirect $\left(\left(\hat{\delta}_{h, 2}+\delta_{s, k}, \delta_{s}\right) . \delta_{k}\right.$ ) IT effects (see equation (4.6)).

See chapler 3 or vain Meijl and van Zon (1993).
} 
included in the contract of sales, which influences the demand curves. The essential difference from advertising is that changes in quality enter into variable costs".

For example, an increase in product variety can be expected to increase unit production costs. Brooke (1991) stated that increases in product variety reduced economies of scale which increased unit production costs.

When we consider the variable cost increasing effect of quality we must adjust the production function. ${ }^{\text {s? }}$

$Y=A \cdot L^{1-\alpha} \cdot K^{\alpha} \cdot Q_{p}^{-\xi}$

The quality adjusted total factor productivity is $T F P^{\prime}=A^{\prime}=A / Q_{p}^{5}$. A higher quality level decreases a firm's productivity level.

\subsubsection{Cost Reducing Effects of IT}

The second major impact of IT is that IT goods are used to "smooth" the process of production. Computers, CAM, faxes, etc, deliver services which directly influence the efficiency of the production process. The productivity generation process is specified in the same way as the quality generation process. ${ }^{60}$

$\frac{d A}{d t}=\eta \cdot S_{c}^{\theta_{2}} \cdot\left(I_{c} \cdot E_{c}\right)^{\theta_{s}} \cdot R_{c}^{\theta} \quad ; 0 \leq \theta_{r}, \theta_{1}, \theta_{2}<\mathbb{1}$

A firm can invest in process-oriented IT $\left(I_{c}\right)$ and process-oriented $R \& D\left(R_{c}\right)$ to increase it's efficiency level. The process spillover stock $\left(S_{c}\right)$ again increases the productivity of $R \& D$.

\footnotetext{
59 The general idea is that with a certain amount of inputs (labour and capilat) at certain amount of quality characteristics can be produced: the number of goods produced $\left(y_{i}\right)$ times the unit quadity levell $\left(Q_{p i}=Q_{p, i}^{\prime \prime} / y_{i}\right.$, where $Q_{p, i}^{\prime \prime}$ is the total number of quality units). There cire constam retums to scale with respect to the number of products produced $(y)$ and decreasing retums with respect to the unit quatily tevel. tho achicve a two times ats high unit quality level you must increase your total production factors per unit output by more than two times. Assuming a linear homogenous Cobb Douglas function:
}

$$
Q_{p i}=\frac{Q_{p i}^{\prime \prime}}{y_{i}}=\left[F\left(\frac{L_{i}}{y_{i}}, \frac{K_{i}}{y_{i}}\right)\right]^{\frac{1}{5}}=\left[A_{i} \cdot\left(\frac{L_{i}}{y_{i}}\right)^{1-\infty} \cdot\left(\frac{K_{i}}{y_{i}}\right)^{\infty}\right]^{\frac{1}{t}}, \quad 0<\frac{1}{\xi} \leq 1
$$

The production funcuion based on this condition is:

$$
y_{i}=A_{i}, K_{i}^{\alpha \alpha} \cdot L_{i}^{i-x} \cdot Q_{i, i}^{-i, ~}
$$

60. Similar to the product IT elasticity $\left(\delta_{1}\right)$, the process IT elasticity $\theta_{\xi}$ embodies the direct $\left(\theta_{i, 1}\right)$ and indirect $\left.\left(\theta_{1.2}+\theta_{3, r} \theta_{3}\right) \cdot \theta_{k}\right)$ IT effects (see equation (4.6)). 


\subsubsection{An Endogenous Technological Change Model}

A firm can maximize it profits by choosing its price level, productivity level, and perceived quality level. It can influence these by engaging in product and process R\&D or by buying product and process oriented IT goods. The inter-temporal profit maximisation problem for a firm is: ${ }^{61}$

$\operatorname{Max}_{P_{t} R_{s} R_{s} I_{s}} \pi(0)=\int_{0}^{\infty} e^{-r \cdot t} \cdot\left[P_{0} Y-T C-R_{c} \cdot q_{c}-R_{d d} q_{d}-l_{c} \cdot p_{c}-l_{d d} p_{d}\right] \cdot d t$

where

$Y=X_{0} \cdot Q_{p}^{r(a-1)} \cdot p^{-i a} ;$

$T C=\frac{Q_{p}^{\xi}}{A} \cdot Y \cdot Z_{1} ; Z_{1}=\left(\frac{w^{I}}{1-\alpha}\right)^{1-\alpha} \cdot\left(\frac{v}{\alpha}\right)^{\alpha}$

$d A / d t=\eta \cdot R_{c}^{\theta} \cdot\left(I_{c} \cdot E_{c}\right)^{\theta_{1}} \cdot S_{c}^{\theta_{3}}$

$d Q_{p} / d t=\gamma \cdot R_{d t}^{z} \cdot\left(l_{d} \cdot E_{d}\right)^{\delta_{t}} \cdot S_{a l}^{\delta_{2}}-w \cdot Q_{j}$

where $q_{c} q_{d \prime} p_{c} p_{d}$ are respectively the price level of process $\mathrm{R} \& \mathrm{D}$, product $\mathrm{R} \& \mathrm{D}$, process $I T$ and product $I T, w^{\prime}$ and $w$ are the wage level and the user cost of capital. We assume that all these prices, the exogenous scale of demand $\left(X_{y}\right)$ and the efficiency index of process and product oriented $\mathrm{T}$ goods have a constant growth rate. In general, the prices of $R \& D$ had a positive growth rate while the prices of IT goods declined in the last decades. The growth rate of the efficiency level of the new general purpose technology, IT, was extremely high during this. period.

The solution to this dynamic optimization problem is given in appendix $4 \mathrm{~A}$. The state variables are a firms' productivity level $(A)$ and its perceived quality level $\left(Q_{p}\right)$ and the choice variables are its price level $(P)$, process $R \& D$ level $\left(R_{c}\right)$, product $R \& D$ level $\left(R_{d}\right)$, process-oriented IT level $\left(l_{c}\right)$ and product-oriented IT level $\left(l_{i f}\right)$. We discuss some of the characteristics of any potential equilibrium.

First, marginal costs have to be equal to marginal revenue for the five choice variables. For the price level this condition is:

$$
P=\left[\frac{a}{a-1}\right] \cdot \frac{Q_{p}^{z} \cdot Z_{1}}{A}
$$

The price level will be determined as a mark-up over the marginal costs. The markup is dependent on the price elasticity of demand and can be used to cover the

\footnotetext{
5l As in chapter 3 we use the tocal variable cost function instaad of the production function to save two control wariables (capital and tabour).
} 
fixed IT and R\&D costs. The marginal costs of a firm are not only dependent on the productivity level of the production process (as in chapter 3) but also on the quality level of a product. The higher the quality level, the higher the unit costs and the higher the productivity level, the lower the unit costs.

For the other control variables, the current marginal costs are equal to the marginal benefits which are spread over the future. This condition for processoriented IT is:

$p_{c}=\mu \cdot\left[\frac{\theta_{1} \cdot(d A / d t)}{l_{c}}\right]$

The left hand side of this equation represents the current marginal cost of an investment in process-oriented $\mathrm{rT}$ and is equal to its price $\left(p_{i}\right)$. The right hand side of this equation represents the marginal benefits which are spread over the future. These marginal benefits can be divided into two effects. The first one is represented by $\mu$ which is the co-state variable of the optimal control problem and can be interpreted as the marginal value of $A$. The second one is the change in the productivity level caused by one unit of process-oriented IT, which is represented by the term within brackets.

We also study this condition for process-oriented R\&D:

$\eta_{c}=\mu \cdot\left[\frac{\theta \cdot\left(\eta \cdot E_{c}^{\theta_{1}} \cdot I_{c}^{\theta_{1}} \cdot S_{c}^{\theta_{z}}\right)}{R_{c}^{1-\theta}}\right]$

If we look at the marginal increase in the productivity level, which is the specification within brackets, we identify three important determinants. First, we notice decreasing marginal returns to the $\mathbb{R} \& D$ level $(0 \leq \theta<1)$; therefore the higher a firm's R\&D level, the lower the marginal increase in its productivity level. Second, the higher the value of the process $\mathbb{R} \& D$ elasticity $(\theta)$ the greater the increase in the productivity level. Third, the higher the productivity level of own process R\&D efforts the greater the marginal benefits. The productivity level of process R\&D is dependent on the value and elasticity of the spillover variable and the elasticity, efficiency and number of process-oriented IT goods. The last determinants include the effect that higher spillover effects increase the productivity of a firm's own R\&D and that a larger IT capital stock also increases the productivity of a firm's own R\&D efforts.

The dynamic conditions which describe the development of the marginal value of $A_{j}$ and $Q_{p, i}$ are given by equation (4A.9) and equation (4A.10).

$-\frac{d \mu}{d t}+\mu \cdot r=\frac{T C}{A}$ 


$$
-\frac{d \lambda}{d t}+(r+w) \cdot \lambda=(b-\xi) \cdot \frac{T C}{Q}
$$

The marginal value of the productivity and quality level depreciates at the rate at which productivity and quality are contributing to the current profits (this is represented by the term at the right hand side in both equations). The marginal value appreciates at the rate of the marginal opportunity costs of investing in productivity and quality (this is represented by the second term at the left hand side in both equations).

The steady state growth rates are calculated in appendix 4A. Equation (4A.22) gives the steady state growth rate of product $\mathrm{R} \& \mathrm{D} .^{62}$

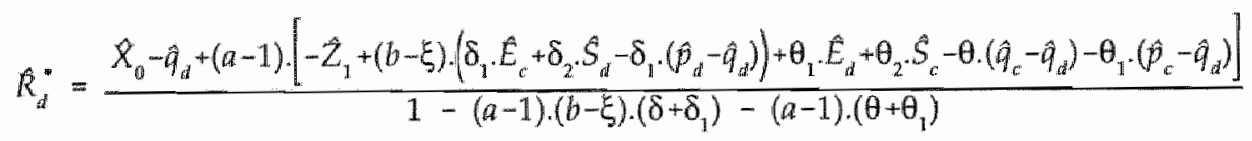

The growth rate multiplier $(M)$ of this system is:

$$
M=\frac{1}{1-(a-1) \cdot(b-\xi) \cdot\left(\delta+\delta_{1}\right)-(a-1) \cdot\left(\theta+\theta_{1}\right)}
$$

The growth rate multiplier again contains a cost reducing, $(a-1) \cdot\left(\theta+\theta_{1}\right)$, and demand creating, $(a-1) \cdot\left(b-\xi_{)}\right)\left(\delta+\delta_{1}\right)$, component which indicates that cost-reducing and demand-creating activities reinforce each other. It is interesting to note that the process IT elasticity $\left(\theta_{1}\right)$ augments the cost-reducing influence and the product IT elasticity $\left(\delta_{1}\right)$ augments the demand-creating influence on the growth rate multiplier. Both IT elasticities increase the value of the growth rate multiplier. This can be seen by putting the process and product elasticity of IT (respectively $\theta_{1}$ and $\delta_{1}$ ) equal to zero. This implies that given a certain positive growth rate of the exogenous variables, the growth rate of product $R \& D$ increases through the introduction of process and product oriented. IT. IT has a direct contribution to technological change and an indirect contribution as it stimulates $\mathbb{R} \& D$ investrnents by increasing the productivity of R\&D efforts.

The cost-increasing effect of an enhanced quality level ( $\xi$ ) has a negative influence on the demand-creating component and therefore on the growth rate multiplier. The incentives to invest in quality improvements are reduced through the cost increasing effects of these improvements.

We now discuss the influences of the exogenous growth rates. We concentrate on the effects that are related to IT and spillover effects. A positive growth rate of

\footnotetext{
62 We assume that $b>\xi$, it is otherwise not profitable to invest in quality improvements. Furthermore, we must rementer that $a>1$.
} 
R\&D and $I T$ prices has a negative influence on the steady state product $R \& D$ growth rate. However, the prices of IT goods fall after their invention. This induces a positive influence on the steady state growth of product R\&D. Product-oriented IT $\left(\hat{p}_{d}<0\right)$ and process-oriented IT $\left(\hat{p}_{c}<0\right)$ price reduction stimulates investments in IT goods, and since these investments increase the productivity of $R \& D$, they create new incentives for $R \& D$ investments. Although the prices of IT goods decreased, the efficiency or quality level of IT goods increased rapidly (see chapter 1). More efficient computers create possibilities for $R \& D$ and we obtain therefore the expected positive influence of a higher efficiency level of process-oriented IT $\left(\hat{E}_{c}>0\right)$ and product-oriented IT $\left(\hat{E}_{d}>0\right)$ on the steady state product R\&D level. What really matters is the price per efficiency unit. The influence of the price per efficiency units of process-oriented IT goods is $\left((a-1) \cdot \theta_{1} \cdot\left(\hat{E}_{c}-\hat{p}_{c}\right)\right)$ and of productoriented IT goods is $\left((a-1) \cdot(b-\xi) \cdot \delta_{1} \cdot\left(\hat{E}_{a}-\hat{p}_{d}\right)\right)$ on the steady state product R\&D growth rate. The decline in IT prices and the strong increase in the efficiency units of IT goods caused the price in efficiency units of IT goods to fall rapidly. Therefore it is a combination of the special characteristics of IT (e.g. IT creates incentives for other firms R\&D) and the strong decline in the price per efficiency units, that enable IT goods to enhance technological change in sectors. Furthermore, the pervasiveness of IT implies that it can create growth for the whole economy.

Spillovers have a positive influence (in this chapter) because their only influence is that a firm or sector can use knowledge generated by other firms. This increases the productivity level of internal $R \& D$ which induces further investment in $R \& D$. In chapter 5 we also introduce a disincentive effect of spillovers; larger spillovers imply that appropriability diminishes. The total effect of knowledge spillovers on internal $R \& D$ efforts will be less clear in that case.

The steady state growth rate of product oriented IT is given in equation (4A.24).

$$
\hat{I}_{d}^{*}=\frac{\hat{X}_{0}-\hat{p}_{d}+(a-1) \cdot\left[-\hat{Z}_{1}+(b-\xi) \cdot\left(\delta_{1} \cdot \hat{E}_{d}+\delta_{2} \cdot \hat{s}_{d}-\delta_{1} \cdot\left(\hat{\theta}_{d}-\hat{p}_{d}\right)\right)+\delta_{1} \cdot \hat{E}_{c}+\theta_{2} \cdot \hat{S}_{c}-\theta \cdot\left(\hat{q}_{c}-\hat{p}_{d}\right)-\theta_{1} \cdot\left(\hat{p}_{c}-\hat{p}_{d}\right)\right]}{1-(a-1) \cdot(b-\xi) \cdot\left(\delta+\delta_{1}\right)-(a-1) \cdot\left(\theta+\theta_{1}\right)}
$$

\section{$I T$ and RED Intensities}

In this sub-section we investigate what determines a firm's IT and R\&D intensity. These $R \& D$ and IT intensities can also be seen as important indicators of technological change. These intensities are calculated with a four step procedure in appendix $4 \mathrm{~A}$ (equations (4A.27) - (4A.30)). The steady state product IT intensity is:

$$
\frac{I_{d}^{*} \cdot p_{w^{*}}}{Y^{*} \cdot P^{*}}=(b-\xi) \cdot\left(1-\frac{1}{a}\right) \cdot\left(\delta_{1}\right) \cdot\left(\frac{\hat{Q}^{*}+w}{r+w-\lambda^{*}}\right)
$$


The steady state product-oriented IT intensity is a constant ratio because the steady state growth rate of the product IT budget $\left.\left(p_{a^{1}}\right)^{\prime}\right)$ is equal to the steady state growth rate of the value of production $(P . Y)$. Therefore firms will choose a constant product IT intensity, the value of which is determined with a simple rule given in equation (4.21). The determinants of this constant product IT intensity are: First, the $I_{\text {\# }}$ intensity will be higher when quality is valued more by consumers (higher $b$ ). Second, the increase in unit costs from an increase in the quality level is less (lower $\xi$ ). Third, the price competition is stronger on the market (higher value of a). Fourth, the product IT elasticity of the quality generation process is higher $\left(\delta_{11}\right)$. Fifth, more IT investments are needed when the growth rate of quality and the depreciation rate of quality are higher and sixth, the opportunity costs are lower $(\gamma-\lambda+w)$.

The process IT intensity is given by equation (4A.30).

$$
\frac{Y_{c}^{*}{ }^{*} p_{c}}{Y^{*} \cdot P^{*}}=\left(1-\frac{1}{a}\right) \cdot\left[\theta_{1}\right] \cdot\left(\frac{\hat{A}^{*}}{r-\hat{\mu}^{*}}\right)
$$

When we compare the process IT intensity with the product IT intensity there is one significant difference, namely there is no influence of the quality sensitivity of consumers (b) and the cost increasing effect of quality $(\xi)$.

\subsubsection{Implications for TFP Growth Estimations}

Many empirical studies exist on the relationship between IT and productivity growth (see Brynjofsson 1993, for a review of the literature). Most of the empirical studies could not find the expected positive link between IT investments and productivity. There was no relationship (or even a negative one) between IT and productivity. "This is called the "IT productivity paradox". What can our model, which includes both the cost-reducing and demand-creating role of $\mathrm{IT}$, contribute to this discussion? To answer" this question we derive the implied TFP growth equation of this model.

First, we put the production function lequation (4.9)) in growth rates:

$\hat{Y}=\hat{A}+\alpha \cdot \hat{K}+(1-\alpha) \cdot \hat{L}-\xi \cdot Q$

Second, we take the steady state growth rates of the two dynamic conditions (equation (4.7) and equation $(4.10))^{6}$

\footnotetext{
63 To concentute on the IT effect we assume uat the growth rates of spillowers $\left(S_{c}\right.$ and $S_{d}$ and the efficiency level af process and product IT $\left(E_{\text {; }}\right.$ and $\left.E_{d}\right)$ are equal to zero.
} 


$$
\begin{aligned}
& \hat{A}=\hat{\eta}+\theta \cdot \hat{R}_{c}+\theta_{1} \cdot \hat{I}_{c} \\
& \hat{Q}_{p}=\hat{\gamma}+\delta \cdot \hat{R}_{d}+\delta_{1} \cdot \hat{I}_{d t}
\end{aligned}
$$

Third, the definition of TFP growth is:

$$
T \hat{F P}=\hat{Y}-(1-\alpha) \cdot \hat{L}-\alpha \cdot \hat{K}
$$

Substituting the last three equations in equation (4.23) yields:

$$
T \hat{F} P=\hat{\eta}-\xi . \hat{\gamma}+\theta \cdot \hat{R}_{c}-\xi \cdot \delta \cdot \hat{R}_{d}+\theta_{1} \cdot \hat{l}_{c}-\xi \cdot \delta_{1} \cdot \hat{l}_{d}
$$

It is virtually impossible to separate IT expenditures into process-oriented and product-oriented IT goods in empirical studies. The best we can do is to assume that the ratio of product-oriented to process-oriented IT goods remains constant. An implication of this assumption is that $\hat{l}_{c}=\hat{l}_{r}$. Substitution of this result in the last equation yields:

$T A P=\pi_{0}+\theta \cdot \hat{R}_{c}-\xi \cdot \delta \cdot \hat{R}_{d}+\left[\theta_{1}-\xi \cdot \delta_{1}\right] \cdot \hat{I}$

where $\pi_{0}=\hat{\eta}-\xi . \hat{\gamma}$. The IT coefficient contains two terms. The first term, $\theta_{1}$, measures the cost reducing impact of process-oriented IT and the second term, $\xi . \delta_{1}$, measures the cost-increasing aspect of product-oriented IT. The overall effect of IT is dependent on the strength of both effects. When the cost reducing effect of IT is larger than the second effect, the estimated coefficient will be positive. However, when the most important impact of IT is demand creation, the estimated coefficient will be negative.

We can conclude that studies which estimate the direct relationship between productivity and IT (measuring the value $\theta_{1}$ ) measure the cost-reducing impact of IT less the cost increasing impact of demand creating IT $\left(\theta_{1}-\delta_{1}, \xi\right)$. These studies therefore obtain an under-measurement of the real efficiency impact of IT. A correct estimation of the influence of IT on productivity requires the division of IT goods into process-oriented and product-oriented IT goods.

Our simple model provides a possible explanation for the "disappointing" results of the empirical studies on the relationship between productivity and IT. When the total IT stock is used as the explanatory variable, no relationship between productivity and IT can also indicate that the cost-reducing role of process-oriented IT is equal to the cost increasing role of product-oriented IT. A negative relationship implies that the latter effect is the most important of the two.

\subsection{The Influence of Market Segmentation on Technological Change}

In this section we investigate the influence of market characteristics on technological change. The two market characteristics which we focus on are the 
degree of competition and the quality preferences of consumers. Interesting questions in this context are whether or not a sophisticated (quality loving) domestic demand stimulates technological change and if a more competitive export market stimulates domestic technological change. To investigate these questions further we divide the market into a domestic and a foreign or export market. These two markets differ in the degree of competition and in the sophistication of their demand. We assume that the markets are segmented so that firms can choose a different price level for each market. The domestic demand is given by:

$F=(1-m) \cdot F_{0} \cdot Q_{p}^{b_{f}\left(a_{f}-1\right)} \cdot P_{f}^{m a_{f}}$

where $F$ is the final domestic demand, w reflects the degree of import penetration, $P_{f}$ is the price charged on the domestic market and $F_{0}$ is an exogenous scale parameter of the domestic demand. The degree of domestic market competition will be reflected in the domestic price elasticity of demand $\left(a_{f}\right)$. The sophistication of the domestic demand is reflected in the parameter $b_{f}$. A higher value of $b_{f}$ means that consumers prefer products with a high quality level. The export demand is:

$E=E_{0} \cdot Q_{p}^{b_{s} \cdot\left(a_{t}-1\right)} \cdot P_{e^{*}}^{* a_{e}}$

where $E_{0}$ is an exogenous scale parameter of the export demand and $P_{e}$ is the price charged on the export markets. $a_{e}$ and $b_{e}$ represent the export price elasticity of demand and the "quality loving" export indicator.

In this section we study the influence of the introduction of segmented markets. Our model will therefore be as simple as possible; firms only engage in product R\&D to increase a product's quality level. The profit maximization problem is:

$$
\begin{aligned}
& \underset{R_{s} P_{n} R_{a}}{\operatorname{Max}}=\int_{0}^{\infty} \cdot e^{-r t} \cdot\left[P_{f} \cdot F+P_{e} \cdot E-T C-R_{d} q_{d}\right] \cdot d t \\
& \text { where } \\
& Y=F * E \\
& F=(1-n n) \cdot F_{0} \cdot Q_{p}^{b_{j}\left(\left\{n_{j}-1\right)\right.} \cdot P_{f}^{-u_{f}} \\
& E=E_{0} \cdot Q_{p}^{b\left(a_{c}-1\right)} \cdot P_{c}^{-H_{c}} \text {; } \\
& T C=\frac{Q_{p}^{\prime}}{A} \cdot Y \cdot Z_{1} \\
& T C=T C_{j}+T C_{d}=F \cdot\left[\frac{Q_{p}^{\xi} Z_{1}}{A}\right]+E \cdot\left[\frac{Q_{p}^{k} Z_{1}}{A}\right] \\
& d Q_{n} / d t=\left(\gamma \cdot R_{i}^{\delta}-w \cdot Q_{p}\right)
\end{aligned}
$$


A firm maximizes the present value of its profits by choosing its domestic and export price level and its product $R \& D$ level. A full description of the solution of this problem is given in appendix $4 \mathrm{~B}$.

The static marginal costs are equal to marginal revenue conditions for the domestic and foreign price level and are:

$$
\begin{aligned}
& \frac{\partial H_{c}}{\partial P_{f}} \Rightarrow\left[1-\frac{1}{a_{f}}\right] \cdot P_{f}=\frac{Q_{p} \cdot Z_{1}}{A} ; a_{f}>1 \\
& \frac{\partial H_{c}}{\partial P_{e}} \Rightarrow\left[1-\frac{1}{a_{e}}\right] \cdot P_{e}=\frac{Q_{p} \cdot Z_{1}}{A} ; a_{\mathrm{e}}>1
\end{aligned}
$$

The price will be determined as a mark-up over unit costs. The unit costs are the same for domestic and export goods and are equal to the right hand side of both equations. The mark-up is dependent on the price elasticities of demand. A higher degree of competition in the export market $\left(a_{e}>a_{f}\right)$ leads to the familiar conclusion that the export price level will be lower.

The development of the marginal value of the perceived quality level is given by (see equation (4B.6):

$$
-\frac{d \lambda}{d t}+(r+w) \cdot \lambda=\left(b_{f}-\xi\right) \cdot \frac{T C_{f}}{Q_{p}}+\left(b_{e}-\xi\right) \cdot \frac{T C_{e}}{Q_{p}}
$$

As we discussed in section 4.2 , the marginal value of the quality level depreciates at the rate at which quality is contributing to the current profits. This contribution, which is represented by the right hand side of the last equation, is now divided into two terms; one representing the home market and one representing the foreign market.

We now discuss the main results of this analysis. We first discuss the influence of market segmentation on the steady state product $R \& D$ growth level and then discuss the influence of market segmentation on the product R\&D intensity. The steady state growth rate for $R_{d}$ is (see equation (4B.16)):

$$
\begin{aligned}
\hat{R}_{d}^{*} & =M \cdot\left[\left(\frac{F}{Y}\right) \cdot\left(\hat{E}_{0}-\hat{m}+\left(a_{f}-1\right) \cdot\left(\hat{A}-\hat{Z}_{1}\right)\right)+\left(\frac{E}{Y}\right) \cdot\left(\hat{E}_{0}+\left(a_{e}-1\right) \cdot\left(\hat{A}-\hat{Z}_{1}\right)\right]\right. \\
M & =\frac{1}{1-\delta \cdot\left(\frac{F}{Y} \cdot\left(b_{f}-\xi\right) \cdot\left(a_{f}-1\right)+\frac{E}{Y} \cdot\left(b_{e}-\xi\right) \cdot\left(a_{e}-1\right)\right)}
\end{aligned}
$$


The steady state growth rate of product R\&D contains two building blocks. The first one is the growth rate multipler $(M)$ and the second one is a weighted set of domestic and foreign exogenous growth rates. We concentrate on the growth rate multiplier and therefore assume that the exogenous growth rate of both markets is equal. If, for example, the two markets differ only by a higher degree of competition in the export market, $a_{e}>a_{f}$, then the larger the share of exports in total production ( $E / Y$ ), the larger the value of the growth rate multiplier. If, however, the only difference between the two markets is that the domestic demand is more sophisticated, $b_{>} b_{e^{*}}$ then the value of the multiplier will be higher if the domestic market share is higher. If we combine both examples, then the market which best stimulates technological change will depend on the strength of both effects. In conclusion we can say that a larger market share in a competitive market or a market with a more sophisticated demand stimulates technological progress when the exogenous growth rates of both markets are equal.

Next, we study the steady state product $R \& D$ intensity. The steady state product R\&D intensity is calculated in appendix $4 \mathrm{~B}$ (equation (4B.17))

$$
\frac{R_{i f}^{*} \cdot g_{d}}{F^{*} \cdot P_{f}^{*}+E^{*} \cdot P_{e}^{*}}=\delta \cdot\left[s_{f}^{*} \cdot\left(b_{f}-\xi\right) \cdot\left(1-\frac{1}{a_{f}}\right)+s_{E}^{*} \cdot\left(b_{c}-\xi\right) \cdot\left(1-\frac{1}{a_{c}}\right)\right] \cdot\left(\frac{\hat{Q}^{*}+w^{*}}{r+w-\hat{\lambda}^{*}}\right)
$$

where $s_{F}^{*}$ is the value share of domestic production: $s_{F}{ }^{*}=P_{j}^{*} \cdot F^{*} /\left(P_{f}^{*} \cdot F^{*}+P_{e}^{*} E^{*}\right)$ and $s_{E}^{*}$ is the value share of exports: $s_{E}^{*}=P_{*}^{*} \cdot E^{*} /\left(P_{f}^{*} \cdot F^{*}+P_{*}^{*} \cdot E^{*}\right)$.

The product R\&D intensity once again shows that a large market share in a sophisticated market (people value quality more) or a more competitive market boosts the technologicall intensity of production.

\subsection{Conclusion}

In this chapter we discussed the influence of the current general purposetechnology, information technology, on technological progress. We distinguished between a direct and an indirect infiuence of IT on technological progress. The "direct" effect stems from the special nature of IT goods; IT goods are special because they are used to "smooth" the production process while other capital goods are used together with labour to produce output. However, IT goods also influence the knowledge creation process and have therefore an "indirect" influence on technological progress. We identified two influences of IT on the knowledge creation process. On the one hand, IT goods increase the productivity of $R \& D$ directly (computer simulations instead of expensive and time consuming physical experiments) and on the other hand IT increases the usability of knowledge spillovers that increases the productivity of internal $R \& D$ indirectly (researchers are forced to translate their thoughts into computer programmes and translate tacit or 
context dependent knowledge in codified knowledge and information technology creates information networks).

IT also has some special characteristics. IT is pervasive, so it can be used in the whole economy. In addition, the price of IT goods declined in the last decades while the efficiency of IT products has increased rapidly.

In section 4.2 we made an endogenous technological change model driven by investments in process-oriented IT and R\&D to obtain efficiency gains and investments in product-oriented IT and R\&D to enhance the quality level. ITrelated parameters showed up in the important equations of the model, (e.g. in the growth rate multiplier). IT goods increased the value of the growth rate multiplier because the demand-creating and cost-reducing mechanisms were enriched. The combination of the special influences of IT together with the special characteristics of IT may enhance the growth of the economy.

Our model implies that standard empirical studies which investigate the relationship between productivity growth and $I T$, underestimate the value of the efficiency increasing effect of IT. This is because a part of IT is directed at increasing the perceived quality of a firm's product, which may cause higher unit production costs. Dividing IT into process-oriented IT and product-oriented IT can give accurate estimates of the cost-reducing and cost-increasing impacts of IT. However, such a division is not possible in practise.

The analysis of market segmentation showed us the importance of demand characteristics for technological progress. A larger market share in a more competitive market and/or a more sophisticated demand boosts technological change. 


\section{Appendix 4A: The influence of process-oriented and product-oriented IT goods}

In this appendix we derive the steady state growth rates for the model in section 4.1 . The current value hamiltonian is:

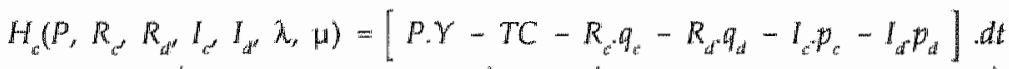

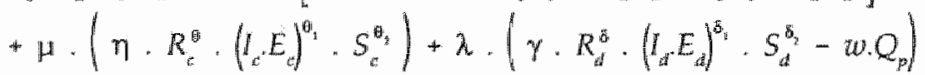

where:

$Y=X_{0} \cdot Q_{p}^{p(a-1)} \cdot p^{-t a}$;

$\Upsilon C=\frac{Q_{p}^{z}}{A} \cdot Y Z_{1} \quad ; \quad Z_{1}=\left(\frac{w v^{\gamma}}{1-\alpha}\right)^{\|-\alpha}\left(\frac{v}{\alpha}\right)^{\alpha}$

$d A / d t=\eta \cdot R_{c}^{*} \cdot\left(I_{c} \cdot E_{c}\right)^{\theta_{i}} \cdot S_{u^{*}}^{\theta_{z}}$

$d Q_{p} / d t=\gamma \cdot R_{d}^{\delta} \cdot\left(I_{d} \cdot E_{a}\right)^{\delta_{1}} \cdot S_{d}^{b_{2}}-w \cdot Q_{p}$

First Order Conditions:

The first order conditions with respect to the controll variable; $P, R_{\varepsilon^{\prime}} R_{d^{\prime}} l_{t}$ and $I_{d}$ are respectively:

$$
\begin{aligned}
& \frac{\partial H_{c}}{\partial P}=\gamma+P \cdot \frac{\partial Y}{\partial P}-\frac{\partial T C \cdot \frac{\partial Y}{\partial P}}{\partial Y} \Rightarrow P=\left(\frac{a}{a-1}\right) \cdot \frac{T C}{\gamma} ; a>1 \\
& \frac{\partial H_{u}}{\partial R_{c}} \Rightarrow q_{c}=\mu \cdot\left[\frac{\theta \cdot(d A / d t)}{R_{t}}\right] \\
& \frac{\partial H_{c}}{\partial R_{u}} \Rightarrow q_{d}=\lambda \cdot\left(\frac{\delta \cdot\left(d Q_{p} / d t+w \cdot Q_{p}\right)}{R_{d}}\right) \\
& \frac{\partial H_{c}}{\partial I_{c}} \Rightarrow p_{c}=\mu \cdot\left[\frac{\theta_{1} \cdot(d A / d t)}{I_{i}}\right] \\
& \frac{\partial H_{i}}{\partial I_{t}} \Rightarrow p_{i}=\lambda \cdot\left[\frac{\delta_{1} \cdot\left(d Q_{p} / d t+w \cdot Q_{p}\right)}{I_{d}}\right)
\end{aligned}
$$

The two "Transversality conditions (TVC) are:

$$
\begin{aligned}
& T V C_{\lambda}=\lim _{n \rightarrow \infty} \lambda_{i} e^{x(n) !}=0 \\
& T V C_{\mu}=\lim _{l \rightarrow \infty} \mu_{t} e^{(-r) \cdot h}=0
\end{aligned}
$$

The first order conditions with respect to the state variables $Q_{p}$ and $A$ are respectively:

$$
\begin{aligned}
\frac{\partial H}{\partial A} & \Rightarrow-\frac{d \mu}{d t}+\mu \cdot r=\frac{T C}{A} \\
\frac{\partial H}{\partial Q_{p}} & \Rightarrow-\frac{d \lambda}{d t}+r \cdot \lambda=P \cdot b \cdot(a-1) \cdot \frac{Y}{Q_{p}}-\zeta \frac{T C}{Q_{p}}-b \cdot(a-1) \cdot \frac{T C}{Q_{p}}-w \cdot \lambda \\
& \Rightarrow-\frac{d \lambda}{d t}+(r+w) \cdot \lambda=(b-\xi) \cdot \frac{T C}{Q}
\end{aligned}
$$

In the last equation we used the result of equation (4A.3). The two dynamic constraints given in equation (4.A.2) are the other first order conditions. 


\section{Calculation of Steady State Growth Rates}

The growth rate of the demand function is:

$\hat{Y}=\hat{X}_{0}+b \cdot(a-1) \cdot Q_{p}-a \cdot P$

The growth rate of the total variable costs ( $T C$ ) is:

$T C=\hat{\gamma}+\xi \cdot Q_{p}+\hat{Z}_{1}-\hat{A}$

The constant growth rates of the first order conditions are respectively:

$\hat{P}=\hat{T C}-\hat{y}$

$Q_{\text {c. }}=\hat{\mu}+A-\hat{R}$

$\hat{q}_{i t}=\hat{\lambda}+\hat{Q}_{p}-\hat{R}_{d}$

$\hat{p}_{c}=\hat{\mu}+\hat{A}-\hat{l}$

$p_{d}=\hat{\lambda}+\hat{Q}_{p}-\hat{l}_{d}$

$\hat{\mu}=T \hat{C}-\hat{A}$

$\lambda=T C-Q_{p}$

The constant growth rates of the productivity and quality generation process are:

$A=\theta \cdot R_{c}+\theta_{1} I_{i}+\theta_{1} \cdot E_{c}+\theta_{2} \cdot S_{0}$

$Q_{p}=\delta \cdot R_{d}+\delta_{1} \cdot P_{d}+\delta_{1} \cdot \hat{E}_{d}+\delta_{2} \cdot S_{d}$

We are left with eleven unknowns (the growth rates of $Y, T C, P, R, R_{d}, I, I_{d,}, \lambda, \lambda, A$ and $Q$, ) and eleven independent equations (equation (4A.11) till equation (4A.21)) which makes the solution unique. Straightforward substitution gives the steady state growth rate for $R_{d}$ :

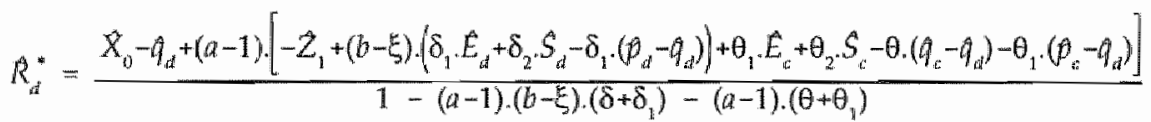

Substituting equation ( $4 \mathrm{~A}, 17)$ in equation (4A.15) we get:

$\hat{f}_{d}=q_{d}+R_{d}-p_{d}$

Substituting the steady state growth rate of $R_{d}$ in this equation we get the steady state growth rate of $l_{d}$.

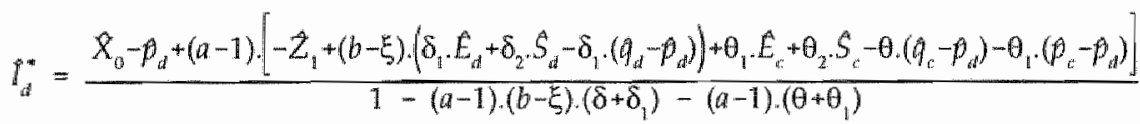

The other steady state growth rates can be found by straight forward substitution. We now investigate the general growth rate of the system. In the steady state the revenues, total variable costs, process and product R\&D and IT budgets all grow at the same general growth rate. This general growth rate is:

$\sigma=p_{a}+\hat{l}_{a^{*}}^{*}=q_{c}+\hat{R}_{c}^{*}=\hat{p}^{*}+\hat{Y}^{*}$ 
A constant steady state growth rate for $\lambda$ and $\mu$ implies that the transversality condition are satisfied when:

$T V C_{\hat{n}} \Rightarrow r-\hat{h}^{*}>0, T V C_{\mu} \Rightarrow r-\hat{\mu}^{*}>0$

Steady State RED and IT Interisities:

To calculate the steady state R\&D and IT intensities we use the first order conditions. We start by calculating the steady state product R\&D intensity. Firstly, we solve equation (4A.3) for TC. Secondly, we solve equation (4A.5) for $\lambda$. Thirdly, we divide both sides of equation $(4 A .10)$ by $\mathcal{A}$. Fourthly, we substitute the results of steps one to three into equation (4A.10). The steady state product $R \& D$ intensity is:

$\frac{R_{a}^{*} \cdot a_{a}}{\gamma^{*} \cdot P^{4}}=(b-\xi) \cdot\left(1-\frac{1}{a}\right) \cdot(\delta) \cdot\left(\frac{Q_{p}^{*}+w}{\gamma+w-\hat{\lambda}^{*}}\right)$

Using the same four step procedure we can derive the steady state product IT intensity. Only the second step differ's because we solve equation (4A.7) for $\lambda$.

$$
\frac{r_{b^{*}}^{*} \cdot p_{a}}{\gamma^{*} \cdot P^{*}}=(b-\xi) \cdot\left(1-\frac{1}{a}\right) \cdot\left(\delta_{i}\right) \cdot\left(\frac{Q_{p}^{*}+w}{r+w-\hat{\lambda}^{*}}\right)
$$

To calculate the process R\&D intensity we solve equation (4A.4) for $\mu$ in the second step. divide equation (4.A.9) by $\mu$ in the third step and substitute all the results in equation (4A.9).

$$
\frac{R_{i}^{*} \cdot q_{c}}{Y^{*} \cdot P^{*}}=\left(1-\frac{1}{a}\right) \cdot[\theta] \cdot\left(\frac{A^{*}}{r-\mu^{*}}\right)
$$

To calculate the process IT intensily we solve equation (4A.6) for $\mu$ in the second step.

$$
\frac{y_{0}^{*} p_{u}}{r^{*} p^{*}}=\left(1-\frac{1}{a}\right) \cdot\left[\theta_{1}\right] \cdot\left(\frac{A^{*}}{\gamma-\hat{p}^{*}}\right)
$$

\section{Appendix 4B: Market Segmentation: Domestic and Foreign Markets}

In this appendix we derive the steady state growth rates for the model in section 4.2 . A firm can only engage in product R\&D. However, it faces a domestic and a foreign market in which the degree of price and quality competition is different. The current value Hamiltonian is:

$$
H_{i}\left(P, R_{w} u^{2}\right)=\left[P_{f} F+P_{e} \cdot E-T C-R_{d} q_{d}\right] \cdot d t+\lambda \cdot\left(\gamma R_{d}^{8}-\tau w \cdot Q_{p}\right)
$$

where: 


$$
\begin{aligned}
& \gamma=F+E
\end{aligned}
$$

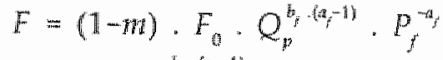

$$
\begin{aligned}
& E=E_{0} \cdot Q_{p}^{b_{p}\left(a_{x}-1\right)} \cdot P_{e}^{-i z} ; \\
& T C=\frac{Q_{p}^{z}}{A} \cdot Y Z_{1} ; Z_{1}=\left(\frac{w}{1-\alpha}\right)^{1-\alpha}\left(\frac{v}{\alpha}\right)^{\alpha} \\
& T C=T C_{f}+T C_{e}=F \cdot\left[\frac{Q_{p}^{\xi} Z_{1}}{A}\right]+E \cdot\left[\frac{Q_{p}^{\xi} \cdot Z_{1}}{A}\right] \\
& d Q_{p} / d t=\left(\gamma \cdot R_{d}^{\delta}-w Q_{p}\right)
\end{aligned}
$$

First Order Conditions:

The first order conditions with respect to the control variable $P_{f}, P_{g}$ and $R_{d}$ are respectively:

$$
\begin{aligned}
& \frac{\partial H_{c}}{\partial P_{f}} \Rightarrow P_{f}=\left(\frac{a_{f}}{a_{f}-1}\right) \frac{T C_{f}}{F} ; a_{f}>1 \\
& \frac{\partial H_{c}}{\partial P_{e}} \Rightarrow P_{e}=\left(\frac{a_{e}}{a_{c}-1}\right) \frac{T C_{c}}{E} ; a_{e}>1 \\
& \frac{\partial H_{c}}{\partial R_{d}} \Rightarrow q_{d}=\lambda \cdot\left[\frac{\delta \cdot\left(d Q_{p} / d t+w Q_{p}\right)}{R_{d}}\right]
\end{aligned}
$$

The first order conditions with respect to the state variables $Q_{p}$ is:

$$
\begin{aligned}
\frac{\partial H}{\partial Q_{p}} & \Rightarrow-\frac{d \lambda}{d t}+r \cdot \lambda=P_{f} b_{f}\left(a_{f}-1\right) \cdot \frac{F}{Q_{p}}+P_{e} \cdot b_{e} \cdot\left(a_{e}-1\right) \cdot \frac{E}{Q_{p}}-\zeta \frac{T C}{Q_{p}}-b_{j}\left(a_{f}-1\right) \cdot \frac{T C_{f}}{Q_{p}}-b_{i} \cdot\left(a_{e}-1\right) \frac{T C_{e}}{Q_{p}}-w \cdot \lambda \\
& \Rightarrow-\frac{d \lambda}{d t}+(r+w) \cdot \lambda=\left(b_{f}-\xi\right) \cdot \frac{T C_{f}}{Q_{p}}+\left(b_{e}-\xi\right) \cdot \frac{T C_{e}}{Q_{p}}
\end{aligned}
$$

In the last equation we used the results of equation (4B.3) and equation (4B.4).

The fifth first order condition is the dynamic constraint given in equation (4B.2).

Calculation of Steady State Grouth Rates

The growth rate of domestic demand is:

$\hat{F}=F_{0}-\hat{m}+b_{i} \cdot\left(a_{j}-1\right) \cdot Q_{p}-a_{f} \cdot P_{f}$

The growth rate of export demand is:

$\hat{E}=\hat{E}_{0}+b_{a} \cdot\left(a_{e}-1\right) \cdot \hat{Q}_{p}-a_{e} \cdot \hat{P}_{\mathrm{e}}$

The growth rate of the total variable costs (TC) is:

$T C=\left[\frac{F}{Y}\right] \cdot T C_{Y}+\left[\frac{E}{Y}\right] \cdot T C_{r}$

where the growth rates of $T C_{f}$ and $T C_{p}$ are: 
$T C_{f}=\hat{F}+\xi_{p} Q_{p}-A ; T C_{c}=E+E Q_{p}+Z_{p}-\hat{A}$

The constant growth rates of the first order conditions are respectively:

$\hat{p}_{f}=\mathrm{T}_{f}-\hat{f}$

$P_{e}=T C_{i}-E$

$q_{d}=\hat{\lambda}+Q_{p}-R_{d}$

$\hat{\lambda}=\left[\frac{F}{Y}\right] T C_{\gamma}+\left[\frac{E}{Y}\right] T C_{e}-Q_{p}$

The constant growth rates of the quality generation process is:

$Q_{p}=8 R_{d i}$

There are ten unknowns (the growth rate of $F, E, T C, T C_{a^{\prime}} T C_{r^{\prime}} P_{p^{\prime}} P_{\bar{a}^{t}} R_{d^{\prime}} \lambda$ and $Q_{p}$ ) and ten equations, straightforward substitution gives the steady state grow th tate for $R_{d}$ :

$$
R_{d}^{*}=\frac{\left[\frac{F}{Y}\right] \cdot\left(F_{0}-\gamma+\left(a_{f}-1\right) \cdot\left(A-Z_{1}\right)\right)+\left[\frac{E}{\gamma}\right] \cdot\left(\hat{E}_{0}+\left(a_{c}-1\right) \cdot\left(A-Z_{1}\right)\right.}{1-\delta \cdot\left[\frac{F}{\gamma} \cdot\left(b_{j}-\xi\right) \cdot\left(a_{j}-1\right)+\frac{E}{\gamma} \cdot\left(b_{e}-\xi\right) \cdot\left(a_{e}-1\right)\right]}
$$

Steady State RED Intensities:

To calculate the steady state R\&D intensities we use the four step procedure described in appendix 4A. Firstly, we solve equation (4B.3) for $T C$ and equation (4B.4) for $T C_{e}$. Secondly, we solve equation (4B.5) for $\lambda$. Thirdly, we divide equation (4B.6) by $\lambda$. Fourthly, we substitute the results of step one to three in equation (4B.6). The steady state product $\mathbb{R} \& \mathrm{D}$ intensity is:

$$
\frac{R_{i}^{*} \cdot g_{i d}}{F^{*} \cdot P_{f}^{*}+E^{*} \cdot P_{*}^{*}}=\delta\left[s_{p}^{*}\left(b_{j}-\xi\right) \cdot\left(1-\frac{1}{a_{f}}\right)+s_{E}^{*} \cdot\left(b_{e}-\xi\right) \cdot\left(1-\frac{1}{a_{e}}\right)\right] \cdot\left(\frac{Q^{*}+w}{r-\lambda^{*}}\right)
$$

where $s_{F}^{*}$ is the value share of domestic production: $s_{f}^{*}=P_{j}^{*} \cdot F^{*} /\left(P_{j}^{*} \cdot F^{*}+P_{*}^{*} \cdot E^{*}\right)$ and $s_{E}^{*}$ is the value shane of exports: $s_{E}^{*}=P_{e}^{*} \cdot E_{*}^{*} /\left(P_{j}^{*} \cdot F^{*}+P_{*}^{*} \cdot E^{*}\right)$ 


\section{Inter- and Intra- Industry Spillovers, Product Differentiation and Entry}

Firms perform R\&D activities in order to improve their competitiveness. Benefits occur in the form of cost-reducing and demand-creating innovations. These benefits are, however, imperfectly appropriable. A part of the knowledge generated by one firm can also be used by other firms. The R\&D of a firm improves, therefore, not only its own technological level but also the technological level of its competitors. The degree of appropriability therefore influences a firm's incentive to invest in R\&D because it knows that a part of its own efforts will be used by other firms and it can use the R\&D results of other firms. Spence (1984), in particular, finds that a decrease in appropriability, creating larger spillovers, reduces the incentives of individual firms to invest in $R \& D{ }^{64}$ We neglected this disincentive effect of knowledge spillovers in chapters 3 and 4 . Knowledge spillover effects, create on the one hand, incentives for R\&D investments because knowledge generated by other firms increases the productivity level of own R\&D efforts. On the other hand, however, knowledge spillovers create a disincentive effect because competing firms can use a part of the internally created knowledge. An important question in this chapter is therefore whether larger knowledge spillover effects do enhance or temper R\&D investments. This question is also relevant with respect to the influences of IT on technological progress, as one of the influences of IT is that a large part of the created knowledge can be used by other firms.

The model developed in chapter 3 concentrated on the technological behaviour of a certain firm, whereas activities of competing firms were hidden behind the demand function or reflected in the exogenous depreciation factor of the perceived quality level. In this chapter we introduce competing firms, which are able to use a part of the knowledge developed by other firms. The model from chapter 3 will include intra-industry spillover effects, product differentiation, and entry. We also

(64) Many other authors have examined the relationship between incentiwes for R\&D investment and the degree of appropriability in a shatic context (e.g. Lonry 1979, Lee and Wilde 1980, Levin and Reiss 1988, Cower and Levinthal 1989. De Bondi et al 1992). 
incorporate the cost-increasing effect of quality improvements and inter-industry knowledge spillovers developed in chapter 4 .

With regard to expected demand, we assume that people are not only interested in the quantity of a good but also in the quality of a good. We distinguish between "Love of Variety" and "Good Characteristics" preferences. The difference between these preferences is that love-of-variety-people value variety in its own right and good-characteristic people do not (Spence 1976, Dixit and Stiglitz 1977). These two kinds of preferences imply different demand characteristics and influence the decision making process of a firm regarding technological investments.

Another important determinant of the technological and economic behaviour of a firm is entry. The number of firms limits the appropriability of $R \& D$ but also increases the industry knowledge stock. Furthermore, entry has an important influence on the perceived price and quality demand elasticity. The influence of entry on welfare is dependent on consumer preferences.

This chapter investigates the influence of spillover effects, consumer preferences, and entry on the technological performance and profit level of individual firms and the general welfare level.

In section 5.1 we derive the demand functions which display "Love of Variety" or "Good Characteristics" preferences. Section 5.2 describes the technology generation functions and the modelling of spillovers. Section 5.3 describes the total endogenous technological change model and the characteristics for any potential equilibrium. In section 5.4 we discuss the steady state growth rates. Section 5.5 shows the dynamic behaviour of the model and section 5.6 discusses the influence of spillovers, entry and product differentiation on the steady state R\&D intensities, technological performance and welfare.

\subsection{Derivation of Demand Functions with Product Differentiation}

In our model consumers not only value the quantity of a good but also the quality. They enjoy utility of the amount of characteristics which are present in a good. We consider the situation in which there are two kinds of goods: differentiated products and homogenous products. Consumer preferences are represented by a two level utility function:

$$
U=\left[u_{1}(D), u_{2}(H)\right]=\frac{X_{0}}{\zeta} D^{\zeta}+H, \quad \zeta<\mathbb{1}
$$

where $u_{2}(H)$ is the sub-utility function of the homogenous good $(H)$ and $u_{1}(D)$ the sub-utility function of the differentiated good $(D)$. We assume that the utility of the homogenous good depends only on the quantity of the good consumed $\left(u_{2}(H)=H\right)$ while the utility of the differentiated good depends on the quantity and quality of 
each variety consumed. The overall utility function (U[.]) adds the two sectoral utility levels together.

The utility function of the differentiated good is an elaborated Spence-DixitStiglitz utility function (Spence 1976, Dixit and Stiglitz 1977). A quality index for each good is included.

$$
D=\left[\sum_{i=1}^{n}\left(y_{i}, Q_{p, i}^{b}\right)^{p}\right]^{\frac{1}{p}}, \quad a=\frac{1}{1-p}>1
$$

where $D$ is the quality characteristic index, $y_{i}$ is the consumption of good $i, Q_{p, i}$ is the perceived quality level of good $i, n$ is the number of varieties of a certain good and $a$ is the elasticity of substitution between differentiated products. ${ }^{65}$ The perceived quality level of a product is the result of a firms decision making process which is described in sections 5.3 and 5.4. The equilibrium quality levels will be equal across sectors, $Q_{p, i}=Q_{p, j}$, because we assume that each firm confronts the same decision problem and that we study only symmetric equilibria.

It is easy to show that this elaborated Spence-Dixit-Stiglitz sub-utility function contains a love of variety preference. Assume that the expenditures for the differentiated goods are $I_{D}$. In the symmetric case the quantities and prices of all varieties are equal. The demand for one variety is therefore $y_{i}=I_{D} /\left(n . p_{i}\right)$. The level of the quality characteristic index (D) obtained from $I_{D}$ in the symmetric case is:

$$
D=\left[n \cdot Q_{p, i}^{b, p} \cdot\left(\frac{I_{D}}{n \cdot p_{i}}\right)^{p}\right]^{\frac{1}{\rho}}=n^{\frac{1-p}{p}} \cdot Q_{p, i}^{b} \cdot \frac{I_{D}}{p_{i}}=n^{\frac{1}{\alpha-1}} \cdot Q_{p, i}^{b} \cdot \frac{I_{D}}{p_{i}}, \quad a>1
$$

The index $D$ depends positively on the number of varieties ( $n$ ). Equation (5.1) shows that a higher index $D$ provides a higher utility level. The utility level therefore increases as the number of varieties increases. This confirms that the demand specification allows for "Love of Variety" preferences. The love of variety effect declines when the elasticity of substitution between pairs of varieties (a) increases. The influence of the number of different varieties $(n)$ on $D$ and therefore the utility level becomes neglible when this elasticity $(a)$ becomes large. People derive utility from the total amount of good characteristics; the quality level $\left(Q_{p, i}\right)$ times the total number of goods $\left(I_{D} / p_{i}\right)$ purchased (the number of product varieties plays no role). Variety is not valued per se anymore, people are only interested in the characteristics of the good.

65 The substimtion elasticity $(a)$ has to be larger than one so that the price elasticity of demand, perceived by a fim, will be larger than one (see equation (5.16)). This is required to avoid negative marginal revenue in a monopolistic situation. 
The utility maximization problem of the consumer can be solved in a two-stage budgeting procedure (Dixit and Stiglitz 1977). ${ }^{66}$ This procedure is described in appendix 5.A. The demand function for each differentiated good implied by the utility function, equation (5.1) is given by equation (5A.15).

$y_{i}=X_{0}^{\varepsilon} \cdot Q_{p, i}^{b \cdot(a-1)} \cdot p_{i}^{-a} \cdot P_{D}^{a-\varepsilon}$

where $a=1 /(1-\rho)>1$ and $\varepsilon=1 /(1-\zeta)>1$.

The definition of $P_{D}$ is stated in equation (5A.16):

$P_{D}=\left[\sum_{i=1}^{n} Q_{p, i}^{b(a-1)} \cdot p_{i}^{1-a}\right]^{\frac{1}{1-a}}$

The elasticity of substitution is given by parameter $a$ and the overall price elasticity of demand is given by $\varepsilon$. It is logical to assume that the elasticity of substitution within a sector is larger than the overall price elasticity of demand $(a>E)$. The demand of a differentiated product will positively depend on the quality level and the overall price index and negatively depend on its own price level. In the symmetric equilibrium the demand function for each variety is:

$y=X_{0}^{e} \cdot Q_{p}^{b \cdot(e-1)} \cdot p^{-\varepsilon} \cdot n n^{\frac{a-e}{1-a}}$

\subsection{Cost Reducing and Demand Creating R\&D with Inter- and Intra-industry R\&D Spillovers}

\subsubsection{Efficiency Improvements}

As in chapters 3 and 4 , efficiency improvements are directed at increasing the productivity level of the production process. We again use a linear homogenous production function for each firm:

$y_{i}=A_{i} \cdot F\left(K_{i^{\prime}} L_{i}\right)=A_{i} \cdot K_{i}^{\alpha} \cdot L_{i}^{1-\infty}$

where $y_{i}$ is output of firm $i, K_{i}$ is capital input of firm $i, L_{i}$ the is labour input of

\footnotetext{
(66) The two stage maximisation process is allowed because the separability of the total utility function (equation $(5.1)$ ) and the linear homogeneity imposed by equation $(5.2)$.
} 
firm $i$ and $A_{i}$ is the total factor productivity or efficiency level of the production process of firm $i$.

As in chapters 3 and 4 , firms engage in process R\&D to improve the efficiency of their production process (see also Dasgupta and Stiglitz 1980, Sato and Suzawa 1983) since a part of the knowledge generated by own R\&D spills over to other firms and industries. We assume that the efficiency of the production process is not only influenced by own process $R \& D$ but also by process $R \& D$ of other firms in the same industry and process R\&D performed in other industries (as Levin and Reiss 1988 assumed). ${ }^{67}$ The "use" of R\&D from firms operating in the same industry is called an intra-industry $R \& D$ spillover effect and the "use" of $R \& D$ from firms operating in different industries is called an inter-industry spillover effect.

It can be expected that knowledge that spills over to firms in the same industry generates a disincentive effect for R\&D efforts. What is especially important in this context is therefore the intra-industry pool of knowledge. It can be modelled as: ${ }^{.68}$

$$
T_{c, i}=R_{c, i}+\omega_{i} \sum_{j \neq i}^{n} R_{c, j}, \quad 0 \leq \omega_{s} \leq 1
$$

where $n$ is the number of competing firms in the same industry. The relevant intra-industry pool of knowledge for firm $i\left(T_{c, i}\right)$ consists of the total amount of knowledge created with own $R \& D$ efforts $\left(R_{c, j}\right)$ and a part of the knowledge created by all other firms in the industry. The part of the competitors R\&D that a firm can use is represented by parameter $\omega_{a}$ and can be called the "extent" of process R\&D spillovers. A low value of $\omega_{i}$ implies that a large part of the knowledge is tacit and can be kept inside the firm while a high value implies that a large part of the generated knowledge is of the "blue-print" type and can be used by other firms. The expected increased codification of knowledge (see chapter 1) implies therefore that the value of $w_{n}$ will increase in the IT era.

We elaborate on the previous mentioned studies by putting this process into a dynamic context and assuming that the efficiency level has a positive influence on the productivity of $R \& D$. The current efficiency level is the result of previous process $R \& D$ and is therefore the result of accumulated knowledge. A higher

67 R:BD from other industries can only be obtaned from industries which also produce differentiated products. In the simple utility function of section two (equation $(5.1)$ we astumed only one differentiated good which make inter-industry spill-overs impossible. The semarability condition of this utility function, our specifications of the product differentivtion process (equations (5.2)) and the presence of the homogenous good, make it possible to introduce more differentated goods wilnout affecting the demand functions of the differentiated good atready present.

68 This way of modelling the knowledge stock is also used in Spence (1984) and Levin and Reiss (1988). 
knowledge stock implies a higher productivity of the R\&D process. ${ }^{6}$ In our specification of the productivity generation process we include the specifications of the earlier contributors:

$$
\frac{d A_{i}}{d t}=\left(\eta \cdot A_{i}^{m} \cdot T_{c, i}^{\theta_{i}} \cdot S_{i}^{\theta_{i}}\right), \mathbb{R}_{c, i}^{\theta} \quad 0 \leq \theta, \theta_{1}, \theta_{2} \leq 1, m_{a}<1
$$

The own level of process $R \& D\left(R_{c, i}\right)$ is the principal determinant of the change in the productivity level $\left(d A_{i} / d t\right)$ of firm $i$. The other three variables - the intraindustry pool of knowledge $\left(T_{c, j}\right)$, the inter-industry pool of knowledge $(S)$, and the productivity level itself $\left(A_{i}\right)$, influence the productivity of own process $\mathbb{R} \& D$. We assume diminishing returns to own R\&D and the two pools of R\&D. The interindustry pool of knowledge is assumed to be exogenous and the intra-industry pool of knowledge is given by equation (5.8). It is important to distinguish between the extent $\left(\omega_{A}\right)$ and the productivity $\left(\theta_{1}\right)$ of process R\&D spillovers. The "extent" represents the part of rival $R \& D$ a firm can use, while the productivity represents the effectiveness of rival R\&D (see Levin and Reiss 1988).

Several special cases of this productivity generating function are used in static studies. By only considering the influence of own process $\mathrm{R} \& \mathrm{D}, \theta_{1}=0, m_{\text {in }}=0$ and $\theta_{2}=0$, one obtains the Dasgupta and Stiglitz (1980) specification. When $\theta=0, m_{a}=0$, $\theta_{2}=0$ and $\theta_{1}=1$, we derive the Spence (1984) specification which is also used by de Bondt et al. (1992). The Levin and Reiss specification, which is most similar to the model specification, can be obtained when $m_{a}=0, \theta_{2}=0$. The specification in the dynamic Sato and Suzawa (1983) analysis will be obtained when $\theta_{1}=0, \theta_{2}=0$ and $m_{a}=1$.

We have two remarks on earher specifications of the productivity generation process. First, Levin and Reiss (1988) separately include own $\mathbb{R} \& D\left(R_{c, i}\right)$ and a pool consisting of own and rival $R \& D\left(T_{i, i}\right)$ which causes double counting of own $R \& D$. They argue that justifying the specification in this manner will "emphasize that own R\&D contributes to a firm's idiosyncratic capabilities as well as to an industry pool of knowledge (Levin and Reiss, p. 540). This logically implies that when a firm engages in own R\&D it creates new knowledge which increases its productivity level. The double counting method, however, implies that when the same new knowledge is added to the general existing knowledge pool, it increases the productivity level of the firm once again although there is no new knowledge available to the firm. We will include this "double counting" method in our specification of the productivity generation process to study its influence on the model results. We can also avoid this double counting by putting the parameter $\theta$

69 In the endogenous growth theories it is also assumed that the knowledge stock adds to the productivity of the research sector (see for example Romer 1990, Grossman and Helpman 1991a, 1991b, and Aghion and Howit 1992). Similar reasoning in the context of human capital accumulation can be found in Lucas (1988). 
equal to zero. Second, other dynamic models (such as Sato and Suzawa 1983) assume that $m$ is equal to $1^{7 a}$ This implies that the same level of R\&D expenditures is required to achieve a certain productivity level growth rate irregardless if one possesses a low or a high productivity level. Is it not more intuitive, however, to assume that more resources are needed if one possesses a higher productivity level.

$$
\begin{array}{ll}
\frac{\frac{d A_{i}}{d t}}{A_{i}}=\eta \cdot f\left(R_{c, i^{\prime}} T_{c, i^{r}} S_{j}\right) \cdot A^{-\beta_{s}} & \beta_{a}>0 \\
\frac{d A_{i}}{d t}=\eta \cdot f\left(R_{c, i^{r}} T_{c, i^{r}} S_{c}\right) \cdot A^{1-\beta_{a}} & m_{a}=1-\beta_{a}<1
\end{array}
$$

\subsubsection{Quality Improvements}

Perceived quality improvements are mainly completed to increase expected demand. Section 5.1 showed us that when quality is positively valued by consumers it has a positive influence on the demand functions. Quality improvements are changes in the real characteristics of a good. It can therefore be expected that a higher quality level increases unit production costs (see e.g. Dorfman and Steiner 1954). When we take into account the variable cost increasing effect of quality we have to adjust our production function (see section 4.2.1).

$$
y_{i}=A_{i} \cdot L_{i}^{1-\alpha} \cdot K_{i}^{\alpha} \cdot Q_{p, i}^{-\xi}
$$

This cost increasing effect of quality is neglected in Levin and Reiss (1988) and in van Mejil and van Zon (1993).

We treat the perceived quality generation process in much the same way as we treated the productivity generation process. Perceived quality improvements can be created by engaging in own product $R \& D\left(R_{d, i}\right)$. The productivity of own product $R \& D$ is dependent on the intra-industry pool of product knowledge $\left(T_{d, i}\right)$ and the inter-industry pool of product knowledge $\left(S_{d}\right)$ and is dependent on the quality level itself $\left(Q_{p, i}\right)$, which can be seen as the knowledge stock of product $R \& D$. The quality generating process is: ${ }^{71}$

70. This is also atn assumption which is characteristic of the "new" growth theories (see chaplex 2 ).

78 When product ReD is interpreted ass averising and the guality level ass goodwill this function contains several specifications which have been used in prewious studies. The Nerlove and Arrow (1962) specification cin be oblained when $m_{2}=\delta_{1}=\delta_{2}=\delta_{3}=0$ and $\delta=\gamma=1$. The Gould (1970) speciffcation differs from this Nerlove and Arrow specifications in that $8 \neq 1$. 


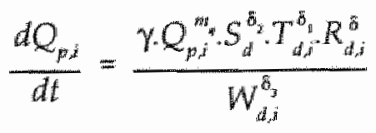

The inter-industry knowledge stock is again exogenous to the firm. The intraindustry knowledge stock is constructed in the same manner as in the case of process $R \& D$ and contains own product $R \& D$ and a fraction of the product $R \& D$ of all other firms. "This fraction, $\alpha_{n}$, symbolizes the "extent" of the quality spillover effect and we expect that this fraction has increased during the information technology area through increased codification.

$$
r_{d, j}=R_{d, i}+\omega_{i,} \sum_{j, i}^{s t} R_{d, j}
$$

This situation is again characterised by double counting of own product R\&D. Again we will compare the influence of this double counting method with the method which avoids double counting by setting $\delta$ equal to zero. Another aim of this study is to investigate the consequences of a higher value of $\omega_{\text {, }}$ caused by increased codification, on the technological and economical performance of firms.

At this point the similarities with the productivity generation process ends. As Levin and Reiss (1988) did, we use perceived quality, which is the quality level of a product relative to the quality level of similar or competing products. An increase in the product $R \& D$ level of competing products may therefore reduce the perceived attractiveness of a firm's product. In chapters 3 and 4 we introduced the "perceivedness" with a constant exponential decay of the perceived quality level. However, in this chapter we also consider a firm's competitors in the same industry. We assume that the perceived quality level of a product diminishes if competing products in the same industry enhance their quality level. Quality enhancing R\&D activities by competitors generates a threat to the perceived quality level. This threat is treated as endogenous in this model. Levin and Reiss assumed that the intramindustry spillover pool, own R\&D and a part of other firms R\&D $\left(T_{d, j}\right)$, is also the relevant pool of available knowledge which has a negative influence on the perceived-quality generation process. The parameter $\delta_{1}$ can therefore be positive or negative in their model. However, the relevant knowledge pool which threatens (reduces) a firm's perceived quality level is not its own R\&D and a part of other firms R\&D but other firms R\&D and a part of its own R\&D. The threatening intra-industry knowledge pool, $W_{d, i^{*}}$ can therefore be defined as:

$$
W_{d, i}=\sum_{j \neq i}^{n}\left[R_{d i, j}+\omega_{i, j} \sum_{i \neq j}^{t h} R_{d t, j}\right]
$$


This threatening intra-industry pool of knowledge demonstrates clearly the disincentive effect of higher knowledge spillovers caused in the information technology area. If other firms can use a larger part of internally created knowledge to increase their quality level, then the perceived quality level of the firm that created the knowledge will be less valued. The incentives to invest in product R\&D diminish through the introduction of the threatening intra-industry pool.

\subsection{An Endogenous Technological Change Model}

Consider a firm in a differentiated product industry that chooses its price level, productivity level and quality level so as to maximize the present value of its profits. It can influence its productivity level by engaging in process R\&D and its quality level by engaging in product $R \& D$. The inter-temporal profit maximisation problem for a firm is:

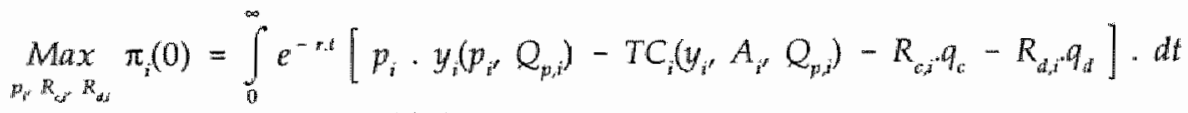

$$
\begin{aligned}
& \text { s.t. } y_{i}=X_{0}^{e} \cdot Q_{p, i}^{b, j a-1)} \cdot p_{i}^{-a} \cdot P_{D}^{a-\varepsilon} \quad, a>\varepsilon>1 \\
& P_{D}=\left[\sum_{i=1}^{n} Q_{p, i}^{b \cdot(a-1)} \cdot p_{i}^{1-a}\right]^{\frac{1}{1-a}} \\
& T C_{i}=\frac{Q_{p, i}^{\xi}}{A_{i}} \cdot y_{i} \cdot w^{1-\alpha} \cdot v^{\alpha} \cdot(1-\alpha)^{\alpha-1} \cdot \alpha^{-\alpha}, 0 \leq \alpha \leq 1 \\
& d A_{i} / d t=\eta \cdot A_{i}^{m_{s}} \cdot R_{c, i}^{\theta} T_{c, i}^{\theta_{1}} \cdot S_{c}^{\theta_{2}} \quad, 0 \leq \theta_{i}, \theta_{1}, \theta_{2} \leq \mathbb{1}, m_{a}<1 \\
& d Q_{p, i} / d t=\left(\gamma \cdot Q_{p, i}^{m_{q}} \cdot R_{d, i}^{\delta} \cdot T_{d, i}^{\delta_{i}} S_{d i}^{\delta_{i}}\right) / W_{d, i}^{\delta_{3}}, 0 \leq \delta, \delta_{1}, \delta_{2,} \delta_{3} \leq 1, m_{q}<1 \\
& A_{i=0}=A_{0}, Q_{p, t=0}=Q_{p, 0}
\end{aligned}
$$

where $w$ is the wage level and $v$ is the user cost of capital. The presence of subscripts $c$ or $d$ in a variable implies that this variable is related to process R\&D and product $R \& D$ respectively. The subscript $a$ in a parameter indicates a relationship with productivity and the subscript $q$ indicates a relationship with quality.

We assume that the firm takes the price and R\&D strategy of other firms as given. The analysis is limited to the symmetric case: costs, technological opportunities, initial quality and productivity level and demand conditions for each firm within an industry are equal. The number of firms $(n)$ is exogenous, which enables us to investigate the effects of market concentration on technological progress and welfare. Furthermore, we assume that wages $(w)$, the user cost of capital $(v)$, the process- and product R\&D prices (respectively $q_{c}$ and $q_{d}$ ), the interindustry process- and product R\&D stocks (respectively $S_{c}$ and $S_{d}$ ) and the autonomous scale of demand $\left(X_{0}\right)$ grow at a constant exogenous growth rate. 
We use optimal control theory to solve this problem. The state variables are a firms" productivity level $\left(A_{i}\right)$ and its quality level $\left(Q_{p, i}\right)$ and the control variables are its price level $\left(p_{i}\right)$, process $R \& D$ level $\left(R_{c, i}\right)$ and product $R \& D$ level $\left(R_{d, i}\right)$. The solution of the current value Hamiltonian associated with this problem is given in appendix $5 \mathrm{~B}$ and appendix $5 \mathrm{C}$. In this section we use the results calculated in appendix $5 \mathrm{~B}$.

\section{Characteristics of Potential Equilibrium}

First, marginal costs have to be equal to marginal revenue for the three choice variables. With respect to the price level, marginal costs are equal to the "perceived" marginal revenue (see equation (5B.3) and equation (5B.4) in appendix B).$^{72}$

$$
\left(1-\frac{1}{a_{p}}\right) \cdot p_{i}=\frac{T C\left(A_{i,}, Q_{p, i}\right)}{y_{i}} ; a_{p}=a-\left(\frac{a-\varepsilon}{n}\right)>1
$$

where $a_{p}$ is the perceived price elasticity by a firm. It is called the perceived price elasticity because it is calculated under the assumption that other firms keep their price and R\&D levels fixed, which is not necessarily the case. The perceived price elasticity increases with the number of firms/varieties $(n)$, the elasticity of substitution between varieties within an industry $(a)$ and the general price elasticity of demand $(\varepsilon)$. It approaches the elasticity of substitution $(a)$ as the number of firms becomes large and it is equal to the general price elasticity $(\varepsilon)$ in a monopolistic situation. The price is determined as a mark-up above marginal costs. The specification of the production function implies that marginal costs are independent of the production level and equal to the variable production costs. The mark-up is only dependent on the perceived price elasticity.

The static marginal cost-is-equal-to-marginal revenue conditions for process and product $\mathbb{R} \& D$, and are calculated in equation (5B.6) and equation (5B.7), respectively.

$$
\begin{aligned}
& q_{c}=\mu \cdot \frac{\partial\left(d A_{i} / d \mathrm{i}\right)}{\partial R_{c, i}} \\
& q_{d}=\lambda \cdot \frac{\partial\left(d Q_{p, j} / d t\right)}{\partial R_{d, i}}
\end{aligned}
$$

72 Helpmatr and Krugman (1989, p. 90) describe the perceived marginal tevenue as "perceived marginat reverue-the increase in revenue that a fim expects to receive by producing one more unit, which is always less than the price (because of the effect on intra-marginal sales) but may exceed the true marginal revenue that would prevail if the industry acted in concert". 
where $\mu$ and $\lambda$ are the co-state variables of the optimal control problem. They can be interpreted as the marginal value of $Q_{p, i}$ and $A_{i}$ respectively or the increase in future profits resulting from an increase in $Q_{p, i}$ and $A_{i}$ respectively at time $t$. With this in mind, it is easy to see that the right hand side of these two equations depicts the marginal profits of $R \& D$ in the future, while the left side represents current marginal R\&D costs. To identify the influence of, for example, spillover effects on marginal profits, we must calculate the partial derivatives. To simplify the expressions we directly substituted the equilibrium values. Because we consider only the symmetric equilibrium this implies e.g. that $R_{c j,}=R_{c, j}$ (see equation (5B.6) and equation (5B.7)):

$$
\begin{aligned}
& q_{c}=\mu \cdot\left[\eta \cdot A^{m_{x}} \cdot(1+(1) \cdot(n-1))^{\theta_{1}} \cdot R_{c}^{\theta_{1}} \cdot S_{c}^{\theta_{2}}\right] \cdot\left[\theta+\frac{\theta_{1}}{1+\omega_{i} \cdot(n-1)}\right] \cdot R_{c}^{\theta-1}
\end{aligned}
$$

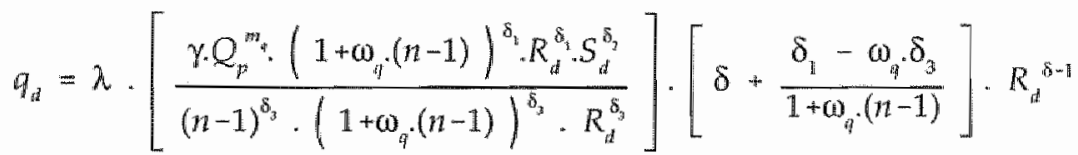

The first term between brackets on the right hand side of both equations represents the productivity level of own $R \& D$ expenditures and the second term between brackets on the right hand side of both equations represents the R\&D elasticity in the symmetric equilibrium. The extent of process R\&D $\left(\omega_{\Omega}\right)$ and product R\&D spillovers $\left(\omega_{q}\right)$, which reflect the part of knowledge that spills over to other firms, has a negative influence on the process and product $R \& D$ elasticity. This reflects, therefore, the disincentive effect of knowledge spillovers (less appropriability). However, an incentive effect in the case of process R\&D also exists because the extent of process R\&D spillovers has a positive influence on the productivity level of internal process $R \& D$. This is due to the fact that a firm can use more knowledge generated by competing firms (see equation (5.19)). In the case of product R\&D (equation (5.20)), the influence of the extent of product R\&D spillovers is ambiguous. Similar to the extent of process R\&D spillovers it increases the intra-industry spillover pool $\left(T_{d, i}\right)$ which has a positive influence, but also increases the threatening intra-industry spillover pool $\left(W_{d, j}\right)$, which has a negative influence on the productivity of product $R \& D$. If $\delta_{1}>\delta_{3}$, then the first effect dominates the second and $\omega_{i j}$ has a positive affect on the productivity level of product $R \& D$. The total affect of the extent of spillovers on the marginal benefits of internal $R \& D$ is therefore also ambiguous.

We now consider other influences on the R\&D elasticities depicted in the second term between brackets in equation (5.19) and equation (5.20). It is important to note the different influences on these elasticities by the extent $\left(\omega_{1}, \omega_{4}\right)$ and productivity $\left(\theta_{1}, \delta_{1}, \delta_{3}\right)$ of spillovers. The productivity $\left(\delta_{3}\right)$ of the threatening knowledge pool $\left(W_{d, j}\right)$ has a negative influence on the product $R \& D$ elasticity whereas the 
productivities $\left(\theta_{1}, \delta_{1}\right)$ of the intra-industry spillover pool $\left(T_{c, i}, T_{d i}\right)$ have a positive influence on these elasticities. If we compare the elasticity of product $R \& D$ with that in the Levin and Reiss specification, in which $W_{c, i}$ is equal to $T_{c, j^{r}}$ then the elasticity of product $\mathbb{R} \& D$ is $\left[\delta+\left(\delta_{1}-\delta_{3}\right) /\left(1+\omega_{4} \cdot(n-1)\right]\right.$. With regard to this threatening intra-industry spillover pool our specification recognises that only a part of our own R\&D will be used by other firms.

The perceived quality elasticity of demand $\left(b_{p}\right)$ is calculated in equation (5B.10):

$b_{p}=b \cdot(a-1)-\frac{(a-\varepsilon) \cdot b}{n} \quad, b \cdot(\varepsilon-1) \leq b_{p}<b \cdot(a-1)$

The first term on the right hand side is the direct influence of quality on demand and the second term is the influence of quality on $P_{p}$; the indirect effect on demand. Interestingly the perceived quality elasticity increases as the number of firms increase. A higher perceived quality elasticity means that the benefits of investing in quality improvements are perceived to have a greater influence on expected demand. This means that entry stimulates technological progress.

\subsection{Steady State Growth Rates}

The steady state growth rates are calculated in appendix 5B. Equation (5B.25) states the steady state growth rate of process and product $R \& D .^{73}$

$$
\hat{R}^{*}=M \cdot\left[\varepsilon \cdot \hat{X}_{0}-\hat{q}_{c}-(\varepsilon-1) \cdot z_{1}+(\varepsilon-1) \cdot(b-\xi) \cdot\left(\frac{\delta_{2}}{1-m_{q}}\right) \cdot \hat{S}_{d}+(\varepsilon-1) \cdot\left(\frac{\theta_{2}}{1-m_{a}}\right) \cdot \hat{S}_{c}\right]
$$

where $Z_{1}=(1-\alpha) \cdot \hat{w}+\alpha \cdot \hat{0}$ and $M$ is the steady state growth rate multiplier.

$$
M=\frac{1}{1-(\varepsilon-1) \cdot(b-\xi) \cdot\left(\frac{\delta+\delta_{1}-\delta_{3}}{1-m_{i}}\right)-(\varepsilon-1) \cdot\left(\frac{\theta+\theta_{1}}{1-m_{n}}\right)}
$$

*3 To simplify the steady state growth rate equation we made the not uncommon assumption that the growth rattes of the prices of process and product R\&D are equal. As a result the $\hat{q}_{d}-\hat{q}_{c}$ term in equation (5B.25) cancels cut. Another result of this assumption is that the growth tates of process and product R\&D are equal, see equation (5B.27), i.e. $\hat{R}_{e, i}^{*}=\hat{R}_{d, i}^{*}=\vec{R}^{*}$. 
For expository convenience we have dropped the subscript $i$. This can be done because firms take the same decisions in a symmetric equilibrium. Before equation (5.22) is discussed, a few assumptions must be made. First, the value of the multiplier, $M$, is positive and second, $b>\xi$. The first assumption is made to derive a meaningful situation in which, for example, the growth rate of the price of $\mathbb{R} \& D$ has a negative influence on the growth rate of $R \& D{ }^{74}$ The second assumption is required to make product $R \& D$ profitable; and states that the demand creating effect $(b)$ is larger than the cost increasing effect of quality $(\xi)$.

Nested within equation (5.22) is the result derived by van Meijl and van Zon (1993) which can be achieved when $\xi=m_{a}=m_{d}=\theta_{1}=\delta_{1}=0$ and $\hat{S}_{c}=\hat{S}_{d}=0$. The discussion of the steady state growth rates is therefore especially geared toward these parameters and growth rates.

The influence of the various spillover effects is quite different. The "extent" of intra-industry spillovers $\left(\omega_{a}\right.$ or $\left.\omega_{q}\right)$ has no influence on the steady state growth rate of R\&D. The negative influence of this effect on the R\&D level found by Spence (1984) and Levin and Reiss (1988), cannot, therefore, be observed for the R\&D growth rates. The "productivity" $\left(\theta_{1}, \delta_{1}\right)$ of the intra-industry process and product $R \& D$ spillovers increases the value of the multiplier and therefore strengthen the positive or negative effect of exogenous growth rates. The productivity of the threatening spillover pool $\left(\delta_{3}\right)$ decreases the value of the growth rate multiplier. The productivity and growth rate of inter-industry spillovers have a positive influence on the steady state R\&D growth. Finally, it is apparent that product (process) inter-industry $\mathrm{R} \& \mathrm{D}$ spillovers also have a positive influence on the growth rate of process (product) $R \& D$.

The denominator of the growth rate multiplier includes two terms that describe the influence of product and process $\mathbb{R} \& D$. We first explain the influence of process $R \& D$, the third term in the denominator. A firm engages in process $R \& D$ to increase productivity. Own process $R \& D$ is productive in three ways; 1 ) direct productivity effect $(\theta), 2)$ indirect productivity effect via intra-industry $R \& D\left(\theta_{1}\right)$, and 3) indirect productivity effect via the own knowledge stock $\left(m_{t}\right)$. The total influence of process $R \& D$ on productivity is therefore given by $\left(\theta+\theta_{1}\right) /\left(1-m_{A}\right)$. The increased productivity, induced by process $R \& D$, decreases the price level and increases revenue by $(\mathrm{E}-1)$ times the productivity change. The total third term therefore characterises the cost-reducing effect.

A similar explanation can be found for the second term which includes the product $R \& D$ effect. A marginal increase in product $R \& D$ leads to a change in quality of $\left(\delta+\delta_{1}-\delta_{3}\right) /\left(1-m_{q}\right)$. This quality change influences the demand directly by $b .(\varepsilon-1)$ and indirectly via higher costs $\rightarrow$ higher price level by $\xi \rightarrow$ lower demand by $\xi . \varepsilon \rightarrow$ lower revenue by $\xi .(\varepsilon-1)$. The second term therefore represents the demand creating effect.

74 In our analyses of the dynanics of the system we also find that when this condition holds we have a saddle point stable situation and when this is not the case the system becomes unstable, see appendix $5 \mathrm{D}$. 
Both the cost-reducing and demand creating effects increase the value of the growth rate multiplier. The value of the multiplier is therefore higher than in a situation where one can engage in either product $R \& D$ or process $R \& D$. The cost reducing and demand creating effects reinforce each other rather then compete with each other.

Finally, the growth rate of the autonomous scale of demand has a positive influence and the growth rates of unit costs and the price of process R\&D have a negative influence on $\hat{R}_{*} \cdot 75$

\subsection{Dynamics}

The dynamic behaviour of this system can be studied by analyzing the four differential equations. These are non-linear and dependent on time ${ }^{76}$, which makes the system aralytically intractable. As in section 3.3 we used a qualitative graphic or phase-diagram analysis to study dynamics. A condition for this analysis is that the differential equations are not dependent on time. Appendix $5 C$ therefore uses a time elimination method to make the system autonomous: the endogenous variables will be deflated with their steady state growth rates.

The two dynamic constraints in terms of the redefined or deflated variables are:

$$
\begin{aligned}
& \frac{d A^{\prime \prime}}{d t}=\eta \cdot A^{\prime \prime m_{0}} \cdot R_{c}^{\prime \prime \theta} \cdot T_{c}^{\prime \prime \theta_{1}} \cdot S_{c}^{\prime / \theta_{\alpha}}-\sigma_{s} \cdot A^{\prime \prime}
\end{aligned}
$$

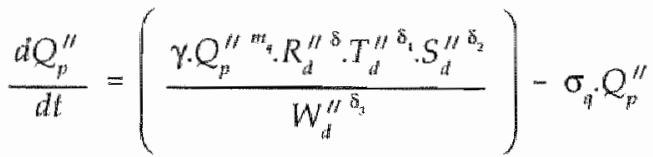

where $x^{\prime \prime}=$ the deflated value of variable $x^{77}$, and $\sigma_{x}$ is the steady state growth rate of variable $x$. In comparison with the dynamic constraints of the original system stated in equation (5.9) and equation (5.12), a depreciation factor exists in the new constraints, which is equal to the steady state growth rate.

The $d R_{c}^{\prime \prime} / d t$ and $d R^{\prime \prime} / d t$ differential equation are given by equation (5C.16) and equation (5C.19) respectively in appendix $5 \mathrm{C}$.

75 The wige and user cost of the capital growth rate have a negative inlluence on the R\&D staady state growth rate, which seems counter intuitive bectuse higher input prices imply that the potential benefits of process RQD per mit of output increases. But in st situation with a price-elastic demand curve $(a, \varepsilon>1)$ higher input prices also imply that the price level will increase, which decreases demand more than proportionaly.

76 The time dependency is ctused by the assumption that several exogenous variables, which enter the dillerential equations, have a constant growth rate.

77 The mathematical expression of this dellation method is stated in equation (5C.1) in appendix $5 \mathrm{C}$. 


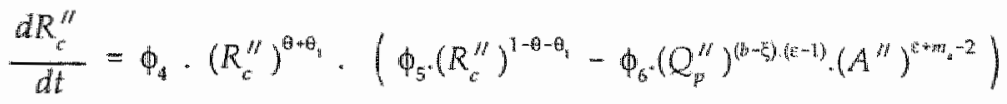

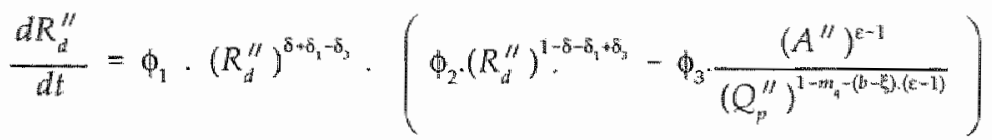

where the $\phi_{i}$ s are described in appendix $5 \mathrm{C}$ and only depend on a number of parameters and exogenous variables.

As in section 3.3, we divide the total system into two parts. One sub-system describes the relationship between process $R \& D$ and productivity given the quality level and the other characterises the relationship between product R\&D and quality given the productivity level.

\section{Quality Generation}

Let us assume that productivity improvements are not possible. A firm only engages in product $R \& D$ to enhance its quality level and considers its productivity level as given. The dynamics of this system are characterised by equation (5.25) and equation (5.27). These differential equations together describe the movement of $Q_{p \prime}^{\prime \prime}$ and $R_{d}^{\prime \prime}$ in the phase diagram. The two demarcation lines, $d Q_{p}^{\prime \prime} / d t=0$ and $d R_{d}^{\prime \prime} / d t=0$, which describe any potential equilibrium are:

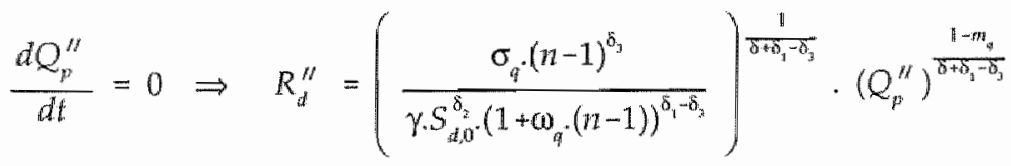

$$
\begin{aligned}
& \frac{d R_{d}^{\prime \prime}}{d t}=0 \Rightarrow R_{d}^{\prime \prime}=\left[\left(\frac{\phi_{3}}{\phi_{2}}\right) \cdot \frac{\left(A^{\prime \prime}\right)^{c-1}}{\left(Q_{p}^{\prime \prime}\right)^{\left.1-n_{i}-(p-\xi)^{\prime}\right)((-1)}}\right]^{\frac{1}{1-\delta-S_{1}+\delta_{3}}}
\end{aligned}
$$

The $d Q_{p}^{\prime \prime} / d t=0$ is drawn in figure 5.1 and is positively sloped $\left(m_{q}<1\right.$ and we assume $\left.0<\delta+\delta_{2}-\delta_{3}<1\right)$. The slope of the $d R_{d}^{\prime \prime} / d t=0$ locus is negative if $1>m_{q}+(b-\xi)$. $(\varepsilon-1)$ which is most likely to be the case. This siruation, which is drawn in figure 5.1 , is familiar to us because we have seen it in chapter 3. This configuration of horizontal and vertical arrows therefore implies saddle point stability. These trajectories are illustrated in figure 5.1 by the dotted lines which lead to the equilibrium $\left(Q_{p}^{\prime \prime} ", R_{d}^{\prime \prime}\right)$. This unique or saddle path is also the optimal path,

78 There are exacty one peir of trajectories which lead to the equilibrituris $t \rightarrow \infty$ (see chapter 3 ). 
because all other paths ultimately lead to an infinitely large level of $Q_{p}^{\prime \prime}$ and $R_{d}^{\prime \prime}$ or to a zero level of perceived quality. ${ }^{79}$ Given the firms initially quality level, $Q_{y, k}^{\prime \prime}$, the optimal product $R \& D$ level to be chosen is the corresponding point on the saddle path, $R_{d, 0}$.

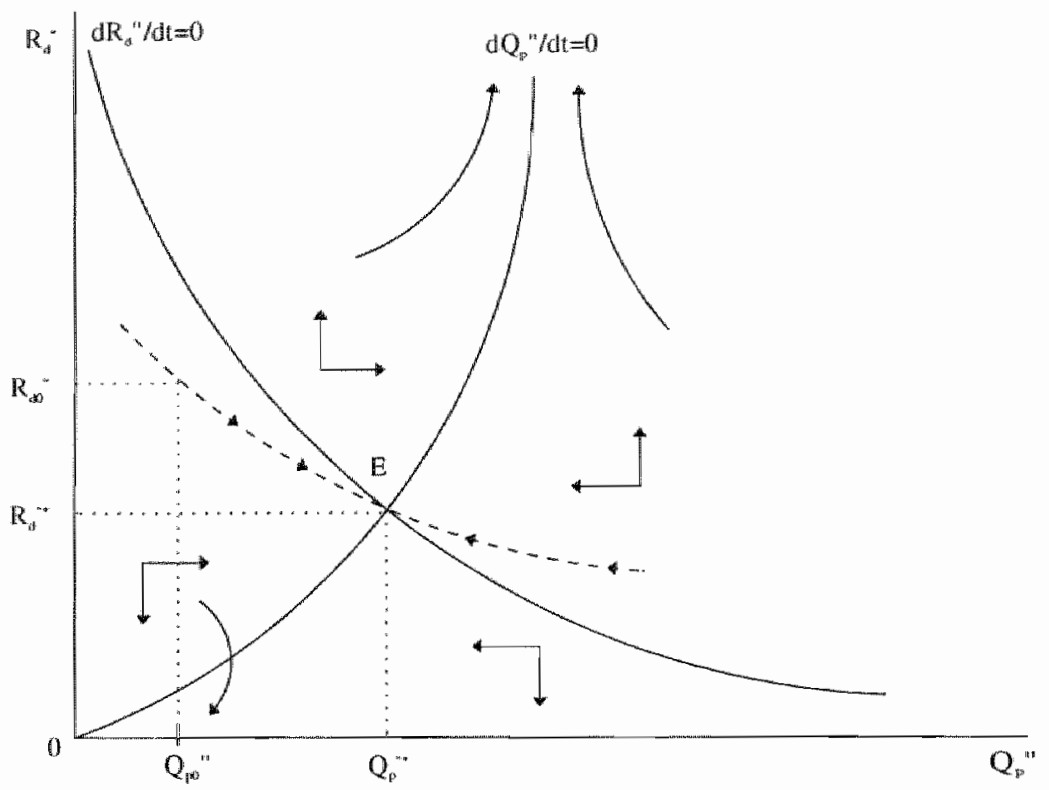

Figure 5.1. Phase diagram: Product R\&D and Demand Creation.

Lets continue the discussion about the slope of the $d R_{d}^{\prime \prime} / d t=0$ locus. When $1=m_{i}+(b-\xi) \cdot(c-1)$ the locus is horizontal and when $1>m_{t}+(b-\xi) \cdot(\varepsilon-1)$ the slope of the locus becomes positive. According to the arrow configurations, the system saddlepoint remains stable if the slope of the $d A^{\prime \prime} / d t=0$ locus is steeper than the slope of the $d R_{i}^{\prime \prime} / d t=0$ locus. The system is therefore saddle point stable as

79 "The intuition for the non-optimality of an intinitely large $\left(Q_{p}^{\prime \prime}, R_{d}^{\prime \prime}\right)$ or a zero level of perceived qualty lewel is that if your quality level is perceived to be zero by consumers, your expected demand will also be zero, which is not an economically viable situation. An infinitely large level of product $\mathbb{R} \& D$ and perceived quality is also not optimal because there are diminishing returns to product R\&D in the productivity generation process and to demand creating effects of quality. For a technical discussion of these statements see chapter 3 . 
$1>m_{q}+(b-\xi) \cdot(\varepsilon-1) \cdot\left(\delta+\delta_{1}-\delta_{3}\right)$ and unstable as $1 \leq m_{q}+(b-\xi) \cdot(\varepsilon-1) \cdot\left(\delta+\delta_{1}-\delta_{3}\right) .50$ The system is therefore stable as long as the quality generating opportunities are not too large.

We now examine what happens when the given productivity level changes. If the value of $A^{\prime \prime}$ changes, then the $d R_{d}^{\prime \prime} / d t=0$ locus, moves upwards (downwards) as the productivity level is higher (lower). These movements have no influence on the stability characteristics. The $d R_{d i}^{\prime \prime} / d t=0$ locus shifts because the marginal costs of performing product R\&D are equal to the marginal benefits. A higher (lower) productivity level increases (decreases) the benefits while costs stay the same. This results in an upward (downward) shift of the $d R_{d}^{\prime \prime} / d t=0$ locus.

\section{Productivity Improvement}

We will now assume that only productivity improvements are possible. The dynamics of this system are characterised by equation (5.24) and equation (5.26), which describe the movement of $A^{\prime \prime}$ and $R_{c}^{\prime \prime}$ in the phase diagram. The two demarcation lines are:

$$
\begin{aligned}
& \frac{d A^{\prime \prime}}{d t}=0 \Rightarrow R_{c}^{\prime \prime}=\left(\frac{\sigma_{a}}{\eta \cdot S_{c, 0^{\prime}}^{\theta_{2}}\left(1+\omega_{a^{\prime}} \cdot(n-1)\right)^{\theta_{1}}}\right)^{\frac{1}{\theta+\theta_{1}}} \cdot\left(A^{\prime \prime}\right)^{\frac{1-m_{c}}{\theta+\theta_{1}}}
\end{aligned}
$$

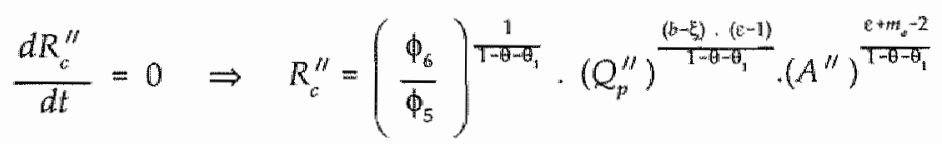

${ }^{80}$ We checked the saddle point stability by a first order Taylor expansion of the non-linear differential system around its equilibrium. The Jacobean matrix evaluated at the stealy-state point (E) is:

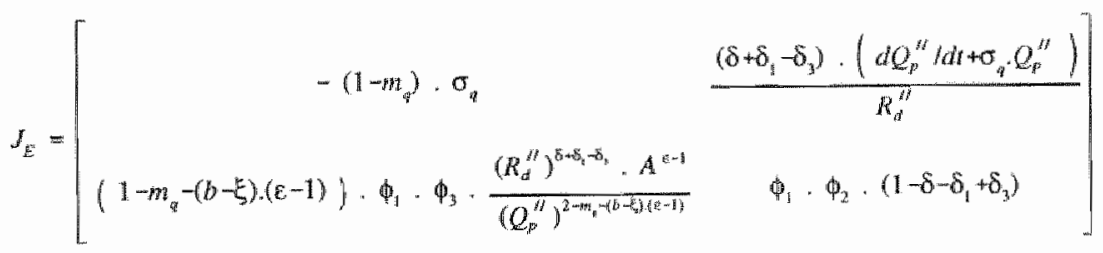

It is simple to werify that the determinant of the Jacobean matrix can eventually be written as:

$$
r_{1} \cdot r_{2}=\left|J_{E}\right|=-\phi_{1} \cdot \phi_{2} \cdot \sigma_{q} \cdot\left[1-m_{q}-(b-\xi) \cdot(\varepsilon-1) \cdot\left(\delta+\delta_{1}-\delta_{3}\right)\right]
$$

The value of the determinant is negative as $1>m_{q}+(b-\xi)(\varepsilon-1) \cdot\left(0+\delta_{1}-\delta_{3}\right)$. This cnables us immediately to conclude that the system is saddlepoint stable because the two characteristic roots, $r_{1}$ and $r_{2}$, have opposite signs. When $1 \leq m_{3}+(b-\xi)(\varepsilon-1) \cdot\left(\delta+\delta_{1}-\delta_{3}\right)$ the determinant of the Jacobean natrix is positive and we are not able to draw conclusions about the local stability of the system. To make such an inference we must calculate the trace of the Jacobean matrix: tr $J_{E}=r_{1}+r_{2}=r-\sigma$. The trace is positive if $r a \sigma$. This condition is fulfulled if the transwersality condition is satisfied (see equation (5C.12)). Positive values for both the determinant and the trace of the Jacobean matrix implies a locally unistable equilibrium. For more details of the procedure of linearization of a non-linear system, see A.C. Chiang (1984) section 18.6. 
The $d A^{\prime \prime} / d t=0$ is positively sloped, $m_{i t}<1$. The slope of the $d R_{z}^{\prime \prime} / d t=0$ locus is dependent on the value of the general price elasticity, $\varepsilon$ and the value of the knowledge stock elasticity of the productivity generation process, $m_{a}$. When $\varepsilon+m_{a}<2$ the $d R_{c}^{\prime \prime} / d t=0$ locus has a negative slope. The locus is horizontal when $\varepsilon+m_{a}=2$ and has a positive slope if $\varepsilon+m_{i b}>2$. This situation is analogous to the quality generation case and the phase diagram associated with this system looks similar to figure 5.1. When the price elasticity of demand gets higher, the $d R_{c}^{\prime \prime} / d t=0$ locus rotates counter-clockwise. The system stays saddle point stable as long as the slope of the $d A^{\prime \prime} / d l=0$ locus is steeper than the slope of the $d R_{c}^{\prime \prime} / d t=0$ locus. The system is therefore saddle point stable as long as the price elasticity of demand is $1<\varepsilon<\left(1-m_{4}+\theta+\theta_{1}\right) /\left(\theta+\theta_{1}\right)$ and unstable if $\varepsilon \geq\left(1-m_{2}+\theta+\theta_{1}\right) /\left(\theta+\theta_{1}\right)$. $^{81}$

\section{Quality and Productionty Generation}

By integrating the two sub-systems, the dynamics of a situation where both quality and productivity changes are possible can be examined. Productivity improvements created by process $R \& D$ increase the marginal benefits of product $R \& D$ while quality improvements induced by product R\&D increase the marginal benefits of process R\&D. In section 3.3 we used two phase diagrams to explain this process, and deduced that the total system was saddle point stable under certain conditions. This implied a unique long run adjustment path if the initial values of the state variables were not equal to the steady state values (see figure 3.11 and 3.12). We will not repeat this analysis but will only determine the conditions under which the model developed in this chapter is saddle point stable. In appendix 5D we checked the conditions of Feichtinger and Hartl for a saddle point plane and concluded that this system is saddle point stable when the following condition holds:

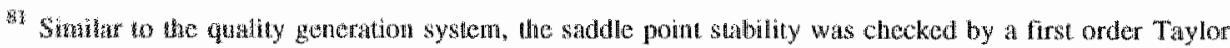

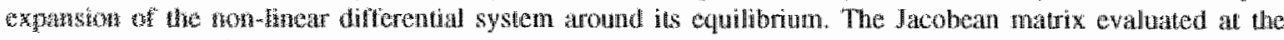

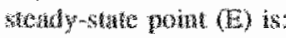

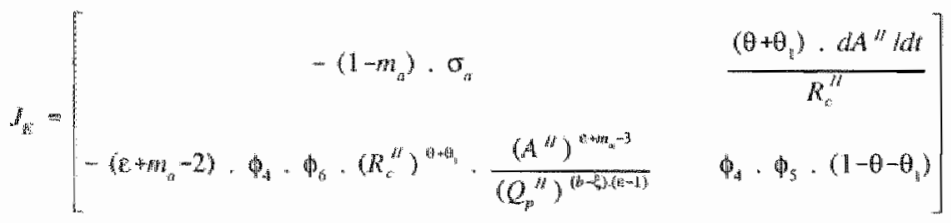

is simple to verify that the cletermintut of dhe Jocobean matrix can eventually be written as:

$$
v_{\mathrm{i}} \mathrm{a}_{2}=\left|d_{\mathrm{t}}\right|=-\phi_{4} \cdot \phi_{5} \cdot \sigma_{4} \cdot\left[1-m_{6}-(\varepsilon-1) \cdot\left(\theta+\theta_{1}\right)\right]
$$

The value of the determinam is negative as $1>m_{4}+(c-1) \cdot\left(\theta+\theta_{1}\right)$, which imples saddle point stability. When $1 \leq m+(\varepsilon-1)\left(\theta+\theta_{1}\right)$ the deterninant is positive. The value of the trace is again $r-\sigma$ which together with the postuve determinatimplies a locully unstable equilibrium. 


$$
1>(b-\xi) \cdot(\varepsilon-1) \cdot\left(\frac{\delta+\delta_{1}-\delta_{3}}{1-m_{4}}\right)+(\varepsilon-1) \cdot\left(\frac{\theta+\theta_{1}}{1-m_{4}}\right)
$$

This expression is familiar as it is the denominator of the growth rate multiplier (see equation (5.23)).

\subsection{Steady State}

The steady state values of the redefined systern can be obtained by putting the four differential equations equal to zero. Appendix $5 \mathrm{C}$ shows these calculations.

\subsubsection{RED mtensities}

An important measure of technological change is the $R \& D$ intensity of production. The $R \& D$ intensity is defined as the $R \& D$ expenditures to total revenue ratio. In this paragraph we investigate which factors favour or temper this technological change measure. The steady state product and process $R \& D$ intensities are given by equation (5C.27) and equation (5C.28) respectively.

$$
\begin{aligned}
& \phi_{c}=\left(1-\frac{1}{a_{p}}\right) \cdot\left(\theta+\frac{\theta_{1}}{1+\omega_{a} \cdot(n-1)}\right) \cdot\left(\frac{\sigma_{a}}{\gamma-\sigma+\left(1-m_{a}\right) \cdot \sigma_{a}}\right) \\
& \phi_{d q}=(b-\xi) \cdot\left(1-\frac{1}{a_{p}}\right) \cdot\left(\delta+\frac{\delta_{1}-\omega_{q} \delta_{3}}{1+\omega_{q} \cdot(n-1)}\right) \cdot\left(\frac{\sigma_{q}}{r-\sigma+\left(1-m_{q}\right) \cdot \sigma_{q}}\right)
\end{aligned}
$$

where $\phi_{c}$ denotes the process R\&D intensity and $\phi_{d}$ denotes the product R\&D intensity.

It is apparent that the "extent" of the intra-industry process (product) R\&D spillovers has a negative influence on the steady state process (product) R\&D intensity. Therefore information technology, which induces a higher value of $\omega_{A^{\prime}}$ lowers internal $R \& D$ intensities, ceteris paribus. The perceived price elasticity, $a_{p}=a-(a-\varepsilon) / n$, has a positive influence on both $\mathrm{R} \& \mathrm{D}$ intensities. For a given number of firms, this generates the interesting result that the $R \& D$ intensity is higher for products which are characterised by a higher elasticity of substitution (a) or a higher general price elasticity $(c)$. The explanation for this is that a higher perceived price elasticity, which implies a higher perceived quality elasticity, means that productivity and quality improvements are expected to result in larger changes in demand. 
A higher elasticity of substitution means that the "Love of Variety" effect is lower (see section 5.1). Variety per se is valued less by consumers which implies that the products are perceived as less differentiated by consumers and that competition between different varieties increases. We interpret the elasticity of substitution as an indicator of the degree of product differentiation. The higher the elasticity of substitution, the lower the degree of product differentiation. This implies that products which have a higher degree of product differentiation have a lower process and product $R \& D$ intensity.

However, the "correct" price elasticity for a firm in a symmetric industry is the inter-industry price elasticity ( $\mathrm{E})$, which is smaller than the perceived elasticity. The assumption of the competitor's given price and quality level, which is the cause of the higher perceived price elasticity, therefore favours technological change. If firms considered the reactions of other firms, they would have $\varepsilon$ as price elasticity and would have invested less in $R \& D$. Without spillover effects, price setting firms over-invest in process and product $R \& D$ with respect to their optimal profit level. ${ }^{\text {s2 }}$

With spill-over effects there is an under-investment in process R\&D. Firms determine their optimal process $R \& D$ level while taking the process $R \& D$ level of competitors as constant. The elasticity on the industry pool of process $R \& D$ is perceived as $\theta_{1} /\left(1+\omega_{n}(n-1)\right)$ but is $\theta_{1}$ in a symmetric industry. The perceived elasticity on the industry pool of R\&D is therefore lower than the "correct" elasticity, which leads to an under-investment in process $R \& D$ with respect to optimal profits.

In the case of product $R \& D$ we also obtain an under-investment in $R \& D$ if we only consider the influence of the intra-industry spillover pool, $T_{d, i}$, because the elasticity of this industry pool is perceived as $\delta_{1} /\left(1+\omega_{p}(n-1)\right)$ while it is $\delta_{1}$ in a symmetric industry. However the perceived elasticity of the threatening intraindustry spillover pool, $W_{d, j,}$, is $-\delta_{3} \cdot \omega_{q} /\left(1+\omega_{q}(n-1)\right)$ but $-\delta_{3}$ in a symmetric equilibrium. The negative influence of the threatening pool is therefore underestimated causing an over-investment in product $R \& D$. The total effect of both the intra-industry spillover pools on product $R \& D$ is therefore ambiguous.

The perceived price elasticity implies an over-investment, while the productivity spillover effect implies under-investment in the R\&D intensity and the extent of the quality spillover effect is indeterminate. The net effect is ambiguous and dependent on the strength of the three effects.

We will now consider the effects of changes in the parameters on the R\&D intensities. Differentiating the process $R \& D$ elasticity $\left(\phi_{c}\right)$ with respect to the number of firms $(n)$ we find: ${ }^{83}$

\footnotetext{
82. See also Delbono and Dentcollo (1990).

(2) Remember that $a_{p}$ is also dependent on $n: a_{p}=a-(a-\varepsilon) / n$.
} 


$$
\frac{\partial \omega_{c}}{\partial n}=\phi_{c} \cdot\left(\frac{-\omega_{a} \cdot \theta_{1}}{\theta \cdot\left(1+\omega_{n} \cdot(n-1)\right)^{2}+\theta_{1} \cdot\left(1+\omega_{a} \cdot(n-1)\right)}+\frac{a-\varepsilon}{(n \cdot(a-1)-a+\varepsilon) \cdot(n a-a+\varepsilon)}\right)
$$

The first term between brackets has a negative value and represents the disincentive effect of entry when a part of a firms knowledge can be used by other firms and when it can also use knowledge of other firms. It can use R\&D of other firms without costs. This is therefore a disincentive effect for R\&D intensity. On the other hand, a greater number of firms implies a higher perceived price elasticity, which increases the perceived change in demand by a productivity improvement. This effect, which is represented by the second term between brackets, favours R\&D intensity and is positive. This effect increases with the value of the intraindustry price elasticity.

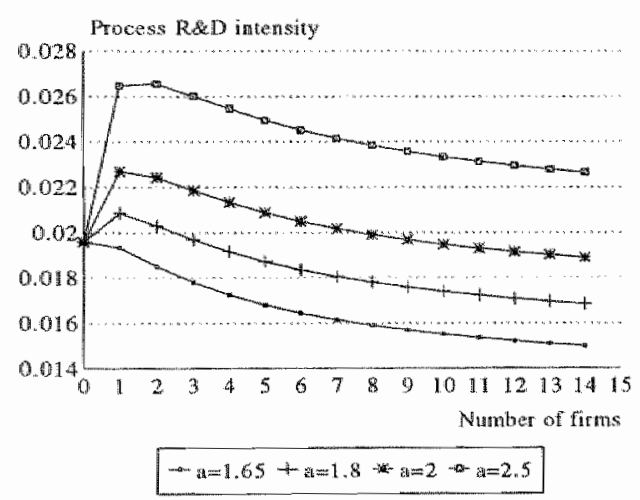

Figure 5.2. The infuence of entry on the process $R \& D$ intensity given various degrees of product differentiation and $\omega_{a}=(2.5$.

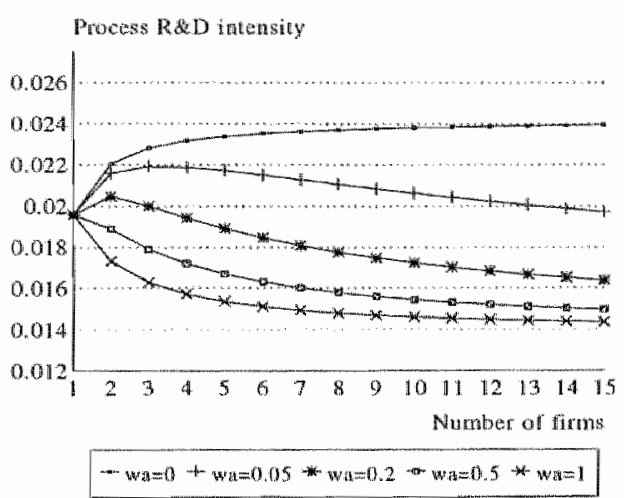

Pigure 5.3. The influence of entry on the process RoD intensity given different spillover levels and $y=1.7$. (wa in legend is $\omega_{p}$ )

The net effect of an increase in the number of firms on the R\&D elasticity is dependent on the strength of the two opposite effects. The R\&D intensity decreases with $\mathrm{n}$ if and only if the sum of the two terms between brackets is negative. This is more likely to be the case when $\omega_{6}$ is large or the difference between a and $\varepsilon$ is small as illustrated in figure 5.2 and figure 5.3. All the figures in this section are obtained from simulations executed with the values of the parameters and exogenous variables described in appendix $5 \mathrm{E}$. When different values for some parameters are used, these values are reported. 
Table 5.1: Derivatives of process and product R\&D intensity.

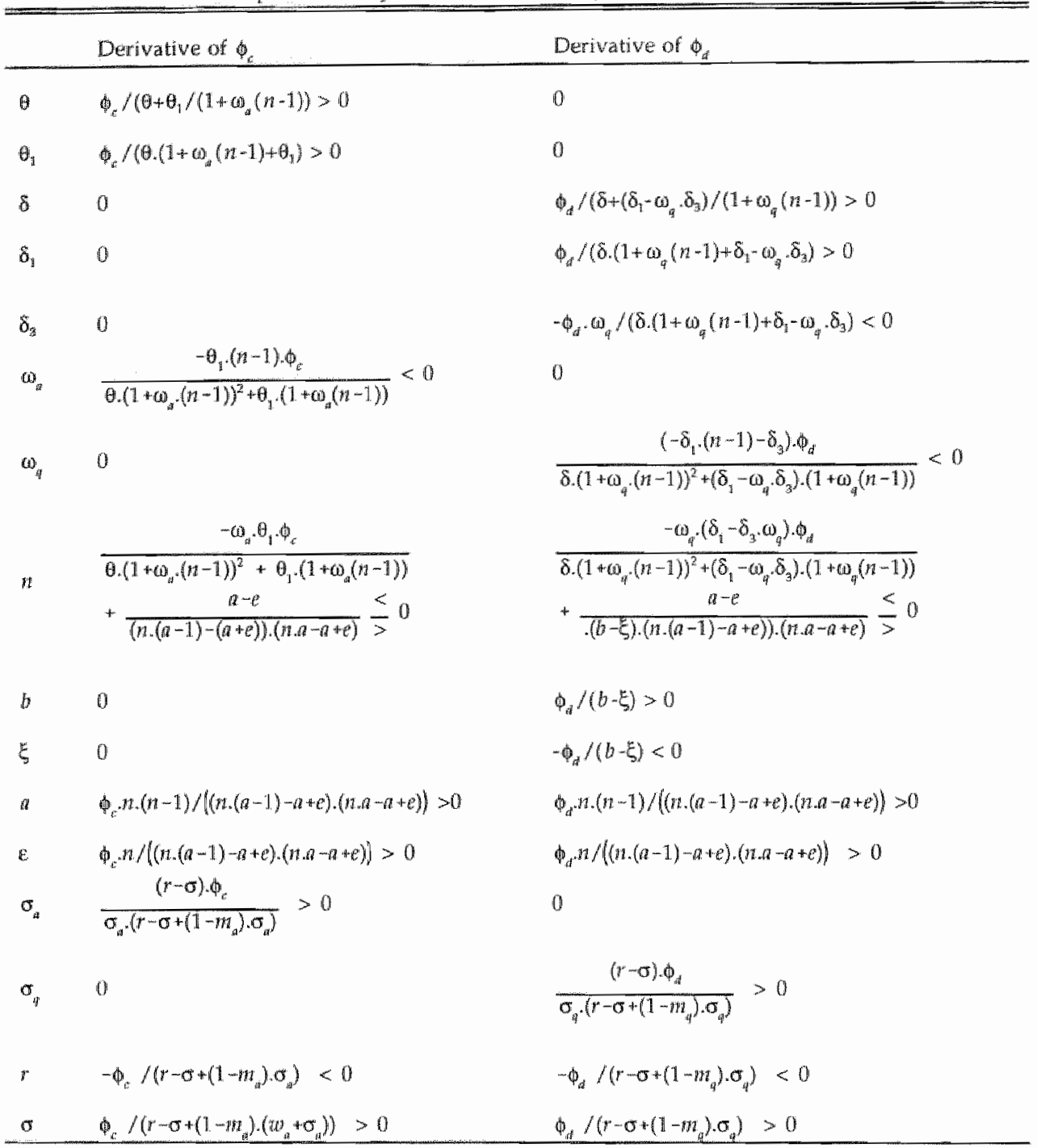

Figure 5.2 shows that given the inter industry price elasticity $(\varepsilon)$ and a moderate extent of spillovers $\left(\omega_{i 1}=0.5\right)$, a higher elasticity of substitution $(a)$ causes the R\&D intensity to rise. When the elasticity of substitution is low the R\&D intensity decreases with entry. With a higher intra-industry price elasticity the R\&D intensity first increases and then decreases with entry.

Figure 5.3 shows the influence of entry on R\&D intensity for various levels of the extent of spillovers $\left(\omega_{4}\right)$. When a larger part of foreign R\&D can be used (higher value of $\left.\omega_{a}\right)$ the R\&D intensity decreases. When spillovers are not present $\left(\omega_{a}=0\right)$, 
then the R\&D intensity increases with entry. If the spiliovers are perfect $\left(\omega_{a}=1\right)$, then the $R \& D$ intensity decreases with entry. For levels in between 0 and 1 , the $R \& D$ intensity first increases and then decreases.

The derivatives of the $R \& D$ intensity with respect to the other parameters are given in table 5.1. Larger technological opportunities represented by $\theta_{s} \theta_{1}, \delta_{r} \delta_{1}$ and $b$ increase the process or product $R \& D$ intensity. The productivity of the threatening spillover pool has a negative influence on the product $R \& D$ intensity. Cost increasing effects such as the discount rate $(r)$ and quality elasticity of costs ( $\xi$ ) decrease the $R \& D$ intensity as in the case of extent of spillover effects. Higher steady state growth rates $\left(\sigma_{i}, \sigma_{a^{\prime}} \sigma_{p}\right)$ increase the R\&D intensity. These results are not dependent on the "double counting" method which is easily checked by setting $\theta$ and $\delta$ equal to zero.

\subsubsection{RED Levels and Spillower Effects}

In this sub-section we will investigate the relationship between the steady state process and product $R \& D$ level and the extent of process and product $R \& D$ spillovers. The steady state levels of process and product $R \& D$ are calculated in appendix $5 \mathrm{C}$ and are stated in equation (5C.20) and equation (5C.24) respectively. We first consider the case of process R\&D spillovers. In general, we expect that larger spillovers tend to decrease cost reducing innovations (Spence 1984). De Bondt et al (1992) state that this tendency may be reversed in moderately to highly differentiated oligopolies with low R\&D costs (high technological opportunities) and that this is more likely to occur if leakages and the number of firms are not too high. Their argument is that "the benefits of cost reduction can better be appropriated in these circumstances and high technological opportunities make sure increases in investments are not very costly".

Differentiating $R_{c}^{\prime \prime}$ " with respect to the extent to which knowledge spills over to rival firms $\left(\omega_{a}\right)$ we find:

$\operatorname{sign}\left(\frac{\partial R_{c}^{\prime \prime}}{\partial \omega_{a}}\right)=\operatorname{sign}\left[\frac{\varepsilon-1}{1-m_{a}} \cdot\left(1+\omega_{a} \cdot(n-1)\right)-\frac{1-(b-\xi) \cdot(\varepsilon-1) \cdot\left(\frac{\delta+\delta_{1}-\delta_{3}}{1-m_{q}}\right)}{\theta+\frac{\theta_{1}}{1+\omega_{a} \cdot(n-1)}}\right]$

The net effect of $\omega_{a}$ on the process $R \& D$ level is ambiguous and dependent on two effects. First, a larger extent of spillovers increases the industry pool of knowledge. This raises the productivity of own $R \& D$ which results in higher marginal benefits of R\&D. This positive effect is represented by the first term between the brackets. Second, a larger extent of spillovers means that the appropriability of own R\&D 
declines. The elasticity of process R\&D, $\theta+\theta_{1} /\left(1+\omega_{q} \cdot(n-1)\right)_{*}$ decreases when $\omega_{a}$ increases. This negative effect is shown by the second term between brackets.

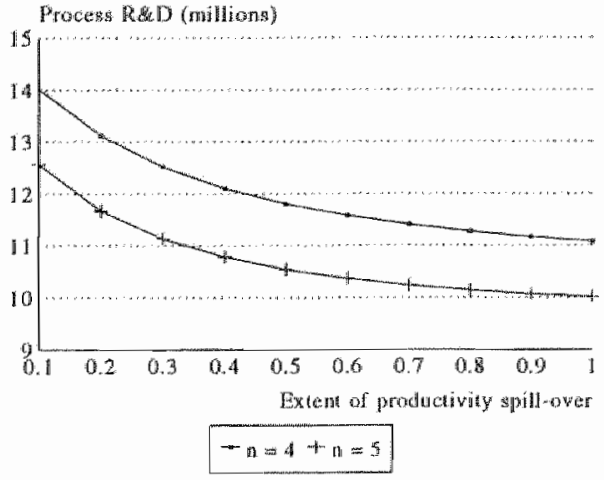

Figure 5.4. The influence of the extent of the productivity spillover on the level of process $R \& D$, givern a few firms.

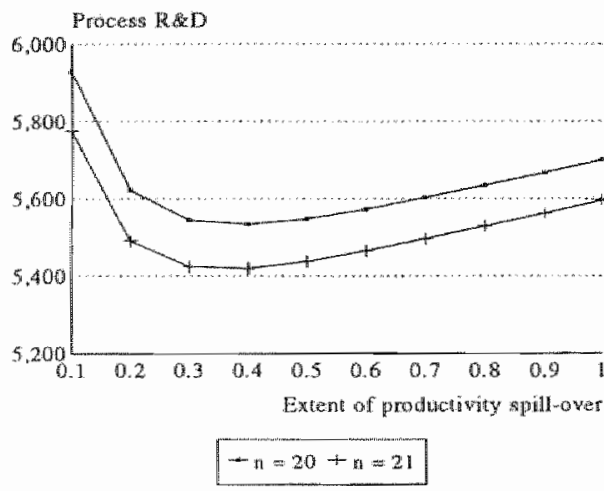

Figure 5.5. The influence of the extent of the productivity spillover on the level of process $R \& D$ with many firms.

Both effects are stronger when the number of firms is larger and the extent of spillovers is higher. Multiplying both expressions by $\theta+\theta_{1} /\left(1+\omega_{a} \cdot(n-1)\right)$ and using the definition of the growth rate multiplier (equation (5.23)) we obtain:

$\operatorname{sign}\left(\frac{\partial R_{c}^{u *}}{\partial \omega_{i t}}\right)=\operatorname{sign}\left[-\frac{1}{M}+\frac{E-1}{1-m_{a}} \cdot \theta_{1} \cdot\left(\omega_{a} \cdot(n-1)\right]\right.$

Condition (5.32) requires that $M$ is positive. Since $M$ is independent of $n$ and $\omega_{a}$, it is clear that more competition and less appropriability increase the chances that the influence of $\omega_{a}$ on $R^{* *}$ is positive. This result is opposite to the findings of De Bondt et al which is expected because they do not consider the first effect: a higher knowledge stock increases the productivity of a firm's own R\&D (see the specification of their technological generation process on page 5). Figure 5.4 illustrates the case when the number of firms is small and figure 5.5 depicts the case when the number of firms is larger.

Equation (5.37) shows that with the Spence (1984) specification $(\theta=0)$, the second term on the right hand side vanishes and the influence of the extent of spillovers on the process R\&D level is always negative. The parameter $\theta$ is therefore of crucial importance to obtain the possible positive effect of $\omega_{i}$ on $R_{c, i}$. The "double counting" method of Levin and Reiss therefore causes the possible positive influence of $\omega_{i}$ on $R_{i, i}^{\prime \prime}$. 
Another important determinant of the sign of the derivative is the inter-industry or general price elasticity of demand (e). The value of this inter-industry price elasticity is an indicator for the degree of product differentiation with regard to the homogenous good $(H)$. A higher level of $\varepsilon$ or less differentiated products increases the possibility of a positive influence of $\omega_{a}$ on $R_{v}^{\prime \prime}$. This effect also differs from the findings of $\mathrm{De}$ Bondt $e t$ al who conclude that a positive effect is more likely when product differentiation is moderate to high. In their model, moderate to high product differentiation implies that appropriability is larger. In our model, thigher product differentiation leads to a lower perceived price elasticity of demand.

Our analysis confirms the result of De Bondt et al that high technological opportunities (high $\theta$ and high $\theta_{1}$ ) will make a positive influence of $\omega_{c}$ on $R_{c}^{\prime \prime}$, more likely. The influence of the extent of product $R \& D$ spillovers on the steady state process $R \& D$ level is again ambiguous.

$\operatorname{sign}\left(\frac{\partial R_{c}^{\prime \prime}}{\partial \omega_{q}}\right)=\operatorname{sign}\left[\left(\delta_{1}-\delta_{3}\right) \cdot(n-1)-\frac{\left(\delta+\delta_{1}-\delta_{3}\right) \cdot\left(\delta_{3}+(n-1) \cdot \delta_{1}\right)}{\delta \cdot\left(1+\omega_{q^{*}}(n-1)\right)+\delta_{1}-\delta_{3} \cdot \omega_{q}}\right] \geq 0$

When the productivity of the threatening spillover pool is zero $\left(\delta_{3}=0\right)$ then the influence of $\omega_{\theta}$ on $R_{c}^{\prime \prime}$ is positive. This is always the case if one uses the Levin and Reiss specification of the threatening knowledge pool $\left(\delta_{3}=0\right.$ and $\delta_{1}$ can be positive or negative). If there is no "double counting" $(\delta=0)$ then the influence of $\omega_{i p}$ on $R_{c i}$ is negative if $\delta_{1}>\delta_{3}$ and positive if $\delta_{1}<\delta_{3}$. If there is no "double counting" and in addition $\delta_{3}$ is equal to zero, then there is no influence of $\omega_{4}$ on $R_{c}^{\prime \prime}$.

The influence of the extent of product $R \& D$ spillovers on the steady state product R\&D level is again ambiguous. The possibility of a positive influence is higher when the productivity of the intra-industry knowledge pool $T_{d, i}^{\prime \prime}\left(\delta_{i}\right)$ is higher and when the productivity of the threatening pool $W_{a, i}^{\prime \prime}\left(\delta_{3}\right)$ is lower. Interestingly, the influence of the extent of process spillovers, $\omega_{a}$, always positively effects the steady state product R\&D level.

We conclude that the influence of a larger extent of R\&D spillovers, which may have arisen in the IT era on the process and product R\&D steady state level, is ambiguous.

\subsubsection{Cost Reducing and Demand Creating Innovations and Spillovers}

The technological performance of a firm can be measured by its steady state productivity and quality level. These are stated in equation $(5 \mathrm{C} .25)$ and equation (5C.26) respectively. In this sub-section we investigate the relationship between technological performance and the extent of spillovers. The sign of the derivative of the steady state quality and productivity level with. respect to $\omega_{q}$ is given by equation (5.38). Our statements regarding the influence of $\omega_{q}$ on $R_{c, i}^{\prime \prime}$ are also valid 
in these cases. The influence of $\omega_{q}$ on $A_{i}^{\prime \prime}$ and $Q_{p, i}^{\prime \prime}$ is again ambiguous. The influence of the extent of productivity spillovers $\left(\omega_{a}\right)$ on the steady state productivity is:

$\operatorname{sign}\left(\frac{\partial A_{i}^{\prime \prime}}{\partial \omega_{a}}\right)=\operatorname{sign}\left(\frac{\partial Q_{p}^{\prime \prime}}{\partial \omega_{a}}\right)=\operatorname{sign}\left[1-\frac{\theta+\theta_{1}}{\theta \cdot\left(\mathbb{1}+\omega_{a} \cdot(n-1)\right)+\theta_{1}}\right]>0$

In contrast to the influence of $\omega_{q}$, the influence of $\omega_{a}$ on $A_{i}^{\prime \prime}$ and $Q_{p, i}^{\prime \prime}$ is always positive.

We can conclude that the extent of productivity spillovers stimulates the innovative performance of a firm and that the extent of quality spillovers tempers or stimulates innovative performance.

\subsubsection{Cost Reducing and Demand Creating Innovations and Entry}

In this section we investigate the influence of entry on the steady state productivity and quality level. We first consider the influence of entry on the productivity level. De Bondt et al (1992) find that, in a homogeneous oligopoly, entry reduces the innovative output. In a differentiated industry the effect is dependent on the level of spillovers. Low spillovers decrease, moderate spillovers increase, and high spillovers first increase and then decrease innovative output with entry.

The derivative of the steady state productivity level with respect to the number of firms is:

$$
\begin{aligned}
\operatorname{sign}\left(\frac{\partial A^{\prime \prime}}{\partial n}\right)= & -\frac{(a-\varepsilon) \cdot\left(1-m_{q}\right)}{n \cdot(a-1)}-\frac{\delta_{3} \cdot(b-\xi) \cdot(\varepsilon-1)}{n-1} \\
& +\frac{\left(1-m_{q}\right) \cdot \varepsilon \cdot(a-\varepsilon)}{(n \cdot(a-1)-a+\varepsilon) \cdot(a \cdot n-a+\varepsilon)} \\
& +\omega_{i \cdot} \cdot \theta_{1} \cdot \frac{\left(1-m_{q}-(b-\xi) \cdot(\varepsilon-1) \cdot\left(\delta+\delta_{1}-\delta_{3}\right)\right)}{1+\omega_{i \cdot} \cdot(n-1)} \cdot\left[\frac{1}{\theta+\theta_{1}}-\frac{1}{\theta \cdot\left(1+\omega_{u \cdot} \cdot(n-1)\right)+\theta_{1}}\right] \\
& +\frac{\omega_{q} \cdot(b-\xi) \cdot(\varepsilon-1)}{1+\omega_{i} \cdot(n-1)} \cdot\left[\delta_{1}-\delta_{3}-\frac{\left(\delta+\delta_{1}-\delta_{3}\right) \cdot\left(\delta_{1}-\delta_{3} \cdot \omega_{q}\right)}{\delta \cdot\left(1+\omega_{i q} \cdot(n-1)\right)+\delta_{1}-\delta_{3} \cdot \omega_{q}}\right]
\end{aligned}
$$

The first term on the right hand side states that more competitors reduce market shares which make strategic investments in R\&D less profitable. The second term implies that more rivals will cause a larger threat to a firm's perceived quality level which discourages demand creating innovations. Section 5.5 showed that demand creating and cost reducing innovations reinforce each other which also discourages cost reducing innovations. The third term shows that more competitors cause a 
higher perceived price elasticity which encourages $R \& D$ investments. The fourth term describes that more competitors increase the intra-industry process R\&D spillover stock which stimulates $R \& D$ investments. The fifth term describes the effect of entry on the product R\&D spillover pool which can be positive or negative. The net effect of these live effects is ambiguous.

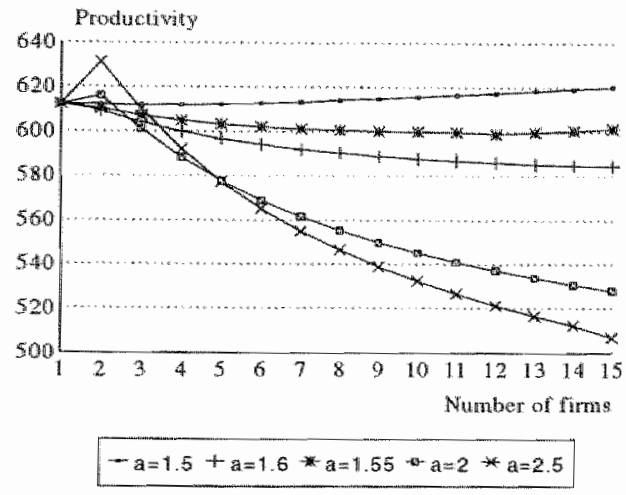

Figure 5.6. The influence of entry on the productivity level given various degrees of product differentiation and a low level of productivity spillovers $\left(\omega_{a}=0.1\right)$.

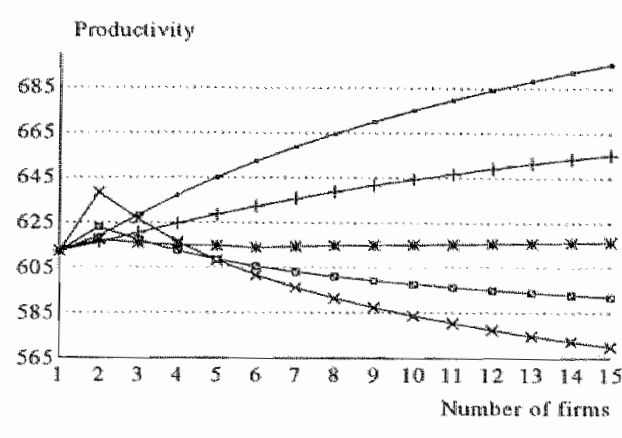

$-a=1.5+a b=1.6 * a+1.75+a=2+a=2.5$

Figure 5.7. The inl?uence of entry on the productivity level given various degrees of product differentiation and a high level of productivity spillovers $\left(\omega_{a}=1\right)$.

A few simulations help to illustrate the complex interplay between market structure and technological change in our model. We will discuss two important determinants of the net effect. The first one is the difference between the elasticity of substitution and the inter-industry price elasticity $(a-\varepsilon)$. This effect is dependent on the perceived differentiation of a product on the intra- and inter-industry level. The second one is the extent of the process R\&D spillovers $\left(\omega_{n}\right)$. The influence of the first determinant on the relationship between entry and the productivity level is illustrated in figure 5.6 and figure 5.7 given a low and a high level of the extent of productivity spillovers respectively.

In all of the simulations the value of $\varepsilon$ is 1.5 . We can identify four patterns. 1) When the difference between $a$ and $\varepsilon$ is equal to zero or small, entry may encourage the innovative efforts independently of the spillover level and degree of product differentiation. 2) If the difference between $a$ and $\varepsilon$ is not too large and the extent of spillovers are low or moderate, a monopolistic situation yields the highest productivity level. With high spillovers a duopoly obtains the highest productivity level. 3) If the difference between $a$ and $\varepsilon$ is large a duopoly is the best 
independent of the level of spillovers and product differentiation. The fourth pattern, $a=1.55$ in figure 5.6 and $a=1.75$ in figure 5.7, is an interesting one and to our knowledge a new pattern to the literature. To further illustrate this pattern we depicted it, given low and high spillovers, once more in figure 5.8. The productivity level first decreases and then increases with entry in the case of low spillovers. In the case of high spillovers, the productivity level increases from monopoly to duopoly, then decreases until it reaches a minimum value for an intermediate level of rivalry, and entry after this point increases the productivity level again. The intuition behind these results is that the first effect (lower market shares) drives the productivity level down. This negative effect decreases when the number of firms increases and at a certain intermediate level of rivalry the fourth and fifth effect (positive influence of a larger intra-industry knowledge stock) become more important.

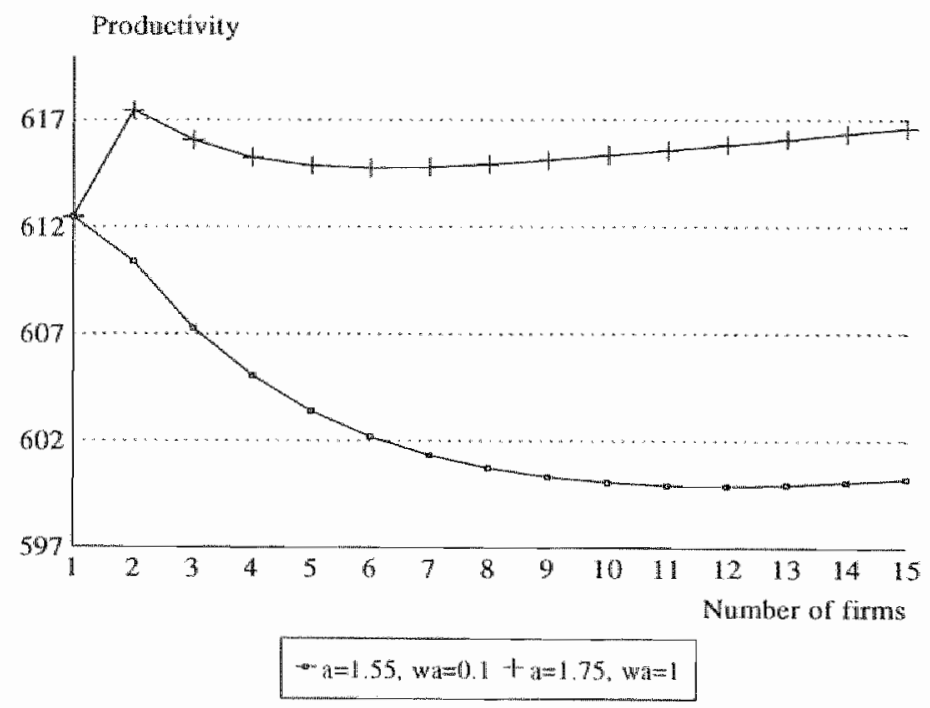

Figure 5.8. The influence of entry on productivity, given a small difference between $a$ and $\mathrm{E}$ for a high and a low tevel of spillovers.

These results differ from the findings of De Bondt et al by the newly observed pattern and furthermore that low and moderate spillovers can decrease, increase or first increase and then decrease the innovative output with entry. Another difference is that even with high spillovers the innovative output may increase with entry.

\subsubsection{Welfare, Entry and Spillowers}

Welfare (W) is the sum of consumer surplus (CS) and total profits. 


$$
W=C S+n \cdot \pi
$$

The consumer surplus can be defined as the utility derived from the differentiated good less the total expenditures.

$$
C S=U(D)-n \cdot p \cdot y
$$

The utility function is given by equation (5.1). Using equations (5.1), (5.2), (5.6) and the first order condition (5C.6) it is easy to show that:

$$
C S=T C^{\prime \prime} \cdot\left[1-\frac{1}{a_{p}}\right] \cdot \frac{1}{\varepsilon-1}
$$

where

$$
T C^{\prime \prime}=n^{\frac{\varepsilon-1}{n-1}} \cdot X_{a, \theta}^{\varepsilon} \cdot\left(Q_{p}^{\prime \prime}\right)^{(a-\xi)(\varepsilon-1)} \cdot\left(A^{\prime \prime}\right)^{e-1} \cdot Z_{a}^{1-\varepsilon} \cdot\left[1-\frac{1}{a_{p}}\right]^{\varepsilon}
$$

The consumer surplus is positively dependent on the technological performance of firms. A higher quality and productivity level increases the consumer surplus. The direct effect of the number of firms on consumer surplus is also positive, which reflects the love of variety. The love of variety is large when products are perceived as very differentiated (low level of $a$ ) and small when product differentiation is not very important (high level of $a$ ). Finally, a higher perceived price elasticity also has a positive influence on $C S$ because this decreases the mark-up of prices over costs.

Profits, $\pi$, are defined as revenue less total costs.

$$
\pi=p^{\prime \prime} \cdot y^{\prime \prime}-T C^{\prime \prime}-q_{c, 0} R_{c}^{\prime \prime}-q_{d, a} \cdot R_{d \prime}^{\prime \prime}
$$

Using the first order conditions (5C.6)-(5C.10) in combination with equation (5.6) we find that:

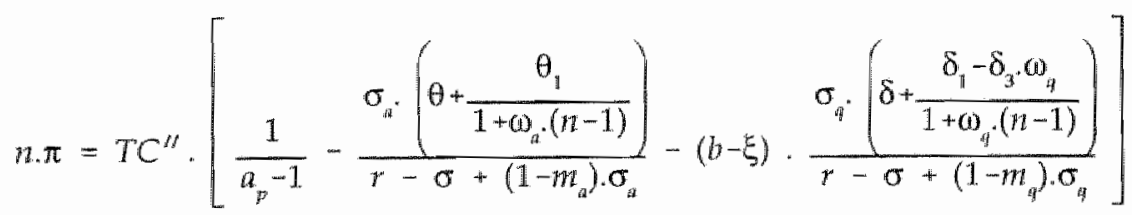

where $n . \pi$ are total profits. Note that total profits are, like a consumer surplus, positively dependent on technological performance (the quality and productivity level). The term between brackets is the net profit to total variable cost ratio (net 
profit ratio). The first term between brackets represents the gross profit ratio and the second and third term between brackets represents the process and product R\&D fixed costs ratio's respectively. The net profit ratio can be positive or negative depending on the strength of the three effects. Hence, a negative net profit ratio also implies negative profits which is not a viable situation in the long run. We will first investigate which factors make a viable situation less likely.

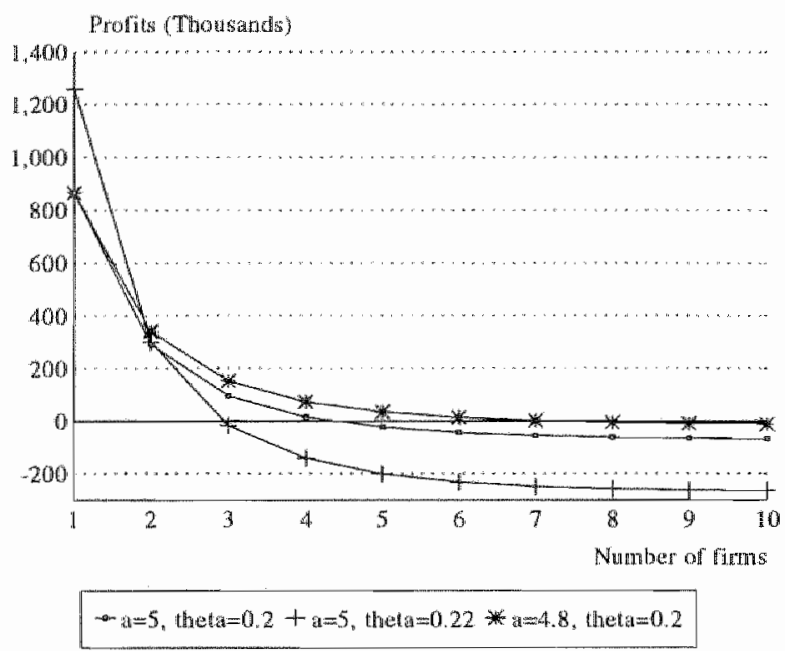

Figure 5.9. The Influence of higher technological opportunities (a higher value of $\theta$ ) and a lower degree of product differentiation (a higher level of $a$ ) on the number of viable firms in an industry

Entry increases the perceived price elasticity, which lowers the gross profit ratio, and decreases the two R\&D ratio's. The first effect decreases the net profit ratio but the second effect increases this ratio. The derivative of the net profit ratio with respect to entry is therefore ambiguous. We will discuss two cases. First, the traditional or normal case: the first effect dominates the second effect such that entry reduces the net profit ratio. We will first discuss this traditional or normal case, which is illustrated in figure 5.9 (there are two modifications of the base run

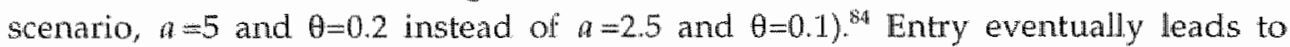
negative profits, thus limiting the number of viable firms in an industry. The appearance of a negative net profit ratio is also more likely when technological opportunities $\left(\theta, \theta_{1}, b, \delta, \delta_{1}\right)$ are larger. This argument, together with the finding that entry traditionally reduces the net profit ratio, implies that industries with

${ }^{34}$ We checked the value of the net-profit ratio for all the other simulations which are described in section 5.6. In all of the cases, the value of the nel-protil ratio wats positive. The number of firms were therefore not limited in the other simulations. In this case the number of firms is restricted because $\theta$ and $a$ are both higher decreasing the net profint rate. 
larger technological opportunities tend to be more concentrated. This relationship is also found by Dasgupta and Stiglitz (1980) and is illustrated in figure 5.9. When the own R\&D elasticity increases from $\theta=0.2$ to $\theta=0.22$, the number of viable firms in the industry decreases.

A higher extent of process or product R\&D spillovers decreases the fixed R\&D costs ratio's which reduces the probability of a negative profit rate. In the traditional or normal case this implies that the number of viable firms in a market increases. If information technology generates a higher extent of spillovers, then number of viable firms in a market will increase.

A higher degree of product differentiation (lower level of a) causes a decrease in $a_{p^{\prime}}$ which makes a negative profit ratio less likely. Traditionally, the number of viable firms increases in this market. The modiel therefore predicts that industries with a higher degree of differentiated goods are normally less concentrated than the industries with more homogenous goods (see figure 5.9). When a decreases from 5 to 4.8 the number of viable firms increases.

Combining these findings allows us to conclude that traditional industries with high technological opportunities, a low extent of R\&D spillovers, and a low degree of product differentiation, are very concentrated while highly differentiated industries with low technological opportunities and high spillovers are characterised by many firms.

We will now discuss the exceptional case, which is illustrated in figure 5.10. Entry first reduces the net profit rate but at a certain level of competition increases the net profit-rate. This means that after a certain level of competition the influence of entry on the R\&D fixed costs ratio's is greater than the influence on the gross profit ratio (perceived price elasticity). In other words, entry means smaller market shares, thus reducing own $\mathbb{R} \& D$ expenditures, because entry also increases the knowledge stock, which implies that a firm's own R\&D is more productive. This implies that only a concentrated industry is viable or an industry which is quite competitive (a large number of firms). An intermediate level of competition is unviable. Figure 5.10 also shows that industries with higher technological opportunities (higher vallue of $\theta$ ) increase the range of unviable market situations which permits either more concentrated industries or more competitive markets. This figure also shows that a higher level of product differentiation (lower value of a) has the opposite effect. We can conclude that in comparison with the results in the traditional situation, industries with a low level of product differentiation or with a high level of technological opportunities are more concentrated than industries with a high level of product differentiation or with a low level of technological opportunities when the number of firms in the industry is small. The opposite is the case when the number of firms is large. 


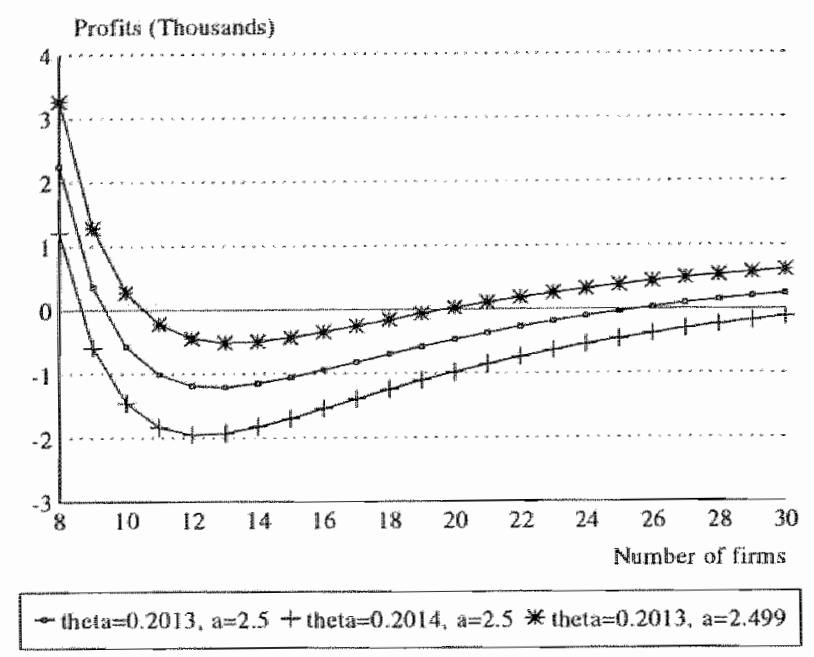

Figure 5.10. An exceptional relation between entry and profits

\section{Welfare}

We investigated the influence of entry and the extent of spillovers on welfare. The influence of the extent of process R\&D spillovers on wellare is positive. First, a higher extent of process $R \& D$ spillovers increases the net profit ratio (see equation (5.46)). Secondly, according to section 5.6.3, it also increases the productivity and quality level. This has a positive effect on both the consumer surplus and the total profit level. Therefore, the extent of process $R \& D$ spillovers increases the welfare level.

The influence of entry and the extent of product R\&D spillovers on welfare is again ambiguous. We study the influence of entry. First, entry increases the perceived price elasticity which increases the consumer surplus and decreases or increases total profits. Second, the influence of entry on the productivity and quality level is ambiguous (see paragraph 5.6.4). The difference between $a$ and $\varepsilon$ and the extent of the spillover effects determine whether the influence is positive or negative. Third, when people possess love of variety, entry increases the consumer surplus directly, since every firm produces its own variety. Such a situation is illustrated in figure 5.11.

When consumers possess "good characteristic" preferences (large a) the welfare typically increases first and then decreases with entry. With less differentiated goods (high a), an intermediate level of rivalry stimulates most technological change (see section 5.6.4). This high level of technological change results in a higher

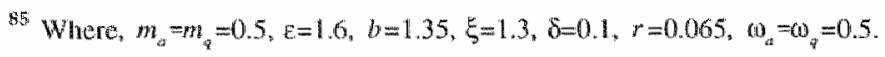


quality and productivity level which is valued by consumers and producers. This situation is illustrated in figure 5.12 .

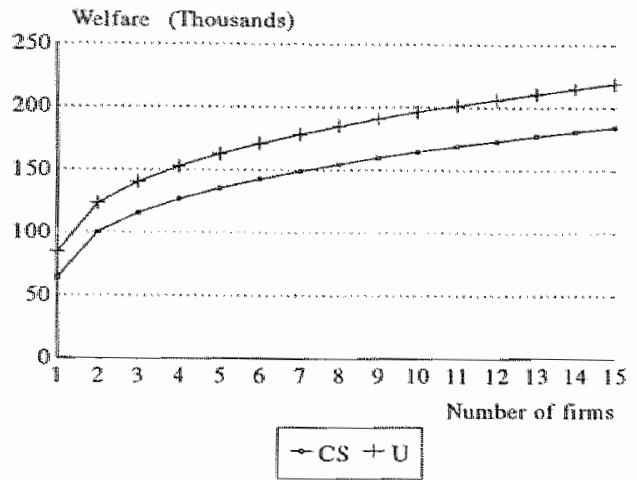

Figure 5.11. The influence of entry on consumer surplus and total welfare given "Love of Variety" $(a=2.5)$.

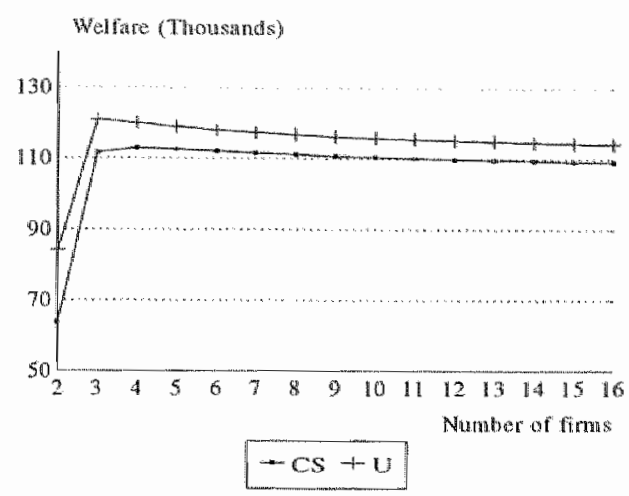

Figure 5.12. The influence of entry on consumer surplus and total welfare given a lower "Love of variety" $(a=7.5)$.

If we combine welfare characteristics with the viability of a market, we can identify an interesting case for government policy. Figure 5.10 showed us that an intermediate level of rivalry is not viable. If we also assume that profits have to be zero in the equilibrium, then only two levels of rivalry satisfy this condition (for the scenario with $a=2.5$ and $\theta=2.014$ are this 9 and 26 firms, see figure 5.10). The situation described in figure 5.10 is characterised by love of variety which implies that the welfare level positively depends on the number of firms. Therefore if this industry possesses 9 firms the welfare level is lower than in the equilibrium with 26 firms. This industry is "locked in" an inferior industry structure. A temporary government policy, for example a $\mathbb{R} \& D$ subsidy, is now necessary to bridge the unviable levels of rivalry.

\subsection{Conclusion}

The background for this chapter is the discussion of the importance of general or public knowledge versus tacit or private knowledge in the knowledge creation process. General or public knowledge implies that a firm can use knowledge created by other firms and that internally created knowledge can also be used by other firms and is of less value to the innovator. Economists such as David and 
Foray (1994), Nelson (1992), Arora and Gambardella (1994), Ergas (1994), argue that generic public knowledge becomes more important in the knowledge creation process within the information technology era. The central question is whether this tempers or stimulates technological progress.

This chapter addressed this important question in a simple model with endogenous technological change. The most important conclusion of this study is that the relationship between spillovers and technological change is by no means a simple one. We cannot conclude that higher spillovers temper a firms incentive to invest in research and development as was the case in the Spence (1984) model and stimulate technology investments (chapter 4). Spence obtained his well-known result because he did not consider that increased spillovers enthance the productivity level of internal technology investments. Chapter 4 did not consider that internally created knowledge also increases the economic performance of competitors. This chapter combines both effects which makes the general influence of spillovers on technology investment incentives dependent on a variety of other determinants of technological change. Besides the degree of knowledge spillovers, consumer preferences and entry also have an important influence on technological progress. It is therefore the complex interplay between spillover effects, product differentiation and entry that determine the level of technological change. One has to know the exact values of the inter- and intra-industry level of product differentiation, the value of the extent and productivity of spillover effects, and the number of firms, in order to draw conclusions about the influence of a change in one of these variables on incentives to invest in technological change. Nonetheless some conclusions can be drawn about some general influences of spillovers and entry on technological change.

A larger value of the extent of process and product $R \& D$ spillovers reduces the process and product $R \& D$ intensity. The influence on internal process and product R\&D level is ambiguous. The extent of process R\&D spillovers on the steady state process R\&D level is in general negative, however a large number of firms, large spillovers, high technological opportunities and a high degree of inter-industry price elasticity increases the possibility of a positive relationship. The influence of the extent of product R\&D spillovers on the steady state process $R \& D$ level is dependent on, for example, the value of the elasticities of the productivity enhancing intra-industry spillover pool and the threatening intra-industry spillover pool. Irregardless that the extent of the process $R \& D$ spillover can reduce internal $R \& D$ investments, its influence on the level of technological change (productivity or quality level) is positive. However, a larger extent of product $R \& D$ spillovers can reduce private incentives to invest in process or product $R \& D$ as well as the quality or productivity level achieved in the steady state. In generall we can say that a higher degree of spillovers tends to reduce private incentives to invest in R\&D but it also tends to increase the obtained technological level because firms use more knowledge created by other firms. 
The influence of entry on the R\&D intensities is ambiguous. On the one hand nore firms implies a higher spillover stock which reduces the R\&D intensity and on the other hand, more firms means a higher perceived price elasticity of demand which increases the R\&D intensity. Entry may increase the R\&D intensity given a low degree of spillovers, it tends to increase first and then to decrease this intensity given moderate spillovers and it tends to induce a lower R\&D intensity given high spillovers. The influence of entry on the productivity level is even more complicated. An interesting pattem is that, given a small difference between the elasticity of substitution and the inter-industry price elasticity of demand, entry first decreases and then increases the productivity level. It decreases the productivity level because entry implies smaller market share which creates a disincentive effect to invest in technology. The more firms there are the smaller this effect will be. However, entry may create larger industry pools of knowledge that may increase the productivity level. If this happens then it is possible that this effect is able to dominate the "smaller market share effect" when the number of firms increases.

In general it can be expected that entry reduces profits. The occurrence of negative profits limits the number of firms in an industry. High technological opportunities and a low level of differentiated products usually lead to more concentrated markets. We also found that higher knowledge spillovers leads to a larger number of viable firms in a market. With high knowledge spillovers, firms will spend less on internal R\&D but use more of knowledge generated by other firms.

This model also allows us to examine an interesting relationship between entry and profits. High technological opportunities and a low level of differentiated products can create an industry structure that is characterised by either a few competitors or a large number of competitors; an intermediate levell of rivalry is not viable. Depending on the welfare implications, a concentrated or a competitive industry is desirable. Temporary government policy is necessary if one industry is locked in the wrong industry structure.

Welfare is enhanced by productivity spillover effects. The influence of the extent of quality spillovers is ambiguous. In general we can conclude that when consumers show love of variety, entry increases welfare. When consumers are only interested in product characteristics, however, an intermediate level of rivalry generates the highest wellare level. 


\section{Appendix 5A. Derivation of Demand Functions}

Two Stage Utility Maximization subject to a Budget Constraint: "Love of Variety" Approach.

First Stage: Minimisation of expenditures to achieve a certain level of quality chatacteristics of differentiated goods.

The total quality characteristics index is:

$D=\left[\sum_{i=1}^{n}\left(y_{i} Q_{j, j}^{b} j^{p}\right]^{\frac{1}{\beta}}=\left[\sum_{i=1}^{n} c_{i} y_{i}^{\rho}\right]^{\frac{1}{p}}\right.$

where $c_{i}=Q_{p, i}^{b}$. The quality level of a product is given for a consumer. We limit attention to symmetric equilibria which implies that the quality level of different products will be the same. Consumers minimize expenditures to achieve a certain level of utility.

$L_{y_{i}}=\sum_{i=1}^{n} p_{i} y_{i}-\lambda\left[\left[\sum_{i=1}^{n} c_{i} y_{i}^{\rho}\right]^{\frac{1}{\beta}}-D\right]$

The first order conditions associated with this problem are:

$$
\begin{aligned}
& \frac{\partial L}{\partial y_{i}} \Rightarrow \frac{p_{i}}{\lambda}=c_{i}\left(\frac{y_{i}}{D}\right)^{\beta-i} \\
& \frac{\partial L}{\partial \lambda} \Rightarrow 1=\sum_{i=1}^{n} c_{i}\left(\frac{y_{i}}{D}\right)^{p}
\end{aligned}
$$

Taking equation (5A.3) to the power $\rho /(\rho-1)$ yields:

$$
\left(\frac{p_{i}}{\lambda_{i} c_{i}}\right)^{\frac{\rho}{p-1}}=\left(\frac{y_{i}}{D}\right)^{p}
$$

Substitution of equation (5A.5) in equation (5A.4) yields:

$$
1=\sum_{i=1}^{n} c_{i}\left(\frac{p_{i}}{\lambda c_{i}}\right)^{\frac{p}{p-1}}
$$

Solving equation (5A.6) for $\lambda$ yields:

$$
\lambda=\left[\sum_{i=1}^{n} c_{i}^{1} \frac{1}{p-p} p_{i}^{p-p}\right]^{\frac{p-1}{p^{2}}}
$$

Using totall expenditures (C) and equation (5A.5), it is easy to verify:

$C=\sum_{i=1}^{n} p_{i} y_{i}=\lambda \cdot \sum_{i=1}^{n} y_{i} c_{i}\left(\frac{y_{i}}{D}\right)^{p-1}=\lambda \cdot \sum_{i=1}^{n}\left[c_{i} y_{i}^{p}\right] \cdot D^{1-p}=\lambda \cdot D$

$\lambda$ is therefore the price index $\left(P_{D}\right)$ for the quality characteristic index $(D)$. 
Using Shephard's lemma we find:

$$
\frac{\partial C}{\partial p_{i}}=y_{i}=\frac{\partial \lambda}{\partial p_{i}} D
$$

The demand function conditional on the quality characteristic index can be derived using equation (5A.7) and equation (5A.9).

$y_{i}=\left[\sum_{i=1}^{m} c_{i}^{\frac{1}{1-p}} \cdot p_{i}^{\frac{p}{p-1}}\right]^{-\frac{1}{p}} \cdot c_{i}^{\frac{1}{1-p}} \cdot p_{i}^{\frac{1}{\beta-1}} \cdot D=c_{i}^{\frac{1}{1-p}} \cdot\left(\frac{p_{i}}{P_{p}}\right)^{\frac{1}{p+1}} \cdot D$

Since $P_{D}=\lambda$.

Second Stage: Choose allocation of expenditures across goods $D$ and $H$ to maximize total utility subject to the overall budget constraint.

The maximisation problem is:

$$
L_{D, H}^{\prime \prime}=\frac{X_{6}}{\zeta} \cdot D^{\zeta}+H-\mu \cdot\left(P_{D} \cdot D+H-D\right)
$$

where $I=$ income in terms of the numerary, $H$ =homogenous good (numerary)

The first onder conditions ane:

$$
\begin{aligned}
& \frac{\partial L^{\prime \prime}}{\partial D}=X_{a} D^{\zeta-1}-\mu P_{b}=0 \\
& \frac{\partial L^{\prime \prime}}{\partial H}=1-\mu=0
\end{aligned}
$$

Combining these first order conditions we derive the level of the quality characteristics dependent on the price index.

$D=\left(\frac{P_{D}}{X_{0}}\right)^{\frac{1}{\zeta-1}}$

The result of the first stage (equation (5A.10)) and the result of the second stage (equation (5A.14) together give the demand equation for each differentiated good $\left(c_{i}=Q_{p, i}^{p}\right)$.

$y_{i}=X_{i j}^{\varepsilon} \cdot Q_{p, i}^{b(a-1)} \cdot P_{i}^{-a} \cdot P_{D}^{a-n}$

where $a=1 /(1-p)>1$ and $E=1 /(1-\zeta)>1$.

The definition of $P_{\mathrm{D}}$ now becomes (see equation (5A.7))

$P_{D}=\left[\sum_{i=1}^{n} Q_{p, i}^{b(a-1)} \cdot p_{i}^{1-a}\right]^{\frac{1}{1-a t}}$

\section{Appendix 5B. Calculation of Steady State Growth Rates}

In this appendix we derive the steady state growth rates for the model in subsection 5.4 . 
The current value Hamiltonian associated with the maximization in this section is:

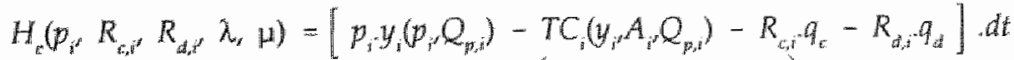

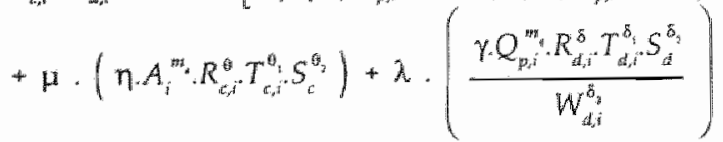

where:

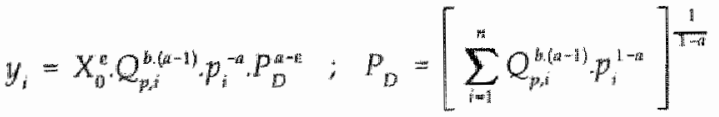

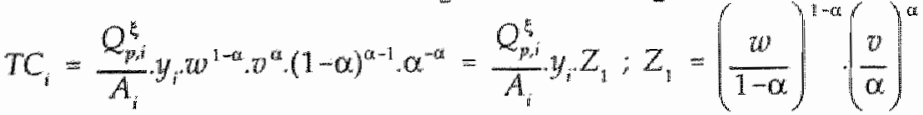

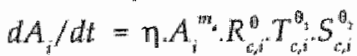

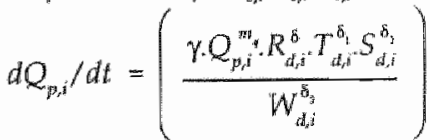

First Order Conditions:

$$
\frac{\partial H_{c}}{\partial p_{i}}=y_{i}+p_{i} \frac{\partial y_{i}}{\partial p_{i}}-\frac{\partial T C_{i}}{\partial y_{i}} \frac{\partial y_{i}}{\partial p_{i}} \Rightarrow p_{i}=\left(\frac{a_{p}}{a_{p}-1}\right) \cdot \frac{T C_{i}}{y_{i}} ; a_{p}>1
$$

where $a_{p}$ is the perceived price elasticity. The derivation of this elasticity is:

$$
a_{p}=-\frac{\partial y_{i}}{\partial p_{i}} \cdot \frac{p_{i}}{y_{i}}=a-(a-\varepsilon) \cdot \frac{Q_{p, i}^{b(a-1)} \cdot p_{i}^{i-a}}{\sum_{i=1}^{n} Q_{p, i}^{k,(a-1)} \cdot p_{i}^{1-a}} \cdot \frac{n}{n}=a-\frac{(a-\varepsilon)}{n}
$$

Using the dynamic constraint, and equation (5.8), $T_{b, i}=R_{c, i}+(i t) \sum R_{i, j}$, and the assumption that firms presume that other firms will not change their $R_{\mathrm{C} D} \mathrm{strategy:} \partial T_{c, d} / \partial R_{c, i}=1$.

The first order condition with respect to $R_{r}$ becomes:

$$
\frac{\partial H_{c}}{\partial R_{i, i}} \Rightarrow q_{i}=\mu \cdot\left[\frac{\theta \cdot\left(d A_{i} / d i\right)}{R_{r, i}}+\frac{\theta_{1} \cdot\left(d A_{i} / d t\right)}{T_{i, i}}\right]
$$

In a symmetric equilibrium the $R \& D$ expenditures are equal across firms $\left(R_{c i j}=R_{c i}\right)$. Equation (5B.5) becomes in the symmetric equilibrium:

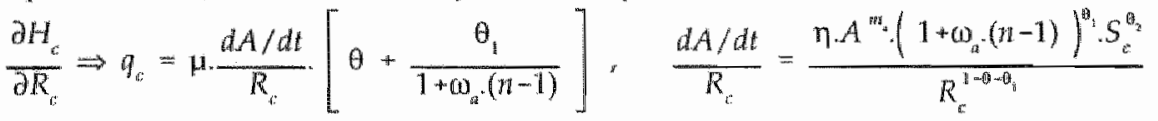

In the same way we can calculate the derivative with respect to $R_{d, i}$ (use equation (5.14) and equation $(5.13)$ ):

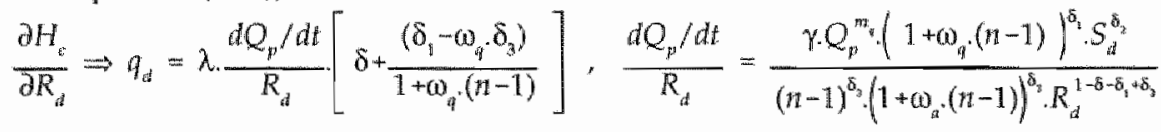


The first order conditions with respect to the state variables $Q_{m i}$ and $A_{i}$ are:

$$
\begin{aligned}
& \frac{\partial H_{\varepsilon}}{\partial A_{i}} \Rightarrow-\frac{d \mu}{d t}+\mu \cdot r=\frac{T C_{i}}{A_{i}}+\mu \cdot m_{a^{*}}\left(\frac{\frac{d A_{i}}{d t}}{A_{i}}\right) \Rightarrow-\frac{d \mu}{d t}=\frac{T C_{i}}{A_{i}}-\mu \cdot\left(r-m_{a} \cdot A_{i}\right) \\
& \frac{\partial H}{\partial Q_{p, i}} \Rightarrow-\frac{d \lambda}{d t}+r \cdot \lambda=-\zeta \frac{T C_{i}}{Q_{p, i}}-\frac{d T C_{i}}{d y_{i}} \frac{d y_{i}}{d Q_{p, i}}+p_{i} \frac{d y_{i}}{d Q_{i}}+\left(\frac{\frac{d Q_{p, i}}{d t}}{Q_{p, i}}\right) m_{q} \cdot \lambda
\end{aligned}
$$

As in the case with the perceived price elasticity of demand we can calculate the perceived quality elasticity of demand $\left(b_{p}\right)$.

$$
\begin{aligned}
b_{p} & =\frac{\partial y_{i}}{\partial Q_{p, i}} \cdot \frac{Q_{p, i}}{y_{i}}=b \cdot(a-1)+\left(\frac{a-\varepsilon}{1-a}\right) \cdot b \cdot(a-1) \cdot\left[\frac{Q_{p, i}^{b(a-1)} p_{i}^{[i-a}}{\sum_{i=1}^{m} Q_{p, i}^{k, a-1)} \cdot p_{i}^{i-a}}\right] \cdot \frac{n}{n} \\
& =b \cdot(a-1)-\frac{(a-\varepsilon) \cdot b}{n}=b \cdot\left(a_{p}-1\right)
\end{aligned}
$$

Combining the last two equation yields:

$$
\begin{aligned}
\frac{\partial H_{c}}{\partial Q_{p, i}} & \Rightarrow-\frac{d \lambda}{d t}+\lambda \cdot\left(r-m_{q} Q_{p, i}\right)=-\zeta \frac{T C_{i}}{Q_{p, i}}-\frac{T C_{i}}{y_{i}} \cdot b_{p} \cdot \frac{y_{i}}{Q_{p, i}}+p_{i,} \cdot b_{p} \cdot \frac{y_{i}}{Q_{p, i}} \\
& =\left(-b_{p}-\zeta\right) \cdot \frac{T C_{i}}{Q_{p, i}}+b_{j} ;\left[\frac{a_{p}}{a_{p}-1}\right] \cdot \frac{T C_{i}}{Q_{p, i}}=\left[\frac{b_{p}-\zeta \cdot\left(a_{p}-1\right)}{a_{p}-1}\right] \frac{T C_{i}}{Q_{p, i}}=(b-\zeta) \cdot \frac{T C_{i}}{Q_{p, i}}
\end{aligned}
$$

where use has been made of equation (5B.3).

The two Transversality Conditions (TVC) are:

$$
\begin{aligned}
& T V C_{k}=\lim _{t \rightarrow \infty} \lambda_{t} e^{-x, t}=0 \\
& T V C_{\mu}=\lim _{t \rightarrow \infty} \mu_{t} e^{-x t}=0
\end{aligned}
$$

The dynamic constraints equation (5.9) and equation (5.12) are the other first order conditions.

\section{Calculation of Steady State Growth Rates in a Symmetric Equilibrium}

The growth rate of the demand function (i.e. equation (5.6)) in a symmetric situation is: $y_{i}=\varepsilon \cdot X_{0}+b \cdot(\varepsilon-1) \cdot Q_{p, i}-\varepsilon \cdot p_{i}$

The growth rate of the total variable costs $(T C)$ is:

$$
T C_{i}=g_{i}+\xi \cdot Q_{p, i}+Z_{i}-A_{i}
$$

The constant growth rates of the first order conditions (equations (5B.3), (5B.6), (5B.7), (5B.8) and (5B.11)) are respectively: 


$$
\begin{aligned}
& p_{i}=T C_{i}-Q_{i} \\
& Q_{c}=\hat{H}+A_{i}-R_{c i} \\
& Q_{d}=\hat{\lambda}+Q_{p, i}-R_{d, i} \\
& \hat{\mu}=T C_{i}-A_{i} \\
& \lambda=T C_{i}-Q_{p, i}
\end{aligned}
$$

The constant growth rates of the productivity (equation 3.2) and quality generation (equation 3.5 ) process are respectively:

$0=\theta \cdot R_{c, i}+\theta_{1} \cdot R_{i, j}+\theta_{2} \cdot S_{i}-\left(m_{i}-1\right) \cdot A_{i}$

$$
0=\delta \cdot \hat{R}_{d, i}+\delta_{1} \cdot R_{d, i}-\delta_{3^{\prime}} R_{d, i}+\delta_{2^{2}} S_{d i}-\left(m_{t i}-1\right) \cdot Q_{p, i}
$$

Combining equations (5B.16) and (5B.18) yields:

$q_{c}=T_{C}-R_{c i}$

Combining equations (5B.17) and (5B.19) yields:

$q_{i t}=T C_{i}-\hat{R}_{l i, i}$

We first substitute the growth rate of $p_{i}$ from equation (5B.15) in equation (5B.13) and solve this result for $y_{i}$. Substituting this result in equation (5B.14) we get:

$T C_{i}=\varepsilon \cdot X_{0}+(b-\xi) \cdot(\varepsilon-1) \cdot \hat{Q}_{p, i}+(1-\varepsilon) \cdot Z_{1}+(\varepsilon-1) \cdot A_{i}$

Equation (5B.24) is then substituted in respectively equation (5B.22) and equation (5B.23). We solve one of the two resulting equations for $R_{i t, i}$ and substitute the result in the other equation. The steady state growth ratte for $\mathbb{R}_{i, i}$ is:

$$
R_{i, i}^{*}=\frac{\varepsilon \cdot X_{0}-q_{c}+(\varepsilon-1) \cdot\left[-Z_{1}+(b-\xi) \cdot\left(\frac{\delta_{2}}{1-m_{q}} S_{i}-\frac{\delta+\delta_{1}-\delta_{a}}{1-m_{q}} \cdot\left(q_{i}-q_{c}\right)\right)+\frac{\theta_{2}}{1-m_{a}} \cdot S_{c}\right]}{1-(\varepsilon-1) \cdot(b-\xi) \cdot\left(\frac{\delta+\delta_{1}-\delta_{3}}{1-m_{q}}\right)-(c-1) \cdot\left(\frac{\theta+\theta_{1}}{1-m_{q}}\right)}
$$

Using equation (5B.20) and equation (5B.25) we can derive the steady state growth rate for the productivity level $\left(A_{i}\right)$ :

$A_{i}^{*}=\left(\frac{\theta+\theta_{1}}{1-m_{*}}\right) \cdot R_{c, i}^{*}+\left(\frac{\theta_{2}}{1-m_{c B}}\right) \cdot S_{i}$

Equation (5B.22) and equation (5B.23) together show that the steady state growth rates of the product and process R\&D budget are equal. The steady state growth rate for product R\&D is then:

$R_{i, i}^{*}=q_{c}+R_{i, i}^{*}-q_{d}$

Equation (5B.21) gives the steady state growth rate for the quality level $\left(Q_{p, i}\right)$

$Q_{p, i}^{*}=\left(\frac{\delta+\delta_{1}-\delta_{3}}{1-m m_{q}}\right) \cdot R_{d, i}^{*}+\left(\frac{\delta_{2}}{1-m_{\text {q }}}\right) \cdot S_{d}$ 


\section{Appendix $5 C$. Time Elimination}

As in van Meijl and van Zon (1993) we deflate all variables with their steady state growth rates. Two examples are:

$A_{i j}^{d y}=A_{i} \cdot e^{-a_{u} t}$
$Q_{p, i}^{\nu / i}=Q_{p, i} \cdot e^{-\sigma_{i t} t}$

where $\sigma_{q, a}$ and $\sigma_{a}$ are the steady state growth rates of $Q_{p, i}$ and $A_{i}$ respectively. We defined $R_{c_{s},}^{\prime \prime}, R_{d, i}^{\prime \prime s}, y_{i}^{p /}$ and $p_{i}^{\prime \prime}$ in the same way.

The two dynamic constraints with the deflated variables become:

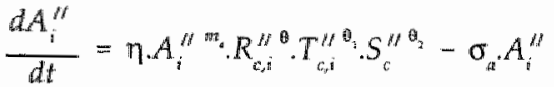

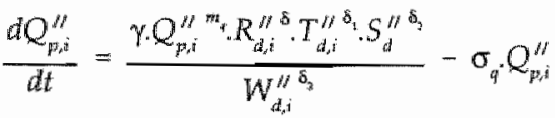

The Hamiltonian of the profit maximization problem with the redefined variables is: $H_{c}\left(p_{;}^{\prime \prime}, R_{e, i}^{\prime \prime}, R_{d, i}^{\prime \prime}, \lambda^{\prime \prime}, \mu^{\prime \prime}\right)=$

$$
\begin{aligned}
& \int_{0}^{\infty} e^{(\omega-\tau), t} \cdot\left[p_{i}^{\prime \prime} \cdot y_{i}^{\prime \prime}\left(p_{i}^{\prime \prime}, Q_{p, i}^{\prime \prime}\right)-T C_{i}^{\prime \prime}\left(y_{i}^{\prime \prime}, A_{i}^{\prime \prime}, Q_{p, i}^{\prime \prime}\right)-R_{c, i}^{\prime \prime} \cdot q_{c, 0}-R_{d, i}^{\prime \prime} \cdot q_{d, 0}\right] \cdot d t
\end{aligned}
$$

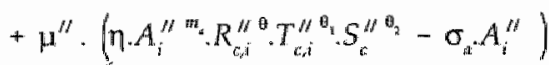

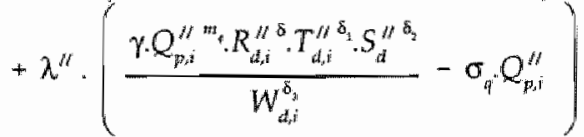

$y_{i}^{\prime \prime \prime}=X_{0,0}^{E} \cdot Q_{p, i}^{\prime b \cdot(a-1)} \cdot p_{i}^{\prime \prime-a} \cdot P_{D}^{\prime \prime a-\varepsilon}$

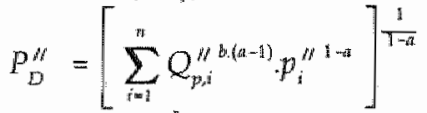

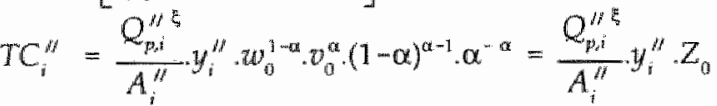

Where $\sigma$ is the steady state growth rate of revenues, $R \& D$ budgets and profits and $q_{c, 0^{*}} q_{d, 0}$. $X_{0,0,} w_{0}$ and $\tau_{0}$ are the initial values of respectively the price of process $R \& D$, the price of product $R \& D$, the exogenous scale of demand, wages and unit cost of capital.

The first order conditions are:

$$
\frac{\partial H_{c}}{\partial p_{i}} \Rightarrow p_{i}^{\prime /}=\frac{a_{p}}{a_{F^{\prime}}-1} \cdot \frac{T C^{\prime \prime}}{y_{i}^{\prime \prime}} ; a_{p}>1
$$

The perceived price elasticity $\left(a_{p}\right)$ is give by equation (5B.4).

$$
\frac{\partial H_{\varepsilon}}{\partial R_{c, i}^{\prime \prime}} \Rightarrow q_{c, 0}=\mu^{\prime \prime} \cdot \frac{\left(d A_{z}^{\prime \prime} / d t+\sigma_{i x} \cdot A_{i}^{\prime \prime}\right)}{R_{c, i}^{\prime \prime}} \cdot\left[\theta+\frac{\theta_{1}}{1+\omega_{\alpha}^{\prime \prime} \cdot(n-1)}\right]
$$




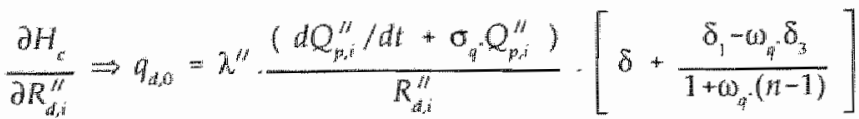

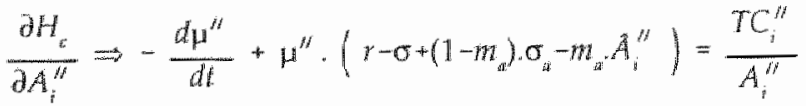

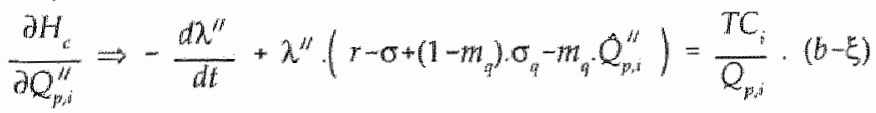

The two Transwersality Conditions (TVC) are:

$$
\begin{aligned}
& T V C_{\chi^{*}}=\lim _{t \rightarrow \infty} \lambda_{p}^{\prime \prime} \cdot e^{(s-r) t}=0 \\
& T M C_{z^{* t}}=\lim _{t \rightarrow \infty} \mu_{t}^{i t} \cdot e^{(\alpha-t) t}=0
\end{aligned}
$$

The dynamic constraints equation (5C.2) and equation (5C.3) are the other first order conditions. When we put these first order conditions in growth rates and calculate the steady state growth rates of the redefined variables we find that the steady state growth rates of $A_{i}^{\prime \prime}, Q_{p, i,}^{\prime \prime} R_{d, i, i}^{\prime \prime}, R_{i, \lambda^{\prime}} \lambda^{\prime \prime}$ and $\mu^{\prime \prime}$ are equal to zero. A zero growth rate for $\lambda^{\prime \prime}$ and $\mu^{\prime \prime}$ implies that the transversality condition is satisfied when $r>\sigma$.

$T V C \Rightarrow r-\sigma>0$

\section{Deriontion of the Fout Differential Equations (Symmetric Equilibrium)}

The $d A_{i}^{\prime \prime} / d t$ and $d Q_{p, i}^{\prime \prime} / d t$ differential equations are given by respectively equation (5C.2) and $\left(5 \mathrm{C} 3\right.$ ). To derive the $d R_{t, i}^{\prime \prime} / d t$ differential equation we first solve equation $(5 \mathrm{C} .7)$ for $\mu^{\prime \prime}$ :

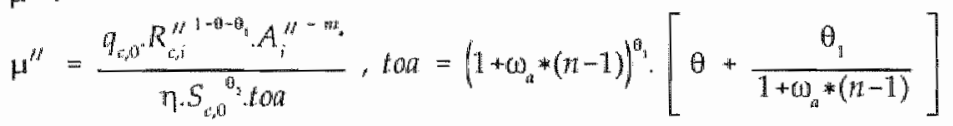

When we differentiate $\mu^{\prime \prime}$ with respect to time we get:

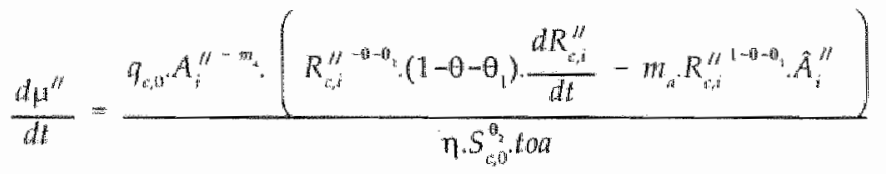

When we substitute $\mu^{\prime \prime}$ and $d \mu^{\prime \prime} / d t$ in equation (5C.9) we get the $d R_{c ; i}^{\prime \prime} / d t$ differential equation:

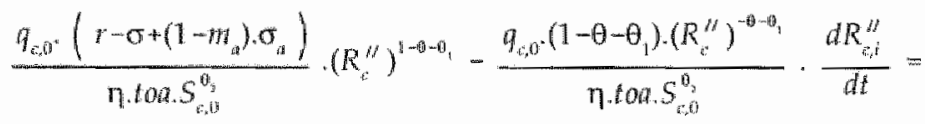

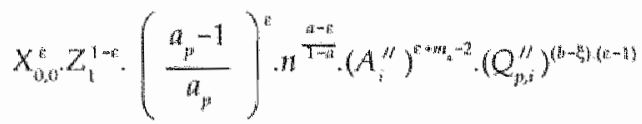

we can simplify this equation to: 


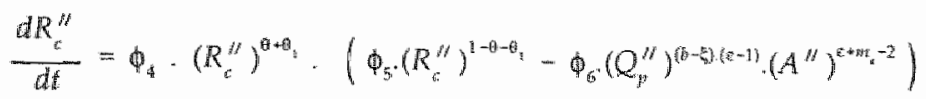

where

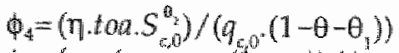

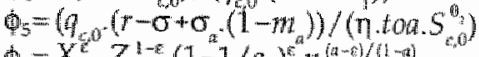

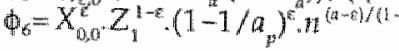

We can also derive the $d R_{d i}^{i / d} / d$ differential equation in the same manner. We solve equation ( $5 C .8$ ) for $\lambda^{\prime \prime}$ and take the time derivative of this equation. Substituting $\lambda^{\prime \prime}$ and $d \lambda^{\prime \prime} / d t$ in equation $(5 \mathrm{C} .10)$ gives:

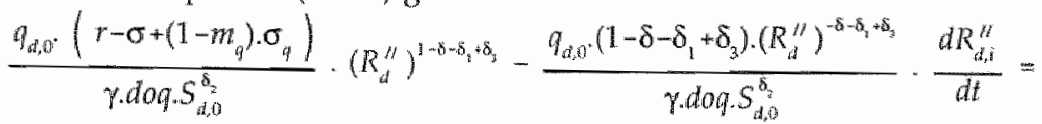

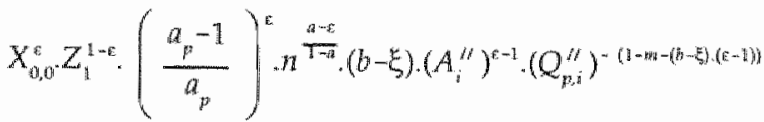

where:

$d o q=\frac{\left(1+\omega_{i q} \cdot(n-1)\right)^{\delta_{0}-\delta_{2}}}{(n-1)^{\delta_{1}}} \cdot\left[\delta+\frac{\delta_{1}-\omega_{q^{7}} \delta_{3}}{1+\omega_{q^{2}}(n-1)}\right]$

We can simplify equation $(5 \mathrm{C}, 15)$ to:

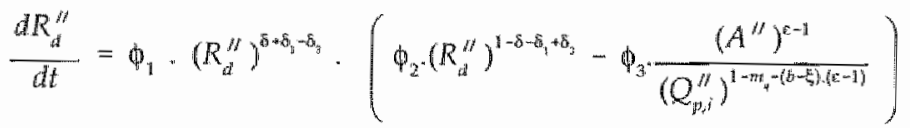

where:

$\phi_{1}=\left(\gamma \cdot \log S_{3,0}^{\delta_{3}}\right) /\left(q_{1,0}\left(1-\delta-\delta_{1}+\delta_{3}\right)\right)$

$\phi_{2}=\left(q_{d, 0}\left(r-\sigma+\sigma_{1} \cdot\left(1-m_{0}\right)\right) /(\gamma / d o g) S_{d, 0}^{\delta_{2}}\right)$

$\phi_{3}=X_{0,0}^{\varepsilon} \cdot Z_{1}^{1-\varepsilon} \cdot\left(1-1 / a_{p}\right)^{e} \cdot n^{(i n-\varepsilon) /(1-a)} \cdot(b-5)$

In the steady state are $d A_{i}^{\prime \prime} / d t, d Q_{p, i}^{\prime \prime} / d t, d R_{c, i}^{\prime \prime} / d t$ and $d R_{d, i}^{\prime \prime} / d t$ equal to zero.

The steady state level of process $\mathbb{R} \& D$ is:

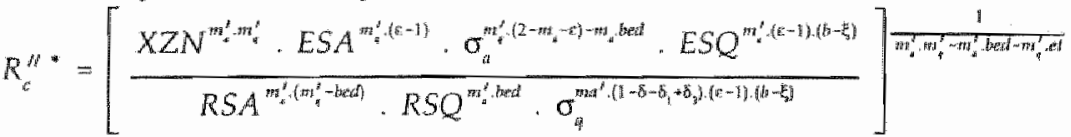

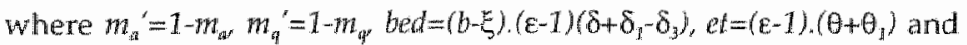

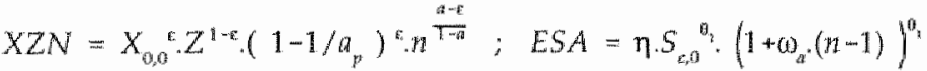




$$
\begin{aligned}
& R S A=\frac{\left(\gamma-\sigma+\left(1-m_{a}\right) \cdot \sigma_{a}\right) \cdot q_{c, n}}{\left[0+\frac{\theta_{1}}{1+\omega_{a} \cdot(n-1)}\right]}: R S Q=\frac{\left(r-\sigma+\left(1-m_{q}\right) \cdot \sigma_{n}\right) \cdot q_{d, 0}}{\left[\delta+\frac{\delta_{1}-\omega_{a} \delta_{1}}{1+\omega_{n}(n-1)}\right]} \cdot(b-\xi) \\
& E S Q=\gamma S_{d, 0}^{\sigma_{3}} \cdot\left(1+\omega_{q} \cdot(n-1)\right)^{\hat{b}_{2}-\hat{b}_{3}} /(n-1)^{\delta_{1}}
\end{aligned}
$$

The steady state level of product R\&D is:

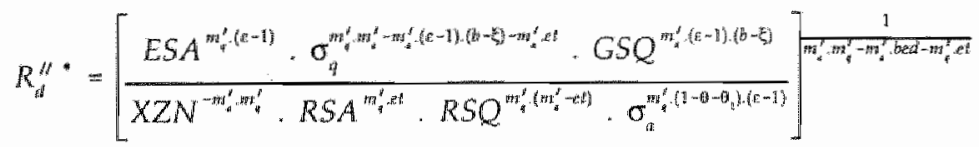

The steady state productivity level can be obtained by substituting the steady state process R\&D level in equation (5C.2):

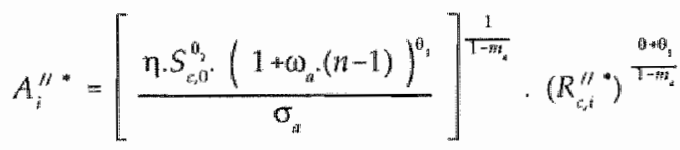

The steady state product R\&D level and equation (5C.4) provide the steady state quality level:

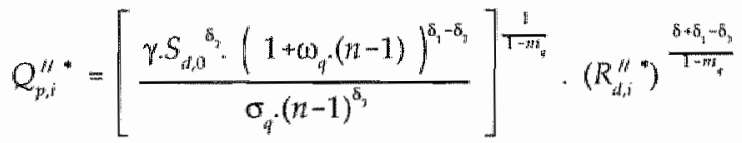

Steady State RGD Intensities:

In order to calculate the steady state $R \& D$ intensities we use the first order conditions. First, we solve equation (5C.6) for $T C$. Second, we solve equation (5C.2) for $d A_{i}^{\prime \prime} / d t+\sigma_{\mu} \cdot A_{i}^{\prime \prime}$. Third, we solve equation $(5 C .7)$ for $\mu^{\prime \prime}$. Fourth, we notice that $d \mu^{\prime \prime} / d t=0$ is in the steady state. Fifth, we substitute the results of step one to four in equation (5C.10). The steady state product R\&D intensity is:

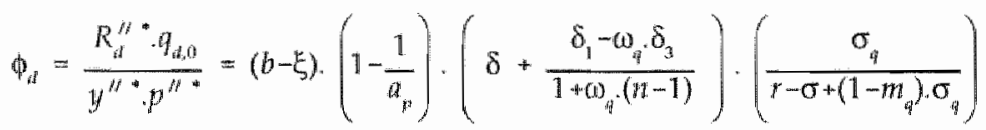

Using the same four step procedure we can derive the steady state process intensity. In the second step we solve equation $(5 \mathrm{C} .3)$ for $d Q_{p, i}^{\prime \prime} / d t+\sigma_{i q,} Q_{p, 4}^{\prime \prime}$. In the third step we solve equation (5C.8) for $\lambda^{\prime \prime}$. The fourth step is substituting all results in equation (5C.9).

$$
\Phi_{t}=\frac{R_{i}^{\prime \prime} \cdot q_{i, 0}}{y^{\prime \prime *} \cdot p^{\prime \prime}}=\left(1-\frac{1}{a_{p}}\right) \cdot\left[\theta+\frac{\theta_{1}}{1+\omega_{a} \cdot(n-1)}\right] \cdot\left(\frac{\sigma_{a}}{r-\sigma+\left(1-m_{a}\right) \cdot \sigma_{a}}\right)
$$




\section{Appendix 5D. Checking Saddle Point Stability}

As in appendix $3 C$ we use the method of Feichtinger and Hartl to check the saddle point stabillity characteristics. First, we have to compute the determinant of the Jacobean matrix evaluated at the steady-state point (E):

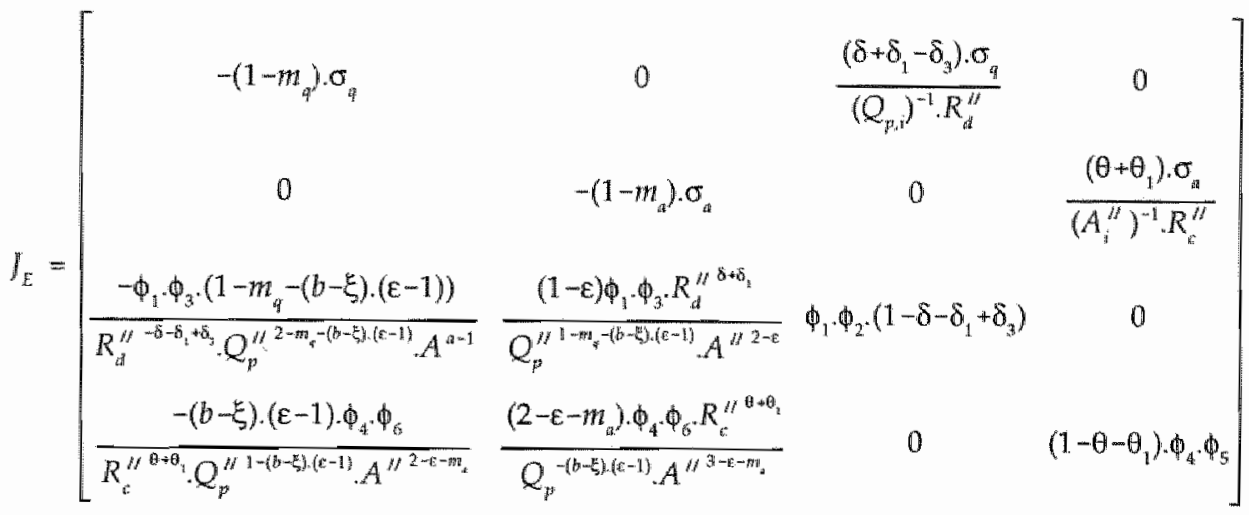

The determinant of the Jacobean matrix can be simplified to:

$$
\|=\phi_{1} \cdot \phi_{4} \cdot \phi_{2} \cdot \phi_{5}, \sigma_{a} \cdot\left(1-m_{z}\right) \cdot \sigma_{q} \cdot\left(1-m_{q}\right) \cdot\left[1-(b-\xi) \cdot(\varepsilon-1) \cdot\left(\frac{\delta+\hat{\sigma}_{1}-\delta_{3}}{1-m_{q}}\right)-(\varepsilon-1) \cdot\left(\frac{\theta+\theta_{1}}{1-m_{a}}\right)\right]
$$

The value of the determinant is positive when:

$$
1>(b-\xi) \cdot(\varepsilon-1) \cdot\left(\frac{\delta+\delta_{1}-\delta_{3}}{1-m_{q}}\right)+(\varepsilon-1) \cdot\left(\frac{\theta+\theta_{1}}{1-m_{a}}\right)
$$

Therefore, when this condition holds the first condition, a positive determinant for the Jacobean matrix is met. Second, the value of variable $K$ in this model is: ${ }^{86}$

$$
K=-\phi_{1} \cdot \phi_{2} \cdot \omega_{q^{*}}\left[1-m_{4}-(b-\xi) \cdot(\varepsilon-1) \cdot\left(\delta+\delta_{1}-\delta_{3}\right)\right]-\phi_{4} \cdot \phi_{5} \cdot \sigma_{a}\left[1-m_{a}-(\varepsilon-1) \cdot\left(\theta+\theta_{1}\right)\right]
$$

The second condition, a negative value for $K$, is satisfied when the condition described in equation (5D.2) holds. The third condition, $0<$ det $\| \leq K^{2} / 4$, is also satisfied if the first condition holds. We can conclude that this system is saddle point stable when the condition described in equation (5D.2) holds. 
Appendix 5E. Values of Parameters and Exogenous Variables used in Base Run Scenario

\begin{tabular}{|c|c|c|c|}
\hline Parameters & Vallues & Parameters & Values \\
\hline$a$ & 2.500 & $S_{0}$ & 2.000 \\
\hline$\varepsilon$ & 1.500 & $S_{\text {d }}$ & 2.000 \\
\hline 8 & 0.150 & $w$ & 0.800 \\
\hline$\delta_{\sharp}$ & 0.075 & $v$ & 0.600 \\
\hline$\delta_{2}$ & 0.050 & $X_{0}$ & 100,000 \\
\hline$\delta_{3}$ & 0.050 & 9 & 0.800 \\
\hline$\theta$ & 0.100 & $q a$ & 0.800 \\
\hline$\theta_{1}$ & 0.075 & $r$ & 0.070 \\
\hline$\theta_{2}$ & 0.050 & $\omega_{\text {i }}$ & 0.300 \\
\hline$b$ & 1.400 & $\omega_{i f}$ & 0.300 \\
\hline v & 1.100 & $\mathrm{X}_{0}$ & 0.040 \\
\hline$m_{\mathrm{at}}$ & 0.050 & $\hat{w}$ & 0.020 \\
\hline$m_{4}$ & 0.050 & 0 & 0.020 \\
\hline$\tau$ & 1.000 & $q_{c}$ & 0.020 \\
\hline$\eta$ & 1.000 & $q_{z}$ & 0.020 \\
\hline \multirow[t]{2}{*}{$\alpha$} & 0.250 & $S_{i}$ & 0.020 \\
\hline & & $S_{i}$ & 0.020 \\
\hline
\end{tabular}




\section{PART III}

MEASUREMENT 



\section{The Influence of R\&D and R\&D Spillovers on Productivity Growth: A Survey of Econometric Studies}

It is widely recognised that the knowledge stock of a firm or industry has a significant impact on its productivity. However, this knowledge stock is not observable. Empirical studies have looked for observable determinants of this stock. Many studies considered R\&D and its spillovers as important inputs to the generation of knowledge. The empirical studies surveyed in this chapter study the influence of R\&D and spillovers on productivity growth via the unobservable knowledge stock. A considerable number of surveys exist in the field of R\&D and R\&D spillovers (see for example, Griliches 1979, 1992, 1994, Nadiri 1990, Mohnen 1992a, Mairesse and Sassenou 1991 and Mairesse and Mohnen 1994). This survey differs from previous ones as it studies in detail the two types of spillovers effects (embodied in purchased goods and knowledge spillover effects) distinguished by Griliches (1979), and it reviews the empirical evidence on the different roles of process - oriented and product-oriented $R \& D$ with respect to productivity growth.

We first discuss the general framework that is used in most studies and the empirical results on the relationship between "own" $\mathrm{R} \& \mathrm{D}$ and productivity growth. Section 6.2 explains the concepts of knowledge and rent spillovers and the empirical evidence on both types of spillovers. Section 6.3 investigates the empirical evidence on the roles of process-oriented and product-oriented R\&D. Finally, we conclude with a discussion and summary of the main effects.

\subsection{R\&D Investment and Productivity Growth}

The relationship between productivity growth and $R \& D$ investment has attracted considerable interest and analysis in the econometric literature. The majority of these studies use the extended production function approach, which builds on the work of Tinbergen (1942) who added a time trend representing the level of "efficiency" to the production function developed by Cobb and Douglas (1928). Solow (1957) integrated this approach with the national accounts (record of measured inputs 
such as employed capital and labour) and found that the growth rate of this "efficiency" measure was the most important contributor to economic growth. This efficiency measure became know as the "Solow residual" and represents all sources of economic growth that are not caused by the growth of factors of production. The residual is usually interpreted as a reflection of technological progress. The studies investigated in this chapter attempt to explain a part of this residual or technological progress by introducing "own" R\&D and/or outside R\&D (spillovers) as separate factors in the production function.

Some empirical studies use the dual approach, which is based on a dual representation of the technology and a flexible functional form. Instead of estimating the production function directly, this approach estimates the factor demand functions and infers the underlying technological structure (Varian 1984). A disadvantage of this approach is that it has to make some behavioral (cost minimization or profit maximization) or market structure assumptions which can lead to misspecification biases. This advantage of the extended production function approach vanishes if one calculates total factor productivity growth by using the factor cost shares as proxy for the factor elasticities. Mairesse and Mohnen (1994) discuss the advantages and disadvantages of the two approaches in more detail. Moreover, with respect to the magnitude of the estimated marginal product or rates of return to $R \& D$, they conclude that the results obtained with the dual approach are consistent with those of the extended production function approach.

In this study we focus on the main approach or the extended production function approach as it is used in the empirical section of the thesis (chapters 7 and 8). We first describe the general framework and then discuss the empirical results obtained using this framework. ${ }^{8}$

The theoretical framework for the empirical studies that use the production function approach is the Cobb-Douglas production function:

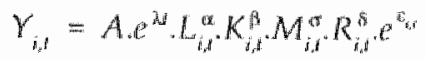

where $\gamma_{i, i}$ is the output of the $i^{h_{1}}$ industry at time $t, L$ is a measure of labour input, $K$ measures the plant and equipment capital stock, $M$ is materials usage, $R$ is the research capilal stock, $\lambda$ is the rate of disembodied technical change and $\varepsilon$ is an error term. One advantage of using a Cobb-Douglas function is that it can be estimated in a linear regression if expressed in terms of logarithms or growth rates.

$y_{i, i}=a+\lambda \cdot l+\alpha \cdot i_{i, 1}+\beta \cdot k_{i, l}+\sigma \cdot m_{i, i}+\delta \cdot r_{i, t}^{*}+\varepsilon_{i, l}$

\footnotetext{
\$7 The dwal wpprach is poswible becanse of maximization of protit is equivalent to minimization of cost

94. For readers who are interested in lho dual approxch we refer to the surveys of Mohnen (1992a) and Matrose and Mohnen (1994) and the articles cited in those surveys.
} 


$$
\widehat{\Upsilon}_{i, t}=\lambda+\alpha \cdot \hat{L}_{i, t}+\beta \cdot \hat{K}_{i, t}+\sigma \cdot \hat{M}_{i, t}+\delta \cdot \hat{R}_{i, t}+\varepsilon_{i, t}
$$

where $x_{i, t}$ denotes the logarithm of variable $X_{i, k}$ and $\hat{X}_{i, \Lambda}$ is the growth rate of a variable $X_{i, t}$

The parameter of interest in most studies is the estimated elasticity of research capital ( $\delta$ ). The research capital stock is usually calculated by means of the perpetual inventory method:

$$
R_{i, t}=R \& D_{i, t}+(1-\gamma) \cdot R_{i,-1}
$$

where $R \& D_{i, t}$ are the deflated $R \& D$ expenditures for industry $i$ during year $t$, and $\gamma$ is the rate of R\&D depreciation, which is assumed to be constant. The problem with calculating the research capital stock in this way is that it requires data as R\&D expenditures over an extended period. Many studies circumvented this problem by using the Terleckyj (1974) transformation. Noting that $\delta=(\partial Y / \partial R) .(R / Y)$, Terleckyj has shown that $\delta . \hat{R}$ in equation (6.3), can be written as $(\partial Y / \partial R) \cdot(\dot{R} / Y)$, where the first ratio can be estimated as a regression coefficient and represents the marginal product of $R \& D$ or rate of return to $R \& D$. Disregarding $R \& D$ depreciation implies that $k$ can be approximated by the $R \& D$ expenditures, which in contrast to the $R \& D$ capital stock, is easily available. Equation (6.3) can therefore be estimated as:

$\hat{Y}_{i, 1}=\lambda+\alpha \cdot L_{i, t}+\beta \cdot \hat{K}_{i, t}+\sigma \cdot \hat{M}_{i, t}+p \cdot\left(\frac{R \& D}{Y}\right)_{i, t}+\varepsilon_{i, t}$

The parameter of interest is the rate of return $\rho$ instead of the elasticity $\delta$.

We can simplify equation (6.5) by using the Divisia index of total factor productivity growth (TFP).

$$
\operatorname{TPP} P_{i, t}=\hat{Y}_{i, i}-\alpha \cdot \hat{L}_{i, i}-\beta \cdot \hat{K}_{i, t}-\sigma \cdot \hat{M}_{i, t}
$$

The production elasticities can be replaced with the corresponding cost shares if we make the additional assumptions that there are constant returns to scale to the traditional factors of production $(\alpha+\beta+\sigma=1)$, competitive behaviour and optimal choices of factors of production. The production elasticities, $\alpha$ and $\sigma$, can be calculated by using the share of the costs of labour and intermediate goods in total costs, respectively. The production elasticity $\beta$ can then be calculated as $1-\alpha-\sigma$.

Substitution of equation (6.6) in equation (6.3) and equation (6.5) gives the two specifications which are estimated in most studies:

$$
T \hat{F} P_{i, d}=\lambda+\delta \cdot \hat{R}_{i, i}+\varepsilon_{i, s}
$$


$T \hat{F} P_{i, 1}=\lambda+\rho \cdot\left(\frac{R \& D}{Y}\right)_{i, i}+\varepsilon_{i, 1}$

Equation (6.7) estimates the elasticity of $R \& D$ using constructed research capital stock data and equation (6.8) estimates the rate of return to R\&D using R\&D intensities.

It is possible to derive an estimate of $\rho$ from that of $\delta$ and vice versa, but one should remember that in equation (6.7), $\delta$ is assumed to be constant and in equation (6.8), $\rho$ is assumed to be constant.

Next we discuss the empirical results of studies that investigate the relationship between "own" R\&D and productivity growth. Table 6.1 shows the estimated. elasticities and rates of returns to "own" R\&D of a few studies which are representative of the vast number of studies in this field. We discuss only a few studies since our main interests are in the field of spillovers and process and product R\&D. ${ }^{89}$

Most of the studies found a strong positive influence of R\&D on productivity growth, either at the firm level, the industrial level or the economy level. The estimated elasticities range from $0 \%$ (Griliches 1980b) to 33\% (Cunéo-Mairesse 1984) and the estimated rates of return range from $0 \%{ }^{90}$ (Griliches 1980b) to $78 \%$ (Hall and Mairesse 1992). Most of the studies present a rate of return ranging from $10 \%$ $50 \%$. The studies are based on cross-section estimates or time series estimates. In general the cross-section estimates of the elasticity of production to R\&D are higher than their time series counterparts (see, for example, the study of Minasian 1969 and Hall-Mairesse 1992). The main problem in time series estimates is the collinearity of R\&D and other variables related to time. In another finding, not reflected in table 6.1, the estimated rate of return in high-tech sectors tends to be higher than those in low-tech sectors.

\section{2 "Pure" Rent and Knowledge Spillover Effects}

An important feature of the knowledge creation process is the existence of externalities or spillover effects. When firms are unable to capture all the benefits of their innovations, a part of the created knowledge spills over to other firms. With regard to R\&D spillover effects, Griliches (1979) distinguished two types of research and development spillovers. On the one hand, externalities may occur because downstream users do not pay the full value of the input. This kind of spillover can be called a "pure rent spillover". On the other hand, "knowledge spillovers" are ideas discovered in one sector that can be used in other sectors.

89 Some excellent reviews on this subject can be found in Mairesse and Sassenou (1991) and Mohnen $(1992 a)$.

90 An outher in these findings is the negative rate of return found by Odagiri (1985). 
Table 6.1: Direct rates of return and elasticities of $\mathbb{R} \& D$

\begin{tabular}{|c|c|c|c|}
\hline Sundy & Sample & Elasticity & Rate of recurn \\
\hline \multicolumn{4}{|l|}{ United States } \\
\hline \multirow[t]{2}{*}{ Miriasian (1969) } & Firm, "48-'57" cross & $26^{6 / \%}$ & $54 \%$ \\
\hline & : bime & $8 \%$ & \\
\hline Griblithes (1980a) & Firm, $57-^{\prime} 65$ & $7 \%$ & $27 \%^{\circ}$ \\
\hline \multirow[t]{2}{*}{ Griliches (1980b) } & Industry, '59-"68 & $7 \%$ & $42 \%$ \\
\hline & $69-77$ & $0 \%$ & $0 \%$ \\
\hline Marusfield (1980) & Firm, ${ }^{\prime 60} 6-76$ & & $28 \%$ \\
\hline Schankerman (1981) & Firm, 63 & & $24 \%-73 \%$ \\
\hline Griliches-Mairesse (1984) & Firm, $73-80$ & $5 \%-9 \%$ & $35 \%-64 \%$ \\
\hline Griliches (1986) & Firm, " $72-79$ & $9 \%-11 \%$ & $33 \%-39 \%$ \\
\hline Patel-Soete (1988) & Total Economy, "56-"85 & $6 \%$ & \\
\hline Lichtenberg-Slegel (1989) & Firm, $72-85$ & & $13 \%$ \\
\hline Griliches-Mairesse (1990) & Firrn, $73-80$ & & $25 \%-41 \%$ \\
\hline \multicolumn{4}{|l|}{ France } \\
\hline Grilliches-Mairesse (1983) & Firm, '73-'78 & & $310 \%$ \\
\hline Cunê-Mairesse (1984) & Firm, $72-77$ & $22 \%-33 \%$ & $55 \%$ \\
\hline Patel-Soete (1988) & Totall economy, '56-"87 & $13 \%$ & \\
\hline \multirow[t]{2}{*}{ Hall-Mairesse (1992) } & Firm, '80-87: cross & $25 \%$ & $78 \%$ \\
\hline & : lime & $7 \%$ & $22 \%$ \\
\hline \multicolumn{4}{|l|}{ Japan } \\
\hline Odagini (1985) & Industry, "60-777 & & $(-) 66-24 \%$ \\
\hline \multirow[t]{2}{*}{ Odagiri-lwata (1986) } & Firm, $66-73$ & & $17 \% \%-20 \%$ \\
\hline & ${ }^{4} 74-182$ & & $110 \% 17 \%$ \\
\hline Patell-Soete (1988) & Total economy, $56-87$ & $13 \%$ & \\
\hline \multicolumn{4}{|l|}{ Germany } \\
\hline Hathoff $(1994)$ & Firm, " $77-89$ & $9 \%-1.3 \%$ & \\
\hline Patel-Soete (1988) & Total econony, '56.87 & $21 \%$ & \\
\hline
\end{tabular}




\subsubsection{Rent Spillovers}

Quality improvements, which are the result of product $R \& D$, may not be reflected completely in new prices for two main reasons: competitive pressure in the innovation producing industry and statistical methods used by statistical agencies to calculate price indices are rarely corrected for quality improvements. In this subsection we investigate the effect of an enhanced quality level on the measurement of the productivity level in the innovation producing and innovation using industries.

A correct productivity measure includes quality or efficiency improvements. In order to obtain the measurement in efficiency units, it is assumed that the number of physical units $\left(X_{f}\right)$ can be multiplied by an efficiency index $\left(E_{p}\right)$.

$$
X_{i}=X_{i} E_{i}
$$

where $X_{i}$ is the number of computers in efficiency units. A quality improvement is reflected in an increase of the efficiency index.

The efficiency index $\left(E_{t}\right)$ can be calculated using a variety of methods:

1) The conventional method, used by statistical agencies, i.e. the "chain system" proposed by Marshall (1887). "The essence of the conventional method is to translate quality into quantity by reference of market prices. If a new variety of a good is introduced, one physical unit of the new good is not simply equated to one physical unit of the old good. Instead, one unit of the new good is regarded as equivalent to one unit of the old good times the ratio of the price of the new good to that of the old good in an overlap period" (Jaszi 1964, p. 405)." According to Triplett (1990), however, we usually only have the price of the old good in the base period and the price of the new good in the current period $(t=1)$. The "conventional" efficiency index in this situation is therefore:

$$
\frac{E_{i}}{E_{0}}=\frac{P_{i}}{P_{0}}
$$

This measure is sometimes termed a "matched models" index. When one compares only prices for models that can be "matched" exactly in the two periods one obtains a "matched-models-only" "ndex (Triplett 1990).

2) Hedonic indices (Waugh 1929, Court 1939, Griliches 1961, 1971. Triplett 1987, 1990). In the hedonic literature it is assumed that goods are bundles of product characteristics, and economic behaviour is related to these characteristics. A value is assigned to each characteristic and aggregation of the number of characteristics times their value gives a proxy for the quality level. A comparison of the value of 
the bundle of characteristics before and after the innovation provides the hedonic efficiency index $\left(E_{i}^{h} / E_{0}^{h}\right)$.

3) Welfare indices: "Quality adjusted" price indices calculated on the basis of welfare gains (consumer surplus) of product innovations (Trajtenberg 1989, 1990). Just as the Hedonic literature uses this approach the characteristic approach to demand, leading to estimates of the preferences for the attributes of products, and from there to value measures of quality change. Trajtenberg (1990) concludes that this method, based on welfare gains, yields a better approximation of quality increases in newly emerging sectors than the hedonic indices. The hedonic indices systematically understate the quality improvements in technologically progressive sectors of the economy but may provide a reasonable first approximation for wellestablished sectors. The problem with a method based on welfare gains is the large requirement of data that are difficult to come about. The quality index based on welfare gains is $\left(E_{t}^{w} / E_{\theta}\right)$.

Beside the quality adjustment method used by statistical agencies, the degree of competition is important for the existence of pure rent spillovers. The degree of competition determines whether quality improvements can be appropriated, i.e. whether quality improvements lead to a higher price level. For simplicity, we assume that the quality and price level in the base period are equal to one $\left(P_{0}=Q_{0}=1\right)$. The relationship between quality and price can then be given by:

$$
P=\varphi \cdot E
$$

With no or a low level of competition, quality improvements will be fully reflected in prices, $\varphi=1 \rightarrow P=E$. If competition is vigourous, then quality improvements will not be reflected in prices, $\varphi=1 / E \rightarrow P=\mathbb{1}$ (i.e. $P$ is unchanged with regard to the money level). With an intermediate degree of competition, quality improvements will be partly reflected in prices, $1 / E<\varphi<1$.

We examine the influence of the degree of competition and the qualityadjustment method on productivity levels of the innovation producing and innovation using sectors. First, we examine the situation when quality improvements are taken into account, using a computer producing sector as an example. We assume that this sector improves the quality level of its products and produces computers against constant unit costs. The productivity level for the computer producing sector in efficiency units $\left(\pi_{p}{ }^{\circ}\right)$ can be represented by:

$$
\pi_{p}^{*}=\frac{X^{p}}{X \cdot C}=\frac{E}{C}
$$

where $\mathrm{C}$ represents the unit production costs for computers which, for ease of exposition, are assumed to be constant over time.

A computer wsing sector exists that needs exactly $X^{r}$ efficiency units of computers. Its productivity level in efficiency units $\left(\pi_{u}{ }^{8}\right)$ can be represented by: 


$$
\pi_{t}^{*}=\frac{R}{T+X^{e}}=\frac{R}{T+X \cdot E}
$$

where $R$ is the constant level of total revenue for the computer using firm and $T$ are the total other costs of this sector, which are also assumed to be constant.

The correct quality adjusted productivity index reflects that the productivity in the computer industry increases if the quality level of a computer increases ( $E$ increases) and the productivity level of the computer using sector stays the same.

The most common procedures used by the statistical agencies involve no correction for quality improvements or "quality adjustment" according to the "conventional" method, i.e. method 1. We investigate the influence of these two methods on productivity under various degrees of competition. With the "conventional" quality adjustment method (method 1) or the use of values (current prices) quality improvements are taken into account as far as they are reflected in the price level $(E=P)$. The productivity index for the computer producing and using firm becomes:

$$
\pi_{p}=\frac{P}{C} \quad, \quad \pi_{u}=\frac{R}{T+X^{*} \cdot \frac{P}{E}}
$$

With vigourous competition $(P=1)$ the productivity index of the computer producing sector stays the same and this index for the computer using sector improves when $E$ increases. So, a quality improvement of computers benefits the productivity index of the using firm instead of that of the producing firm.

With no competition $(P=E)$, quality improvements will be reflected in prices and the conventional index measures the productivity levels correctly. The productivity index of the producing industry increases while this index remains the same for the computer using industry. With an intermediate level of competition $(1<P<E)$ the productivity index of both the producing and using sectors improve.

When the productivity indexes are calculated with constant prices without quality adjustment these indices are:

$$
\pi^{\infty}{ }^{\infty}=\frac{1}{C} \quad, \quad \pi^{\infty}{ }^{\infty}=\frac{R}{T+\frac{X^{e}}{E}}
$$

The productivity levels are independent of the degree of competition with this method. A quality improvement in computers will enhance the productivity level of the computer using industry and will not affect the productivity level of the producing industry. This frequently used method therefore measures the productivity gains systematically in the wrong industries.

The hedonic efficiency index and the welfare efficiency index should give us proxies for the real efficiency index. If these efficiency indexes correctly reflect 
quality/efficiency changes, then the measured productivity increase is equal to the real productivity increase.

$$
\frac{E_{p}}{E_{0}}=\frac{E_{y}^{u}}{E_{0}^{w j}}=\frac{E_{t}^{h}}{E_{0}^{h}}
$$

The measured productivity level in the producing sector is given by equation (6.12) and the measure productivity level of the using industry is given by equation (6.13) after the innovation. When the hedonic indices and welfare indices undervalue the efficiency increase, however, then the productivity increase will be under-measured in the producing sector and over-measured in the using industry. According to Trajtenberg (1990), this will be the case when one uses hedonic indices in newly emerging industries. The problem with both these methods is that they require a lot of data, which are usually unavailable. These methods are therefore seldom used in official data. However, the differences can be large, when price indices are adjusted for quality improvements using the conventional or hedonic method in sectors characterised by rapid technological development. For example, Cole et al. (1986) calculated a conventional and a hedonic price index for processors. The conventional index decreased by an annual rate of $8.5 \%$ and the hedonic index decreased by approximately $18.5 \%$ each year during the 1972-1984 period (see figure 6.1).

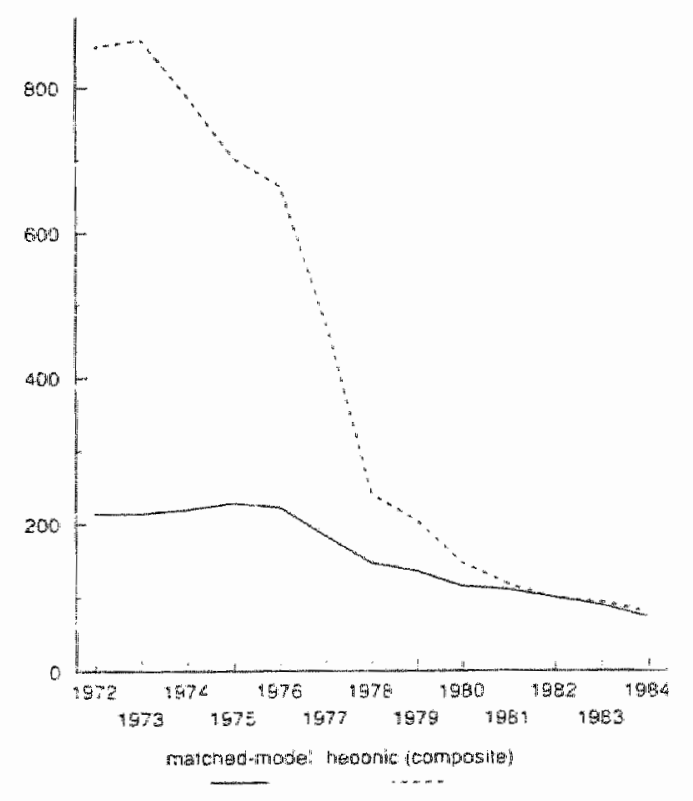

Figure 6.1. Comparison of conventional-model and hedonic price-indices 
Table 6.2 The influence of quality improvement on the productivity level of computer producing and using sectors

$\begin{array}{ll}\text { Vigourous Competion } & \text { No Competition } \\ \phi=1 / E, P=\varphi, E, P=1 & (Q=1, P=Q, E, P=E\end{array}$

Constant Prices adjusted for Quality with Chain Index or Current Prices

Producing Sector: $\pi_{p}=\frac{p}{C}$

Using Sector: $\pi_{*}=\frac{R}{T+X^{*} \cdot \frac{T^{2}}{E}}$

\section{Constant Prices}

Producing Sector: $\pi_{p}^{\text {tix }}=\frac{X . P_{i}}{X . C}=\frac{1}{C}$

$\frac{1}{C}$

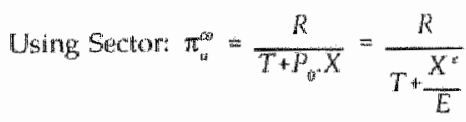

Productivity Index adjusted with correct efficiency index
Producing Sector: $\pi_{p}^{*}=\frac{X^{*}}{X . C}=\frac{E}{C}$

Using Sector: $\pi_{i:}^{r}=\frac{R}{T+X^{r}}$

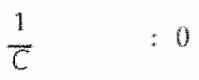

$\frac{R}{T+\frac{X^{e}}{E}}:+$
$\frac{E}{C}$

$\frac{R}{T+X^{E}}: 0$
0

$\frac{1}{\mathrm{C}} \quad: 0$

$\frac{R}{T+\frac{X^{*}}{E}}$

$:+$

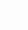

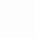


called "pure rent spillowers". It is important to recognise that these spillovers are due to conoentional measurement problens.

In general quality improvements are badly reflected in prices so the innovation using industries get more inputs (in efficiency units) than is measured. Although we do not know the quality or efficiency increase in products $\left(E_{0} / E_{0}\right)$, we can estimate its importance. The production function, which incorporates efficiency indexes for it"s purchased inputs, is:

$Y_{j, t}=A \cdot e^{\lambda, t} \cdot L_{i, t}^{\alpha} \cdot\left[\frac{E_{k, j, t}}{E_{k, j, 0}} K_{i, k}\right]^{\beta} \cdot\left[\frac{E_{m, j, t}}{E_{m, j, 0}} \cdot M_{i, d}\right]^{\infty}$

In growth rates we get:

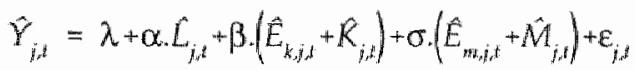

Using the definition of Total Factor Productivity, equation (6.6), gives:

$T \hat{F P} P_{j, t}=\lambda+\beta \cdot \hat{E}_{k j, i}+\sigma \cdot \hat{E}_{m, i, t}+\varepsilon_{i, l}$

The TFP growth in a sector is therefore dependent on the growth rates of the efficiency indexes of capital and intermediate goods. It can be assumed that the growth rate of the efficiency index of purchased goods is dependent on the amount of product R\&D performed in the producing industries. Process R\&D is not important in this context because it does not change the quality level or efficiency index of a product." Product R\&D is aimed at improving the quality level or efficiency index of a product. The amount of product R\&D embodied in a product can therefore be a direct, although imperfect, measure of this efficiency index. Brown and Conrad (1967) have built an efficiency index on the basis of intermediate purchases and total R\&D intensities.

$\hat{E}_{n i s, j}=\gamma_{n+1} \cdot \sum_{i=1}^{n} X_{i j, t} \cdot \frac{R \& D_{i, 1}}{Y_{i, 1}^{\prime}}$

where $X_{i, j}$ are the intermediate goods purchased by sector $j$ from sector $i$ and $R \& D_{i}$ are the R\&D expenditures of sector $i$.

Next to this measure of R\&D embodied in intermediate goods Terlecky) (1974, 1980) used a measure built with capital flows and total R\&D intensities:

91 Process R\&D can be important. howewer, when a product is "linked in" at its introdnctory (or subsequent) price in the official price indices. Process R\&D, which lowers cosls and probably prices, leads again to a produchivity increase in the asing sector. 
$\hat{E}_{k, j}=\gamma_{k} \cdot \sum_{i=1}^{n} I_{i, j} \cdot \frac{R \& D_{i, j}}{\gamma_{i, j}}$

where $I_{i, j}$ are the investment goods purchased by sector $j$ from sector $i$.

All the empirical studies that used purchased goods flows to build the efficiency indexes used total $R \& D$ instead of product $R \& D$. The general model that these studies estimate is a combination of the model developed in section 6.1, in which own R\&D influences TFP growth, and the model described in this section.

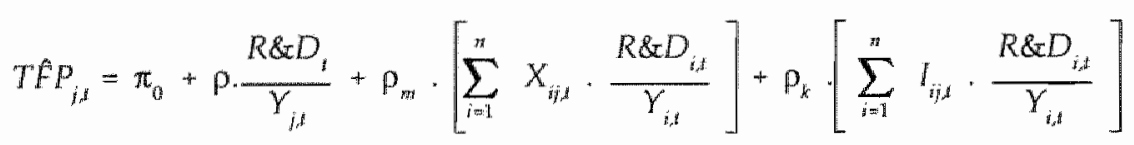

where $p$ is the rate of return to own $R \& D, \rho_{m}$ is the rate of return to rent spillovers of intermediate goods and $\rho_{k}$ is the rate of return to rent spillovers of capital goods. We will now discuss the empirical results of rent spillovers. Table 6.3 shows the rates of return of own $R \& D$ and three rent spillover variables; rent spillovers embodied in purchased intermediate goods or investment goods or a variable which adds these two together. The rate of return to own R\&D is, as in section 6.1 , in the range of 0 (not significant) to $60 \%$, with most estimates between $10 \%$ and $30 \%$. The rates of return on rent spillovers show a much greater dispersion. The largest range that can be constructed for all kinds of rent spillovers is 0 (not significant, Odagiri 1985) to $861 \%$ (Sveikauskus 1981). The rates of return to rent spillovers in intermediate goods range from $0.5 \%$ till $53 \%$, with an outlier of $360 \%$ (Odagiri 1985). The evidence on the rates of return of rent spillovers embodied in investment goods is mixed. Terleckyj (1974) and Sveikauskus (1981) found very high rates of returns in the range of $(262 \%-861 \%)$, while Wolff and Nadiri (1993) found only modest rates of return $(9 \%-11 \%)$. The Terlecky) (1974) study is the only study that estimated both rates of return to rent spillovers in one model. He found that the rates of return to rent spillovers in capital goods were substantially higher than those of intermediate goods. The estimated range for the rates of return, when capital and intermediate rent spillovers are combined, is $45 \%-187 \%$. In general it can be said that $R \& D$ embodied in purchased goods has a positive influence on the productivity growth in the buying sectors. The estimated rates of return to rent spillovers are substantially higher than the estimated rates of return to own R\&D. Another conclusion is that the estimated rate of return to rent spillovers embodied in investment goods is higher than the rate of return to these spillovers embodied in purchased intermediate goods.

Terleckyj (1974) found that the rate of return to rent spillovers in the nonmanufacturing sectors exceeded the rate in the manufacturing sectors and that the rate of return to rent spillovers of privately financed $R \& D(75 \%)$ was higher than that of publicly-financed R\&D ( $45 \%$ ). The last result was also found by Wolff and 


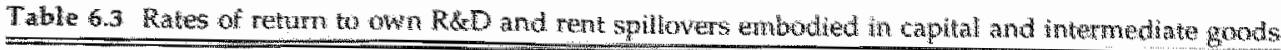

\begin{tabular}{|c|c|c|c|c|c|}
\hline Study & Sample & "OWn" RKD & \multicolumn{2}{|c|}{$\begin{array}{l}\text { Rent Spillowers } \\
\text { Internadiate Goods }\end{array}$} & $\begin{array}{l}\text { Rent Spillovers } \\
\text { Capltal Goods }\end{array}$ \\
\hline Terleckyj & $48-66$ US & & & & \\
\hline \multirow[t]{6}{*}{$(1974)$} & All 33 ind. & $-16 \%$ & & $62 \%$ & \\
\hline & 20 mantif. ind. & $28 \%$ & & $45 \%$ & \\
\hline & 20 manuf. (private) & $29 \%$ & & $78 \%$ & \\
\hline & 13 non-manuf ind. & $-18 \%$ & & $187 \%$ & \\
\hline & All 33 ind. & $6 \%$ & $17 \%$ & & $262 \%$ \\
\hline & 20 manuf. indu. & $10 \%$ & $53_{\not 2 x}^{m p}$ & & $762 \%$ \\
\hline Terleckyi & $48-66$ US & & & & \\
\hline$(1980)$ & 20 nanut ind. & $20 \%$ & & $183 \%$ & \\
\hline Sweikauskas & $59-89$. US & & & & \\
\hline$(1981)$ & $144 \mathrm{ind}$. & $16 \%-25 \%$ & & & $844 \%-867 \%$ \\
\hline Odagiri & $60-77: J P N$ & & & & \\
\hline \multirow[t]{2}{*}{$(1985)$} & 15 manuf. ind. & $30 \%-60 \%$ & 1.. $-360 \%$ & & \\
\hline & 15 manuf. ind. & $-60 \%-24 \%$ & n.s. & & \\
\hline Goto-Suzulki & $78-83: \sqrt{P N}$ & & & & \\
\hline \multirow[t]{2}{*}{ (1989) } & 50 manuf. ind. & $26 \%$ & & $80 \%$ & \\
\hline & 50 manuf. ind. & $28 \%$ & (electronics) & n.s. & \\
\hline Sterlacchini & '54-"84: UK & $14 \%-19 \%$ & $7 \%-12 \%$ & & \\
\hline$(1989)$ & 15 mantef. ind. & & & & \\
\hline Fecher & $81-83: B E L$ & & & & \\
\hline (1990) & 292 firms & n.,.s. & $0.5 \%$ & & \\
\hline Wolff and Nadiri ${ }^{3}$ & $470^{\circ} 77:$ US & & & & \\
\hline \multirow[t]{4}{*}{ (1993) } & 19 marvuf. ind. & $10 \%$ & $14^{6 / 6 x^{4}}$ & & \\
\hline & 19 marnuf. (private) & $38 \%$ & $17 \%$ & & \\
\hline & all 55 ind. & $21 \%$ & & & $9 \%$ \\
\hline & all 55 ind. (private) & $59 \%$ & & & $11 \%$ \\
\hline
\end{tabular}

\footnotetext{
Not significant

"Uses only one variable which includes rent spillovers of capital and intermediate goods.

2 Includes effect of output grow th (learning) on productivity growth.

${ }^{3}$ The R\&D spillovers in the investment goods variable is insignificant among manufacturing sectors and the R\&D spillovers embodied in intermediate goods variable is insignificant across all sectors of the econorny.
}

Nadiri (1993), 11\% versus 9\%. Another interesting finding of these two studies is that rent spillovers of intermediate goods were only significant when only manufacturing industries were included in the sample. Wolff and Nadiri found that 
rent spillovers of investment goods were only significant when manufacturing and non-manufacturing industries were included in the sample. Terleckyj, however, found that rent spillovers of investment goods were significant in both types of samples. These results suggest that rent spillovers embodied in intermediate purchases are only important for manufacturing sectors and that spillovers embodied in investment goods play a role in the non-manufacturing (service) sectors.

Goto and Suzuki (1989) investigated whether the rate of return to rent spillovers of electronic goods (e.g. computers) was higher than the rate of return to other goods. The rent spillovers embodied in all capital and intermediate goods were significant and equal to $80 \%$. When only rent spillovers embodied in electronic goods were considered, the estimated rate of return became insignificant. This indicated no "special" influence by electronic goods.

\subsection{2 "Pure" Knowledge Spillowers}

The second type of spillovers are called "pure knowledge spillovers" and exist when jideas discovered in one sector can be used in other sectors. "Pure" knowledge spillovers occur because a discovery in one firm/sector can provoke a discovery in another sector when new ideas pop up, new avenues of research are undertaken, or previous efforts become economical to pursue or to bring to full fruition. It can be expected that knowledge generated by various sectors differs in its usefulness with regard to own R\&D efforts. Knowledge generated in a sector which is "technologically" close to own sector is probably of more value than knowledge generated in sectors which draw on a totally different knowledge base. "An aircraft manufacturer is more likely to benefit from the $R \& D$ in the "precision instruments" than from R\&D in the wood industry" (Mairesse and Mohnen 1994, p. 838). To create a measure of a useful knowledge pool for each sector we have to weigh, therefore, the knowledge generated by other sectors.

$$
S_{i, t}=\sum_{i, t} \omega_{i, j} R_{i}
$$

$S_{j}$ is the total amount of useful knowledge for industry $j$ and $\omega_{i, j}$ is a weighting factor which represents the technological closeness of sectors $i$ and $j$.

As Griliches $(1979,1992)$ stated, the technological closeness of sectors need not to be related with input purchases as are rent spillovers. "The photographic equipment industry and the scientific instruments may not buy much from each other but may be, in a sense, working on similar things and hence benefiting much from each other's research (Griliches 1979, p. 104)". There are various approaches to identify the technological closeness of industries (see also Griliches 1992). Some of these are listed below. 
1) Give equal weight to all the R\&D stocks of different industries or sum up the various industrial R\&D stocks (see e.g. Bernstein 1988). This method assumes that knowledge generated by different industries is of equal value to an industry.

2) Use STC classification as a measure of technological closeness. Knowledge generated in industries with the same four-digit industry code is most valuable to that industry and the technological distance between these industries is the smallest. Knowledge generated in industries which share in the same thee-digit industry code is less valuable and the technological distance is therefore greater. This argument can be extrapolated to industries that are in the same two digit industry code. The problem with this approach is the extension across other twodigit industries. This approach is followed by, for example, Raines (1968) and Schankerman (1981).

3) Use patent data classified by industry of production and industry of use to construct an inter-industry technology flow matrix or techflow matrix (Scherer 1982. Englander et al. 1988, Evenson et al. 1988, Mohnen and Lépine 1991).

4) Use innovation data classified by industry of production and industry of use to construct an inter-industry technology flow matrix (Robson et al. 1988, Geroski 1989, Sterlacchini 1989).

5) Identify the 'technological position' of a firm with the help of the technological classes in which it patents. It is assumed that two firms/industries benefit more from each others' research when the overlap in technological classes in which it patents is larger. A statistical proximity technique measures the proximity of the firms in 'technological space'. The impact of other firms' R\&D is weighted according to this proximity (Jaffe 1986, 1988, 1989, Goto and Suzuki 1989).

6) Instead of using a concept for technological closeness, one may use the R\&D stocks of different industries as separate variables (Bernstein 1989). The problem with this approach is the multicollinearity between the various R\&D stocks.

7) It is also possible to build a knowledge stock for each industry by using article count data in each scientific field and weighting them by the composition of scientists for each industry. The spillover variable is constructed as a weighted sum of the industry knowledge stocks. The weights are computed by the statistical proximity technique developed by Jaffe (1986) which measures the proximity of industries in the employment of scientists by field (Adams 1990).

The general model to be estimated in these studies includes a measure of own. R\&D and the weighted knowledge stock

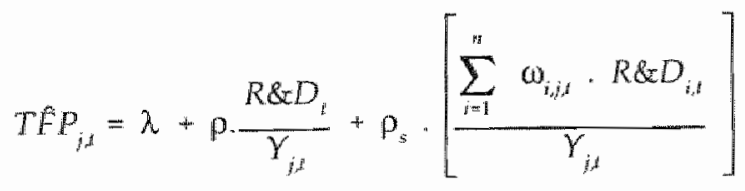


where $p$ is the rate of return to own $\mathrm{R} \& \mathrm{D}$ and $p_{s}$ is the rate of return to knowledge spillovers.

Before we discuss the estimation results of these studies we must first discuss the techflow matrix used by Scherer (1982) and Griliches and Lichtenberg (1984). This matrix is constructed with a base of patents that are investigated and assigned to an industry of origin and an industry of use, $42 \%$ of all patents were classified in one specific industry of use (Scherer 1984). In 17\% of all patents, innovations were classified in two and three industries of use. The division of such a patent across these two or three industries of use is done in proportion to their purchases of the sector which produces this patent. Wide spread innovations $29 \%$ of total innovations) are allocated in proportion to sales (intermediate and investment goods) of the origin industry to using industries (use of transactions and capital flows input-output tables). According to our definition of rent spillovers (embodied in goods) and "pure" knowledge spillovers, this techflow matrix includes a mixture of rent and "pure" knowledge spillovers. We classify it as "pure" knowledge spillovers because it also includes spillovers which are not embodied in or go along with good purchases and because the smaller part, $29 \%$ is based only on good purchases.

The estimated rates of return to own R\&D and knowledge spillovers found by previous empirical studies in this field are shown in table 6.4. As with the studies described in section 6.1 and the studies regarding rent spillovers, the rate of return to own R\&D is statistically significant in almost all cases and the estimates range from 2\% (Sterlacchini) to 56\% (Mohnen and Lépine). Again, most estimates are in the range of 10\%-30\%. Most studies found that "pure" knowledge spillovers had a statistically significant influence on the productivity growth of sectors. The estimated rate of return is dependent on the weighting matrices. Studies that used the "proximity in technological research space" as a weighting matrix found relatively low values for the rate of return to "pure" knowledge spillover. The estimated rate of return lies between $4 \%$ (Goto and Suzuki) and $40 \%$ (Adams). Studies that used a techflow matrix based on patent information found relatively high rates of return to "pure" knowledge spillovers. Most of these studies found estimates that are in the range of $86 \%$ (Mohnen and Lépine) to $147 \%$ (Scherer), however, an exception is the study by Griliches and Lichtenberg (1984), which also found insignificant values for the rate of return to knowledge spillovers. Studies that used a techflow matrix based on innovations or didn't weight the $\mathbb{R}_{\&} \mathrm{D}$ stocks found intermediate values for the estirnated rate of return to knowledge spillovers. Estimates ranged from $7 \%$ (Sterlacchini) to 58\% (Bernstein 1989).

The choice of the weighting matrices also influences the relative values of the rates of return to own $R \& D$ and "pure" knowledge spillovers. In general, the rates of return to "pure" knowledge spillovers are higher than the rates of return to internal R\&D. However, if one uses the "proximity in technological research space" 
Table 6.4 Rates of return to own RaD and "pure" knowledge spillovers

\begin{tabular}{|c|c|c|c|c|}
\hline Study & Sample & $\begin{array}{l}\text { Weighting } \\
\text { matrix }\end{array}$ & Own R\&D & $\begin{array}{l}\text { Knowledge } \\
\text { Spillovers }\end{array}$ \\
\hline Raines (1968) & $24 \mathrm{ind}$. & $\begin{array}{l}\text { proximity in technological } \\
\text { research space (product } \\
\text { fields) }\end{array}$ & & $0.56^{4}$ \\
\hline Scherer $(1982)^{1}$ & $\begin{array}{l}' 64-78: \text { USA } \\
20-87 \text { ind. }\end{array}$ & $\begin{array}{l}\text { origin/use pattent matrix } \\
\text { (input-output tables) }\end{array}$ & $19 \%$ & $118 \%-147 \%$ \\
\hline $\begin{array}{l}\text { Grilliches- } \\
\text { Lichtenberg }(1984)^{1}\end{array}$ & $\begin{array}{l}59-78: \text { USA } \\
193 \text { ind. }\end{array}$ & $\begin{array}{l}\text { origin/use patent matrix } \\
\text { (input-output tables) }\end{array}$ & $11 \%-31 \%$ & $0 \%-90 \%$ \\
\hline $\begin{array}{l}\text { Jaffe } \\
(1986)\end{array}$ & $\begin{array}{l}73-79 . \text { USA } \\
432 \text { firms }\end{array}$ & $\begin{array}{l}\text { proximity in technological } \\
\text { research space (patents) }\end{array}$ & $27 \%$ & $10 \%$ \\
\hline $\begin{array}{l}\text { Bernstein } \\
1988)\end{array}$ & $\begin{array}{l}78-81: \text { CAN } \\
7 \text { ind. } 170 \text { firms }\end{array}$ & $\begin{array}{l}\text { unweighed: sum of outside } \\
\text { R\&D }\end{array}$ & $12 \%$ & $20-26 \%$ \\
\hline $\begin{array}{l}\text { Bermstein-Nadini } \\
\text { (1988) }\end{array}$ & $\begin{array}{l}58-81 \\
5 \text { high-tech ind. }\end{array}$ & $\begin{array}{l}\text { unweighed: } \\
\text { individ ual industry R\&D } \\
\text { stocks }\end{array}$ & $\begin{array}{l}17 \% \\
19 \%-27 \% 1\end{array}$ & $\begin{array}{l}50 \% \\
{[11 \%-161 \%]}\end{array}$ \\
\hline $\begin{array}{l}\text { Jaffe } \\
(1988)\end{array}$ & $\begin{array}{l}\text { "72-77: USA } \\
434 \text { firms }\end{array}$ & $\begin{array}{l}\text { proximity in technological } \\
\text { space }\end{array}$ & $10 \%-115 \%$ & $10 \%$ \\
\hline $\begin{array}{l}\text { Bernstein' } \\
(1989)\end{array}$ & $\begin{array}{l}\text { "63-83: CAN } \\
9 \text { ind. }\end{array}$ & $\begin{array}{l}\text { unweighed: } \\
\text { individual industry R\&D } \\
\text { stocks }\end{array}$ & $\begin{array}{l}33 \% \\
{[24 \%-47 \%]}\end{array}$ & $\begin{array}{l}58 \% \\
|29 \%-94 \%|\end{array}$ \\
\hline $\begin{array}{l}\text { Goto-Suzuki } \\
(1989)\end{array}$ & $\begin{array}{l}78-^{-} 83: \sqrt{ } \mathrm{PN} \\
50 \text { manuf. ind. }\end{array}$ & $\begin{array}{l}\text { proximity in technological } \\
\text { research space }\end{array}$ & $29 \%$ & $4 \% \%^{2}$ \\
\hline $\begin{array}{l}\text { Sterlacdimi } \\
(1989)\end{array}$ & $\begin{array}{l}\text { "54 " } 84: \text { UK } \\
15 \text { manuf. ind. }\end{array}$ & origin/use innovation matrix & $2 \%-14 \%$ & $7 \%-32 \%$ \\
\hline $\begin{array}{l}\text { Adams } \\
(1990)\end{array}$ & $53-80:$ USA & $\begin{array}{l}\text { technological proximity } \\
\text { (scientific articles) }\end{array}$ & $35 \%$ & $40 \%{ }^{3}$ \\
\hline $\begin{array}{l}\text { Mohnen-Lépine* } \\
(1991)\end{array}$ & $\begin{array}{l}75-83: \text { CAN } \\
12 \text { manuf. ind. }\end{array}$ & origin/use patent matrix & $\begin{array}{l}56 \% \\
{[5 \%-144 \%]}\end{array}$ & $\begin{array}{l}86 \% \\
111 \%-319 \%\end{array}$ \\
\hline
\end{tabular}

"Cost-function or dual approach.

The results for process oriented and product oriented R\&D found in this study are described in table 6.5. In Scherer's techflow matrix, widespread innovations are allocated in proportion to sales of the origin industry to using industries. This matrix therefore measures rent and knowledge spillovers.

"Includes only knowledge spillovers from electronic industries. This regression also includes a variable which measures rent spllovers of electronic capital and intermediate goods. However, this variable is not significant.

${ }^{3}$ Mean elasticity based on table 11 of Adams (1990), page 699. " Output elasticity 
as a weighting matrix, then the rate of return to own $R \& D$ exceeds this rate of "pure" knowledge spillovers.

\subsubsection{Theoretical versus Empirical Implementation of Rent and Knowledge Spillowers}

In theory it is possible to make a distinction between rent and knowledge spillovers. Rent spillovers exist because inputs are not correctly measured and are therefore not "pure" knowledge spillovers. "Pure" or real knowledge spillovers reflect ideas discovered in one sector that can be used by research teams in other sectors. The empirical implementation discussed in sub-sections 6.2 .1 and 6.2 .2 suggests that rent spillovers can be measured as (product) R\&D embodied in purchased inputs and knowledge spillovers can be measured with some indicator of the technological closeness of sectors. However, there is a caveat in this line of reasoning. In reallity it can be expected that some "pure" knowledge or ideas flow together with input purchases. Input purchases mean that people communicate and can therefore learn from each other. Measures constructed with input purchases include not only "pure" rent spillovers but also knowledge spillovers that go along with these purchases. The measured rent spillovers in sub-section 6.2 .1 should therefore be carefully interpreted.

From this point onward "rent spillovers" will be defined as all spillovers that are obtained together with input purchases. Rent spillovers therefore include "pure" rent spillovers (quality measurement errors) and knowledge spillovers related to input purchases. The rates of return depicted in table 6.3 reflect both of these effects. The knowledge spillover concepts treated in section 6.2 .2 reflect all knowledge flows that are not related to input purchases and will be called "pure" knowledge spillovers. "Pure" simply means that they are not related to inputs.

\subsection{Process R\&D, Product R\&D and R\&D Spillover Effects}

The division between process-oriented $R \& D$ and product-oriented R\&D may provide a useful distinction leading to better insights into the process of technologicall change. It can be expected that process R\&D, which attempts to reduce production costs, increases the productivity of the performing sector. Product-oriented R\&D attempts to increase the quality or efficiency level of products. As described in section 6.2 , an increase in the quality or efficiency level of a product is often poorly reflected in price indices and therefore in the measurement of productivity. If a statistical agency uses the "conventional" or "chain" method then an increase in the quality level of a product is only reflected in an improvement of the productivity level when the quality change is reflected in the product's price. The degree to which quality is reflected in prices is dependent on the degree of competition in an industry. The relationship between product $\mathbb{R}: D$ and productivity is therefore dependent on the degree in which quality 
improvements are measured by the productivity measures, which is dependent on the degree of competition and the statistical methods used. If quality changes are fully reflected in the productivity measurement, then product R\&D has a positive influence on the performing sector. If quality changes are not reflected in productivity measurements, then product $\mathrm{R} \& \mathrm{D}$ tends to raise productivity of industries purchasing the improved product and tends to have no influence on the productivity level of the performing industry. If only a part of the quality change is reflected in productivity levels, then product $R \& D$ tends to raise the productivity level of both the performing and using industry.

Table 6.5 Rates of return to process and product R\&D and used R\&D

\begin{tabular}{|c|c|c|c|c|c|}
\hline Sudy & $\begin{array}{l}\text { Process R\&D } \\
+ \text { Product R\&D }\end{array}$ & $\begin{array}{l}\text { Process } \\
\text { RRD }\end{array}$ & $\begin{array}{l}\text { Product } \\
\text { RAD }\end{array}$ & $\begin{array}{l}\text { Process ReD + } \\
\text { product RifD } \\
\text { spillovers }\end{array}$ & $\begin{array}{l}\text { Product ReD } \\
\text { spillowers }\end{array}$ \\
\hline
\end{tabular}

Limik (1982)

$74-78: 275$ firms

: high tech

: medium tech

: low kech

$\begin{array}{ll}22 \% & 1 \% \\ 34 \% & 4 \% \\ 1 \% & (-2 \% \% \\ 6 \% & (-) 2 \%\end{array}$

Sclveren (1982, 1984)

"48-69:20 ind. $\quad 19 \%$

$64-69.87$ ind.

$13 \%$

"73-78: 87 ind.

$29 \%$

$64 \%$

$73-78: 37-87$ ind

$37 \%-93 \% \quad 0 \%-32 \%$

$87 \%-147 \%$

Griliches-Lichtenberg (1984)

'64-'69: 193 ind.

$73-78$ : 193 ind.

"59-78: 193 and." $11 \%$ \% $31 \%$

59-68: 193 ind. 29\%

$69-78: 193$ ind. $\quad 31 \%$

\begin{tabular}{|c|c|}
\hline $11 \%-31 \%$ & $38 \%-76 \%$ \\
\hline $29 \%$ & $76 \%$ \\
\hline $31 \%$ & $38 \%$ \\
\hline
\end{tabular}

\footnotetext{
Not significant.
}

Estimated range across all specifications and sub-periods.

In theory, the division of $R \& D$ into process-oriented and product-oriented R\&D is useful. However, there are only a few empirical studies that have tested the different roles of process and product $R \& D$ in relation to productivity growth. The 
is mainly due to the unavailability of this kind of data. In addition, it is extremely difficult to divide total $R \& D$ expenditures into process and product $R \& D$ expenditures. First, "It should be remembered, however, that the distinction between product and process innovation is relative to the organizational detail reflected in the data. Product improvements in producer goods become process improvements for their users (Terleckyj 1982, p. 86)". Second, R\&D devoted to the design or development of new products often requires accompanying process improvements because the production process has to be adopted to produce the new product and vice versa. "Not only are process and product efforts jointly pursued on a project basis, and thus difficult to disentangle, but even pure product development can change the efficiency of a process. A new product design, for example, may lead to a reduction in the number of operations required or in simplification of tasks, so that labour input is reduced even without any capital investment (Clark and Griliches 1984, p. 407)".

Next, we discuss the results of the few empirical studies that separated R\&D expenditures into process-oriented and product-oriented $R \& D$ expenditures. The first approach used questionnaires while the second approach used the diagonal elements in a techflow matrix as proxies for process R\&D.

Link (1982) created a data set of 275 manufacturing firms that responded to a mailed questionnaire concerning the allocation of $R \& D$ to product and process innovation. He first examined the influence of profits and the technological environment on the inter-firm differences in the ratio of process versus product $R \& D$. The more profitable firms were relatively more process intensive while firms in a technological environment characterised by lucrative opportunities for new product breakthroughs and by complex product configurations were relatively more product intensive. Link also examined the relationship between product and process $\mathrm{R} \& \mathrm{D}$, and productivity growth. Table 6.5 shows the estimated results of this study which suggest that process $R \& D$ has the greatest influence on productivity growth. Link found no significant influence of product R\&D on productivity. The rate of return to process R\&D was $22 \%$ when all firms were included in the sample. Allowing for industry differences shows that the rate of return to process $\mathrm{R} \& \mathrm{D}$ is high $(34 \%)$ for high-tech firms and low for medium- (1\%) and low-tech firms $(6 \%)$. Terleckyj (1982) studied the influence of total R\&D expenditures versus process $R \& D$ expenditures on the productivity growth of an industry. He found that process $\mathbb{R} \& D$ had contributed to the productivity of the industry in which it was conducted, whereas the total $\mathbb{R} \& D$ expenditures had not. Clark and Griliches (1984) found that an increase in product R\&D's share in total R\&D investment is associated with a lower rate of productivity growth. These studies, based on questionnaires, all suggest that process $\mathbb{R} \& D$ is more important than product $R \& D$ with respect to productivity growth.

Another way to obtain the percentage of process $R \& D$ in total $R \& D$ is to use a techflow matrix. Schmookler (1966) postulated a kind of input-output matrix of 
invention flows. The rows of such a matrix represent the industries which produce the invention and the columns represent the sectors which use the innovation. An interesting feature of such a techflow matrix is that the diagonal elements can be interpreted as process innovations. Division of this diagonal element by the row totals delivers the percentage of process $R \& D$ in total $R \& D$. This method is used by Scherer $(1982,1984)$ and Griliches and Lichtenberg (1984). Scherer investigated the following hypothesis; because quality improvements are poorly reflected in prices, it can be expected that "used" R\&D which consists of "own" process R\&D and product $R \& D$ embodied in good purchases, has a larger influence on the productivity level of a sector than total "own" R\&D (internal process and product R\&D). Table 6.5 shows that indeed "used" R\&D was more important than total "own" R\&D in the studies of Scherer and Griliches and Lichtenberg $(G-L)$. The rate of return to "own" $R \& D$ was $19 \%\left(r^{2}=0.22\right)$ and that of "user" $R \& D$ was $87 \%$ $\left(r^{2}=0.44\right)$ in the study of Scherer. The study by G-L estimated a range of $11 \%-31 \%$ for the rate of return to total "own" R\&D and a range of $41 \%-62 \%$ for "user" R\&D. These results indicate that product $R \& D$ performed by other sectors embodied in goods is more important than "own" product R\&D with respect to a sectors productivity level.

These studies also estimated the influence of "own" product $R \& D$ versus "used" R\&D on productivity growth for two time periods (1964-1969 and 1973-1978). Used R\&D was more important than "own" product R\&D for productivity growth in both studies for both periods. However, there is a clear trend that product R\&D is becoming more important. The rate of return to product R\&D increases from $13 \%$ (insignificant) to $29 \%$ (significant) in the study by Scherer and from $15 \%$ to $28 \%$ in the study by G-L. However, the studies found different results with respect to the most important determinant of "used" R\&D and with respect to the influence of "used" R\&D over time. Scherer found that the spillover variable (embodied product $R \& D$ ) was the most important determinant to "used" R\&D and that the influence of "user" $R \& D$ is becoming more important over time. The latter is caused by an increase in the influence of the spillover variable. G-L on the other hand found that "own" process R\&D contributed most to "used" R\&D and that the influence of "user" R\&D declined over time. This decline was caused by a decrease in the rate of return to "own'" process $R \& D$.

\subsection{Conclusion}

It is not easy to investigate the relationship between $R \& D, R \& D$ spillovers and productivity as the relationships between these variables are highly complex. The benefits of $R \& D$ are uncertain and occur with long lags. The amount of knowledge and the usefulness of this amount of knowledge for other firms is dependent on the nature of the producing and using sectors, their technological and economical relationship, time lags and sometimes coincidence. In addition to all these complex 
relationships, the quality of the available data is questionable. One important data problem in this context is that quality improvements are not or only partly reflected in price indices. In econometric studies the complex relationships have to be reduced to simple models that can be estimated. "It is therefore an agreeable surprise to discover that most studies have managed to produce statistically significant and frequently plausible estimates of the elasticity of $R \& D$ or the rate of return to R\&D" (Mairesse and Sassenou 1991). We can conclude the same for the studies that measured $R \& D$ spillover effects.

The studies in section 6.1 found a positive influence of "own" or internal R\&D on productivity growth. Section 6.2 added that spillover effects also had an influence on the productivity level. A part of the "residual" can be explained by R\&D performed in other sectors. Firms are not islands but are captured in economic and technological networks. Spillover effects can be related or unrelated to input purchases. Measured spillovers related to inputs, the so-called rent spillovers, reflect both quality mis-measurement ("pure" rent spillovers) and real knowledge spillovers that come along with imput purchases. On the other side, knowledge spillovers exist that are not related to input purchases, the so-called "pure" knowledge spillovers. An empirical model that deals with spillover effects has to consider all these effects, otherwise it only measures a part of the spillover effects and it neglects some determinants of technological change.

Considering the econometric studies on R\&D and R\&D spillovers, there is a need for a theoretical/empirical model that treats "own" $R \& D$ and the various spillover effects in one framework. The endogenous technological change model developed in the theoretical chapter of this thesis can serve as a starting point. The model should be elaborated with the fact that quality improvements are badly reflected in prices. Therefore, it should also incorporate "pure rent spillovers". In chapter 7 we develop a model that integrates rent and knowledge spillovers in one framework and can handle quality improvements which may or may not be reflected in the data.

Section 6.3 studied the importance of process and product R\&D with regard to productivity growth. Despite the straightforward theoretical division of R\&D into process-oriented and product-oriented, it is a very dangerous job in an empirical sense. Empirical results on both kinds of R\&D have to be carefully interpreted. The expected positive influence of process R\&D on productivify is found in almost all studies, the influence of product $\mathbb{R} \& D$ on productivity was in general not significant. However, there is a slight indication that the influence of product $R \& D$ on productivity is increasing over time. This may be connected to the IT area in which pure product development diminishes the number of components (e.g. integrated circuits replace many parts) or tasks to produce a product. 


\section{$R \& D$ and}

\section{R\&D Spillovers}

"Why don't we know more about the sources of productioity growth? Why does it feel as if the glass is still half-empty?--The metaphor of the glass halfempty is also misleading. As we fill it, the glass keeps growing. A major aspect of learning is that the unknown keeps expanding as we learn. This showld be looked at positively. It is much better this way-especially for those of us who are engaged in research!" (Zvi Griliches 1994, pp. 17-18).

This chapter measures the influence of the R\&D process throughout the economy. It estimates the most important parameters of the endogenous technological change model developed in the theoretical part of this thesis. We investigate the importance of internal R\&D efforts as well as the influence of several spillover effects. Other important elements of the theoretical model, such as the "special" influence of the current general purpose technology, will be investigated in the next chapter. We use the "extended production function approach" in this empirical chapter. Despite the fact that this approach is commonly used, our study differs in a number of ways from the standard approach.

First, R\&D expenditures are divided into process-oriented R\&D and productoriented R\&D. These types of R\&D have different influences on own and other sectors' productivity (Scherer 1982) and on demand. The explicit distinction between efficiency gains and demand creation effects of various R\&D concepts is a second feature which differs from the existing literature. We follow the argument of Scherer (1982) that industries engage in process $R \& D$ to achieve cost-reducing innovations. The main impact of intermal process $R \& D$ can therefore be expected on the industries own productivity level. Product R\&D is especially oriented to increase the quality of its products. The main effect of increasing the quality of a product is to increase expected demand by expanding the size of the total market or increasing market share. We formulate several models. In the first model process $\mathrm{R} \& \mathrm{D}$ influences the efficiency level, and product $\mathrm{R} \& \mathrm{D}$ influences the expected demand through a higher quality level. In the second model a higher quality level also changes the cost structure of a product. In this context, we distinguish two wews. The traditional view assumes that a higher quality level can only be achieved at higher production costs (see for example Dorfman and Steiner (1954)). 
Another view is that current product development is aimed at developing a new product which is better and cheaper than the old product. Which of these two views gets empirical support is a third contribution. In the first two models we take the common assumption that quality improvements are not reflected in price indices. The third model will relax this assumption and investigate whether this does influence the regressions to be estimated or the interpretation of the regression coefficients.

The fourth contribution of this chapter is the integration of various spillover concepts into one framework. In chapter 6 we discussed the two kinds of research and development spillovers distinguished by Griliches (1979): "pure rent spillovers" and "knowledge spillovers". We identify two varieties of knowledge spillovers; knowledge spillovers that come along with input purchases and "pure" knowledge spillovers that are not-related to input purchases.

The spillover variables are all a weighted index of R\&D performed in other sectors. The "pure" rent spillovers and input-related knowledge spillovers consist of R\&D "embodied" in purchased intermediate and capital inputs of other industries. We therefore weigh these spillovers by input-output coefficients and investment coefficients (Terlecky) 1974). Knowledge spillovers need not be related with input purchases (Grifliches 1979). As an imperfect proxy for these non-input related knowlecge spillovers we use the Yale techflow matrix to weigh the R\&D performed by other sectors (Evenson et al 1989). This matrix is constructed on the basis of patent information available from the Canadian Patent Office. For each patent, the industries of manufacture and industries of use are known. The final techflow matrix predicts, given the level of $R \& D$ expenditures in a sector, the part of this R\&D which can be used in other sectors.

A fifth point which this chapter investigates is whether all these effects differ for different groups of industries. We distinguish between high-tech industries, services and other industries.

A last contribution of this chapter is the investigation of the assumptions underlying the TFP growth calculation. This study investigates the influence of the assumptions of optimal choices of production factors and constant returns to scale on the regression results. Another question in this context is whether the use of a two-factor or a three-factor TFP growth rate does influence the regression results.

We use mainly French INSEE sectoral data to investigate these questions. Our database contains time-series data for thirty sectors, during the period from 1977 to 1992. These data are recent compared to other contributions in this field, which could be important with regard to spillover effects as it is suspected that intersectoral spillovers have increased during the 1980s due to the paradigmatic shift from electrochemical automation to information technologies (see chapter 1).

In the next section the three models of endogenous technological change caused by cost-reducing and demand-creating innovations in the presence of rent and knowledge spillovers are developed. Section 7.2 contains a data description of the 
most important variables and section 7.3 and 7.4 show the estimation results. Section 7.3 estimates the model with gross value added and actual production, with or without constant returns to scale, for the whole economy. Section 7.4 differs from this section because it investigates whether the influence of own R\&D or the various spillovers are different for different groups of sectors.

\subsection{A Model of Technology Generation by Cost Reduction and Demand Creation with Spillovers}

The theoretical model underlying the present analysis treats technological change as an endogenous process in which industries invest in cost-reducing and demandcreating innovations. This model is related to the static model of Levin and Reiss (1988) and their dynamic counterpart developed in chapter 3 (without spillovers) and 4 (with knowledge spillovers). These models will be elaborated on by introducing "pure" rent spillovers of intermediate and capital goods.

In this section we formulate the equations to be estimated in the third section of this chapter. The empirical implementation of the endogenous technological change model developed in chapter 3 and 4 has some data problems. The most important problem is that quality improvements are not or are poorly reflected in price indices. The situation where price indices are not adjusted for quality improvements is described in sub-section 7.1.1 and sub-section 7.1.3.A. In these sub-sections we introduce rent spillovers to include the effect that inputs are undervalued. In sub-section 7.1.1 we define equations to be estimated when industries perform process $R \& D$ to increase their productivity level and product $R \& D$ has no influence on unit production costs. In sub-section 7.1.3.A product $R \& D$ may influence unit production costs in a positive or negative direction. Sub-section 7.1.3.B investigates the situation where a part of the quality improvements are reflected in price indices and whether this influences the interpretation of the parameters. Sub-section 7.1.2 deals with demand creation.

\subsubsection{Cost Reducing Innovations}

In this model the production function is represented by:

$$
Y_{j}=A_{i}, F\left(L_{j}, K_{j}^{e}, M_{j}^{e}\right)=A_{i} L_{j}^{\alpha} \cdot\left(K_{j}^{e}\right)^{\beta} \cdot\left(M_{j}^{e}\right)^{\alpha}
$$

where $Y_{j}, A_{j}$ and $L_{j}$ are respectively the output, the total factor productivity (TFP) and the labour input of industry $j$. The other two inputs, capital $\left(K_{j}^{*}\right)$ and intermediate goods $\left(M^{*}\right)$, are measured in efficiency units. We measure these purchased products in efficiency units to account for pure rent spillovers (see subsection 6.2.1). 
The purchased amount of efficiency units by an industry is the number of physical units times an efficiency index. It can be expected that the efficiency index is dependent on the amount of embodied product R\&D.

$$
\begin{aligned}
& K_{i}^{e}=K_{j} \cdot E_{k, j}^{\bar{\lambda}} \quad ; \quad E_{k, j}=\int_{0}^{1} \sum_{i=1}^{n} \frac{I_{i, T}}{Y_{i, T}} R D_{i, T} \cdot d T+E_{k, j, 0} \\
& M_{j}^{e}=M_{j} \cdot E_{m, j}^{\mu} \quad ; \quad E_{m, j}=\int_{0}^{1} \sum_{i=1}^{n} \frac{X_{i, T}}{Y_{i, T}} R D_{i, T} \cdot d T+E_{m i, 0}
\end{aligned}
$$

where $R D_{i}$ is the amount of product $R \& D$ expenditures in sector $i, X_{i, j}$ is the intermediate delivery of good $i$ to sector $j, l_{i, j}$ is the investment delivery of good $i$ to sector $j$ and $n$ is the number of sectors.

The productivity generation process for an industry is:

$$
A_{i}=\left[\eta_{i} e^{\eta \cdot r} \cdot S_{i}^{\theta_{1}} \cdot S_{k, j}^{\theta_{2}} \cdot S_{m, j}^{\theta_{s}}\right] \cdot R_{c, j}^{\theta}
$$

Industries can increase their productivity by engaging in process $R \& D\left(R_{c, j}\right.$ is the process R\&D stock of sector $j) .{ }^{92}$ The productivity of own process $R \& D$ with regard to the productivity generation process is dependent on the accessibility to a general knowledge pool. We divide the knowledge spitlovers into knowledge spillovers that are related to capital $\left(S_{k_{i}}\right)$ and intermediate $\left(S_{m_{i}}\right)$ input purchases and "pure" knowledge spillovers that are not related to input purchases $\left(S_{j}\right)$. In chapter 4 and 5 an industry uses knowledge generated in other industries to increase the productivity of internal $R \& D$ efforts. $\theta, \theta_{1}, \theta_{2}$ and $\theta_{3}$ are the elasticities of productivity generation with regard to process $R \& D$ and the three kinds of knowledge spillovers. The constant $\eta_{0}$ is the productivity in the base year and the parameter $\eta$ represents its exogenous growth rate.

The "pure" knowledge spillover variable unrelated to inputs is a weighted R\&D stock of other sectors R\&D, industries "use" different amounts of knowledge from other industries dependent on their technological distance from them:

$$
S_{j}=\sum_{j=1}^{n} \omega_{i, j} R_{i}
$$

$S_{j}$ is the total amount of aggregated knowledge used by industry $j$ from all other industries. $\omega_{i, j}$ is a weighting factor that represents the technological closeness of the sectors $i$ and $j$.

\footnotetext{
92 Notice that the derinition of $R_{c, j}$ llas changed in comparison to chapters 3 to 5 . In these chapters $R_{c, j}$ denoted the process R\&D expenditures of sector $j$.
} 
The knowledge spillover variable related to inputs are associated with the purchase of goods and are therefore closely linked to the concept of pure rent spillovers. The most important difference is that pure rent spillovers are mainly caused by product $R \& D$ and knowledge spillovers are mainily caused by total $R \& D$. We can identify knowledge spillovers related to investment $\left(S_{k_{i}}\right)$ and intermediate $\left(S_{m, j}\right)$ purchases.

$$
S_{k, j}=\sum_{j=1}^{n} \frac{l_{i, j}}{Y_{i}} \cdot R_{i}, S_{m, i}=\sum_{j=1}^{n} \frac{X_{i, j}}{Y_{i}} \cdot R_{j}
$$

We calculated the growth rates of the production function (equation(7.1)):

$$
\hat{Y}_{i}=\hat{A}_{i}+\alpha \cdot \hat{L}_{j}+\beta \cdot\left(\hat{R}_{i}+\lambda \cdot \frac{E_{k, j}}{E_{k, j}}\right)+\sigma \cdot\left(\hat{M}_{i}+\mu \cdot \frac{\dot{E}_{m, j}}{E_{m, j}}\right)
$$

where hats represent growth rates and dots depict the derivative with respect to time. Using the time derivative of equation (7.2) and equation (7.3) together with equation (7.7) we get:

$$
\hat{Y}_{j}=\hat{A}_{j}+\alpha \cdot \hat{L}_{j}+\beta \cdot \hat{K}_{j}+\sigma \cdot \hat{M}_{i}+\beta \cdot \lambda \cdot \frac{\sum_{i=1}^{n} \frac{l_{i, j}}{Y_{i}} R D_{i}}{E_{k, j}}+\sigma \cdot \mu \cdot \frac{\sum_{i=1}^{n} \frac{X_{i, j}}{Y_{i}} R D_{i}}{E_{m, j}}
$$

The partial output elasticities of capital and intermediate consumption are:

$$
\beta . \lambda=\frac{\partial Y_{i}}{\partial E_{k, j}} \cdot \frac{E_{k, i}}{Y_{i}} \quad, \quad \sigma \cdot \mu=\frac{\partial Y_{i}}{\partial E_{m, j}} \cdot \frac{E_{w z, j}}{Y_{i}}
$$

Using these definitions in combination with equation (7.8) and the Terleckyi transformation, we obtain: ${ }^{93}$

$$
\hat{Y}_{i}=\hat{A}_{j}+\alpha \cdot \hat{L}_{i}+\beta \cdot \hat{K}_{j}+\sigma \cdot \hat{M}_{i}+\frac{\partial Y_{j}}{\partial E_{k, j}} \cdot \frac{\sum_{i=1}^{n} \frac{l_{i, j}}{Y_{i}} R D_{i}}{Y_{j}}+\frac{\partial Y_{i}}{\partial E_{m, j}} \cdot \frac{\sum_{i=1}^{n} \frac{X_{i j}}{Y_{i}} \cdot R D_{i}}{Y_{i}}
$$

Taking the growth rate of the technology generation process (equation (7.4)) we obtain: 


$$
A_{i}=\eta+\theta \cdot \hat{R}_{c, i}+\theta_{1} \cdot S_{j}+\theta_{2} \cdot \hat{S}_{k, j}+\theta_{3} \cdot \hat{S}_{m, j}
$$

Substituting equation (7.11) in equation (7.10) we get the first general equation which can be estimated as:

$$
\hat{\gamma}_{j}=\eta+\theta \cdot \hat{R}_{c, j}+\theta_{1} \cdot \hat{S}_{j}+\theta_{2} \cdot \hat{S}_{k, j}+\theta_{3} \cdot \hat{S}_{m, j}+\alpha \cdot \hat{L}_{i}+\beta \cdot \hat{K}_{i}+\sigma \cdot \hat{M}_{i}+\rho_{e k j} \frac{\sum_{i=1}^{n} \frac{I_{i, j}}{Y_{i}} R D_{i}}{Y_{j}}+\rho_{e m} \frac{\sum_{i=1}^{n} \frac{X_{i, j}}{Y_{i}} \cdot R D_{i}}{Y_{j}}
$$

where $\rho_{e k}=\partial Y_{j} / \partial E_{k, j}$ and $\rho_{e m i}=\partial Y_{j} / \partial E_{m, j}$

One problem is that $R \& D$ and spillover stocks require data on $R \& D$ expenditures over an extended period. We can avoid this problem by considering the marginal productivity or "rate of return" instead of the elasticity with regard to $R_{c, j}, S_{j,} S_{k, i}$ and $S_{n, i, j}$ (see section 6.1). Equation (7.12) can therefore be estimated as:

$$
\begin{aligned}
& \hat{Y}_{i}=\eta+\alpha \cdot \hat{L}_{i}+\beta \cdot \hat{R}_{i}+\sigma \cdot \hat{M}_{i}+\rho_{r c} \cdot \frac{R C}{Y_{i}}+\rho_{w c} \cdot \frac{\sum_{i=1}^{n} \omega_{i, j} R \& D_{i}}{Y_{i}}+\rho_{s c k} \cdot \frac{\sum_{i=1}^{n} \frac{l_{i, j}}{Y_{i}} \cdot R \& D_{i}}{Y_{j}} \\
& +\rho_{s e m i} \frac{\sum_{i=1}^{n} \frac{X_{i, j}}{Y_{i}} R \& D_{i}}{Y_{i}}+\rho_{e k} \cdot \frac{\sum_{i=1}^{n} \frac{Y_{i, j}}{Y_{i}} R D}{Y_{i}}+\rho_{\varepsilon m m} \cdot \frac{\sum_{i=1}^{n} \frac{X_{i j}}{Y_{i}} R D}{Y_{i}}
\end{aligned}
$$

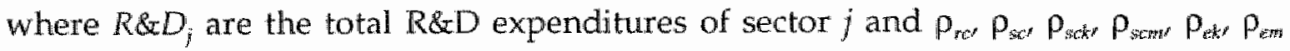
are, respectively, the rates of return to process $R \& D$, non-input related knowledge spillovers, capital related knowledge spillovers, intermediate goods related knowledge spillovers and rent spillovers of capital goods and rent spillovers of intermediate goods. The subscript $c$ indicates that this variable is related to the productivity generation process in which process $R \& D$ plays a key role.

A second problem is the expected high correlation between product and total R\&D expenditures.4. This causes identification problems between input-related knowledge spillovers and "pure" rent spillovers. As described in sub-section 6.2.3 we redefine the spillover concepts and include both "pure" rent spillovers and input-related knowledge spillovers in one concept called rent spillovers. In our

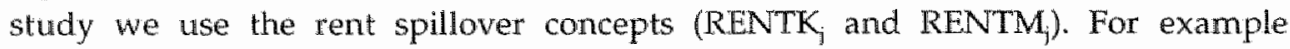
RENTK $_{\mathrm{i}}$ reflects that quality improvements are not reflected in prices of investment goods ("pure" rent spillovers, $\rho_{e k}$ ), and an approximation of knowledge spillovers that come along with the good $\left(\rho_{\text {scak }}\right)$.

\footnotetext{
94 The correlation coefficicat in our sample is tor example 0.93 (see table 7.6 ).
} 
A third well known problem associated with estimating equation (7.12) is multcollinearity between the two inputs, capitall and labour. This problem can be circumvented by subtracting the labour or capital growth rate from both sides.

$$
\begin{aligned}
& \left(\frac{\hat{Y}_{i}}{L_{j}}\right)=\eta+\pi_{c o s}+\beta\left(\frac{\hat{R}_{i}}{L_{i}}\right)+\sigma \cdot\left(\frac{\hat{M}_{i}}{L_{i}}\right)+p_{n:} \cdot \frac{R C}{Y_{j}}+p_{s e} \cdot \frac{K N O W_{i}}{Y_{i}} \\
& +\left(\rho_{e k}+\rho_{s c k}^{\prime}\right) \cdot \frac{R E N T K_{i}}{Y_{i}^{\prime}}+\left(\rho_{e m}+\rho_{s c m}^{\prime}\right) \frac{R E N T M_{i}}{Y_{i}}
\end{aligned}
$$

where $\pi_{c r s}=\alpha+\beta+\sigma-1, \hat{\gamma}_{j} / L_{i}, R_{j} / L_{j}, \hat{M}_{j} / L_{j}$ are respectively the growth rate of labour productivity, the growth rate of the capital-labour ratio and the growth rate of intermediate goods-labour ratio. $K N O W=\sum_{i} \omega_{i, j} R n D_{i}$ represent the non-input related knowledge spillovers, $R E N T K_{i}=\sum_{i}\left(I_{i, j} / Y_{j}\right) \cdot R D_{i}$ represents the "pure" rent spillovers and input-related knowledge spillovers of investment goods and $R E N T M_{j}=\sum_{i}\left(X_{i, j} / Y_{i}\right) \cdot R D_{i}$ represents these spillovers related to intermediate goods. This equation can also be used to test constant returns to scale. When the estimated coefficient, $\pi_{c r s}$ of $L_{j}$ is not significantly different from zero then there are constant returns to scalle, while a negative value implies decreasing returns to scale and a positive value implies increasing returns to scalle. It is also possible to divide equation (7.13) by the growth rate of capital, the dependent variable then becomes the capital productivity growth rate.

If we assume constant returns to scale $(\alpha+\beta+\sigma=1)$ and optimal choices of factors of production, we can directly measure the TFP growth rate by calculating the labour and intermediate good elasticities ( $\alpha$ and $\sigma$ ) by the share of the costs of labour (wages and related charges) and intermediate goods in total variable costs. In that case the growth rate of TFP is given by:

$$
T \hat{F P}_{j}=\hat{\gamma}_{i}-\alpha \cdot L_{i}-(1-\alpha-\sigma) \cdot \hat{K}_{j}-\sigma \cdot \hat{M}_{j}
$$

When we combine equation (7.12) and equation (7.15) we get the following general form of the TFP equations estimated in this chapter:

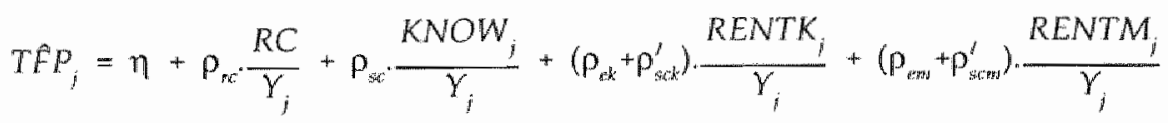

The total factor productivity growth is therefore dependent on the own process R\&D expenditures, "pure" or non-input-related knowledge spillovers, rent embodied in purchased intermediate goods, rent spillovers embodied in investment goods, and an exogenous trend term. 


\subsubsection{Demand Crealing Inwootions}

As in chapter 3 , we postulate the following demand function:

$Y_{j, 1}^{d}=X_{j, 1}^{0} \cdot Q_{j, k}^{b} \cdot P_{j, i}^{\cdots}$

where $X^{w}$ is an autonomous scale parameter of demand, $P_{j}$ is the price level and $Q_{j}$ is the quality level.

The quality of an industry's product can be raised by performing internal product $R \& D\left(R_{d, j}\right)$. The productivity of own product $R \& D$ is dependent on the input-related $\left(S_{k_{k j},} S_{m, j}\right)$ and non-input related $\left(S_{j}\right)$ knowledge spillovers and an exogenous trend term $(\gamma)$. The quality generation process becomes:

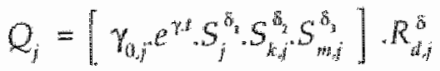

The growth rates of these two equations are:

$$
\begin{aligned}
& \hat{Y}_{j}=\hat{X}_{a, j}+b \cdot \hat{Q}_{j}-a \hat{P}_{j} \\
& \hat{Q}_{j}=\gamma+\delta \cdot \hat{R}_{d, j}+\delta_{1} \cdot S_{j}+\delta_{2} \cdot S_{k, j}+\delta_{3} \cdot \hat{S}_{m, i}
\end{aligned}
$$

Substitution of the second equation in the first equation gives the general form of the demand equations to be estimated in this study:

$$
\hat{Y}_{j}=\hat{X}_{0, j}+b \cdot \gamma+b . \delta \cdot \hat{R}_{d j}+b \cdot \delta_{j} \cdot \hat{S}_{j}+b \cdot \delta_{2} \hat{S}_{k, j}+b \cdot \delta_{3} \hat{S}_{m, j}-a \cdot \hat{P}_{j}
$$

As in the case with process $R \& D$ we can avoid constructing product $R \& D$ and spillover stocks by estimating the rates of return instead of elasticities. The explanatory variables become R\&D intensities (instead of R\&D stocks):

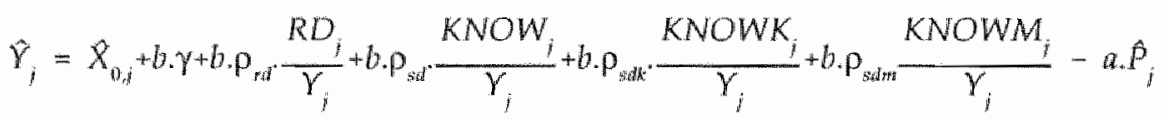

The supscript $d$ is related to the quality generation process in which product R\&D plays an important role.

We are unable to identify the various rates of returns $\left(\rho_{\text {rid }} \rho_{s d^{\prime}} \rho_{s d k}\right.$ and $\rho_{\text {sdm }}$ ) because we cannot separate these effects from $b$ in the estimated coefficients. However, the estimated coefficients can give us an indication of the relative magnitude of the various rates of returns because the value of $b$ is the same for all the estimated coefficients. 


\subsubsection{Demand Creating Imowations and Productionty Growth.}

\section{A) Dewand Creating Innovations Infuence Unit Production Costs}

In sub-section 7.12 we assumed that product R\&D only influenced the quality level and had no influence on the unit costs. It can be expected, however, that a new quality level also changes unit production costs (see sub-section 4.2.1). It is traditionally thought that a higher quality level can only be achieved with higher unit production costs (see e.g. Dorfman and Steiner 1954). The other view is that pure product devellopment may also lead to a reduction in inputs because it either reduces the required number of operations or components or it results in a simplification of tasks (see e.g. Clark and Griliches 1984). Examples of such industries are the computer and photographic industries where the quality of products has improved while the unit production costs have fallen over the last few decades. Product R\&D can therefore increase or decrease unit costs. When we take into account the cost effect of quality we have to adjust the production function. Equation (7.1) becomes (see equation (4.9)):

$$
Y_{j}=A_{j}\left(Q_{j}\right) \cdot F\left(L_{j} K_{j}^{e}, M_{j}^{e}\right)=\left[A_{j} \cdot Q_{j}^{\xi}\right] \cdot L_{j}^{\alpha} \cdot\left(K_{i}^{e}\right)^{\beta} \cdot\left(M_{i}^{e}\right)^{\alpha}
$$

Where $\xi$ is negative in the traditional view and positive in the other view. We calculated the growth rates of this production function as:

$$
\hat{Y}_{i}=\hat{A}_{i}+\alpha \cdot \hat{L}_{i}+\beta \cdot\left(\hat{K}_{j}+\lambda \cdot \hat{E}_{i, j}\right)+\sigma \cdot\left(\hat{M}_{i}+\mu \cdot \hat{E}_{m, i}\right)+\xi \cdot \hat{Q}_{i}
$$

Substituting the time derivatives of equation (7.2) and equation (7.3) in this equation we obtain the equivalent of equation (7.10):

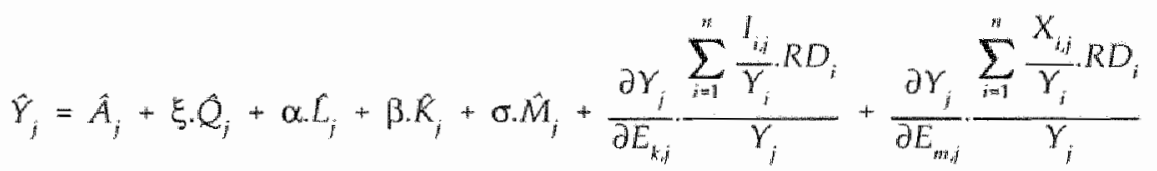

Substituting the growth rates of the technology generation process (equation(7.4)) and the quality generation process (equation (7.19)) in this equation we get:

$$
\begin{aligned}
& \hat{Y}_{i}=\hat{\eta}+\hat{\gamma} \cdot \xi+\theta \cdot \hat{R}_{\theta, i}+\xi \cdot \delta \cdot \hat{R}_{d, i}+\left(\theta_{1}+\delta_{1} \cdot \xi\right) \cdot \hat{S}_{j}+\left(\theta_{2}+\delta_{2} \cdot \xi\right) \cdot \hat{S}_{k_{i}}+\left(\theta_{3}+\delta_{3} \cdot \xi\right) \cdot S_{m, i} \\
& +\alpha \cdot \hat{L}_{j}+\beta \cdot \hat{K}_{j}+\sigma \cdot \hat{M}_{j}+\rho_{\mathrm{ek}} \frac{R E N T K_{j}}{Y_{j}}+p_{\mathrm{ew}} \cdot \frac{\operatorname{RENTM} M_{j}}{\gamma_{i}}
\end{aligned}
$$


Next we use the Terleckyj transformation to avoid the construction of R\&D stocks and we combine "pure" rent and knowledge spillovers embodied in purchased capital and intermediate goods to avoid the problem of multicollinearity between these spillovers. Together with the definition of TFP growth we obtain:

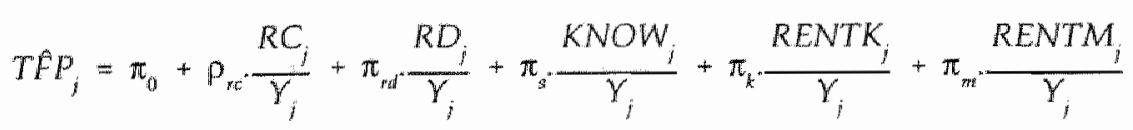

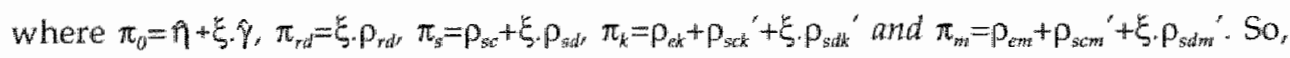
for example the regression coefficient for spillovers embodied in capital purchases $\left(\pi_{k}\right)$ measures 1) "pure" rent spillovers of capital goods $\left.\left(\rho_{e k}\right), 2\right)$ knowledge spillovers embodied in capital goods that increase the productivity of the productivity generation process $\left(\rho_{s c k}\right)$ and 3) knowledge spillovers embodied in capital goods that increase the productivity of the quality generation process $\left(p_{\text {sdd }}^{\prime}\right)$ if quality influences unit production costs $(\xi<>0)$.

If we estimate this equation in combination with equation (7.20) we can get an indication of almost all the important parameters dependent on the value of $b$. Equation (7.20) provides the values of $\rho_{v i d}, \rho_{s i d}$ and $\rho_{s w k}$ (dependent on the value of $b$ ) and we are able to identify $\xi$ and $p_{s c}$ (also dependent on the value of $b$ ). We are not able to separate the various spillover effects that are embodied in goods. We obtain onlly the aggregate effects $\pi_{k}$ and $\pi_{m m^{\prime}}$. This is due to the problem of multicollinearity between product and total $R \& D$. We are therefore unable to measure the influence of "pure" rent and knowledge spillovers embodied in goods separately.

\section{B) Quality Partly Reflected in Price Indices}

Until now we assumed that quality improvements were not reflected in the price indices. If output and inputs correctly adjusted for quality or efficiency improvements, then we obtained the following "ideal" quality adjusted TFP measure:

$$
T P P_{i}^{\prime}=\left[\hat{\gamma}_{i}+\hat{Q}_{j}\right]-\alpha \cdot \hat{L}_{i}-\beta \cdot\left[\hat{R}_{i}+\lambda \cdot \hat{E}_{\mathrm{k}, j}\right]-\sigma \cdot\left[\hat{M}_{i}+\mu \cdot \hat{E}_{m, i}\right]
$$

When, the quality level of product $j\left(Q_{j}\right)$ has been set equal to the efficiency level of product $j\left(Q_{j}=E_{y, j}\right)$. In sub-section 6.2 .1 we argued that this correct $T F P$ index could be approximated if price indices were adjusted using the hedonic or welfare efficiency index or the conventional or chain index if quality improvements were fully reflected in prices. However, the welfare efficiency index is not used in official statistics and the hedonic price index is used only for computers in a few countries (e.g. US, Canada). Competition between firms also causes quality improvements to be only partly reflected in prices. We can conclude that at best only a part of the 
quality improvements is reflected in the price indices. The measured productivity index is therefore:

$$
\operatorname{TPP} P_{i}^{*}=\left[\hat{Y}_{j}+s_{y} \cdot \hat{Q}_{j}\right]-\alpha \cdot \hat{L}_{j}-\beta \cdot\left[\hat{K}_{j}+s_{k} \cdot \lambda \cdot \hat{E}_{k, j}\right]-\sigma \cdot\left[\hat{M}_{j}+s_{m} \cdot \mu \cdot \hat{E}_{m, i}\right]
$$

where $s_{y}$ reflects the part of the quality or efficiency improvement of product $j$ that is reflected in the price indices, $s_{m}$ and $s_{k}$ represents these parts for intermediate and capital inputs, respectively.

Substitution of equation (7.23) in this measured productivity index gives a keyequation for TFP measurement in a world that is characterised by quality improvements:

$$
T \hat{F}_{j}^{*}=\hat{A}_{j}+s_{y} \cdot \hat{Q}_{j}+\left(1-s_{k}\right) \cdot \beta \cdot \lambda \cdot \hat{E}_{k, j}+\left(1-s_{m}\right) \cdot \sigma \cdot \mu \cdot \hat{E}_{m, j}-\xi \cdot Q_{j}
$$

where $\hat{A}_{j}$ reflects the total factor productivity growth when quality or efficiency improvements are not possible $\left(\hat{Q}_{j}=\hat{E}_{k, j}=\hat{E}_{\mathrm{in}, j}=0\right)$. This situation is usually assumed in empirical studies. However, when quality improvements are possible the measured TFP growth is dependent on whether these quality improvements are taken into account (this is dependent on the method used by statistical agencies and/or the degree of competition at the industry level). Until now we assumed that quality improvements were not reflected in prices, i.e. $s_{y}=s_{k}=s_{t t}=0$. If we substitute these values in equation (7.29), we obtain the situation of sub-section 7.1.1 $(\xi=0)$ or the situation described in sub-section 7.1.3A. These are the situations where inputs are under-valued and therefore mismeasured and sectors that use the improved product obtain a growth in their measured productivity level while the sector that improved the product shows no measured productivity gain. It is therefore important to correct for these "pure rent spillovers" if one wants to obtain reliable estimates for the importance of R\&D activities. With regard to the influence of process-oriented and product-oriented R\&D on TFP growth, this situation implies that product-oriented $R \& D$ has no influence on TFP growth unless it has a direct effect on unit production costs $\left(\xi . \hat{Q}_{i}\right)$.

We will now discuss the opposite situation where all quality improvements are reflected in the price indices (for example "ideal" hedonic price indices), i.e. $s_{y}=s_{k}=s_{x n}=1$. If we assume that quality doesn't influence unit production costs $(\xi=0)$ equation (7.29) is reduced to:

$$
T \hat{F}_{i}^{*}=\hat{A}_{j}+\hat{Q}_{j}
$$

In this case the measured productivity index is the correct index. Inputs are corrected for quality improvements; we therefore have no miss-measurement on the input side. Outputs are also corrected for quality improvements so quality 
improvements are reflected in TFP growth. The correct TFP grow th index therefore reflects quality and efficiency improvements and process R\&D, as well as product R\&D which is expected to influence TFP growth. "Pure" rent spillovers are expected to have no influence on TFP growth in this situation. This enables us to measure knowledge spillovers that are embodied in inputs since the "pure" rent spillover effect is zero.

Now we discuss the case when only a part of the quality improvement is reflected in the prices, i.e. $0<s_{y^{\prime}} s_{k^{\prime}} s_{m}<1$. Substitution of this condition in equation (7.29) implies that the measured productivity consists of 1) the measured productivity when there are no quality improvements, 2) the part of own quality improvements of the produced product that is reflected in the price indices $\left(s_{y}\right)$ times the growth rate of the quality improvement $\left.\left(\hat{Q}_{j}\right), 3\right)$ the part of input quality improvements that is not reflected in the input price indices (1-s $s_{m}$ for intermediate goods and $1-s_{k}$ for capital goods) times the growth rates of these improvements and 4) if quality improvements directly influence unit production costs then the TFP index also measures this effect $\left(\xi \cdot \hat{Q}_{j}\right)$.

If we substitute $\hat{A}_{\text {; }}$ as depicted in equation (7.11) and $\hat{Q}$, as shown in equation (7.19) in equation (7.29) and perform the Terleckyj transformations for the various $R \& D$ concepts we obtain:

$$
\begin{aligned}
& T \hat{F P}_{i}=\left[\hat{\eta}+\left(s_{y}+\xi\right) \cdot \hat{\gamma}\right]+\rho_{r c} \cdot \frac{R C_{j}}{Y_{j}}+\left[\left(s_{y}+\xi\right) \cdot \rho_{r d}\right] \cdot \frac{R D_{j}}{Y_{j}}+\left[\rho_{s c}+\left(s_{y}+\xi\right) \cdot \rho_{s d}\right] \cdot \frac{K N O W_{j}}{Y_{j}} \\
& +\left[\left(1-s_{k}\right) \cdot \rho_{e k}+\rho_{s c k}+\left(s_{y}+\xi\right) \cdot \rho_{s d t}\right] \cdot \frac{R E N T K_{j}}{Y_{j}}+\left[\left(1-s_{m}\right) \cdot \rho_{e m}+\rho_{s c m u}+\left(s_{y}+\xi\right) \cdot \rho_{s i d m s}\right] \cdot \frac{R E N T M_{j}}{Y_{j}}
\end{aligned}
$$

This is the general TFP equation that contains all other TFP specifications as special cases. If $s_{y}=s_{k}=s_{m}=\xi=0$, then we obtain the result derived in sub-section 7.1.1 (equation (7.16)) and if $s_{y}=s_{k}=s_{m i}=0$, then we obtain the result of sub-section 7.1.3.A (equation (7.26)).

When we compare the regression coefficients of this equation with those of equation (7.26) we must adjust our interpretation of these coefficients. First, when a part of the quality improvements are reflected in the price indices, product R\&D has two influences on TFP growth. On the one hand, a part of an enthanced quality level is directly reflected in the TFP measures $\left(s_{y} \cdot \rho_{s_{i j}}\right)$ and on the other hand, quality improvements may influence unit production costs $\left(\xi . \rho_{\text {rid }}\right)$. We are not able to separate these effects and identify $s_{y}$ and $\xi$, separately. However, if we estimate this equation and equation (7.20) together, we are then able to obtain the value of $s_{y}+\xi$ (dependent on the value of b). Second, the rate of return to capital and intermediate 
Table 7.1 Growth in Sectoral Output and lts Sowrces, 1978-1992 (Average Annual Rates)

\begin{tabular}{|c|c|c|c|c|c|}
\hline Industry & $\begin{array}{l}\text { Rate of } \\
\text { Ontput } \\
\text { Growth }\end{array}$ & $\begin{array}{l}\text { Intermediate } \\
\text { Input }\end{array}$ & $\begin{array}{l}\text { Capital } \\
\text { Imput }\end{array}$ & $\begin{array}{l}\text { Labour } \\
\text { Input }\end{array}$ & $\begin{array}{c}\text { Rate of } \\
\text { Total Factor } \\
\text { Productivity } \\
\text { Growth }\end{array}$ \\
\hline Agriculture $(A G \mathbb{R})$ & .0194 & .0105 & .0222 & -.0257 & .0219 \\
\hline Energy $(E N G)$ & .0044 & -.0057 & .0323 & .0122 & .0030 \\
\hline Metals (MET) & -0011 & -.0037 & .0018 & -.0444 & .0092 \\
\hline Non-metallic mineral (NMM) & .0027 & .0077 & .0092 & -.0259 & .0046 \\
\hline Basic chemicals (CHES) & .01178 & .0177 & .0281 & -.0246 & .0050 \\
\hline Phamaceuticals (FAR) & .0652 & .0564 & .0552 & .0183 & .0169 \\
\hline Para-chemicals (CHTS) & .0300 & .0290 & .0320 & .0035 & .0066 \\
\hline Fabricated metal products (FAM) & .0055 & .0093 & .0302 & -.0112 & .0004 \\
\hline Machinery (MAC) & .0018 & .0068 & .0152 & -.0172 & .0018 \\
\hline Office \& data processing machinery (TT) & .0586 & .0678 & .0826 & .0087 & .0064 \\
\hline Electrical machinery (ELMS) $)^{1}$ & .0247 & .0244 & .0386 & -.0149 & .0115 \\
\hline Tellecommunication equipment (IT2) & 0376 & .0384 & .0629 & -0011 & .0101 \\
\hline Electronic equipment (IT3S) & .0582 & 0336 & .0511 & -0279 & .0347 \\
\hline Transport industry (TRA) & .0192 & .0298 & .0437 & -.0222 & .0023 \\
\hline Instruments (INS) & .0204 & .0265 & .0154 & -.0027 & .0064 \\
\hline Food industry (FOO) & .0187 & .0204 & .0353 & .0004 & .0002 \\
\hline Textlles (TEX) & -.0083 & -.0033 & .0146 & -.0432 & .0053 \\
\hline Wood and other industries (OTH) & .0051 & .0077 & .0316 & -.0148 & .0014 \\
\hline Paper and Printing (PAP) & .0289 & .0380 & .0622 & .00003 & -.0012 \\
\hline Rubber and Plastics (RUB) & .0298 & .0379 & .0276 & -.0017 & $.004 \mathrm{~m}$ \\
\hline Building and construction (BUD) & .0107 & .0122 & .0218 & -.0141 & .0079 \\
\hline Commercial sector (COM) & .0238 & .0405 & .0419 & .0067 & .0003 \\
\hline Recovery and Repair (REP) & .0138 & .0243 & .0291 & .0077 & -.0057 \\
\hline Lodging (HOT) & .0155 & .0184 & .0614 & .0332 & -.0151 \\
\hline Transport services (TRS) & .0293 & .0322 & .0275 & .0102 & .0074 \\
\hline Telecommunication services (TEL) & .0784 & .041 .5 & .0399 & .0072 & .0537 \\
\hline Other market services (OMS) & .0474 & .048 & .0625 & .0308 & -.0007 \\
\hline Enterprise market services (SME) & .0443 & .0622 & .0246 & .0405 & .0037 \\
\hline Finance and insurance (FIN) & .0775 & .1508 & .0694 & .0088 & -.0123 \\
\hline Non-market services (NMS) & .0248 & .0312 & .0426 & 0148 & .0058 \\
\hline
\end{tabular}

Except IT2 and IT3S. 
rent spillovers depends on the part of quality improvements that is reflected in price-indices. When quality improvements are fully reflected in prices, we can then measure knowledge spillovers. When this is not the case we measure a mixture of "pure" rent spillovers (mis-measurement) and input-related knowledge spillovers.

\subsection{Data}

The importance of internal R\&D and R\&D spillovers with regard to productivity growth and demand growth will be assessed with cross-sectional data on thirty French sectors which cover the total economy (industrial as well as service sectors). ${ }^{95}$ These data are drawn from several sources. The core data are from French INSEE data. Production, gross value added, investment supply and inputoutput data are available in constant and current prices for 1977-1992. Investment matrices are available in constant prices from 1977-1988. ${ }^{96}$ The SEC2 database of EUROSTAT contains labour and investment demand data from 1967-1991. Capital stocks are constructed with these investment demand data in the conventional way. ${ }^{97}$ The total R\&D expenditures by sector are taken from OECD (STAN).

In table 7.1 we show the average sectoral output growth rate and its sources for the thirty French sectors over the 1978-1992 period. As sources of growth, we identified the average anuual growth rates of intermediate, capital and labour inputs and changes in productivity at the sectoral level. Among the ten sectors with the highest sectoral output growth rates are four service sectors (telecommunications $7.84 \%$, the financial sector $7.75 \%$, other market services $4.7 \%$ and enterprise market services $4.4 \%$ ) and six high-tech sectors ${ }^{98}$ (pharmaceuticals $6.5 \%$, the three information technology sectors respectively $5.9 \%, 5.8 \%$ and $3.8 \%$, para-chemicals $3 \%$ and rubber and plastics $3 \%$ ). When we compare these results with the sectoral growth rates of the United States for the 1947-1985 period calculated by Jorgenson (1990), we notice a striking resemblance. The seven sectors with the highest growth rates in the US are telecommunications $(6.4 \%)$, elec-

\footnotetext{
95 A detailted description of these thiry sectors is given in appendix $7 \mathrm{~A}$.

96 The construction of the investment matrices for the period $89-92$ is described in appendix 78 .

97 Capital Stocks are constructed in a conventional manner:

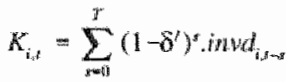

where $T$ is economic lifetime (10 years) and invd is investment demand by sector ${ }^{*} \delta^{*}$ is the depreciation rate $\left(8^{\prime}=0.1\right)$. The initial capital stock $(1977)$ is constructed in this manther with investment demand data from 1967-1977 from the SEC2 domain of the CRONOS dabase available from EUROSTAT.

\footnotetext{
98. High tech sectors in this thesis are defined as all the sectors that have a R\&D intensity higher than $1 \%$ of: actual production (sec table 7.2).

We compare seven U.S. sectors with ten Firench sectors because in the Jorgenson stady the IT technology sectors and the chemical sector are not divined into in several sub-sectors.
} 
Table 7.2 R\&D, Actual Production and Gross Value Added (constant prices (1980=100), Average value period 1978-1992)

\begin{tabular}{|c|c|c|c|c|c|c|}
\hline & $\mathbb{R \& D}$ & PROD & GVAMP & $\begin{array}{l}\text { Cumulative } \\
\% \text { in total } \\
\text { R\&D }\end{array}$ & $\begin{array}{l}\text { Cumulative } \\
\text { of in total } \\
\text { production }\end{array}$ & R\&D/PROD \\
\hline TRA & 11970 & 236249 & 77455 & 0.30 & 0.043 & 0.051 \\
\hline $\operatorname{TT} 2$ & 7834 & 65734 & 34774 & 0.49 & 0.055 & 0.119 \\
\hline CHES & 3724 & 100528 & 29683 & 0.58 & 0.074 & 0.037 \\
\hline FAR & 3222 & 40951 & 15269 & 0.66 & 0.081 & 0.079 \\
\hline AT1. & 1672 & 27013 & 11857 & 0.70 & 0.086 & 0.062 \\
\hline ELMS & 1464 & 71390 & 32206 & 0.74 & 0099 & 0.021 \\
\hline$M A C$ & 1448 & 128689 & 52261 & 0.78 & 0.123 & 0.011 \\
\hline IT3S & 1166 & 9633 & 3915 & 0.80 & 0.125 & 0.1121 \\
\hline RLB & 1125 & 64638 & 24865 & 0.83 & 0.136 & 0.017 \\
\hline ENG & 1034 & 305522 & 127854 & 0.86 & 0.192 & 0.003 \\
\hline MET & 829 & 126095 & 32778 & 0.88 & 0.215 & 0.007 \\
\hline FOO & 662 & 348108 & 93384 & 0.89 & 0.279 & 0.002 \\
\hline OMS & 561 & 193788 & 143521 & 0.91 & 0.3115 & 0.003 \\
\hline SME & 530 & 606283 & 430744 & 0.92 & 0.426 & 0.001 \\
\hline CHTS & 504 & 52270 & 20229 & 0.93 & 0.435 & 0.010 \\
\hline NMM & 475 & 71307 & 34266 & 0.95 & 0.448 & 0.007 \\
\hline INS & 473 & 19001 & 10113 & 0.96 & 0.452 & 0.025 \\
\hline AGR & 418 & 271679 & 135262 & 0.968 & 0.501 & 0.002 \\
\hline FAM & 406 & 123704 & 54066 & 0.976 & 0.524 & 0.003 \\
\hline BUI & 294 & 408972 & 195727 & 0.98 & 0.599 & 0.001 \\
\hline TEX & 224 & 127146 & 50857 & 0.990 & 0.622 & 0.002 \\
\hline OTH & 136 & 83948 & 35253 & 0.994 & 0.638 & 0.002 \\
\hline TRS & 1.27 & 204228 & 122957 & 0.997 & 0.675 & 0.001 \\
\hline PAP & 125 & 123123 & 44352 & 1 & 0.697 & 0.001 \\
\hline NMS & 0 & 684165 & 374350 & 1 & 0.823 & 0 \\
\hline FIN & 0 & 249376 & 128025 & 1 & 0.868 & 0 \\
\hline TEL & 0 & 92867 & 80417 & 1 & 0.885 & 0 \\
\hline REP & 0 & 96338 & 52338 & 1 & 0.903 & 0 \\
\hline $\mathrm{COM}$ & 0 & 417913 & 325660 & 1 & 0.979 & 0 \\
\hline HOT & 0 & 112105 & 644146 & 1 & 1 & 0 \\
\hline TOT & 40424 & 5462762 & 2838854 & 1 & 1 & 0.0074 \\
\hline
\end{tabular}


tric utilities $(5.4 \%)$, electrical machinery $(5.3 \%)^{100}$, instruments $(5.1 \%)$, chemicals $(4.6 \%)$, rubber and plastic products $(4.5 \%)$ and the financial sector $(4.1 \%)$. We can conclude that the sectors with the highest growth rates are mainly in the high-tech and service sectors.

The productivity growth rate is calculated as the growth rate of output less the with their value shares weighted growth rates of the three inputs (see equation (7.15)). Six sectors have an annual productivity growth rate greater than $1 \%$. These sectors are telecommunications $(5.4 \%)$, two information technology sectors (respectively $3.5 \%$ and $1 \%$ ), agriculture $(2.2 \%$ ), pharmaceuticals $(1.7 \%$ ) and electrical machinery $(1,2 \%)$. Almost all of these are high-tech sectors. We will again compare these results with the results obtained by Jorgenson for the United States. The six sectors with the highest (three-factor) productivity growth rate in the US are telecommunications $(2.2 \%)$, electrical machinery including IT (1.6\%), agriculture $(1.6 \%)$, electric utilities $(1.5 \%)$, instruments $(1.2 \%)$ and chemicals including pharmaceuticals (1.2\%). Again we observe similarities between the sectors with high productivity growth in the United States and France.

With regard to the growth rate of inputs it should be noticed that: First, several high-tech sectors and a few service sectors have an anmual growth rate of capital stock higher then $5 \%$. Second, employment growth is negative in seventeen of the thirty sectors. In general it can be said that employment growth is positive in service sectors because output growth is high and productivity growth is low, more or less equal to zero in the high-tech sectors (high output and high productivity growth) and negative in the other sectors.

Table 7.2 shows the average annual values of $R \& D$ expenditures, actual production (PROD) figures and the gross value added at market prices (GVAMP). The values of PROD and GVAMP indicate the size of a sector and therefore its importance in the total economy. The first column shows the sectorall R\&D expenditures. It is apparent that almost all R\&D is performed in only a few sectors. Columns four and five provide some concentration measures. Column four indicates that the sector with the highest R\&D expenditures, the transport industry (TRA) performs $30 \%$ of the total R\&D activities in France. Column five indicates that this $30 \%$ of total R\&D is performed by only $4.3 \%$ of actual production. When we look at these figures for the two largest R\&D performers (transport and telecommunication equipment) it appears that these two sectors perform almost $50 \%$ of total R\&D and represent only $5.5 \%$ of total actual production. If we include more sectors it turns out that the sectors responsible for $10 \%$ of actual production perform $74 \%$ of total $R \& D$ activities. In conclusion we can say that the R\&D activities are very concentrated.

The R\&D intensity is the last measure we included in table 7.2 (R\&D expenditures/actual production). The information technology sectors (IT1, IT2 and

100 Includes information technology sectors. 
Rent Spillowens (intermediate product flows)

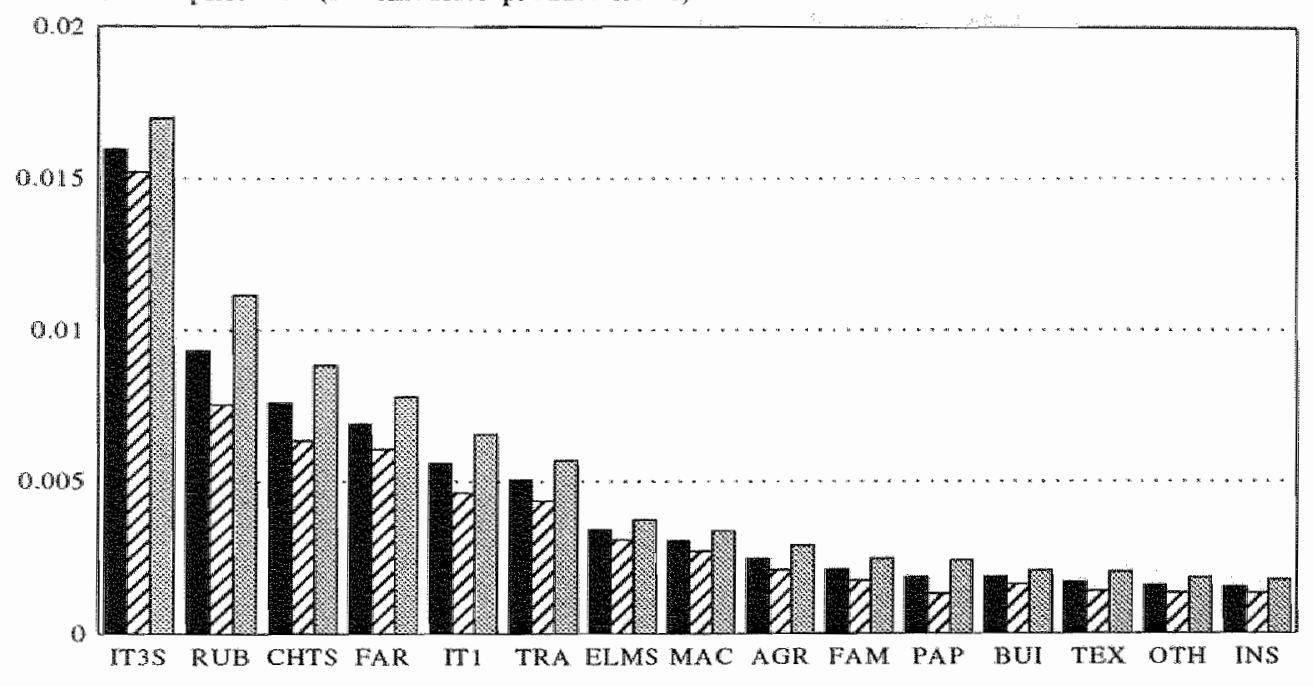

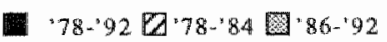

Figure 7.1. R\&D received by purchased intermediate inputs (high spillovers)

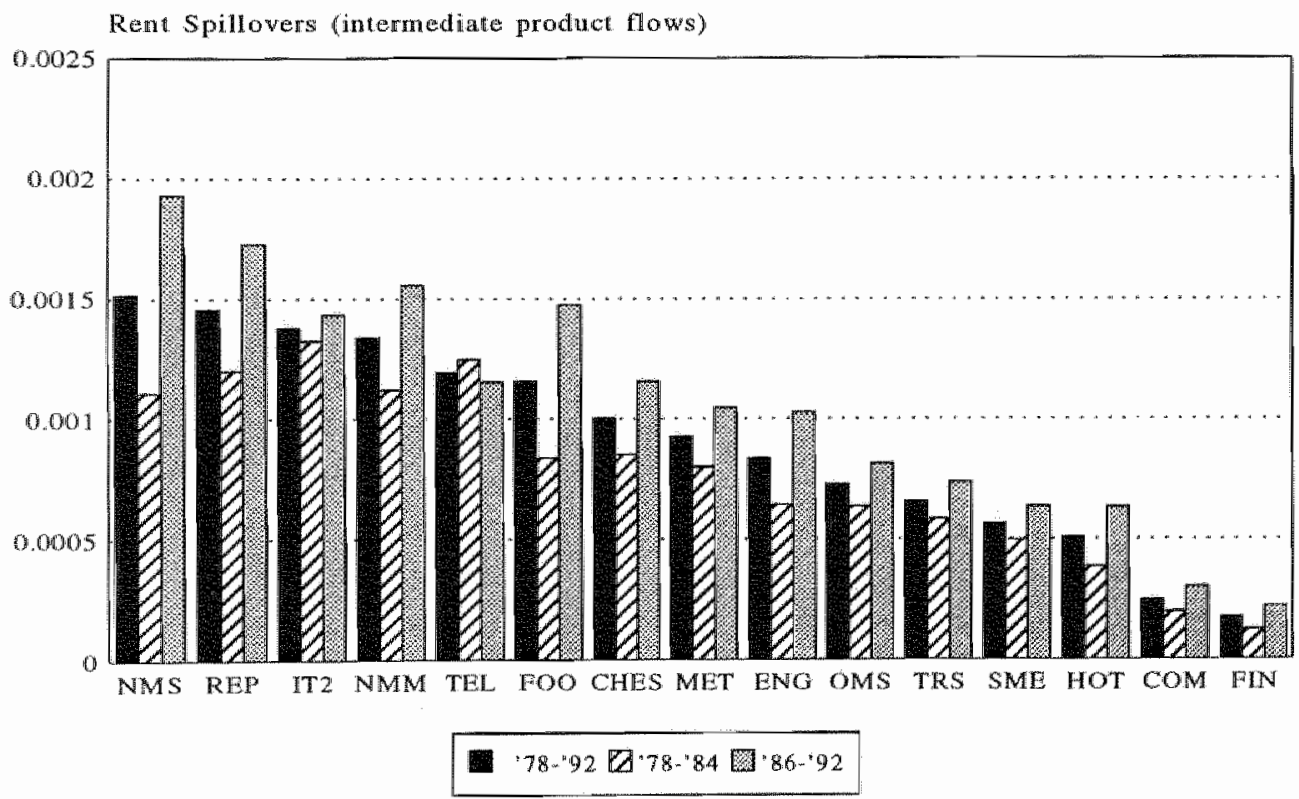

Figure 7.2. R\&D receiwed by purchased intermediate inputs (low spillovers) 
IT3) possess the highest R\&D intensities (12.1\%, $11.9 \%$ and $6.2 \%$ respectively) together with the pharmaceutical sector (FAR, 7.9\%).

However, sectors perform not only R\&D themselves but also benefit from R\&D performed in other sectors (see section 7.1). We identified two types of R\&D spillowers, rent or input-related spillovers and "pure" (non-input-related) knowledge spillovers. We will now discuss how we measured these spillover effects and their magnitude for the thirty French sectors.

The rent spill-overs linked to intermediate and investment goods are constructed as defined, for example in equation (7.14). The rent spillover intensity of intermediate goods for sector $j\left(R E N T M_{-} Y_{j}\right)$ is calculated as:

$\operatorname{RENTM}_{-} Y_{i}=\frac{\sum_{i \neq i}^{30} X_{i, j} \cdot \frac{R D_{i}}{Y_{i}}}{Y_{j}}$

where $X_{i j}$ represents the quantity of output of industry $i$ purchased by industry $j$. $Y_{i}$ is the actual production of industry $i$ and $R D_{i}$ is the "own" or internal product R\&D of industry $i$.

"Pure" rent spillovers exist because quality improvements are not fully reflected in prices (see sub-section 6.2.1). According to our model, we can expect that product $R \& D$ expenditures in particular are aimed at improving the quality level or efficiency index of a product. Our calculation of rent spillovers in equation (7.32) implies that sector $j$ receives more rent spillovers from sector $i$ if it purchases more intermediate products from this sector (thus increasing the value of $X_{i, j}$ ) and when the R\&D intensity of these purchased products is higher.

The rent spillover intensities for the thirty French sectors over the total period (1978-1992) and over two sub-periods (1978-1984 and 1986-1992) are depicted in figure 7.1 and figure 7.2. IT3S is the sector that receives the highest rent spillovers embodied in intermediate goods relative to its own production (RENTM_ $Y=1.5 \%$ ). It is easy to illustrate how this percentage is composed, as this sector purchases most of its intermediate inputs in the R\&D intensive IT2 sector. The IT2 deliveries to the IT3S account for $25 \%$ of the actual production of the IT3S sector $\left(X_{m 2, r 3} / Y_{n 35}=0.25\right)$. Table 7.2 shows that the R\&D intensity in the TT2 sector is $12 \%$. Together with the percentage product R\&D in the IT2 sector, that is $50 \%$, this implies that the product R\&D intensity is $6 \%$ in the IT2 sector $\left(R D_{T T 2} / Y_{T T 2}=0.06\right)$. The rent spillovers that the IT3S sector receives from the IT2 sector by buying intermediate goods are therefore $1.5 \%(0.06 * 0.25)$. So the IT3S sector receives almost all of its rent spillovers from the IT2 sector. It receives relatively more rent

101 Process $R \& D$ expenditures are aimed at improving the elticiency of the production process without changing the product characteristics. 


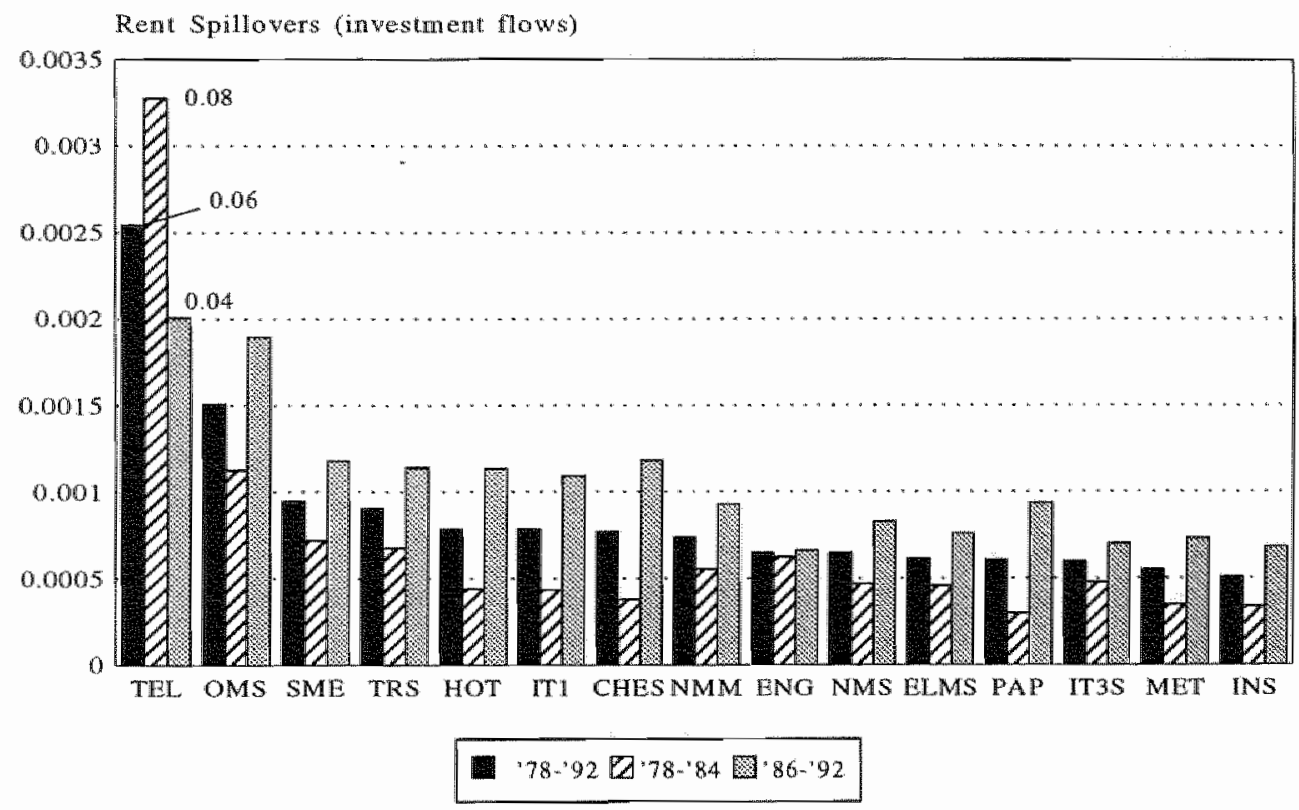

Figure 7.3. R\&D received by purchased investment inputs (high spillovers)

Rent Spillovers (investment flows)

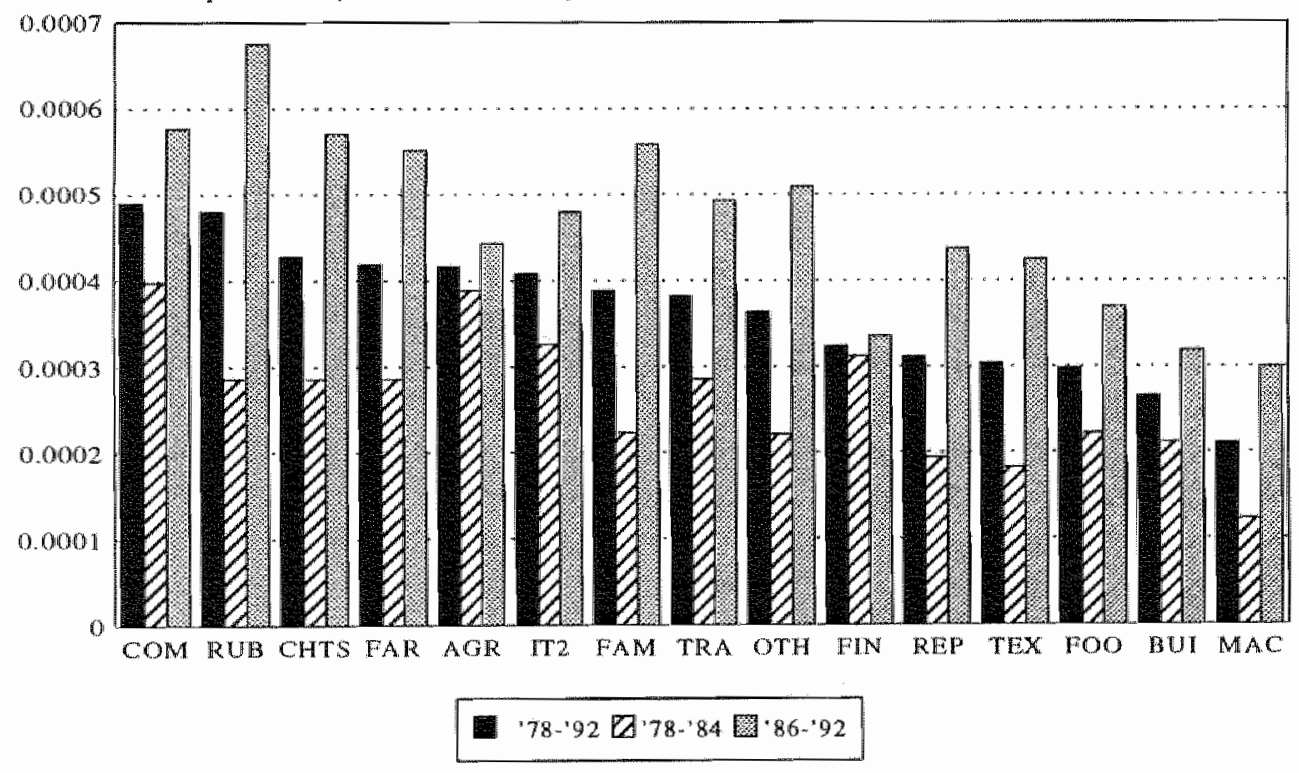

Figure 7.4. R\&D received by purchased investment inputs (low spillovers) 
spillovers of intermediate product than other sectors because it purchases most of its intermediate products relative to its total production and this sector purchases them from a sector with a very high product $R \& D$ intensity.

It is apparent that the high-tech sectors do not only perform a large amount of R\&D (table 7.2), but also receive relatively the highest rent spillovers of intermediate products (figure 7.1). When we compare the rent spillover intensities from the first sub-period (1978-1984) with those of the more recent period (19861992) it is apparent that all sectors received relatively more rent spillovers in the more recent period. The average annual growth rate of the rent spillovers intensity is $2.2 \%$ in the first period. In this period the average product R\&D intensity had an annual growth rate of $3 \%$ and the intermediate good intensity of production (total intermediate deliveries/actual production) decreased by $0.4 \%$. Compositional changes are responsible for a decrease of $0.4 \%(2.2 \%=3 \%-0.4 \%-x \%)$. In the second period the annual growth rate of the rent spillover intensity increased to $3.9 \%$. The average product $R \& D$ intensity growth rate contributed $2.1 \%$ (instead of $3 \%$ in first period), while the intermediate good intensity increased by $0.9 \%$ per year. Compositional changes in the second period are therefore responsible for another $0.9 \%$ grow th in the rent spillover. This implies that the rent spillovers" intensity grows faster than before, not because industries R\&D intensity increases faster but because firms buy relatively more intermediate inputs and more inputs from $R \& D$ intensive sectors.

The rent spill-overs related to investment goods are constructed using the same method as rent spillovers of intermediate goods:

$$
\text { RENTK_ } Y_{j}=\frac{\sum_{i * j}^{3} l_{i, j} \cdot \frac{R O_{i}}{Y_{i}}}{Y_{j}}
$$

where $I_{i, j}$ represents the quantity of capital purchases by industry $i$ from industry $j$ and RENTK $Y_{j}$ is the investment rent spillover intensity of sector $j$.

Figure 7.3 and figure 7.4 show the investment rent spillover intensities of the French sectors. It is interesting to note that the service sectors have the highest rent spillover intensities from investment goods instead of the high-tech sectors which are characterised by the highest own $R \& D$ intensities and the highest rent spillover intensities of intermediate goods. The service telecommunications sector receives, relative to actual production, the largest amount of rent spillover's. Not surprisingly, these spillovers come mainly from the telecommunications equipment sector (IT2). The enterprise market services (SME) invest mainly in office and data processing equipment (TT1), in the other market services (OMS), in instruments (INS) and in telecommunications (IT2). The transport service sector (TRS) receives its investment rent spillovers from the transport industry (TRA).

If we compare the investment rent spillover intensity in the first and second per- 


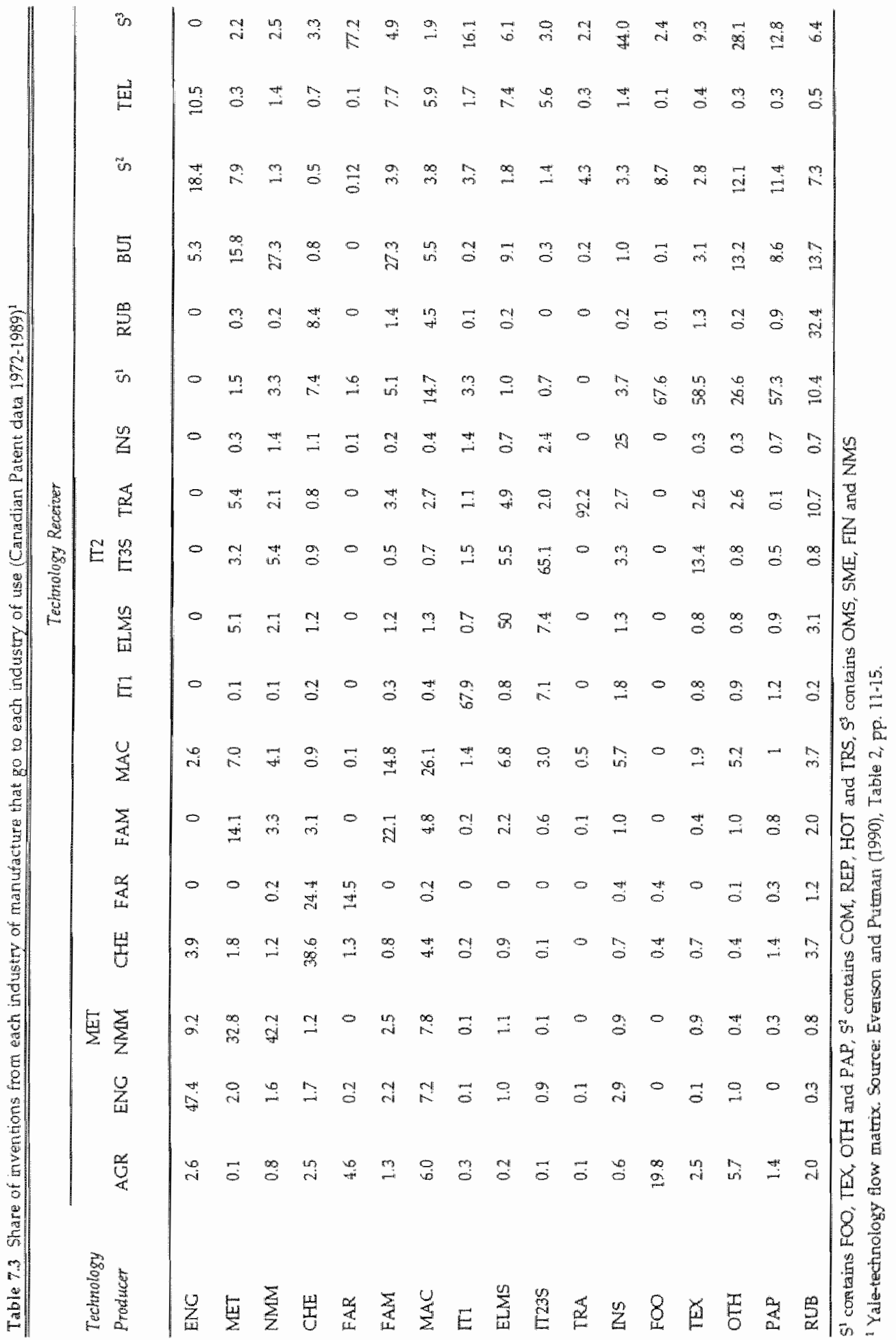


iod, we observe that in 29 of 30 sectors this intensity has increased, which indicates a positive growth rate during the second period (a notable exception is the telecommunications service sector). The growth rate of the investment rent spillover intensity was negative $(-0.4 \%)$ in the first period and positive $(1.6 \%)$ in the second period. This was partly due to a negative growth rate of the investment intensity in the first period $(-1.83 \%)$ and a positive growth rate of this intensity in the second period $(0.28 \%)$. The R\&D intensity grew by $3 \%$ in the first period and by $2 \%$ in the second period. These figures imply that compositional changes were responsible for $-1.63 \%$ in the first period and $0.21 \%$ in the second period. The increase in rent spillovers of investment goods is therefore caused by a higher investment intensity, higher product R\&D intensities and by compositional changes in favour of more R\&D intensive goods.

The available pool of "pure" knowledge spillovers (not related to input purchases) is constructed using the Yale technology flow matrix set up by Evenson et al (1988), based on approximately 200.000 patents granted in Canada during the 1972-1989 period. Canadian patent data are cross-classified by industry of manufacture and industry of potential use. The Yalle matrix predicts technology flows to user industries, given the industry of manufacture of the patent. We constructed an aggregated version of this Yale technology flow matrix in table 7.3. Each row represents a single industry of manufacture (technology producer), and the elements of the row show the fraction of the industry's inventions that are used by each using industry. The elements in each row add up to one. ${ }^{102}$ For example given a patent manufactured in the energy sector, this matrix predicts that $2.6 \%$ of this patent will be used in the agricultural sector (AGR), $47.4 \%$ in the own industry, $9.2 \%$ in the aggregated metal and non-metallic industry (MET and NMM) etc. For our estimation purposes, we have assumed that these probabilities are the same for patents granted in France. ${ }^{10 \%}$

The "pure" knowledge spillover intensity (KNOW_Y $Y_{j}$ ) for sector $j$ is calculated as (see equation $(7.13)$ ):

$K N O W_{--} \gamma_{j}=\frac{\sum_{i \neq i}^{30} \omega_{i, i} \cdot R \& D_{i}}{Y_{i}}$

The weighting factor which represents the technological closeness, $\omega_{i j}$ is approximated by the Yale technology flow coefficients. For example $\omega_{\mathbb{E N G}, A C R}$ is equal to 0.026 and $\omega_{\mathrm{FAR}, A G R}=0.046$ (see table 7.3 ). Multiplying the Yale matrix with

\footnotetext{
${ }^{102}$ The elements in the technology flow matrix depicted in table 7.3 do not add up to exactly one due to the exclusion of the petrochemical industry (not present in French INSEE data) and rounding errors.

103 This is not an unusual assumption. For example, Evenson fund Putman (1993) have used the Yale tochfow matrix for Italy, and Basant (1993) has used it for $\amalg$ ndia.
} 


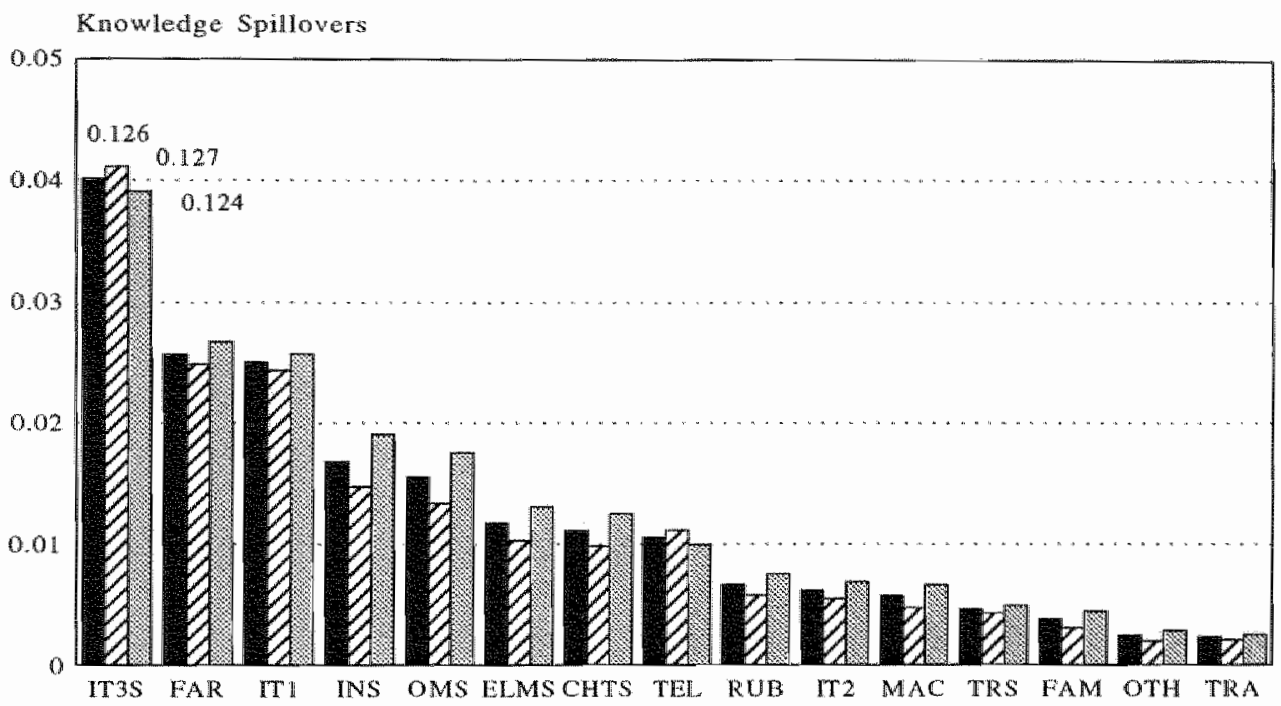

Figure 7.5. R\&D received by knowledge spillovers (high spillovers)

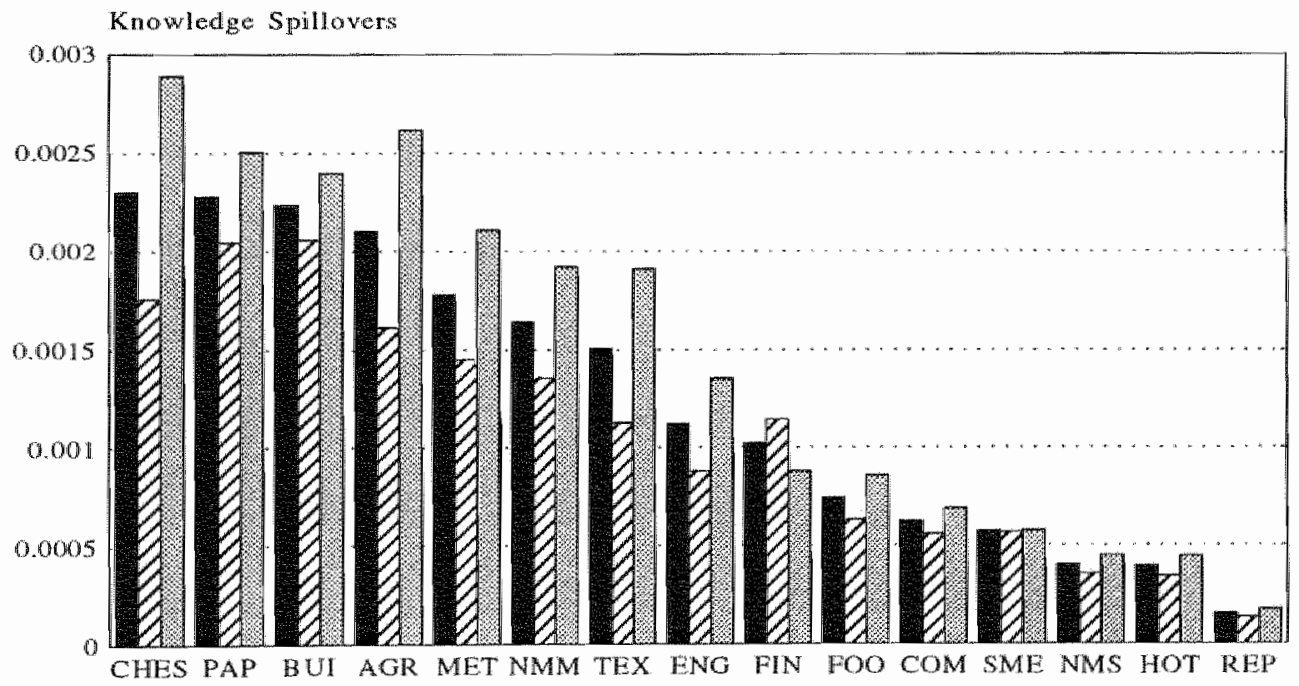

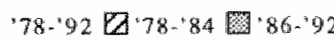

Figure 7.6. R\&D received by knowledge spillovers "low spillovers) 
R\&D expenditures provides the R\&D used by sectors.

Besides providing us with coefficients that should indicate the technological closeness of sectors, the Yale matrix also yields the division of total R\&D expenditures in process-oriented and product-oriented $\mathrm{R} \& \mathrm{D}$. The diagonal elements of table 7.3 represent those inventions which the inventing industry uses itself and may be broadly considered as process $\mathbb{R} \& D$. For example the share of process $R \& D$ in the energy sector (ENG) is $47,4 \%$. The share of process innovations ranges from $14,5 \%$ (FAR) to $92.2 \%$ (TRA). All elements of the Yale technology flow matrix are average values over the 1972-1989 period; we will therefore assume that all elements (including the percentage of process R\&D) of this matrix are constant for our estimation period.

Figure 7.5 and figure 7.6 show the non-input related knowledge spillover intensities for the French sectors for the total period and two sub-periods. The high-tech sectors possess the highesit non-input-related knowledge spillover intensities. This follows our theoretical model in which it is assumed that knowledge spillovers are used to enhance the productivity level of internally performed $R \& D$. The knowledge spillover intensity of the electronic equipment sector (IT3S, includes television sets) is very high. This is due to the fact that this sector is very small (see table 7.2) and uses much of the knowledge created by the seven times larger and technologically close telecommunications sector (IT2). It is logical that a small high-tech sector will not perform all the R\&D itself but relies heavily on the knowledge created by their large technological sisters. In this sense the degree of disaggregation of the data is very important. Disaggregated data imply large knowledge spillovers, while aggregated data usually imply that technologically close sectors are added together, so that a large part of the knowledge spillovers become internal and will not show up in the knowledge spillover callulations.

Apparent is the large knowledge spillover intensity of the other market services (OMS). Why does a firm use a lot of knowledge when it performs only a little amount of R\&D itself? The answer is that this sector has some special characteristics. For example it includes health services that use a large amount of drugs created by the pharmaceutical sector (FAR). This example shows that the Yale technology flow matrix is not a perfect measure for "pure" non-input-related knowledge spillovers but also incorporates some spillovers that are "embodied". The Yale proxy can therefore only serve as an approximation of real non-inputrelated knowledge spillovers.

Finally, we investigate the relative magnitude of the various knowledge sources for the various sectors. A sector can perform R\&D itself or use R\&D performed in other sectors via rent spillovers of intermediate and capital inputs and knowledge spillovers. If we assume that $\mathbb{R} \& D$ received from the various sources is equally important we can simply add up the own R\&D intensity and the various spillover 


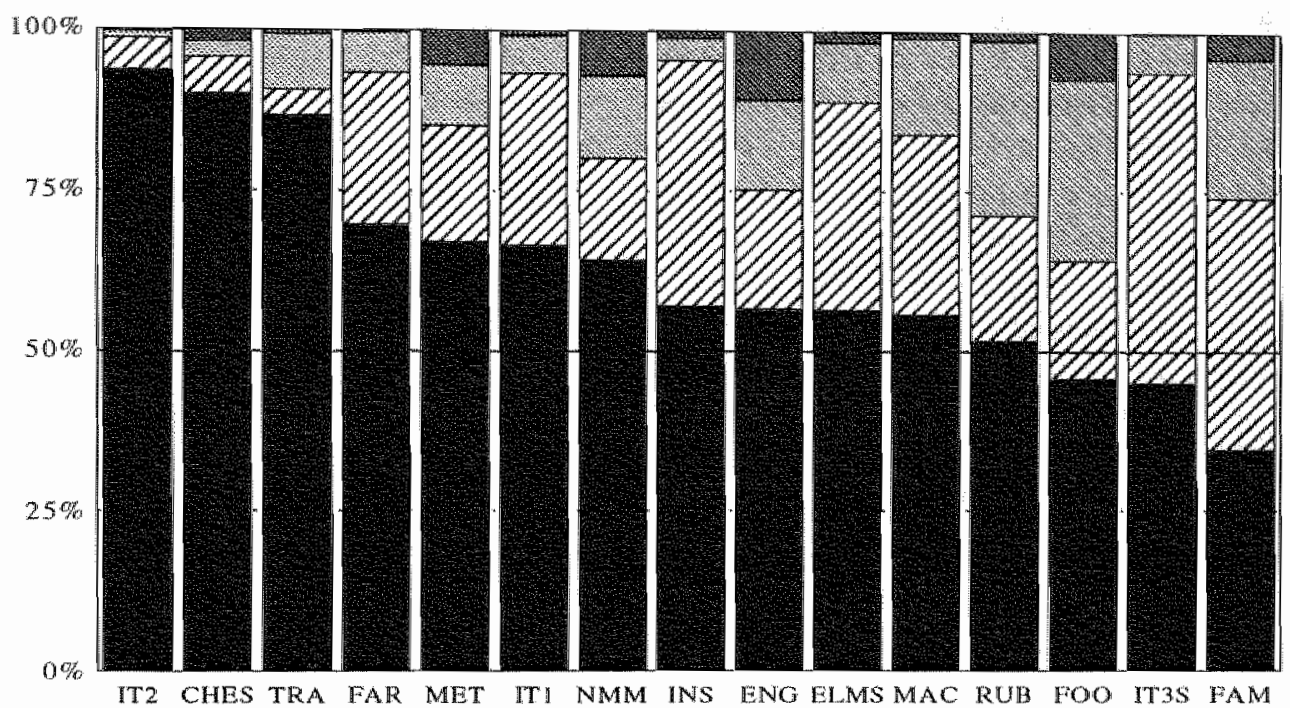

OWN RdeD $\triangle \mathrm{KNOW}$ RENTM RENTK

Figure 7.7. Shares of own R\&D and the various spillover sources in total R\&D used by a sector

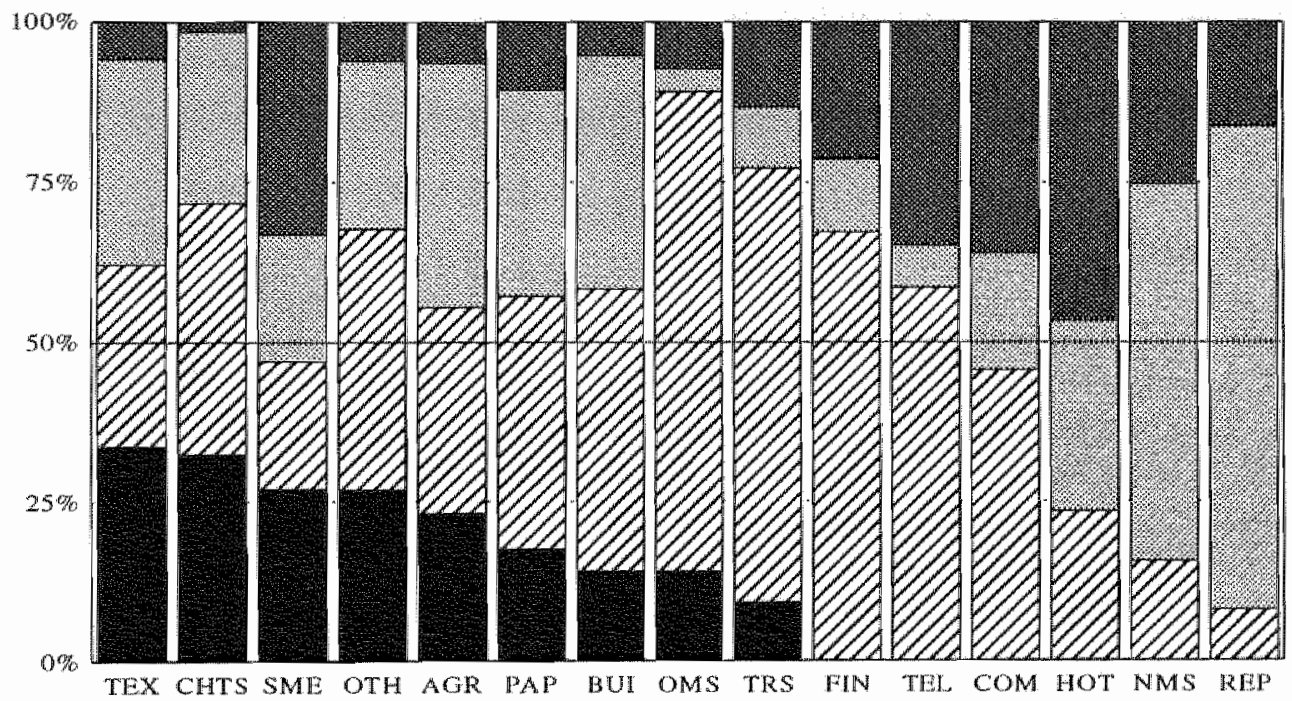

OWN R\&D Z KNOW 圈 RENTM

Figure 7.8. Shares of own R\&D and the various spillover sources in total R\&D used by a sector 
intensities to obtain the total "used" R\&D intensity of a sector. ${ }^{104}$ Figure 7.7 and figure 7.8 illustrate the relative importance of the four R\&D sources in the total amount of $\mathbb{R} \& D$ used by a sector. So, for example the office and data-processing machinery industry (IT1) relies on internal $R \& D(66 \%)$, knowledge spillovers $(27 \%)$, rent spillovers embodied in intermediate goods $(6 \%)$ and rent spillovers embodied in investment goods $(1 \%)$.

The black areas represent the importance of own or internal R\&D in total used $R \& D$. The figures show that the amount of dependency on internal $R \& D$ differs greatly between sectors. High-tech sectors perform the largest part of their R\&D activities themselves, whereas the other sectors rely primarily on $R \& D$ generated in other sectors. When we define sectors that perform more than $50 \%$ of their used R\&D themselves as "net-technology exporters" and sectors that receive more than $50 \%$ from other sectors as "net-technology importers", then twelve industries can be classified as net-technology exporting industries, while the other eighteen industries are net-technology importing industries.

Knowledge spillover's that are not related to goods purchased are the second most important source of used R\&D for the high-tech sectors. Internal R\&D and knowledge spillovers represent more than $90 \%$ of all used $R \& D$ in nine of the ten high-tech sectors. The exception is the rubber and plastics industry (RUB) in which own $\mathbb{R} \& \mathrm{D}$ and knowledge spillovers add up to $72 \%$; this sector receives $27 \%$ of its used R\&D through rent spillovers embodied in intermediate goods (mainly from the chemical sectors). For the medium and low-tech industries ${ }^{105}$, such as NMM, FOO, FAM, TEX, CHTS, PAP and MET, rent spillovers embodied in intermediate goods are relatively more important, while in the service sectors rent spillovers embodied in investment goods are relatively more important.

\subsection{Estimation Results}

The productivity and demand equations, which are estimated on cross-sectional French data, include a measure of the industry"s internal $R \& D$ intensity and some spillover measures. We have estimated the rates of return (marginal productivities) for these types of $R \& D$. Internal $R \& D$ will be divided into process and product R\&D. According to the model, the main influence of process $R \& D$ is on productivity (see sub-section 7.1.1) and the main influence of product $R \& D$ is on demand (see sub-section 7.1.2). The influence of product R\&D on productivity is not straightforward (see sub-section 7.1.3).

In sub-section 7.3 .1 we estimate the estimations derived in section 7.1 with gross value added and in sub-section 7.3 .2 we do the same but with actual production.

\footnotetext{
144 This is a simple accounting method. We estimate the importance of the internal R\&D and the warions spillover concepts in section 7.3 .

105 R\&D intensity is less than $1 \%$ of actual production.
} 
This enables us to investigate whether it makes a difference to use gross value added or actual production or whether certain assumptions such as constant returns to scale are more harmful in one of the two situations. The definitions of the variables used as regressors in the estimations are described in table 7.4.

Table 7.4 Definition of variables

\begin{tabular}{|c|c|}
\hline Symbo: & Description \\
\hline$y^{\prime}$ & actual production (growth rate) \\
\hline$G$ & gross value added (growth rate) \\
\hline $\mathbb{L}$ & labour (growth rate) \\
\hline $\mathbb{K}$ & capital stock (growth rate) \\
\hline$M$ & intermediate deliveries (growth rate) \\
\hline$Y / K$ & capital productivity (growth ate) \\
\hline$L / K$ & labour/capital ratio (growth robe) \\
\hline$M / K$ & inter mediate goods/capital ratio (growth rate) \\
\hline $\mathrm{TPP} Y$ & three factor Total Factor Productivity (labour, capital and intermediate goods.) \\
\hline $\mathrm{TPP} G$ & two factor Total factor Productivity (labour and capital) \\
\hline$R_{-} X$ & "own" R\&D intensity (R\&D expenditures divided by actual production) \\
\hline$R_{-} G^{106}$ & "own" R\&D intensily (R\&D expenditures divided by gross value added) \\
\hline $\mathbb{R C} Y$ & "own" process R\&D intensity \\
\hline RD_Y & "own' product R\&D intensity \\
\hline RENTM_Y & "Pure" rent spillovers and knowledge spillovers related to intermedate goods. \\
\hline RENTK $Y$ & "Pure" rent spillovers and knowledge spillovers related to inverment goods. \\
\hline KNOW_Y & "Pure" or non-input-related knowledge spillovers \\
\hline
\end{tabular}

Other empirical studies on the relationship between spillover effects and productivity growth included either a measure that captures embodied spillovers or a measure that captures non-input related knowledge spillovers. These studies therefore do not measure all spillover effects. This study goes one step further by including both input-related spillovers and "pure" knowledge spillovers.

106 Conditional on the dependent variable, actual production (Y) or gross value added (O) are the various $\mathbb{R} \& \mathrm{D}$ intensities expressed as a $\mathrm{R} \& \mathrm{D}$ variable divided by actud production, postscript $\mathrm{Y}$ or gross value added postscript $\mathrm{G}$. 


\subsubsection{Cross-Section: Gross Value Added}

This section discusses the estimation results for the general cost reduction equations (c.f. equation (7.16), equation (7.26) and equation (7.31)) and the general demand creating equation (equation (7.21)). In this section we discuss the dimension across sectors. We avoid time variation by calculating the mean values over the period 1978-1992 for every sector. This reduces the number of observations from 450 to 30 . In this section we use gross value added (GVA) instead of actual production.

Table 7.5 Mean walues of key variables, cross-section, gross value added, $N=30,1978$-1992

\begin{tabular}{llll}
\hline $\begin{array}{l}\text { Crowth } \\
\text { Rates }\end{array}$ & $\begin{array}{l}\text { R\&D } \\
\text { Intensities }\end{array}$ \\
\hline TFP_G & 0.015 & R_G & 0.053 \\
THPU_G & 0.016 & RC_G & 0.024 \\
GVA & 0.024 & RD_G & 0.029 \\
& & RENTM_G & 0.018 \\
& & RENTK_G & 0.003 \\
& & KNOW_G & 0.024 \\
\hline
\end{tabular}

Table 7.5 presents the mean values of the various variables. The average growth rate across sectors of GVA is $2.4 \%$. The Solow residual or TFP growth, which can be seen as a proxy for technological change, is $1.5 \%$. If one corrects for the rate of capacity utilisation then the TFP increases to $1.6 \%$ (TFPU). Technological change determines about $65 \%$ of output (GVA) growth and is therefore the most important determinant of this output growth. The R\&D intensity is on average $5.3 \%$ of GVA. About $55 \%$ of this R\&D is product oriented R\&D. The magnitude of the various spillover effects is very different. The rent spillovers embodied in investment goods are small and equal to $0.3 \%$ of GVA. Rent spillovers embodied in intermediate goods are six times larger and equal to $1.8 \%$. The "pure" knowledge spillovers are larger than the two kinds of rent spillover together and equal to $2.3 \%$. However, the magnitude of the various spillover effects says nothing about their influence and relevance for sectoral productivity growth.

It can be expected that the various R\&D measures are correlated to each other. The simple correlation coefficients measure the strength of association between two variables and provide an indication with regard to the probability of multicollinearity. The correlation coefficients are given in table 7.6. All R\&D measures have a positive correlation with TFP; the coefficient ranges from 0.36 for rent spillovers embodied in investment goods to 0.75 for the "pure" knowledge 
spillovers. As expected, there is a high correlation between total $R \& D$ and process and product $R \& D$ (because $R \& D$ is concentrated in a few sectors; see table 7.2).

Table 7.6 Correlation Matrix

\begin{tabular}{|c|c|c|c|c|c|c|c|c|}
\hline & TFP & GVA & $\mathrm{R}$ & $R C$ & $\mathrm{RD}$ & RENTK & RHNTT & KNOM \\
\hline$T F \mathbb{P}$ & 1 & & & & & & & \\
\hline GVA & 0.74 & 1 & & & & & & \\
\hline $\mathbb{R}$ & 0.70 & 0.64 & 1 & & & & & \\
\hline $\mathbb{R C}$ & 0.58 & 0.49 & 0.92 & 1 & & & & \\
\hline RD & 0.70 & 0.68 & 0.93 & 0.71 & 1 & & & \\
\hline RENTKK & 0.36 & 0.46 & -0.03 & -0.03 & -0.03 & 1 & & \\
\hline RENTT & 0.54 & 0.44 & 0.64 & 0.55 & 0.63 & -0.1 & 1 & \\
\hline KNOW & 0.75 & 0.68 & 0.85 & 0.79 & 0.78 & 0,02 & 0.65 & 1 \\
\hline
\end{tabular}

An interesting feature exists in the relationship between spillovers and own R\&D. The correlation between "pure" knowledge spillovers, rent spillovers embodied in intermediate goods and own R\&D is positive, but there is no relationship between rent spillovers embodied in capital and own $\mathbb{R} \& D$. This finding is not surprising after reviewing the data (see section 7.2). This section illustrated that own R\&D, rent spillovers of intermediate goods and "pure" knowledge spillovers were mainly concentrated in the high-tech sectors, while rent spillovers of investment goods were the highest in the service sectors. Between the spillover variables themselves we see the same pattern; a positive correlation between rent spillovers embodied in intermediate goods and "pure" knowledge spillovers and no (or a slightly negative) correlation between these two spillover measures and rent spillovers embodied in capital goods. These results differ from the findings of Wolff and Nadiri (1993) where they found a positive correlation (0.7) between capital and other spillovers. In our finding we can use capital spillovers as an additional variable in a regression without multi-collinearity.

What is also interesting is the strength of the correlation between the various spillover effects and own R\&D. The correlation of own R\&D and "pure" knowledge spillovers is the strongest (0.85). On the one hand this implies a multi-collinearity problem between own R\&D and "pure" knowledge spillovers, and also confirms our model in which "pure" knowledge spillovers increase the value of own R\&D and can therefore be expected in sectors which perform a lot of $R \& D$.

\section{Intemal RED, Process-oriented RED and Product-oriented RED}

First, we investigate whether product R\&D does (sub-section 7.1.3) or does not (sub-section 7.1.1) influence TFP growth. We therefore estimate equation (7.16) and 
equation (7.26) without the spillover variables. Regression. 7.1, which is reported in table 7.7, discusses the influence of self performed or internal R\&D. The rate of return to total own $R \& D\left(R \_G\right)$ is $19 \%$ and statistically significant at the 0.002 level. This result is comparable with results found in other studies. After our survey of the empiricall studies on the relationship between TFP growth and own R\&D, we concluded that most studies predicted a value between $10 \%$ and $50 \%$ for the rate of return of total $\mathbb{R} \& D$ (see section 6.1 ). When we look at the rate of return to own process $R \& D$ ( $R C$ C_ $G$ ) in regression 7.2 the rate of return is $30 \%$ but the explanatory power of this equation with only process $\mathbb{R} \& D$ is less than that of regression 7.1 with total $R \& D$. This indicates a role for product $R \& D$ with respect to TFP growth.

Table 7.7 Regression of Total Factor Productivity growth rate on internal R\&D intensity, N=30. (t-ratio's in parentheses)

\begin{tabular}{|c|c|c|c|c|}
\hline $\begin{array}{l}\text { Regression } \\
\text { number }\end{array}$ & coristant & R\&D intensity & $\begin{array}{l}\text { Product } \\
\text { R\&D intensity }\end{array}$ & $\frac{D W}{R^{2}}$ \\
\hline $7.1 \mathrm{TFP}$ & $\begin{array}{l}0.007 \\
(1.63)\end{array}$ & $\begin{array}{l}0.19^{+41} R_{-} G \\
(5.27)\end{array}$ & & $\begin{array}{l}2.12 \\
0.48\end{array}$ \\
\hline 7.2 TFP & $\begin{array}{l}0.009^{\circ} \\
(2.04)\end{array}$ & $\begin{array}{l}0.30^{* *} \mathrm{RC} G \mathrm{G} \\
(3.87)\end{array}$ & & $\begin{array}{l}2.27 \\
0.32\end{array}$ \\
\hline $7.3 \mathrm{TFP}$ & $\begin{array}{l}0.007 \\
(1.73)\end{array}$ & & $\begin{array}{l}0.33^{n+m} \mathrm{RD} G \mathrm{G} \\
(5.21)\end{array}$ & $\begin{array}{l}1.90 \\
0.47\end{array}$ \\
\hline $7.4 \mathrm{TFP}$ & $\begin{array}{l}0.006 \\
(1.59)\end{array}$ & $\begin{array}{l}0.09 R_{1} C_{-} \\
(0.88)\end{array}$ & $\begin{array}{l}0.28^{*} \text { RD_G } \\
(3.10)\end{array}$ & $\begin{array}{l}2.01 \\
0.48\end{array}$ \\
\hline
\end{tabular}

Significant at the 0.10 level (two-tailed test).

". Significant at the 0.01 level (two-tailed test).

significant at the 0.002 level (two-tailed test).

Regression 7.3 measures the influence of product R\&D (RD_G) on TFP growth and shows that product $R \& D$ has a positive influence. The estimated rate of return to product R\&D is 33\%. This result differs from the result found in studies based on questionnaires; namely that process-oriented R\&D is more important with respect to productivity growth than product-oriented R\&D (Link 1982, Terleckyj 1982, Clark and Griliches 1984, see section 6.3).

It is important, however, to keep in mind that it is difficult to distinguish between process and product $R \& D$ in empirical data, as process and product $R \& D$ are often jointly pursued on a project basis and pure quality improvements may also imply a reduction in inputs. The Scherer (1982) and Griliches and Lichtenberg (1984) studies indicated that product $R \& D$ is becoming increasingly important over time. Their estimated values for the 1973-1978 period were higher and more significant than those based on the $1964-1969$ period. Our estimations of the rate of return to product $\mathbb{R} \& D$ are based on the $1978-1992$ period and indicate a clear 
positive influence of product $R \& D$ on productivity. The results of these three studies lead to a similar conclusion: product $\mathbb{R} \& D$ is, with regard to productivity growth, becoming more important over time. This indicates that product R\&D, which is aimed at improving the quality of a product, also decreases costs or that quality improvements are partly reflected in prices (see section 7.1.3).

The importance of product $R \& D$ is reflected in the two variable regression of process and product $R \& D(7.4)$. The regression coefficient of the product $R \& D$ variable is significant while the process $R \& D$ variable becomes insignificant. This indicates a stronger relationship between product R\&D and TFP growth. However, the insignificance of the process $R \& D$ coefficient does not indicate that there is no relation with TFP growth, since it is due to multicollinearity between process and product $R \& D$. Because the relationships between process and product $R \& D$ are blurred by multicollinearity, we will estimate equation $(7.26) /(7.31)$ by using total own $R \& D$ instead of process and product $\mathbb{R} \& D$ as separate variables. The only difference between estimating equation (7.16) and equation (7.26) is that in equation (7.16) we use the own process $R \& D$ concept and in equation $(7.16) /(7.31)$ we use the total R\&D concept.

Table 7.8 Regression of Total Factor Productivity growih rate on used R\&D intensity, $N=36$, (t-ratio's in parentheses)

\begin{tabular}{|c|c|c|c|c|}
\hline $\begin{array}{l}\text { Regression } \\
\text { number }\end{array}$ & Constant & Product R\&D & $\begin{array}{l}\text { Used } R \& D \\
\text { intensity }\end{array}$ & $\frac{\mathrm{DW}}{\mathrm{R}^{2}}$ \\
\hline \multirow[t]{2}{*}{8.1 TFP } & 0.005 & & $0.26^{*-1}$ RCU_G & 2.16 \\
\hline & $(1.01)$ & & $(4.56)$ & 0.41 \\
\hline \multirow[t]{2}{*}{8.2 TFP } & 0.005 & & $0.16^{* *}$ RCUT_G & 1.86 \\
\hline & $(1.37)$ & & $(5.71)$ & 0.52 \\
\hline \multirow[t]{2}{*}{$\mathrm{A} .3 \mathrm{TFP}^{\mathrm{H}}$} & 0.004 & & $0.17^{n+1}$ RTU $G$ & 2.05 \\
\hline & $(1.01)$ & & $(5.55)$ & 0.51 \\
\hline \multirow[t]{2}{*}{ 8.4 TFP } & 0.005 & & $0.12^{\cdots}$ RTUT_C & 1.98 \\
\hline & $(1.29)$ & & $(6.17)$ & 0.56 \\
\hline \multirow[t]{2}{*}{$8.5 \mathrm{TFP}$} & 0.005 & $0.24^{* *}$ & $0.11 \mathbb{R C U} G$ & 1.99 \\
\hline & (1.14) & $(2.51)$ & $(1.29)$ & 0.50 \\
\hline \multirow[t]{2}{*}{ 8.6 TFP } & 0.005 & 0.16 & $0.10^{\circ}$ RCUT_G & 1.89 \\
\hline & $(1.29)$ & $(1.62)$ & $(2.17)$ & 0.55 \\
\hline
\end{tabular}

,", Significant at the 0.10 level, 0.01 level and 0.002 level (two-talled test), respectively.

Used R\&D and Productivity Growth.

Table 7.8 shows the results of the regressions with "used" R\&D concepts. In these concepts internal R\&D and spillover effects are added together and assumed to 
have an equal influence on productivity growth In regression 8.1 and 8.2 we use the Scherer (1982) definition of used R\&D. Used R\&D consists of own process R\&D and spillover effects. In regression 8.1 we used $R C L, G$, own process $R \& D$ and rent spillovers of capital and intermediate goods. The explanatory power of this "used" $\mathrm{R} \& \mathrm{D}$ concept, $\vec{R}^{2}=0.41$, is higher than the explanatory power when we only used process $R \& D\left(\vec{R}^{2}=0.32\right.$, table 7.7$)$. This indicates that rent spillovers have an effect on productivity. The indusion of "pure" knowledge spillovers in this used $R \& D$ concept (RCUT_G) increases the explanatory power until $\vec{R}^{2}=0.52$ (regression 8.2 ). This indicates that "pure" knowledge spillovers have something to add in addition to own R\&D and rent spillovers.

We compare these findings with the results of the Scherer and GrilichesLichtenberg (S-G-L) studies that concluded that "used" R\&D is more important than own $\mathrm{R} \& \mathrm{D}$ with regard to productivity growth. When we compare the explanatory power of regression 7.1 (total internal $R \& D, \bar{R}^{2}=0.48$ ) with the explanatory power of regression 8.1 and 8.2 , our findings contradict the $S-G-L$ conclusion if we include only rent spillovers in the "used" R\&D concept $\left(\bar{R}^{2}=0.41\right)$, but confirm this conclusion if we include both rent and "pure" knowledge spillovers $\left(\bar{R}^{2}=0.52\right)$,

Since the Scherer flow matrix consists of a greater mixture of rent and knowledge spillovers than the Yale flow matrix (see section 6.2) the results of the three studies are comparable. However, our results indicate that, next to rent spillovers embodied in purchased goods as stressed by the S-G-L studies, "pure" knowledge flows that are not connected with such flows are also responsible for this result.

When we include total own $R \& D$ instead of process $R \& D$ in the used $R \& D$ concept, the explanatory power increases from $R^{-\frac{2}{2}}=0.48$ (regression 7.1 , only own R\&D), to $\vec{R}^{2}=0.51$ (regression 8.3 , own $R \& D$ and rent spillovers) to $\vec{R}^{2}=0.56$ (regression 8.4 own R\&D, rent spillovers and "pure" knowledge spillovers). These regressions again show the importance of spillovers and also indicate the importance of product $\mathrm{R} \& \mathrm{D}$ with regard to TFP growth as the explanatory power of total R\&D is higher than that of only process $R \& D$. In regression 8.5 and 8.6 we included both internal product R\&D as well as "used" R\&D a la Scherer. With only rent spillovers, the estimated coefficient of product $R \& D$ is significant and that of "used" R\&D is insignificant, the results reverse if we unclude both rent and knowledge spillovers in the "used" R\&D concept. However, we have to be cautious with respect to the result of these two regressions because the explanatory variables are correlated (correlation coefficient between explanatory variables is about 0.8 in both regressions).

\section{The Influence of the Various Spillower Concepts on TFP Growth}

We will investigate the influences of the various spillover variables by including the spillover effects separately in equation (7.16) and equation $(7.26)$. The regressions of equation (7.16) are depicted in table 7.9 and are labelled with odd 
numbers, while even numbers represent the results of equation (7.26) (process and product $R \& D$ are combined in one total $R \& D$ concept to avoid multicollinearity).

Table 7.9 Regression of Total Factor Productivity growth rate on internal R\&D and spillovers, $N=30,(t-$ ratio's in parentheses)

\begin{tabular}{|c|c|c|c|c|c|c|}
\hline & CONST & $\begin{array}{l}\text { "OWN" } \\
\text { R\&D }\end{array}$ & RENTK_G & RENTM_G & KNOW $G$ & $\begin{array}{l}\bar{R}^{2} \\
D W\end{array}$ \\
\hline 9.1 & $\begin{array}{l}-0.007 \\
(-1.25)\end{array}$ & $\begin{array}{l}0.02 \mathbb{R E} C_{-} G \\
(0,02)\end{array}$ & $\begin{array}{l}4.25^{\circ} \\
(3.29)\end{array}$ & $\begin{array}{l}0.20 \\
(1.16)\end{array}$ & $\begin{array}{l}0.28^{10} \\
(3.24)\end{array}$ & $\begin{array}{l}0.66 \\
1.71\end{array}$ \\
\hline 9.2 & $\begin{array}{l}-0.008 \\
(-1.47)\end{array}$ & $\begin{array}{l}0.07 R_{-} G \\
(1.23)\end{array}$ & $\begin{array}{l}4.35^{24} \\
(3.46)\end{array}$ & $\begin{array}{l}0.16 \\
(0.91)\end{array}$ & $\begin{array}{l}0.20^{\circ} \\
(2.17)\end{array}$ & $\begin{array}{l}0.68 \\
1.80\end{array}$ \\
\hline 9.3 & $\begin{array}{l}-0.012^{*} \\
(-1.74\rangle)\end{array}$ & $\begin{array}{l}0.21^{\circ} \mathrm{RC} \_\mathrm{G} \\
(2.64)\end{array}$ & $\begin{array}{l}4.80^{* *} \\
(3.20)\end{array}$ & $\begin{array}{l}0.47^{*} \\
(2.40)\end{array}$ & & $\begin{array}{l}0.53 \\
2.30\end{array}$ \\
\hline 9.4 & $\begin{array}{l}-0.011 \\
(-1.89)\end{array}$ & $\begin{array}{l}0.15^{* * 1} \text { R_G } \\
(3.98)\end{array}$ & $\begin{array}{l}4.69^{\circ} \\
(3.52)\end{array}$ & $\begin{array}{l}0.26 \\
(1.45)\end{array}$ & & $\begin{array}{l}0.63 \\
2.16\end{array}$ \\
\hline 9.5 & $\begin{array}{l}0.009^{*} \\
\left(2.344^{*}\right)\end{array}$ & $\begin{array}{l}-0.013 \mathrm{RC}_{-} \mathrm{G} \\
(-0.12)\end{array}$ & & & $\begin{array}{l}0.34^{4 *+1} \\
(3.77)\end{array}$ & $\begin{array}{l}0.54 \\
1.79\end{array}$ \\
\hline 9.6 & $\begin{array}{l}0.007 \\
(1.97)\end{array}$ & $\begin{array}{l}0.06 \mathbb{R} G \\
0.97\end{array}$ & & & $\begin{array}{l}0.25^{\circ} \\
(2.41)\end{array}$ & $\begin{array}{l}0.56 \\
1.83\end{array}$ \\
\hline 9.7 & $\begin{array}{l}-0.008 \\
(-1.49)\end{array}$ & $\begin{array}{l}0.16^{* \cdots} \text { RCRSET_G } \\
(6.63)\end{array}$ & $\begin{array}{l}4.39^{44} \\
(3.37)\end{array}$ & & & $\begin{array}{l}0.64 \\
1.92\end{array}$ \\
\hline 9.8 & $\begin{array}{l}-0.01^{*} \\
(-1.76)\end{array}$ & $\begin{array}{l}0.12^{* *} \text { RRSET_G } \\
(7.38)\end{array}$ & $\begin{array}{l}4.43^{* *} \\
(3.65)\end{array}$ & & & $\begin{array}{l}0.69 \\
1.91\end{array}$ \\
\hline
\end{tabular}

Significant at the 0.10 level (two-tailed test).

"Significant at the 0.01 level (woutailed test).

significant at the 0.002 level (womtailed test).

When we examine the regressions in table 7.9, it is apparent that the rate of return to rent spillovers embodied in capital goods is very high, ranging from $425 \%$ to $480 \%$, and it is significant in all specifications. These results indicates that purchased capital goods are important for the TFP growth of a sector. Product innovations in capital producing industries have increased the efficiency of capital goods, which has benefitted the purchasing industries. These results are not unusual and are consistent with the findings of Terleckyj (1974) and Sveikauskas (1981) whose estimates of the rate of return were within the $262 \%$ to $861 \%$ range (see table 6.3). The rate of return to rent spillovers of intermediate goods is much lower and ranges from $16 \%$ to $47 \%$ and is only significant in equation 9.3. This estimated range is within the predicted range $(0.5 \%$ to $53 \%)$ which we constructed 
for the rate of return to rent spillovers of intermediate goods on the basis of our survey of the empirical literature (see section 6.2.1). "Pure" knowledge spillovers are significant in all the regressions and the rate of return ranges from $20 \%$ to $34 \%$. The highest values are achieved when we do not include rent spillovers (spillovers related to input purchases). This supports our hypothesis that the Yale-knowledge proxy includes some input-related rent spillovers and that the inclusion of another variable, which is expected to be related to rent spillovers, purifies the "pure" or non-input-related knowledge spillover concept from input-related rent spillovers. Our estimated range for "pure" knowledge spillovers is lower than most other studies that used a techflow matrix based on patents; Scherer (1982) estimated a range from $118 \%$ to $147 \%$ and Mohnen-Lépine (1991) found an average rate of $87 \%$ (range 11\%-319\%). However, our estimates are more consistent with the estimated results of the studies that constructed knowledge spillovers by the "proximity in technological research space" (range 4\% (Goto-Suzuki 1989) to $40 \%$ (Adams 1990)) or by using a technology flow matrix based on innovations or no weights of R\&D stocks (range $7 \%$ (Sterlacchini 1989) to 58\% (Bernstein 1989)). These methods are sometimes considered to be better measures of "pure" knowledge spillovers than a techflow matrix based on patents, which is often considered as a mixture of rent and "pure" knowledge spillovers. This is obviously true in the case of Scherers" techflow matrix as he uses input-output matrices to distribute important inventions over sectors. The Yale matrix does not use input-output matrices and may therefore be considered a better tool to measure "pure" knowledge spillovers. The estimated range of "pure" knowledge spillovers confirms this since it is consistent with estimates of studies that used a measure expected to better measure "pure" knowledge spillovers.

The large differences in the rate of returns do not imply that the influence of the various spillover variables on TFP growth are significantly different. The quasielasticities associated with these rates of returns are 1.28-1.44 for capital rent spillovers, $0.29-0.85$ for intermediate rent spillovers and $0.48-0.82$ for the "pure" knowledge spillovers. ${ }^{10 \%}$

When we introduce the various spillover variables sequentially in the model we can discover whether this contributes anything to the explanatory power of the model. First, we introduce rent spillovers in regression 7.1 and 7.2 and obtain regressions 9.3 and 9.4. The F-values of the test for the marginal or incremental contribution of an explanatory variable are $F_{2,26}=6.5$ and $F_{2,26}=5.78$ for the addition of rent spillovers in regression 7.1 and 7.2 , respectively. These $F$ values are significant beyond the $1 \%$ level of significance, so the addition of rent spillovers in both equations is worthwhile. The following question is whether the introduction of "pure" knowledge spillovers to this model with own R\&D and two kinds of rent spillovers is still worthwhile. Now we compare the $R^{2}$ of regressions 9.3 and 9.4

107 Quasi-elasticities can be calculated as the measured rate of return (estimnted coefficients in table 7.9) times the average value of the related spillover intensity (see table 7.5 ). 
with regressions 3.1 and 3.2 respectively. The values of the $F$ statistics are respectively $\mathrm{F}_{1,25}=9,38$ and $\mathrm{F}_{1,25}=5$. These values are significant at respectively the $1 \%$ and the $5 \%$ level of significance. We get the same results if we reverse this procedure: we first add "pure" knowledge spillovers and then rent spillovers. This analysis implies that our measures for the various spillover variables have a significant contribution to the model and should hence be added to the model. Own R\&D, rent spillovers and "pure" knowledge spillovers all play a significant role in TFP growth. Studies that include either input-related or non-input related spillovers do not include all spillover effects.

The addition of "pure" knowledge spillovers increases the explanatory power significantly but at the cost of insignificance of the coefficients of own R\&D and rent spillovers embodied in intermediate goods. The fact that "pure" knowledge spillovers make the coefficient of own R\&D insignificant can be seen from a comparison of regressions 9.5 and 9.6 (or 9.3 and 9.4 ) with regressions 7.1 and 7.2 (9.1 and 9.2). The reason for this is the very high correlation coefficient between own $R \& D$ (process $R \& D$ ) and "pure" knowledge spillovers, the value of this coefficient is $0.85(0.79)$ (see table 7.6). The correlation coefficient between own $R \& D$ (process $R \& D$ ) and rent spillovers embodied in intermediate goods is also high with a value of $0.64(0.55)$, which indicates a multi-collinearity problem. We can avoid this problem by adding together the variables which are positively correlated. We constructed therefore a variable, RRSET_G (RCRSET_G), which contains own $R \& D$ (own process $R \& D$ ), rent spillovers of intermediate goods and "pure" knowledge spillovers. Regressions 9.7 and 9.8 show the results. Both the coefficient of this constructed variable and the coefficient of rent spillovers of capital goods are significant and the explanatory power of these equations is relatively high.

\section{Testing the Assumptions Underlying TFP Growth Calculations}

All our estimations are done on a prior measurement of TFP growth. As we have indicated in section 7.1, this method depends on two assumptions: first, the constant returns to scale assumption and second the optimal choice of production factors. These assumptions can imply an over- or under-estimation of the rates of return. Especially constant returns to scale is expected to have a positive bias on the rates of return to the various R\&D concepts (See Mohnen 1990). We will check whether relaxing both assumptions has an important influence on the rates of return. The most important problem of the optimal choices of production factors is that a part of the capital stock is not used all the time. We can address this problem by using the utilization rate to calculate the utilized capital stock. We can relax the assumption of constant returns to scale by estimating equation (7.13). 
Table 7.10 Regression of Gross Value Added growth rate on factor inputs, internal R\&D and spillovers.

\begin{tabular}{|c|c|c|c|c|c|c|c|c|}
\hline & CONST & $\begin{array}{l}\mathbb{L A B} \\
\mathrm{gr}\end{array}$ & $\begin{array}{l}\text { CAP } \\
\text { gr. }\end{array}$ & "OWN" R\&\&D & RENTK & RENTM & KNOW & $\begin{array}{l}\vec{R}^{2} \\
D W\end{array}$ \\
\hline \multirow[t]{2}{*}{10.1} & $0.026^{\circ+4}$ & $0.41^{\circ}$ & & & & & & 0.09 \\
\hline & $(5.19)$ & $(1.71)$ & & & & & & 1.91 \\
\hline \multirow[t]{2}{*}{10.2} & -0.00 & & $0.62^{\circ}$ & & & & & 0.17 \\
\hline & $(-0.05)$ & & $(2.60)$ & & & & & 2.11 \\
\hline \multirow[t]{2}{*}{10.3} & 0.00 & & $0.65^{\circ} \mathrm{U}$ & & & & & 0.17 \\
\hline & $(0.04)$ & & $(2.66)$ & & & & & 2.12 \\
\hline \multirow[t]{2}{*}{10.4} & 0.00 & 0.16 & $0.54^{\circ}$ & & & & & 0.15 \\
\hline & $(0.26)$ & $(0.59)$ & $(1.93)$ & & & & & 2.08 \\
\hline \multirow[t]{2}{*}{10.5} & $0.02^{x}$ & $0.66^{\circ}$ & -0.4 & $0.21^{\cdots \cdots} \mathrm{R}$ & & & & 0.55 \\
\hline & $(2.16)$ & $(3.04)$ & $(-0.56)$ & $(5.02)$ & & & & 1.82 \\
\hline \multirow[t]{2}{*}{10.6} & $0.02^{\circ}$ & $0.61^{\circ *}$ & $-0.06 \mathrm{U}$ & $0,20^{\cdots \cdots} \mathrm{R}$ & & & & 0.54 \\
\hline & $(1.92)$ & $(2.78)$ & $(-0.22)$ & $(4.95)$ & & & & 1.82 \\
\hline \multirow[t]{2}{*}{10.7} & $0.02^{\circ}$ & $0.64^{\circ}$ & -0.06 & $0.33^{* *} \mathrm{RC}$ & & & & 0.37 \\
\hline & $(1.74)$ & $(2.34)$ & $(-0.21)$ & $(3.22)$ & & & & 2.10 \\
\hline \multirow[t]{2}{*}{10.8} & 0.02 & $0.60^{\circ}$ & $-0.00 \mathrm{U}$ & $0.32^{* \prime R C}$ & & & & 0.36 \\
\hline & $(1.63)$ & $(2.17)$ & $(-0.00)$ & $(3.19)$ & & & & 2.11 \\
\hline \multirow[t]{2}{*}{10.9} & -0.00 & $0.56^{4+*}$ & -0.03 & $0.10^{\circ} \mathrm{R}$ & $5.32^{n+4}$ & 0.08 & $0.18^{\circ}$ & 0.81 \\
\hline & $(40.01)$ & $(3,93\}$ & $(-0.70)$ & $(2,07)$ & $(5.35)$ & $(0.66)$ & $(2.48)$ & 1.71 \\
\hline \multirow[t]{2}{*}{10.10} & -0.00 & $0.47^{*}$ & 0.12 & $0.01 \mathrm{RC}$ & $5.17^{\circ 6}$ & 0.15 & $0.29^{\text {nten }}$ & 0.78 \\
\hline & $(-0.47)$ & $(2.79)$ & $(0,63)$ & $(0.11)$ & $(4.78)$ & $(1.03)$ & $(3.93)$ & 1.75 \\
\hline \multirow[t]{2}{*}{10.11} & -0.00 & $0.58^{\prime \prime \prime}$ & -0.66 & $0.18^{* \cdots} \mathrm{R}$ & $5.63^{\cdots}$ & 0.77 & & 0.77 \\
\hline & $(-0.17)$ & $(3.68)$ & $(-0.35)$ & $(4.93)$ & $(5.19)$ & $(1.16)$ & & 2.02 \\
\hline \multirow[t]{2}{*}{10.12} & -0.01 & $0.55^{* *}$ & 0.03 & $0.22 \mathrm{RC}^{*}$ & $5.73^{\cdots}$ & $0.40^{\circ}$ & & 0.64 \\
\hline & $(-0.49)$ & $(2.60)$ & $(0.12)$ & $(256)$ & $(4.20)$ & $(2,32)$ & & 2.28 \\
\hline \multirow[t]{2}{*}{10.13} & $0.02^{\circ}$ & $0.59^{* *}$ & 0.00 & $0.08 \mathrm{R}$ & & & $0.23^{\circ}$ & 0.61 \\
\hline & $(1.91)$ & (2.89): & $(0.02)$ & $(1.21)$ & & & $(2.27)$ & 1.59 \\
\hline \multirow[t]{2}{*}{10.14} & $0.01^{*}$ & $0.48^{* \prime}$ & 0.15 & $-0.24 \mathrm{RC}$ & & & $0.35^{\cdots}$ & 0.59 \\
\hline & $(1.39)$ & $(2,12)$ & $(0.60)$ & $(-0.30)$ & & & $(3.86)$ & 1.66 \\
\hline \multirow[t]{2}{*}{10.15} & -0.00 & $0.58^{m * *}$ & -0.05 & $0.12^{\cdots *}$ RRSET & $5.43^{\cdots *}$ & & & 0.82 \\
\hline & $(-0.07)$ & $(4.22)$ & $(-0.32)$ & $(8.55)$ & $(5.70)$ & & & 1.73 \\
\hline \multirow[t]{2}{*}{$10: 16$} & -0.04 & $0.58^{* *}$ & -0.03 & $0.17^{\cdots \cdots}$ RCRSET & $5.37^{\cdots}$ & & & 0.76 \\
\hline & $(-0.01)$ & $(3.60)$ & $(-0.16)$ & $(6.85)$ & $(4.83)$ & & & 1.83 \\
\hline
\end{tabular}

"," "Nignificant at the 0.10 level, 0.01 level and 0.002 level (two-tailed test), respectively, 
We will first study the results if we use the utilised capital stock instead of the total capital stock. The results of the utilised capital stock are stated in table 7.10 by regressions $10.3,10.7$ and 10.9 which have to compared with the corresponding regressions with the total capital stock, respectively regressions $10.2,10.6$ and 10.8 . This comparison show's us that the correction on the capital stock does not significantly influence the regression results. The rates of returns to R\&D are only slightly lower. A reason for this minor influence can be that our data are: 1) crosssectional (all sectors are in the same phase of the business cycle) and 2) data points are mean values over 15 years.

We will now check the assumption of constant returns to scale by estimating equation (7.13). Regression 10.1 and 10.2 show that both labour growth and capital growth have a positive link with gross value added growth and are both significant. If we add both elasticities, 0.41 and 0.61 we obtain constant returns to scale. However, coefficients in separate regressions which exclude other important variables can be misleading. If we combine labour and capital growth in one regression, however, the labour coefficient becomes insignificant through multicollinearity between labour and capital (see regression 10.4). Introduction of the various R\&D variables in the model changes these results - the labour elasticity becomes significant ( $\alpha= \pm 0.65$ ) and the capital elasticity becomes insignificant. It is therefore impossible to say whether there are decreasing, constant or increasing returns to scale. We are not so much interested in this feature per se, but we are interested in whether the assumptions of constant returns to scale influences the value and significance of the rates of return to $R \& D$.

We have to compare the rates of return in regressions with the constant returns to scale assumption (table 7.7 and 7.9 ) with those regressions without this assumption (table 7.10). We will first consider the influence of relaxing the constant return assumption on the rates of returns. It is apparent that the rate of return to rent spillovers of investment goods has increased from $450 \%$ to $530 \%$. The rate of return to total own R\&D has increalsed in all cases (for example from 19\% (7.1) to $21 \%(10.5)$ and from $15 \%(9.4)$ to $18 \%(10.11))$. They have also increased in the case of own process $\mathrm{R} \& \mathrm{D}$ (for example $30 \%$ in regression 7.2 and $33 \%$ in regression 10.7). There is mixed evidence for the rate of return to "pure" knowledge spillovers whille the rate of return to rent spillovers embodied in intermediate goods is slightly lower. Nevertheless, we can conclude that contrary to our expectations, the constant returns to scale assumption had a negative influence on the rate of return to capital spillovers and a negative influence on the rate of return to own R\&D. Rent spillovers on intermediate goods had the expected over-statement of the rate of return (but only slightly) and there was a mixed influence on "pure" knowledge spillovers.

A second step is to investigate the influence of relaxing the constant returns to scale assumption on the significance of the various rates of return coefficients. A comparison of the regressions in table 7.9 with the regressions 10.9-10.16 reveals 
that the significance of the estimated rates of return to own $R \& D$, rent spillovers of capital goods, and "pure" knowledge spillovers, has increased. A good example is the estimated rate of return to total own R\&D which is insignificant in regression 9.2 but becomes significant in regression 10.9. Only the significance of the rate of return to rent spillovers in intermediate goods has declined.

Table 7.11 Regression of capital productivity growth (gross value added/capital) and labour productivity growth rate (gross value added/labour) on factor inputs, internal R\&D and spillowers, $N=30$, (t-ratio's in parentheses)

\begin{tabular}{|c|c|c|c|c|c|c|c|c|}
\hline $\begin{array}{l}\text { GVA/K } \\
\text { gr. }\end{array}$ & CONST & $\begin{array}{l}\mathrm{L} / \mathrm{K} \\
\mathrm{gr} .\end{array}$ & $\begin{array}{l}\mathrm{K} \\
\mathrm{gr} .\end{array}$ & "OWN" R\&D & RENTK & RENTM & KNOW & $\begin{array}{l}\bar{R}^{2} \\
\mathrm{DW}\end{array}$ \\
\hline 11.1 & $\begin{array}{l}-0.00 \\
(-0.01)\end{array}$ & $\begin{array}{l}0.56^{\prime \ldots} \\
(3.93)\end{array}$ & $\begin{array}{l}-0.46^{+7 x} \\
(-3.53)\end{array}$ & $\begin{array}{l}0.10^{*} \mathrm{R} \\
(2.07)\end{array}$ & $\begin{array}{l}5.32^{\cdots} \\
(5.35)\end{array}$ & $\begin{array}{l}0.08 \\
(0.68)\end{array}$ & $\begin{array}{l}0.18^{\circ} \\
(2.48)\end{array}$ & $\begin{array}{l}0.78 \\
1.71\end{array}$ \\
\hline 11.2 & $\begin{array}{l}-0.00 \\
(-0.07)\end{array}$ & $\begin{array}{l}0.58^{\circ} \\
(4.22)\end{array}$ & $\begin{array}{l}-0.47^{*+1} \\
(-3.73)\end{array}$ & $\begin{array}{l}0.12^{\text {n*** }} \text { RRSET } \\
(8.55)\end{array}$ & $\begin{array}{l}5.43^{\cdots} \\
(5.70)\end{array}$ & & & $\begin{array}{l}0.79 \\
1.73\end{array}$ \\
\hline $\begin{array}{l}\text { GVA/L } \\
\text { gr. }\end{array}$ & CONST & $\begin{array}{l}\mathrm{K} / \mathrm{L} \\
\mathrm{gr} .\end{array}$ & & "OWN" R\&ED & RENTK & RENTM & KNOW & $\begin{array}{l}\bar{R}^{2} \\
D W\end{array}$ \\
\hline 11.3 & $\begin{array}{l}-0.00 \\
(-0.01)\end{array}$ & $\begin{array}{l}-0.03 \\
(-0.17)\end{array}$ & $\begin{array}{l}-0.46^{* *+} \\
(-3.53)\end{array}$ & $\begin{array}{l}0.10^{\circ} \mathrm{R} \\
(2.07)\end{array}$ & $\begin{array}{l}5.22^{\cdots} \\
(5.35)\end{array}$ & $\begin{array}{l}0.08 \\
(0.60)\end{array}$ & $\begin{array}{l}0.18^{\circ} \\
(2.48)\end{array}$ & $\begin{array}{l}0.83 \\
1.71\end{array}$ \\
\hline 11.4 & $\begin{array}{l}-0.00 \\
(-0.07)\end{array}$ & $\begin{array}{l}-0.05 \\
(-0.32)\end{array}$ & $\begin{array}{l}-0.47^{+\cdots} \\
(-3.73)\end{array}$ & $\begin{array}{l}\text { 0.12"w RRSET } \\
(8.55)\end{array}$ & $\begin{array}{l}5.43^{m *} \\
(5.70)\end{array}$ & & & $\begin{array}{l}0.75 \\
1.83\end{array}$ \\
\hline 11.5 & $\begin{array}{l}-0.00 \\
(-0.64)\end{array}$ & $\begin{array}{l}0.10 \\
(0.64)\end{array}$ & $\begin{array}{l}-0.46^{\circ} \\
(-3.88)\end{array}$ & $\begin{array}{l}-0.06 \mathrm{RC} \\
(-0.81) \\
0.21^{*} \mathrm{RD} \\
(3.38)\end{array}$ & $\begin{array}{l}5.25^{\cdots} \\
(5.86)\end{array}$ & $\begin{array}{l}0.05 \\
(0.36)\end{array}$ & $\begin{array}{l}0.20^{\circ} \\
(3.06)\end{array}$ & $\begin{array}{l}0.86 \\
1.56\end{array}$ \\
\hline
\end{tabular}

$\because ", "$, Significant at the 0,10 level, 0.01 level and 0.002 level (two-tailed test), respectively.

We conclude that in general a negative influence exists on the values and significance of the estimated rates of return to internal $R \& D$ and the various spillover concepts, indicating decreasing returns to scale. We will investigate the constant returns to scale assumption and its influence on the rates of return again by estimating equation (7.14). Table 7.11 depicts the results. Regression 11.3 and 11.4 correspond with equation (7.14) and regression 11.1 and 11.2 correspond to this equation expressed in terms of capital instead of labour. All these regressions indicate decreasing returms to scale. Their predictions are consistent and show a labour elasticity ( $\alpha$ ) from 0.56 to 0.59 and a megative capital elasticity $(-0.06<\beta<-$ 0.09 ). These values and the values of the various rates of return are also consistent with the results in table 7.10 without multi-collinearity between labour and capital. The conclusion that the constant returns to scale assumption had a negative 
influence on the predicted rates of return to own R\&D and rent spillovers in capital goods remains therefore valid.

\section{Predicted Parameter Ranges:}

Examining the regressions in tables 7.7 to 7.11 allows us to specify the ranges for the estimated coefficients of section 7.1.1 and 7.1.3.A (see table 7.12, with regard to the interpretation of the regression coefficients we assume that quality improvements are not reflected in price indices).

Table 7.12 Predicted parameter ranges of rate of returns and quasi-elasticities

\begin{tabular}{|c|c|c|c|}
\hline Parameter & Predicted Range" & Parameter & Predicted Range \\
\hline$\rho_{x}$ & $21 \%-33 \%$ & 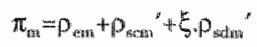 & $40 \%-47 \%$ \\
\hline$\pi_{r d}=E, p_{r d}^{* *}$ & $20 \%-33 \%$ & $\pi_{\mathrm{s}}=\rho_{\mathrm{sx}}+\xi \cdot \rho_{\mathrm{sd}}$ & $18 \%-25 \%$ \\
\hline$\rho_{r}$ & $10 \%-21 \%$ & $p_{\text {sec }}$ & $28 \%-35 \%$ \\
\hline 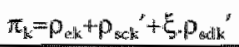 & $425 \%-573 \%$ & & \\
\hline
\end{tabular}

"Only significant values

* We oryly examined estinations of equation (7.26) in which we explicilly used product R\&D (Regressions $7.3,7.4$ and 11.5 ).

\section{Estimation Results: Demand Creating R\&D}

According to the model in sub-section 7.1.2 we would expect that the main influence of product $R \& D$ on demand. This section investigates the relationship between product R\&D and demand by estimating equation (7.21). First, notice that the price elasticity is of demand $(a)$ is inelastic in most cases, $a= \pm 0.8$, and elastic in regression 13.2, a=1.08. Regression 13.1 estimates equation (7.21) without the knowledge spillover effect. The rate of return to product R\&D is highly significant, has the expected sign and is equal to $31 \%$. The exogenous or unexplained growth of demand is insignificant. Our model states that process R\&D does not influence demand. The coefficient of process $\mathrm{R} \& \mathrm{D}$ in regression 13.2 and 13.4 is insignificant and the exogenous growth of demand becomes highly significant when we consider only process R\&D. A regression with the total R\&D concept, regression 13.3, has less explanatory power than when we considered only product R\&D. The exogenous or unexplained growth of demand again becomes significant. On the basis of these results we can conclude that product $R \& D$ is indeed the relevant variable with regard to demand creation. Another implication of our model is that knowledge spillovers are expected to have a positive influence on demand and "pure" rent spillovers play no role (if correctly measured). Regression 13.5 and 13.6 show that the coefficients of all spillovers are not significant. 
Table 7.13 Regression of Demand Equation on internal $R \& D$ and spillovers, $N=30$, (2-ratio's in parrentheses)

\begin{tabular}{|c|c|c|c|c|c|c|c|}
\hline & Const & $\begin{array}{l}\text { Grown } \\
\text { rate of } \\
\text { prices }\end{array}$ & $\begin{array}{l}\text { Product RaD } \\
\text { intensity } \\
\text { (RD } \mathrm{C})\end{array}$ & $\begin{array}{l}\text { Own R\&o } \\
\text { intensity }\end{array}$ & Spillovers & $p^{*}$ & $\begin{array}{l}\vec{R}^{2} \\
D W\end{array}$ \\
\hline 13.1 & $0.0 \mathrm{H}$ & $-0.78^{*}$ & $0.31^{* * 4}$ & & & 0.53 & 0.71 \\
\hline GVA & $(1.57)$ & $(-3.26)$ & $(4.51)$ & & & $(3.21)$ & 221 \\
\hline 13.2 & $0.017^{\circ+20}$ & $-1.08^{4 *}$ & & $0.10 R C_{-} G$ & & & 0.43 \\
\hline$G V A$ & $(3.91)$ & $(-3.37)$ & & $(1.19)$ & & & 1.64 \\
\hline 133 & $0.00^{\circ}$ & $-0.85^{\circ}$ & & $0.15^{4 *} R_{-} G$ & & 0.41 & 0.61 \\
\hline GVA & $(1.93)$ & $(-3.02)$ & & $(3.06)$ & & $(2.32)$ & 2.19 \\
\hline 13.4 & 0.01 & $-0.81^{m}$ & $0.33^{405}$ & $-0.05 R_{0} \mathrm{G}$ & & 0.53 & 0.69 \\
\hline GVA & (1.64) & $(-3.24)$ & $(4.17)$ & $(-0,54)$ & & $(2.99)$ & 2.22 \\
\hline 13.5 & 0,01 & -0.74 & $0.29^{* *}$ & & 0.03 KNOW_G & 0.51 & 0.69 \\
\hline GVA & $(1.65)$ & $(-2.72)$ & $(3.11)$ & & $(0.32)$ & $(2.99)$ & 2.22 \\
\hline 13.6 & 0.07 & $-0.72^{* *}$ & $0.29^{\text {twan }}$ & & 0.14 RENT_G & 0.56 & 0.71 \\
\hline GVA & $(1.47)$ & $(-2.93)$ & (3.79) & & $(0.90)$ & $(3.40)$ & 222 \\
\hline
\end{tabular}

;"* "* Significant at the 0.10 level, 0.01 level and 0.002 level (two-tailed test), respectively.

Regressions adjusted for auto-correlation: the value of $p$ with corresponding $t$ value.

\section{Predicted Parameter Ranges:}

Table 7.14 summarizes the ranges for the coefficients of section 7.3.1. Combining the parameter ranges from table 7.12 and table 7.14 enables us to calculate the implied values for the parameters; $\xi$ and $\rho_{r d}$ (dependent on the value of $b$ ). In chapter three we assumed the value of $b$ between 0 and 1 . This assumption together with the predicted values provides an indication that the estimated rate of return to product $\mathrm{R} \& \mathrm{D}, 29 \% / b-33 \% / b$, is higher than the predicted rate of return to process $\mathrm{R} \& \mathrm{D}, 19 \%-33 \%$. The value of the parameter $\xi$, which indicates the influence of quality on production costs, varies between $[0.61$ times $b]$ and $[1.14$ times $b$. Together with the assumption that $0 \leq b<1$ this implies that sectors which have a higher quality level also thave a higher productivity level.

We have to realise, however, that these interpretations are based on the assumption that quality improvements are not reflected in price indices. It can be expected that normally part of the quality improvement is captured by the TFP measure implying that the range $[0.61$ times $b]$ and $[1.14$ times $b]$ represents $s_{y}+\xi$ instead of $\xi$ (see section 7.1.3.B). 
Table 7.14

\begin{tabular}{ll}
\hline Parameter & Predicted Range \\
\hline at & $0.72-1.08$ \\
b. $\rho_{\mathrm{rdi}}$ & $29 \%-33 \%$ \\
b. $\rho_{\mathrm{sst}}$ & $0 \%$ \\
$\rho_{\mathrm{rdi}}=\mathrm{b} \cdot \rho_{\mathrm{rd}} / \mathrm{b}$ & $29 \% / \mathrm{b}-3.3 \% / \mathrm{b}$ \\
$\xi=\pi_{\mathrm{rd}} / \rho_{\mathrm{rd}}{ }^{1}$ & $0.61 . \mathrm{b}-1.14 \mathrm{~b}$ \\
\hline Represents $\xi+s_{\gamma}$ if part of the quality improvement is reflected in the price indices.
\end{tabular}

\subsubsection{Cross-Section: Actual Production}

In this section we use gross output (including intermediate inputs) instead of gross value added. We investigate whether the inclusion of intermediate inputs changes the value and significance of the coefficients. Terleckyj (1980) found that with a two factor TFP-index the coefficient for own R\&D is 0.27 and is significant at the $1 \%$ level, a three factor TFP index resulted in a statistically insignificant coefficient of 0.20 . Wolff and Nadiri (1993) predict that the estimates of sectoral TFP growth with intermediate goods included, are about half those based on value added.

Table 7.15 Mean values of key variables, Cross-section, Actual Production, $N=30$.

\begin{tabular}{|c|c|c|c|}
\hline Growth Rates & & R\&D Intensities & \\
\hline TFP_Y & 0.0065 & $R_{-} Y$ & 0.0192 \\
\hline TFPU_Y & 0.0070 & $\mathrm{RC}_{-} \mathrm{Y}$ & 0.0088 \\
\hline \multirow[t]{4}{*}{ Y } & 0.0268 & RD $Y$ & 0.0104 \\
\hline & & RENTM_Y & 0.0028 \\
\hline & & RENTK $Y$ & 0.0008 \\
\hline & & KNOW_Y & 0.0098 \\
\hline
\end{tabular}

The mean values of the key variables are given in table 7.15. The three-factor TFP growth is $0.65 \%$ instead of $1.5 \%$ as in the two-factor TFP case (see table 7.5 ). Adjusted for the utilisation rate of capital, these growth rates are $0.7 \%$ and $1.6 \%$, respectively. The various $R \& D$ intensities are now expressed in terms of actual production instead of gross value added. For example the total R\&D intensity is $1.9 \%$ from gross output and $5.3 \%$ from gross value added. Simple regressions between the three factor TFP growth rates and internal. R\&D measures, regressions 16.1-16.3, show that the value and statistical significance of the coefficients have 
decreased in comparison with these in the two-factor TFP case, which are given in table 7.7. Only the coefficient of total R\&D is significant at the $10 \%$ level of significance and has a value of $13 \%$. However, in the two-factor case the value is $18 \%$ and is significant at the $0.2 \%$ level of significance. These results are similar to the results of Terleckyj (1980) and support the view of Nadiri and Wolff that the estimated rates of returns are lower with three-factor TFP than with two-factor TFP growth. The change from a two-factor to a three-factor TFP index has a remarkable negative influence on the results. A possible explanation is that the assumptions underlying the TFP grow th calculations are much more harmful in the three-factor case. The assumption of constant returns comes to mind.

Table 7.16 Regression of three-factor Total Factor Productivity growth rate (adjusted for utilisation rate) on internal $R \& D$ and spillovers, $N=30$, (t-ratio's in parentheses)

\begin{tabular}{|c|c|c|c|c|c|c|}
\hline & CONST & "OWN" R\&D & RENTKYY & RENTM_Y & KNOW_Y & $\begin{array}{l}\vec{R}^{2} \\
D W\end{array}$ \\
\hline 16.1 & $\begin{array}{l}0.005^{\circ} \\
(2.07)\end{array}$ & $\begin{array}{c}0.20 R_{-} Y \\
(1.52)\end{array}$ & & & & $\begin{array}{l}0.04 \\
2.15\end{array}$ \\
\hline 16.2 & $\begin{array}{l}0.004^{2} \\
(1.73)\end{array}$ & $\begin{array}{c}0.13^{\circ} R_{-} Y \\
(1.96)\end{array}$ & & & & $\begin{array}{l}0.09 \\
2.11\end{array}$ \\
\hline 163 & $\begin{array}{l}0.04 \\
(1.67)\end{array}$ & $\begin{array}{c}0.03 R_{-} Y \\
(0.18) \\
0.22 R_{-} Y \\
(1.33)\end{array}$ & & & & $\begin{array}{l}0.09 \\
2.11\end{array}$ \\
\hline 16.4 & $\begin{array}{l}-0.005^{\circ} \\
(-2.31)\end{array}$ & $\begin{array}{c}0.08 R_{-} \mathrm{Y} \\
(1.64)\end{array}$ & $\begin{array}{l}8.7)^{\cdots} \\
(6.89)\end{array}$ & $\begin{array}{l}1.29^{*} \\
(2.55)\end{array}$ & & $\begin{array}{l}0.67 \\
1.92\end{array}$ \\
\hline 16.5 & $\begin{array}{l}0.004^{2} \\
(1.74)\end{array}$ & $\begin{array}{c}0.01 \mathrm{R}_{-} \mathrm{Y} \\
(0.15)\end{array}$ & & & $\begin{array}{l}0.26^{\circ} \\
(2.12)\end{array}$ & $\begin{array}{l}0.19 \\
2.04\end{array}$ \\
\hline 16.6 & $\begin{array}{r}-0.0044^{*} \\
(-1.74) \\
\end{array}$ & $\begin{array}{c}0.07 \mathrm{R}_{-} \mathrm{Y} \\
(1.19) \\
\end{array}$ & $\begin{array}{l}8.43^{\cdots} \\
(6.42) \\
\end{array}$ & $\begin{array}{c}0.88 \\
(1.24) \\
\end{array}$ & $\begin{array}{l}0.09 \\
(0.85)\end{array}$ & $\begin{array}{l}0.67 \\
1.80\end{array}$ \\
\hline
\end{tabular}

" "." Significant at the 0.10 level, 0.01 level and 0.002 level (two-tailed test), respectively.

Before we investigate the assumption of constant returns, we should highlight that regressions 16.4-16.6 indicate that spillovers, and especially rent spillovers of capital goods, remain an important determinant of TFP growth. The value and statistical significance of the rate of return to rent spillovers of capital goods is two times larger than in the case with a two-factor TFP and the value of the coefficient to rent spillovers of intermediate goods is five times higher and slightly more statistically significant (compare with regressions in table 7.9). The higher value of rentspillovers in a three-factor TFP was also found by Terleckyj (1980) but in his study the significance declined while in our estimates the significance has increased. 
Table 7.17 Regressions of capital productivity growth rate (actual production/capital) and labour productivity growth rate (actual production/labour") on factor ratios, internal $R \& D$ and spillovers, $N=30$. (i-ratio"s in parentheses)

\begin{tabular}{|c|c|c|c|c|c|c|c|c|c|}
\hline $\begin{array}{c}\mathrm{Y} / \mathrm{K} \\
\mathrm{gr} .\end{array}$ & CON & $\begin{array}{c}\mathrm{L} / \mathrm{K} \\
\mathrm{gr} .\end{array}$ & $\begin{array}{c}M / K \\
g r . \\
\end{array}$ & $\begin{array}{l}\mathrm{K} \\
\mathrm{gr}\end{array}$ & $\begin{array}{l}\text { "Own" } \\
\text { R\&D* }\end{array}$ & RENTK & RENTM & KNOW & $\begin{array}{l}\bar{R}^{2} \\
\text { DW }\end{array}$ \\
\hline $17.1^{4}$ & $\begin{array}{c}0.03 \\
(0.95)\end{array}$ & $\begin{array}{l}0.23^{*} \\
(3.65)\end{array}$ & $\begin{array}{c}0.56^{4 .} \\
(12.44)\end{array}$ & $\begin{array}{l}-0.24 \\
(-4.25)\end{array}$ & $\begin{array}{c}0.14 \mathrm{RC} \\
(1.60)\end{array}$ & $\begin{array}{l}7.95^{\circ} \\
(9.16)\end{array}$ & $\begin{array}{l}0.95^{+} \\
(2.31)\end{array}$ & $\begin{array}{l}0.21^{\ldots+} \\
(3.62)\end{array}$ & $\begin{array}{l}0.91 \\
1.84\end{array}$ \\
\hline $17.2^{4}$ & $\begin{array}{c}0.01 \\
(0.19)\end{array}$ & $\begin{array}{l}0.38^{* *} \\
(5.62)\end{array}$ & $\begin{array}{l}0.60^{n \times} \\
(10.92)\end{array}$ & & $\begin{array}{c}0.02 \mathrm{RC} \\
(0.23)\end{array}$ & $\begin{array}{l}8.24^{40} \\
(7.90)\end{array}$ & $\begin{array}{l}0.90^{\circ} \\
(1.86)\end{array}$ & $\begin{array}{l}0.21^{\circ} \\
(3.19)\end{array}$ & $\begin{array}{l}0.86 \\
1.74\end{array}$ \\
\hline $17.3^{4}$ & $\begin{array}{c}0.02 \\
(0.97)\end{array}$ & $\begin{array}{l}0.19^{* e x} \\
(3.92)\end{array}$ & $\begin{array}{l}0.55^{\circ \cdots} \\
(14.96)\end{array}$ & $\begin{array}{l}-0.29^{* *} \\
(-6.19)\end{array}$ & $\begin{array}{c}0.15^{6 *} \mathrm{R} \\
(3.96)\end{array}$ & $\begin{array}{l}8.09^{\circ+} \\
(11.19)\end{array}$ & $\begin{array}{l}0.92^{*} \\
(2.67)\end{array}$ & $\begin{array}{l}0.16^{*} \\
(3.19)\end{array}$ & $\begin{array}{l}0.94 \\
1.91\end{array}$ \\
\hline $17.4^{\text {\# }}$ & $\begin{array}{c}0.00 \\
(0.10)\end{array}$ & $\begin{array}{l}0.38^{4+4} \\
(6.24)\end{array}$ & $\begin{array}{c}0.59^{\text {na* }} \\
(11.17)\end{array}$ & & $\begin{array}{l}0.05 \mathrm{R} \\
(0.98)\end{array}$ & $\begin{array}{l}8.26^{*} \\
(7.98)\end{array}$ & $\begin{array}{l}0.87^{*} \\
(1.84)\end{array}$ & $\begin{array}{l}0.200^{*} \\
(2.95)\end{array}$ & $\begin{array}{l}0.87 \\
1.82\end{array}$ \\
\hline $\begin{array}{l}Y / L \\
\mathrm{gr} .\end{array}$ & CON & $\begin{array}{l}\mathrm{K} / \mathrm{L} \\
\mathrm{gr} .\end{array}$ & $\begin{array}{l}\mathrm{M} / \mathrm{L} \\
\mathrm{gr} .\end{array}$ & $\begin{array}{l}\mathrm{L} \\
\mathrm{gr} .\end{array}$ & $\begin{array}{l}\text { "OWn" } \\
\text { R\&D }\end{array}$ & RENTK & RENTM & KNOW & $\begin{array}{l}\vec{R}^{2} \\
\text { DW }\end{array}$ \\
\hline 17.5 & $\begin{array}{c}-0.00 \\
(-0.63)\end{array}$ & $\begin{array}{c}0.09 \\
(0.94)\end{array}$ & $\begin{array}{l}0.48^{0+x} \\
(9.47)\end{array}$ & $\begin{array}{l}-0.28^{\operatorname{sx}} \\
(-4.22)\end{array}$ & $\begin{array}{c}0.07 \mathrm{RC} \\
(0.78)\end{array}$ & $\begin{array}{l}8.47^{+6 x} \\
(7.89)\end{array}$ & $\begin{array}{c}1.12^{\circ} \\
(1.95)\end{array}$ & $\begin{array}{l}0.16^{\circ} \\
(1.82)\end{array}$ & $\begin{array}{l}0.92 \\
1.83\end{array}$ \\
\hline 17.6 & $\begin{array}{c}-0.00 \\
(-0.45)\end{array}$ & $\begin{array}{c}0.04 \\
(0.48)\end{array}$ & $\begin{array}{c}0.49^{4 *} \\
(10.95)\end{array}$ & $\begin{array}{l}-0.31^{+4} \\
(-5.32)\end{array}$ & $\begin{array}{l}0.12 \mathrm{R}^{*} \\
(2.75)\end{array}$ & $\begin{array}{l}8.77^{\text {nax }} \\
(9.28)\end{array}$ & $\begin{array}{l}0.96^{\circ} \\
(1.91)\end{array}$ & $\begin{array}{c}0.11 \\
(1.36)\end{array}$ & $\begin{array}{l}0.94 \\
1.89\end{array}$ \\
\hline
\end{tabular}

;"," "' Significant at the 0.10 level, 0.01 level and 0.002 level (two-tailed test), respectively.

All intensities are expressed in terms of actual production, "Regressions adjusted for auto-correlation

We will now test the constant returns to scale assumption underlying the TFP growth calculations. Regressions 17.2 and 17.4 imply constant returns to scale and regressions 17.1 and 17.3 allow for increasing or decreasing returns to scale. As in the case with two production factors, the production process is characterised by decreasing returns to scale because the coefficient of capital growth in regression 17.1 and 17.3 is negative. The value of this coefficient is large, 0.24-0.29, and statistically significant at the $0.2 \%$ level. A comparison of the first four regressions shows that the assumption of constant returns to scale, when there are in fact decreasing returns to scale, lowers the statistical significance of all the rates of returns to the various kinds of $R \& D$. The values of the rates of returns to own R\&D and rent spillovers of intermediate goods are also lower with constant returns to scale, however, the rates of return to capital rent spillovers or "pure" knowledge spillovers are higher. We compare the results of the two-factor equation which allows for non-constant returns to scale (regression 11.1 and 11.3) and the corresponding three-factor equations (regression 17.3 and 17.6). The rates of return to own R\&D and the two kinds of rent spillovers are higher and more significant in the three-factor case. The value of the coefficient of rent spillovers in intermediate goods is ten times higher in the three-factor case and significant in comparison 
with the two-factor case. An exception is the value of the predicted rate of return to "pure" knowledge spillovers which is lower in the three-factor regressions.

We can therefore conclude that the lower and insignificant results with a threefactor TFP in comparison with a two-factor TFP are due to the constant returns to scale assumption; which has more harmful implications in the three-factor TFP case. Releasing the constant returns to scale conditions turns things around: the rates of returns and their significance are higher when three-factors are included than when two-factors are included.

Table 7.18 Regression of capital productivity growth rate (actual production/capital) on factor inputs. internal R\&D and spillowers, $N=30$, (t-ratio's in parentheses)

\begin{tabular}{|c|c|c|c|c|c|c|c|c|c|}
\hline $\begin{array}{l}\mathrm{Y} / \mathrm{K} \\
\mathrm{gr} .\end{array}$ & $\mathrm{CON}$ & $\begin{array}{l}\mathrm{L} / \mathrm{K} \\
\mathrm{gr} .\end{array}$ & $\begin{array}{c}\mathrm{M} / \mathrm{K} \\
\mathrm{gr} .\end{array}$ & $\begin{array}{l}\mathrm{K} \\
\mathrm{gr} .\end{array}$ & $\begin{array}{c}\text { "OWN" } \\
\text { R\&D }\end{array}$ & RENTK & RENTM & KNOW & $\begin{array}{l}R^{2} \\
\bar{R}^{2} \\
\end{array}$ \\
\hline 18.1 & $\begin{array}{c}0.01 \\
(1.34)\end{array}$ & $\begin{array}{c}0.19 \\
(1.17)\end{array}$ & $\begin{array}{l}0.53^{* \cdots} \\
(4.74)\end{array}$ & $\begin{array}{c}-0.24 \\
(-1.70)\end{array}$ & $\begin{array}{c}0.34^{\circ} R C \\
(1.90)\end{array}$ & & & & $\begin{array}{l}0.55 \\
0.48\end{array}$ \\
\hline 18.2 & $\begin{array}{l}0.01 \\
(1.55)\end{array}$ & $\begin{array}{c}0.2 \\
(1.39)\end{array}$ & $\begin{array}{l}0.53^{\prime \prime \prime} \\
(5.17)\end{array}$ & $\begin{array}{l}-0.27 \\
(-2.08)\end{array}$ & $\begin{array}{c}0.23^{-\mathrm{R}} \\
(2.91)\end{array}$ & & & & $\begin{array}{l}0.62 \\
0.55\end{array}$ \\
\hline 18.3 & $\begin{array}{c}0.01 \\
(1.09)\end{array}$ & $\begin{array}{c}0.15 \\
(0.98)\end{array}$ & $\begin{array}{l}0.52 \cdots \\
(5.110)\end{array}$ & $\begin{array}{c}-0.26^{\circ} \\
(-1.98)\end{array}$ & $\begin{array}{c}0.05 \mathrm{RC} \\
(0.23) \\
0.37^{\circ} \mathrm{RD} \\
(2.27)\end{array}$ & & & & $\begin{array}{l}0.63 \\
0.55\end{array}$ \\
\hline 18.4 & $\begin{array}{c}-0.00 \\
(-0.01)\end{array}$ & $\begin{array}{l}0.14^{\circ} \\
(2.58)\end{array}$ & $\begin{array}{c}0.52 \% \\
(12.79)\end{array}$ & $\begin{array}{l}-0.32^{m * *} \\
(6.30)\end{array}$ & $\begin{array}{c}0.17^{m+10} \mathrm{R} \\
(4.49)\end{array}$ & $\begin{array}{c}8.80^{* m+} \\
(100.93)\end{array}$ & $\begin{array}{l}1.49^{\prime \prime \prime} \\
(4.66)\end{array}$ & & \\
\hline 18.5 & $\begin{array}{l}0.011^{\circ} \\
(1.75)\end{array}$ & $\begin{array}{l}0.22^{\circ} \\
(1.73)\end{array}$ & $\begin{array}{l}0.52^{*} \\
(5.71)\end{array}$ & $\begin{array}{l}-0.25^{\circ} \\
(-2.23)\end{array}$ & $\begin{array}{l}0.10 \mathrm{R} \\
(1.10)\end{array}$ & & & $\begin{array}{c}0.30^{\circ} \\
(2.72)\end{array}$ & $\begin{array}{l}0.71 \\
0.65\end{array}$ \\
\hline
\end{tabular}

"," '” Significant at the 0.10 level, 0.01 level and 0.002 level (two-tailed test), respectively.

We investigate the marginal or incremental contribution of the various spillover variables using regressions 17.3, 18.2, 18.4 and 18.5. Adding both types of rent spillovers (regression 18.4) to regression 18.2 raises the $\bar{R}^{2}$ from 0.55 to 0.93 . The value of the $\mathrm{F}$ test is $\mathrm{F}_{2,23}=65.56$ which is significant at the $1 \%$ level of significance, and implies that rent spillovers should be added to the model. Also, adding "pure" knowledge spillovers (regression 17.3) delivers a $F$ value of $F_{1,22}=4.26$, which is significant at the 10\% level of significance. Therefore it is worthwhile to add "pure" knowledge spillovers even when rent spillovers are already present. Reversing this procedure, first adding "pure" knowledge spillovers and then rent spillovers, yields $F$ values of $F_{1,24}=7.42$ and $F_{2,22}=56.21$ which are both significant at the $5 \%$ level and therefore imply that both kinds of spillovers should be included. 
Table 7.19 shows the predicted parameter ranges for the various R\&D concepts. The estimated rates of returns significantly differ between the various concepts. However, a high rate of return says nothing about the importance of a $\mathbb{R} \& D$ or spillover category since a high rate of return to a spillover that is very small still has a low influence on the productivity growth of a sector. The importance of a certain $R \& D$ concept for sectoral productivity growth depends on the productivity (rate of return) and quantity of this $R \& D$ concept (the estimated rate of return times the sample mean). This measure is known as the quasi-elasticity. The implied quasi-elasticities are also depicted in table 7.19. A striking resemblance exists between the predicted ranges. The quasi-elasticities of own R\&D, rent spillovers of intermediate goods and "pure" knowledge spillovers range from 0.11 to 0.44 . The influence of rent spillovers of capital goods is larger and ranges from 0.64 to 0.7 .

Tablle 7.19 Estimated ranges for the various rates of return and the corresponding quasinelasticities

\begin{tabular}{|c|c|c|}
\hline Variable & $\begin{array}{l}\text { Prediched Range: } \\
\text { Rate of Remun }\end{array}$ & $\begin{array}{l}\text { Predicted Range: } \\
\text { Quasi-Ebasticity }\end{array}$ \\
\hline 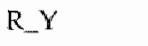 & $12 \%-23 \%$ & $0.23-0.44$ \\
\hline $\mathrm{RC}_{-} \mathrm{Y}$ & $34 \%$ & 0.30 \\
\hline $\mathbb{R D}_{-} \gamma$ & $37 \%$ & 0.38 \\
\hline RENTK_Y & $754 \%-880 \%$ & $0.64-0.70$ \\
\hline RENTM_Y & $90 \%-149 \%$ & $0.25-0.42$ \\
\hline KNOW_Y & $16 \%-30 \%$ & $0.16-0.29$ \\
\hline
\end{tabular}

'Only significant walues are included.

2 Quasi-elasticities are calculated as rate of returns (is equal to marginal productivity) times average value of the related spillover intensity. The average values of the spillover intensities are depicted in table 7.15 .

\subsection{The Role of Technology in Different Groups of Sectors: High-tech, Services and Other Sectors.}

In this section we investigate whether the influence of internal R\&D, rent spillovers and "pure" knowledge spillovers differs between certain groups of sectors. The first group of sectors we distinguish are the "High-tech Sectors", in which it can be expected that internal $R \& D$ plays an important role with regard to cost-reduction and demand creation. The second group are the "Service Sectors" which do not perform a lot of R\&D themselves but rely on technology generated in other sectors. The third group, called the "Other Sectors" are all the sectors which do not belong 
to the first two groups. This group contains mainly medium- and low-tech sectors. ${ }^{168}$

Table 7.20 Mean walues of key wariables, period $1981-1992$ (four-year moving average)

\begin{tabular}{lllll}
\hline \hline All Sectors & $\begin{array}{c}\text { High } \\
\text { Tech }\end{array}$ & Services & $\begin{array}{c}\text { Other } \\
\text { Sectors }\end{array}$ \\
\hline TFP_Y & $0.74 \%$ & $1.06 \%$ & $0.5 \% \%$ & $0.58 \%$ \\
Y & $2.74 \%$ & $3.48 \%$ & $4.02 \%$ & $1.03 \%$ \\
R_Y & $1.92 \%$ & $5.4 \%$ & $0.05 \%$ & $0.34 \%$ \\
RENTM_Y & $0.28 \%$ & $0.53 \%$ & $0.08 \%$ & $0.21 \%$ \\
RENTK_Y & $0.08 \%$ & $0.06 \%$ & $0.13 \%$ & $0.05 \%$ \\
KNOW_Y & $0.99 \%$ & $2.32 \%$ & $0.38 \%$ & $0.28 \%$ \\
LAB & $-0.47 \%$ & $-0.90 \%$ & $1.67 \%$ & $-1.8 \%$ \\
CAP & $3.94 \%$ & $4.77 \%$ & $4.47 \%$ & $2.76 \%$ \\
INTD & $3.14 \%$ & $3.60 \%$ & $5.1 \%$ & $1.12 \%$ \\
PP & $-0.42 \%$ & $-0.98 \%$ & $0.25 \%$ & $-0.46 \%$ \\
\hline
\end{tabular}

A sector is considered to be a high-tech sector when the R\&D expenditures are larger than $1 \%$ of actual production.

In this sub-section we use pooled data instead of pure cross-sectional data. We calculated a four-year moving average to avoid the influence of business cycles. This reduces the period from 1978-1992 to 1981-1992, we are left with twelve observations for each sector which implies 120,108 and 132 observations for the high-tech, services and other sectors, respectively.

The means of the important variables for the various groups of sectors are given in table 7.20. This table shows that the three groups are characterised by different growth performance and technological behaviour. We will be brief in our description of these features for the three sectoral groups because we already discussed both kinds of behaviour for each sector individually in section 7.1. Let us first consider the growth performance. The three-factor TFP growth rate in the high-tech sectors is two times as high as in the other two sectors. However, the growth rate of actual production, $Y$, is highest in the service sectors, still high in the high-tech sectors and relatively low in the other sectors. As a result, the growth

\footnotetext{
108 Diwision of the thirty sectors across the three groups (a description of the abbreviations of the sectors is given in appendix $7 \mathrm{~A}$ ):

Th (High Tech sechors): CHES, FAR, MAC, ITI, ELMS, IT2, IT3, TRA, INS, RUB

$S$ (Service seciors): COM, REP, HOT, TRS, TEL, OMS, SME, FIN, NMS

O (Other sectors): BUI, AGR, ENG, MET, NMM, CHTS, FAM, FOO, TEX, OTH, PAP
} 
rate of labour (LAB) is positive in the service sectors $(1,67 \%)$, negative in the hightech $(-0.9 \%)$ and strongly negative in the other sectors $(-1.8 \%)$. Prices increase in the service sectors but decrease in the other sectors and even strongly decrease in the high-tech sectors.

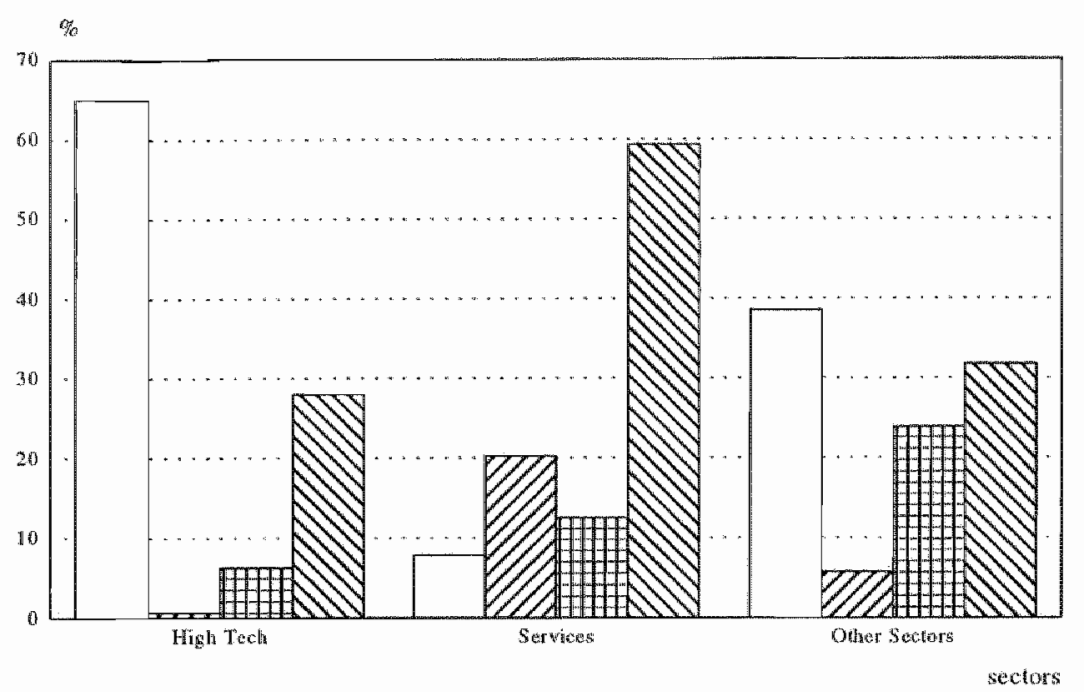

$\square$ Internal R\&D DRENTK BRENTM DKNOW

Figure 7.9. Relative importance of various R\&D sources

We now turn to the technological behaviour of the three sectors. The total R\&D intensity (internal R\&D and all types of spillovers) is very different across sectors. This intensity is $8.31 \%$ in the high-tech sectors, which is ten times higher than that in the other sectors and services which are $0.78 \%$ and $0.64 \%$, respectively. The relative importance of the various sources of knowledge for each sector is given in figure 7.9. The contribution of internal $R \& D$ to the total amount of knowledge in a sector is $59 \%$ in the high-tech sector, $30 \%$ in the other sectors and only $8 \%$ in the service sector. The high-tech sectors generate the largest part of their knowledge themselves but the other sectors, and especially the service sectors, are dependent on the knowledge which is generated in other sectors. Rent spillovers embodied in capital goods are both relatively (figure 7.9) and absolutely (table 7.20) the most important factor in the services sectors. Rent spillovers embodied in intermediate goods are relatively most important in the other sectors but in an absolute sense are more important in the high-tech sectors. "Pure" knowledge spillovers are in an absolute sense the most important contributor in the high-tech sectors $(2.32 \%$ versus $0.38 \%$ and $0.28 \%$ in the services and other sectors, respectively). 
Table 7.21 Regression of the capital productivity growth rate (actual production/capital), results for High-tech, Services and Other Sectors, (i-ratio's in parentheses)

\begin{tabular}{|c|c|c|c|c|c|c|c|c|c|}
\hline $\begin{array}{l}Y / K \\
g r .\end{array}$ & CON & $\begin{array}{c}\mathrm{L} / \mathrm{K} \\
\mathrm{g} g\end{array}$ & $\begin{array}{c}M / K \\
g r .\end{array}$ & $\begin{array}{l}\mathrm{K} \\
\mathrm{gr} .\end{array}$ & $\begin{array}{l}\text { "own" } \\
\text { R\&D }\end{array}$ & RENTK & RENTM & KNOW & $\begin{array}{l}R^{2} \\
N\end{array}$ \\
\hline 21.1 & 0.01 & $0.15^{+\infty}$ & $0.52^{+* x}$ & $-0.33^{* a}$ & $0.21^{+*}$ & $0.64^{\cdots}$ & $0.23^{*-}$ & $0.15^{*}$ & 0.89 \\
\hline All. & $(0.05)$ & $(7.32)$ & $(36.67)$ & $(-15.67)$ & $(5.25)$ & $(17.91)$ & $(3,37)$ & $(4.24)$ & 360 \\
\hline 21.2 & .0 .05 & 0.04 & $0.68^{* 0}$ & $-0.25^{\circ \cdots}$ & $0.55^{\circ}$ & -0.15 & 0.18 & $0.44^{4+\infty}$ & 0.81 \\
\hline Tech. & $(-0.14)$ & $(0.68)$ & $(9.47)$ & $(-4.65)$ & $(2.66)$ & $(-0.58)$ & $(0.82)$ & $(3.30)$ & 120 \\
\hline 21.3 & 0.42 & $0.12^{\circ}$ & $0.48^{* *}$ & $-0.45^{\cdots}$ & -0.09 & $0.97^{* m}$ & -0.06 & $0.38^{\circ}$ & 0.95 \\
\hline Ser. & (1.81) & $(2.56)$ & $(33.06)$ & $(-9.08)$ & $(-0.89)$ & $(8.89)$ & $(-0.44)$ & $(2.34)$ & 108 \\
\hline 21.4 & -0.01 & $0.12^{* *}$ & $0.66^{\ldots *}$ & $-0.19^{*}$ & -0.04 & 0.27 & $0.19^{\circ}$ & & 0.94 \\
\hline Oth. & $(-0.04)$ & $(3.71)$ & $(25.61)$ & $(-5.08)$ & $(-0.32)$ & (1.51) & (1.83) & & 132 \\
\hline
\end{tabular}

" and "Significant at the 0.10 level, 0.01 level and 0.002 level (two-tailed test), respectively.

In this section we investigate the importance of the various spillover concepts for the 3 groups of sectors. The height of the rate of return of a spillover variable does not say much about the influence of this variable on sectoral productivity growth, because the magnitude of this spillover variable can be low. Therefore we will normalize the various $R \& D$ intensities by dividing the observations by their sample means. Instead of estimating the rates of return, we estimate the rates of return times their mean value which is equal to the "quasi-elasticity". This concept indicates the influence of a spillover variable with respect to productivity growth. We now illustrate this for the internal $R \& D$ variable. ${ }^{109}$

$$
\rho_{r c} \cdot \frac{R C}{Y}=p_{r c}^{\prime \prime} \cdot \frac{\frac{R C}{\gamma}}{\frac{R C}{Y}} \Rightarrow \rho_{r c}^{\prime \prime}=p_{r c} \cdot \frac{\overline{R C}}{\gamma}=q e_{r c}
$$

Instead of the rate of return, $\rho_{v}$, we estimate $\rho_{r}$ " which is equal to $\rho_{r}$ times $\bar{R} / Y$ (sample mean of the $R \& D$ intensity) and therefore a "quasi-elasticity". In this section we estimate quasi-elasticities so that we directly can compare the influence of the various spillovers concepts for the 3 sectors.

However, for those who are interested in the estimated values of the rates of returns they can easily obtain these values by dividing the quasi-elasticities by the sample means which are given in table 7.20. The estimation results of equation (7.14) are shown in table 7.21. The quasi-elasticity for "own" R\&D for all sectors

109 Remember that $\rho_{\mathrm{r}}=\frac{\partial Y}{\partial R}$ 
(regression 21.1) is equal to 0.21 and the sample mean for this variable is $1.92 \%$ (see table 7.20 ), the rate of return for all sectors is therefore $0.21 / 1.92=0.109$ or $10.9 \%$.

Now we turn to the discussion of the estimation results. The estimation results for the total economy are given in regression 21.1. Production is characterised by decreasing returns to scale (a negative value of the coefficient for the capital growth rate). This is a typical result of studies that apply panel data to estimate production functions (see e.g. Cunéo and Mairesse 1984, Griliches and Mairesse 1984, 1990). A problem with our findings is that the implied value for the capital elasticity is zero. This result is consistent with the general experience when the estimated equations are in growth rates. "The usual explanation is simple: The quality of our capitall measures is to poor to identify annual variations in capital services" (Klette and Griliches 1992 , p. 31).

The values of the estimated elasticities of the various $R \& D$ concepts based on this pooled database are comparable to the estimates based on pure cross-section data (see table 7.19). The value of the quasi-elasticity of own R\&D is equal to 0.21 and statistically significant at the 0.002 level of significance. This value is somewhat lower than the 0.23 to 0.44 range specified in table 7.19 but it is within the range of 0.05 to 0.25 given by Mairesse and Mohnen (1994) in an extensive review of the literature. The estimated value of the quasi-elasticity of the rent spillovers in capital groods is equal to 0.64 and therefore fits into the $0.64-0.70$ range predicted in table 7.19. R\&D embodied in capital goods again has a large influence on the productivity level of other sectors. The estimated quasi-elasticities of rent spillovers embodied in intermediate goods and "pure" knowledge spillovers both have a positive value, 0.23 and 0.15 respectively, and are both statistically significant. The rate of return to intermediate rent spillovers are both somewhat lower than the predicted ranges in section $7.3 .2(0.25-0.42$ and $0.16-0.29$ respectively). These results confirm the general rule that the time dimension generates lower estimates than the cross-section dimension. Again we can conclude that all three kinds of spillover effects are important for the productivity growth of sectors.

However, the main interest in this sub-section is whether the influence of own R\&D and the various spillover concepts differs between different groups of sectors. In the high-tech sector the estimated influence of "own" R\&D and "pure" knowledge spillovers is definitely important. This is consistent with our model because we expected that knowledge spillovers are particularly relevant when a sector performs R\&D itself and it can use results of other sectors. The quasielasticity of internal R\&D is 0.55 in the high-tech sectors and is therefore two times as thigh as in the case where we included all sectors.

In the service sectors, rent spillovers embodied in capital goods are important. The estimated quasi-elasticity is 0.97 and very significant. We can also look at this case in another way, since capital rent spillovers add $0.97 \%$ to the labour productivity growth of $2.35 \%$ in services which is $41 \%$ of the original growth rate. This large influence of rent spillovers of capital goods in services may be caused by the use of computers or other information technology investment goods in the service sectors. We will investigate this hypothesis in chapter 8 . The positive influence of knowledge spillovers was not expected since service sectors perform 
almost no R\&D. This result is primarily dependent on the "knowledge" spillovers from the pharmaceuticals to the health sectors. However, these spillovers may be considered as rent spillovers and therefore show that the Yalle matrix does not only measure "pure" knowledge spillovers, but also in part rent spillovers. In addition, the estimated quasi-elasticity for "pure" knowledge spillovers is not significant in the service sectors when we use gross value added instead of actual production.

In the "other sectors", it is impossible to distinguish between "pure" knowledge spillovers and rent spillovers of intermediate goods because the simple correlation coefficient is 0.96. We therefore dropped the "pure" knowledge spillover variable in the "other sectors" because it turned out that rent spillovers embodied in intermediate goods yielded a higher explanatory power.

We can conclude that own R\&D and "pure" knowledge spillovers are important in high-tech sectors; spillovers embodied in (information technology) capital goods are important in the service sectors; and spillovers embodied in intermediate goods are important in the other sectors.

\subsection{Conclusion}

In this chapter we measured the influence of internal R\&D and various spillover concepts on productivity and demand using a database for France covering the 1978-1992 period. Similar to other empirical studies, which are based on the 1970 s and early 1980s, we found a statistically significant relationship between the $R \& D$ intensities of a sector and its productivity growth rate. The most important contribution of this empirical. chapter to the existing literature on this field is the treatment of spillover effects.

First, we developed a model that integrated internal R\&D efforts, "pure" rent spillovers, and knowledge spillovers that are related and not related to input purchases, into one framework. We included the possibility that quality improvements are partly reflected in price indices which results in the existence of "pure" rent spillovers or measurement errors.

The empirical implementation of this model generated three spillovers concepts that were expected to influence technological change in a sector. First, "pure" rent spillovers and knowledge spillovers that are related to investment purchases. Second, "pure" rent spillovers and knowledge spillovers that are related to intermediate purchases and third, "pure" knowledge spillovers that are not related to input purchases. Our estimations showed that the coefficients of all the three concepts were positive and statistically significant. This indicates that it is worthwhile to distinguish between the different spillover concepts and that each concept has a measurable influence on technological progress at the sectoral level.

The implied quasi-elasticities based on actual production ranged from 0.23-0.44 for own or internal R\&D. The elasticity for rent spillovers embodied in intermediate goods ranged from 0.25 to 0.42 and the elasticity for "pure" knowledge spillovers ranged from 0.16 to 0.29 . The highest and most significant quasi-elasticities were obtained for rent spillovers in investment goods; these ranged from 0.64 to 0.70 . 
Most previous studies included only one spillover concept, i.e. mostly spillovers embodied in intermediate goods (measured with input-output coefficients). These studies clearly fail to measure the important investment spillover effects and "pure" knowledge spillover effects.

The inclusion of the various spillover effects becomes especially important when one investigates spillover effects for different groups of sectors, since it may be expected that the influence of the various spillover effects differs between sectors (different groups of sectors obtain their knowledge via different channels). Our estimation results supported these expectations. For the high-tech sectors own R\&D in combination with "pure" knowledge spillovers are very important. Services rely on rent spillovers embodied in capital goods while other sectors mainly rely on rent spillovers of intermediate goods.

With regard to the influence of process-oriented and product-oriented $R \& D$ we also obtained some interesting results. In contradiction with most earlier studies we found a positive influence of product R\&D on a sectors productivity growth. This indicates that product R\&D directed at improving products is also directed at making new products with less production factors or that quality improvements are only partly reflected in price indices. If we compare our results with those of Scherer (1982) and Griliches and Lichtenberg (1994), there exists a clear indication exists that product $R \& D$ is getting more important over time. With respect to demand creation, we found the expected influence of product $R \& D$, while process $R \& D$ turned out to be insignificant. Using total $R \& D$ instead of product $R \& D$ detoriated the explanatory power with respect to demand creation.

TFP growth estimations which assume constant returns, had a negative influence on height and significance of predicted rates of returns in our sample (that was characterised by decreasing returns to scale). The constant returns to scale assumption turned out to be more harmful in the three-factor TFP case than in the two-factor TFP case. This implied that regressions based on actual production, which include intermediate inputs, generally yield in general higher and more significant estimates of the rates of returns than those which are based on gross value added if the assumption of constant returns is not included. The opposite is true when the estimates assume constant returns to scale.

In all estimations the coefficient of rent spillovers in capital goods is very high and highly significant. An interesting question in this context is whether it is information technology goods that cause this large influence of capital related spillovers on productivity growth. However, further empirical research is needed to discover if these positive effects are linked to IT capital goods. 


\section{Appendix 7A: Sector Classification and Description}

Table 7A.1: Sector Classification

\begin{tabular}{|c|c|c|c|}
\hline $\mathrm{SEC}$ & T-CLAS IMSEE & SUCLAS IINSEE] & NACEACLIO \\
\hline AGR & rot & $501-503$ & Not \\
\hline $\mathrm{ENC}$ & $\mathrm{TO4-06}$ & $504-508$ & No3-11 \\
\hline MET & T07 -08 & $509-513$ & $\mathrm{NH}_{3}$ \\
\hline MMUM & 709.10 & $514-16$ & $\mathbb{N} 15$ \\
\hline CHES & TI & $517-19,543$ & N17 - N257/N259 \\
\hline FAR & p.o. of T12 & $\operatorname{sig}$ & N257 \\
\hline CHTS & P.0. of T/2 & $\mathrm{S18}$ & N258-259 \\
\hline FAM & $\mathrm{T} 13$ & $S 20-21$ & N19 \\
\hline MAC & T14w534 & $\$ 22-25$ & $\mathrm{~N} 2 \mathrm{I}$ \\
\hline IT1 & p.o. TisA & $\$ 27$ & $N 330$ \\
\hline ELMM & $\begin{array}{l}\text { P.o T15B } \\
\text { p.o T15A }\end{array}$ & $\$ 28,530$ & N25 \\
\hline $\mathrm{TTZ}$ & p.o. T15A & 5291 & N344 \\
\hline 1735 & p.o. T15B & $\$ 292$ & N345 \\
\hline TRA & T16-17 & $526,531-33$ & $N 27-29$ \\
\hline INS & p.o. T.14 & $\mathrm{S34}$ & $N 371-74$ \\
\hline $\mathrm{FOO}$ & $\mathrm{T} 2-3$ & $S 35-42$ & N3:-39 \\
\hline $\operatorname{TEX}$ & T18-19 & $544-47$ & $N 41-43$ \\
\hline OTH & $\mathrm{T} 20$ & $548-49,554$ & $\mathrm{~N} 45, \mathrm{~N} 5 \mathrm{I}$ \\
\hline PAP & T21-22 & $550-5$ & $\mathrm{~N} 47$ \\
\hline RUB & $\mathrm{T} 23$ & $552-53$ & $\mathrm{~N} 49$ \\
\hline BUI & T24 & $\$ 55$ & N53 \\
\hline $\mathrm{COM}$ & $T 25 \times 28$ & $557-64$ & N5t \\
\hline REP & $\begin{array}{l}\text { p.o. T33, T29, } \\
\text { p.o. T34 }\end{array}$ & $\begin{array}{l}556, \$ 65 \\
\$ 66\end{array}$ & N55 \\
\hline HOT & 130 & 567 & N59 \\
\hline TRS & $\mathrm{T} 3 \mathrm{H}$ & $568-\$ 74$ & N6:-65 \\
\hline TEL & $\mathrm{T} 32$ & 575 & $N 67$ \\
\hline OMS & 734 w 566 & $584-87$ & $N 77-79$ \\
\hline SMË & T33 w 556, T35 & $576-83$ & $N 71-75$ \\
\hline $\mathrm{FIN}$ & $\mathrm{T} 36-37$ & $588-89$ & N69 \\
\hline NMS & $\mathrm{T} 38$ & $590-98$ & $\mathrm{NBH}+93$ \\
\hline
\end{tabular}




\section{Siector Description}

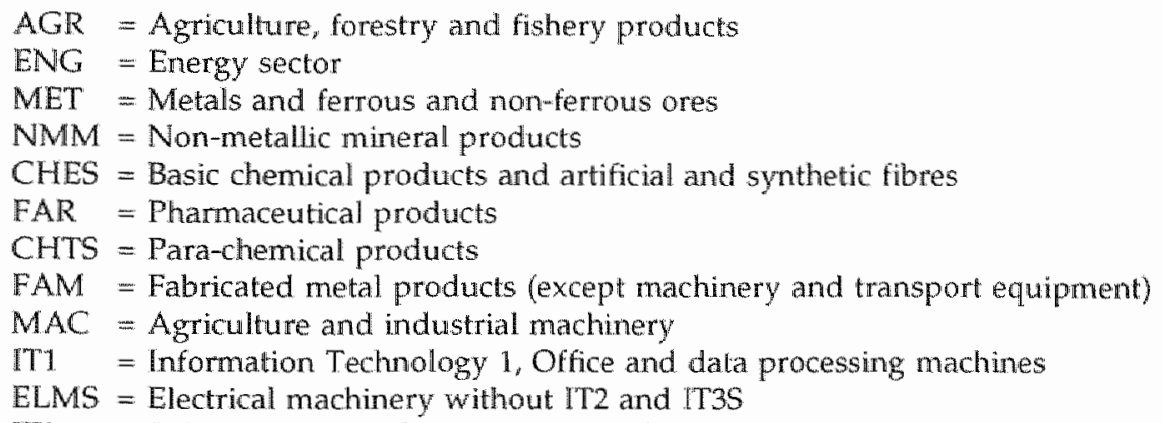

IT2 = Information Technology 2, Telecommunication equipment, meters and measuring equipment, electro medical equipment

IT3S = Information Technology 3 , electronic equipment, television receiving sets, sound reproducing and recording equipment

TRA = Transport industry

INS = Precision and optical instruments

FOO = Food, drink and tobacco products

TEX = Textile, footwear and leather products

$\mathrm{OTH}=$ Wood, cork and furniture and other manufacturing products

PAP = Paper and printing products

RUB = Rubber and plastic products

BUI = Building and construction

$\mathrm{COM}=$ Commercial sector (wholesalle and retail trade)

$\mathrm{REP}=$ Recovery and repair services

HOT $=$ Lodging and catering services

TRS = Transport services

TEL = Telecommunication services

OMS = Other market services (e.g, health, recreational)

SME = Market services for enterprises ( e.g. lawyers, real estate, accountants, tax advisers, management consultants, computer and data processing services and market services of education and research)

FIN = Services of credit and insurance institutions

NMS = Non-market services

\section{Appendix 7B: Construction Missing Investment Matrices 1989-1992}

First, we possess investment matrices in constant prices for 1977-1988 and second we possess investment demand (SEC2) and supply (INSEE) data in constant prices for the period 1977-1992. Construction method:

1) Calculate investment coefficients for 1977-1988.

2) Estimate the possibility of time trends in these investment coefficients. If the time trend is significant, then extrapolate the investment coefficients for the period 1989-1992.

3) Use these corrected investment coefficients as initial data in a RAS procedure in which the column totals are the investment demand data of SEC2 and the row totals are the investment supply data of INSEE. This RAS procedure calculates the investment deliveries of sector $i$ to sector $j$ which are comparable with the row and column totals. 



\section{Information Technology Spillovers: Empirical Evidence}

"We see computers everywhere except in the economic statistics", Robert Solow.

Chapter 7 investigated the existence and magnitude of the R\&D spillower effects in general. In this chapter we investigate the importance of spillover effects related to the current generall purpose technology: information technology. General purpose technologies are expected to generate important spillovers for the entire economy.

In this chapter we investigate the two principal hypotheses of this thesis. First, external knowledge sources become more important in the knowledge generation process. The value of spillover effects increases in this IT era. Second, the IT paradox is loosing its "paradoxical" characteristics.

Most empirical studies are based on data sets mainly covering the 1970 s and the early 1980s. Our database covers the period 1977-1992, allowing us to investigate both hypotheses. We investigate both hypotheses by splitting the different spillover: effects into IT and non-IT spillovers and by examining the importance of IT and non-IT spillovers over time.

Section 8.1 elaborates the empirical endogenous technological change model developed in chapter 7 with IT and non-IT rent and knowledge spillovers. Section 8.2 contains a short description of the data and section 8.3 investigates whether IT influences productivity growth. Section 8.4 investigates whether spillover effects are becoming more important in the IT era. Finally, we examine some other recent empirical studies on the relationship between $I \mathbb{I}^{\prime}$ and productivity.

\subsection{An Empirical Model of Technological Change with Spillovers related to IT- goods and non-IT goods.}

In chapter 7 we presented some empirical models based on the theoretical endogenous technological change models described in chapters 3 to 5 . In chapters 4 and 5 we assumed a special influence of information technology on technological change. The central question this chapter attempts to answer is whether or not the special influence does indeed exist. In this section we derive an empirical model that enablles us to test the influence of IT goods on technological change. 
The empirical model that tests the influence of IT goods is a straightforward elaboration of the empirical models developed in chapter 7 (section 7.11). The ITmodel differs from the models of chapter 7 because it separates the various spillover effects into IT-related spillover effects and non-IT related spillover effects. We extend the general TFP growth equation developed in sub-section 7.1.3.B (equation 7.32). A sector can use "pure" knowledge generated by IT (KNOW, ) and non-IT sectors (KNOW $W_{N,}$ ) and it receives rent spillovers from purchased IT

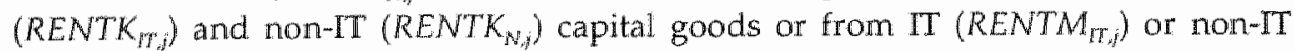
(RENTM $M_{M, j}$ ) intermediate goods.

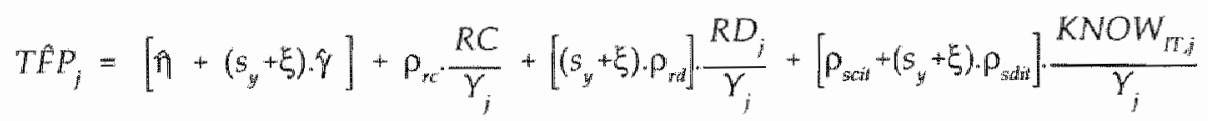

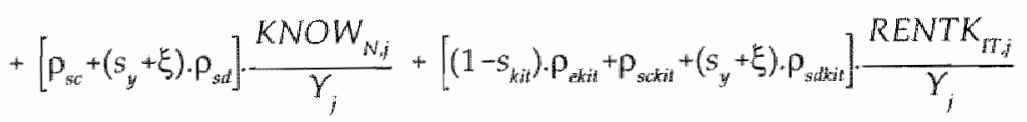

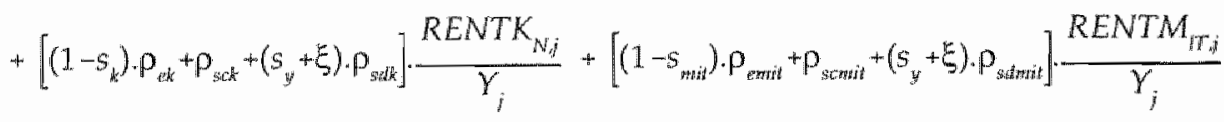

$$
\begin{aligned}
& +\left[\left(1-s_{m}\right) \cdot \rho_{e m}+\rho_{\mathrm{sin} m}+\left(s_{y}+\xi\right) \cdot \rho_{s i t m}\right] \cdot \frac{\operatorname{RENTM} N_{j}}{Y_{j}}
\end{aligned}
$$

We now discuss the determinants of technological progress in this model. The intercept $\left[\hat{\eta}+\left(s_{y}+\xi\right), \hat{\gamma}\right]$ measures the autonomous rate of technological change. Process R\&D directly contributes to the productivity level of a sector and this equation directly measures the rate of return to process $R \& D\left(\rho_{m}\right)$. Product $R \& D$ influences the measured productivity level if quality improvements are reflected in the price indices $(s, 00)^{110}$ or if an enhanced quality level directly increases or decreases the unit production costs $(\xi<0$ or $\xi>0)$. "Pure" knowledge generated by the IT producing sectors may enhance the productivity of own process $\mathbb{R} \& D\left(\rho_{\text {sait }}\right)$ or own product R\&D $\left(\left(s_{y}+\xi\right) \cdot \rho_{\text {sitit }}\right)$ and therefore TFP growth. "Pure" knowledge generated by non-IT producing sectors has a similar influence. Four other determinants of the total factor productivity growth are the IT and non-IT rent spillovers which are embodied in purchased intermediate and investment goods. These rent spillovers consist of "pure" rent spillovers and knowledge spillovers rellated to input purchases. "Pure" rent spillovers are "just" measurement errors because quality improvements are not reflected in the price indices. It is worth noting the difference between rent spillovers and "pure" rent spillovers. We illustrate the various spillovers that are related to good purchases for the rent spillover of IT capital goods concept $\left(R E N T K_{I T, j}\right)$. The term, $\left(1-s_{k i t}\right) \cdot p_{\text {edal }}+p_{\text {sckit }}+\left(s_{y}+\xi\right)$.

\footnotetext{
110 Where $s_{y}$ rellects the pirt of the quality or efficiency improvement of product $j$ that is reflected in the price indexes (see section $7.1 .3 . \mathrm{B}$ ).
} 
$\rho_{\text {sdkit }}$ reflects both "pure" rent spillovers, $\left(1-s_{k i}\right) \cdot \rho_{\text {ekit }}$ and knowledge spillovers embodied in goods, $p_{\text {sdai }}+\left(s_{y}+\xi\right) \rho_{\text {sitki }}$. If quality improvements of IT capital goods are fully reflected in the price indices, $s_{k j i}=1$, then there are no "pure rent spillovers or measurement errors. The knowledge spillovers related to input purchases have the same influence as "pure" (non-input-related) knowledge spillovers. They enhance the productivity of own process R\&D $\left(\rho_{s+d i}\right)$ or own product R\&D $\left.\left(s_{y}+\xi\right) \cdot p_{\text {sdkit }}\right)$

In chapter 7 we concluded that the constant returns to scale assumption underlying the TFP growth equations was rejected. In this chapter we will also estimate models that do not incorporate this assumption. Releasing the constant returns to scale assumption turns equation (8.1) into: 11

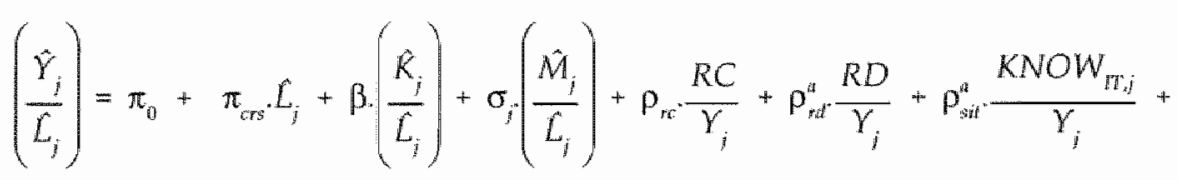

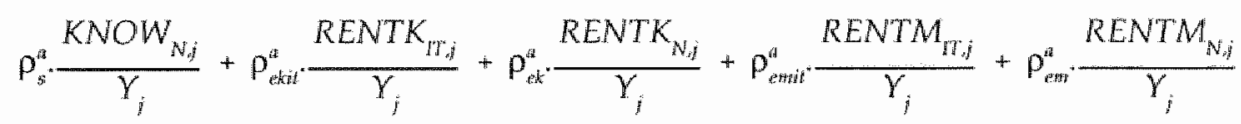

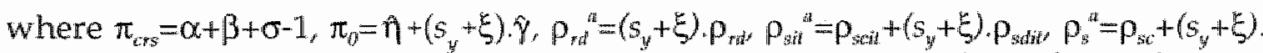

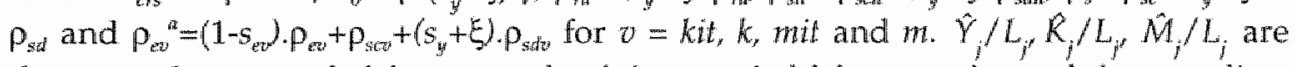
the growth rates of: labour productivity, capital-labour ratio and intermediate goods-labour ratio. This equation can be used to test the constant returns to scale assumption. When the estimated coefficient $\pi_{i r s}$ is zero there are constant returus to scale, a negative value implies decreasing retums to scale and a positive value implies increasing returns to scale.

As in section 7.4 we estimate quasi-elasticities instead of rates of return so that we can compare the influence of the various spillovers over time. ${ }^{12}$

\subsection{Data Description}

The database described in chapter 7 (section 7.1) will also be used to estimate the importance of IT and non-IT spillovers with regard to productivity growth. We identified three $\mathrm{IT}$ producing sectors. The first one is called "TT1" and produces

111 In this empirical shady we did not separate the capital stock into IT and non-IT capilal stock and the internediute goods into IT and non-lT intermediate goods. The main reason for not splitting the capital stock is that we possess no data about the IT and non $1 \mathrm{~T}$ investment demand by a sector for the period $1967-77$ 10 construct the initial capital stock (see section 7.2). Another reason is that our main interest iss on the spillowe: effects, and that the inclusion of wo additional variables increases the problems of multicolinearity.

112 For a more claborated weatment of the quasi-elasticily concept see section 7.4. 
"office and data processing machinery" which is classified as 527 in the INSEE classification and $\mathrm{N} 330$ in the NACE-CLIO classification. The second one is called "IT2" and produces mainly telecommunication equipment (S291 or N344) and the third IT producing sector produces mainly electronic equipment ( $\$ 292$ or $\mathrm{N} 345$ ) and is called "TT35".

In this section we describe some data characteristics of the IT sectors and the importance of the various IT and non-IT spillover variables for the sectors. First, we discuss the INSEE price indices of the three IT sectors to get some indication of whether quality improvements are included. Second, we discuss the magnitude of the IT producing sectors in economic and technological terms and third we describe the relevance of the various IT and non-IT spillovers for the sectors.

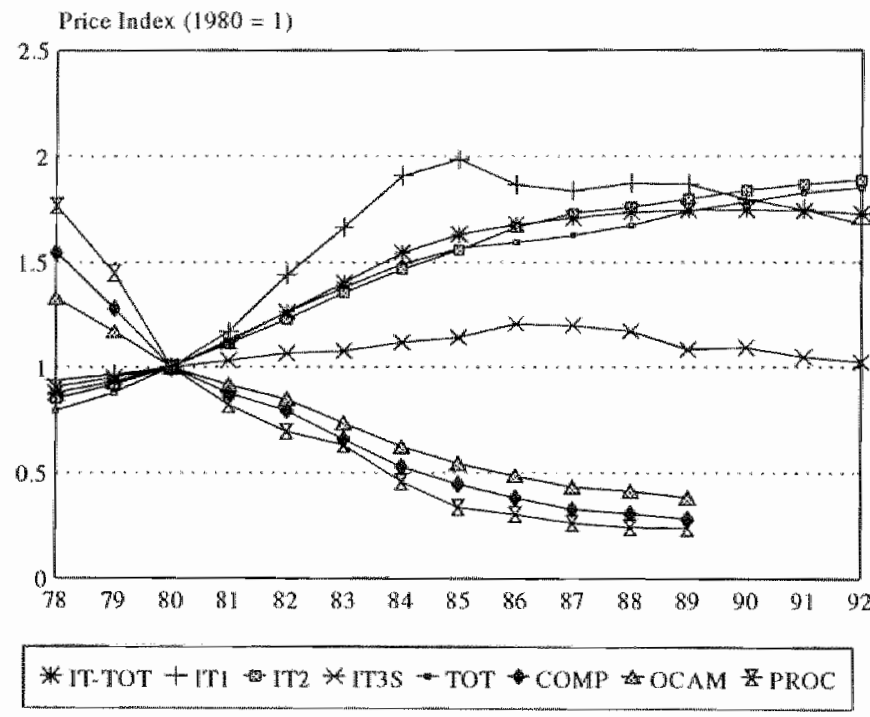

Figure 8.1. Development of French and hedonic price indices for information thonogy sectors Source: hedonic indices, Jorgenson and Stroh 1995, table 2

Price Iridices for the IT sectors

Advances in the computer industry have generated incredible gains in efficiency and a broad array of new products. It is generally assumed that these characteristics are poorly reflected in the traditional "chain" or matched price indices and that there is a need for hedonic price indices (see Griliches 1971, Cole et al. 1986, Triplett 1990). The hedonic price index adjusts price changes for changes in product characteristics and is therefore recognised as a better index for 
industries that are experiencing significant quality changes. ${ }^{113}$ In the case of computers, hedonic price indices take into account characteristics such as the speed of a processor (MIPS) and the main memory (megabytes). The difference between the traditional index and a hedonic index can be quite substantial in the IT sectors. Cole et al. (1986) compared both indices for processors and found that the hedonic index declined twice as much as the traditional index ( $8.5 \%$ versus $18 \%$ ) Dulberger (1989) calculated both indices for computer prices. Hedonic computer prices fell about $90 \%$ over the 1972-1984 period, while the traditional index fell by $67 \%$.

Figure 8.1 depicts seven price indices for IT products and one for the whole economy (TOT). The first four price-indices, as described in the legend, represent the French INSEE price indices for an aggregate of the IT sectors (IT-TOT) and the three IT sectors separately. These price indices are calculated with the traditional method. The other three are hedonic or constant quality investment price deflators as calculated by Jorgenson and Stiroh (1995). ${ }^{114}$ The hedonic price indices are depicted for office, computing and accounting machinery (OCAM), computers (COMP) and processors (PROC). Processors are a component of computers and computers represent an increasingly important part of OCAM.

Figure 8.1 clearly illustrates that price indices based on the traditional and hedonic methods are quite different. While the traditional indices show an increase in IT prices, the quality adjusted hedonic price indices decline (see also Wyckoff, 1995). For example we can compare the price indices for the ITI and OCAM sectors because these sectors cover more or less the same category of production. The IT1 price index shows an annual increase of $4.3 \%$ while the OCAM price index decreased by $10.6 \%$ each year. The enormous increase in the quality of office and computing machinery causes this discrepancy.

We can conclude that the French price indices do not reflect the huge increases in the quality of IT products. This means that output by the IT sectors and the IT inputs purchased by other sectors are under-stated - "pure" rent spillovers therefore exist. This is one of the reasons why measured sectoral TFP growth is positively influenced by IT purchases.

\section{Information Technology Products}

The IT producing sectors together represented an average of $1.87 \%$ of total actual production over the 1978-1992 period in France. In 1978 this percentage was equal to $1.48 \%$ and in 1992 this percentage had increased to $1.95 \%$. Although of little direct importance in a production sense, the IT producing sectors perform $26.2 \%$ of the total R\&D (see table 7.2). This indicates that these small sectors provide an

113 However, Trajtenberg $(1989,1990)$ argues that in technologically progressive sectors of the economy. hedonic price indexes understate quality changes. A quality adjusted price index on the basis of welfare gangs is a more appropriate index in these sectors (see sub-section 6.2.1).

"14 These constant quality investmemt price deflators are based on the Survey of Current Business, BEA and the author"s calculations. 
important contribution to the total amount of knowledge created in an economy. If spillower effects are present and significant, then the IT sector may be important for an economy. It therefore seems that the IT sectors may be small in economic terms but giant in technological terms.

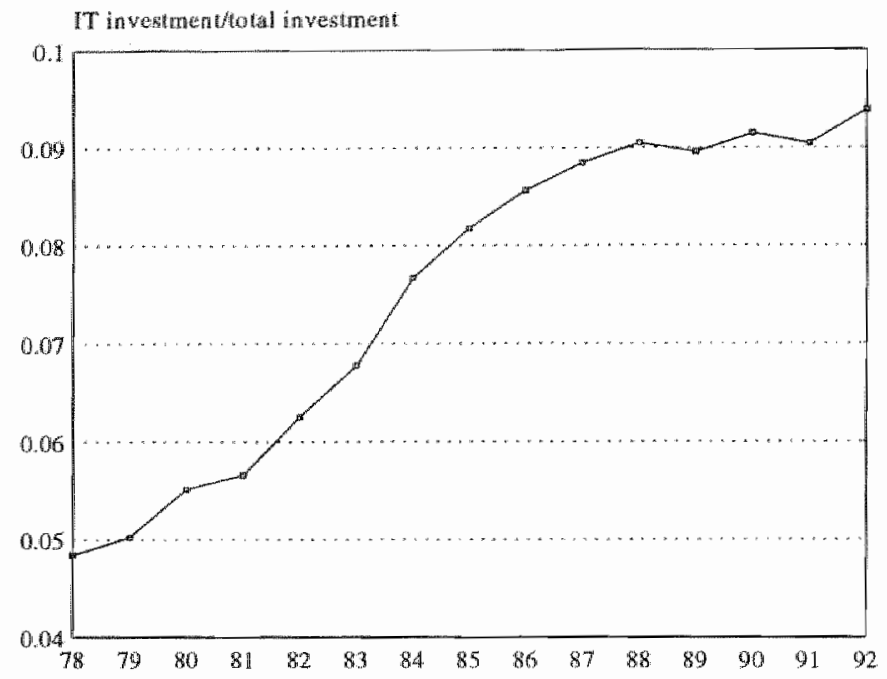

Figure 8.2. Development of IT investment share in total investments Source, INSEE

The increasing importance of IT products can also be seen in the development of IT investments. During the 1978-1992 period, IT investments had an annual growth rate of $6.7 \%$, while total investments grew by $1.84 \%$. This resulted in a doubling of the share of IT investments to total investments. This share is depicted in figure 8.2 and increased from $4.7 \%$ in 1978 to $9.4 \%$ in 1992. However, this increase is understated because quality improvements are under-stated in the price indices used to deflate investments. Finally, we note that the share of IT investments in total production increased from $6.3 \%$ to $11.1 \%$ during the $1978-1992$ period.

The Magnitude of IT and non-IT Rent and Knowledge Spillowers

In section 7.2 we described how rent spillovers of capital and intermediate goads and "pure" knowledge spillovers are calculated (see equation (7.32)-(7.34)). We will not repeat that in this section but only show the division of investment rent spillovers into IT-oriented and non-IT oriented investment goods. 


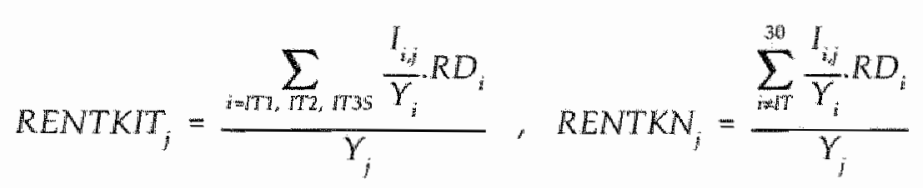

RENTKT, represents rent spillovers received from purchasing investment goods from the three sectors that produce IT goods (IT1, IT2 and IT3S). RENTKN, measures investment rent spillovers received from purchasing all other investment goods. The division of "pure" knowledge spillovers (equation (7.34)) and rent spillovers of intermediate goods (equation (7.32)) into IT and non-IT spillovers is achieved in the same manner.

Table 8.1 Mean values of general purpose variables, 1981-1992 (four-year moving average)"

\begin{tabular}{lllll}
\hline & All Sectors & $\begin{array}{l}\text { High } \\
\text { Tech }\end{array}$ & Services & $\begin{array}{l}\text { Other } \\
\text { Sectors }\end{array}$ \\
\hline R_Y & $1.92 \%$ & $5.40 \%$ & $0.05 \%$ & $0.34 \%$ \\
RENTM_Y & $0.28 \%$ & $0.53 \%$ & $0.08 \%$ & $0.21 \%$ \\
RENTMT_Y & $0.10 \%$ & $0.25 \%$ & $0.03 \%$ & $0.004 \%$ \\
RENTK_Y & $0.08 \%$ & $0.06 \%$ & $0.13 \%$ & $0.05 \%$ \\
RENTKIT_Y & $0.05 \%$ & $0.03 \%$ & $0.10 \%$ & $0.01 \%$ \\
KNOW_Y & $0.99 \%$ & $2.32 \%$ & $0.38 \%$ & $0.28 \%$ \\
KNOWT_Y & $0.62 \%$ & $1.78 \%$ & $0.09 \%$ & $0.02 \%$ \\
\hline
\end{tabular}

The division of the thirty sectors among the three groups is described in section 7.4.

Table 8.1 indicates the relative magnitude of IT spillovers for the high-tech, services and other sectors. Chapter 7 showed that rent spillovers embodied in capilal goods are important in the service sectors. Table 8.1 shows that $77 \%$ of the capital rent spillovers in the service sectors come from the three information technology producing sectors. ${ }^{115}$ In the high-tech and other sectors this is $63 \%$ and $20 \%$, respectively. It is apparent that services and high-tech sectors invest mainly in IT goods while the other (medium and low-tech) sectors invest mainly in non-IT goods.

In chapter 7 , rent spillovers embodied in intermediate goods were the most important in the other sectors category. Only $1.9 \%$ of these spillovers in the other sectors category comes from IT producing sectors; this percentage in the high-tech and services categories are $47 \%$ and $38 \%$ respectively. The importance of IT rent

115 This percentage can be obtained by dividing the rent spillovers from IT captiall goods in serwices (RENTKIT_Y $Y=0.10 \%$ ) by the total capital rent spillovers in services (RENTK_Y $Y=0.13$ ) times $100 \%$, 
spllovers embodied in intermediate goods is therefore lower than it was in capital goods. Again it is apparent that the other sectors use relatively less IT spillovers.

"Pure" knowledge spillovers are in an absolute sense the most important contributor in the high tech-sectors $(2.32 \%)$, while this intensity in the services and other sectors is $0.38 \%$ and $0.28 \%$ respectively. The share of "pure" IT knowledge spillovers in total "pure" knowledge spillovers is $76 \%, 24 \%$ and $7 \%$ respectively. The high-tech sectors receive a large part of their "pure" knowledge from the IT sectors. Chapter 7 showed that "pure" knowledge spillovers had an especially important influence in the high-tech sectors and to a lesser extent in the service sectors.

When we compare the relative magnitude of IT spillovers in the spillover concepts with the estimation results obtained in chapter 7 there is an indication that IT caputal and "pure" knowledge spillovers may be important for productivity growth. Finally, we discuss the relative importance of the six spillover effects for the 30 French sectors. The results are shown in figure 8.3 and figure 8.4 . "Pure" IT knowledge spillovers are relatively important in the IT sectors themselves and in. the instrument sector (INS) and electrical machinery sector (ELMS). This can be expected as "pure" knowledge is especially valuable in industries that are technologically "close". "The IT3S sector receives most of its spillovers from "pure" IT knowledge spillowers. There are two arguments for this fact. First, an IT sector is obviously technologically "close" to other IT sectors. Second, IT3S is a relatively small IT sector so it can use a large amount of "pure" knowledge generated by its big brothers."

Non-IT "pure" knowledge spillovers are linked with two other technologically related clusters. First, the chemical cluster (CHES, FAR and CHTS) and second the mechanical duster (MAC, FAM and MET). The relative importance of non-IT "pure" knowledge spillovers in two service sectors, other market sectors (OMS) and transport services (TRS), is caused by drug deliveries of the pharmaceutical to OMS and transport equipment to the TRS sector. This again shows that the Yale matrix is. no perfect measure of "pure" knowledge spillover in the sense that it is a measure of knowledge flows that are used in other sectors' R\&D. The black areas represent the IT spillovers in intermediate goods. These are important in, for example, the transport industry (TRA) and paper and printing industry (PAP). IT capital spillovers are relatively important in the service sectors (e.g. telecommunication services (TEL), financial services (FIN) and enterprise market services (SME)).

In general we can say that the magnitude of the various knowledge sources is different for each sector. The knowledge base for a sector is a mixture of different types of spillovers. It is important to recognise that a sector is not isolated in the economy but is a part of several inter-related networks. An investigation of the importance of internal R\&D performance for productivity growth must therefore

116 The second argument explains why the $\mathrm{TT} 2$ sector receives relatively less IT spillowers from the othar IT sectors because this sector performs $73 \%$ of total $R \& D$ in IT itselt. 


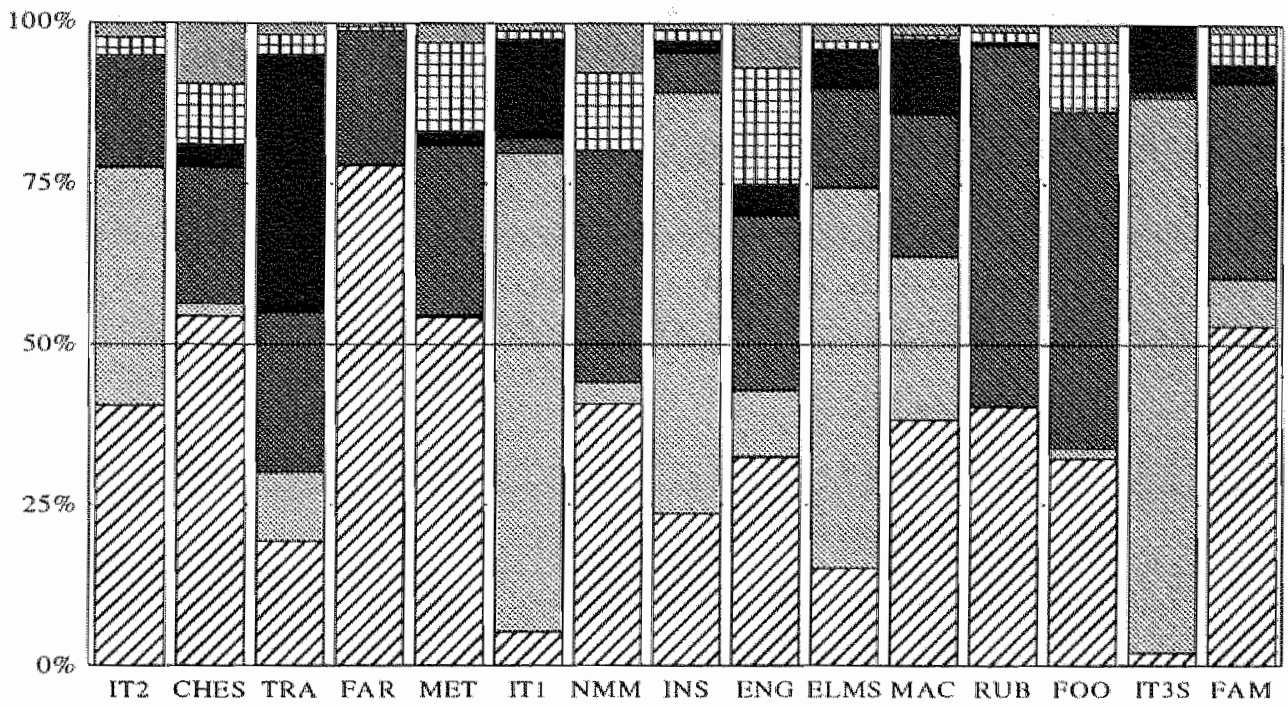

UKNOWN 圈KNOWIT

Figure 8.3. Shares of the various spillover sources in total R\&D received

$100 \%$

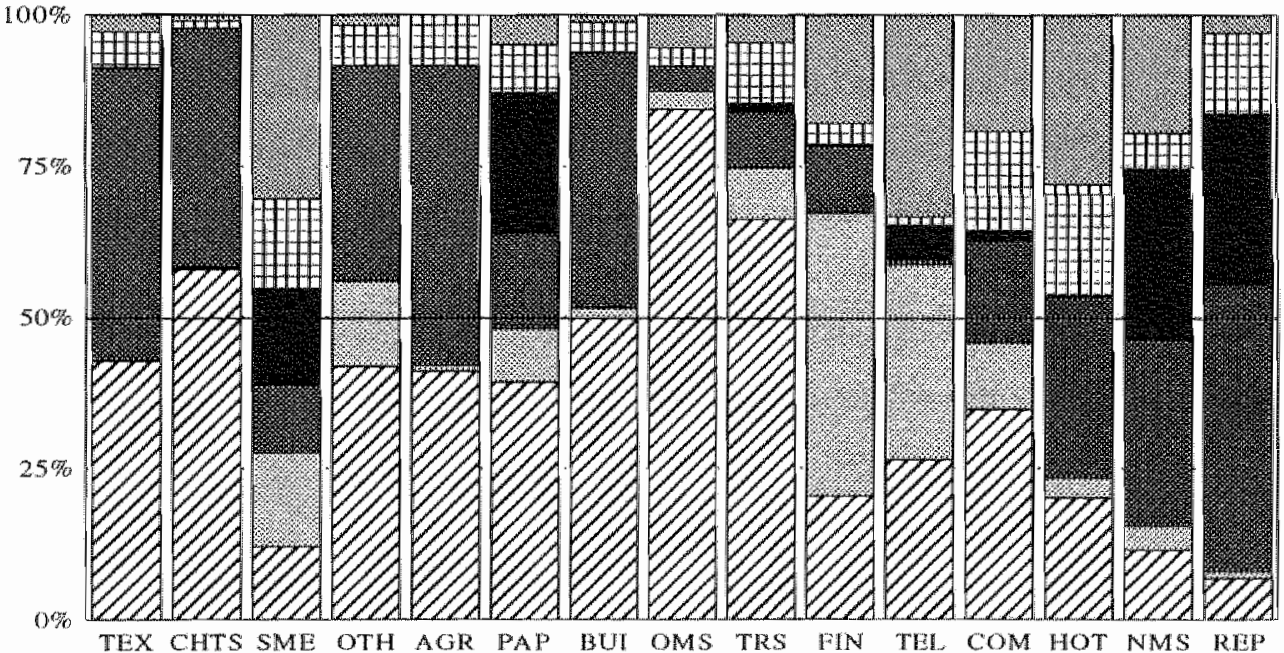

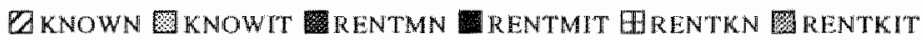

Figure 8.4. Shares of the various spillover sources in total R\&D received 
also take spillover effects into account, because besides a firms" own R\&D expenditures, knowledge generated by other sectors is also important.

Table 8.2 Definition of variables

\begin{tabular}{|c|c|}
\hline Symbol & Description. \\
\hline$Y$ & actual production (growth rate) \\
\hline$G$ & gross walue added (growth rate) \\
\hline L. & labour (growth rate) \\
\hline K & capitall stock (growth rate) \\
\hline$M$ & initermediate deliveries (growth rate) \\
\hline$Y / K$ & capitall productivity (growth rate) \\
\hline $\mathrm{L} / \mathrm{K}$ & labour/capital ratio (growth rate) \\
\hline$M / K$ & intermediate goods/capital ratio (growth rate) \\
\hline TFP_Y & three factor Total Factor Productivity (labour, capital and intermediate goods) \\
\hline $\mathrm{THP}$ G & two tactor Total factor Productivity (labour and capital) \\
\hline $\mathbb{R}_{-} \mathbf{Y}$ & "own" R\&D intensity (R\&D expenditures divided by actual production) \\
\hline $\mathrm{R}_{\mathrm{m}} \mathrm{G}^{117}$ & "own' R\&D intensity (R\&D expenditures divided by gross value added) \\
\hline$R_{C} Y$ & "own'" process R\&D intensity \\
\hline $\mathrm{RD} Y$ & "own" product R\&D intensilty \\
\hline RENTMN_Y & "Pure" rent and knowledge spillowers related to non-IT intermediate goods \\
\hline RENTMIT_Y $Y$ & "Pure" rent and knowledge spillovers related to IT intermediake goods \\
\hline RENTKN_Y & "Pure" rent and knowledge spillovers related to non-lT investment goods \\
\hline RENTKII_Y & "Pure" rent and knowledge spillovers related to IT investment goods \\
\hline $\mathrm{KNOWN} \mathrm{Y}$ & "Pure" non-IT knowledge spillovers \\
\hline KOWIT_Y & "Pure" TT knowledge spillovers \\
\hline RKN_Y & RENTMN_Y + KNOWN_Y \\
\hline RKMT_Y & RENTMIT_Y+KNOWIT_Y \\
\hline RRKIT $Y$ & 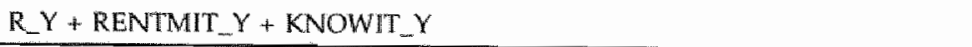 \\
\hline
\end{tabular}

117 Conditionat on the dependent variable, actual production $(Y)$ or gross value added $(G)$ are the various R\&D intensities expressed as a R\&D variable divided by actual production, postscript $Y$ or gross value added, postscript_G. 


\subsection{Estimation Results}

Measuring the importance of the current general purpose technology, information technology, for productivity growth is central in this section. We irvestigate whether the various IT spillover variables contribute to technological change and whether this contribution increases over time. In addition to the IT effects, we measure the influence of internal $R \& D$ and the non-IT spillover effects on productivity growth. The definitions of the variables we use as regressors in the estimations are described in table 8.2 .

Table 83 Correlation Matrix

\begin{tabular}{|c|c|c|c|c|c|c|c|c|c|}
\hline & $\mathrm{TFP}$ & r & $R$ & $\begin{array}{l}\text { RENTK } \\
N\end{array}$ & $\begin{array}{l}\text { RENTK } \\
\mathbb{I T}\end{array}$ & $\begin{array}{l}\text { RENTM } \\
\mathrm{N}\end{array}$ & $\begin{array}{l}\text { RENTM } \\
\text { IT }\end{array}$ & $\begin{array}{l}\text { KNOW } \\
\mathrm{N}\end{array}$ & $\begin{array}{l}\text { KNOW } \\
\text { IT }\end{array}$ \\
\hline TFP & 1 & & & & & & & & \\
\hline$Y$ & 0.42 & 1 & & & & & & & \\
\hline $\mathrm{R}$ & 0.35 & 0.37 & 1 & & & & & & \\
\hline RENTKN & -0.03 & -0.17 & -0.16 & 1 & & & & & \\
\hline RENTKIT & 0.67 & 0.50 & -0.10 & -0.05 & 1 & & & & \\
\hline RENTMN & 0.08 & 0.05 & 0.17 & -0.15 & -0.18 & 1 & & & \\
\hline RENTMTT & 0.42 & 0.33 & $0.62^{\circ}$ & -0.13 & 0.03 & -0.08 & 1 & & \\
\hline KNOWN & $0.2 \pi$ & 0.38 & 0.27 & 0,08 & 0.08 & $0.60^{\prime \prime}$ & -0.08 & 1 & \\
\hline KNOWIT & 0.46 & 0.32 & $0.61^{\circ}$ & -0.12 & 0.03 & -0.06 & 0.96 & $-0,044$ & 1 \\
\hline
\end{tabular}

"possible threat of multicollinearity between explanatory variables

\subsubsection{Influence of IT and non-TT spillovers; Average Values for Total Period: 1978-1992}

The database in this sub-section contains the mean values of the variables over the period 1978-1992 for 30 sectors. The correlation matrix which is given in table 8.3 shows that the IT spillover wariables have the strongest correlation with TFP growth. Rent spillovers of IT capital goods show a correlation coefficient of 0.67 and "pure" knowledge spillovers and rent spillovers in intermediate IT goods have a correlation coefficient of 0.46 and 0.42 respectively. These are all higher than the correlation coefficients of own R\&D and the non-IT spillover variables. These correlation coefficients also give an indication of possible multicollinearity between explanatory variables. Possible candidates are marked with an asterisk in table 8.3. There is a strong positive correlation, 0.96, between "pure" knowledge spillovers from the IT sector and rent spillovers embodied in IT intermediate goods. To avoid 
multicollinearity we add these two spillover effects and create a new variable: RKIT=RENTMIT+KNOWIT. These two kinds of IT spillovers, however, are also closely linked with own R\&D. A new variable RRKIT $=R+R K T T$ is also constructed. It is apparent that almost no correlation exists between own R\&D, IT "pure" knowledge spillovers and IT intermediate rent spillovers, with the rent spillovers of IT capital goods. A possible explanation is that service sectors with high investments IT capital goods do not perform much $R \& D$ themselves. Less pronounced but still a possible caveat is the correlation coefficient of KNOWN and RENTMN, 0.60. We can again create new variable: RKNT=RENTMN+KNOWN.

We tum to the estimates of the equations developed in section 8.1. The estimation results of equation (8.1) are shown in table 8.4 and table 8.5. Regressions 4.1 to 4.3 show the results for the three-factor TFP growth and regressions 5.1-5.3 for the two-factor TFP growth. In regressions 4.1 and 5.1 we included "own" R\&D and all the spillover concepts. In regressions $4.2,4.3,5.2$ and 5.3 we add some concepts together, according to the method proposed earlier, to reduce the problem of multicollinearity. It is apparent that rent spillovers of capital goods from the IT sector (RENTKIT) are highly significant in all cases. The estimated value of the quasi-elasticity is about 0.37 in the case of TFP_Y and about 0.66 in the case of TFP_G. The combined variable RKIT, which represents IT "pure" knowledge spillovers and rent spillovers of IT intermediate goods is also significant in all cases and has an estimated quasi-elasticity of 0.12 in the case of TFP_ $Y$ and 0.38 in the case of TFP_G. The various non-1T spillovers are significant in some cases and insignificant in other cases.

An important assumption behind the TFP growth calculations is the assumption of constant returns to scale. In chapter 7 we found that production was characterised by decreasing returns to scale and that the constant returns to scale assumption in this case had a negative influence on the significance and heights of the various rates of return. We will therefore release the constant returns to scale assumption by estimating equation (8.2). We use the capital productivity growth rate instead of the labour productivity growth rate as the dependent variable. The reason for this change is that the chance for multicollinearity between $L / K$ growth and $M / K$ growth is much smaller than that with $K / L$ and $M / L$. We estimate the equations with "own" process $\mathbb{R} \& D$ or total "own" R\&D (process and product R\&D) to get an indication of whether or not product $R \& D$ also has an influence on the productivity of a sector.

The estimated coefficients of equation (8.2) are given in regressions 4.4-4.7 (actual production) and regressions 5.4-5.7 (gross value added). We can make the following four general observations before discussing spillover effects: First, all equations indicate decreasing returns to scale, because the coefficient of the capital growth $(\mathrm{K})$ is negative. Second, relaxing the assumption of constant returns to scale improves, in almost all cases, the statistical significance of the estimated quasi-elas- 


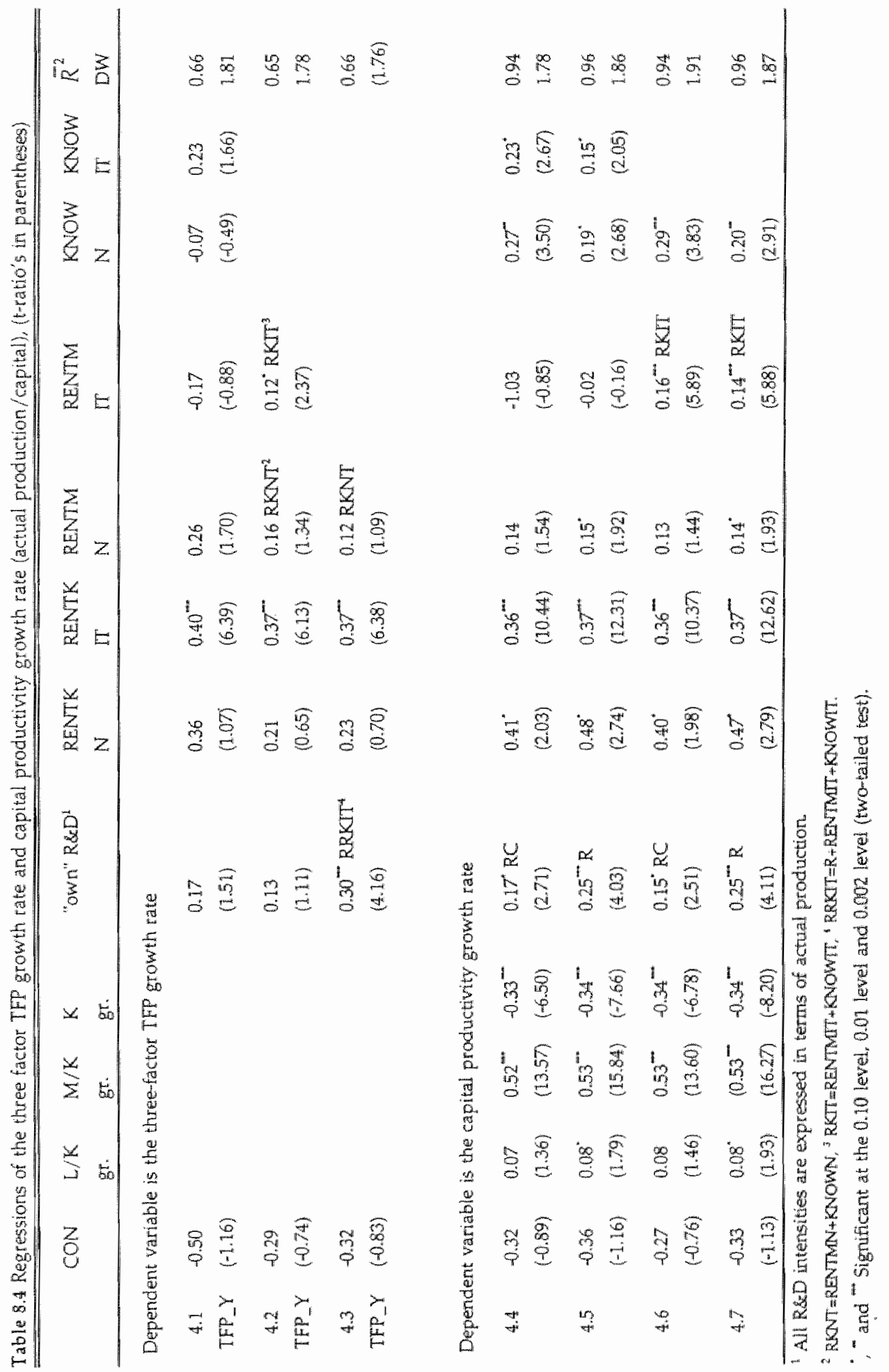




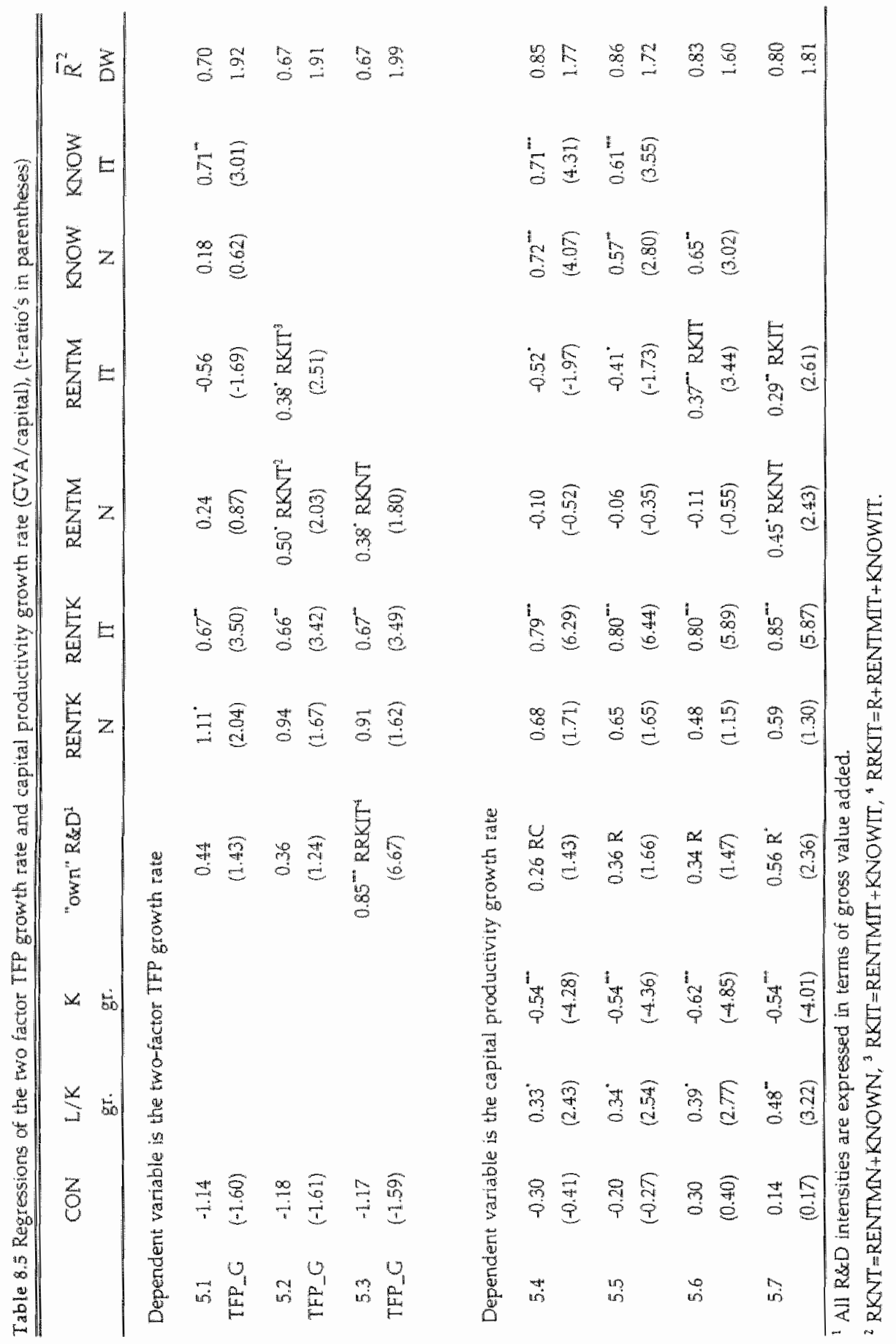


ticities. Third, the explanatory power of the regressions in which we used total "own" R\&D is higher than when we only used process R\&D. This indicates an influence of product $\mathbb{R} \& D$ on the productivity of a sector. Fourth, the values of the estimated quasi-elasticities in the case of actual production are generally about half the value of those with gross value added. Exceptions are the quasi-elasticities of the "pure" knowledge spillovers and RKNT which are one third in the case of actual production of those values in the case of gross value added.

With regard to the spillover effects, the high significance and high value of the estimated quasi-elasticity of rent spillover effects on IT investment goods is apparent. The values are about 0.45 in the case of actual production and 0.8 in the case of gross value added. Compared to the TFP growth estimates the estimated quasi-elasticity of own R\&D has become significant. The value is 0.25 in the case of actual production and 0.56 in the case of gross value added.

Another interesting result is that the rent spillovers of non-IT capital goods are insignificant in the case of gross value added (regression 5.4-5.7) and significant in the case of actual production (regression 4.4-4.7). In the case of actual production the estimated quasi-elasticity is high $(0.41-0.48)$ which also implies a large influence of spillovers on non-IT capital goods. The influence of non-IT capital goods is even higher than the influence of IT capital goods (0.36). However, in this equation the average influence from 1978 to 1992 of rent spillovers on non-IT capital is slightly higher than those of IT capital, which does not say anything about the development of these influences over time. According to our hypotheses developed in chapter 1 it can be expected that spillovers of IT goods will become more pronounced over time, and it is this "lag" hypothesis which we will investigate.

\subsubsection{The Influence of IT and non-IT Spillovers: Two Periods}

We divide the period into two sub periods 1978-1985 and 1985-1992 and calculate the mean values over these periods. For these two periods, which will be called T1 and $\mathrm{T} 2$ respectively, we estimated a two- and a three-factor TFP growth equation and the capital productivity measured with actual production and gross value added. The estimation results are shown in table 8.6. In line with the "lag" hypothesis the quasi-elasticity to rent spillovers of IT capital is much higher in the second period than in the first period. The estimated quasi-elasticity almost doubles in all cases. This suggests that the influence of IT spillovers in capital goods has become more important over time.

The various regressions indicate a weak increase in the quasi-elasticity of non-IT capital rent spillovers. Therefore the influence of IT capital goods becomes more important relative to other capital goods. Other spillovers from the IT producing sectors, "pure" knowledge spillovers and rent spillovers embodied in intermediate goods (RKIT), are also growing more important over time. Their increase, however, 


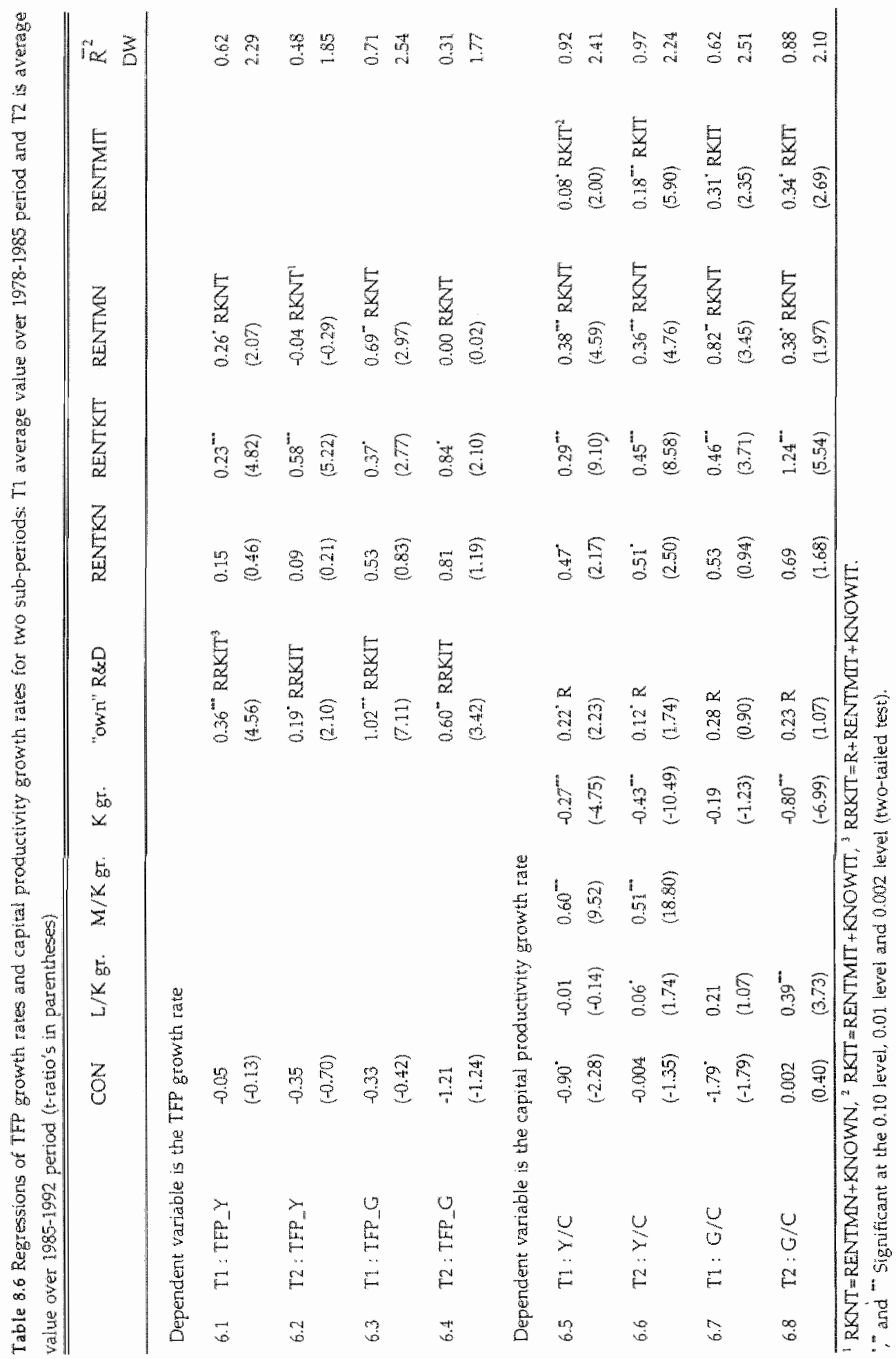


is not as significant as the increase in the rate of return to rent spillovers of IT investment goods. Contrary to the development of the influences of all these spillovers there is a decline in the estimated quasi-elasticity of "pure" knowledge and rent spillovers in intermediate goods (RKNT) of the non-IT sectors.

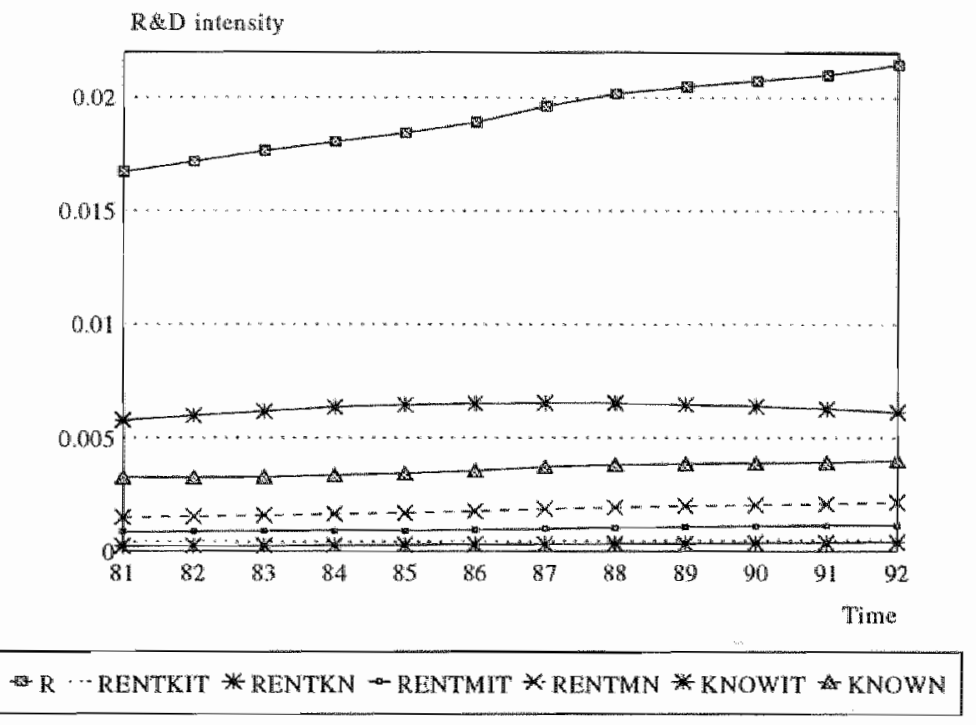

Figure 8.5. Development of own R\&D and various spillover concepts over time

\subsubsection{The Influence of IT and non-IT Spillovers: Yearly Estimates}

In this sub-section we will investigate the development of the influence of the various spillover variables over time. To reduce the influence of business cycles we calculate a four-year moving average for the period 1978-1992 which reduces the estimation period to 1981-1992. We are therefore able to study the influence of spillovers for a period of twelve years.

We first consider the development of the mean values of several $R \& D$ variables. Figure 8.5 indicates the magnitude of the various R\&D concepts. "OWn" R\&D has the largest magnitude and is increasing over time. Next "pure" knowledge spillovers of IT goods have the largest magnitude followed by "pure" knowledge spillovers of non-IT goods. The other types of spillovers are relatively small.

We now discuss the equations which we have estimated for every year in the period from 1981 to 1992 . We performed 7 different regressions to get a broad indication of the development of the quasi-elasticities. The seven regressions are stated in table 8.7. Regressions 1, 2, 3,6 and 7 are straightforward extensions of equation (8.2) and regressions 4 and 5 are extensions of equation (8.1). The results 
of regression 1 and 4 are shown in table 8.8 and 8.9 , respectively " ${ }^{118}$ The regression results are graphed to show the development of the influences of the various spillover effects over time. To the variable names in the graphs, the number of the regression is added as a postscript. So $\mathbb{R} 1$ and $R 7$ represent the estimated quasi-elasticity to own $R \& D$ in regression 1 and 7 , respectively.

Table 8.7 Key equations

\begin{tabular}{|c|c|c|c|c|c|c|c|c|c|c|}
\hline Number & $\begin{array}{c}\text { Dependent } \\
\text { variable }\end{array}$ & Exp & natory & Variat & bles & & & & & \\
\hline 1 & $Y / K$ & C & $L / K$ & $M / K$ & $\mathrm{~K}$ & $\mathrm{R}$ & RENTKIT & RENTKN & RKNT & RKIT \\
\hline 2 & $Y / K$ & $c$ & $L / K$ & $M / K$ & $K$ & RRKT & RENTKIT & RENTKN & RKNT & \\
\hline 3 & $Y / K$ & $C$ & $\mathrm{~L} / \mathrm{K}$ & $\mathrm{M} / \mathrm{K}$ & $\mathrm{K}$ & $\mathrm{R}$ & RENTKIT & RENTKN & $\begin{array}{l}\text { RENTMN } \\
\text { KNOWN }\end{array}$ & $\begin{array}{l}\text { RENTMIT } \\
\text { KNOWIT }\end{array}$ \\
\hline 4 & TFP_Y & $c$ & & & & RRKIT & RENTKIT & & & \\
\hline 5 & $T F P_{-} \mathrm{G}_{\mathrm{S}}$ & C & & & & RRKIT & RENTKIT & & RKNT & \\
\hline 6 & $G / K$ & $\mathrm{C}$ & $L / K$ & & K & RRKIT & RENTKIT & & RKNT & \\
\hline 7 & $G / K$ & $c$ & $\mathbb{L} / \mathrm{K}$ & & K & $\mathrm{R}$ & RENTKIT & & RKNT & RKIT \\
\hline
\end{tabular}

Tables 8.8 and 8.9 both show the growing influence of the rent spillovers of IT capital goods (RENTKIT) over time and therefore support the "lag" hypothesis. The influence of RENTKIT increased from $0.32(0.23)$ to $0.64(0.75)$ in table 8.8 (table 8.9). We depicted the estimated values of the quasi-elasticities of RENTKT for the first four specifications in figure 8.6 (specifications that used actual production).

There is a clear positive time trend in all cases. The marked area contains all the estimated values for the quasi-elasticity of rent spillovers in capital goods. This "band" indicates that the influence of the rent spillovers embodied in IT capital goods (computers) on productivity growth remained more or less constant during the period 1981-1988, but showed a rapid increase during the most recent period (1988-1992). It seems that we have found the expected important influence of the current general parpose technology. The measurable influence of IT capital goods became important in the most recent period. It seems that the IT paradox has wanished.

The development of the influence of "own" R\&D is shown in figure 8.7. With actual production $(q+1)$, the influence of own R\&D is more or less constant over time and equal to 0.2. The picture in the case of gross value added is less clear. The influence of spillovers patterns of non-IT capital goods are depicted in figure 8.8 .

115 The sstimation results of regression 5 and 7 are shown in appendix $8 \mathrm{~A}$. 


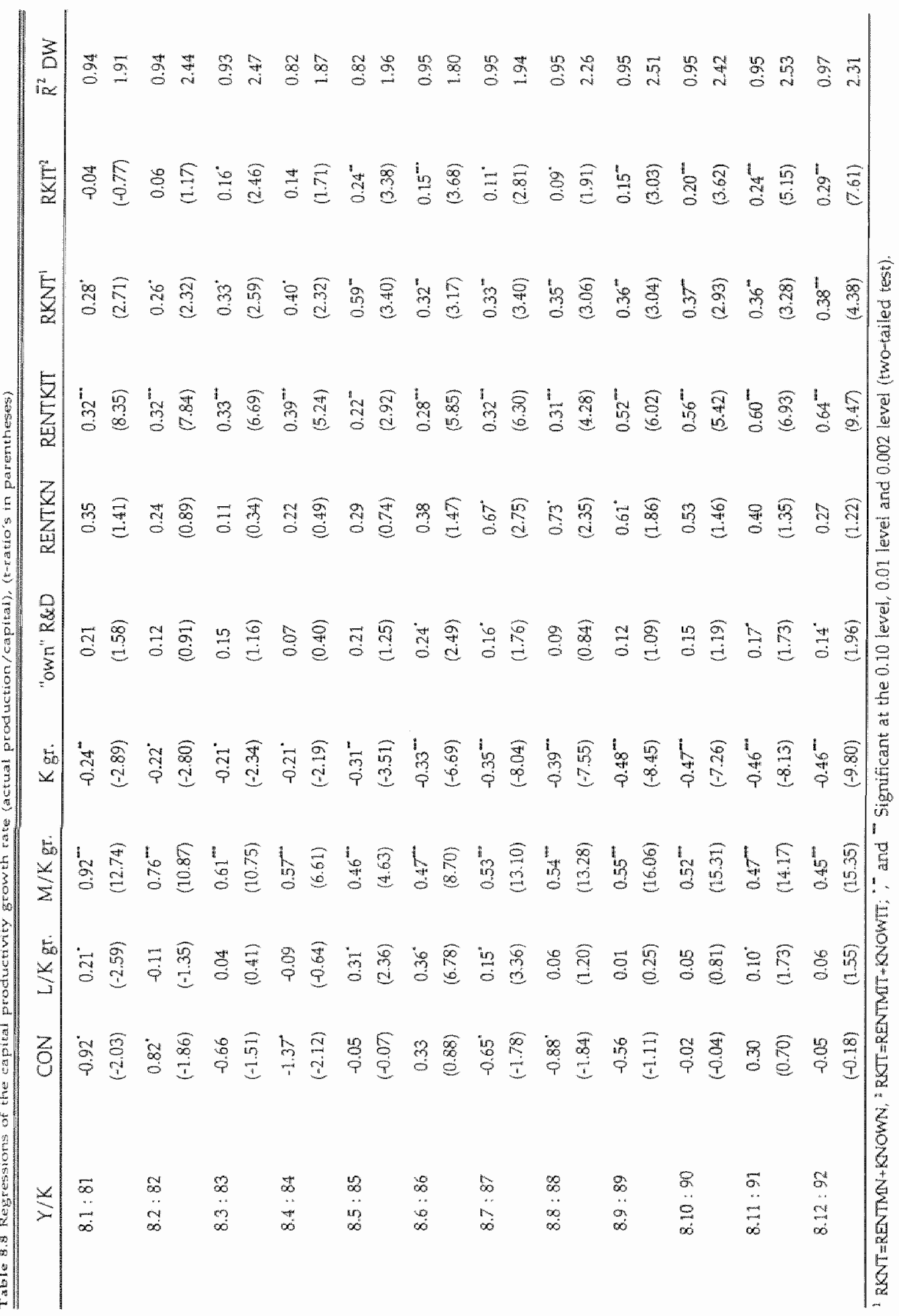


Table 8.9 Regressions of the three factor TFP growith rate (t-ratio's in parentheses)

\begin{tabular}{|c|c|c|c|c|c|}
\hline$T_{-Y}$ & $\mathrm{CON}$ & RRKIT"I & RENTTKIT & RKNT & $\begin{array}{l}R^{2} \\
D W\end{array}$ \\
\hline $91: 81$ & $\begin{array}{c}0.14 \\
(0.51)\end{array}$ & $\begin{array}{l}0.39^{\circ *} \\
(3.79)\end{array}$ & $\begin{array}{l}0.23^{\mathrm{x}} \\
(4.20)\end{array}$ & $\begin{array}{c}0.22 \\
(1.38)\end{array}$ & $\begin{array}{l}0.52 \\
2.12\end{array}$ \\
\hline $9.2: 82$ & $\begin{array}{c}-0.09 \\
(-0.33)\end{array}$ & $\begin{array}{l}0.42^{*} \\
(4.34)\end{array}$ & $\begin{array}{l}0.28^{*} \\
(4.98)\end{array}$ & $\begin{array}{c}0.28^{\circ} \\
(1.79)\end{array}$ & $\begin{array}{l}0.61 \\
2.20\end{array}$ \\
\hline $9.3: 83$ & $\begin{array}{c}-0.32 \\
(-1.33)\end{array}$ & $\begin{array}{c}0.49^{*} \\
(5.56)\end{array}$ & $\begin{array}{l}0.32^{\circ} \\
(5.57)\end{array}$ & $\begin{array}{c}0.26^{\circ} \\
(1.84)\end{array}$ & $\begin{array}{l}0.69 \\
2.07\end{array}$ \\
\hline $9.4: 84$ & $\begin{array}{c}-0.16 \\
(-0.49)\end{array}$ & $\begin{array}{l}0.45^{2 \times 4} \\
(3.78)\end{array}$ & $\begin{array}{l}0.28^{\circ} \\
(3.41)\end{array}$ & $\begin{array}{c}0.32 \\
(1.62)\end{array}$ & $\begin{array}{l}0.48 \\
2.22\end{array}$ \\
\hline $9.5: 85$ & $\begin{array}{c}0.116 \\
(0.41)\end{array}$ & $\begin{array}{l}0.32 \\
(2.79)\end{array}$ & $\begin{array}{c}0.24^{\prime \prime} \\
(2.79)\end{array}$ & $\begin{array}{c}0.29 \\
(1.55)\end{array}$ & $\begin{array}{l}0.35 \\
2.41\end{array}$ \\
\hline $9.6: 86$ & $\begin{array}{c}0.11 \\
(0.41)\end{array}$ & $\begin{array}{c}0.11 \\
(1.18)\end{array}$ & $\begin{array}{l}0.30^{4} \\
(3.90)\end{array}$ & $\begin{array}{c}0.17 \\
(1.13)\end{array}$ & $\begin{array}{l}0.34 \\
2.39\end{array}$ \\
\hline $9.7: 87$ & $\begin{array}{c}0.22 \\
(0.94)\end{array}$ & $\begin{array}{c}-0.03 \\
(-0.29)\end{array}$ & $\begin{array}{l}0.34^{+2} \\
(4.10)\end{array}$ & $\begin{array}{c}0.00 \\
(0.07)\end{array}$ & $\begin{array}{l}0.33 \\
2.41\end{array}$ \\
\hline $98: 88$ & $\begin{array}{c}0.22 \\
(0.77)\end{array}$ & $\begin{array}{c}-0.00 \\
(-0.09)\end{array}$ & $\begin{array}{l}0.44^{* 04} \\
(3.98)\end{array}$ & $\begin{array}{c}-0.02 \\
(-0.15)\end{array}$ & $\begin{array}{l}0.31 \\
2.52\end{array}$ \\
\hline $9.9: 89$ & $\begin{array}{c}-0.02 \\
(-0.07)\end{array}$ & $\begin{array}{l}0.20^{*} \\
(1.80)\end{array}$ & $\begin{array}{l}0.61 \\
(4.33)\end{array}$ & $\begin{array}{c}-0.05 \\
(-0.28)\end{array}$ & $\begin{array}{l}0.39 \\
2.37\end{array}$ \\
\hline $9.10: 90$ & $\begin{array}{l}-0.003 \\
(-0.78)\end{array}$ & $\begin{array}{l}0.34^{* *} \\
(2.96)\end{array}$ & $\begin{array}{l}0.74^{* \cdots} \\
(4.65)\end{array}$ & $\begin{array}{c}-0.00 \\
(-0.02)\end{array}$ & $\begin{array}{l}0.48 \\
1.91\end{array}$ \\
\hline $9.11: 91$ & $\begin{array}{c}-0.46 \\
(-1.32)\end{array}$ & $\begin{array}{l}0.40^{\text {ten }} \\
(3.41)\end{array}$ & $\begin{array}{l}0.79^{+\infty} \\
(4.87)\end{array}$ & $\begin{array}{c}-0.05 \\
(-0.24)\end{array}$ & $\begin{array}{l}0.52 \\
1.78\end{array}$ \\
\hline $9.12: 92$ & $\begin{array}{c}-0.77 \\
(-2.22)\end{array}$ & $\begin{array}{l}0.39^{*} \\
(3.30)\end{array}$ & $\begin{array}{l}0.75^{*} \\
(4.62)\end{array}$ & $\begin{array}{c}-0.06 \\
(-0.33)\end{array}$ & $\begin{array}{l}0.49 \\
1.60\end{array}$ \\
\hline
\end{tabular}

${ }^{1}$ All R\&D intensities are expressed in terms of actual production

${ }^{2}$ RKNT=RENTMN +KNOWN, "RRTTERENTMIT+KNOWIT, "RTKIT=R+RENTMIT+KNOWTT

;." and "'." Significant at the 0.10 level, 0.01 lewel and 0.002 level (two-tailed testy.

The patterns indicate that the influence of RENTKN increased until 1988 and decreased during the most recent period indicating that these non-IT capital spillovers loose their importance over time. The development of the influence of IT "pure" knowledge spillovers, in combination with IT intermediate goods spillovers (RKIT), is depicted in figure 8.9. In 1981 the influence was nihil but the influence increased over time. We can also conclude that the variability in the quasielasticities is much bigger when gross value added is used then when actual 


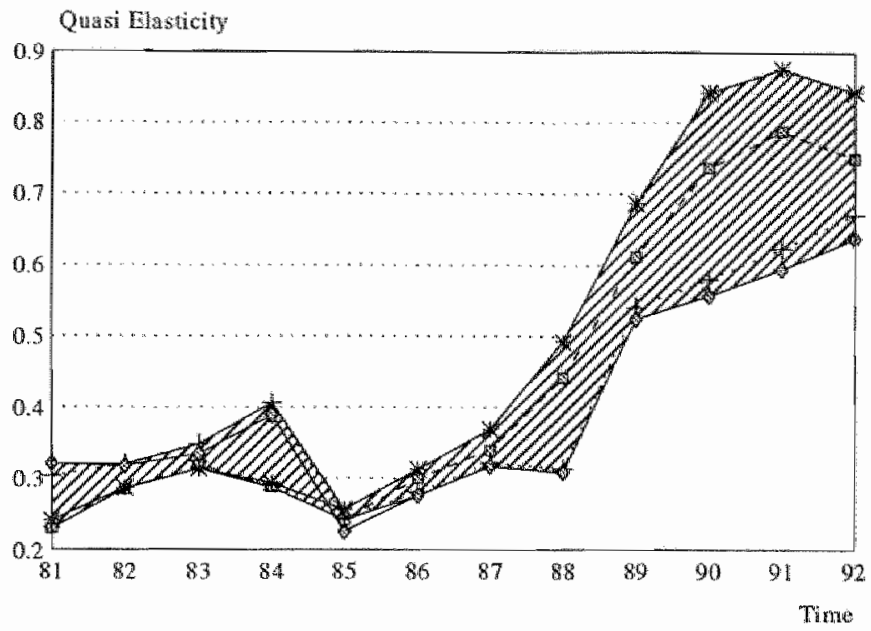

\$ q_RENTKIT1 +q_RENTKIT2 * q_RENTKIT3-q_RENTKIT4

Figure 8.6. Estimated influence of rent spillovers in IT capital goods

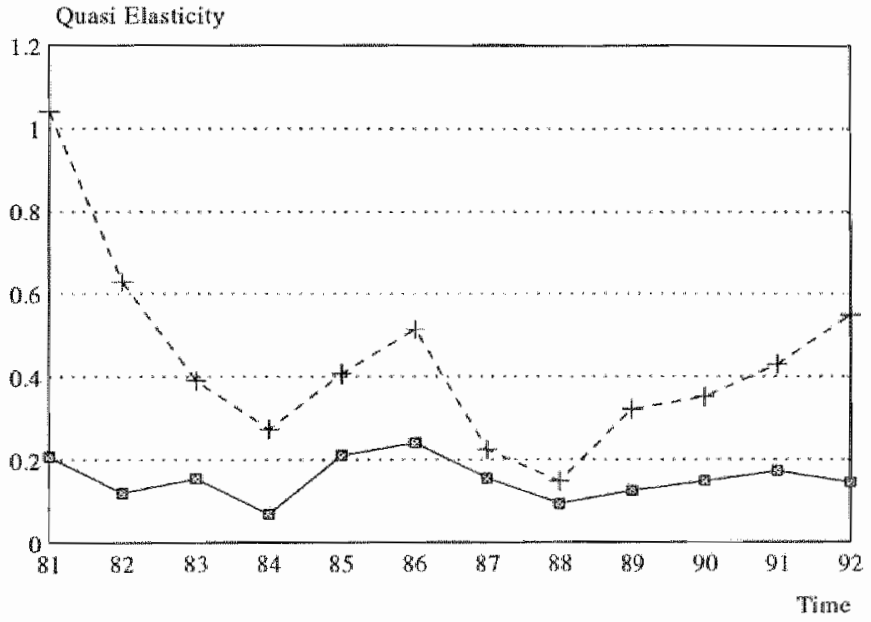

$$
+q_{-} R I+q_{-} R 7
$$

Figure 8.7. Estimated influence of own R\&D 


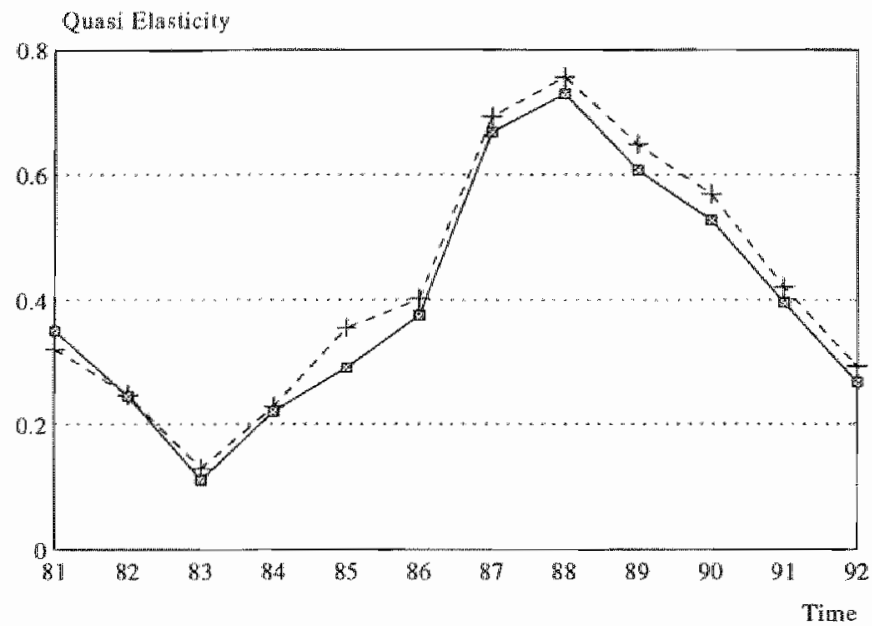

- q RENTKN + q RENTKN2

Figure 8.8. Estimated quasi-elasticities of RENTKN

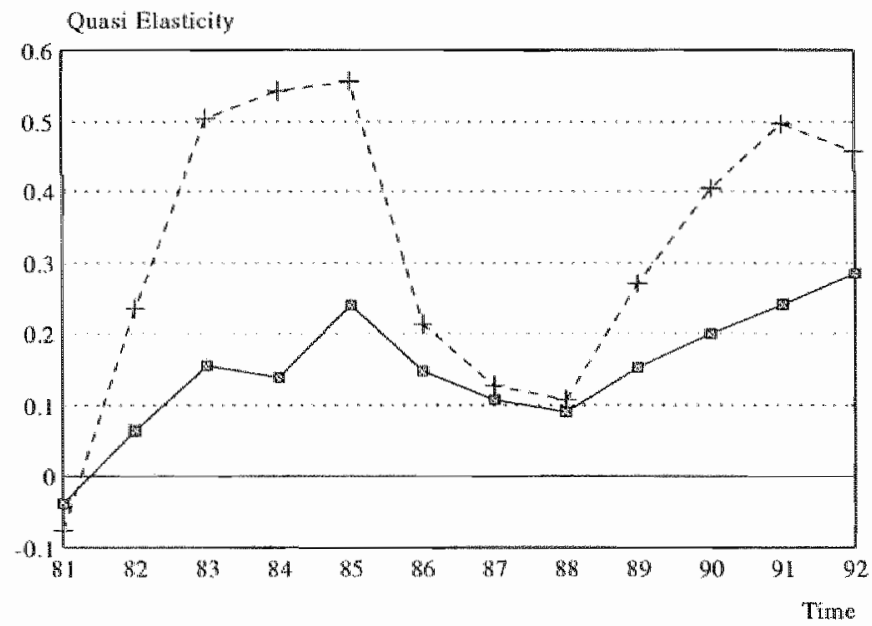

G_RKITI + q_RKITJ

Figure 8.9. Estimated influence of RKTT 
production is used. Figure 8.10 depicts the effects of the various spillover effects in one graph. It is clear that the importance of the various spillover effects has changed. A general conclusion which can be drawn from this graph is that in the 1980 s the spillover effects of non-IT goods were most important, but the spillovers of IT goods and especially IT capital goods are becoming more important over time.

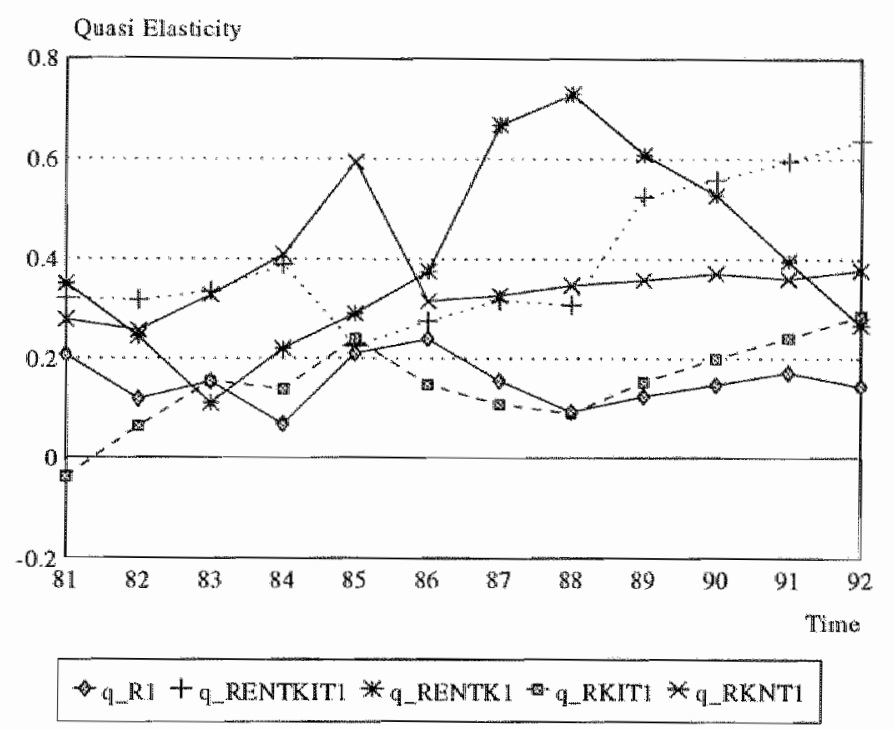

Figure 8.10, Estimated quasi elasticities of regression 1

We next check the existence of statistically significant time trends in the various quasi-elasticities. ${ }^{119}$ The results are shown in table 8.10 . The trend in the value of the quasi-elasticity of rent spillovers of IT capital good is significant and has a value of about 0.03, which is remarkably high. Again this is evidence for the growing importance of IT capital goods. The quasi-elasticity of "own" R\&D has no significant time trend but a significant constant value of 0.17 . This indicates that the influence of own R\&D is more or less constant over time. The quasi-elasticity of the other IT spillovers, i.e. IT "pure" knowledge spillovers and rent spillovers of intermediate IT goods (RKIT), possesses a statistically significant trend which is equal to 0.017. This again indicates that the importance of IT is growing. However, when we add "own" R\&D and other TT spillovers (RKIT) the trend is significant and equal to 0.03 which is almost twice as high as the trend when we only considered RKIT. This means that the joint effect of own R\&D and IT "pure"

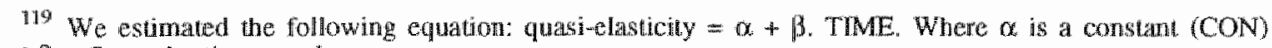
and $\beta$ renlects the time trend. 
knowledge/intermediate IT goods spillovers is larger than the separate effects which indicates synergy effects. Finally, we can conclude that the spillovers of nonIT goods show no significant time trends. However, there is a constant influence of RKNT (non-IT "pure" knowledge spillovers and rent spillovers in non-IT intermediate goods capital goods) which is significant $(0.29)$.

Table 8.10 Time trends in quasi-elasticities

\begin{tabular}{|c|c|c|c|}
\hline & $\begin{array}{l}\text { Dependent } \\
\text { variable }\end{array}$ & CON & Trend \\
\hline 10.1 & QE RENTKITI & $\begin{array}{l}0.159 \\
(1.67)\end{array}$ & $\begin{array}{l}0.034 " \\
(2.68)\end{array}$ \\
\hline 10.2 & QE_RENTKIT2 & $\begin{array}{l}0.186 \\
(1.60)\end{array}$ & $\begin{array}{l}0.032^{\prime \prime} \\
(2.01)\end{array}$ \\
\hline 10.3 & $\mathrm{QE} R \mathrm{RI}$ & $\begin{array}{l}0.173^{n+w} \\
(5.36)\end{array}$ & $\begin{array}{r}-0.002 \\
(-0.57)\end{array}$ \\
\hline 10.4 & QE_RRKIT2 & $\begin{array}{l}0.145^{\circ} \\
(1.91)\end{array}$ & $\begin{array}{l}0.03^{\circ *} \\
(3.47)\end{array}$ \\
\hline 10.5 & QE_RENTKNI & $\begin{array}{l}0: 01.63 \\
(0.66)\end{array}$ & $\begin{array}{r}-0.078 \\
(-0.54)\end{array}$ \\
\hline 10.6 & QE_RKNTI & $\begin{array}{l}0.29^{4 \times} \\
(7.77)\end{array}$ & $\begin{array}{l}0.007 \\
(1.54)\end{array}$ \\
\hline 10.7 & QE_RKITI & $\begin{array}{l}0.035 \\
(0.48)\end{array}$ & $\begin{array}{l}0.0117^{\circ} \\
(2.09)\end{array}$ \\
\hline
\end{tabular}

The estimated quasi-ellasticities are weighted with their variances.

Corrected for auto correlation (Cochrane-Orcutt iterative technique).

\subsection{The Importance of Spillover Effects over Time}

In this section we examine the second hypothesis: "External knowledge sources will become more important in the knowledge generation process. The value of spillover effects will increase in the IT era".

As a proxy for the influence of all kinds of spillovers on the productivity level of sectors we can calculate a weighted quasi-elasticity.

$$
q d\left(S_{i o s}\right)=\sum_{i} q e\left(X_{i}\right) \frac{m\left(X_{i}\right)}{\sum_{i} m\left(X_{i}\right)}
$$


where $X_{i}=R E N T K N, R E N T K I T, R K N T$ and $R K I T$ in the case of regression $i, g e\left(X_{j}\right)$ is the quasi-elasticity of variable $i$ and $m(X)$ is the mean value of variable $i$. This measure indicates that a $1 \%$ increase in the tatal spillovers increases the productivity by $g e\left(S_{t}\right) * 100 \%$. We depicted the weighted quasi-elasticity of total R\&D spillovers (q_spiltot) based on the estimation results of regression 1 in table 8.7. The weighted elasticity increases from 0.1 in 1981 to 0.33 in 1992 . The estimated time-trend equation is :qe_spiltot $1=0.16(4.27)+0.014(2.74)$ * time. The t-values are given in parentheses and imply that the constant and slope are both significant. This indicates that the influence of spillovers and therefore external knowledge sources are becoming more important over time.

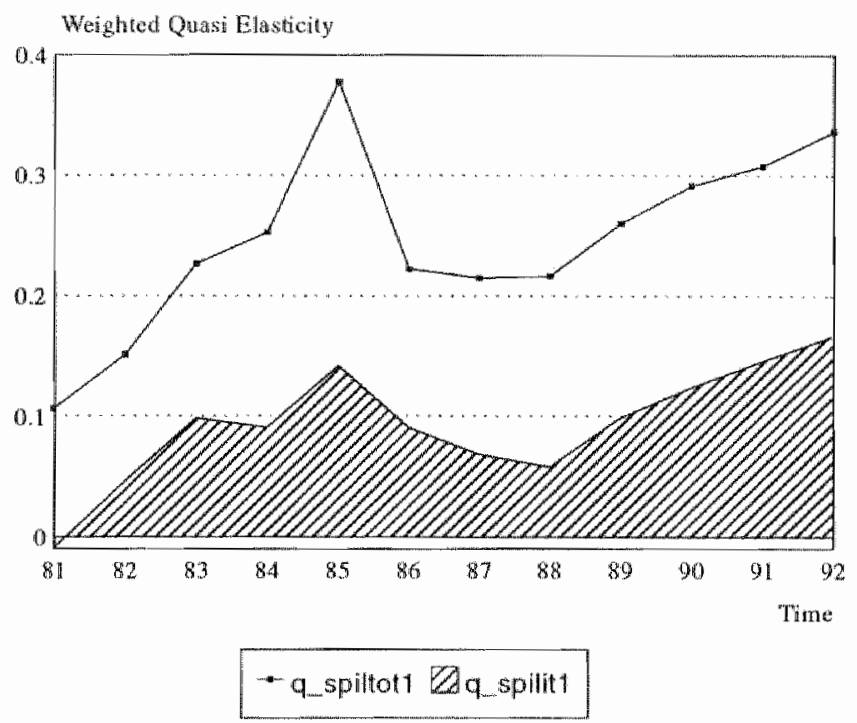

Figure 8.11. Weighted quasi-elasticity of total and $\mathrm{TT}$ spillovers (calculated from results of regression 1)

We also separated the spillover effect in a weighted IT and non-IT quasi-elasticity. The marked area in figure 8.11 represents the weighted influence of IT spillovers and the area between the IT spillovers (q_spiliti) and the total spillovers (q_.spiltot1) represent the non-IT spillovers. The estimated time trend equation for the weighted IT spillovers is: qe_spilit1 $=0.03(1.40)+0.01(3.26)$ * time, and for the non-IT spillovers is: qe_spilnt1 $=0.13(6.48)+0.004(1.53)$ time. The influence of IT spillovers is increasing significantly over time while the influence of non-IT spillovers is more or less constant over time. The trend term in the total spillovers is therefore caused by IT spillovers.

A measure which can indicate the usefulness of a piece of knowledge generated by other industries for the own industry is the marginal productivity of knowledge. 
Increased codification may imply that this measure should have increased over time. The weighted marginal productivity for total R\&D spillovers is:

$$
m p\left(S_{\text {toto }}\right)=\sum_{i} \frac{q e\left(X_{i}\right)}{m\left(\frac{X_{i}}{Y}\right)} \frac{m\left(X_{i}\right)}{\sum_{i} m\left(X_{i}\right)}=\sum_{i} \frac{q d\left(X_{i}\right)}{\sum_{i} m\left(\frac{X_{i}}{Y}\right)}
$$

where $X_{i}=$ RENTKN, RENTKIT, RKNT and RKIT in the case of regression $i, m p\left(X_{j}\right)$ is the marginall productivity or rate of return of variable $i$ and $m\left(X_{i} / Y\right)$ is the mean value of variable $i$.

A graphical illustration of the development of the weighted marginal productivity of total spillovers is given in figure 8.12. The marginal productivity is increasing over time which indicates that each unit of knowledge generated by other sectors is more valuable to a sector.

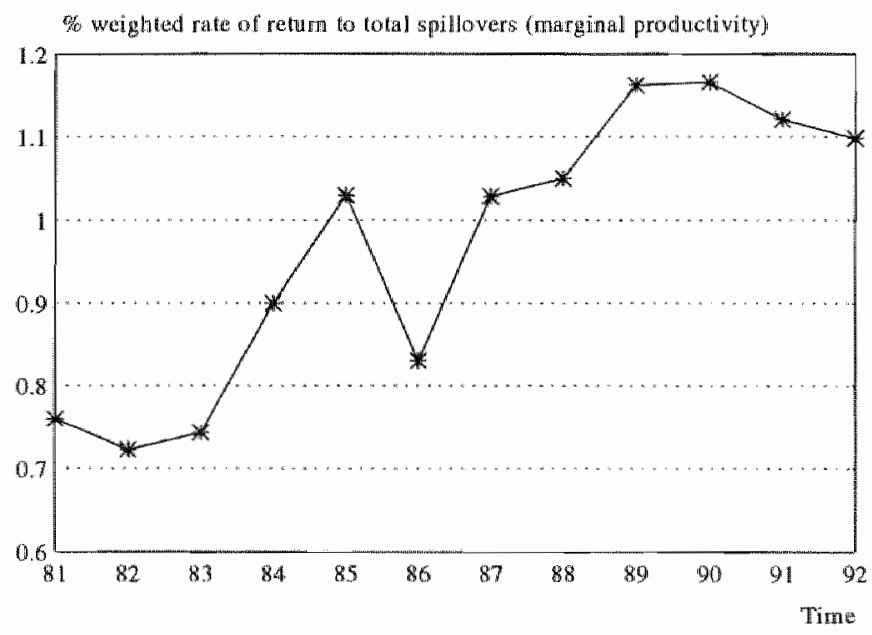

* Total Spillovers

Figure 8.12. Weighted rate of return to all spillovers (calculated from results of regression 1)

When we put equation (8.5) in growth rates it can be stated that the growth rate of the quasi-elasticity is equall to the growth rate of the marginal productivity and the growth rate of the total spillover intensity. Assuming a constant growth rate over the total period implies that the growth rate of the quasi-elasticity is $4.6 \%$ each year; $3.1 \%$ is caused by an increase in the marginal productivity each year and $1.5 \%$ by an increase in the use of the amount of spillovers per unit output or spillover 
intensity. Therefore two thirds of the increased influence of spillovers is caused by an increase in the marginal productivity and one third by the use of more spillovers per unit of output.

In conclusion we can say that our empirical results support the view that spillovers are becoming more important in the current information technology era.

\subsection{Reconsidering the "IT Productivity Paradox"}

The empirical results in sections 8.4 and 8.5 indicate that IT has a significant influence on the productivity level of the whole economy. These results suggest that the "TT Productivity Paradox" has lost its "paradoxical" characteristics. In this section we examine whether our results are exceptional.

Table 8.10 Recent Empinical Studies and Productivity

\begin{tabular}{|c|c|c|c|}
\hline Study & Sample & IT & Remarks \\
\hline Bresnathan (1986) & $\begin{array}{l}\text { Financial } \\
\text { services } \\
\text { USA:'52-79. }\end{array}$ & & $\begin{array}{l}\text { High spillovers from } T \text { to financial } \\
\text { service sectors. }\end{array}$ \\
\hline $\begin{array}{l}\text { Siegel-Griliches } \\
\text { (1992) }\end{array}$ & $\begin{array}{l}\text { Industry } \\
\text { USA: '88-'911 }\end{array}$ & & $\begin{array}{l}\text { positive correlation between } \\
\text { productivity growth and } \| T\end{array}$ \\
\hline Latu-Tokutsu (1992) & $\begin{array}{l}\text { Aggregate: } \\
\text { USA: '60-'90 }\end{array}$ & $\beta_{\mathrm{IT}}=7 \%$ & $\begin{array}{l}50 \% \text { of real output growth can be } \\
\text { attributed to IT }(\vec{Y}=3.1 \% \text {, } \\
\left.\hat{R}_{T T}=21.5 \%\right) \text {. }\end{array}$ \\
\hline Lichtenberg (1993) & $\begin{array}{l}\text { Firm } \\
\text { USA: ' } 88-91\end{array}$ & $\begin{array}{l}\beta_{\mathrm{rr}}=10 \%-13 \% \\
\alpha_{\mathrm{TI}}=6 \%-18 \%\end{array}$ & $\begin{array}{l}\text { Positive and "excess" returns to IT } \\
\text { capital and IT labour }\end{array}$ \\
\hline $\begin{array}{l}\text { Brynjolfsson-Hitt } \\
(1993)\end{array}$ & $\begin{array}{l}\text { Firm } \\
\text { USA: } 87-91\end{array}$ & $\begin{array}{l}\beta_{\mathrm{II}}=0.6 \% \\
\alpha_{4 I}=2.7 \%\end{array}$ & $\begin{array}{l}\text { Positive and "excess" returns to IT } \\
\text { capital and IT labour }\end{array}$ \\
\hline & $\begin{array}{l}\text { Manuf. } \\
\text { Manuf. + serv. }\end{array}$ & $\begin{array}{l}\rho_{\mathrm{II}}=54 \%, \rho_{\mathrm{K}}=4 \% \\
\rho_{\mathrm{IT}}=69 \%, \rho_{\mathrm{KC}}=7 \%\end{array}$ & \\
\hline Bernstein (1995a) & $\begin{array}{l}\text { Manuf. } \\
\text { CAN:"66-91 }\end{array}$ & $\begin{array}{l}66-70: 0.025 \\
71-75: 0.021 \\
76-80: 0.016 \\
71-85: 0.063 \\
86-91: 0.077\end{array}$ & $\begin{array}{l}\text { Contribution of spillover from } \\
\text { communication equipment to } \\
\text { average annual TFP growth rates } \\
\text { manufacturing increased over time. }\end{array}$ \\
\hline
\end{tabular}

Bresnahan (1986) found that the spillovers from computers to the financial services were high during the 1958-1972 period. The financial service sectors can be seen as 
one of the first sectors in which computers are particularly valuable. Bresnahan calculated the welfare gains from using computers in the financial service sector for the period 1958-1972 using a different methodology than we used in this chapter. He showed that the area under the derived demand curve for an intermediate input (adjusted for income effects) is a welfare index as well. Bresnahan's results suggest that the gains from using computers in financial services were high: 3 to 6 times as high than the expenditures for the finamcial services when the expenditure elasticity of demand is one and 11 to 19 times as high when this elasticity is thee.

Some other empirical studies used the general production function to estimate the productivity of IT capital. They separate the total capital stock into an IT and a non-IT capital stock in a Cobb-Douglas production function and estimate the marginal product or output elasticity of IT capital.

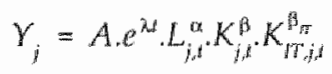

$K$ measures the non-IT capital stock and $K_{\text {ar }}$ measures the IT capital stock. The parameter of interest in these studies is $\beta_{V T}$, the output elasticity of IT capital or the related marginal product of IT capital $\left(\rho_{t T}=\beta_{1 T}\left(Y / K_{T I}\right)\right)$. The empirical studies investigate whether or not IT has a contribution to output growth, or whether or not $\beta_{i T}$ is positive. Whereas studies based on the 1970 s and early 1980 s, such as Loveman (1988), did not find a positive value for $\beta_{\mathbb{V}}$, studies based on recent data did find a positive influence of IT capital (see table 8.11). Lichtenberg (1993) and Brynjolfsson and Hitt (1993) used detailed US firm data from the late 1980s and early 1990s. They found a positive and significant value for the output elasticity of IT capital $\left(\beta_{r T}=10 \%-13 \%, \beta_{r T}=0.6 \%\right.$, respectively).

A second objective is to investigate whether the return to IT investment is higher than the return to non-IT investment. ${ }^{121}$ The results found by Lichtenberg and Brynjolfsson and Hitt (1993) also imply excess returns to IT capital. Another interesting result of the Bryrjolfsson and Hitt (1993) study is that the rates of returns to IT capital $\left(\rho_{\text {in }}\right)$ are higher when one considers manufacturing and service firms $\left(\rho_{r r}=69 \%\right)$ than when one only considers manufacturing firms $\left(\rho_{m}=54 \%\right)$. This indicates that the rates of returns to IT capital are higher in the services than in manufacturing sector.

The Lichtenberg and Brynjolfsson and Hitt studies also separated also labour into IT-oriented labour and non-IT-oriented labour and found that the IT-labour elasticities $\left(\alpha_{1 T}\right)$ were positive and there were excess returns to IT labour, although the size and significance of the excess return to IT capital is larger.

120 Tho second bypothesis is that the ratio of the marginal products of IT and non-IT capitall is less than the ratio of their nental prices (see Lichtemberg $1993, \mathrm{p}, 6$ ). 
Two other studies also found IT to have a positive influence. Siegel and Griliches (1992) found a positive correlation between productivity growth and IT. The LauTokutsu (1992) study found that $50 \%$ of real output growth can be attributed to IT.

Bernstein (1995a) studied the spillovers from the communication sector to the manufacturing sector in Canada for the 1966-1991 period. Consistent with our results Bernstein concluded that the importance of spillovers increased over time: "Moreover, the importance of the communication equipment industry grew in the post slowdown era. From 1966 to 1973 around $2 \%$ of annual TFP growth is contributed by the spillover from communicating equipment. The contribution increased to about 22\% from 1974 to $1991^{\prime \prime}$ (pp. 20-21).

Some evidence suggest that the "IT Productivity Paradox" is loosing its paradoxical characteristics. Bresnahan (1986) found a positive influence of IT in financial services during the 1958-1972 period. However, funancial services is a sector in which IT diffused rather early. Lau and Tokutsu (1992), Lichtenberg (1993), Brynjolfsson and Hitt (1993), and Bernstein (1995a) found a positive influence of IT on productivity for the whole economy and their results are therefore consistent with our findings. It seems that IT has diffused on a large enough scale in the current period and that its influence is actually measurable.

\subsection{Conclusion}

In this chapter we tested two important hypotheses underlying our theoretical endogenous technological change model. First, we investigated the expected "special" influence of information technology on productivity growth and second, we studied whether R\&D spillover effects are becoming more important during the IT era.

The data description showed that the three IT sectors are small in economic terms. They only represent $1.87 \%$ of actual production. However in technological terms they are quite important because they perform $26.2 \%$ of total R\&D. The data also showed that $\mathrm{IT}$ investments and production grows much faster than non-IT investments and production. The IT share in total investments and actual production doubled in 15 years. This increase is under-stated because the huge quality improvements in the IT sectors are not reflected in the price indices.

In the second part of this chapter we measured the importance of rent and "pure" knowledge spillover effects related to IT and non-IT sectors and studied their influence over time. The influence of "own" R\&D remained more or less constant over time while the influence of spillover effects increased over time. The increase in the spillover effects is mainly caused by an increase in the IT spillover effects, and especially spillover effects of IT capital goods (e.g. computers). The large influence of IT spillovers is especially apparent since 1988 - it seems that the "IT productivity paradox" vanished in the most recent period. This supports the 
"lag" hypothesis - it takes time before the new general purpose technology (IT) has a measurable influence on all sectors of the economy.

Bresnahan (1986) found that the spillovers from computers to the financial services were high during the 1958-1972 period. The financial service sectors can be seen as one of the first sectors in which computers are particularly valuable. Our findings, which are consistent with the results of some other recent studies, indicate that the influence of computers/information technology has spread over the whole economy in the last lew decades. Information technology has become a real general purpose technology which can boost technological change in the whole economy.

Our estimates also support the view that spillovers are becoming more important in the IT era. One-third of the influence of codified knowledge or spillovers is caused by the use of more spillovers per unit of output while two-thirds are caused by the increased productivity of spillovers. 
Appendix 8A: TT spillowers and productivity: Estimates based on Gross value added

Table 8.A.1 Regressions of the two factor TFP growth rate. (t-ratio's in parentheses)

\begin{tabular}{|c|c|c|c|c|c|}
\hline$T F P_{-} C_{1}$ & CON & RRKTT & RENTKIT & RKNT & $\begin{array}{l}\mathrm{R}^{2} \\
\mathrm{DW}\end{array}$ \\
\hline A1.1:81 & $\begin{array}{c}0.46 \\
(0.84)\end{array}$ & $\begin{array}{l}0.94^{\text {nan }} \\
(5.42)\end{array}$ & $\begin{array}{l}0.23 \\
(2.34)\end{array}$ & $\begin{array}{l}0.65^{*} \\
(2.23)\end{array}$ & $\begin{array}{l}0.59 \\
2.20\end{array}$ \\
\hline$A 1.2: 82$ & $\begin{array}{c}-9.27 \\
(-0.48)\end{array}$ & $\begin{array}{l}1.22^{4} \\
(6.84)\end{array}$ & $\begin{array}{l}0.44^{*} \\
(2.75)\end{array}$ & $\begin{array}{l}0.78^{\circ} \\
(2.64)\end{array}$ & $\begin{array}{l}0.69 \\
2.31\end{array}$ \\
\hline$A 1.3: 83$ & $\begin{array}{c}-0.82 \\
(-1.58)\end{array}$ & $\begin{array}{l}1.50^{3} \\
(9.02)\end{array}$ & $\begin{array}{l}0.52^{\circ} \\
(3.19)\end{array}$ & $\begin{array}{c}0.63^{\prime \prime} \\
(2.34)\end{array}$ & $\begin{array}{l}0.78 \\
2.09\end{array}$ \\
\hline A1.4:84 & $\begin{array}{c}-0.49 \\
(-0.69)\end{array}$ & $\begin{array}{l}1.52^{n+m} \\
(6.47)\end{array}$ & $\begin{array}{l}0.47^{\circ} \\
(1.88)\end{array}$ & $\begin{array}{c}0.71^{*} \\
(1.92)\end{array}$ & $\begin{array}{l}0.62 \\
2.26\end{array}$ \\
\hline A1.5:85 & $\begin{array}{c}0.03 \\
(0.05)\end{array}$ & $\begin{array}{l}1.07^{2 \ldots} \\
(4.58)\end{array}$ & $\begin{array}{c}0.36 \\
(1.40)\end{array}$ & $\begin{array}{c}0.70^{\prime} \\
(1.96)\end{array}$ & $\begin{array}{l}0.48 \\
2.53\end{array}$ \\
\hline A1.6: 86 & $\begin{array}{c}0.28 \\
(0.45)\end{array}$ & $\begin{array}{l}0.47^{*} \\
(2.29)\end{array}$ & $\begin{array}{c}0.43 \\
(1.66)\end{array}$ & $\begin{array}{c}0.40 \\
(1.26)\end{array}$ & $\begin{array}{l}0.20 \\
2.38\end{array}$ \\
\hline A $1.7: 57$ & $\begin{array}{c}0.57 \\
(0.92)\end{array}$ & $\begin{array}{c}0.15 \\
(0.76)\end{array}$ & $\begin{array}{c}0.47 \\
(1.52)\end{array}$ & $\begin{array}{c}0.51 \\
(0.17)\end{array}$ & $\begin{array}{l}0.00 \\
2.36\end{array}$ \\
\hline A.1.8:88 & $\begin{array}{c}0.62 \\
(0.88)\end{array}$ & $\begin{array}{c}0.19 \\
(0.92)\end{array}$ & $\begin{array}{c}0.57 \\
(1.44)\end{array}$ & $\begin{array}{c}0.10 \\
(0.29)\end{array}$ & $\begin{array}{l}0.01 \\
2.37\end{array}$ \\
\hline A1.9:89 & $\begin{array}{c}0.26 \\
(0.32)\end{array}$ & $\begin{array}{l}0.65^{\circ} \\
(2.93)\end{array}$ & $\begin{array}{c}0.73 \\
(1.45)\end{array}$ & $\begin{array}{c}0.82 \\
(0.22)\end{array}$ & $\begin{array}{l}0.23 \\
2.34\end{array}$ \\
\hline A1:10:90 & $\begin{array}{l}-0.21 \\
(-0.25)\end{array}$ & $\begin{array}{l}0.90^{n+4} \\
(4.24)\end{array}$ & $\begin{array}{c}0.94^{\circ} \\
(1.79)\end{array}$ & $\begin{array}{c}0.20 \\
(0.55)\end{array}$ & $\begin{array}{l}0.41 \\
1.77\end{array}$ \\
\hline A1.11:91 & $\begin{array}{c}-0.46 \\
(-0.51)\end{array}$ & $\begin{array}{l}1.04^{5+\infty} \\
(4.51)\end{array}$ & $\begin{array}{c}0.88 \\
(1.52)\end{array}$ & $\begin{array}{c}0.41 \\
(0.10)\end{array}$ & $\begin{array}{l}0.41 \\
1.73\end{array}$ \\
\hline A.1.12:92 & $\begin{array}{l}-1.11 \\
(-1.19) \\
\end{array}$ & $\begin{array}{l}0.96^{\circ} \\
(4.11)\end{array}$ & $\begin{array}{r}0.82 \\
(1.34) \\
\end{array}$ & $\begin{array}{r}0.08 \\
(-0.21) \\
\end{array}$ & $\begin{array}{l}0.35 \\
1.68 \\
\end{array}$ \\
\hline
\end{tabular}

All the R\&D intensities are expressed in terms of gross value added.

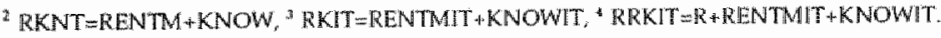

$\because$ and ${ }^{2}$ Significant at the 0.10 level, 0.01 level and 0.002 level (two-taniled reat). 


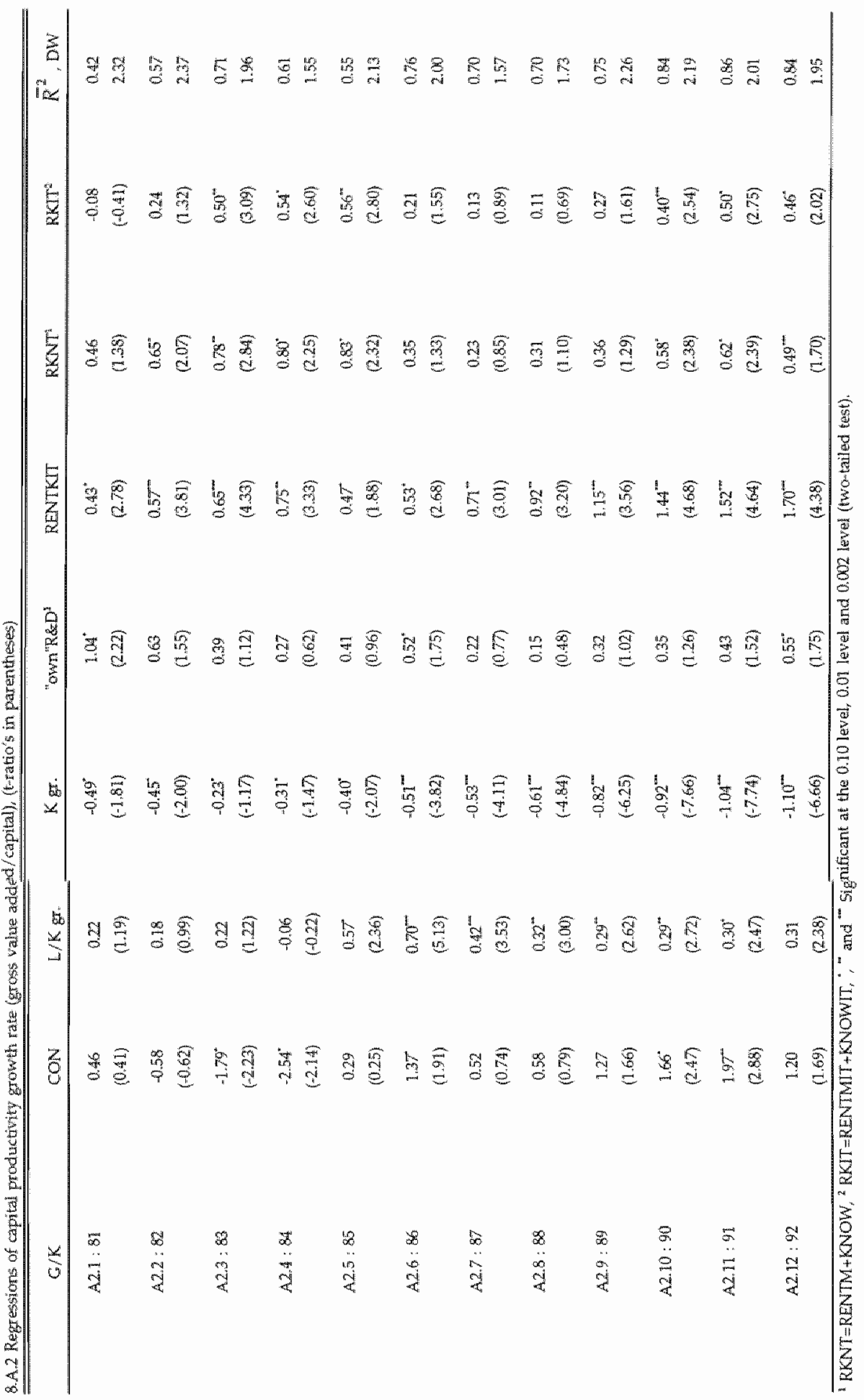


PART IV

SUMMARY 



\section{Summary}

This thesis examines the nature of the technology generation process in a theoretical and empirical way. We concentrated on three determinants of technological change: internal R\&D efforts, knowledge spillover effects and information technology, which is the current general purpose technology. This thesis consisted of 3 parts. The first part introduced the main concepts of this thesis and gave a selective survey of the literature on technological change. This part concluded with the identification of six elements of an endogenous technological change model. The second part developed a theoretical endogenous technological change model based on these elements. The third part was devoted to some empirical tests of the model developed in part II. Crucial hypotheses tested in part III were whether internal $R \& D$ efforts, $R \& D$ spillover effects and information technology have a positive influence on technological change and whether the influence of spillover effects and information technology increased over time.

This chapter summarizes the thesis and reviews the main findings. Each part will be discussed in a separate section.

\subsection{The Process of Technological Progress is Changing}

Chapter 1 provided a short overview of current developments in the field of technological change. We observed that the contemporary world is characterised by rapid technological change. A clear example is the enormous rate of technological change in the information technology (IT) sector. This high rate of technological change in $\mathbb{I T}^{\circ}$ influences the whole economy and it induces changes in the production process, knowledge creation process, pattern of employment and social and institutional infrastructure. These changes are often called the "information technology revolution". These adjustments are not easy and instantaneous: people have to acquire different skills, production processes have to be adjusted, some sectors decline while other sectors emerge, some people loose their job while others obtain a job, etc. However, we have not concentrated on these important adjustments. Instead we have focused on some fundamental forces that drive economic growth and how they are affected by the information technology revolution. 
In this study we focused on the dual nature of technological change. The gentration and distribution of knowledge were accepted as two fundamental forces of the process of technological change. For many years internally performed R\&D (knowledge generation) was the principal source of knowledge for industrial firms. Knowledge was mainly obtained by trial-and-error procedures based on physical experiments. Knowledge was firm specific, local and context dependent and therefore mainly of the "tacit" nature. However, the information technology revolution affected the knowledge generation process. Enormous improvements in computer power, together with an improved theoretical understanding of problems and improved instrumentation, changed the nature of knowledge from tacit to more codified knowledge (Ergas 1994, Arora and Gambardella 1994). This implies that knowledge created by other firms can be more easily transferred (knowledge distribution) and that knowledge becomes more valuable to other firms. This increases the value of spillover effects and external knowledge sources. One important hypothesis in this thesis was that spillover effects are becoming more important in the IT area and that the relative importance of internal R\&D diminishes relative to external knowledge sources.

Larger spillover effects have two major influences on the knowledge creation process. First, the productivity of own research efforts increases if a firm can use more of the knowledge created by other firms. This is an incentive effect for internal $R \& D$ efforts. Second, the appropriability of internal $\mathbb{R} \& D$ declines, which means a disincentive effect for technological change.

Besides the important influence of IT on the vallue of spillovers, we argued that IT becomes an important tool in the knowledge creation process. Computer simulations substitute for physical experiments because they are often cheaper and faster. IT therefore improves the productivity of internal R\&D efforts. IT also influences the process of technological change directly, because IT products are used to "smooth" the process of production.

The appearance of IT promised therefore huge productivity gains (Freeman and Soete 1990). However, these huge productivity gains were not found by studies that actually measured the relationship between IT and productivity. Bailey and Gordon (1988) commented upon this situation by asking: "where is the "black hole" into which all those computers are disappearing?". The discrepancy between the promised and the actually measured productivity gains became known as the "IT productivity paradox".

Besides measurement errors, redistribution, and mismanagement a possible explanation for this paradox is the "lag" hypotheses (Brynjolfsson 1993). It may take decades before the potential benefits of a new general purpose technology appear because the plain diffusion, the development of complementary assets, and the change of the social and economic "way of live" are difficult (Freeman and Perez 1988, Helpman and Trajtenberg 1994). An interesting historical analogy to the case of IT is the experience with the introduction of electric power (Freeman and Soete 
1987, David 1992). Contemporary developments with regard to the price (rapid decline) and diffusion (rapid increase) of IT may indicate that the time is coming closer that we are able to measure the influence of IT and the "IT productivity paradox" is loosing its "paradoxical" characteristics. The latter is our second hypothesis.

Chapter 2 gave a selective survey of the literature on technological change and growth. We reviewed the endogenous growth theories and some contributions which model the influence of a key or general purpose technology. From these contributions we obtained six basic elements for an endogenous growth model. First, a model that has to deal with growth should treat technology as endogenous. Second, technology does not appear as a side-product of other activities but is the intentional outcome of economic behaviour. Resources have to be "invested" to create knowledge and technological change. Third, a part of the knowledge created should be appropriable to earn back the resources of knowledge creation (private properties of knowledge). Fourth, knowledge also has some public (non-rival) features - a part of the knowledge spills over to other firms (externalities). Fifth, some dominant, key or general purpose technologies exist that influence all sectors of the economy. Sixth, these general purpose technologies are special because they are characterised by large technological opportunities and they may create incentives for other sectors to invest in technological change. In chapters 3 to 5 we attempted to incorporate these basic elements into a model.

\subsection{Theoretical Considerations on Endogenous Technological Change}

In chapter 3 we developed a simple model to treat technological change. Firms "invest" in knowledge creation to generate technological change and obtain benefits in the future. We only considered the influence of internal $R \& D$ expenditures on knowledge creation. This model describes the traditional knowledge generation model in which internal R\&D was the foremost principal knowledge source and knowledge spillovers and IT were of minor importance. Our model differs from most models, such as, for example, Dasgupta and Stiglitz (1980), Spence (1984), and De Bondt et al (1992) because we identified two different kinds of knowledge. First, knowledge which is directed at increasing the efficiency of the production process or "cost-reducing" knowledge. It can be expected that this kind of knowledge is generated by performing process-oriented R\&D. Second, knowledge that is directed at enhancing the quality level of a product or "demand-creating" knowledge. Demand creating knowledge can be generated by engaging in product $\mathrm{R} \& \mathrm{D}$. This is in line with the Levin and Reiss (1988) model. Our model, however, treated the knowledge process in a dynamic context and recognised that the benefits of current R\&D expenditures are spread over time. Because of the inter-temporal effects of $R \& D$, we used optimal control theory to solve the models. The latter usually 
implies that the models themselves are (over-) simplified representations of economic behaviour/constraints.

An interesting feature of this model is our treatment of the relationship between quality and demand. We assumed that it is the "perceived" quality level that influences demand and not the "pure" quality level. Perceived implies that it is the quality level relative to other products that is important for demand. In this simple single firm model we treated the behaviour of competitors exogenously. The perceived quality level depreciated each year through product improvements by competitors. This catches the Schumpeterian idea that benefits to innovations are temporary and that a firm should continually innovate to survive. In chapter 5 we endogenised the "perceivedness" of the quality levell by introducing the behaviour of competitors. The depreciation of the perceived quality level was dependent on the $R \& D$ investments of competitors.

Technological progress (innovations) is generally favoured by factors which increase demand or by factors which increase the efficiency of the perceived quality or productivity generation process. Cost factors, such as the interest rate and the price of $\mathbb{R} \& D$, temper technological progress. An interesting result was that the steady state R\&D intensities turned out to be dependent on only a few structural parameters. Again high technological opportunities and favourable demand conditions lead to a high R\&D intensity. We discovered that vigourous quality competition, reflected in a rapid depreciation of a firms' perceived quality increases the product $R \& D$ intensity. Product and process $R \& D$ turned out to be complements rather than substitutes in our analysis because they reinforce each other (the total $\mathbb{R} \& D$ budget can be freely chosen).

Chapter 4 elaborated this model with some of the special influences of information technology and the incentive aspect of the "public" nature of knowledge. The purpose of IT goods is to process information implying that its role in the production process differs from the role of other capital goods. IT investment goods are used to "smooth" the process of production while other capital goods are used together with labour to produce output and therefore possess a direct cost-reducing role (Van Zon 1991). IT capital goods are aimed at improving perceived quality. This occurs when "pure" quality increases (e.g. better design by using $C A D$, better monitoring of manufacturing), service improves (better and faster communication channels), shorter delivery time (better communication) and more product variety (flexible production). It can be expected that IT helps to increase demand; therefore IT goods also possess demand creating characteristics. The model developed in chapter 3 is well suited to treat the special characteristics of IT (i.e. the cost-reducing and demand creating aspects of IT).

We also introduced the influence of IT on the knowledge creation process. On the one hand IT improves the tools in the knowledge creation process and on the other hand is IT linked to the fundamental shift in the nature of knowledge (increased codification). Spillovers are becoming more important. Knowledge 
spillovers increase the productivity of internal R\&D efforts in this chapter. One firm can benefit from another firms" R\&D because a part of the R\&D "spills over:" to other firms.

The decline in IT prices and the strong increase in the efficiency units of IT goods caused the price in efficiency units of $\mathrm{T}$ goods to decline rapidly. We investigated the influence of this rapid decline in the price per efficiency units on technological change. It turned out that the combination of the special characteristics of IT (e.g. direct and indirect influence on technological change) and the rapid decline in the price per efficiency units allowed $T$ to enhance technological change in sectors.

We also studied the influence of market segmentation on technological progress. A larger market share in a more competitive market or a more sophisticated demand boosts technological change.

In chapter 5 we consider the appropriability diminishing effect of knowledge spillovers next to the incentive effect of spillovers (chapter 4 ). The central question in this chapter was therefore whether spillover effects tempers or stimulates technological progress. This question becomes really important in this 17 era because it is closely linked to the hypothesis that knowledge becomes more of the "blueprint" kind than of the tacit kind.

Chapter 5 addressed this important question in a simple model with endogenous technological change. The most important finding from this study is probably that there is no simple relationship between spillovers and technological change. It is not possible to conclude that higher spillovers temper a firms incentives to invest in R\&D (the Spence (1984) model) or stimulate technology investments (as was the case in chapter 4). Spence obtained his well-known result because he did not consider that more spillovers enhance the productivity level of internal technology investments. Chapter 4 neglected that internal created knowledge also increased economic performance of competitors. This chapter combined both effects which makes the general influence of spillovers on technology investment incentives dependent on a number of other determinants of technological change. Apart from the degree of knowledge spillovers, consumer preferences and entry have an important influence on technological progress. It is therefore the complex interplay between spillover effects, product differentiation and entry that determine the level of technological change. One has to know the exact values of the inter- and intraindustry level of product differentiation, the value of the extent and productivity of spillover effects and the number of firms to draw conclusions about the influence of a change in one of these variables on incentives to invest in technological change. However, there is one general tendency in our results. Larger spillover effects tend to reduce the internal $R \& D$ efforts but also tend to increase the obtained technological level because firms use more knowledge created by other firms. 
This general tendency also implies that higher knowledge spillovers lead to a Larger number of viable firms in a market. With high knowledge spillovers, firms spend less on internal R\&D but use more of knowledge generated by other firms. This increases the number of viable firms in a market. This implies that in this IT era the number of viable firms in a market may increase. Technological change will not depend on a few firms that create the largest part of knowledge themselves but will depend on a larger number of firms that create a minor part of knowledge themselves and mainly rely on knowledge created by other firms. Furthermore, high technological opportunities and lowly differentiated products normally lead to more concentrated markets.

This model also determined an interesting relationship between entry and profits. High technological opportunities and lowly differentiated products cause an industry structure, that is characterised by a few competitors or a large number of competitors; an intermediate level of rivalry is not viable. Entry increases the degree of competition and therefore lowers the gross profit rate (mark-up over variable costs) which is necessary to cover fixed R\&D costs. This traditional effect limits the number of viable firms in a market. In our model entry also means that in general the external knowledge stock increases and that the internal R\&D efforts decline. If the traditional effect dominates when the number of firms is small and the second effect when the number of firms increases then the described industrial structure is possible. Depending on the welfare implication a concentrated or a competitive industry is desirable. Temporary government policy is necessary if one industry is locked in the wrong industry structure.

\subsection{Empirical Results}

In the first part we developed an endogenous technological change model. Knowledge and information technology were considered as two important determinants of technological change. In the second part we empirically investigated the importance of these determinants. Central in the empirical chapters are two hypotheses. First, knowledge created by other sectors is important for the level of technologicall change in a sector and this influence is increasing in the IT area. Second, information technology has a special influence on the process of technological change. Chapter 6 gave a survey of the results of previous empiricall studies. In chapter 7 we measured the importance of internal R\&D and various spillover concepts and in chapter 8 we investigated the influence of IT and the development of the various spillover effects over time.

The impact of the knowledge stock on technological change was the central focus of a large number of the empirical studies, reviewed in chapter 6. Instead of measuring the influence of the knowledge stock directly these studies concentrated on the determinants of the knowledge stock because it is itself unobservable. The principal determinant in most studies are the internal or own R\&D efforts. Most 
studies found a statistically significant and often plausible relation between $R \& D$ and productivity at all levels of aggregation (firm, sector, country and world level).

We hypothesized that studies that only consider internal R\&D efforts as explanations of technological change are characterised by a specification bias which importance is expected to increase in the IT era. The specification bias is caused by neglecting the various spillover concepts. It can be expected that external knowledge sources becoming increasingly important due reasons explained earlier. There are various external knowledge sources whose importance differs for different sectors. The problem in econometric studies is always how R\&D efforts of other sectors or firms should be weighted to build a sector specific knowledge stock.

According to Griliches (1979) spillover effects may occur for two reasons. "Pure rent spillovers" exist because purchasing firms pay less than the full quality price while "knowledge spillovers" exist because knowledge created in one industry can be used in other industries. Knowledge spillovers may be related or unelated to input purchases. We argued that this theoretical division of spillovers is not possible in an empirical sense. However, another meaningful division can be made in econometric studies. On the one hand we can identify spillovers that are related to input purchases. These spillovers include "pure rent spillovers" and knowledge spillovers that are linked to intermediate and investment purchases. We define these spillovers as rent spillovers. It may be expected that these rent spillovers are more important the larger the input purchases from a sector and the more R\&D is embodied in a product. Input-output matrices and investment matrices can be used to measure these spillovers. Rent spillovers are therefore linked to the "economic network" of a sector. On the other hand knowledge spillovers that are unrelated to input purchases ("pure knowledge spillovers") exist. According to Griliches (1979, 1992), knowledge spillovers are larger the more closely connected sectors ane in technological terms. The weights of the R\&D of other sectors may be approximated by a patent or innovation-base technology-flow matrix (for example Scherer 1984. Evenson et all 1988) or the proximity in technological space (for example Jaffe 1986. 1988). "Pure" knowledge spillovers are therefore linked to the "technological network" of a sector. In chapter 7 and 8 we used the Yale technology flow matrix developed by Evenson et al (1988) to approximate the technological network of a sector. An econometric model that includes all these spillovers should therefore include a measure for investment related rent spillovers, intermediate goods related rent spillovers and "pure knowledge spillovers".

The survey of econometric studies that includied R\&D spillover concepts showed that all econometric studies included either a measure for rent spillover or a measure for "pure knowledge spillovers". All of these studies therefore only included a part of the total spillovers and neglected some determinants of productivity growth. In general, these empirical studies found a positive significant influence for the spillover variable. In general, rent spillover of capital goods had a 
higher rate of return than those linked to intermediate goods. The magnitude of "pure" knowledge spillovers is dependent on the method used to approximate the technological closeness of sectors.

Chapter 7 began with the development of an econometric technological change model. This model incorporates four main features that are usually neglected in other empirical studies. First, R\&D expenditures are divided into process-oriented and product-oriented $R \& D$. Second, product improvements may influence unit production costs directly. Third, the model recognises the data problem that quality improvements are badly reflected in price indices and fourth, the model is unique in the sense that it includes rent spillovers of investment goods, rent spillovers of intermediate goods and non-input related knowledge spillovers in one framework. The database consists of thirty French sectors which covered the whole economy for the recent 1978-1992 period.

The model predicts that process R\&D has a direct influence on productivity and that product $R \& D$ expenditures influence these measures when quality is reflected in price indexes or when quality improvements directly influence unit production costs. In general, despite the straightforward theoretical division of R\&D into process-oriented and product-oriented $R \& D$, it is a difficult job in an empirical sense. Empirical results on both kinds of R\&D must be carefully interpreted. The survey in chapter 6 showed that the expected positive influence of process $R \& D$ on productivity is found in most studies while the influence of product R\&D on productivity was generally insignificant. However, there is a slight indication that the influence of product $R \& D$ on productivity is increasing over time. In contradiction with most earlier studies we found that product $R \& D$ has a positive influence on a sectors productivity growth. This indicates that product R\&D directed at improving products is also directed at making new products with less production factors or that quality improvements are partly reflected in price indexes. We concluded that quality improvements continue to be poorly reflected in prices which indicated that product $R \& D$ is also directed at reducing costs. We also believe that it becomes increasingly difficult to separate $R \& D$ expenditures into product-oriented and process-oriented $R \& D$ because these activities have become more and more interrelated in the IT area.

An important finding in chapter 7 was that in addition to internal $R \& D$, all our three spillover concepts were statistically significant. The largest influence came from rent spillovers related to capital goods. Rent spillovers related to intermediate goods and "pure knowledge spillovers" were also important and their magnitude was comparable to the influence of internal R\&D efforts. The inclusion of all these spillover concepts was really important when one investigates the influence of the various R\&D variables for different groups of sectors. In the high-tech sectors own $R \& D$ in combination with "pure" knowledge spillovers are very important. Services rely on rent spillowers from capital goods while other sectors mainly use rent 
spillovers from intermediate goods. We can conclude that studies who neglect one of the spillover concepts miss one determinant of technological change.

Chapter 8 may be considered as the key chapter in this thesis because it investigated two important hypotheses. First, it investigated the expected positive influence of IT on productivity. Second, it investigated the nagnitude of spillovers over time. With regard to the latter hypothesis, it turned out that the magnitude of spillovers is increasing over time while the influence of internal $R \& D$ efforts remained more or less constant over our estimation period. This indicates that external knowledge sources are getting more important for productivity growth in comparison with internal $R \& D$ efforts over time.

The first hypothesis was also supported because it turned out that IT-related spillovers were important for productivity growth and that the measured influence increased rapidly over time. Our data indicated that the "IT productivity paradox" has disappeared. This result is consistent with some other empirical studies that used recent data such as Brynjolfsson and Hitt (1993) and Lichtenberg (1993).

In particular the magnitude of IT investment spillovers (related to e.g. computers) increased very fast. Our opinion is that this large and very significant influence is caused by several features. The first is the expected direct productivity influence of IT. Second, the expected influence of IT on the productivity of internal R\&D (better tools). Third, the predicted influence of IT on spillovers. IT enhances existing communication chanuels and makes networks more important. Furthermore, IT is related to the fundamental shift in the nature of knowledge. Knowledge becomes more of the codified than of the tacit kind, which makes spillover more valuable. Fourthly, this shift in the nature of knowledge is related not only to IT but also to a better theoretical understanding of problems and improved instrumentation (Arora and Gambardella 1994). IT investments are closely connected with the "changing technology of technical change" (Rosenberg in Arora and Gambardella 1994). In conclusion we can say that the large estimated influence of IT spillovers reflects not only the influence of IT goods in separation, but reflects that the whole system of production and knowledge creation is changing. 



\section{References}

Abramovitz, M., 1956, Resource and output trends in the United States since 1870, American Economic Review, Papers and Proceedings, Vol. 46, No. 2, pp. 5-23.

Acharya, R. and T. Ziesemer, 1994. A Closed Economy Model of Horizontal and Vertical Product Differentiation, Innovation in Biotechnology, Paper presented at Eunetic Conference on "Evolutionary Economics of Technological Change", Strassbourg, October. Acs, Z., D. Audretsch and M. Feldman, 1992, Real effects of academic research: comment, American Economic Review, Vol. 82, No. 1, pp. 363-367.

Adams, J.D., 1990. Fundamental stocks of knowledge and productivity growth, Joumal of Political Economy, Vol. 98, No. 41, pp. 673-702.

Aghion, P, and P. Howitt, 1992, A Model of Growth Through Creative Destruction, Econometrica, Vol. 60, pp. 323-351.

Amable, B., 1993, Radical and Incremental Innovation: A Model of Endogenous and Unsteady Growth, mineo, January.

Amable, B. and R. Boyer, 1992, The R\&D-productivity relationship in the context of new growth theories, mimeo.

Armington, P.S., 1969, A theory of demand for products distinguished by place of production, International Monetary Fund Staff Papers, Vol. 16, pp. 159-178.

Arora, A. and A. Gambardella, 1994, The changing technology of technological change: general and abstract knowledge and the division of innovative labour, Research Policy, Vol. 23 , pp. $523-532$.

Arrow, K.J., 1962. The Economic Implications of Leaming by Doing, Review of Economic Studies, Vol. 29, pp. 155-173.

Baily, M.N. and R.J. Gordon, 1988, The Productivity Slowdown, Measurement Issues, and the Explosion of Computer Power, Brookings Papers of on Economic Activity, Vol. 192. pp. $347-420$.

Barro, R.J, and X. Sala-i-Martin, 1992, Public Finance in Models of Economic Growth, Review of Economic Studies, Vol. 59. No. 4, pp. 645-661.

Barro, R.J. and X. Sala-i-Martin, 1995, Economic Growth, Mc Graw Hill, New York.

Basant, R., 1993, R\&D, Foreign technology purchase and technology spillovers in Indian industry: some explorations, UNU/INTECH Working Paper, No. 8.

Berndt, E.R. and C.J. Morrison, 1994. Hightech Capital Formation and Economic Performance in U.S. Manufacturing Industries: An Exploratory Analysis, Journal of Econometrics.

Berndt, E.R., C.I. Morrison and L. Rosenblum, 1992, High-tech capital formation and labor 
composition in U.S manufacturing industries: an exploratory analysis, NBER Working Paper, No. 4010.

Berndt. E.R. and J.E. Triplett, 1990, Fify Years of Economic Measurement: The Jubilee of the Conference on Research in Income and Wealth, Studies in Income and Wealth, Voll. 54, The University of Chicago Press, Chicago.

Bemstein, J.I., 1988, Costs of production, intra- and interindustry R\&D spillovers: Canadian evidence, Canadian Joumal of Economics, Vol. 212, pp. 324-347.

Bernstein, J.I., 1989. The structure of Canadian interindustry R\&D spillovers, and the rates of retum to R\&D, Journal of Industrial Economics, Vol. 373, pp. 315-328.

Bernstein, J.I. 1995a, The Canadian communication equipment industry as a source of R\&D spillowers and productivity growth, Paper presented at the Conference of Implications of Knowledge-Based Growth for Micro-Economic Policies, March 30-31, Ottawa, Ontario.

Bernstein, J.I., 1995b, International R\&D spillovers between industries in Canada and the United States, social tates of return, and productivity growth, Canadian foumal of Economics, Papers and Proceedings, (fotthcoming).

Bernstein, J.I. and P. Mohnen, 1994, International R\&D spillovers between U.S. and Japanese R\&D intensive sectors, NBER Working Paper, No. 4682.

Bernstein, J.I. and M.I. Nadiri, 1988, Interindustry $\mathbb{R} \& \mathbb{D}$ spillovers, rates return, and production in high-tech industries, American Economic Papers and Proceedings, pp. 429 434.

Bernsteirn, J.I. and M.I. Nadiri, 1989, Research and development and intra-industry spillovers: an empirical application of dynamic duality, Review of Economic Studies, Vol. 56, pp. $249-269$.

Bernstein, J.L and M.I. Nadiri, 1991, Product demand, cost of production, spillovers, and the social rate of return to R\&D, NBER Working Paper, No. 3625.

Brander, J.A. and B.J. Spencer, 1985, Export Subsidies and International Market Share Rivalry, Joumal of Intermational Economics, Vol, 18, pp. 83-100.

Bresnahan, T., 1986, Measuring the spillovers from technical advance: Mainframe computers in financial services, American Economic Review, Vol. 74, No. 4, pp. 743-755.

Bresmahan, T. and M. Trajtenberg, 1992, General Purpose Technologies: 'Engines of Growth?", NBER Working Paper, No. 4148.

Brooke, G., 1991, Information Technology and Productivity: An economic analysis of the effects of product differentiation. Ph.D thesis, University of Minnesota.

Brown, M. and A. Conrad, 1967. The Intluence of Research on CES Production Rellations, un M. Brown (ed.), The Theory and Empirical Analysis of Production, Studies in Income and Wealth. Vol. 3. New York: Columbia University Press for NBER, pp. 275-340.

Brynjolfsson, E., 1993, The Productivity Paradox of Information Technology, Communicarions of ACM, Vol. 36. December, pp. 66-77.

Brynjolfsson, E, and L. Hitt, 1993. Is Information Systems Spending Productive? New Evidence and New Results, Proceedings of the 14th Intemational Conference on Information Systems, Ortando.

Brynjolfsson, E. and L. Hitt, 1994, Information Technology as a Factor of Production: The Role of Differences Among Firms, mimeo.

Caballero, R.J. and R.K. Lyons, 1990, Intemal versus External Economies in U.S. Manufacturing, European Economic Review, Vol. 34, pp. 805-830.

Chiang. A.C., 1984, Fundamental Methods" of Mathematical Economics, Third Edition, 
MoGraw-Hill Book Company, Singapone.

Chiang, A.C., 1992, Elements of Dynawnic Optimization, MoGraw-Hill, New York.

Chow, G.C., 1967, Technological Change and the demand for Computers, American Economic Review, Vol. 57. No. 5. December, pp. 1117-1130.

Clark, J., C. Freeman and L. Soete, 1982, Unemployment and Technical Imonation, Pinter Publishers, London.

Clark, B. and Z. Griliches, 1984, Productivity growth and R\&D at the business level: Results from the PIMS data base, in Z. Griliches (ed.), R\&D, Patents and Productivity, University of Chicago Press, Chicago.

Cobb, C.W. and P.H. Douglas, 1928, A Theory of Production, American Economic Review. Vol. 18, pp. 28-45.

Coe, D.T. and E. Helpman, 1993, International R\&D spillovers, NBER Working Paper, No. 4444.

Coe, D.T., E. Helpman and A.W. Hofmaister, 1995, North-South R\&D spillowers, NBER Working Paper, No. 5048.

Cohen, W.M. and D.A. Levinthal, 1989, Innovation and learning: two faces of R\&D. The Economic Jounal, Vol. 99.pp. 569-596.

Cole, R., Y.C. Chen, J.A. Barquin-Stolleman, E. Dulberger, N. Helvacian and J.H. Hodge, 1986, Quality-adjusted Price-Indexes for computer processors and selected peripheral equipment, Survey of Current Business, Vol. 66, No. 1, pp. 37-75.

Conlisk, J., 1967, A modified Neoclassical model with Endogenous Technical Change, The Southern Economic Journal, Vol. 34, No. 2, October, pp. 199-208.

Court, A.T., 1939, Hedonic Price Indexes with Automotive Examples, in The Dymamics of Automotive Demand, New York, General Motors Corporation, pp. 99-117.

Cunéo, P. and J. Mairesse, 1984, Productivity and R\&D at the firm level in French manufacturing, in $Z$. Grilliches (ed.), $R \& D$, Patents and Productivity, University of Chicago Press, Chicago.

Dasgupta, P. and P.A. David, 1987, Information disclosure and at the economics of science and technology, in G. Feiwel (ed.). Arrow and at the Ascent of Modern Economic Theory, New York University Press, New York.

Dasgupta, P, and P.A. David, 1994, Towards a new economics of science, Research Policy, Vol. 23, pp. 487-521.

Dasgupta, P. and J.E. Stiglitz, 1980, Industrial structure and the nature of innovative activity, Econonic Journal, Vol. 90, pp. 266-293.

David, P., 1992, Computer and Dynamo, The modern productivity paradox in a not-too-distant mirror, in Technology and Productivity: The Challenge for Economic Policy, OECD, Paris, pp. $171-185$.

De Bondt, R. and P. Slaets and B. Cassiman, 1992, The degree of spillovers and the number of rivals for maximum effective $\mathrm{R} \& \mathrm{D}$, International Jomal of Industrial Organisation, Vol. 10, pp. 35-54.

Delbono, F. and $V$. Denicolo, 1990, R\&D investment in symmetric and homogenous oligopoly: Bertrand vs. Cournot, International Joumal of industrial Organisation, Vol. 8 , pp. $297-314$.

De Long, J.B. and L.H. Summers, 1991, Equipment Investment and Economic Growth, Quarterly Journal of Economics, Vol. 106, pp. 445-502.

Denison, E.F., 1962. The Sources of Economic Growth in the United States and the 
Alternatives Before US, Committee for Economic Development.

Deolalikar, A.B. and R.E. Evenson, 1989, Technology production and technology purchase in indian industry: an econometric Anallysis, The Review of Economics and Statisnics, Vol. 71, No. 4, p. $687-692$.

Devine, W., 1983, From shafts to wires: historical perspectives, Journal of Economic History, No. 43 , pp. $347-373$.

Diamond, P., 1990, Growth/Productivity/Unemployment, MTT Press, Cambridge.

Dixit, A.K. and V. Noman, 1980, Theory of International Trade, Cambridge.

Dixit, A.K. and J.E. Stiglitz, 1977 , Monopolistic competition and optimum product diversity, American Economic Review, Vol. 67, pp. 297-308.

Dogramaci, A. and R. Fare, 1988, Applications of Modern Production Theory: Efficiency and Productivity, Kluwer Academic Publishers, Boston.

Dorfman, R., 1969. An Economic Interpretation of Optimal Control Theory, American Economic Review, December, pp. 817-831.

Dorfman, R. and P.O. Steiner, 1954, Optimal Adwertising and Optimal Quallity, American Economic Review, Vol. 44, pp. 826-836.

Dosi, G., C. Freeman, R. Nelson, G. Silverberg and L. Soete, Technical Change and Economic Theory, London/New York, Pinter Publishers.

Dosi, G., K. Pavitt and L. Soete, 1990, The Economics of Technical Change and International Trade, Harvester Wheatsheaf, New York.

Dulberger, E., 1989. The application of a hedonic model to a quality-adjusted Price-Index for computer processors, in D.W. Jorgenson and R. Landau (eds.), Technology and Capital Formation, MIT Press, Cambridge, pp. 37-75.

Eaton, J. and A. Panagariya, 1979, Gains from Trade Under Variable Returns to Scale, Commodity Taxation, Tarifs and Factor Market Distortion, Joumal of Intemational Econonics, Vol. 9, pp. 481-501.

Englander, A.S., R.E. Evenson, and M. Hanazaki, 1988, R\&D, Innovation, and the Total Factor Productivity Slowdown, OECD Economic Studies, No. 11, pp. 7-42.

Ergas, H., 1994. The "new face" of technological change and some of its consequences, mimeo.

Ethier, W., 1979, Internationally Decreasing Costs and World Trade, Journal of Intemational Economics, Vol. 9. pp. 1-24.

Ethier, W., 1982, Decreasing Costs in Intemational Trade and Frank Graham's Argument for Protection, Econometrica, Vol. 50, pp. 1243-1268.

Evenson, R.E., 1993, Patents, R\&D, and invention potential: International evidence, American Economic Review, Papers and Proceeding, Vol, 83, S463-468.

Evenson, R.E., S. Kortum and J. Putnam, 1988, Estimating Patents by Industry Using YaleCanada Patent Concordance, Yale University, mimeo.

Evenson, R.E. and J. Putnam, 1993, Inter-sectoral Technology Flows: Estimates From a Patent Concordance with an application to Italy, mimeo.

Fagerberg, J., 1988, International competitiveness, The Economic Journal, Vol. 98, June, pp. 355-74.

Fecher, F., 1990, Effects directs et indirects de la R-D sur la productivite: une analyse de l'industrie manufacturiere belge, Cahiers Economiques de Bruxelles, Vol. 128, pp. 459 483.

Fecher, F. and S. Perelman, 1992, Productivity growth and technical efficiency in OECD 
industrial activities, in R. Caves (ed.), Industrial Effictency in Six Nations, The MIT Press, pp. $459-488$.

Feichtinger, G. and R.F. Hartl, 1986, Optimale Kontrolle Okonomischer Processe: Anwendungen des Maximum prinzips in den Wirschafwissenschaften, de Grayter, Berlin.

Foray, D. and P. David, 1994, Accessing and expanding the science and technology knowledge-base, OECD Report.

Forester, T., 1985, The Information Technology Revolution, Basil Blackwell, Oxford.

Freeman, C., 1994, The Economics of Technical Change, Cambridge Journal of Economics, 1994, Vol. 18, p. 463.

Freeman, C., J. Clark and L. Soete, 1982, Unemployment and Technical Imovation: A Study of Long Waves and Economic Development, Pinter Publishers, London.

Freeman, C. and C. Perez, 1988, Structural crises of adjustment, Business cycles and investment behaviour in Dosi, G., C. Freeman, R. Nelson, G. Silverberg and L. Soete, Technical Change and Economic Theory, London/New York, Pinter Publishers.

Freeman, C., M. Shatp and W. Walker, 1991, Technology and the Future of Europe: Global competition and the Enviromment in the 1990s.

Freeman, C. and L. Soete, 1987, Technological Change and Full Employment, Blackwell, Oxford.

Freeman, C. and L. Soete, 1990, Fast structural change and slow productivity change: some paradoxes in the economics of information technology, Structural Change and Economic Dynamics, Vol. 1, No. 2, pp. 225-242.

Freeman, C. and L. Soete, 1993, Information technology and employment, UPM, Maastricht.

Freeman, C. and L. Soete, 1994. Work for all or Mass Unemployment? Computerised technical change into the twenty-first centwry, Pinter Publishers, London.

Geroski, P.A., 1989, Entry, innovation and productivity growth, Review of Economics and Statistics', Pp. 572-578.

Gomulka, S., 1990, The Theory of Technological Change and Economic Growth, Routledge, London.

Goto, A and K. Suzuki, 1989, R\&D capital, rate of return on R\&D investment and spillover of R\&D in Japanese manufacturing industries, Review of Economics and Statistics, LXXI4, pp. $555-564$.

Gould, J.P. 1970. Diffusion Processes and Optimal Advertising Policy, in E.S. Phelps (ed.), Microeconomic Foundations of Employnent and Inflation Theory. New York.

Griliches, Z., 1961. Hedonic Price Indexes for Automobiles: An Econometric Analysis of Quality Change in The price Statistics of the Federal Government, NBER Staff Report No. 3, General Series No. 73, New York, pp. 173-196.

Griliches, Z., (ed.), 1964, Notes on the Measurement of Price and Quality Change, in Models of Imcome Determination. NBER Studies in Income and Wealth, Vol. 28. Princeton University Press, Princeton, pp. $301-404$.

Griliches, Z. (ed.), 1971, Price Imdices and Quality Change. Harvard University Press, Cambridge.

Griliches. Z., 1979, Issues in assessing the Contribution of Research and Development to productivity growth, The Bell Joumal of Economics, pp. 92- 116.

Griliches, Z., 1980a, Returns to Research and Development Expenditures in the Private Sector, in $\mathrm{J}$. Kendrick and $\mathrm{B}$. Vaccara eds.. New Developments in Productivity Measurement and Analysis, Unversity of Chicago Press, Chicago, pp. 419-454. 
Griliches, Z, 1980b, R\&D and the productivity slowdown, American Economic Review, Vol. 702, pp. 343-348.

Griliches, Z.,(ed.), 1984, R\&D, Patents and Productivity. The University of Chicago Press, Chicago.

Griliches, Z., 1986, Productivity, R\&D and basic research at the firm level in the 1970 , American Economic Review, Vol. 76, pp. 141-154.

Griliches, Z., 1990, Patent statistics as economic indicators: A survey, Journal of Economic Literature, Vol. 28, No. 4. pp. 1661-1707.

Griliches, Z., 1992, The Search for R\&D Spillovers, Scandinavian Journal of Economics, Voll. 94. supplement, pp. $29-47$.

Griliches, Z., 1994. Productivity, R\&D, and the data constraint, American Economic Review, Papers and Proceedings, Vol. 84, No. 1, pp. 1-23.

Griliches, Z. and F. Lichtenberg, 1984, Interindustry Technology Flows and Productivity Growth: A Reexamination, The Review of Economics and Statistics, pp. 324-329.

Griliches, Z. and J. Mairesse, 1983, Comparing Productivity Growth: An Exploration of French and U.S. industrial and furm data, European Economic Review, Vol. 21, pp. 89-119.

Griliches, $Z$, and J. Mairesse, 1984, Productivity and R\&D at the firm level in $Z$. Griliches (ed.), RdD, Patents and Productivity. The University of Chicago Press, Chicago.

Griliches, Z. and J. Mairesse, 1990a, R\&D and Productivity Growth: Comparing Japanese and U.S. manufacturing firms, in C.R. Hulten (ed.), Productivity Growth in Japan and the United States, NBER Studies in income and Wealth, Vol. 53, University of Chicago Press, Chicago.

Griliches, Z. and J. Mairesse, 1990b, Heterogeneity in panel data: Are there stable production functions? in P. Champsaur et al. (ed.), Essays in honour of Edmond Malinvaud. Vol. 3: Empirical Economics, MIT Press, Cambridge.

Grossman, G.M. and E. Helpman, $1991 \mathrm{a}$, Quality ladders in the Theory of Growth, Review of Economic Studies, Vol. 58, pp. 43-61.

Grossman, G.M. and E. Helpman, $1991 \mathrm{~b}$, Innovation and Growth in the Global Economy, Cambriclge Mass.: MIT Press.

Hall, R.E., 1990, Invariance Properties of Solow's Productivity Residual in P. Diamond, Growth /Productivity /Unemployment, MTT Press, Cambridge.

Hall, B. and J. Mairesse, 1992, Exploring the relationship between R\&D and Productivity Growth in French manufacturing firms, NBER Working Paper, No. 3956.

Harhoff, D., 1994, R\&D and productivity in German manufacturing firms, ZEW Discussion paper, No. 94-01, Zentrum fur Europäische Wirtschaftsforschung, Mannheim.

Helpman, E. and P.R. Knngman, 1985, Market Stracture and Foreign Trade: Increasing Returns, Imperfect Comperition, and The International Economy, Cambridge. MIT Press.

Helpman, E. and P.R. Krugman, 1989. Trade Policy and Market Structure, MIT Press, Cambridge.

Helpman, E. and M. Trajtenberg, 1994, A Time to Sow and a Time to Reap: Growth Based on Ceneral Purpose Technologies, NBER Working Paper, No. 4854.

Hoos, S., 1959, The Adverting and Promotion of Farm Products-Some Theoretical Issues, Journal of Farm Economics, Vol. 41, pp. 349-363.

Jaffe, A.B., 1986, Technological opportunity and Spillovers of R\&D: Evidence from firms' patents, profits and market value, Anerican Economic Review, Vol. 76, No. 5, pp. 9841001. 
Jaffe, A.B., 1988, Demand and supply influences in R\&D intensity and productivity growth, The Review of Economics and Statistics, Vol. 70, No. 3, pp. 957-970.

Jaffe, A.B., 1989. Real Effects of Academic Research, American Economic Rewew, Vol. 79. No. 5. pp. $957-970$.

Jaffe, A.B., M. Trajtenberg and R. Henderson, 1992, Geographic Localization of Knowledge Spillovers as evidenced by patent citations, Quarterly Joumal of Economics, Vol. 63 , No. 3. pp. 577-598.

Jaszi, G., 1964, Comment, in Models of Income Deternmation, NBER Studies of Income and Wealth, Vol. 28, Princeton University Press, Princeton, pp. 404-409.

Jones, H., 1975, An Introduction to Modem Theories of Economic Growth. Nelson and Sons, Nairobi.

Jorgenson, D.W., 1990, Productivity and Economic Growth in E.R. Bernd and J.E. Triplett 1990, Fifry Years of Economic Measurement. The Jubilee of the Conference on Research in Thcome and Weaith. Studies in Income and Wealth, Vol. 54, The University of Chicago Press, Chicago.

Jorgenson, D.W. and K. Stiroh, 1995, Investment in computers and U.S. economic growth. Economics of Immovation and New Technology. (forthcoming).

Judd, K.L., 1985, On the Performance of Patents, Econometrica, Vol. 53. No. 3, pp. 567-585. Kaldor, N., 1961, Capital Accumulation and Economic Growtllw, in Lutz, F. and D. Hague (eds.). The Theory of Capital, Intemational Economic Association, MacMillian, London, pp. 177-222.

Kamien, M.I. and N.L. Schwartz, 1969, Induced Factor Augmenting Technical Progress from a microeconomic Viewpoint, Econometrica, Vol. 37, pp. 668-684.

Kamien, M.I. and N.L. Schwartz, 1982, Market Structure and Innovation, Cambridge University Press, Cambridge.

Kendrick, J.W. and B.N. Vaccara, 1980, New Developments in Productivity Measurement and Analysis, University of Chicago Press, Chicago.

Kennedy, C., 1964, Induced buyers in innovation and the theory of distribution, Economic Journal, Vol. 74.

Klette, T.J., 1994, R\&D, Scope Economies and company Structure: A 'not so fixed effect' model of plant performance, mimeo.

Kulette, T.J. and Z. Griliches, 1992. The inconsistency of common scale estimators when output prices are unobserved and endogenous, NBER Working Paper, No. 4026.

Klundert, T. van de and S. Smulders, 1992, Reconstructing Growth Theory: A Survey, De Economist, Vol. 140, No. 2., pp. 177-203.

Krueger, A.B., 1993, How Computers Have Changed the Wage Structure: Evidence from Microdata, 1984-1989, Quarterly Joumal af Economics, Vol. CVIII 1, pp. 33-60.

Krugman, P.R., 1979, Increasing Returns, Monopolistic Competition, and International Trade, Joumal of International Economics, Vol. 9, pp. 469-479.

Krugman, P.R., 1980. Scale Economies, Product Differentiation and the Pattern of Trade, American Economic Review, Vol. 70, pp. 950-959.

Krugman, P.R., 1981, Intra-Industry Specialization and the Gains from Trade, Joumal of Political Economy, Vol. 89, pp. 959-973.

Krugman, P.R., 1986, Strategic Trade Policy and the New International Economics. Cambridge, MTT Press.

Landes, D., 1969, The Unbound Prometheus, Cambridge University Press, Cambridge. 
Lau, L and I. Tokutso, 1992, The Impact of Computer Technology on the Aggregate Productivity of the United States: An Indirect Approach, mimeo, Stanford University Department of Economics.

Lee, "T. and LL. Wilde, 1980, Market structure and innovation, Quarterly Joumal of Economics, Vol. 93, pp. 395-410.

Leontief, W., 1966, Input Output Economics, Oxford University Press, New York.

Levin, R.C., 1988, Appropriability, R\&D spending, and Technological Performance, American Economic Review, Vol. 78, No. 2, pp. 424-428.

Levin, R.C., W.M. Cohen and D.C. Mowery, 1985, R\&D Appropriability, Opportunity, and Market Structure: New Evidence on some Schumpeterian Hypothesis, American Economic Review, Papers and Proceedings, Vol. 75, No. 2, pp. 20-24.

Levin, R.C., A. Klevorick, R. Nelson and S. Winter, 1987, Appropriating the Results of Industrial Research and Development, Brookings Papers on Economic Acrivity, Vol. 3, pp. $783-831$.

Levin, R.C. and P.C. Reiss, 1988, Cost-reducing and Demand-Creating R.\&D with Spillowers, RAND Journal of Economics, Voll. 19, No. 4, Winter.

Lichtenberg, F.R., 1993, The Output Contributions of Computer Equipment and Personnel: A Firm-Level Analysis, NBER Working Paper, No. 4540.

Lichtenberg, F.R. and D. Siegel, 1991. "The Impact of R\&D Investment on Productivity- New Evidence using Linked R\&D-LRD Data, Economic Mquiry, Vol. 292, pp. 203-228.

Link, A.N., 1982, A Disaggregated Analysis of Industrial R\&D: Product wersus Process Innovation in D. Sagal (ed.), The Transfer and Utilization of Technical Knowledge, Lexinton Books, Lexinton.

Link, A.N., 1983, Interfirm Technology Flows and Productivity Growth, Economic Letrers, Vol. 11, pp. 179-184.

Link, A.N., 1986, Technological Change and Productinity Growth, Harwood Acadlemic Publishers, Chur.

Loury, G.C., 1979, Market structure and inmowation: A reformulation, Quarterly Joumal of Economics, Vol. 93. pp. 395-410.

Loveman, G.W., 1988, An assessment of the productivity impact on information technologies, MIT-Management in the 1990s working paper, No. 88-54.

Loveman, G.W. 1994, An assessment of the productivity impact on information technologies in Allen, T.J. and M.S. Scott Morton ed, Information Technology and the Corporation of the 1990s: Research Studies, MIT Press, Cambridge, MA.

Lucas, R.E.B., 1988, On the Mechanics of Economic Development, Jounal of Monetary Economics, Vol. 22, No. 1, pp. 3-42.

Macdonald, B.N. and Markusen J.R., 1985, A rehabilitation of absolute advantage, Joumal of Polirical Economy, Vol. 93, pp. 277-297.

Mairesse. J. and P. Mohnen, 1994, R\&D and productivity Growth: What have we leamed from Econometric Studies?, paper presented at EUNETIC conference, Strassbourg.

Maresse, J. and M. Sassenou, 1991, R\&D and Productivity: A Survey of Econometric Studies at the Firm Level, STI Review (OECD Paris), No. 8, pp. 9-43.

Mansfield, E., 1965, Rates of Return from Industrial Research and Development, American Economic Review, Vol. 55. Pp. 310-322.

Mansfteld, E. 1980, Basic research and productivity increase in manufacturing, American Econamic Review, Vol. 70, pp. 863-873. 
Mansfield, E., 1988, Industrial R\&D in Japan and the United States: A comparative study, American Econonic Review, Papers and Proceedings, Vol. 78, No. 2, pp. 223-228.

Marshall, A., 1887, Remedies for Fluctuations of General Prices, Contemporary Review, Vol. 51, pp. $355-375$.

Meijers, H., 1994, On the diffusion of technologies in a vintage frawework, theoretical considerations and empirical results, PhD thesis, UPM. Maastricht.

Meijl. H. van, 1994, The Influence of spillovers, product differentiation and entry on technological change, MERTT Research memorandum 94-026. Maastricht.

Meijl, H. van and A.H. van Zon, 1993, Endogenows technological change by cost-reducing and demand-creating Innovations, MERIT Research memorandum 93-027, Maastricht.

Mensch, G., 1975, Das Technologische Patt: Imnovationen wiberwinden Depression, Umschau, Frankfurt.

Meyer-Krahmer, F., 1992. The Effects of New Technologies on Employment, Economics of Innovation and New Technology, Vol. 2, pp. 131-149.

Minasian, J.R., 1969, Research and Development. Production Functions and Rates of Return, American Economic Review, Vol. 59, pp. 80-85.

Mohnen, P., 1990a, New technologies and interindustry spillovers, STI Review, OECD, Vol. 7, pp. 131-147.

Mohnen, P., 1990b. The impact of foreign R\&D on Canadian manufacturing total factor productivity growth.

Mohnen, P., 1992a, Relationslaip between R\&D and productivity growth in Canada and other major industrialized countries, mimeo, May 2 revised, Quebec.

Mohnen, P., 1992b, International R\&D spillovers in selected OECD countries, Cahier de recherche du département des sciences économiques de l'UQAM. No. 9208.

Mohnen, P., 1994, The econometric approach to R\&D Externalities, Cahier de recherche du département des sciences économiques de l'UQAM, No. 9408.

Mohnen, P. and N. Lepine, 1991, R\&D, R\&D Spillovers and Payments for Technology: Canadian Evidence, Structural Change and Economic Dynamics, Vol. 21, pp. 213-228.

Mohnen, P., M.I. Nadiri and I.R. Prucha, 1986, R\&D, Production Structure, and Rate of Return in the US, Japanese and German Manufacturing Sectors: a nonseparable dynamic factor model, European Economic Review, Vol. 30, pp. 749.771.

Morrison, C. and E. R. Berndt, 1991. Assessing the productivity of Information Technology Equipment in the U.S. manutacturing industries NBER Working poper. No. 3582.

Mowery, D. and N. Rosenberg, 1989, Technology and the pursuit of Econowic Growth. Cambridge, Cambridge University Press.

Nadiri, M.I., 1980, Sectoral Productivity Slowdown, American Economic Review, Papers and Proceedings, Vol. 702 , pp. 349-355.

Nadiri, M.I., 1993, Innovations and technical spillovers, NBER Working Paper, no. 4423.

Nadiri, M.I. and I.R. Prucha, 1990, Dynamic factor demand models, Productivity Measurement, and Rates of Return: Theory and an empirical application to the U.S. Bell System, Structural Change and Economic: Dynamics, Vol. I, pp. $263-289$.

Nadiri, M.1. and M.A. Schankerman, 1981, Technical Change, Returns to Scale, and the Productivicy Slowdown, American Economic Review, Papers and Proceedings, Vol. 712 , pp. 314-319.

Nelson, R. 1959. The Simple Economics of Basic Scientific Research, Joumal of Political Economy, Vol. 67. 
Nelson, R. 1992, What is "Commercial" and What is "Public" About Technology, and What Should Be?, in Rosenberg N., R. Landau and D. Mowery, pp. 57-68.

Nelson, R. and S. Winter, 1982, An Evolutionary Theory of Economic Change. Harvard University, Cambridge.

Nerlove, M. and K.J. Arrow, 1962. Optimal Advertising Policy under Dynamic Conditions, Economica, pp. 124-142.

Neumann, M., 1989, Market Size, Monopoly Power and Innowations under Uncertainty, in D.B. Audretsch, L. Sleuwaegen and H. Yamawaki (eds.), The Convergence of International and Domestic Markets, Amsterdan, pp. 295-306.

Nickell, S.J., 1978, The Invesment Decisions of Fims, Cambridge University Press, Oxford.

Nordhaus, W.D., 1972, The recent productivity slowdown, Brookings Papers on Economic Activity, No. 3, pp. 493-537.

Odagiri, H., 1985, Research Activity, Output Growth, and Productivity Increase in Japanese Manufacturing Industries, Research Policy, Vol. 14, pp. 117-130.

Odagiri, H. and $H$. Iwata, 1986, The impact of R\&D on productivity increase in Japanese manufacturing companies, Research Policy, Vol. 15, pp. 13.19.

Patti, P. and L. Soete, 1988, L' évaluation des effects economiques de la technology, STI Review, Vol. 4, pp. 133-183.

Pavitt, K. 1984, Sectoral Patterns of Technical Change: towards a Taxonomy and a theory, Research Policy, Vol. 13, No. 6.

Perez, C., 1983, Structural Change and The Assimilation of New Technologies in the Economic and Social System, Futures, Vol. 15, pp. 357-375.

Perez, C., 1985, Micro-electronics, long waves and world structural change: new perspectives of developing countries, World Development, Vol. 13, No. 3, pp. 441-63.

Pindyck, R.S., 1982, Adjustment costs, Uncertainty and the Behaviour of the Firm, American Economic Review, Vol. 74, pp. 61-66.

Polanyi, M., 1966, The Tacit Dimension, Routledge \& Kegan, London.

Rames. F., 1968, The Impact of Applied Research and Development on Productivity, Washington University Working Paper, No. 6814.

Ramanathan. R., 1982, Introduction to the Theory Economic Growth, Springer-Verlag, Berlin.

Rebelo, S., 1991, Long Run Policy Analysis and Long Run Growth, Joumal of Political Economy, Vol. 99, pp. 500-521.

Roach, S., 1991, Services under Siege - The Restructuring Imperative, Harvard Business Review, September-October, pp. 82-92.

Robson, M. J. Townsend and K. Pavitt, 1988, Sectoral Patterns of Production and Use of Innowations in the U.K.: 1945-83, Reseanch Policy, Vol. 17, No. 1. pp. 1-14.

Romer" P.M., 1986, Increasing Returns and Long Run Growth, Jommal of political Economy, Vol. 94, No. 5, pp. 1002-1037.

Romer, P.M., 1987, Growth based on increasing returns due to specialization, American Economic Review.

Romer, P.M., 1990, Endogenous Technological Change, Joumal of political Economy, Vol. 98, No. 2, pp. S71-S102.

Romer, P.M., 1993, Implementing a national technology strategy with self-organizing industry investment boards, Brooking Papers; Microeconomics, Vol. 2, pp. 345-399.

Romer, P.M., 1994a, New Goods, Old Theory and the Welfare costs of Trade Restrictions, Joumal of Development Economics, Vol. 43, pp, 5-38. 
Romer, P.M., 1994b, Idea Gaps and Object Gaps in Economic Development, Journal of Monetary Economics, Vol. 32, pp. 543-473.

Rosenberg, N., 1982, Inside the Black Box, Technology and Economics, Cambridge University Press, Cambridge.

Rosenberg, N., R. Landau and D. Mowery, 1992, Technology and the Wealh of Nations, Stanford University Press, Stanford.

Sala-i-Martin, X., 1990, Lecture Notes on Economic Growth I and II, NBER Working Papers. No. 3563 and No. 3564.

Sato, R., 1981. Theory of Technological Change and Economic Irvariance, Applicafions of Lie Groups, Academic Press, New York.

Sato, R. and R. Ramachandran, 1974, Models of Optimal Endogenous Technological Progress Scale Effect and Duality of Production Functions, Discussion Paper, Department of Economics, Brown University, May, 1974.

Sato, R. and G.S. Suzawa, 1983, Research and Productivity, Endogenous Technological Change, Auburn House Publishing Company, Boston.

Schankerman, M., 1981, The Effects of Double-Counting and Expending on the Measured Return to R\&D, Review of Economics and Statistics, Vol. 63, No, 3, pp. 454-458.

Scherer, F.M., 1982, Interindustry Technology Flows and Productivity Growth, The Review of Economics and Statisitics, pp. 627-634.

Scherer, F.M., 1984, Using Linked Patent and R\&D Data to Measure Inter-Industry Technology Flows, in Z. Griliches (ed.), R\&D, Patents and Producrivity, University of Chicago Press, Chicago.

Scherer, F.M., 1992, International High-Technology Comperition, Harvard University Press, Cambridge.

Schmookler, J., 1966, Invention and Economic Growth, Harvard University Press, Cambridge.

Schneider, J, and T. Ziesemer, 1994, What's New and What's Old in New Growth Theory: Endogenous Technology, Micro-foundation and Growth Rate Predictions - A Critical Overview, MERIT Research Memorandum, No. 94-029, Maastricht.

Schumpeter, J., 1939, Business Cycles: A Theoretical, Historical and Statistical Analysis, McGraw Hill, New York.

Schumpeter, J., 1942, Capitalism, Socialism and Democracy, Harper and Brothers, New York.

Schumpeter, I., 1961, The Theory of Economic Development, Harvard University Press, Cambridge.

Sheshinski, E., 1967, Optimal Accumulation with Leaming by Doing, in K. Shell (ed.), Essays in the Theory of Optimal Growth, MrT Press, Cambridge.

Siegel, D. and Z. Griliches, 1991, Purchased Services, Outsourcing, Computers and Productivity in Manufacturing, NBER Working Paper, No. 3678.

Soete, L., 1987. The Newly Emerging Information Technology, in C. Freeman and L. Soete, Technological Change and Full Employment, Blackwell, Oxford, pp. 189-220.

Soete, L., 1995, OECD Conference on employment and growth in the knowledge-based economy, Rapporteurs' Report, 7-8 November 1994, Copenhagen, Denmark.

Soete, L. and P. Patel, 1985, Recherche-Developpement, importations technologiques et croissance économique, Revue Economique. Vol. 365, pp. 975-1000.

Solow. R.M., 1956, A Contribution to the Theory of Economic Growth, Quarrerly Journal of Economics, Vol. 70, pp. $65-94$.

Solow, R.M. 1957, Technical Change and the Aggregate Production Function, Review of 
Economics and Statistics, Vol. 39, pp. 312-320.

Spence, A.M., 1976, Product selection, fixed costs, and monopolistic competition, Review of Economic Studies, Vol. 43, pp. 217-235.

Spence, M., 1984, Cost Reduction, Competition and Industry Performance, Econometrica, Vol. 52, pp. 101-122.

Steinmueller, W.E., 1994, The U.S. software industry: an analysis and interpretive history, In D.C. Mowery (ed.), The international computer software industry, Oxford University Press.

Sterlacchini, A., 1989, R\&D, innovations and total factor productivity growth in British manufacturing, Applied Economics, Vol. 21, pp. 1549-1562.

Sveikaukas, L., 1981. Technology Inputs and Multifactor Productivity Growth, Review of Economics and Statistics, Vol. 63, pp. 275-282.

Terleckyj, N.E., 1974, Effects of R\&D on the Productivity Growth of Industries: an Exploratory Study, National Planning Association, Washington, D.C.

Terleckyj, N.E., 1980, Direct and Indirect Effects of Industrial R\&D on the Productivity Growth of Industries, in J. Kendrick and B. Vaccara (eds.), New Developments in Productivity Measurement and Analysis: University of Chicago Press, Chicago.

Terleckyj, N.E., 1982, R\&D and U.S. Industrial Productivity in the 1970s, in D. Sagal (ed.), The Transfer and Utilization of Technical Knowledge, Lexinton Books, Lexinton.

Tinbergen, J., 1942, Zur Theorie der Langfristigen Wirtschaftsentwicklung, Weltwirtschaftliches Archiv, Vol. 55, pp. 511-549.

Trajtenberg, M., 1990, Economic Analysis of Product Innovations, Harvard University Press. Triplett, J.E., 1987, Hedonic Functions and Hedonic Indexes, in J. Eatwell, M. Milgate and P. Newman, The New Palgrave: A Dictionary of Economics, London, pp. 630-634.

Triplett, J.E., 1990, Hedonic Methods in Statistical Agency Environments: An Intellectual Biopsy, in, in E.R. Berndt and J.E. Triplett.

Varian, H.R., 1984, Microeconomic Analysis, Second Edition, W.W. Norton \& Company, New York - London.

Verspagen, B., 1992a, Endogenous Innovation in Neo-Classical Growth Models: A Survey, Journal of Macroeconomics, Vol. 14, No. 4, pp. 631-662.

Verspagen, B., 1992b, Uneven growth between interdependent economies: an evolutionary view on technology gaps, trade and growth, $\mathrm{PhD}$ thesis, UPM, Mastricht.

Waugh, F.V., 1929. Quality as a Determinant of Vegetables Prices, Columbia University Press, New York.

Wolff, E.N. and M.I. Nadiri, 1993, Spillover effects, linkage structure, and research and development, Structural Change and Economic Dynamics, Vol. 4, No. 2, pp. 315-331.

Wyckoff, A.W., 1995, The Impact of Computer Prices on International Comparisons of Labour Productivity, Economics of Innowation and New Technology, (forthcoming).

Yamada, T., T. Yamada and G. Liu, 199 , Labor productivity and market competition in Japan, NBER Working Paper, No. 3800.

Young, A., 1993, Substitution and Complementarity in Endogenous Innovation, NBER Working Paper. No. 4256.

Ziesemer, T., 1991, Human Capital, Market Structure and Taxation in a Growth Model with Endogenous Technical Progress, Joumal of Macroeconomics, Vol. 13, Winter, pp. 47-68.

Zon, A.H. van, 1991, HERMIT - A model describing the macro sectoral employment effects of IT production and IT use, MERIT Research Memorandum, No. 91-017. 


\section{Samenvatting \\ (Summary in Dutch)}

Dit proefschrift handelt over het technologie generatieproces en de invloed hierop van informatietechnologie (IT). Het duale karakter van technologische verandering staat centraal, zowel de generatie als de distributie van kennis zijn belangrijke factoren in het proces van technologische verandering.

In deze studie wordt een model ontwikkeld waarin kennis endogeen gegenereerd wordt. Bedrijven "investeren" in kenniscreatie om hun technologische niveau en daardoor hun concurrentiepositie te verbeteren. In deze context identificeren wij twee soorten kennis. Aan de ene kant onderscheiden wij kennis gericht op het verbeteren van de efficiëntie van het produktieproces, oftewel "kosten-reducerende" kennis, en aan de andere kant onderscheiden wij kenuis gericht op het verbeteren van de kwaliteit van een produkt, oftewel "vraag-creërende" kennis.

Het duale karakter van kennis wordt geintegreerd in dit raamwerk doordat naast interne bronnen van kennis (proces-georiênteerde en produkt-georiënteerde $R \& D$ uitgaven) ook externe bronnen van kennis (kennis gegenereerd door andere bedrijven) een rol spelen. De opkomst van IT heeft echter een grote invioed op de distributie van kennis en de realisatie van potentiële spillover-effecten. De belangrijkste voordelen van IT zijn, niet verwonderlijk, gerelateerd aan het verwerken van informatie. IT verhoogt de snelheid, nauwkeurigheid, opslag en het verzenden van informatie. Een belangrijk en vaak verwaarloosd effect van IT is daarom gerelateerd aan de ard van kennis: kennis wordt steeds meer gecodificeerd ("codified" nature) in plaats van opgeslagen in mensen en organisaties ("tacit" nature, David and Foray 1994. Ergaz 1994, Arora and Gambardella 1994). Dit heeft tot gevolg dat kennis-spillovers belangrijker worden in het huidige ITtijdperk.

IT bevordert enerzijds de integratie tussen bedrijven door het creeren van netwerken terwijl zij ook de toegankelijkheid verhoogt van externe kennisbronnen. zoals patentpublicaties en databanknetwerken. Deze factoren verhogen de waarde van kennis-spillovers net zoals de toegenomen codificatie van kennis.

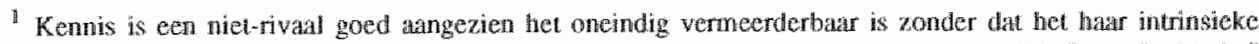
kwaliteiten verliest, zodat het gebruikt kan worden door mecrdere personen tegelifkertijd. Deze" "publieke" eigenschap van kenis belemmert echter datrdoor het terugverdienon wan investerngen in kemiscreatie.
} 
Naast deze invloeden wan IT op kennis-spillovers kan het ook verwacht worden dat IT de produktiviteit van het produktieproces en het kenuis-generatieproces verhoogt. In tegenstelling tot de hoge verwachtingen van $\mathrm{IT}$ hebben empirische studies geen positieve invloed van IT op produktiviteit gevonden. Dit is bekend geworden als de "IT produktiviteits-paradox" of "Solow paradox".

In dit proefschrift wordt een model ontworpen dat de hierboven beschreven kennis generatie- en distributieaspecten beschrijt. Het proefschrift bestaat uit 4 delen. In het eerste deel worden de belangrijkste concepten geintroduceerd en wordt een selectief overzicht gepresenteerd van de literatuur op het gebied van groeitheorieën. Dit deel eindigt met de identificatie van zes basiselementen van een endogeen technologiemodel. In het tweede deel worden theoretische modellen ontwikkeld op basis van deze elementen. In deel m worden deze modellen empirisch getoetst en wordt onderzocht of spillover-effecten inderdaad belangrijker zijn geworden in het IT tijdperk en of de "IT produktiviteits-paradox" bevestigd of verworpen kan worden.

\section{Zes Elementen van een Endogeen Technologie Generatie Model}

Hoofdstuk 2 geeft een selectief overzicht van theorieen op het gebied van technologische verandering en groei. Er wordt aandacht besteed aan de endogene groeitheorieèn en enkele bijdragen welke de invloed van een sleuteltechnologie modelleren. Dit literatuuroverzicht verschaft ons 6 elementen voor een endogeen technologie-generatiemodel. Ten eerste, een model dat groei modelleert moet technologische vooruitgang endogeen behandelen. Ten tweede, technologie is geen nevenprodukt van andere activiteiten, maar de doelbewuste uitkomst van economisch gedrag. Middelen moeten worden geinvesteerd om kennis en technologie te genereren. Ten derde, een deel van de gecreëerde kennis moet men zich kunnen toeëigenen om de geïvesteerde middelen terug te verdienen (private eigenschappen van kennis). Ten vierde, kennis heeft ook enkele publieke (nonrivale) eigenschappen: een deel wan de kennis vloeit over naar andere bedrijven (spillovers). Ten vijfde, er bestaan sleuteltechnologieen die de gehele economie beinvloeden. Ten zesde, deze sleuteltechnologieën worden enerzijds gekenmerkt door grote interne technologische mogelijkheden en anderzijds doordat zij nieuwe technologische mogelijkheden creëren in andere sectoren.

\section{Theoretische Beschouwingen aangaande Endogene Technologische Ontwikkeling}

In hoofdstuk 3 wordt een eenvoudig model, waarin technologische veranderingen worden gegenereerd, ontworpen. Dit model beschrijft een traditioneel kennisgeneratieprocess waarin interne research en development (R\&D) uitgaven de belangrijkste kenuisbron vormen. Dit model verschilt van de meeste modellen, zoals Dasgupta and Stiglitz (1980), Spence (1984) en De Bondt et al (1992), doordat 
het naast kosten-reducerende kennis ook vraag-creerrende kennis genereert. Het is eer dynamisch model, omdat de opbrengsten van huidige R\&D uitgaven verspreid zijn over de tijd. Vanwege de intertemporele effecten van $R \& D$ gebruiken we de "optimal-control" theorie om deze modellen op te lossen.

In hoofdstuk 4 word dit model uitgebreid met enkele speciale invloeden van IT en met een aspect van de publieke eigenschap van kennis. IT-goederen worden gebruikt voor het verwerken van informatie waardoor de rol van deze goederen verschilt van die van normale kapitaalgoederen. IT-kapitaalgoederen worden gebruikt om het produktieproces te stroomlijnen, terwijl andere kapitaalgoederen tezamen met arbeid worden gebruikt om produktie te genereren. IT-goederen hebben daarom een directe kosten-reducerende werking. Echter, IT-goederen hebben ook een vraag-creërende werking, aangezien zij oa. de kwaliteit van een produkt verhogen (bijvoorbeeld d.m.v. van "Computer Aided Design" of een betere "bewaking" van het produktieproces), service verbeteren (betere en snellere communicatiekanalen) en meer produktvariëteiten creëren (flexibele produktie). Het model ontworpen in hoofdstuk 3 is daarom geschikt om de speciale invloeden van IT te modelleren (i.e. vraag-creërende en kosten-reducerende aspecten van IT).

In dit hoofdstuk wordt ook de invloed van IT op het proces van kenniscreatie geïntroduceerd. Aan de ene kant verbetert IT de apparatuur (o.a. computers), waardoor de produktiviteit van het kennis-creatieproces stijgt, terwijl aan de andere kant kennis-spillovers belangrijker worden door o.a. de toegenomen codificatie van kennis. Met betrekking tot kennis-spillovers introduceren we in dit hoofdstuk alleen het interne $R \& D$ produktiviteitsverhogende effect van kennis-spillovers.

De sterke daling van de IT prijzen enerzijds en de sterk toegenomen prestaties van deze goederen anderzijds heeft een enorme daling van de prijs per "efficiency" eenheid veroorzaakt. In combinatie met de hierboven beschreven speciale eigenschappen van IT zorgt deze enorme prijsdaling ervoor dat IT de technologische vooruitgang in vele sectoren kan stimuleren.

In hoofdstuk 5 nemen we in beschouwing dat kennis-spillovers niet alleen de produktiviteit van interne R\&D uitgaven vergroten, maar er ook toe leiden dat kennis-investeringen moeilijker terugverdiend kunnen worden. De centrale vraag in dit hoofdstuk is daarom of spillover-effecten technologische vooruitgang stimuleren of belemmeren. Deze vraag is met name relevant in het huidige IT-tijdperk, waarin de codificatie van kennis snel toeneemt.

De belangrijkste conclusie van hoofdstuk 5 is dat er geen eenvoudige relatie bestaat tussen spillovers en technologische vooruitgang. De invloed van spillovers op technologische verandering is een complex samenspel tussen o.a. de waarde van spillover-effecten, voorkeuren van consumenten, technologische mogelijkheden en marktstructuur. Echter, er is een algemene tendens in onze resultaten. Grotere kennis-spillovers hebben de neiging om interne $R \& D$ uitgaven te reduceren, mat ook om het bereikte technologische niveau te verhogen, aangezien bedrijven meer kennis gebruiken die door andere bedrijven wordt gegenereerd. Deze algemene 
tendens leidt ertoe dat het aantal leefbare bedrijven in een markt toeneemt. Technologische verandering is niet meer afhankelijk van enkele grote bedrijven, die het grootste deel van hun kennis zelf genereren, maar zal steeds meer afhankelijk worden wan meerdere bedrijven die een kleiner deel van hun kennis zelf genereren en voor het grootste deel wich verlaten op kennis die gegenereerd wordt door aridere bedrijuen.

\section{Empirische Resultaten}

In hoofdstuk 6 wordt een literaturoverzicht van empirische studies gepresenteerd. De relatie tussen interne $R \& D$ uitgaven, $R \& D$ spillover-effecten en produktiviteit staat centraal. Studies die alleen de relatie tussen inteme $R \& D$ uitgaven en produktiviteit hebben onderzocht, vonden een statistisch significante en vaak geloofwaardige relatie tussen R\&D en produktiviteit voor elk aggregatieniveau (bedrijf, sector, land en wereld). Aangezien deze studies niet de R\&D spillovereffecten meenemen, worden deze studies gekenmerkt door een specificatie afwijking, die kan toenemen in het IT tijdperk waarin externe kenuisbronnen belangrijker worden.

Met betrekking tot spillovereffecten kunnen we volgens Griliches (1979) onderscheid maken tussen twee soorten. Aan de ene kant zijn er "pure rentspillovers" omdat bedrijven minder dan de volledige kwaliteitsprijs betalen voor intermediaire- en investeringsgoederen." Aan de andere kant bestaan er "kennis" spillovers" omdat in een bepaalde sector gegenereerde kennis gebruikt kan worden in andere sectoren. Deze kennis-spillovers kunnen wel of niet gerelateerd zijn aan goederensitromen.

Echter, deze theoretisch indeling is niet mogelijk in empirische zin. De volgende indeling kan gemaakt worden in empirische studies. Aan de ene kant kan men externe effecten onderscheiden die gerelateerd zijn aan goederenstromen, de zogenaamde "rent-spillovers". Deze rent-spillovers bevatten pure rent-spillovers en kennis-spillovers, gerelateerd aan investerings- en intermediaire goederen, en hun grootte is afthankelijk van de economische afstand tussen sectoren. ${ }^{2}$ Aan de andere kant bestaan er "pure kennis-spillovers", welke ongerelateerd zijn aan goederenstromen en wier grootte afhankelijk is van de technologische afstand tussen sectoren* Een econometrisch model dat spillovers wil modelleren moet daarom zowel rent-spillovers gerelateerd aan investerings- en intermediaire goederen bevatten als pure kennis-spillovers.

2 Dit wordt veroorakat door hevige conchnentie in de innowerende industrie of doondat kwaliteitsm vebeteringen slecht worden weergegeven in de officiële prijsindices van de statistische bureaus.

${ }^{3}$ Investerings en input-ouputmatrices kunnen gebruikt worden om deze rent-spillowers te berekenen.

4 De technologische alstand kan benaderd worclen mat o.at. technologie-stroom-matrices gebaseard op pathent- of innovatiedata (o.a. Scherer 1984 ) of de nabijheid in een technologische ruinte (o.a. Jaffe 1986). 
Het literatumroverzicht van studies die de invloed van spillovers op de produktiviteit meten toont aan dat al deze studies slechts én van de drie spillover concepten in beschouwing nemen. In het algemeen vinden deze studies een positieve significante invloed van externe effecten.

In hoofstuk 7 wordt eerst een econometrisch model aangaande technologische ontwikkeling ontworpen. Dit model heeft 4 kern elementen die over het algemeen verwaarloosd worden in andere empirische studies. Ten eerste, R\&D uitgaven worden gesplitst in proces-georiënteerde en produkt-georiënteerde R\&D uitgaven. Ten tweede, kwaliteitsverbeteringen kunnen de eenheidsproduktiekosten direct beinvloeden. Ten derde, dit model houdt rekening met het feit dat kwaliteitsverbeteringen slecht worden weergegeven in de prijzen en ten vierde is dit model uniek in die zin dat het al de hiervoor beschreven externe effecten in één raamwerk verenigt.

Onze empirische resultaten zijn gebaseerd op 30 Franse sectoren die de gehele economie bestrijken voor de 1978-1992 periode. Een belangrijk resultaat in hoofdstuk 7 is dat, naast interne $R \& D$ uitgaven, al de drie spillover-concepten statistisch significant blijken te zijn. Het opnemen van al de verschillende spilloverconcepten is belangrijk indien men de invloed van de verschillende spillovers voor verschillende groepen sectoren wil bepalen. In de "high tech" sectoren blijken interne R\&D uitgaven en pure kennis-spillovers belangrijk te zijn. De diensten sectoren zijn athankelijk van rent-spillovers gerelateerd aan investeringsgoederen en de overige sectoren zijn afhankelijk van rent-spillovers gerelateerd aan intermediaire goederen.

In hoofdstuk 8 worden twee belangrijke hypothesen getest. Ten eerste wordt in dit hoofdstuk onderzocht of IT een positieve invloed heeft op produktiviteit en ten tweede wordt onderzocht of de waarde van de spillovers toeneemt over de tijd. Met betrekking tot de laatste hypothese blijkt dat de invloed van spillovers inderdaad toeneemt gedurende de 1978-1992 periode en dat de invloed van interne R\&D min of meer constant blift gedurende deze periode. De invloed van externe kennisbronnen op de produktiviteit neemt daarom relatief toe ten opzichte van de invloed van interne R\&D uitgaven. Met betrekking tot de eerste hypothese blijkt dat IT-gerelateerde spillovers (en met name spillovers verbonden aan IT investeringsgoederen) belangrijk zijn voor de produktiviteit en dat de gemeten inwloed zeer snel toeneemt gedurende de schattingsperiode. De resultaten geven daarom een indicatie dat de "TT produktiviteits-paradox" verdwenen is. 



\section{Curriculum Vitae}

Hans van Meijl werd op 5 december 1967 geboren te Gastel. Van 1980 tot 1986 volgde hij Voortgezet Wetenschappelijk Onderwijs aan het Bisscoppelijk College te Weert. Van 1986 tot 1991 studeerde hij Algemene Economie aan de Rijksuniversiteit Limburg te Maastricht. Het doctoraal diploma werd cum laude behaald. Gedurende zijn studie was hij tevens student assistent bij achtereenvolgens de vakgroep Microeconomie en het Maastricht Economic Research Institute on Innovation and Technology (MERIT), onderdeel van de Faculteit der Economische Wetenschappen van de Rijksuniversiteit Limburg. Van 1991 tot 1995 was hij als Assistent in Opleiding verbonden aan MERT. Momenteel is hij als wetenschappelijk onderzoeker verbonden aan het Landbouw-Economisch Instituut (LEI-DLO) gevestigd te Den Haag. 